\title{
EFFECT OF HANFORD PLANT OPERATIONS ON THE TEMPERATURE OF THE COLUMBIA RIVER 1964 TO PRESENT
}
R. T. Jaske and M. O. Sy noground

November 1970

\section{AEC RESEARCH \& DEVELOPMENT REPORT}




\section{LEGAL NOTICE}

This report was prepared as an account of work sponsored by the United States Government. Neither the United States nor the United States Atomic Energy Commission, nor any of their employees, nor any of their contractors, subcontractors, or their employees, makes any warranty, express or implied, or assumes any legal liability or responsibility for the ascuracy, completeness or usefulness of any information, apparatus, product or process disclosed, or represents that its use would not infringe privately owned rights.

\section{PACIFIC NORTHWEST LABORATORY}

RICHLAND, WASHINGTON

operated by

BATIELLE MEMORIAL INSTITUTE

for the

UNITED STATES ATOMIC ENERGY COMMISSION UNDER CONTRACT AT(45-1)-1830 


\section{7}

BNWL -1345

UC- 70 , Waste
Disposal and Processing

EFFECT OF HANFORD PLANT OPERATIONS

ON THE TEMPERATURE OF THE COLUMBIA RIVER

1964 TO PRESENT

By

R. T. Jaske and M. O. Synoground

Water and Land Resources Department Environmental and Life Sciences Division

November 1970

BATTELLE MEMORIAL INSTITUTE

PACIFIC NORTHWEST LABORATORIES

RICHLAND, WASHINGTON 99352 
BNWL -1345

Printed in the United States of America Available from

National Technical Information Service National Bureau of Standards, U.S. Department of Commerce Springfield, Virginia 22151

Price: Printed Copy $\$ 3.00$; Microfiche $\$ 0.65$ 


\author{
EFFECT OF HANFORD PLANT OPERATIONS \\ ON THE TEMPERATURE OF THE COLUMBIA RIVER \\ 1964 TO PRESENT \\ R. T. Jaske and M. O. Synoground
}

\title{
ABSTRACT
}

Compiled and reviewed are significant temperature information and effluent mixing data that have been performed under the study "Effect of Reactor Effluent on the Quality of Columbia River Water," begun in 1962. The report presents basic information needed for a sound view of the temperature modification induced by reactor operation at Hanford and a technical basis for relating these modifications to the ecology. The primary concern here is with physical changes; routine radiological aspects are not included except as they apply to the development of simulation models for effluent transport and dispersion and studies of the mechanics of related food chain transport.

The development of the physical description of the plant effluents includes, among other factors, detailed knowledge of the concentration profiles and travel times of the effluents in the river below the reactors: (1) to determine the extent of interaction between and among operating plants and the city of Richland water plant, (2) to permit prediction of potential exposures from partially diluted effluent to persons downstream, especially in the event of off-standard releases, and (3) to provide a basis for the accurate evaluation and mapping of the extent of zones in which aquatic life might be affected. 


\section{CONTENTS}

INTRODUCTION

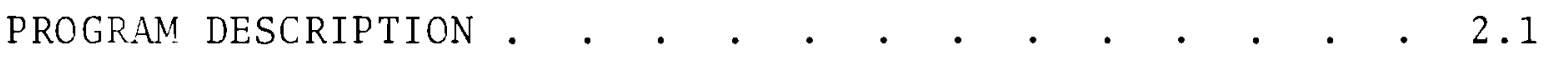

Discussion . • • • • • • • • • • • • • •

Principal Observations Based on Data Collected

in the Program . . . . . . . . . . . . . . . 2.15

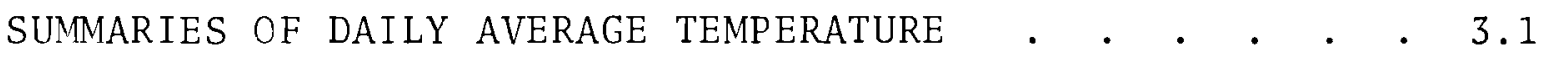

Instrumentation Accuracy . . • • . • • • • • • 3.1

Sensor and Instrument Error Estimates . . . . . 3.1

Data Summaries . . . . . . . . . . . . . . 3.1

Northport, $1966-1969$. . . . . . . . . . 3.3

Grand Coulee, $1964-1969$. . . . . . . . . 3.7

Rocky Reach, 1964 - 1969 . . . • • . . • • . 3.13

Priest Rapids, 1964 - 1969 . . . . . . . . . 3.19

Richland, 1964 - 1969, (Jan. to June 1964 data from

2 mi. above present site . . . . . . . . . . 3.25

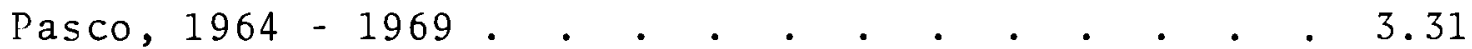

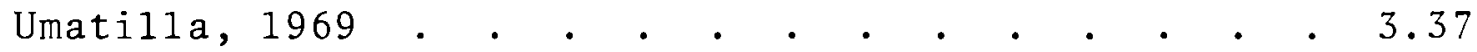

John Day-Biggs, 1969 • • • • • • • . • • . 3.38

MULTI-YEAR Averages • . • . • • • • • • • . 3.39

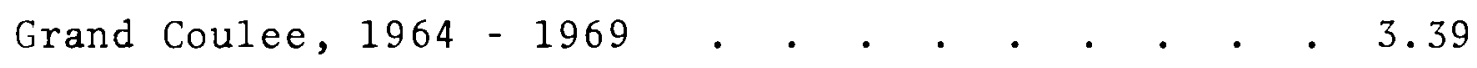

Ice Harbor, $1963-1969$. . . • • . . • . . 3.40

Priest Rapids, $1961-1969$. . . . . . . . . 3.41

LEAST-SQUARES FITTED CURVES • • • • • • • • • • • • •

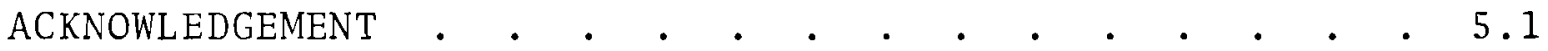

REFERENCES AND BIBLIOGRAPHY • • • • • • • • • • • • 6.1

APPENDIX A - RELATED STUDIES • • • • • • • • • • • • A.1

APPENDIX B - LEAST-SQUARES FITTING PROGRAM LEARN • • • B.1

APPENDIX C - SUMMARY OF EFFLUENT MIXING STUDIES • • • C.1

APPENDIX D

Section I - Effects of Hanford Operations on

Columbia River Temperatures - J. P. Corley . . . . D.I.1

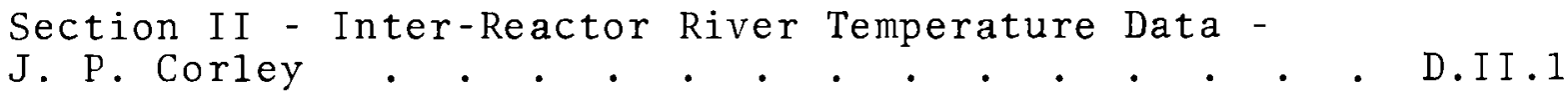

APPENDIX E - ENVIRONMENTAL EFFECTS OF AN EXTENDED

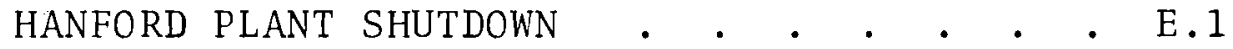




\section{LIST OF FIGURES}

1 General Area Covered by Study Program 2.2

2 Computed Temperature Trends (1938-1969) 2.7

3 Comparative Trends (1960-1969) 2.9

4 Addition of Total Energy Between River Mile 396.0 and River Mile 350.0

5 Comparison of Predicted Average Temperatures at Bonneville Dam With and Without the Addition of Energy in the Hanford Reach

6 Least Squares Fit of Annual River Temperature International Border (Northport) Columbia River Mile 734.1, 1968 . $\mathrm{T}=8.83+6.55 \mathrm{sin}(0.0173 \mathrm{~d}+4.01)$

7 Least Squares Fit of Annual River Temperature International Border (Northport) Columbia River Mile 734.1, 1969.

$\mathrm{T}=9.36+8.14 \sin (0.0172 \mathrm{~d}+3.97)$

4.4

8 Least Squares Fit of Annual River Temperature Grand Coulee Columbia River Mile 596.3, 1968. $\mathrm{T}=10.39+6.88 \mathrm{sin}(0.0166 \mathrm{~d}+3.78)$

9 Least Squares Fit of Annual River Temperature Grand Coulee Columbia River Mile 596.3, 1969. $\mathrm{T}=10.09+7.84$ sin $(0.0172 \mathrm{~d}+3.72)$

10 Least Squares Fit of Annual River Temperature Chief Joseph Columbia River Mile 545.1, 1968. $\mathrm{T}=10.46+6.91 \sin (0.0165 \mathrm{~d}+3.82)$

11 Least Squares Fit of Annual River Temperature Chief Joseph Columbia River Mile 545.1, 1969. $\mathrm{T}=10.76+7.99 \sin (0.0172 \mathrm{~d}+3.73)$

12 Least Squares Fit of Annual River Temperature Rocky Reach Columbia River Mile 473.4, 1968. $\mathrm{T}=10.24+7.07 \mathrm{sin}(0.0167 \mathrm{~d}+3.90)$

13 Least Squares Fit of Annual River Temperature Rocky Reach Columbia River Mile 473.4, 1969. $\mathrm{T}=10.45+8.26 \sin (0.0172 \mathrm{~d}+3.83)$

14 Least Squares Fit of Annual River Temperature Priest Rapids Columbia River Mile 395.6, 1968. $\mathrm{T}=10.56+7.04 \sin (0.0170 \mathrm{~d}+3.94)$

15 Least Squares Fit of Annual River Temperature Priest Rapids Columbia River Mile 395.6, 1969. $\mathrm{T}=10.63+8.24 \sin (0.0172 \mathrm{~d}+3.91)$ 
BNWL -1345

16 Least Squares Fit of Annual River Temperature Richland Columbia River Mile 338.0, 1968.

$\mathrm{T}=11.70+6.87 \sin (0.0171 \mathrm{~d}+3.99)$

17 Least Squares Fit of Annual River Temperature Richland Columbia River Mile 338.0, 1969.

$\mathrm{T}=11.24+8.40 \mathrm{sin}(0.0172 \mathrm{~d}+3.94)$

18 Least Squares Fit of Annual River Temperature

Pasco Columbia River Mile 329.0, 1968.

$\mathrm{T}=11.61+6.82 \sin (0.0169 \mathrm{~d}+4.00)$

19 Least Squares Fit of Annual River Temperature Pasco Columbia River Mile 329.0, 1969.

$\mathrm{T}=11.42+8.34 \sin (0.0172 \mathrm{~d}+3.97)$

4.16

20 Least Squares Fit of Annual River Temperature Ice Harbor Snake River Mile 9.7, 1969.

$\mathrm{T}=11.84+9.65 \mathrm{sin}(0.0172 \mathrm{~d}+3.56)$

21 Least Squares Fit of Annual River Temperature McNary Columbia River Mile 292.0, 1968.

$\mathrm{T}=11.89+7.21 \sin (0.0168 \mathrm{~d}+4.06)$

22 Least Squares Fit of Annual River Temperature McNary Columbia River Mile 292.0, 1969.

$\mathrm{T}=11.75 \pm 8.68$ sin $(0.0172 \mathrm{~d}+3.97)$

23 Least Squares Fit of Annual River Temperature Umatilla Columbia River Mile 290.5, 1969 .

$\mathrm{T}=11.52+8.86 \sin (0.0172 \mathrm{~d}+4.02)$

24 Least Squares Fit of Annual River Temperature John Day - Biggs Columbia River Mile 208.0, 1969. $T=11.6+8.91 \sin (0.0172 \mathrm{~d}+4.04)$

25 Least Squares Fit of Annual River Temperature The Dalles Columbia River Mile 192.8, 1968.

$\mathrm{T}=12.01+7.61 \mathrm{sin}(0.0172 \mathrm{~d}+4.05)$

26 Least Squares Fit of Annual River Temperature

The Dalles Columbia River Mile 192.8, 1969.

$\mathrm{T}=11.76+9.12 \sin (0.0172 \mathrm{~d}+4.03)$

27 Least Squares Fit of Annual River Temperature Bonneville Columbia River Mile 146.1, 1968.

$\mathrm{T}=12.14+7.63 \mathrm{sin}(0.0171 \mathrm{~d}+4.08)$

28 Least Squares Fit of Annual River Temperature Bonneville Columbia River Mile 146.1, 1969.

$\mathrm{T}=11.84+9.10 \mathrm{~s}$ in $(0.0172 \mathrm{~d}+4.04)$

29 Least Squares Fit of Annual River Temperature Warrendale Columbia River Mile 141.0, 1969.

$T=11.51+9.14 \mathrm{sin}(0.0172 \mathrm{~d}+4.06)$

4.26 
BNWL -1345

30 Least Squares Fit of Annual River Temperature Longview Bridge Columbia River Mile 66.0, 1969. $\mathrm{T}=11.51+8.83 \mathrm{~s}$ in $(0.0172 \mathrm{~d}+4.11)$

31 Least Squares Fit of Annual River Temperature Beaver Termina1 Columbia River Mile 53.8, 1969. $\mathrm{T}=11.82+8.64 \sin (0.0172 \mathrm{~d}+4.14)$

A-1 Least Squares Fit of Annual Columbia River F1ow for 1964 at Priest Rapids, River Mile 397.1. $\mathrm{F}=69.5+349.2 \mathrm{e}^{-[(\mathrm{d}-176.7) / 35.1]}$

A-2 Least Squares Fit of Annual Columbia River Flow for 1965 at Priest Rapids, River Mile 397.1.

$L=76.7+236.4 e^{-[(d-165.5) / 46.1]}$

A-3 Least Squares Fit of Annual Solar Radiation in Langleys at Hanford, Washington for 1964 . $L=365.1+283.8$ sin $(0.0168 d+4.91)$

A-4 Least Squares Fit of Annual Solar Radiation in Langleys at Hanford, Washington for 1965 . $\mathrm{L}=367.1+284.2 \sin (0.0167 \mathrm{~d}+5.01)$

A-5 Least Squares Fit of Annual Dewpoint in Degrees Fahrenheit at Hanford, Washington for 1964 . $D_{p}=32.9+10.3 \sin (0.0198 \mathrm{~d}+3.49)$

A-6 Least Squares Fit of Annual Dewpoint in Degrees Fahrenheit at Hanford, Washington for 1965 . $D_{p}=33.8+9.4 \sin (0.0181 d+3.7)$

A-7 Least Squares Fit of Annual Air Temperature in Degrees Fahrenheit at Hanford, Washington for 1964 .

$\mathrm{T}_{\mathrm{A}}=54.4+20.2 \sin (0.0187 \mathrm{~d}+4.07)$

A-8 Least Squares Fit of Annual Air Temperature in Degrees Fahrenheit at Hanford, Washington for 1965 . $\mathrm{T}_{\mathrm{A}}=54.0+21.4 \mathrm{sin}(0.0167 \mathrm{~d}+4.51)$

C-1 Incremental Temperature Above Base River Temperature (Summary of Cross-Section Traverses, River Mile 378-384, Al1 Reactors Operating)

C-2 Incremental Temperature Above Base River Temperature (Summary of Cross-Section Traverses, River Mile 378-384, A11 Reactors Operating)

C-3 Columbia River Surface Temperature Patterns by Imagery $(41,000$ cfs Flow)

C. 5 
C-4 Columbia River Surface Temperature Patterns by Imagery $(80,000 \mathrm{cfs}$ F1ow)

C. 6

C-5 Columbia River Surface Temperature Patterns by Imagery $(110,000$ cfs Flow)

C. 7

C-6 Computer Generated Output of Best Fit to One-Dimensional Dispersion Using LEARN/DI SPERS

C. 10

D.I-1 Temperature Cross Sections at Hanford

D. I. 4

D.I-2 Temperature Cross Sections Above 300 Area

D. I. 5

D. I- 3 Temperature Cross Sections at Pasco

D. I. 6

E-1 Columbia River F1ow Rate at Richland

E. 2

E-2 Equilibrium and Surface Temperatures, and Exchange Coefficients for

Lake Colorado City, Texas

E. 7

E-3 Daily Average Columbia River Temperature

E. 9

E-4 River Traverse Data for July 13, 1966

(River Flow, 254,000 cfs)

E. 11

E-5 Hexavalent Chromium Ion in Columbia River Water

E. 13 


\section{LIST OF TABLES}

1 Values for CY-1969

2.5

2 Comparison of Trends (1935-1969)

2.6

3 Computed Energy Trends for the Reach Between

Priest Rapids and Richland (1965-1969)

2.12

4 Priest Rapids 20-Year Average of Daily Maximum Temperature in Degrees Centigrade for the Years 1947 Through 1967

5 Priest Rapids 20-Year Average of Daily Minimum Temperature in Degrees Centigrade for the

Years 1947 Through 1967

6 Least-Squares Fit Expressions Using the Single Function $\mathrm{T}=\overline{\mathrm{T}}+\mathrm{B} \sin (\mathrm{Cd}+\mathrm{D})$

4.29

A-1 Weather Factors at Hanford

A. 2

A-2 Dewpoint at Hanford Modeled to General Function: $\mathrm{T}=\overline{\mathrm{T}}+\mathrm{B}$ sin $(\mathrm{Cd}+\mathrm{D})$

A. 3

A-3 Columbia River Flow: Trinidad-Priest Rapids

$\mathrm{F} 1 \mathrm{OW}=\mathrm{A}+\mathrm{B}^{-[(\mathrm{d}-\mathrm{C}) / \mathrm{E}]^{2}}$

A. 4

C-1 Summary of Lateral Diffusion Coefficients

C. 12

D.I-1 Flow Time Tests with Dye Releases

D. I. 7

D. I - 2

Cross-Section Mean Versus Recorded Temperatures

D. I. 8

D.I-3 River Temperature Traverses - Priest Rapids Gage (River Mile: 395.4)

D. I. 9

D. I-4 River Temperature Traverses - Reactor Areas

D. I. 10

D.I-5 River Temperature Traverses - Hanford Ferry

(River Mile: 361.6)

D. I .15

D. I- 6 River Temperature Traverses - Ringold

(River Mile: 354.4)

D. I. 23

D.I-7 River Temperature Traverses - Power Line Crossing (River Mile: 350.5)

D. I. 26

D.I-8 River Temperature Traverses - Above 300 Area (River Mile: 344.9)

D. I. 31

D. I-9 River Temperature Traverses - Richland Ferry (River Mile: 340.9)

D. I. 36 

D. I-10 River Temperature Traverses - Pasco Water
Plant (River Mile: 328.7)
D. I. 41
D. I-11 F-Area Fewer Temperatures
D. I. 44
D.I-12 Temperatures in Effluent Bubbles and Plumes
D. I. 45
D. I-13 Equilibrium Dye Concentrations
D. I. 46
D.II-1 Effluent Plume Temperatures
D. II. 4
D. I I - 2
River Temperature Traverses -
400 Yards Below C (River Mile: 383.8)
D. I I. 5
D. I I - 3
River Temperature Traverses - 0.5 Mile
Below C [River Mile: 383.5 (P1ant shore to island on $1 \mathrm{y})$ ]
D. II. 7
D. I I - 4
River Temperature Traverses -
1 Mile Below C (River Mile: 383.0)
D. I I. 8
D. I I - 5
River Temperature Traverses -
400 Yards Below K (River Mile: 381.5)
D. I I 10
D. II -6
River Temperature Traverses -
0.5 Mile Below K (River Mile: 381.3)
D. I I. 13
D.II-7 River Temperature Traverses -
1 Mile Below K (River Mile: 380.8)
D. I I . 20
D. I I - 8
River Temperature Traverses -
Above N (River Mile: 380.0)
D. I I. 26
D. I I - 9
River Temperature Traverses -
100 Yards Below N (River Mile: 379.6)
D. I I. 27
D. I I -10
River Temperature Traverses -
400 Yards Below N (River Mile: 379.4)
D. I I. 28
D. I I - 11
River Temperature Traverses -
0.5 Mile Below $N$ (River Mile: 379.2)
D. II . 35
D. II-12 River Temperature Traverses
1 Mile Below N (River Mile: 378.7)
D. II 37
D. II-13 River Temperature Traverses -
Above 181-D (River Mile: 378.0)
D. I I. 39
D. I I - 14
River Temperature Traverses -
0.5 Mile Below $1904-D R$ (River Mile: 376.8)
D. II 49
D. I I - 15
River Temperature Traverses -
1 Mile Below DR (River Mile: 376.3)
D. I I. 52 
BNWL - 1345

\author{
EFFECT OF HANFORD PLANT OPERATIONS \\ ON THE TEMPERATURE OF THE COLUMBIA RIVER \\ 1964 TO PRESENT \\ R. T. Jaske and M. O. Synoground
}

\title{
INTRODUCTION
}

Since the initiation of operations of the Hanford plant in 1944 , a continuing program of monitoring of the effects of such operations has been conducted under Atomic Energy Commission sponsorship in order to permit a historical evaluation of those operations on the ecology. A great deal of effort and initiative has been taken to assure that the resulting numerical information and derived judgments represent an objective assessment of the events which have taken place. This report is an attempt to review the salient observations gained from the evaluation programs in relation to other thermal modifications of the Columbia River resulting from the aggregate of man controlled activities.

Effluent cooling water from the Hanford reactors and other production related waste streams (including ground seepage) discharges into the main flow of the Columbia River and changes river water temperatures, chemical characteristics, and content of radionuclides. This study is primarily concerned with the physical changes; routine radiological aspects are not included. However, the existence of radionuclides in the effluents is used indirectly in the development of simulation models for effluent transport and dispersion and studies of the mechanics of related food chain transport. River hydrology and effluent distribution data obtained in this program are used for the radiological assessment of plant emergencies and changes in plant operations. 
The information developed by this program is especially valuable in relation to Executive Order 11528 and related legislation involving the establishment of water temperature standards. This covers both increased knowledge of waste inventories and releases and the physical description of waste transport mechanisms.

While prominent importance is given to the measurement, evaluation and prediction of changes in river temperature resulting from plant operation both $10 \mathrm{cally}$ and at distances downstream, other significant chemical parameters are also involved. The development of the physical description of the plant effluents includes, among other factors, detailed knowledge of the concentration profiles and travel times of the effluents in the river below the reactors: (1) to determine the extent of interaction between and among operating plants and the city of Richland water plant, (2) to permit prediction of potential exposures from partially diluted effluent to persons downstream, especially in the event of off-standard releases, and (3) to provide a basis for the accurate evaluation and mapping of the extent of zones in which aquatic life might be affected.

Related progress in mathematical analysis has permitted both better definition of the extent of modification of the river by the heat load from the reactors and improved insight into the distance downstream where significant effects persist. This work is not complete and additional information will be needed from a variety of sources to fully understand and describe the effects of river developments on the water quality. The diurnal, seasonal and hydraulic characteristics of the river, resulting from manipulation of upstream and downstream dams both for power and water quality control purposes, and the effects of impoundments both above and below the Hanford plant are involved. The mixing of the plant effluent with the waters of the Snake River in the McNary reservoir 
confuses to a great extent the ability to determine Hanford plant effects by correlation methods alone. Since the annual thermal cycle of the Snake River is decidedly involved in either offsetting or emphasizing plant effects, it is of significance to have the best possible data and techniques for better understanding the regional river systems.

A number of reports directly related to this program are itemized in section $V$ of this report. This report is a compilation of the more significant temperature information and effluent mixing studies which have been performed under the study "Effects of Reactor Effluent on the Quality of Columbia River Water," Program 02501010 originally authorized by the AEC-Richland Operations Office in 1962. The report attempts to compile in a single document the basic information needed to obtain a sound perspective view of the temperature modifications induced by reactor operation at Hanford and a technical basis for relating these modifications to the ecology. Readers are urged to examine the original source documents for full treatment of individual topics and interpretation by the original investigators. 


\section{PROGRAM DESCRIPTION}

\section{DISCUSSION}

To evaluate the operations of the Hanford project, commencing in 1958, a system of thermal monitoring stations was installed (Figure 1) with particular emphasis on measurement accuracy. The resulting temperature data have beeen carefully adjusted with reference to sensor errors and river sampling deviations to reflect the best estimate of the true bulk river temperature at each station. This was necessary to provide a precise background for the development of thermal simulation models $(1)$ capable of predicting temperature and other associated water quality variables to a high degree of accuracy. Although flow through models of this type are capable of accuracies equal to or better than ordinary temperature measurement practice, statistical analyses were conducted to support conclusions derived from the simulation model, and for trend analyses related to general environmental modification. These analyses were intended to provide a relatively exact basis for making judgments regarding some of the popular concepts of the effects of dams, and the thermal sink capacity in the Columbia River and its tributaries.

The availability of computerized analytical techniques at the Pacific Northwest Laboratory (Battelle-Northwest) made it possible to utilize existing data in a form never before attempted. The general success of this method of least squares analyses has permitted the generation of a series of leastsquares-fitted functional models which form the basis for the conclusions of this presentation.

The need for a reasonably convenient method of making least squares fits of data sets to arbitrary functions has been chronic among experimenters and statisticians everywhere. 


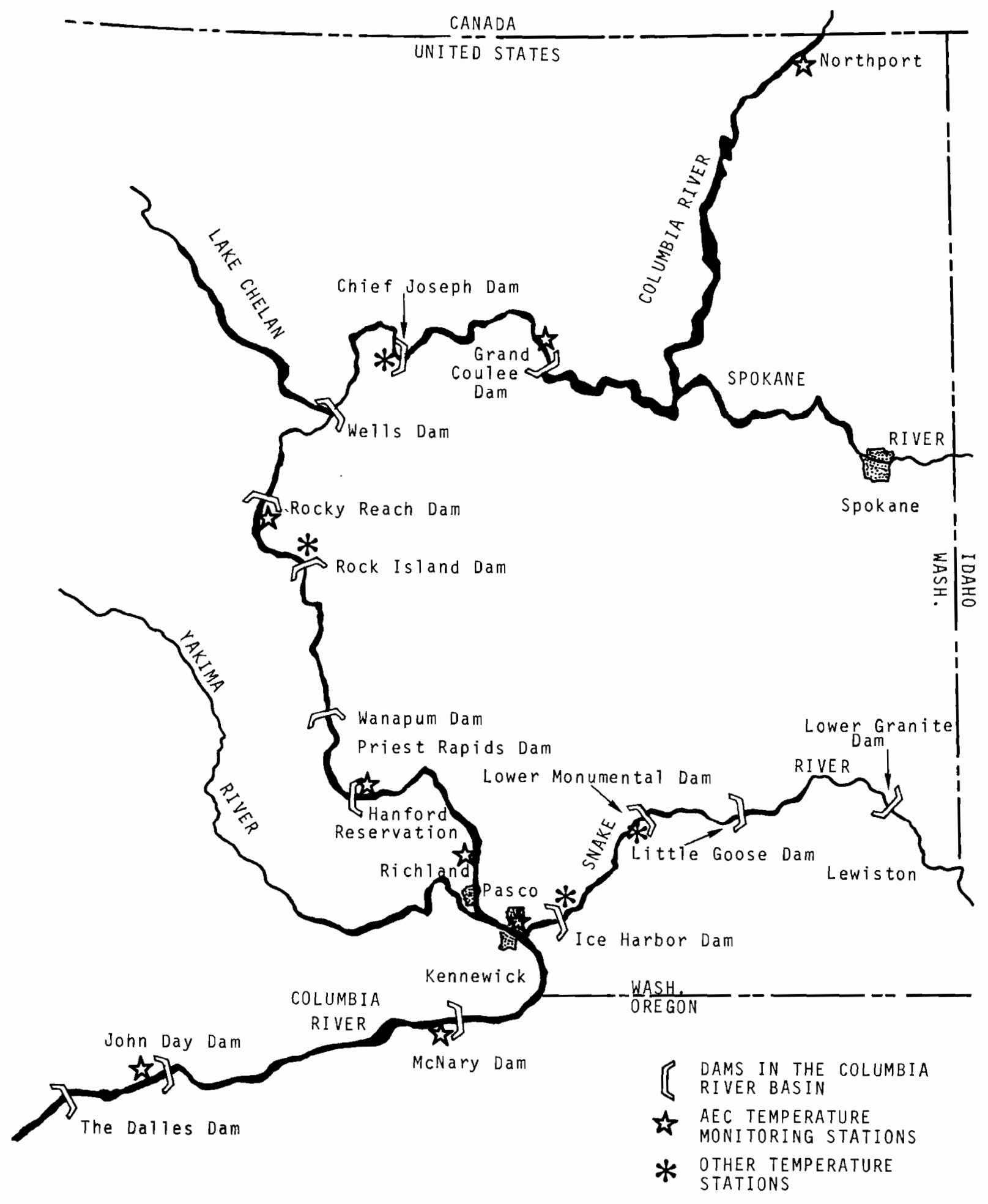

FIGURE 1. General Area Covered by Study Program 
In particular, the solution originally posed by Gauss appeared promising, but its widespread use was prohibited until automatic computing devices became available.

The problem of least squares usually arises from one of two sources:

1. An experimenter desires to represent a set of data by some function. He is not necessarily interested in what the function is; he merely wants a convenient way to express a mass of information. He may be interested in obtaining a calibration curve for comparing future experiments. He may be interested in forming the curve for the purpose of integrating or differentiating the data in a consistent way. He may want only to draw a smooth curve through the data points. But, in each case, he wants the curve to be the same as that obtained by any other person who is willing to assume the same functional relationship between the experimental variables.

2. An experimenter has a definite function in mind--one whose constants (here called parameters) have a definite physical or theoretical meaning. In this situation, he wants to report not only the "best" values for the parameters, but he also wants to be able to attach a measure of the error inherent in the data and, therefore, in the estimates of the parameters. While it is true that not every investigator can match these ideal conditions, it is also true that proper design assists greatly in minimizing the difficulties inherent in the analysis.

In this research, the function $\mathrm{T}=\mathrm{A}+\mathrm{B} \sin (\mathrm{Cd}+\mathrm{D})$ originally used in oceanography and described by Ward (2) was adopted because of the excellence of representation of annual temperature data afforded by its use. In the operations research supporting this study, the data points were grouped 
by year from January 1 through December 31 using 365 or 366 days per year as appropritate. The data were then analyzed by means of a nonlinear, maximum-likelihood, least-squares-fit routine capable of handling nonlinearities and saddle points reliably. This program, developed by the Applied Physics and Electronics Department of Pacific Northwest Laboratory, is called program MISFIT-LEARN and can be conveniently adapted to a variety of computers such as the IBM 7090 or the larger Univac 1108. LEARN makes use of numeric rounding for accuracy, ill-conditioned matrix inversion $\log i c$, and statistical determination of saddle points on the parametric surface. In addition, it contains a two-stage recovery logic. If the usual first order approximation fails, the routine tries the second order terms of the multivariate Taylor expansion. Failing this, the routine branches into a series of approximations best described as "reverse-andhalve the last change" logic. The analysis of the data yields the best-fit parameters and, in addition, produces the rootmean-square error associated with that parameter estimate.

Also included as output is a series of graphs plotted by the CALCOMP plotter from magnetic tapes generated by the computer. These plots include the observed data, the calculated curve, and another pair of curves of plus or minus one standard deviation on each side of the calculated curve. An example is shown in Section IV.

If a function of the form $\mathrm{T}=\mathrm{A}+\mathrm{B}$ sin $(\mathrm{Cd}+\mathrm{D})$ is fitted to annual temperature data, then $C \approx 2 \pi / 365.25 \approx 0.017$. As an added check on the goodness of fit, this parameter was allowed to vary in each case in order to see what value the computer would calculate. Table 1 shows samples of values of all four parameters for a series of data points from the International border to Bonneville dam for the Calendar Year 1969. 
BNWL - 1345

TABLE 1. Values for CY-1969

\begin{tabular}{|c|c|c|c|c|c|}
\hline & $\begin{array}{l}\text { Temp, } \\
{ }^{\circ} \mathrm{C} \\
\end{array}$ & $\begin{array}{l}\text { Mode } 1 \\
\text { Mean } \\
\mathrm{A}-{ }^{\circ} \mathrm{C} \\
\end{array}$ & $\begin{array}{l}\text { Comp. } \\
\text { Extreme - } \\
\mathrm{B}-{ }^{\circ} \mathrm{C} \\
\end{array}$ & $\begin{array}{l}\text { Daily } \\
\text { Shift-C } \\
\text { Radians } \\
\end{array}$ & $\begin{array}{l}\text { Peak } \\
\text { Shift-D } \\
\text { Radians } \\
\end{array}$ \\
\hline International Border & $\mathrm{T}=$ & 9.36 & 8.14 & 0.0172 & 3.97 \\
\hline Grand Coulee & $\mathrm{T}=$ & 10.09 & 7.84 & 0.0172 & 3.92 \\
\hline Rocky Reach & $\mathrm{T}=$ & 10.45 & 8.26 & 0.0172 & 3.83 \\
\hline Priest Rapids & $\mathrm{T}=$ & 10.63 & 8.24 & 0.0172 & 3.94 \\
\hline Richland & $\mathrm{T}=$ & 11.24 & 8.39 & 0.0172 & 3.94 \\
\hline McNary Dam & $\mathrm{T}=$ & 11.75 & 8.69 & 0.0172 & 3.97 \\
\hline Bonneville Dam & $\mathrm{T}=$ & 11.84 & 9.11 & 0.0172 & 4.04 \\
\hline
\end{tabular}

A fuller treatment of the information for the earlier years and specifically those related to the effects of the construction of Grand Coulee Dam is contained in References 3 and 4. The conclusions of this preliminary analysis were:

1. The use of the data plotters with suitable least-squares fitted model functions provides a basis for the processing of significant amounts of stream temperature and other water quality data into promptly available reports with consistent analytical perspective.

2. The erection of low head reservoirs on the main stem of the Columbia River has not produced significant change in the average temperature of the river.

3. The erection of Grand Coulee Dam creating Lake Roosevelt has resulted in a 30-day delay in the transport of water through the reservoir system. This delay and additional delays associated with the expected construction of the Canadian Treaty Dams, when combined, may be significant ecological1y.

4. The erection of dams and reservoirs decreases fluctuations in the water temperatures. The ecologic effects of changes 
resulting from Columbia River impoundments are not, however, expected to be directly comparable to projects with large storage and relatively small steam flow, such as Lake Mead or some of the TVA impoundments.

Additional computations made during the most recent fiscal year support the conclusions of the preliminary study. Leastsquares fitted models have been generated for all of the major projects for which data have been collected over several years. These are detailed in Section IV.

TABLE 2. Comparison of Trends (1935-1969)

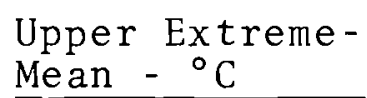

Rock Is land -
Rocky Reach

$$
\begin{gathered}
18.0 \\
\sigma=0.95
\end{gathered}
$$

Bonneville

$$
\begin{gathered}
19.0 \\
\sigma=0.88
\end{gathered}
$$

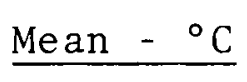

Lower ExtremeMean $-{ }^{\circ} \mathrm{C}$

Several of the trends observed in comparing the results of extended analyses (Table 2) are of great significance in understanding the overall effects of the combined construction of dams and other industrial plants involving thermal waste discharges. A careful review of the data in Figure 2 which compares the values computed for the A (annual average) and $B$ (computed extremes) terms yields the following observations:

1. The annual mean temperature computed for Rock Island-Rocky Reach for the period 1934 to present shows a slight increase of about 0.2 to $0.4{ }^{\circ} \mathrm{C}$. This is about one-half of a standard deviation for the period studied. The annual mean computed for Bonneville during the period 1939 to present shows a lesser overall effect; however, it reflects a definite upturn for the period 1960 to present after the peak 


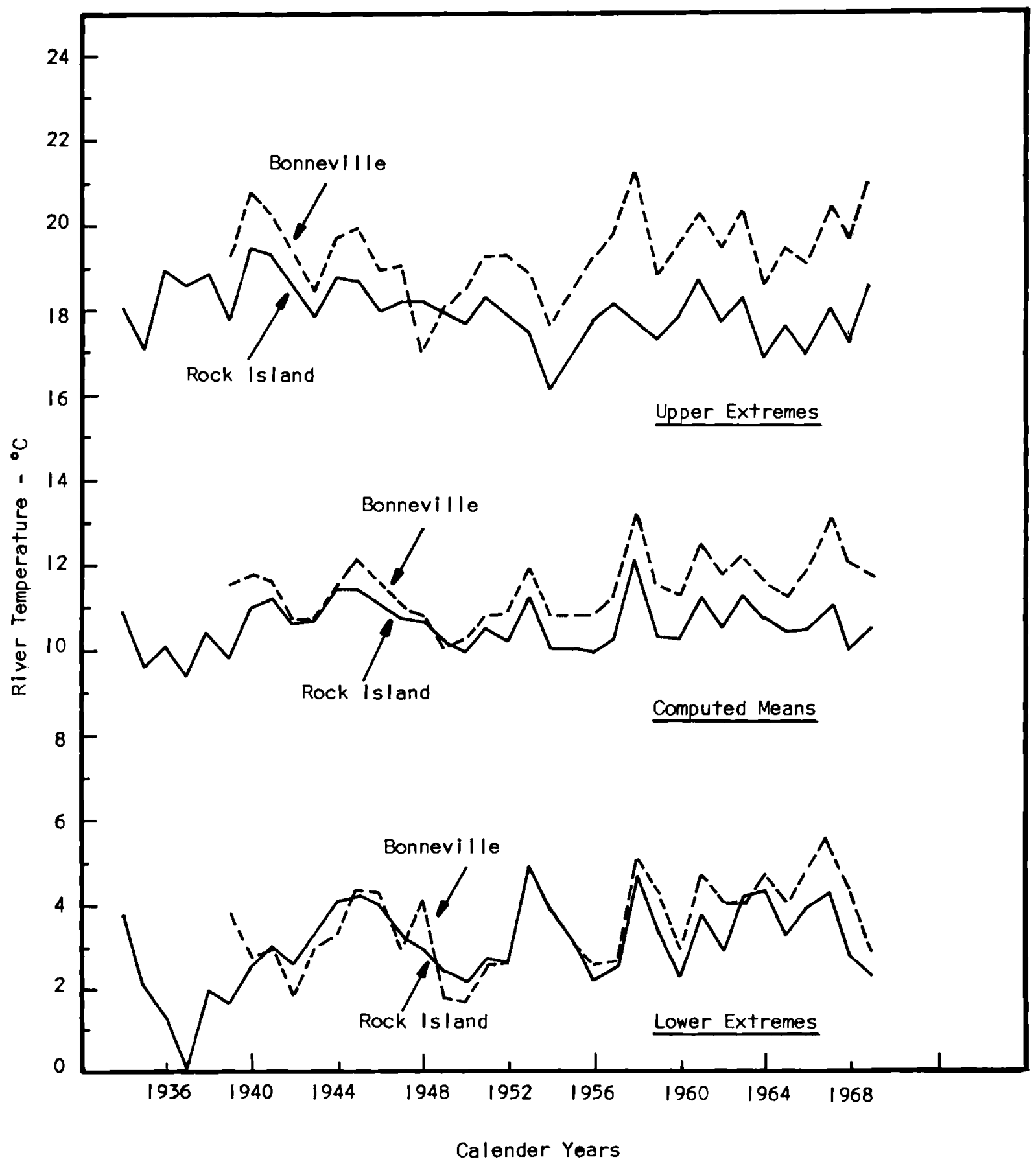

Neg 703803-1

FIGURE 2. Computed Temperature Trends (1938-1969) 
of 1958. The relationship between the computed means for the two data sets shows a significant departure for the period 1944-1948 and, again, 1950 to present. The combination of AEC operations and the Snake River in adding heat to the river in this reach is apparant.

2. The computed upper and lower extremes with the exception of 1948 , the flood year, show a close relationship and consistently rise and fall together. The two groups of data tend to separate in the case of the upper extreme by values about equal to the separation of the means, while the upper extreme of Rock Island continues a steady decrease. During the recent period of 1960 to present both the upper and lower extremes show a sharp tendency to converge on the mean. Again, it is plain that a strong influence has affected the historic trend and sharply reduced the departure from the mean. This timing coincides with the period of greatest dam building activity on the upper Columbia.

To more fully understand the fine structure involved in these differences, additional runs were made in order to add the effects' of intermediate projects. Figure 3 illustrates the computed parameters for six projects for which data are available since 1961. Inspection of these data yield further observations:

1. The values for the means and the extremes fall into distinct groups--those above the Hanford Project and those below it. Further, the mean values for Ice Harbor are essentially equal to the McNary values and could account for much of the difference between the upper and lower sets as related to the flow from the Snake River.

2. The values of the means for the period 1960 to present show no significant trend or departure from the group response to individual yearly weather patterns. 


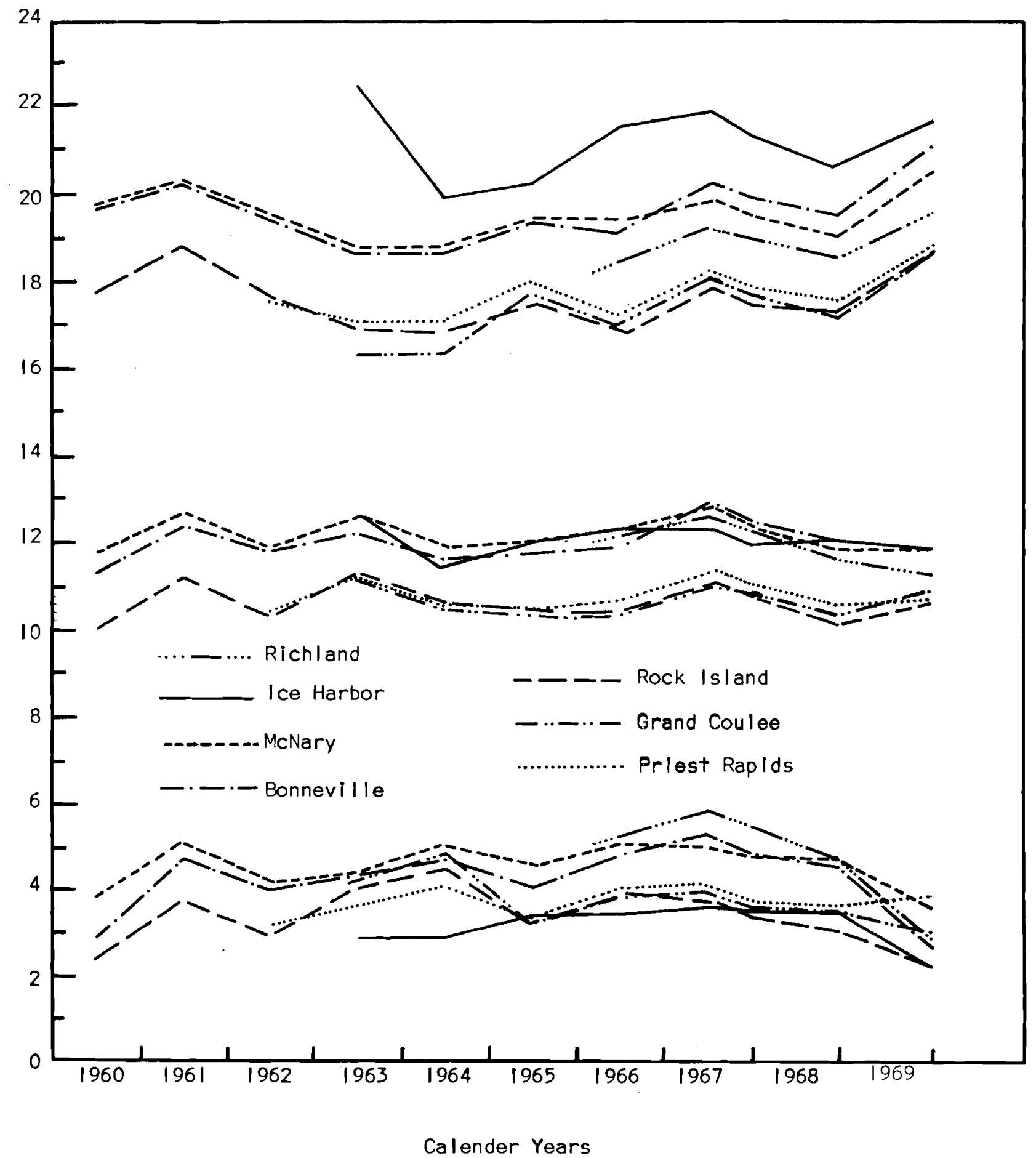

Neg 703803-2

FIGURE 3. Comparative Trends (1960-1969) 
3. The upper and lower extremes for both project sets show a common convergence toward the mean. This is more pronounced for the upstream locations than those downstream indicating that a nonweather related influence is overriding the average convergence to a slight extent.

4. The upper and lower computed extremes for the Snake River are significantly higher and lower than the same Columbia River values and similar to the historic values for Bonneville before the erection of Grand Coulee Dam.

5. The data for the projects upriver from Hanford have closely related values with a tendency for a slight reduction in values of the extremes. On the other hand, the McNary and Bonneville data groups show effects peculiar to a cooling trend with the Bonneville Project showing significant lower values for both the annual mean and the lower extremes.

Since these two data groupings would tend to create the impression that the erection of the Columbia River dams have had little or no effect on the water temperature, review of Reference 4 is necessary to visualize the trends. Reference 4 indicates:

1. A sharp and permanent change in the timing of the peak temperature at Rock Island commencing with the filling of Lake Roosevelt in 1941-1942. The permanent change amounts to about 30 days, the average transport time for the mean annual flow through Lake Roosevelt.

2. This sharp and permanent shift is also observed at Bonneville Dam although to a lesser extent. The two projects reveal surprisingly similar shifts in response to annual weather cycles. While the Rock Island peak has stabilized at about September 20, the Bonneville peak has continued to occur later and later as longer and longer flow times result from the addition of upstream projects. The close relationship of McNary and Bonneville indicate little or no cooling takes place in the reach between these projects. 
BNWL - 1345

3. The values for the Priest Rapids Project closely resemble peak temperature patterns for Rock Island. The data differences of about 7 to 10 days appear to be related to the trave 1 time through Wanapum and Priest Rapids reservoirs in about the same manner as Grand Coulee.

4. A11 projects have strong indications of responding in unison to regional weather influences. For example, all projects show early peak arrivals on high temperature years. Clearly, influences beyond the scope of man's activity affect all water temperatures to a surprisingly similar extent.

The only major industrial factor influencing the analysis is the Hanford complex, whose effect upon the system can be modeled in terms of the net difference between existing temperature records at an upstream and a downstream location. Reference is made to two points, one above and the other below the Hanford Complex, and total addition of natural as well as man-made energy is compared. Once again employing the LEARNLIKELY computer routine (MISFIT) discussed previously, a means of obtaining an unbiased least squares fit to water temperature on an annual basis can be determined.

Between the two points of Priest Rapids and Richland there occurs a singular deviation which is associated with the operation of the Hanford reactors. As mentioned before, the average temperature computed by the program is the first term of the mathematica 1 mode $1 \mathrm{~T}=\mathrm{A}+\mathrm{B}$ sin $(0.0172 \mathrm{~d}+\mathrm{C})$. Summarizing, we are able to calculate the information in Table 3 from unclassified and published river data.

The 19,300 MW from 1966 (see Table 3) was used to estimate the perturbation of the system between Priest Rapids and Richland as a combination of AEC operations and natural weather conditions. Therefore, using the relationship (energy = const $\Delta T \times Q)$ the total energy added to a system between the two points is determined as a function of flow and temperature. 
TABLE 3. Computed Energy Trends for the Reach Between Priest Rapids and Richland (1965-1969)

$\begin{array}{llllllll}\text { Year } & \text { Richland } \bar{A} & \text { Priest R. } \bar{A} & \text { Diff. } & \text { Mean F1ow } & \text { Equiv. MW } \\ 1965 & 12.12{ }^{\circ} \mathrm{C} & 10.64^{\circ} \mathrm{C} & 1.48{ }^{\circ} \mathrm{C} & 129,000 & 23,000 \\ 1966 & 12.04{ }^{\circ} \mathrm{C} & 10.65^{\circ} \mathrm{C} & 1.39{ }^{\circ} \mathrm{C} & 115,000 & 19,300 \\ 1967 & 12.63{ }^{\circ} \mathrm{C} & 11.31{ }^{\circ} \mathrm{C} & 1.32^{\circ} \mathrm{C} & 131,000 & 20,800 \\ 1968 & 11.70{ }^{\circ} \mathrm{C} & 10.56{ }^{\circ} \mathrm{C} & 1.10{ }^{\circ} \mathrm{C} & 118,500 & 15,700 \\ 1969 & 11.24{ }^{\circ} \mathrm{C} & 10.63^{\circ} \mathrm{C} & 0.61{ }^{\circ} \mathrm{C} & 134,000 & 9,680\end{array}$

As a result of the modified hydrology originating with daily flow regulation, this temperature differential in the reach has in the past, before 1968, exceeded the maximum allowable temperature increase recently adopted by the Washington state temperature standards. In these cases the total perturbation of $19,300 \mathrm{MW}$ was reduced to conform to the Washington state standards using the formula

$$
\Delta t=\frac{33.95}{\mathrm{~T}+9.44} \text { in }{ }^{\circ} \mathrm{C}
$$

where $\Delta t=$ allowable increase

$\mathrm{T}=$ final downstream temperature.

The allowable increase will range between 1.15 and $3.60^{\circ} \mathrm{C}$ in the river temperature between 0 and $20^{\circ} \mathrm{C}$. Both of these constraints have been represented graphically in Figure 4. As indicated in Figure 4, the allowable system increase is a function of both flow and Priest Rapids temperatures.

The resulting thermal increments were then routed down the river to Bonneville Dam using the COL HEAT simulation model. (1) By superposition, the remaining increment at the dam was determined and plotted on a day by day basis. The remaining increment averaged about 0.2 to $0.3{ }^{\circ} \mathrm{C}$, about 20 to $30 \%$ of the energy increment added to the simulation in the Hanford Reach, Figure 5.

In a recent study of specific thermal effects of the Hanford plant, which determined the residual fraction of thermal modification remaining from an entire year's operation of the Hanford plant at the Washington-Oregon border, (5) a similar set of values 
ว。‘ $\exists y \cap \perp \forall y \exists d W \exists \perp \quad 7 \forall I \perp I N I$

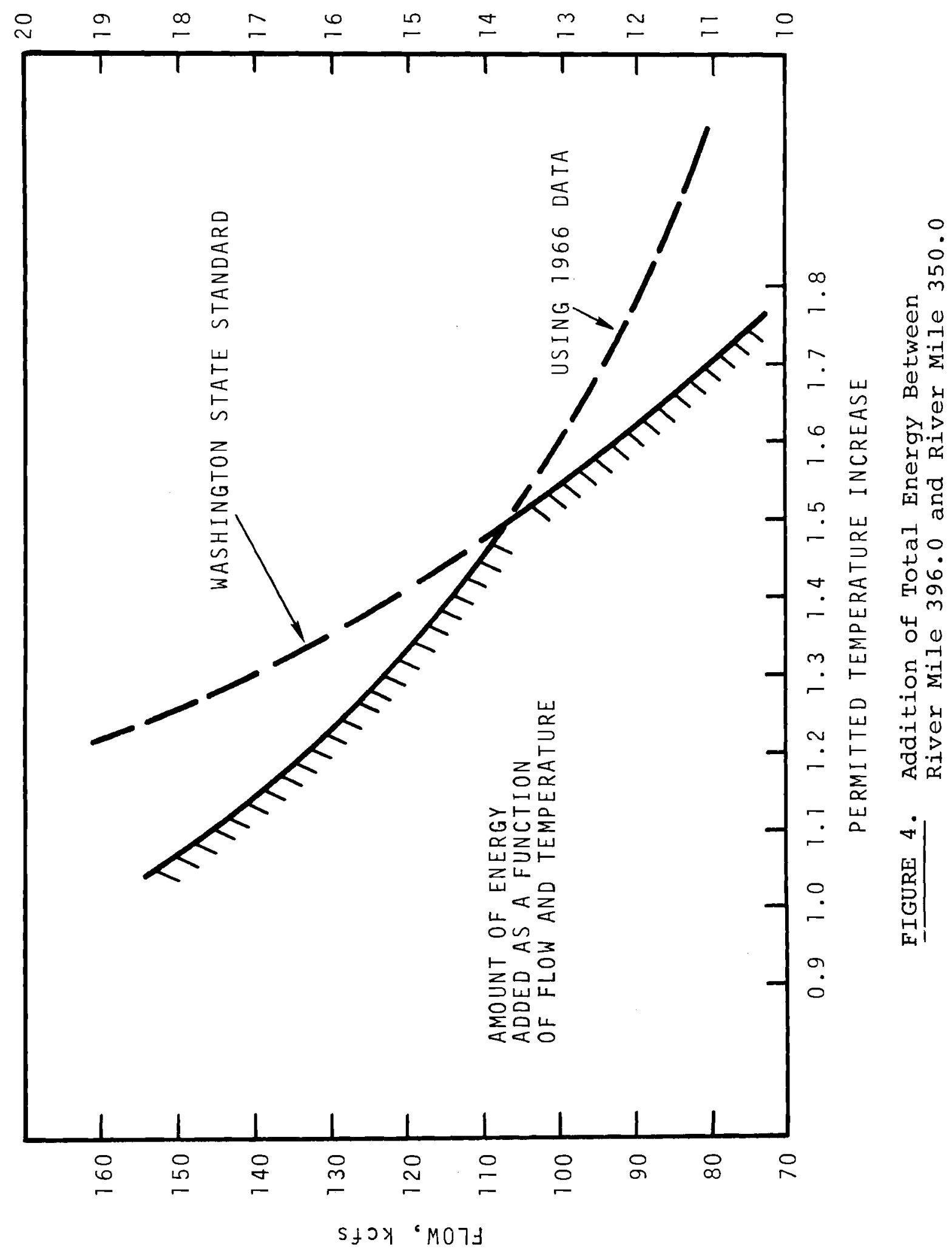




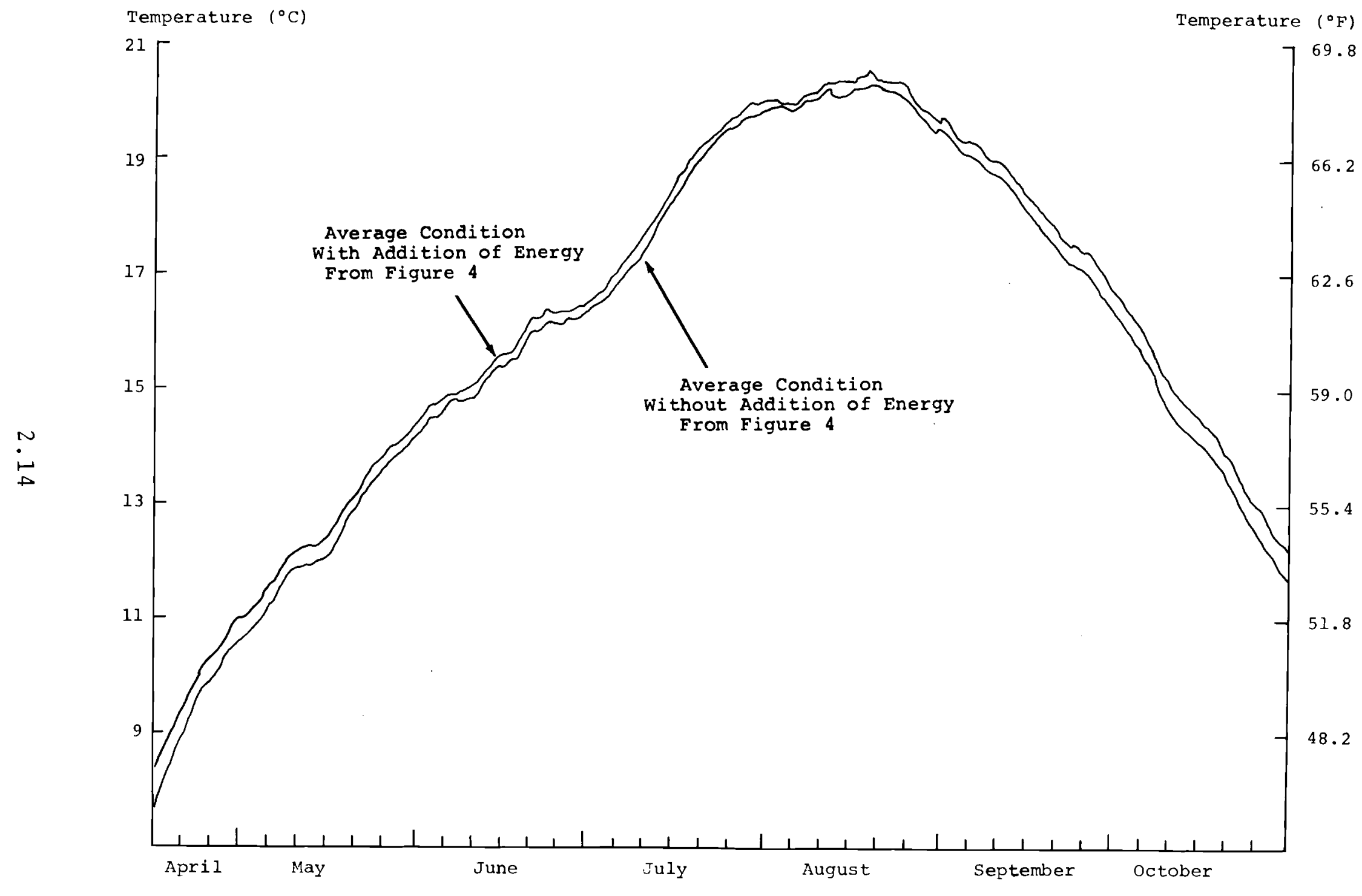

FIGURE 5. Comparison of Predicted Average Temperatures at Bonneville Dam With and Without the Addition of Energy in the Hanford Reach 
BNWL -1345

was determined. From the report, Reference 5, p. 4:

"River temperature modifications introduced by natural environmental effects and operation of reactors at 1969 power levels would be approximately $65 \%$ dis sipated at the Washington-Oregon border. The extent of this dissipation is nearly independent of Priest Rapids water temperature and weather conditions affecting the Columbia River between Priest Rapids Dam and the Washington-Oregon border. The numerical values of the remaining increment ranged from essentially zero to a momentary high of $0.5{ }^{\circ} \mathrm{C}$, the average being $0.20{ }^{\circ} \mathrm{C}$ for the April through October period used in the study.

"On the basis of the transects taken in the summer and fall of 1969 and using water temperature as a tracer, the McNary reservoir at the WashingtonOregon border is sufficiently well mixed that a single point monitoring station could be appropriately located for the purpose of thermal auditing at that point. Such stratification as exists is very weak, and the isotherms appear to be more subject to modification by flow conditions of the Snake and the Columbia than by heat transfer across the water surface in the reservoir. The study suggests that, on the basis of the samples collected and previous transects made before 1962 (Soldat et al., unpublished), it appears that the day-to-day peaking operations of Ice Harbor Dam in relation to Columbia River peaking operations may bear as important a relationship in creating patches of warmer and cooler water within the reservoir flow system as residual Hanford effects. Additional study is needed to verify this conclusively."

PRINCIPAL OBSERVATIONS BASED ON DATA COLLECTED IN THE PROGRAM

On the basis of the information detailed in the discussion and other related studies reported in Section VI, "References and Bibliography," the following principal observations regarding the temperature regime of the Columbia River and its relationship to operations of the Hanford facility appear appropriate:

1. The erection of hydraulic power dams and reservoirs on the Columbia River has had significant effects on the timing 
and extent of annual temperature cycles of the Columbia River. Where annual peak temperatures in the natural, undeveloped river once occurred in mid-August, the construction of reservoirs has altered the timing about in proportion to the increased travel time through the river system. The persistence of this effect verifies earlier predictions of the effect. (6) Reference 6 summarizes the Columbia River cooling program carried out for AEC between 1958 and 1965. The moving of a relatively identical annual cycle laterally on the time scale creates the appearance of warmer water in later fall months and leads to the conclusion by relatively uninformed observers that dam construcion is heating the river system on a general basis. In actuality the reservoir tends to slightly cool the river in more cases than not, and the net effect is the time displacement of a relatively identical thermodynamic history. This is especially true for reservoirs with large ratios of capacity-to-mean flow. Unstratified reservoirs of large capacity such as John Day tend to slightly warm the river in summer due to the increase in travel time and the approach to equilibrium between the river and weather conditions.

2. The erection of hydraulic power dams and reservoirs on the Columbia River has had only a minimal effect on the annual mean temperature of the entire system. This effect is less than $1{ }^{\circ} \mathrm{C}$ over the 34 year period from 1936-1970. The extent of this change could easily be attributed to long-term weather changes due to causes beyond the scope of this study.

3. The temperature history of the Columbia River since 1936 suggests that temperatures are related to river management practices to a large extent and that optimization of the total resource must of necessity include consideration of the integrated temperature effects of water storage and management practices. This is especially true of proposed plans to treat the entire main stem as a moving storage 
BNWL -1345

reservoir for the optimization of power production. Such plans when related to other competitive recreational and industrial uses of the river appear to require additional study to determine related water quality modifications.

4. The reach between Priest Rapids and Richland, the last free flowing portion of the river, responds rapidly to changes in weather and thermal modification from industrial effluents as compared to impounded reaches. Conductive heat transfer rates are higher and evaporation rates are lower than in impounded areas. Summertime temperature increases during periods of reactor shutdown are quite high, ranging from 0.5 to as high a $0.75^{\circ} \mathrm{C}$ in August and early September. A cooling effect of similar magnitude occurs in the winter months. The mean annual temperature differential for the reach has decreased with reactor shutdowns indicating that the thermal modification of the river on a mean annual basis was directly related to the operation of nuclear facilities. This point and the numerically correlated values reported in this section represent important information to the supporting studies of ecological modification carried out through the period 1964 to present.

5. River temperatures between the Hanford plant and the confluence of the Snake River decrease toward an equilibrium value. Studies of the thermal dissipation of reactor effluent indicate that a majority of the heat is dissipated to the atmosphere before the confluence of the Snake and the Columbia. At the Oregon-Washington border, a fraction ranging from $40 \%$ to as $10 \mathrm{w}$ as $5 \%$ and averaging $35 \%$ remains to be dissipated. On this basis, and assuming the entire thermal modification of the reach between Priest Rapids and Richland to be of industrial origin, it can be stated that the residual effect of the Hanford reach in raising river temperatures below McNary dam in the 1965-69 period was 
BNWL - 1345

about equal to the effect of the Snake River. Numerically, the annual mean differential at the confluence of the Snake and Columbia is approximately $0.40{ }^{\circ} \mathrm{C}$, ranging from 0.10 to $0.60{ }^{\circ} \mathrm{C}$. Considering the relative flow ratio of 2.0 between the two streams, the mean modification of the Columbia by the Snake would then be about $0.20{ }^{\circ} \mathrm{C}$ or about the same order of magnitude as the highest differential attributed to the effect of the Hanford reach carried down to the Washington-Oregon border, as reported in BNWL-1345. On this basis, averaged over long time periods, the annual thermal contribution of the Snake to the Columbia is on the order of 4,000 thermal megawatts. This rate of thermal input is about doubled in the August to September period when the average temperature of the Snake is 2.0 to $2.5{ }^{\circ} \mathrm{C}$ higher than the Columbia. This situation is expected to materially worsen after Mica Dam is complete since the differential between the Columbia and the Snake after 1975 during August and September is expected to exceed $4.0{ }^{\circ} \mathrm{C}$ in 9 years out of 10 (Ref.. Bib., p. 6.7, March 1, 1969).

6. Columbia River mean annual temperatures continue to rise slightly at all points upstream of the Columbia Gorge. Passage through the Columbia Gorge modifies the water surface to a more natural condition, and heat transfer rates increase due to increased turbulence thus providing greater cooling. From the Bonneville pool to Warrendale (a distance of 10 miles), a substantial difference in annual average temperature is noted based on provisional records of the Bonneville temperatures. It is concluded that the sensor position at Bonneville does not accurately indicate the bulk river temperature since there is no theoretical basis for the extent of temperature differential between Bonneville and Warrendale. This observation is of prime significance in judging the validity of trends which approach the accuracy limits of the data. It is a conclusion of 
this study that existing temperature records for Bonneville are not of scientific value in judging temperature conditions on the Columbia River at that point. However, Bonneville temperatures are useful as a basis for observing day-to-day trends for simulation and superposition experiments. Using the existing record, it was determined that the residual thermal differential at warrendale from the maximum allowable input of thermal energy in the Priest Rapids-Richland reach under existing Washington State standards would be less than $0.20{ }^{\circ} \mathrm{C}$ during critical summer months and less than $0.35^{\circ} \mathrm{C}$ during winter months. These numerical values indicate that thermal modification in the Priest Rapids-Richland reach is about $80 \%$ dissipated at Warrendale, Mile 140 , about 200 miles below the Richland sensor. This compares with $65 \%$ dissipation at the Washington-oregon border.

7. Detailed measurements in the Hanford plant effluent mixing zone are described in Appendix $C$. These reveal that, during the period when all reactors were operating at the Hanford site, the dispersion patterns were such that in the immediate plant area, significant stream cross-section areas of cooler water were available as migration routes for fish. The Ringold area provides a complete mixing zone for full dispersion of materials above that point and represents a location of maximum average stream temperatures related to the operation of reactors. Cross-section temperature measurements made below Ringold during periods of reactor shutdown reveal consistently higher shoreline temperatures than the essentially uniform midstream or thalweg conditions. A temperature rise of from 0.50 to $0.75{ }^{\circ} \mathrm{C}$ occurs from natural heating in the Hanford reach during August and September which is high compared to impounded reaches. This is the equivalent of 4000 to 6000 thermal megawatts of energy for flows ranging from 
80,000 to $150,000 \mathrm{ft}^{3} / \mathrm{sec}$. The cooling of the river in the Hanford reach is also relatively high compared to the impounded areas. Generally, studies of heat dissipation on the Columbia for thermal effluent and thermal modifications from dam manipulation consistently show that temperature gradients are more conservative in the winter than the summer under average weather conditions. That is, a thermal modification will persist longer in winter than in the summer. This is also shown in the computer simulation of 19,300 thermal megawatts of energy for the Hanford reach in Figure 5 of this discussion.

One of the most significant results of the thermal studies program at Pacific Northwest Laboratory has been the certification of the need for standards of accuracy for stream temperature measurements. Even within a state-of-the-art program, variations in standards exist for reasons of expediency and practicality. It is suggested that the art of stream temperature management. will come of age only when an accepted standard of accuracy and sampling frequency is established for all serious researchers, river users and enforcement personnel to have a common basis in discussion of thermal modification of water quality. 


\section{SUMMARIES OF DAILY AVERAGE TEMPERATURE}

The following tabulations are the daily averages from digitized thermograph records or hourly observations in year, month, day order from Northport (International Border), River Mile 734.1 to River Mile 208.0 below John Day Dam. Included are multiyear averages at Priest Rapids and Grand Coulee on the Columbia River and Ice Harbor on the Snake River.

The daily averages of the maximum and minimum hourly temperature in degrees centigrade observed each day for a period of 20 years, 1947 through 1967 are shown in Tables 4 and Table 5. Temperatures measured at Rock Island, about 56 miles above Priest Rapids, were used for the years prior to 1960.

These data have been adjusted to account for sensor position error and instrument readout error. They represent the best estimate of the actual mean water temperature for the stated period as was technically feasible for the time period involved.

\section{INSTRUMENTATION ACCURACY}

Sensor and Instrument Error Estimates

Nor thport $\pm 0.75^{\circ} \mathrm{C}$
Foxboro thermograph located on bridge pier of Highway 25 , and serviced under a Battelle-Northwest/AEC contract. The instrument is affected by ore trucks using the bridge and by water level fluctuations from dam regulation both above and below the instrument site. Usable accuracy is maintained by comparison to a weekly check made with a certified thermometer and a daily measurement made by United States Geological Survey (USGS). 
Grand Coulee Foxboro the rmograph located on a bridge pier $\pm 0.35{ }^{\circ} \mathrm{C}$ where the highway crosses the river below the dam. Accuracy maintained by periodic checks with an Atkins thermometer.

Priest Rapids Leeds and Northrup thermograph and river stage $\pm 0.20{ }^{\circ} \mathrm{C}$ recorder at the USGS gage site below priest Rapids dam. Historically the most reliable instrument in our reporting network with $0.20^{\circ} \mathrm{C}$ error margin from both river sensor and recorder. Serviced and checked by AEC contractor and Grant County PUD. Periodic boat surveys made.

Rich 1 and Foxboro thermograph at River Mile 338.0. Accuracy $\pm 0.30{ }^{\circ} \mathrm{C}$ of instrument maintained by periodic checks with Atkins thermometer. Upstream islands deter uniform mixing. Monthly boat surveys made.

Pasco $\pm 0.30{ }^{\circ} \mathrm{C}$ Foxboro thermograph (prior to 1969) accuracy maintained by periodic calibration in Battelle-Northwest Instrument Shop. (Readings in 1969 not reliable.)

Umatilla Foxboro thermograph accuracy maintained by $\pm 0.20^{\circ} \mathrm{C}$ weekly check with certified Atkins thermometer and shop calibration when needed.

Biggs-John Day Foxboro thermograph checked periodically with $\pm 0.25{ }^{\circ} \mathrm{C} \quad$ Atkins thermometer.

* The Atkins RTD thermometer has a certified accuracy of $\pm 0.01^{\circ} \mathrm{C}$ with a precision of $\pm 0.005^{\circ} \mathrm{C}$ when tested in a stirred calibration facizity. 


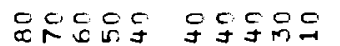

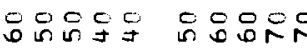

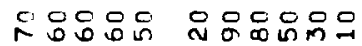

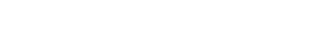

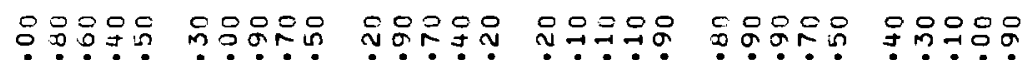

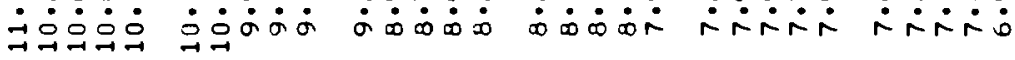

党

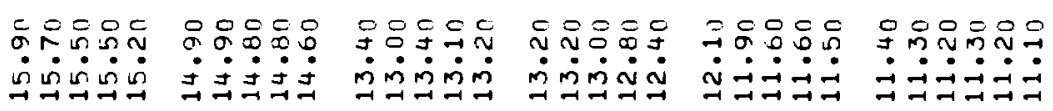

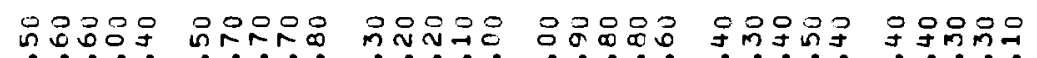

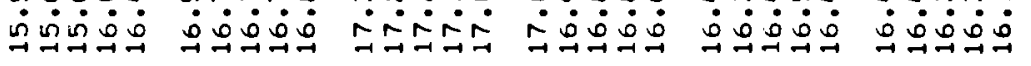

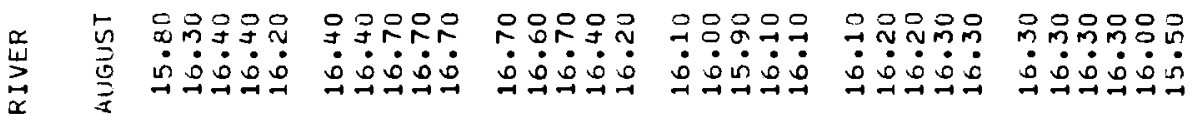

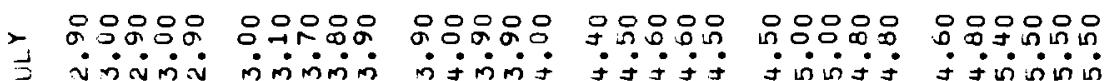

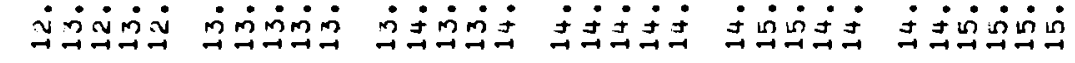

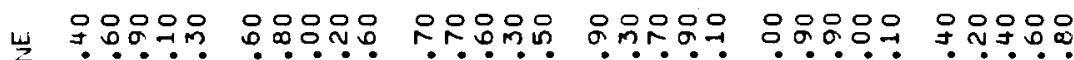

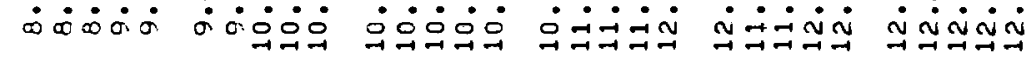

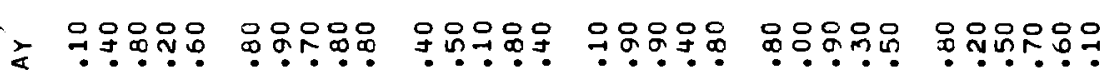

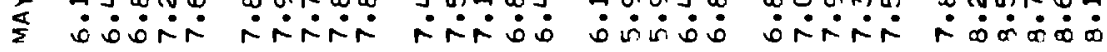

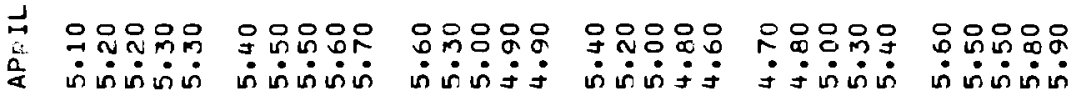

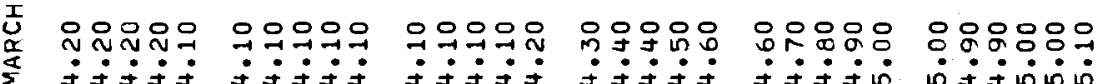

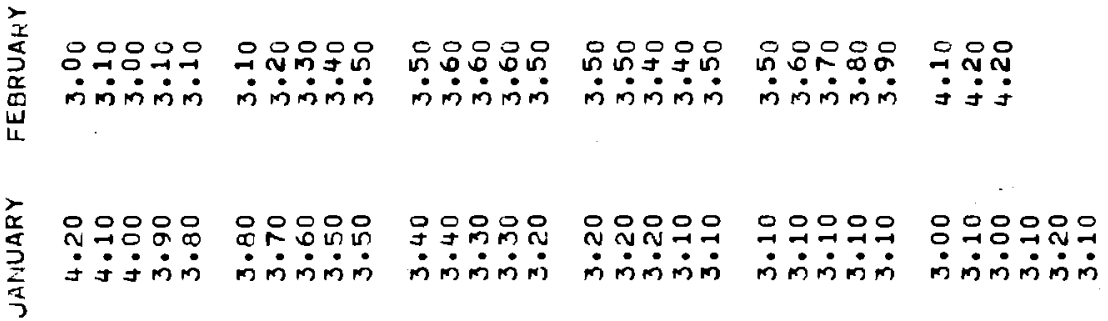

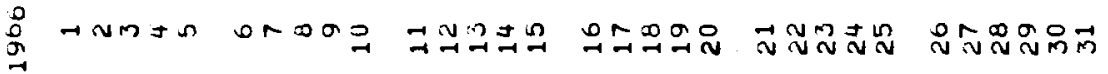


$\frac{2}{4}$

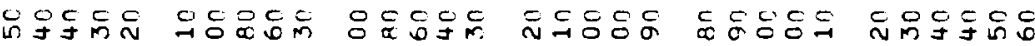

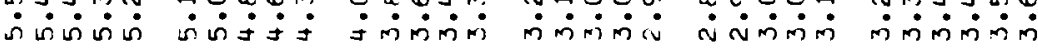

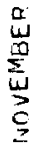

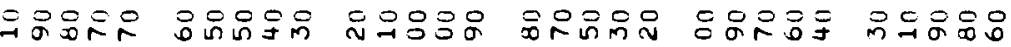

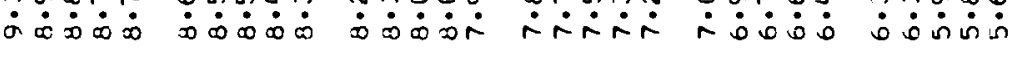

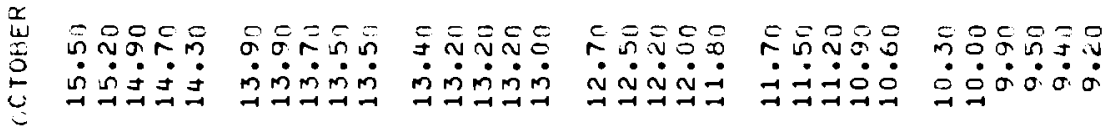

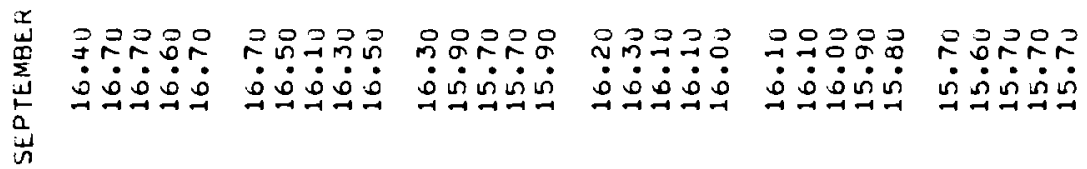

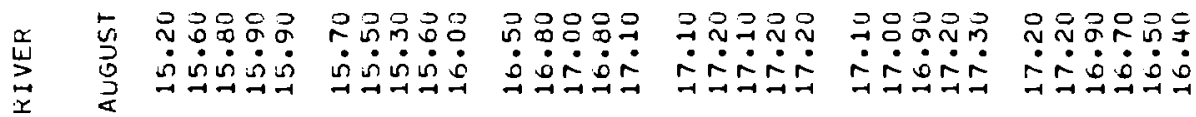

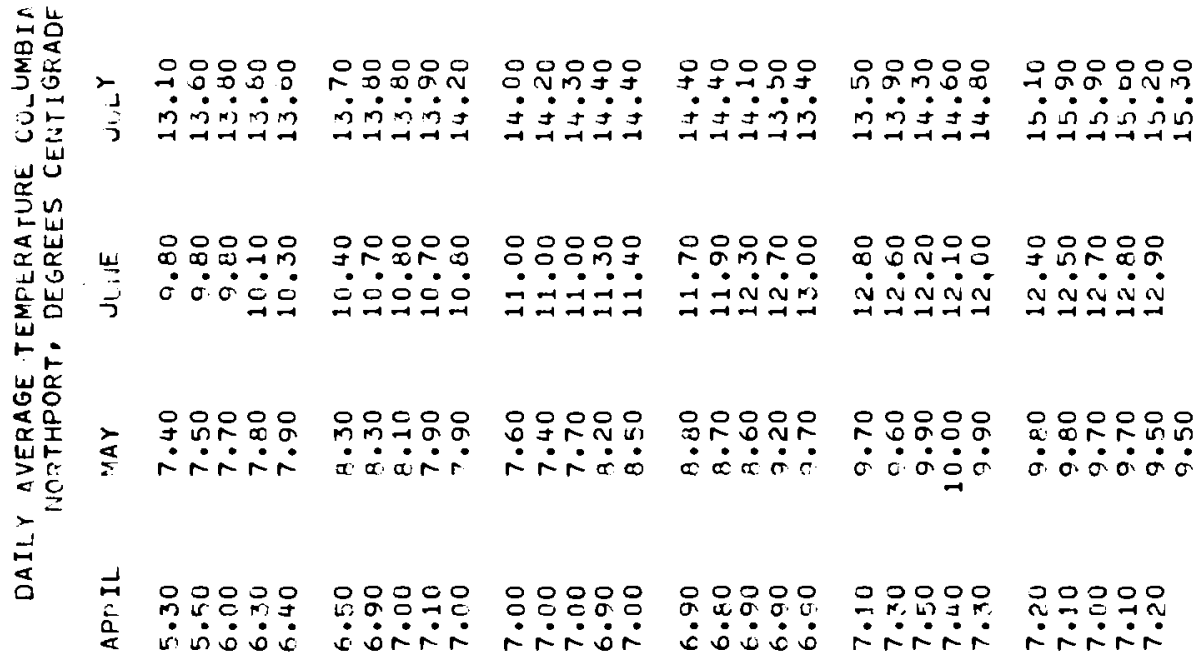

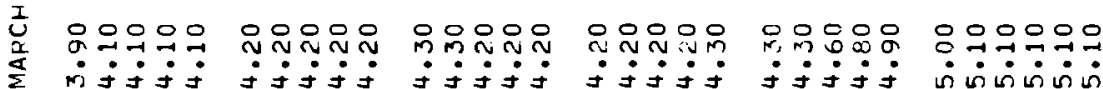

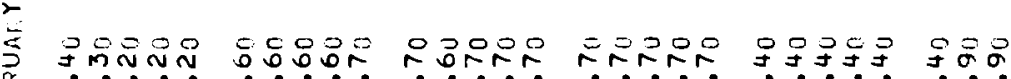

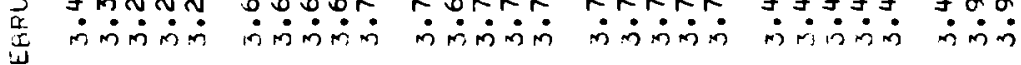

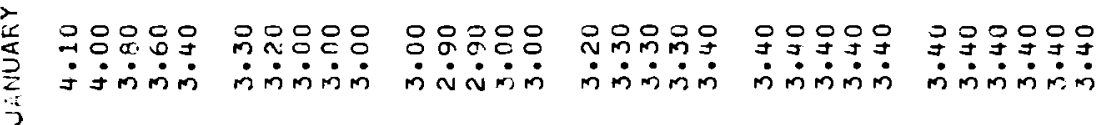

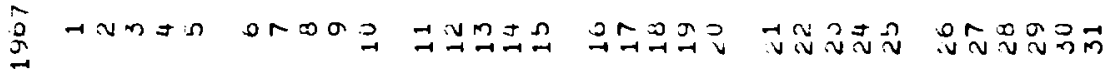




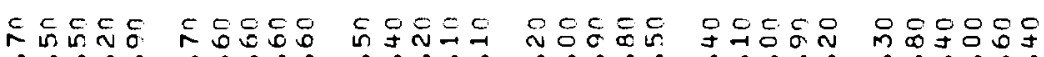

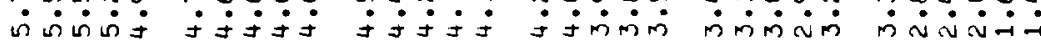

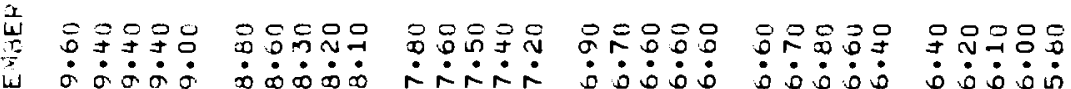

$\stackrel{1}{2}$

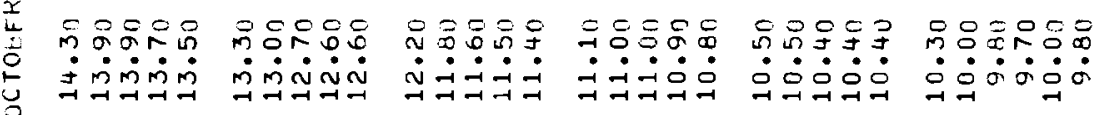

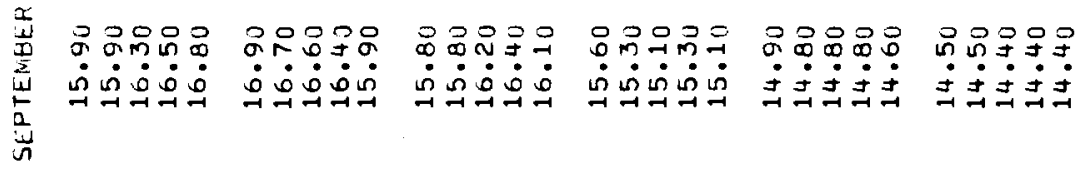

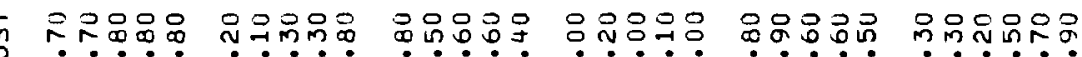
ம்

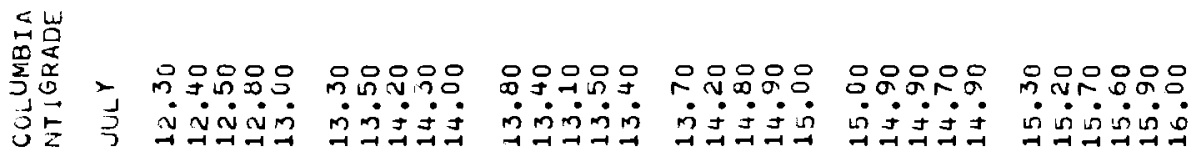

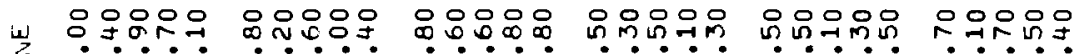

J o îं

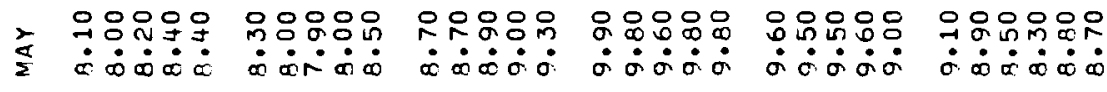

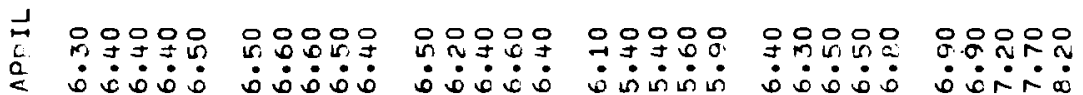

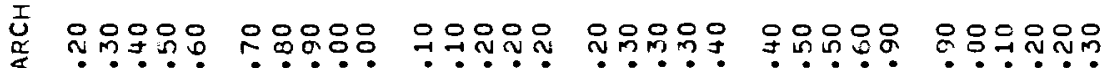

落

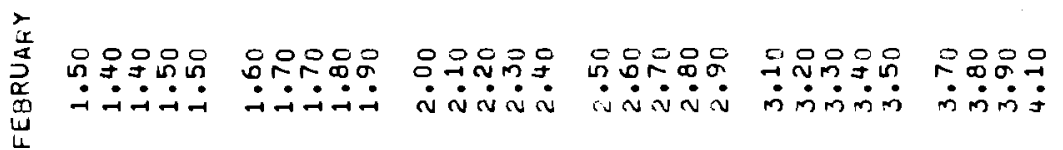

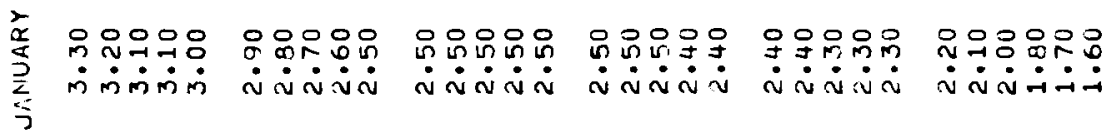

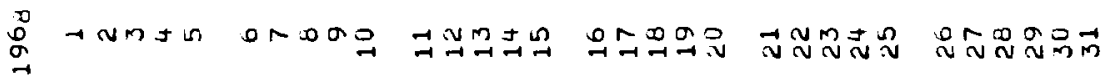




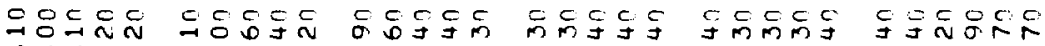

ririr

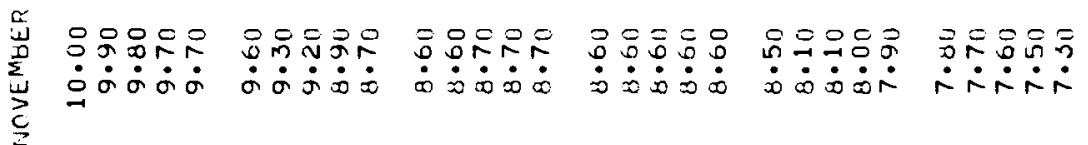

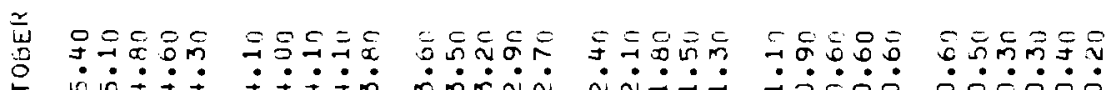

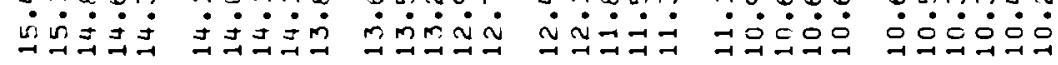

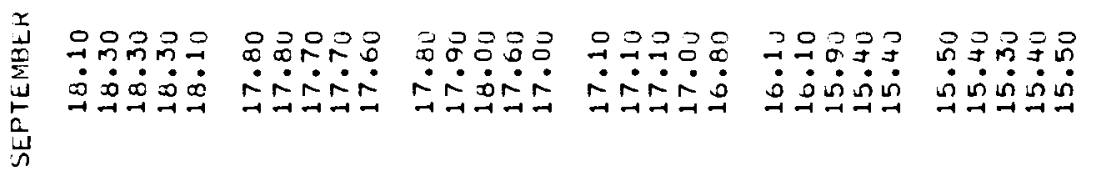

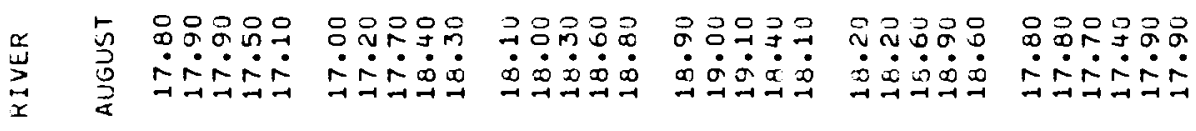

老宏

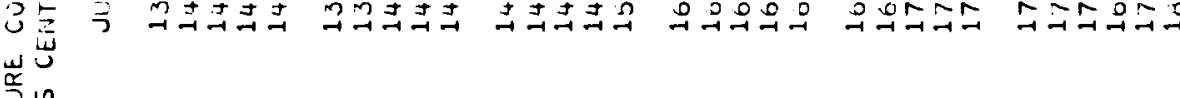

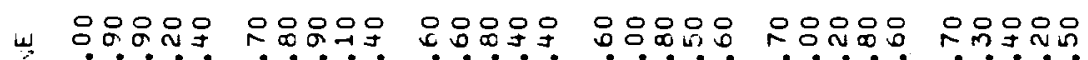

引

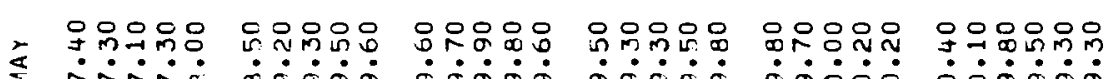

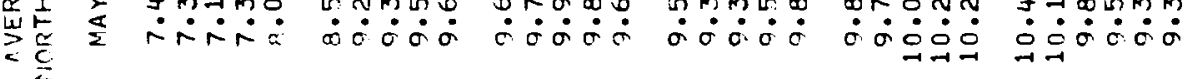

家

南

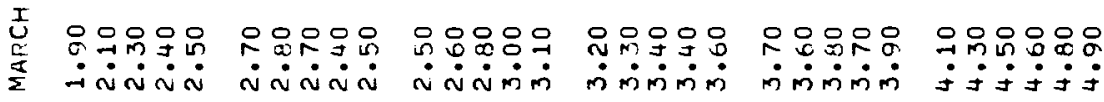

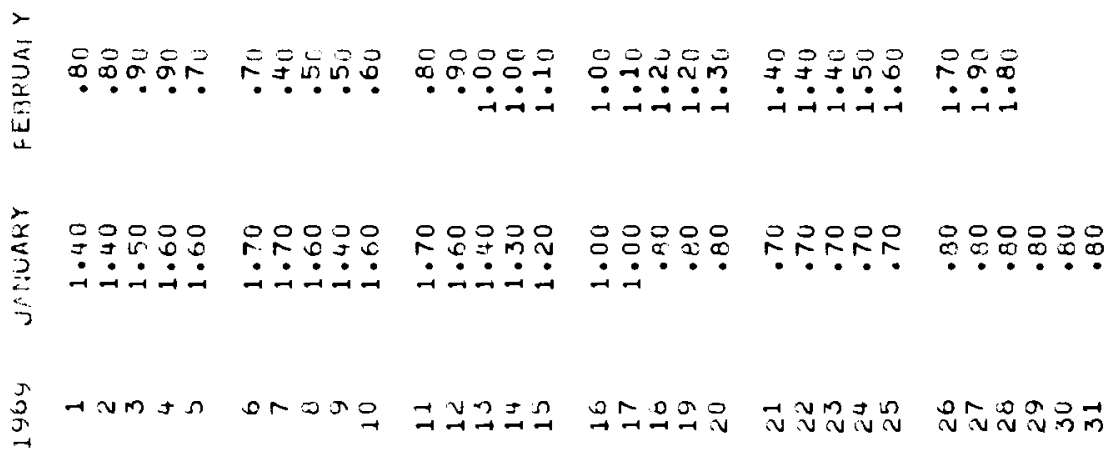




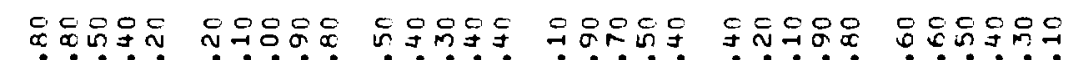

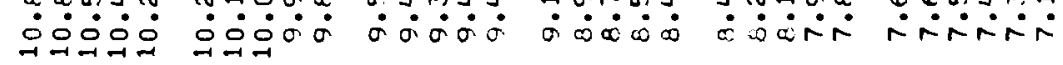

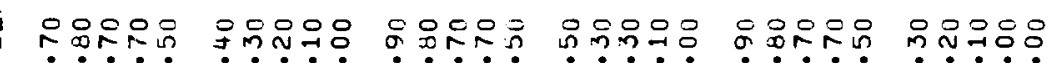

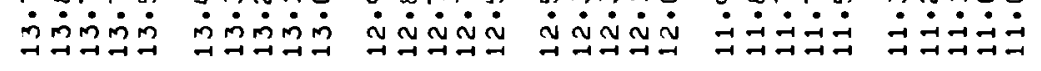

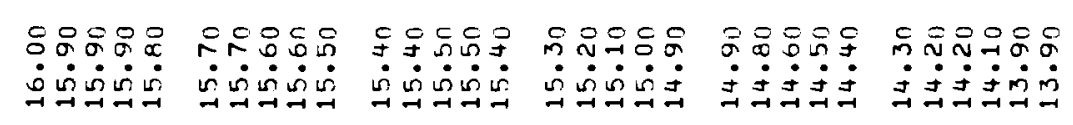

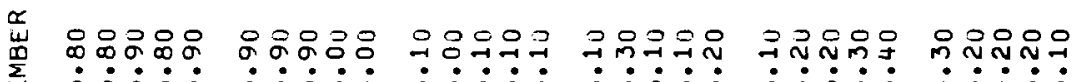

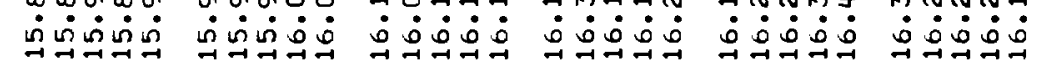

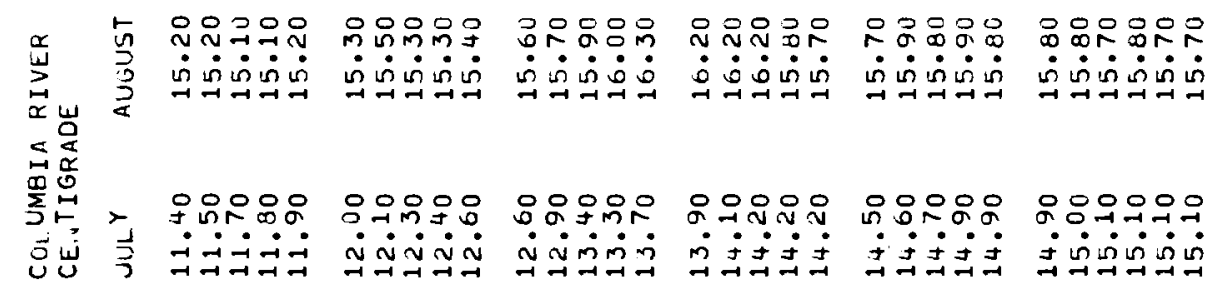

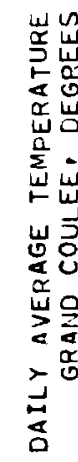

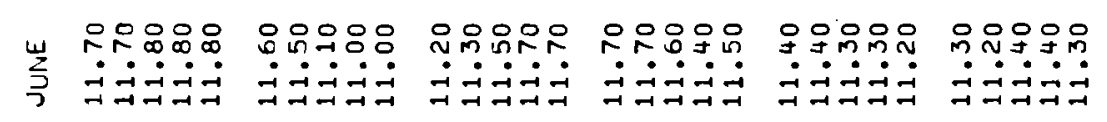

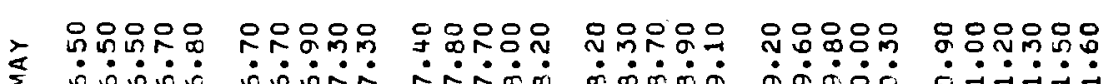

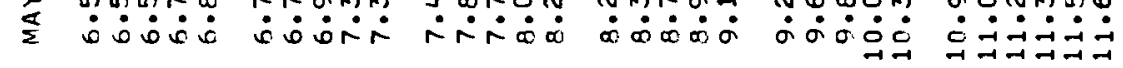

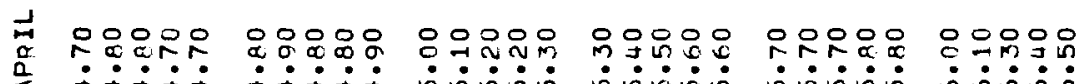

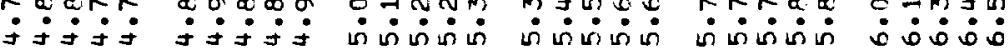

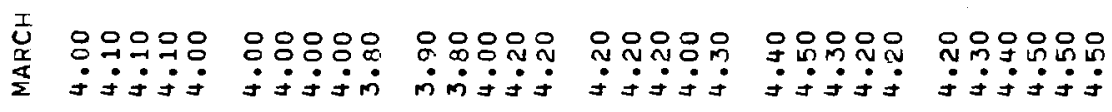

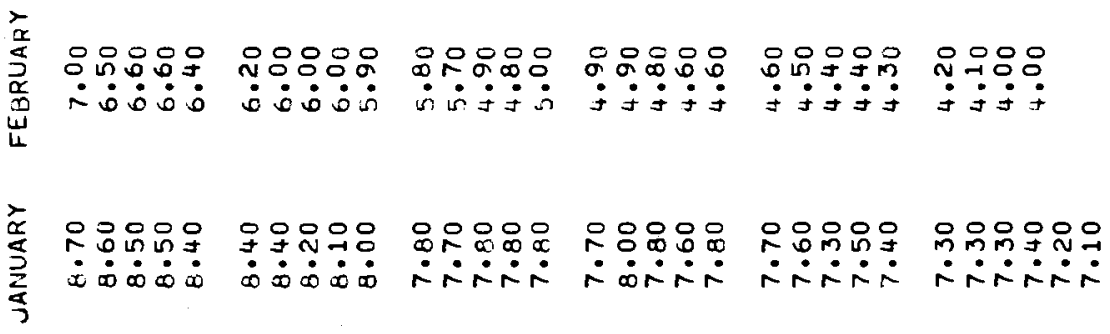

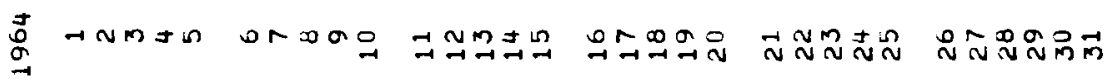




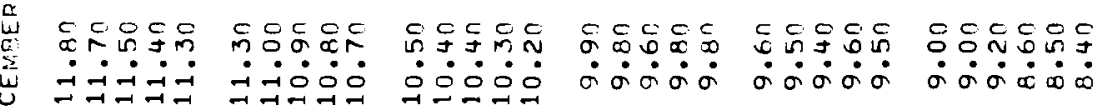

岩

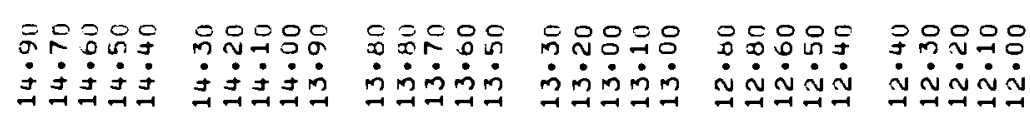

I

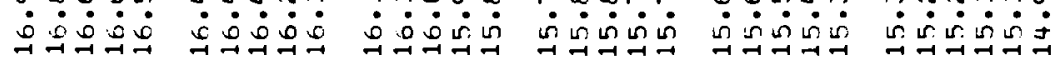

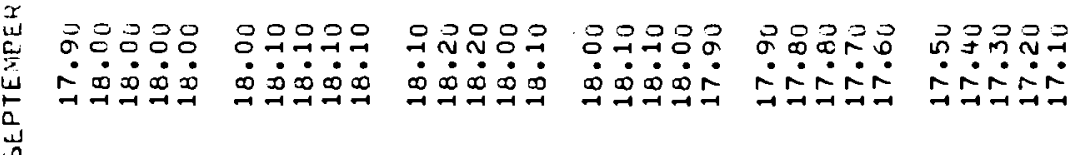

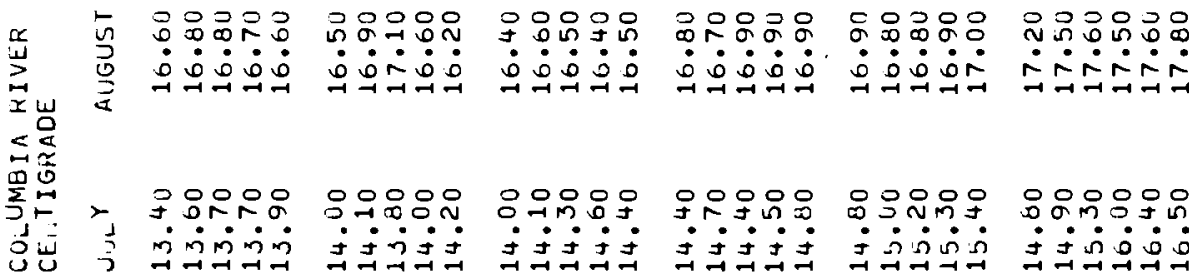

崩

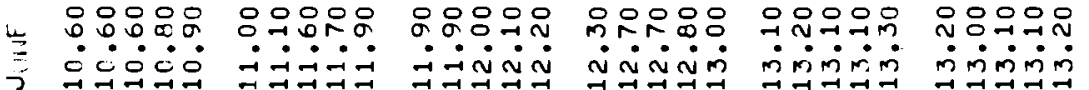

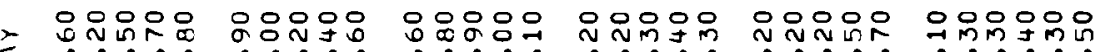

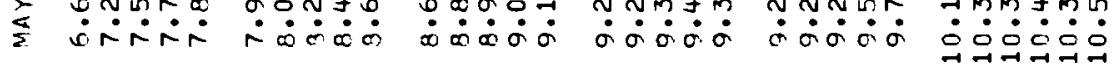

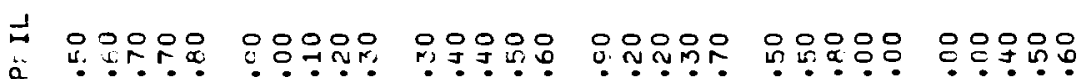

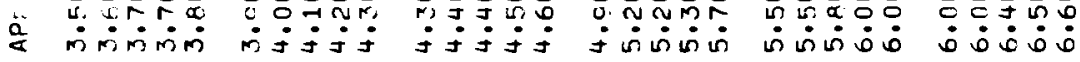

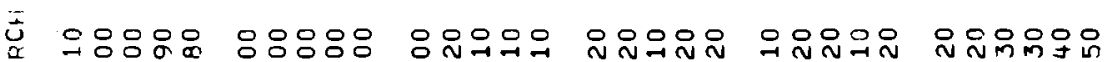

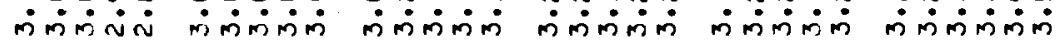

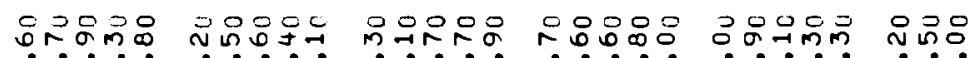

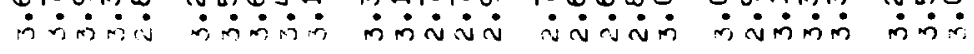

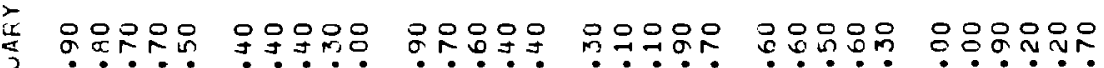

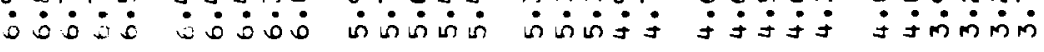

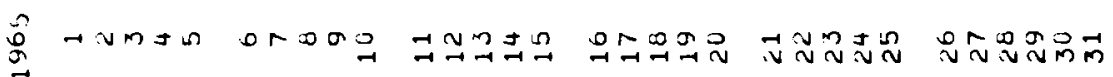


DAILY AVERAGE TEMPERATURE COLUMBIA RIVER GRAND COULEE, DEGREES CE, TIGRADE

\begin{tabular}{|c|c|c|c|c|c|c|c|c|c|c|c|c|}
\hline 1960 & JANUARY & FERRUARY & MARCH & APRIL & MAY & JUNE & JUL.Y & AUGUST & SEPTEMBER & OCTOBER & WOVEMBER & DECENABE? \\
\hline $\begin{array}{l}1 \\
2 \\
3 \\
4 \\
5\end{array}$ & $\begin{array}{l}8.00 \\
8.10 \\
8.20 \\
8.00 \\
7.70\end{array}$ & $\begin{array}{l}4.80 \\
4.70 \\
4.60 \\
4.50 \\
4.40\end{array}$ & $\begin{array}{l}3.10 \\
3.10 \\
3.10 \\
3.00 \\
3.00\end{array}$ & $\begin{array}{l}4.20 \\
4.40 \\
4.50 \\
4.60 \\
4.70\end{array}$ & $\begin{array}{l}7.60 \\
7.70 \\
7.80 \\
7.90 \\
9.00\end{array}$ & $\begin{array}{l}10.40 \\
10.40 \\
10.40 \\
10.40 \\
10.45\end{array}$ & $\begin{array}{l}12.60 \\
12.70 \\
12.80 \\
12.90 \\
12.95\end{array}$ & $\begin{array}{l}14.90 \\
15.00 \\
15.10 \\
15.20 \\
15.45\end{array}$ & $\begin{array}{l}15.90 \\
16.00 \\
16.10 \\
16.20 \\
16.30\end{array}$ & $\begin{array}{l}16.50 \\
16.50 \\
16.70 \\
16.70 \\
16.70\end{array}$ & $\begin{array}{l}15 \cdot 50 \\
15 \cdot 30 \\
15 \cdot 30 \\
15 \cdot 10 \\
14 \cdot 90\end{array}$ & $\begin{array}{l}11.20 \\
11.40 \\
11.30 \\
11.20 \\
11.20\end{array}$ \\
\hline $\begin{array}{r}5 \\
7 \\
8 \\
9 \\
10\end{array}$ & $\begin{array}{l}7.60 \\
7.50 \\
7.40 \\
7.30 \\
7.20\end{array}$ & $\begin{array}{l}4.40 \\
4.30 \\
4.20 \\
4.10 \\
3.80\end{array}$ & $\begin{array}{l}3.00 \\
2.90 \\
2.90 \\
2.90 \\
3.00\end{array}$ & $\begin{array}{l}4.80 \\
4.80 \\
5.00 \\
5.00 \\
5.00\end{array}$ & $\begin{array}{l}8.20 \\
8.20 \\
8.40 \\
3.40 \\
8.40\end{array}$ & $\begin{array}{l}10.60 \\
10.80 \\
10.90 \\
11.15 \\
11.30\end{array}$ & $\begin{array}{l}12.90 \\
13.00 \\
13.10 \\
13.20 \\
13.30\end{array}$ & $\begin{array}{l}15.80 \\
16.10 \\
16.25 \\
15.70 \\
15.90\end{array}$ & $\begin{array}{l}16.20 \\
16.30 \\
16.30 \\
16.30 \\
16.30\end{array}$ & $\begin{array}{l}16.80 \\
16.70 \\
16.70 \\
16.80 \\
16.90\end{array}$ & $\begin{array}{l}14 \cdot 70 \\
14 \cdot 60 \\
14 \cdot 50 \\
14 \cdot 40 \\
14.20\end{array}$ & $\begin{array}{l}10.90 \\
10.80 \\
10.50 \\
10.60 \\
10.50\end{array}$ \\
\hline $\begin{array}{l}11 \\
12 \\
13 \\
14 \\
15\end{array}$ & $\begin{array}{l}7.10 \\
6.90 \\
6.80 \\
6.80 \\
6.60\end{array}$ & $\begin{array}{l}3.50 \\
3.30 \\
3.50 \\
3.30 \\
3.20\end{array}$ & $\begin{array}{l}3.00 \\
3.00 \\
3.00 \\
3.10 \\
3.10\end{array}$ & $\begin{array}{l}5.20 \\
5.20 \\
5.30 \\
5.70 \\
5.60\end{array}$ & $\begin{array}{l}8.70 \\
9.00 \\
8.90 \\
9.30 \\
9.50\end{array}$ & $\begin{array}{l}11.30 \\
11.30 \\
11.30 \\
11.20 \\
11.10\end{array}$ & $\begin{array}{l}13.30 \\
13.60 \\
14.00 \\
14.00 \\
14.00\end{array}$ & $\begin{array}{l}15.90 \\
16.10 \\
16.10 \\
16.30 \\
15.90\end{array}$ & $\begin{array}{l}16.30 \\
16.40 \\
16.40 \\
16.50 \\
16.50\end{array}$ & $\begin{array}{l}16.90 \\
16.80 \\
15.80 \\
16.830 \\
16.80\end{array}$ & $\begin{array}{l}14 \cdot 10 \\
13.90 \\
13.70 \\
13.70 \\
13.00\end{array}$ & $\begin{array}{r}10.30 \\
10.30 \\
10.10 \\
9.90 \\
9.9 n\end{array}$ \\
\hline $\begin{array}{l}16 \\
17 \\
18 \\
19 \\
20\end{array}$ & $\begin{array}{l}6.40 \\
6.40 \\
6.20 \\
6.00 \\
6.00\end{array}$ & $\begin{array}{l}3.30 \\
3.10 \\
3.00 \\
3.00 \\
3.00\end{array}$ & $\begin{array}{l}3.10 \\
3.20 \\
3.20 \\
3.30 \\
3.30\end{array}$ & $\begin{array}{l}5.60 \\
5.60 \\
5.70 \\
6.30 \\
6.40\end{array}$ & $\begin{array}{r}9.60 \\
9.60 \\
9.90 \\
10.10 \\
10.30\end{array}$ & $\begin{array}{l}11.05 \\
11.10 \\
10.80 \\
10.90 \\
11.10\end{array}$ & $\begin{array}{l}14.10 \\
14.30 \\
14.60 \\
14.45 \\
14.40\end{array}$ & $\begin{array}{l}15.40 \\
15.40 \\
15.50 \\
15.80 \\
15.80\end{array}$ & $\begin{array}{l}16.60 \\
16.50 \\
16.50 \\
16.50 \\
16.50\end{array}$ & $\begin{array}{l}16.80 \\
16.50 \\
16.70 \\
16.60 \\
16.60\end{array}$ & $\begin{array}{l}13.40 \\
13.20 \\
13.20 \\
13.10 \\
12.90\end{array}$ & $\begin{array}{l}9.90 \\
9.70 \\
9.50 \\
9.40 \\
9.30\end{array}$ \\
\hline $\begin{array}{l}21 \\
22 \\
23 \\
24 \\
25\end{array}$ & $\begin{array}{l}6.00 \\
5.80 \\
5.80 \\
5.60 \\
5.50\end{array}$ & $\begin{array}{l}2.80 \\
2.80 \\
2.90 \\
3.00 \\
3.00\end{array}$ & $\begin{array}{l}3.30 \\
3.40 \\
3.50 \\
3.50 \\
3.60\end{array}$ & $\begin{array}{l}6.50 \\
6.40 \\
6.50 \\
6.70 \\
6.80\end{array}$ & $\begin{array}{l}10.20 \\
10.20 \\
10.40 \\
10.60 \\
10.70\end{array}$ & $\begin{array}{l}11.30 \\
11.50 \\
11.60 \\
11.70 \\
11.75\end{array}$ & $\begin{array}{l}14.65 \\
14.90 \\
14.95 \\
14.90 \\
14.70\end{array}$ & $\begin{array}{l}15.80 \\
15.80 \\
15.70 \\
15.70 \\
15.75\end{array}$ & $\begin{array}{l}16.50 \\
16.50 \\
16.50 \\
16.50 \\
16.60\end{array}$ & $\begin{array}{l}16.40 \\
16.30 \\
16.10 \\
16.10 \\
16.00\end{array}$ & $\begin{array}{l}12 \cdot 80 \\
12 \cdot 70 \\
12.50 \\
12.40 \\
12.30\end{array}$ & $\begin{array}{l}9.10 \\
9.00 \\
8.90 \\
8.00 \\
8.60\end{array}$ \\
\hline $\begin{array}{l}26 \\
27 \\
28 \\
29 \\
30 \\
31\end{array}$ & $\begin{array}{l}5.40 \\
5.30 \\
5.30 \\
5.10 \\
5.00 \\
4.90\end{array}$ & $\begin{array}{l}3.00 \\
3.10 \\
3.00\end{array}$ & $\begin{array}{l}3.70 \\
3.90 \\
4.00 \\
4.10 \\
4.10 \\
4.20\end{array}$ & $\begin{array}{l}6.60 \\
6.70 \\
7.30 \\
7.60 \\
7.60\end{array}$ & $\begin{array}{l}10.80 \\
10.50 \\
10.40 \\
10.60 \\
10.60 \\
10.60\end{array}$ & $\begin{array}{l}11.80 \\
11.90 \\
12.00 \\
12.10 \\
12.30\end{array}$ & $\begin{array}{l}14.90 \\
15.20 \\
15.30 \\
15.30 \\
15.20 \\
14.80\end{array}$ & $\begin{array}{l}15.80 \\
15.70 \\
15.70 \\
15.75 \\
15.80 \\
15.90\end{array}$ & $\begin{array}{l}16.60 \\
16.50 \\
16.60 \\
16.60 \\
16.60\end{array}$ & $\begin{array}{l}15.90 \\
15.90 \\
15.90 \\
15.80 \\
15.70 \\
15.60\end{array}$ & $\begin{array}{l}12.10 \\
12.00 \\
11.90 \\
11.60 \\
11.30\end{array}$ & $\begin{array}{l}8.50 \\
8.50 \\
8.50 \\
8.60 \\
8.40 \\
8.30\end{array}$ \\
\hline
\end{tabular}




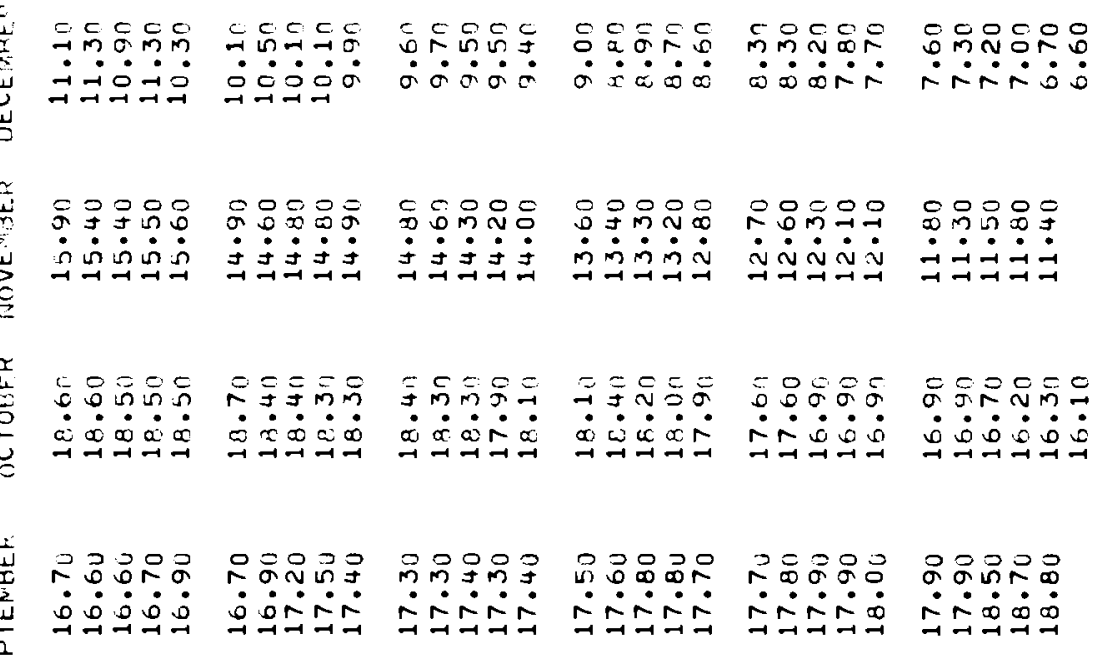

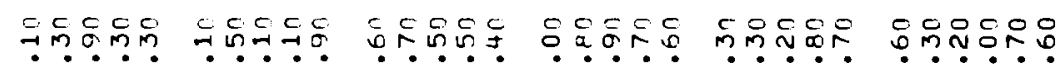

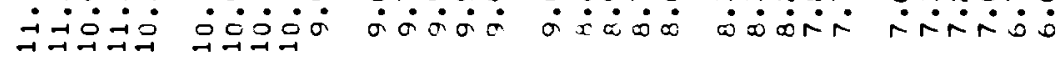

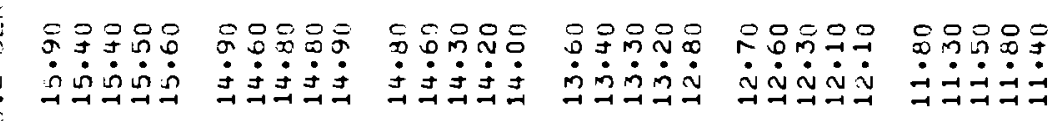

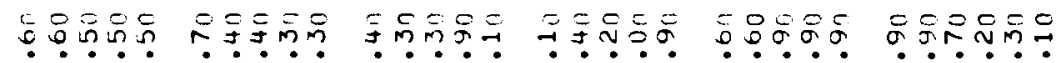

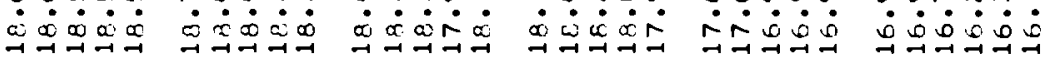

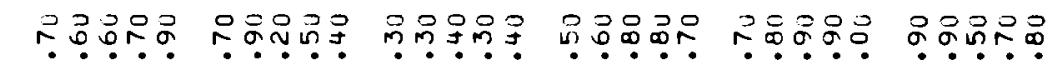

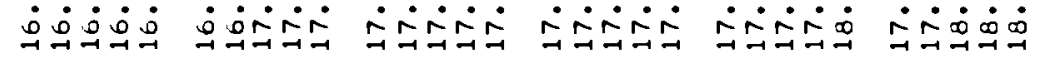

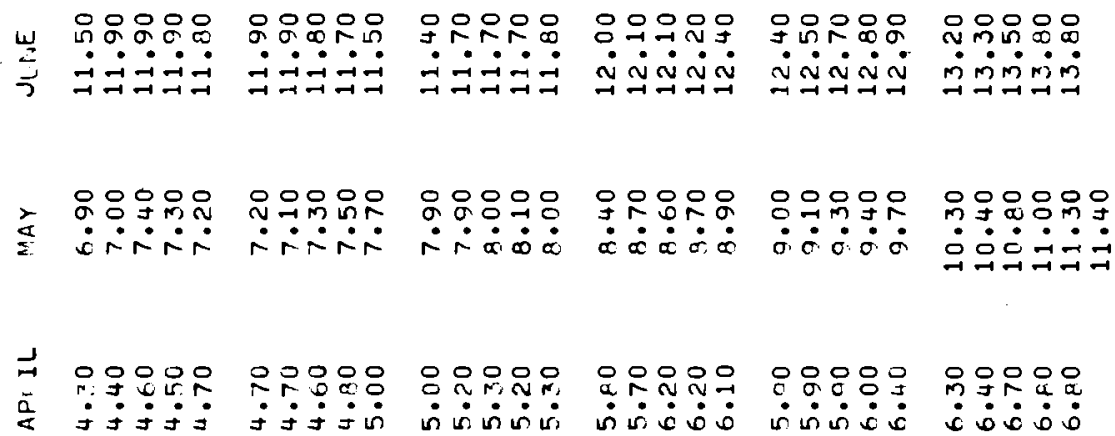

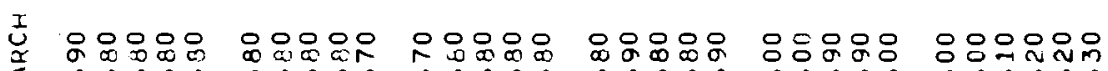

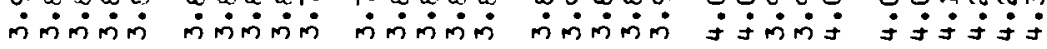

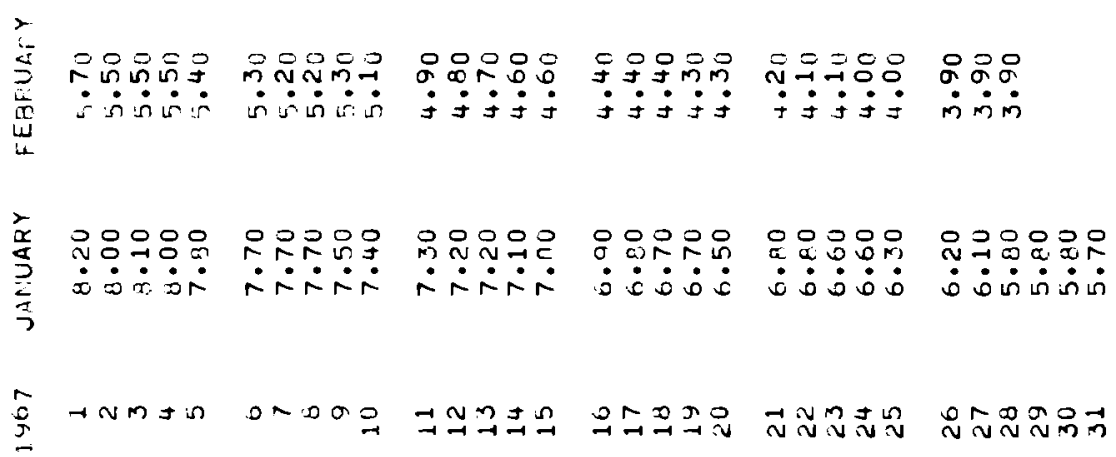




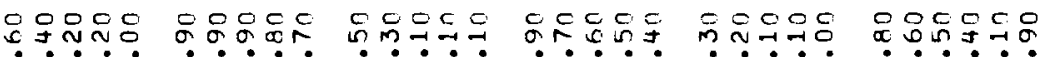

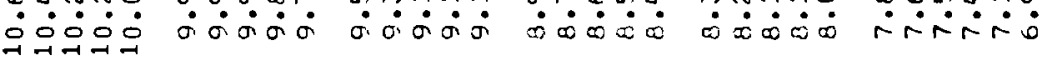

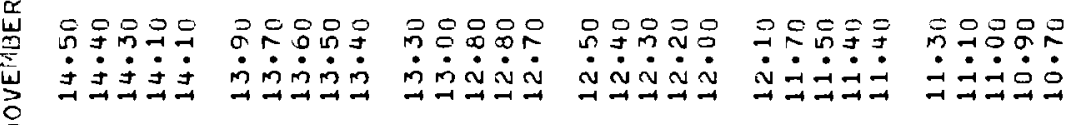

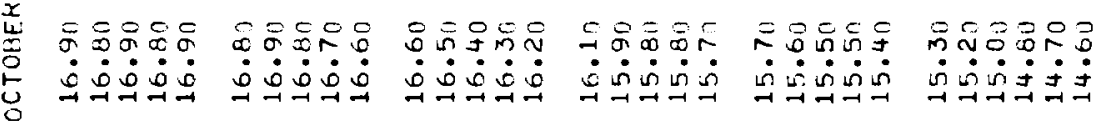

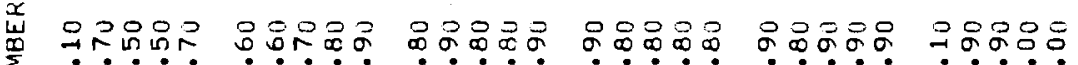

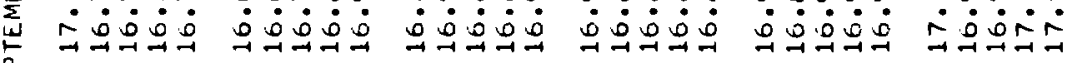

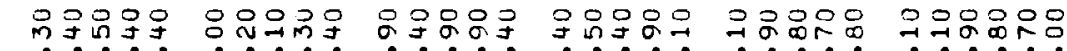

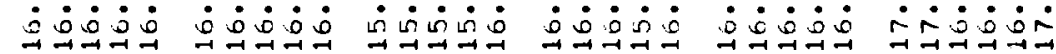

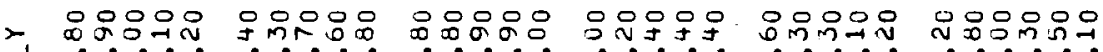

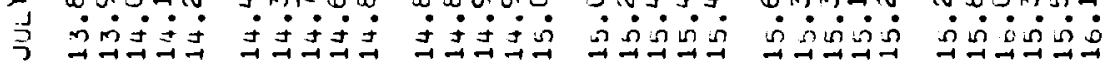

要

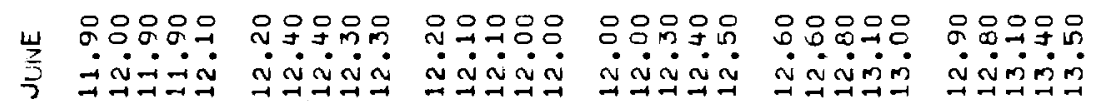

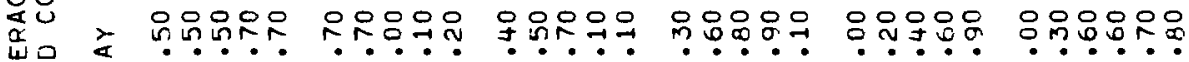

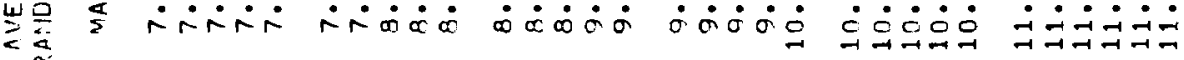
$>$ 定

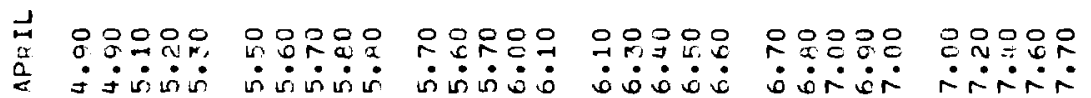

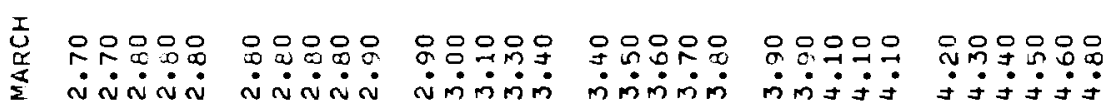

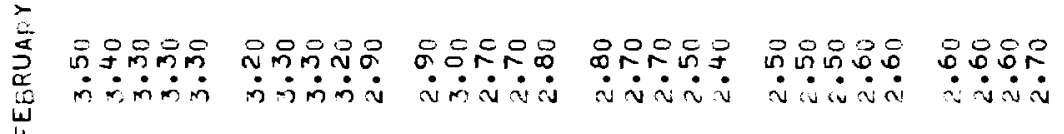

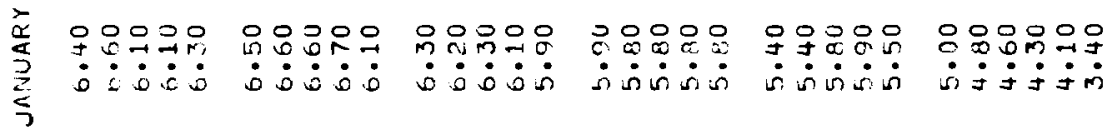

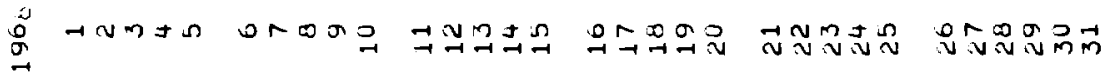




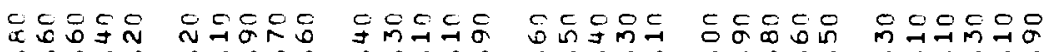

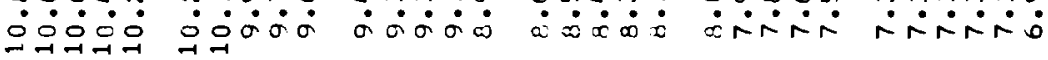

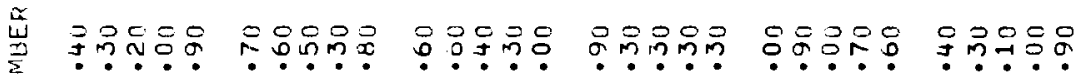

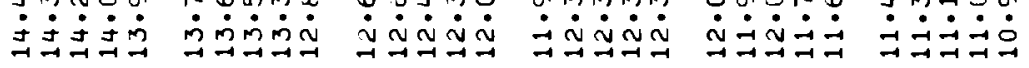

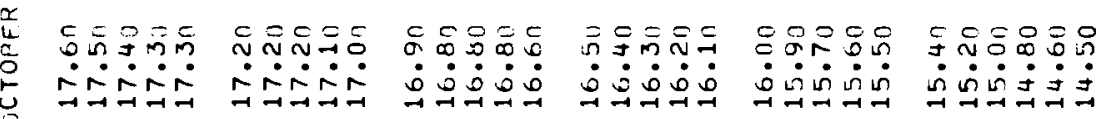
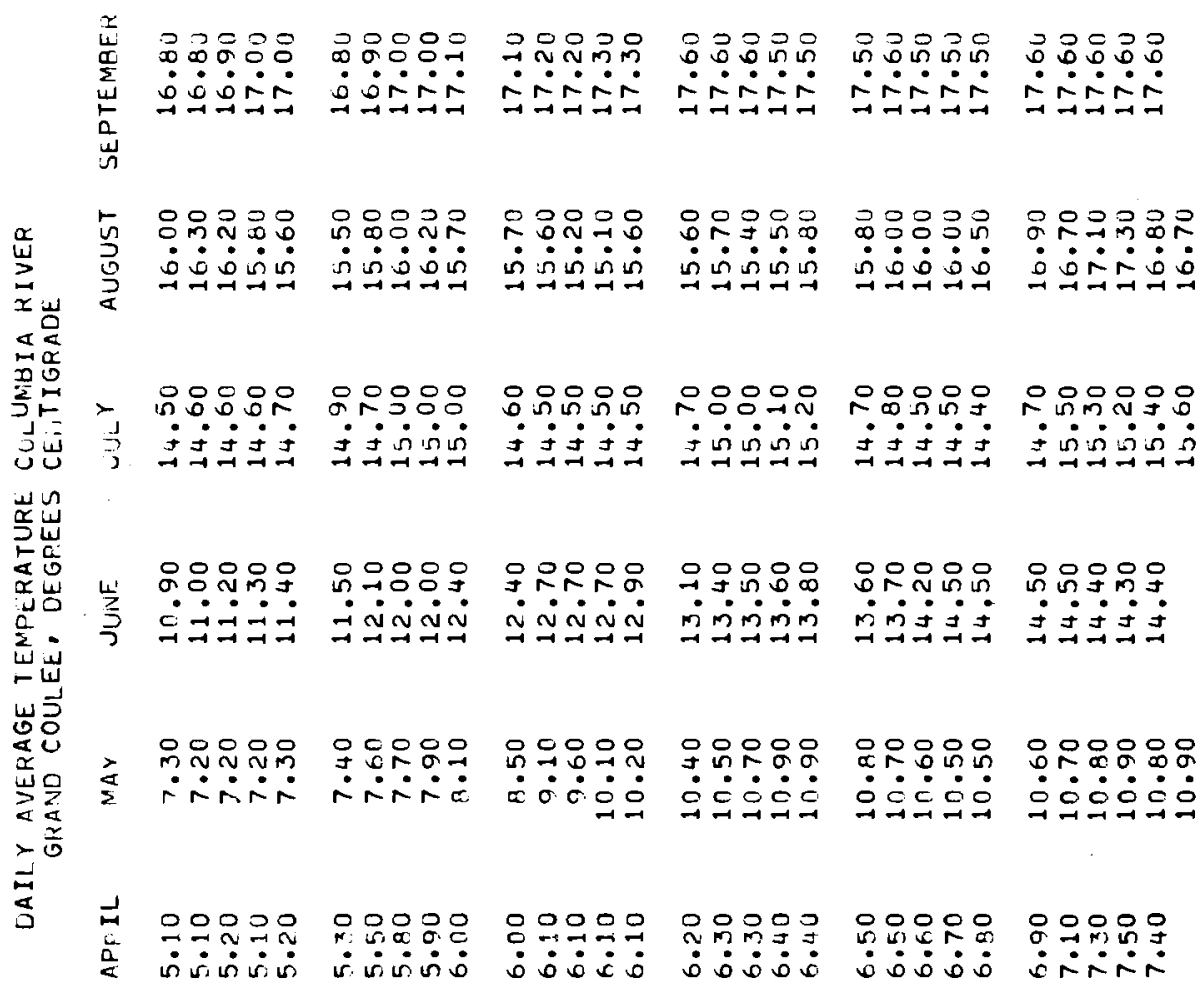

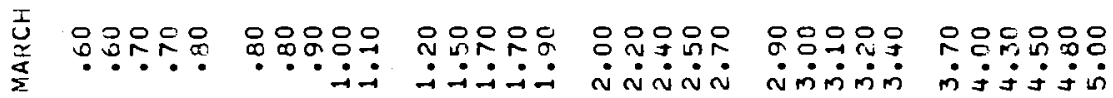

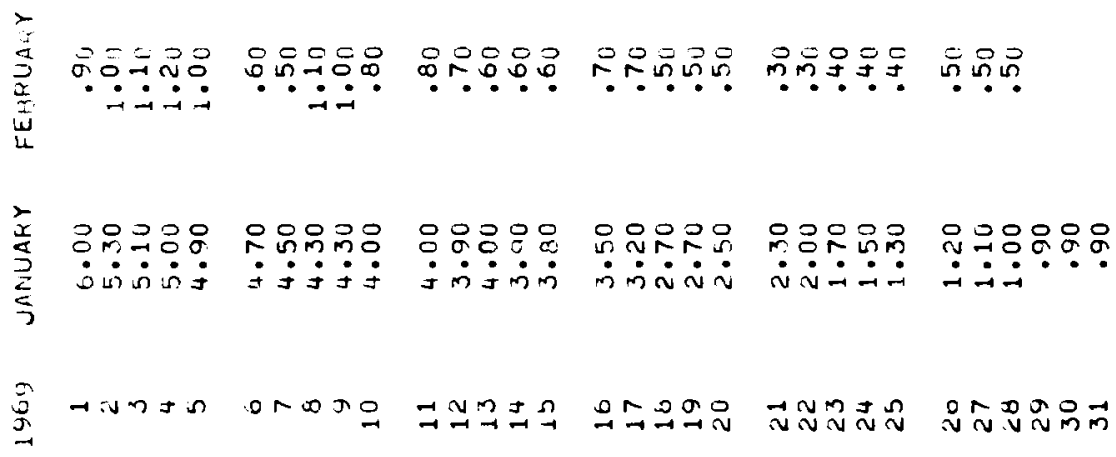




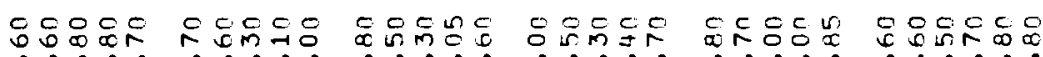

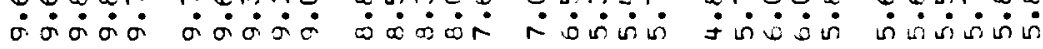

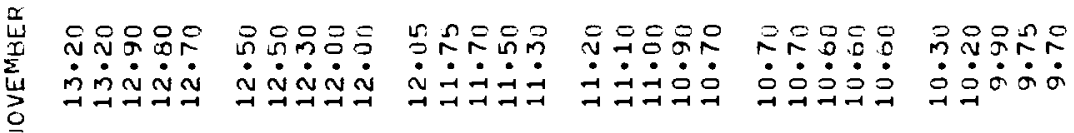

嵌

㟧 㟧

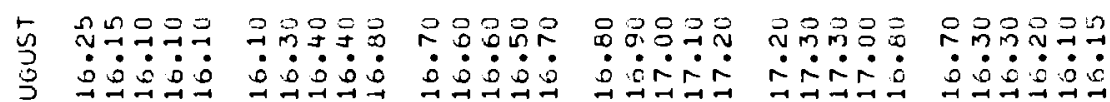

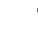

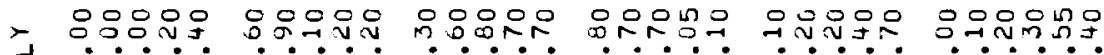

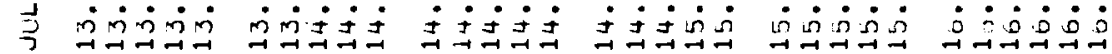

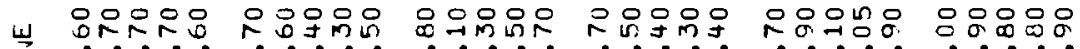

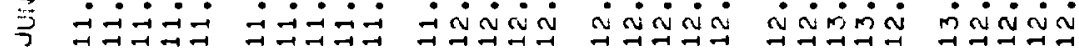

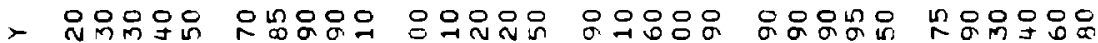
亲

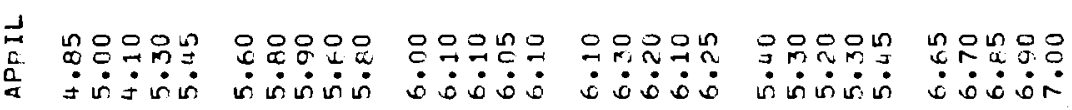

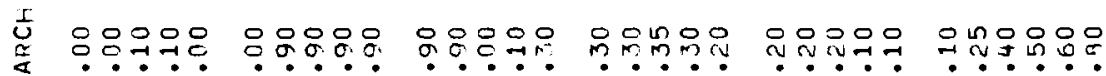

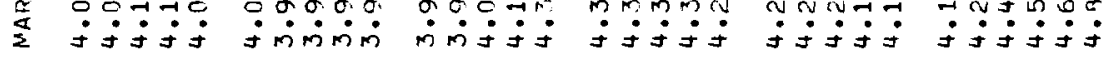

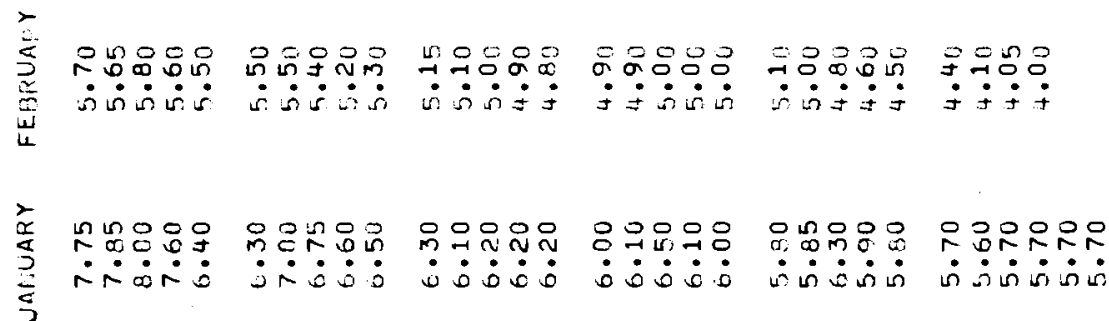
旁 


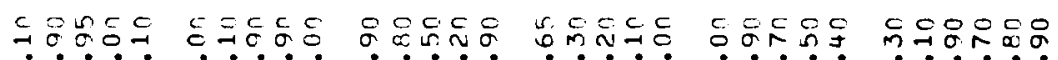

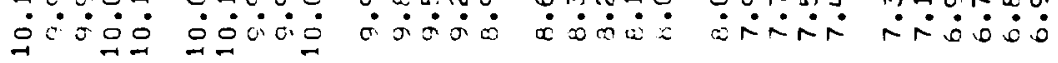

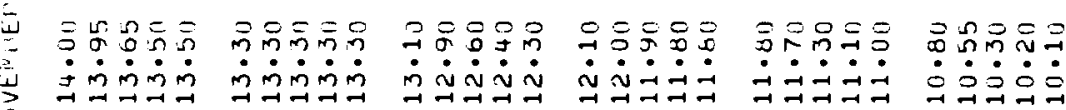

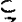

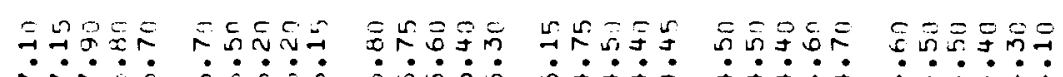

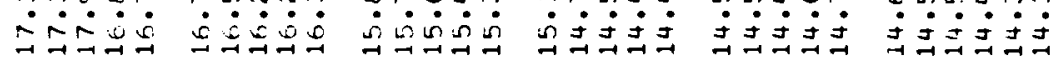

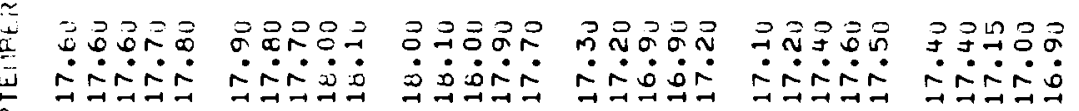
ก

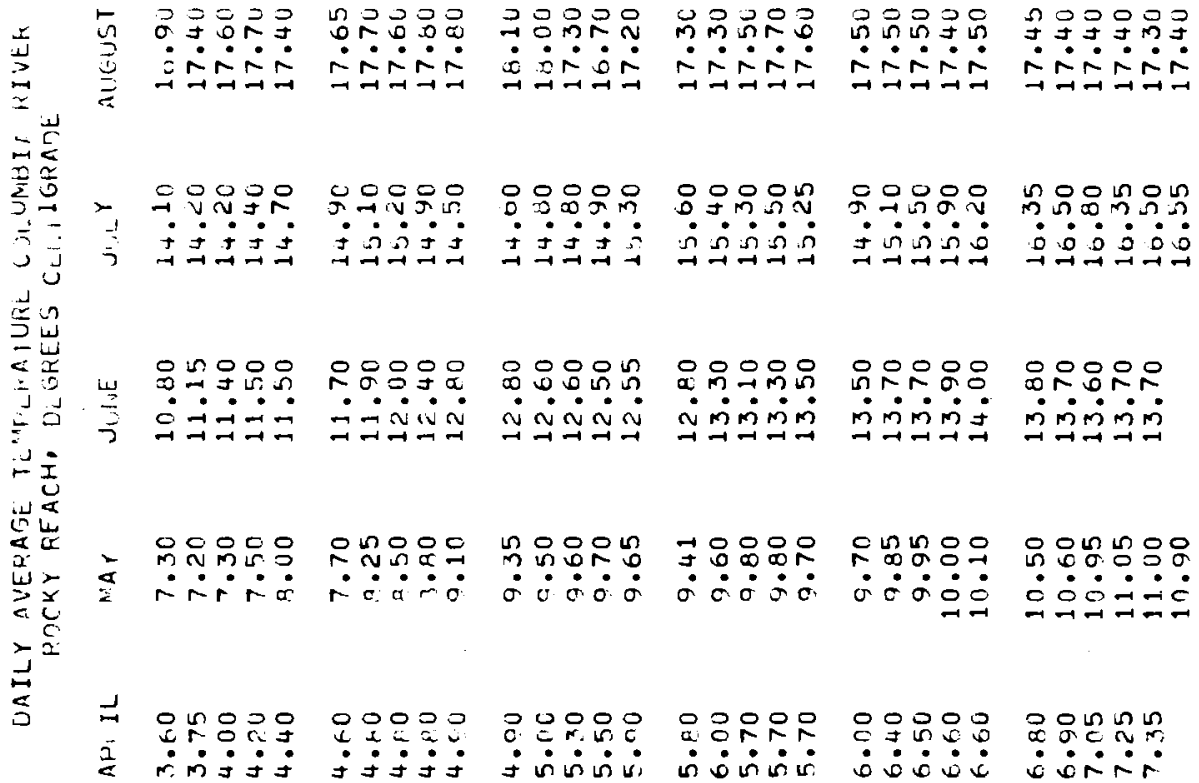

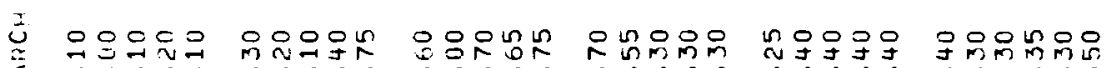

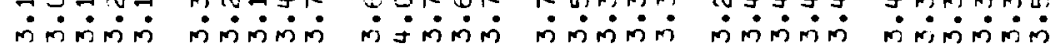

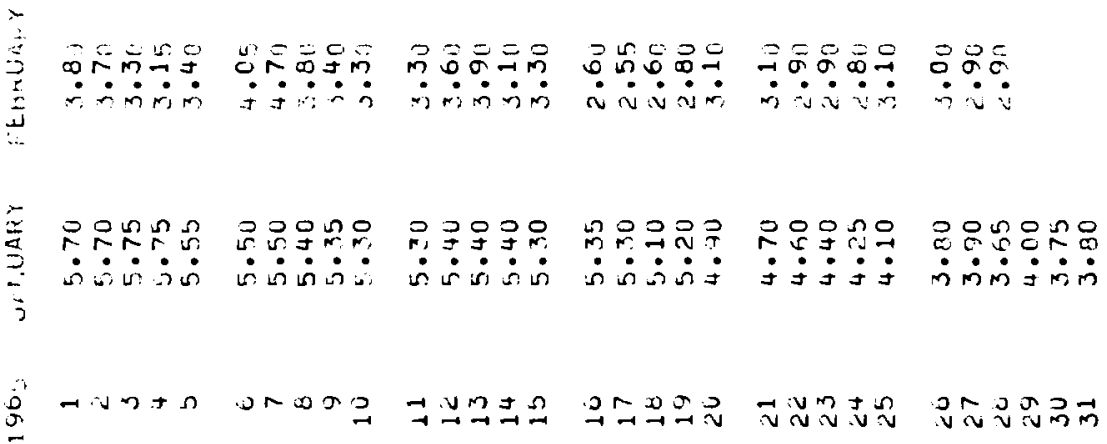




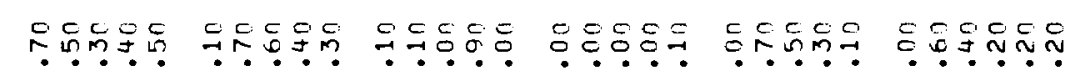

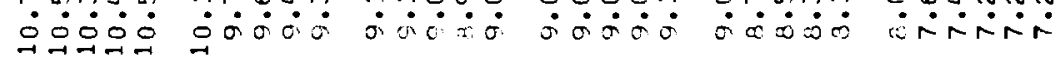

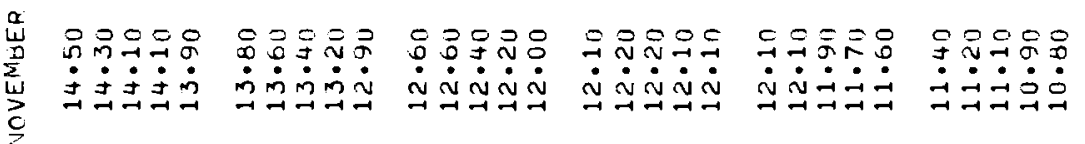

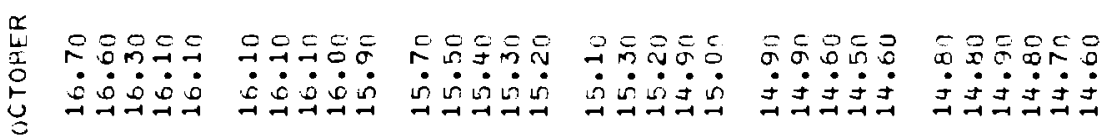

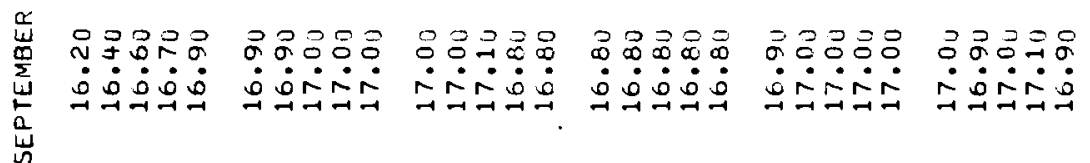

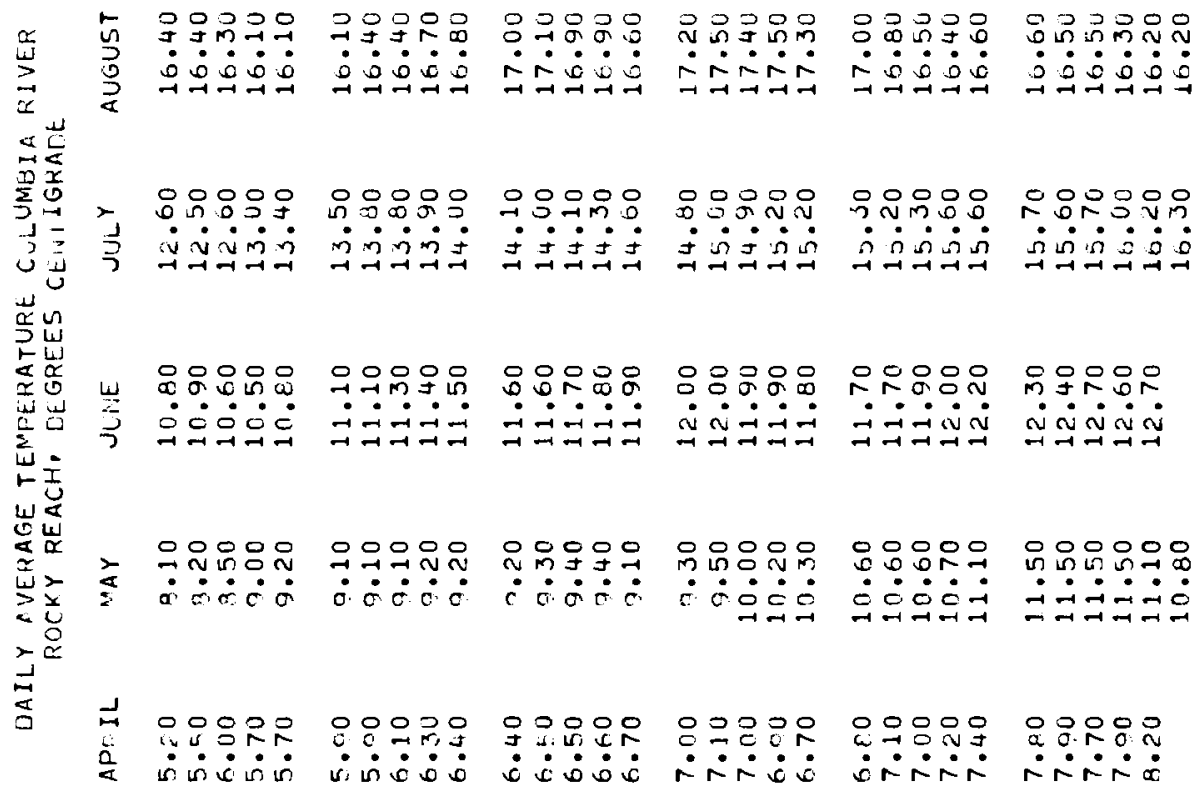

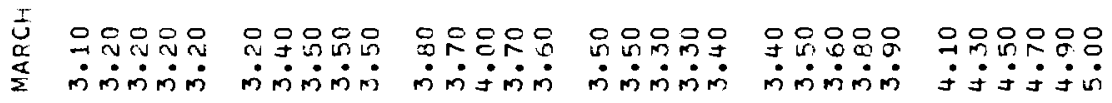

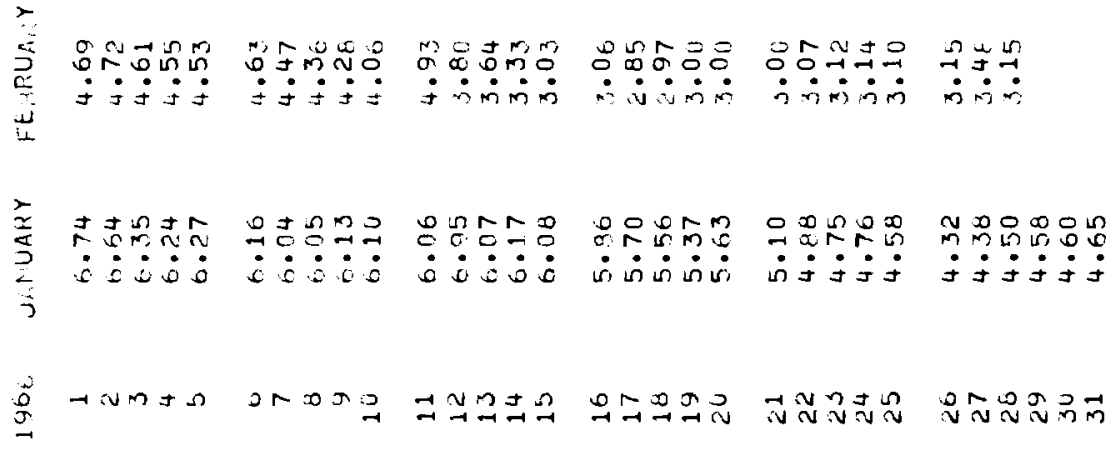




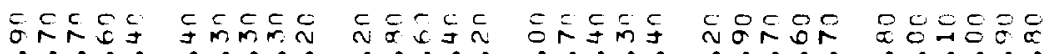

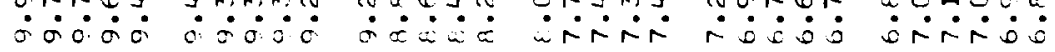

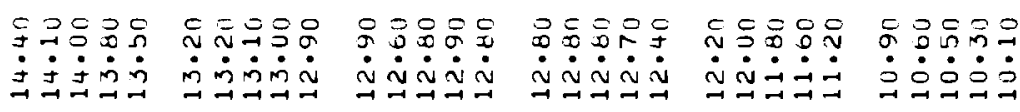

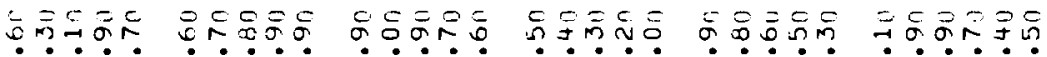

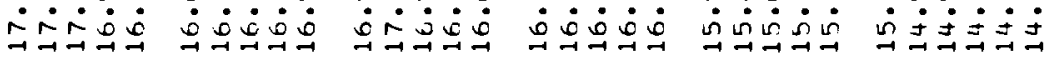

\begin{tabular}{lll}
\hline \\
0
\end{tabular}

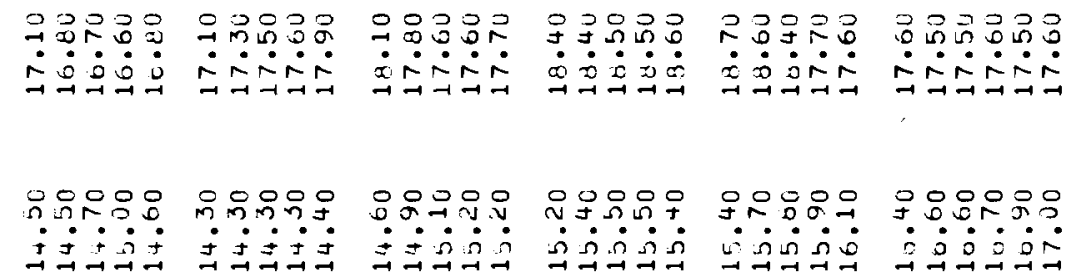

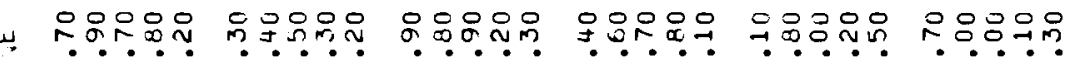

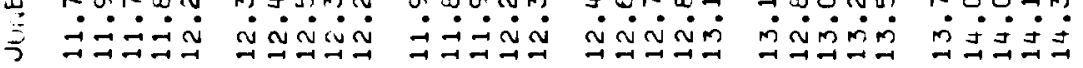

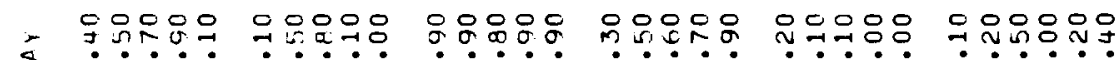

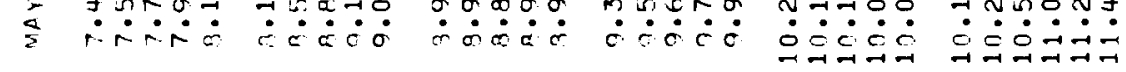

直

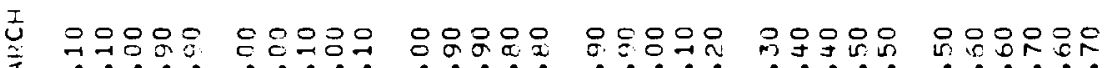

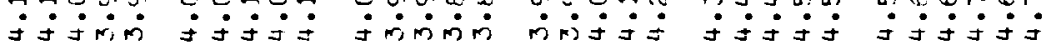

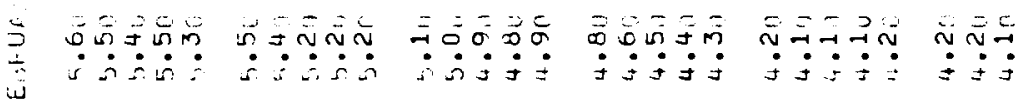

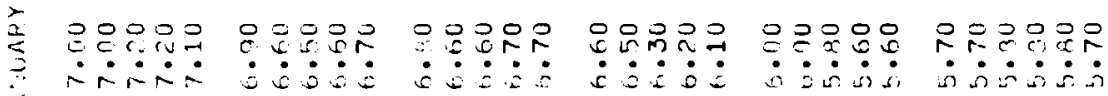

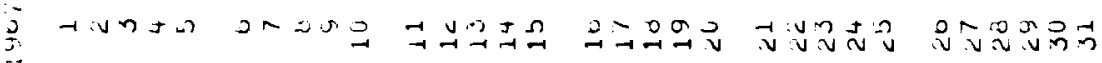




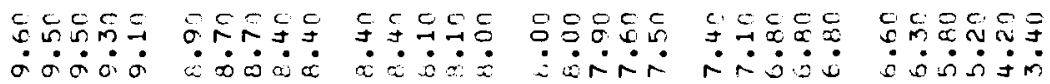

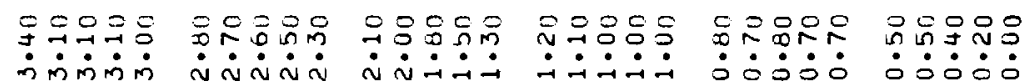

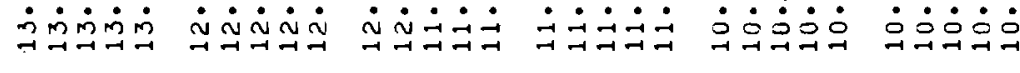

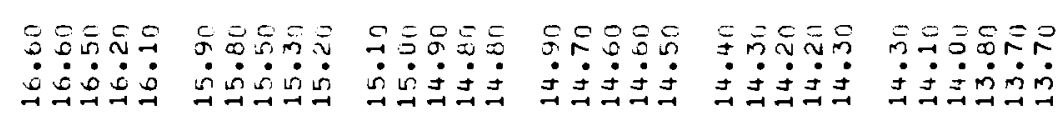

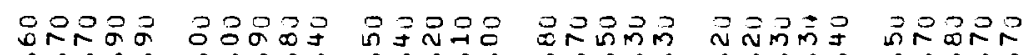

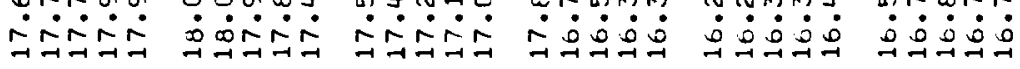

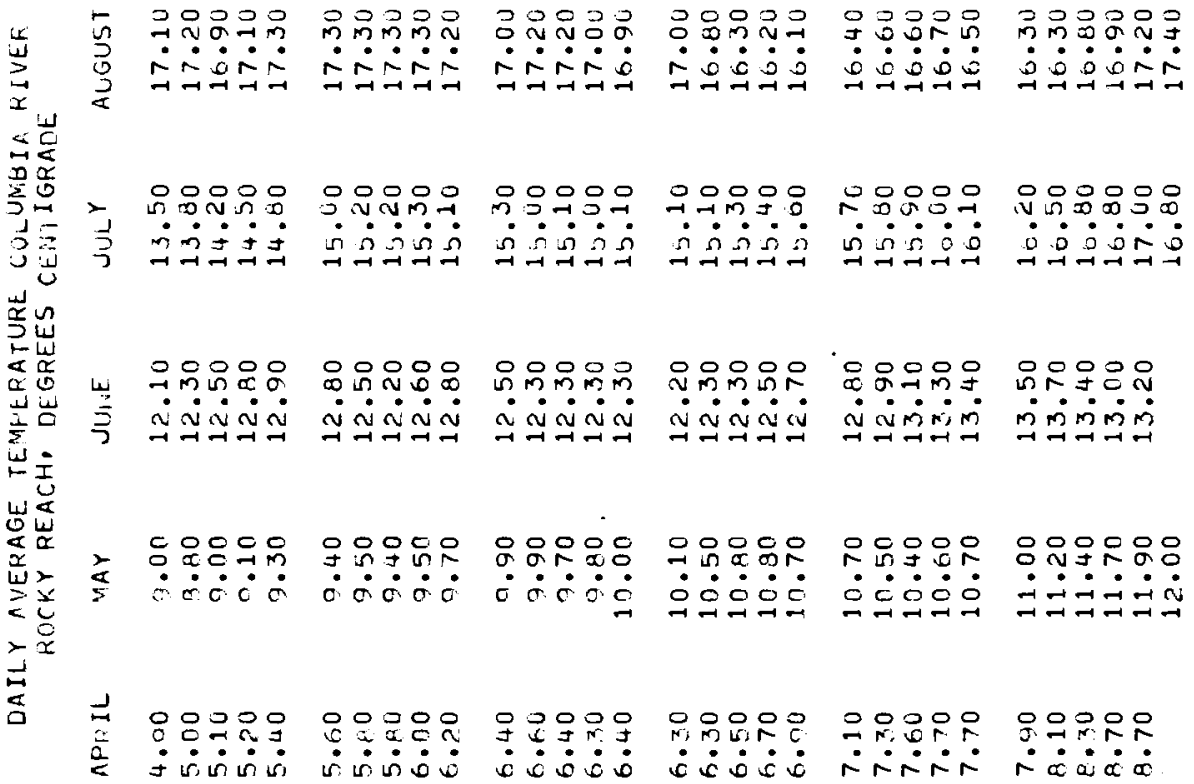

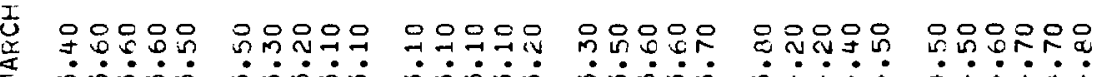

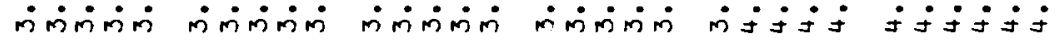

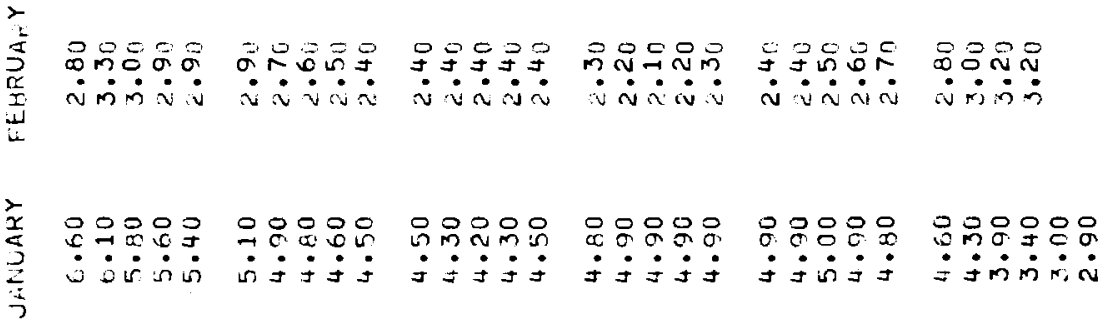

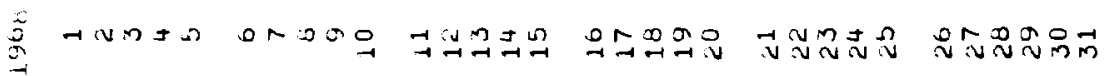




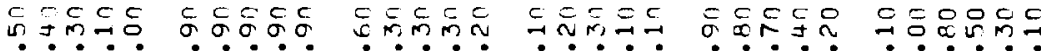

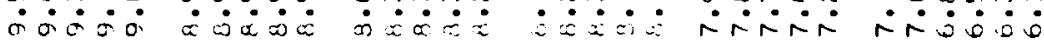

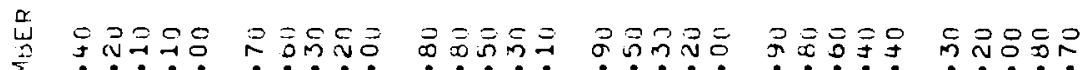

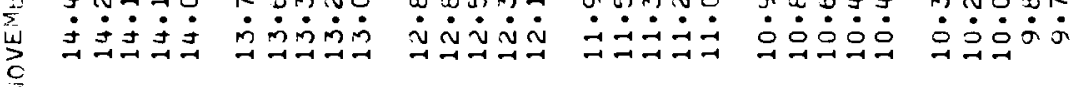

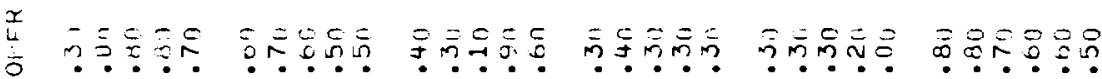

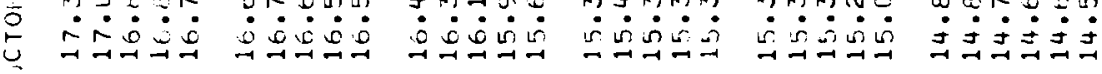

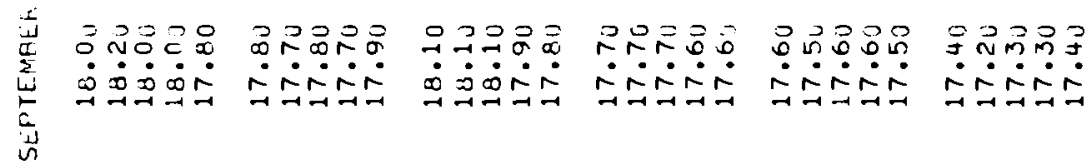

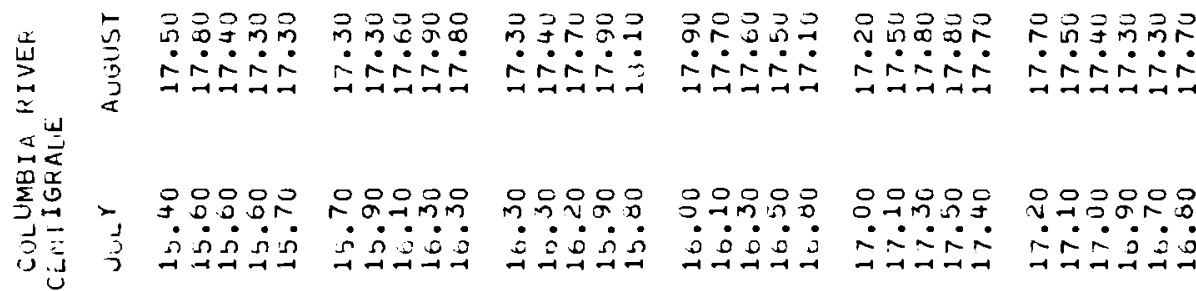

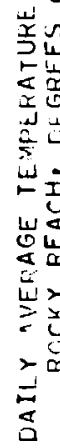

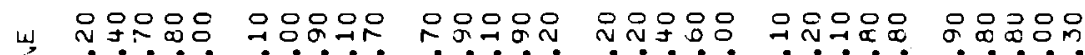

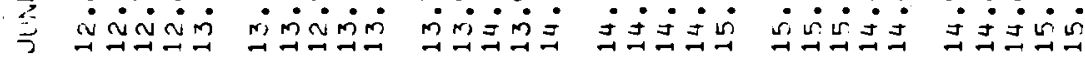

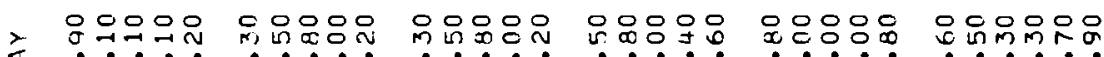

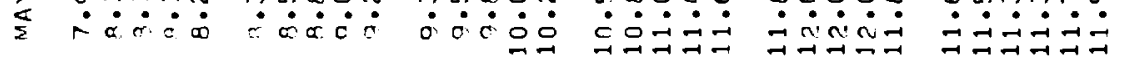

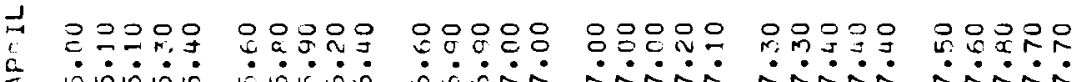
美

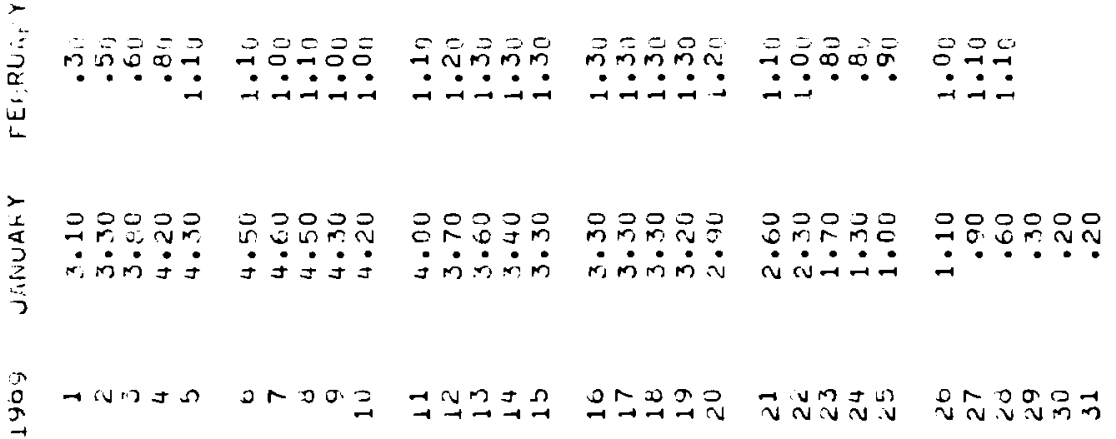




\begin{tabular}{|c|c|c|c|}
\hline $1.40 t_{i}$ & JARUSRRY & FEt I UAOY & MAFECH \\
\hline 1 & 7.20 & 4.60 & 4.70 \\
\hline 2 & 6.70 & 4.60 & 4.70 \\
\hline 3 & 6.70 & 4.60 & 4.80 \\
\hline 4 & 6.60 & 4.60 & 4.70 \\
\hline 5 & 6.60 & 4.40 & 4.60 \\
\hline 6 & 6.40 & 4.50 & 4.40 \\
\hline 7 & 6.30 & 4.40 & 4.50 \\
\hline 8 & 6.20 & 4.50 & 4.40 \\
\hline 9 & 6.10 & 4.60 & 4.20 \\
\hline 10 & 6.20 & 4.60 & 4.10 \\
\hline 11 & 6.20 & 4.70 & 4.50 \\
\hline 12 & 6.00 & 4.70 & 4.50 \\
\hline 13 & $5 \cdot 90$ & 4.50 & 4.50 \\
\hline 14 & 5.80 & 4.50 & 4.60 \\
\hline 15 & 5.60 & 4.60 & 4.70 \\
\hline 16 & 5.40 & 4.50 & 4.70 \\
\hline 17 & $5 \cdot 20$ & 4.40 & 4.70 \\
\hline 18 & 4.80 & 4.70 & 4.80 \\
\hline 19 & 4.70 & 4.80 & 4.70 \\
\hline 20 & 4.80 & $4.8 n$ & 4.80 \\
\hline 21 & $4 \cdot 90$ & 4.70 & 5.00 \\
\hline 22 & 4.80 & 4.80 & 5.00 \\
\hline 23 & $4 \cdot 80$ & 4.90 & 4.50 \\
\hline 24 & 4.50 & 4.80 & 4.60 \\
\hline 25 & $4 \cdot 50$ & 4.80 & 4.70 \\
\hline 26 & 4.40 & 4.90 & 4.90 \\
\hline 27 & 4.40 & 4.90 & 4.90 \\
\hline 26 & 4.70 & 4.80 & 5.10 \\
\hline 29 & 4.70 & 4.60 & 5.60 \\
\hline 30 & 4.70 & & 5.20 \\
\hline 31 & $4 \cdot 60$ & & 5.50 \\
\hline
\end{tabular}

DAILY IVERAGE TEMFERATURE COLUMBIA RIVER P?IEST RAPIOS.OEGREES CENTIGRAUE

\begin{tabular}{|c|c|c|c|c|c|c|c|c|}
\hline$A P: I L$ & MAY & $J_{1} / N E$ & JULY & AUGUST & SEPTENBEF & OCTOKER & NOVE WLE? & DECEMPF? \\
\hline $\begin{array}{l}6.00 \\
6.00 \\
6.00 \\
6.40 \\
6.30\end{array}$ & $\begin{array}{l}8.20 \\
8.00 \\
3.00 \\
3.40 \\
8.60\end{array}$ & $\begin{array}{l}12.40 \\
12.40 \\
12.20 \\
12.10 \\
12.20\end{array}$ & $\begin{array}{l}13.70 \\
13.70 \\
13.80 \\
13.90 \\
13.90\end{array}$ & $\begin{array}{l}16.70 \\
16.70 \\
16.60 \\
16.40 \\
16.20\end{array}$ & $\begin{array}{l}16.40 \\
16.20 \\
16.30 \\
16.30 \\
16.50\end{array}$ & $\begin{array}{l}15.60 \\
15.60 \\
15.30 \\
15.20 \\
15.30\end{array}$ & $\begin{array}{l}13.21) \\
12.90 \\
12.70 \\
12.50 \\
12.40\end{array}$ & $\begin{array}{l}9.10 \\
9.20 \\
9.10 \\
8.90 \\
9.80\end{array}$ \\
\hline $\begin{array}{l}6.10 \\
6.20 \\
6.60 \\
6.60 \\
7.00\end{array}$ & $\begin{array}{l}8.60 \\
8.70 \\
9.10 \\
9.30 \\
9.30\end{array}$ & $\begin{array}{l}12.10 \\
12.30 \\
12.10 \\
12.10 \\
12.10\end{array}$ & $\begin{array}{l}14.10 \\
14.40 \\
14.70 \\
14.70 \\
14.90\end{array}$ & $\begin{array}{l}16.50 \\
16.90 \\
17.00 \\
17.20 \\
17.10\end{array}$ & $\begin{array}{l}16.70 \\
16.70 \\
16.50 \\
16.40 \\
16.30\end{array}$ & $\begin{array}{l}15.30 \\
15.20 \\
15.20 \\
15.20 \\
15.10\end{array}$ & $\begin{array}{l}12.40 \\
12.10 \\
11.90 \\
11.60 \\
11.50\end{array}$ & $\begin{array}{l}8.70 \\
8.50 \\
8.50 \\
8.4 n \\
8.30\end{array}$ \\
\hline $\begin{array}{l}7.20 \\
7.50 \\
7.20 \\
7.30 \\
7.40\end{array}$ & $\begin{array}{l}9.10 \\
9.30 \\
9.30 \\
9.10 \\
8.95\end{array}$ & $\begin{array}{l}12.20 \\
12.40 \\
12.60 \\
12.90 \\
13.10\end{array}$ & $\begin{array}{l}15.10 \\
15.10 \\
15.50 \\
15.60 \\
15.60\end{array}$ & $\begin{array}{l}17.30 \\
17.40 \\
17.10 \\
17.00 \\
17.30\end{array}$ & $\begin{array}{l}16.40 \\
16.60 \\
16.80 \\
16.60 \\
16.50\end{array}$ & $\begin{array}{l}15.20 \\
15.20 \\
15.30 \\
15.20 \\
15.10\end{array}$ & $\begin{array}{l}11.4 n \\
11.30 \\
11.10 \\
10.90 \\
10.80\end{array}$ & $\begin{array}{l}7.90 \\
7.80 \\
7.90 \\
7.50 \\
7.10\end{array}$ \\
\hline $\begin{array}{l}7.40 \\
7.30 \\
7.40 \\
7.80 \\
7.65\end{array}$ & $\begin{array}{r}9.10 \\
9.35 \\
9.55 \\
9.80 \\
10.30\end{array}$ & $\begin{array}{l}13.20 \\
13.10 \\
12.90 \\
12.90 \\
12.90\end{array}$ & $\begin{array}{l}15.40 \\
15.40 \\
15.50 \\
15.30 \\
15.45\end{array}$ & $\begin{array}{l}17.20 \\
17.30 \\
17.40 \\
16.90 \\
17.00\end{array}$ & $\begin{array}{l}16.50 \\
16.60 \\
16.30 \\
16.30 \\
15.90\end{array}$ & $\begin{array}{l}14.90 \\
14.70 \\
14.60 \\
14.40 \\
14.30\end{array}$ & $\begin{array}{l}10 \cdot 40 \\
10.20 \\
10.20 \\
10 \cdot 10 \\
10.10\end{array}$ & $\begin{array}{l}6.20 \\
5.40 \\
5.30 \\
5.00 \\
4.70\end{array}$ \\
\hline $\begin{array}{l}7.60 \\
7.60 \\
7.40 \\
7.75 \\
7.50\end{array}$ & $\begin{array}{l}10.60 \\
10.50 \\
10.30 \\
10.50 \\
10.40\end{array}$ & $\begin{array}{l}12.90 \\
13.10 \\
13.50 \\
13.70 \\
13.75\end{array}$ & $\begin{array}{l}15.80 \\
15.70 \\
15.50 \\
15.80 \\
15.90\end{array}$ & $\begin{array}{l}17.40 \\
17.70 \\
17.90 \\
18.10 \\
18.00\end{array}$ & $\begin{array}{l}15.80 \\
15.90 \\
16.10 \\
16.30 \\
16.40\end{array}$ & $\begin{array}{l}14.10 \\
14.00 \\
13.90 \\
13.80 \\
13.60\end{array}$ & $\begin{array}{r}10.00 \\
9.90 \\
9.70 \\
9.80 \\
9.60\end{array}$ & $\begin{array}{l}4.10 \\
4.20 \\
4.50 \\
4.40 \\
3.60\end{array}$ \\
\hline $\begin{array}{l}8.40 \\
7.60 \\
8.00 \\
8.20 \\
8.20\end{array}$ & $\begin{array}{l}10.60 \\
11.00 \\
11.45 \\
11.50 \\
11.90 \\
12.25\end{array}$ & $\begin{array}{l}13.80 \\
13.60 \\
13.50 \\
13.55 \\
13.60\end{array}$ & $\begin{array}{l}16.10 \\
16.30 \\
16.60 \\
16.80 \\
17.00 \\
17.00\end{array}$ & $\begin{array}{l}17.60 \\
17.30 \\
16.80 \\
16.40 \\
16.30 \\
16.30\end{array}$ & $\begin{array}{l}16.20 \\
16.30 \\
16.10 \\
16.00 \\
15.70\end{array}$ & $\begin{array}{l}13.40 \\
13.20 \\
13.30 \\
13.30 \\
13.30 \\
13.30\end{array}$ & $\begin{array}{l}9.20 \\
8.90 \\
9.20 \\
9.00 \\
9.00\end{array}$ & $\begin{array}{l}3.40 \\
3.30 \\
3.30 \\
3.60 \\
3.60 \\
3.60\end{array}$ \\
\hline
\end{tabular}




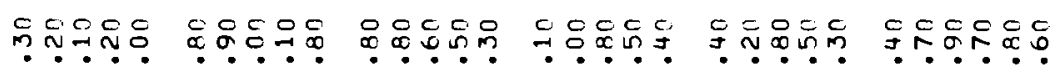

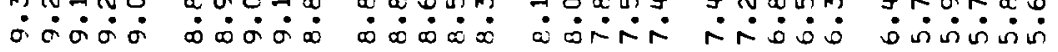

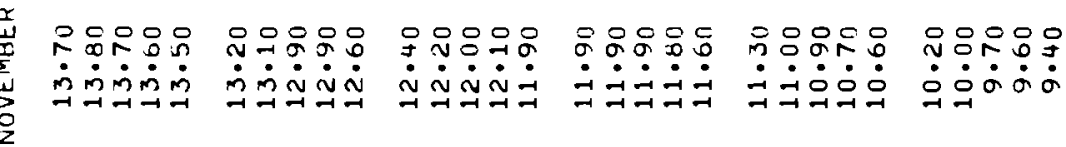

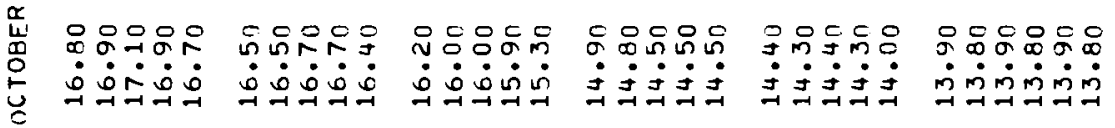

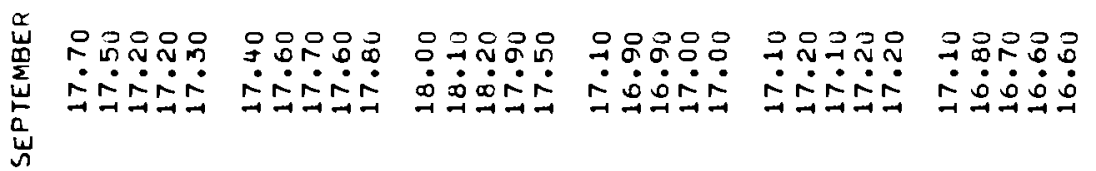

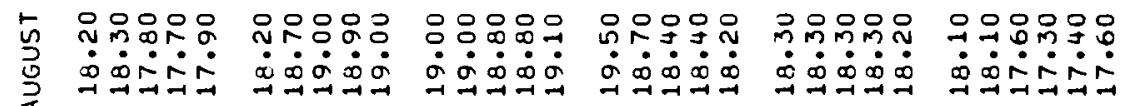

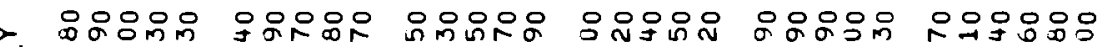

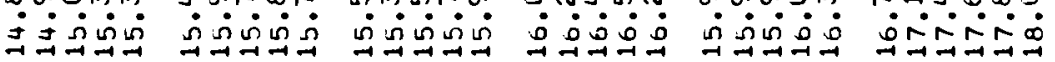

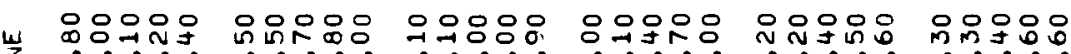

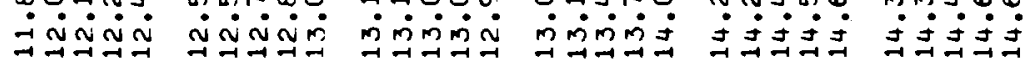

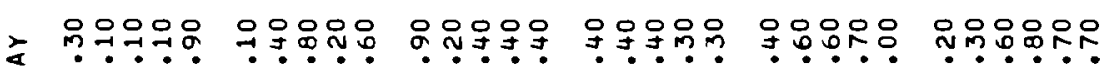

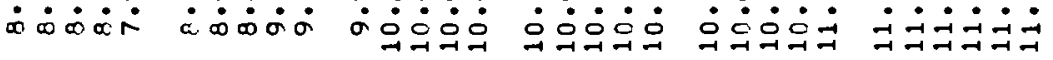

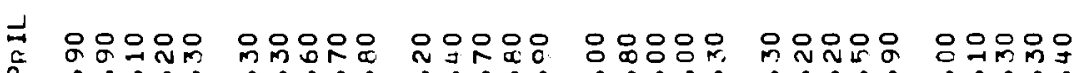

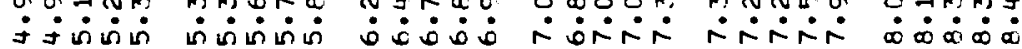

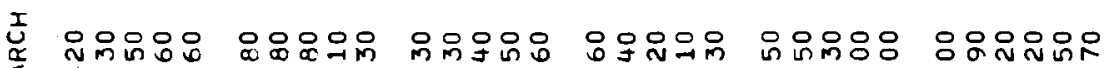

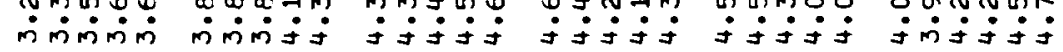

$\frac{2}{5}$

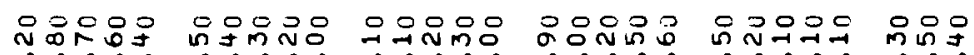

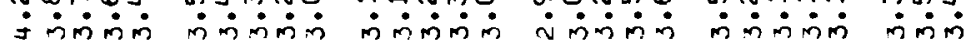

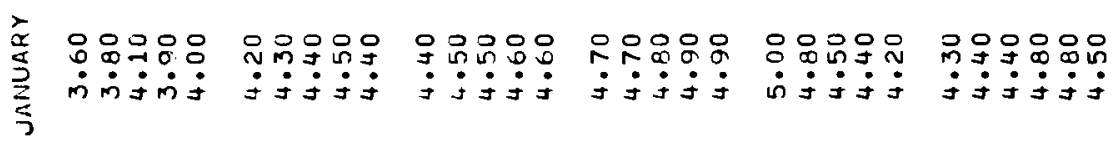

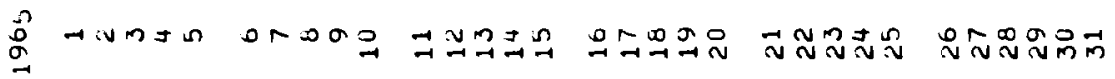




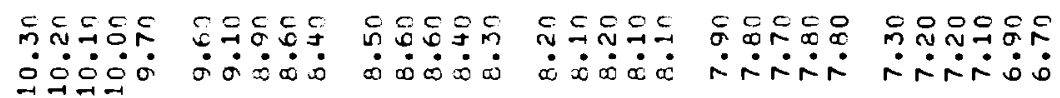

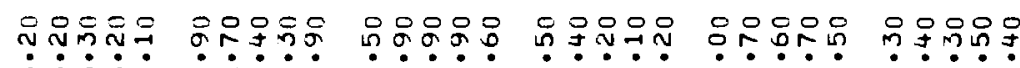

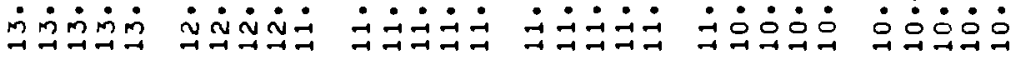

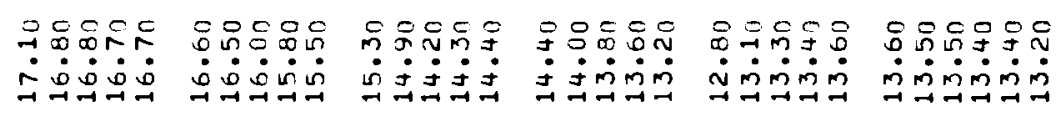

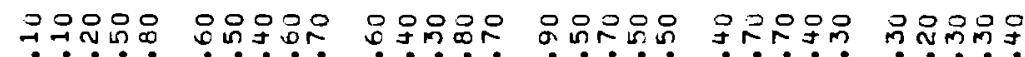

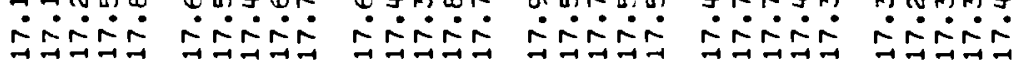

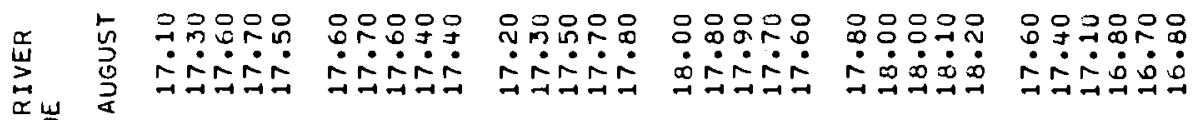

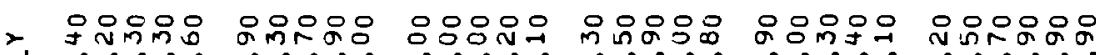

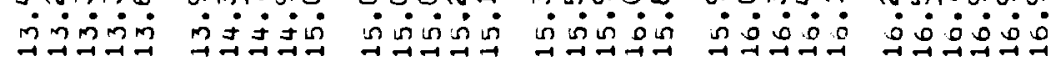

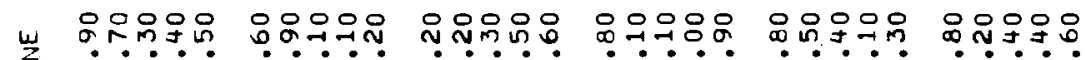

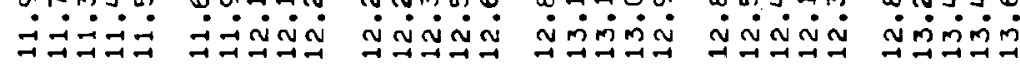

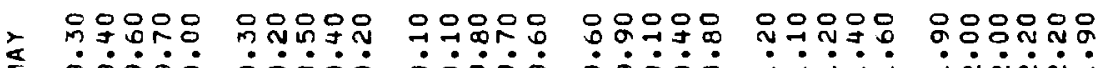

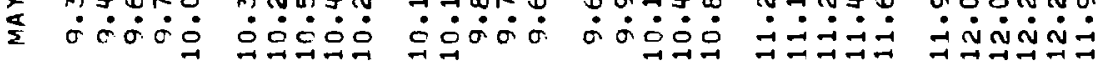

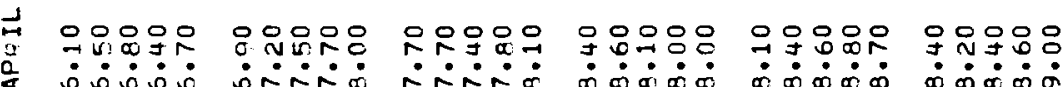

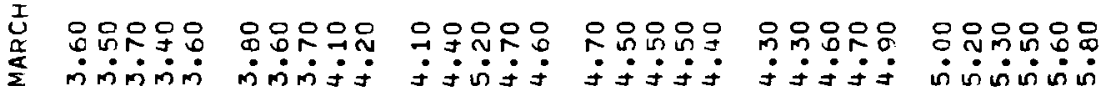

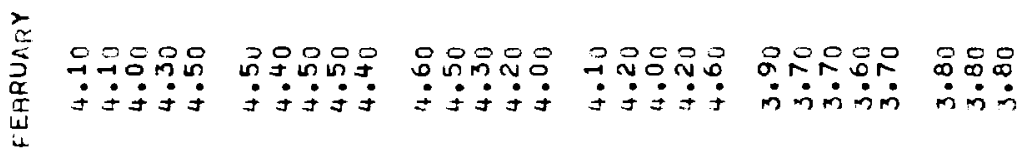

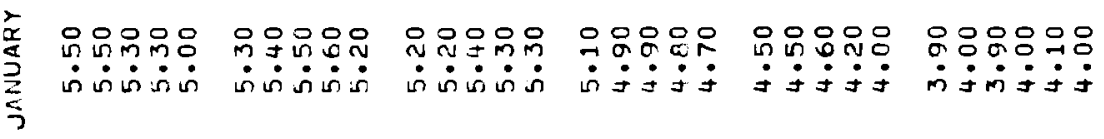

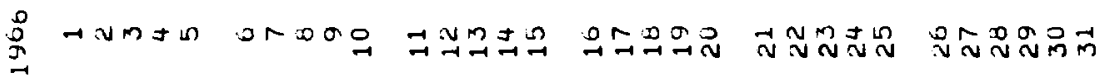




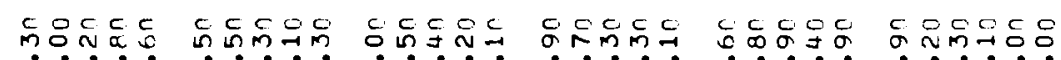

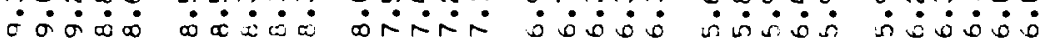

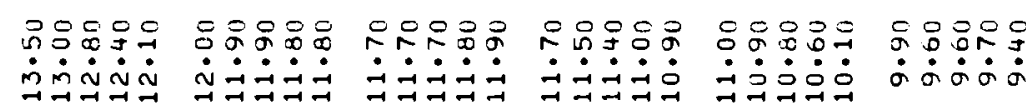

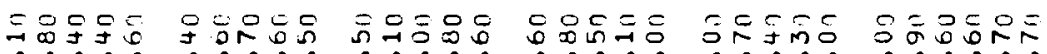

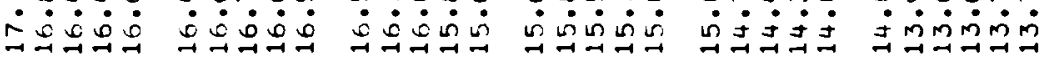

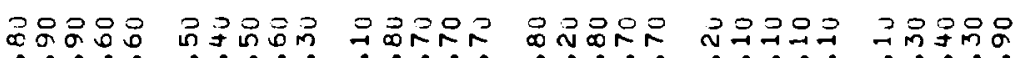

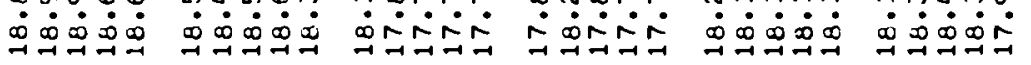

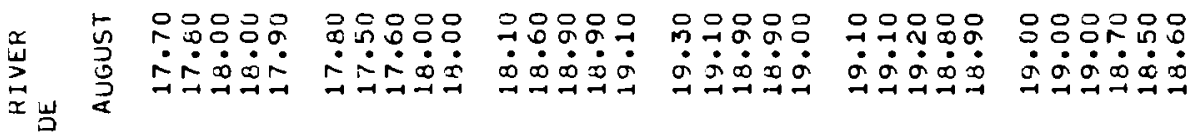

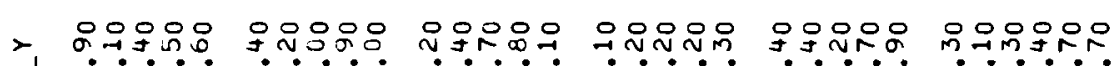

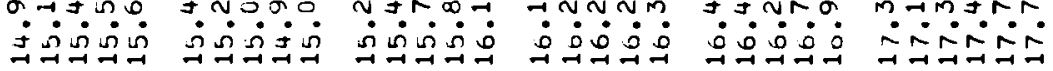

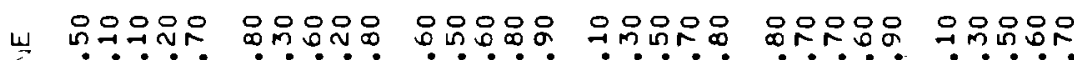

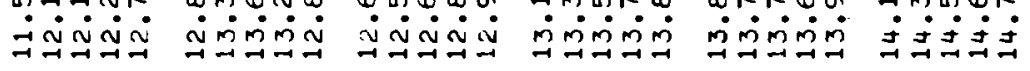

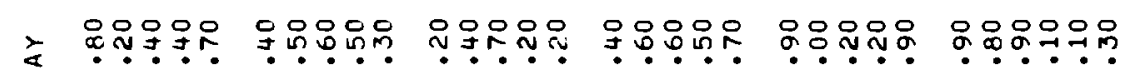

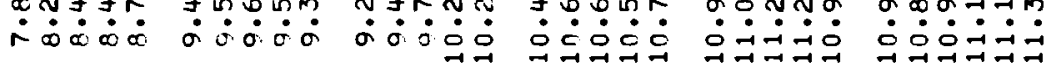

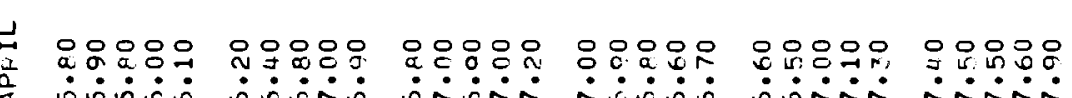

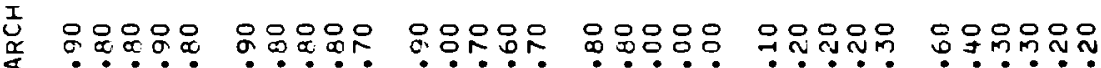

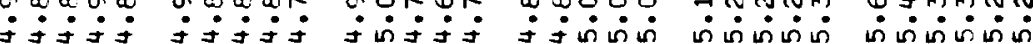

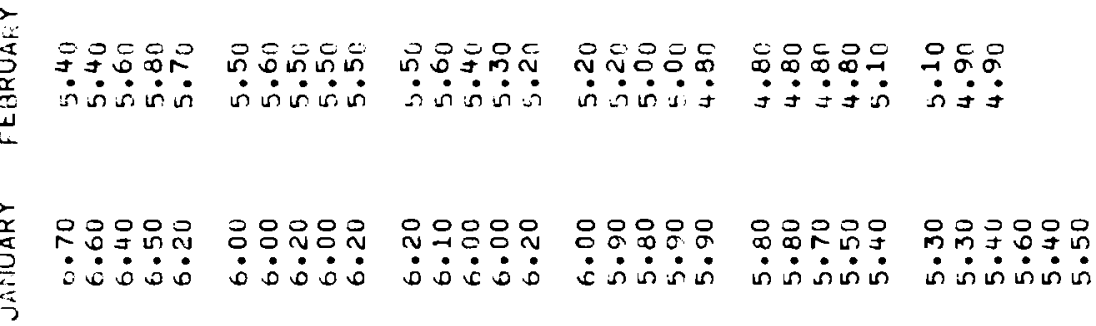

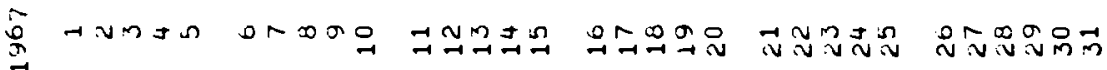




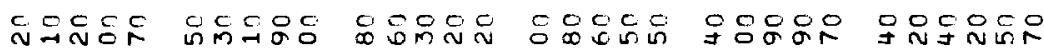

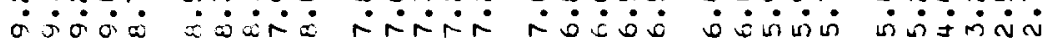

舀 采

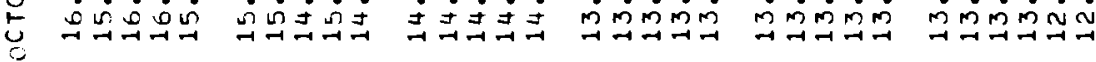
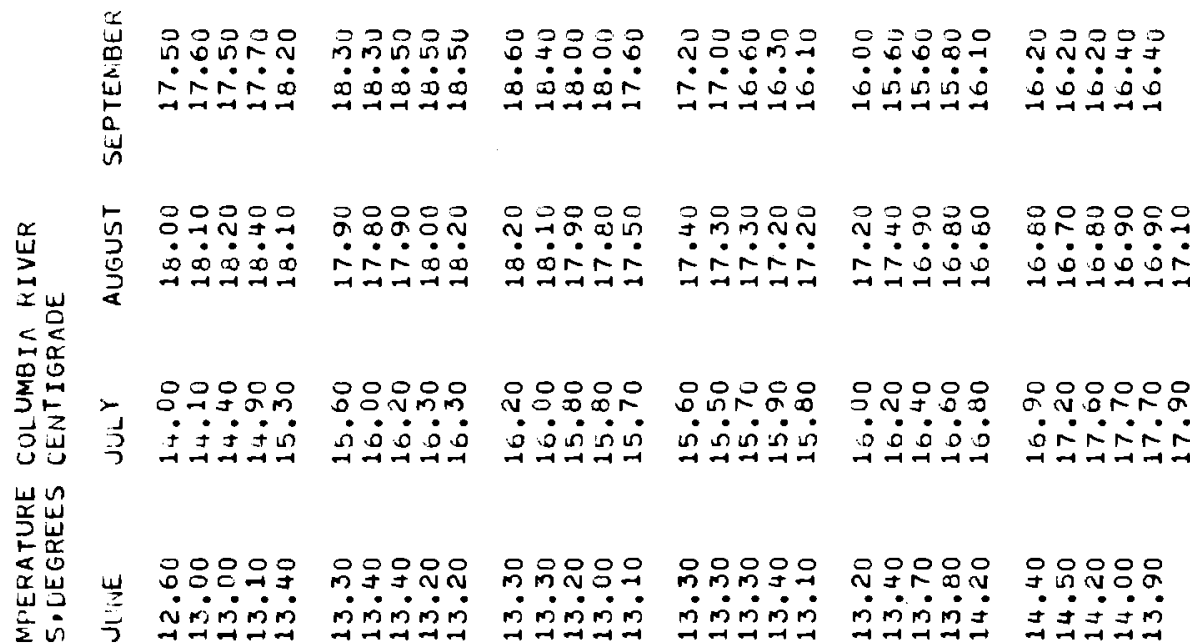

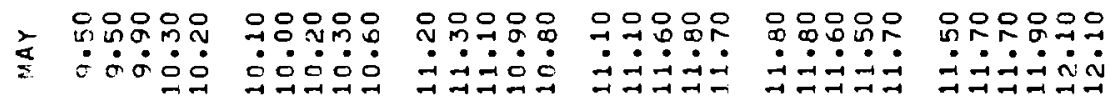

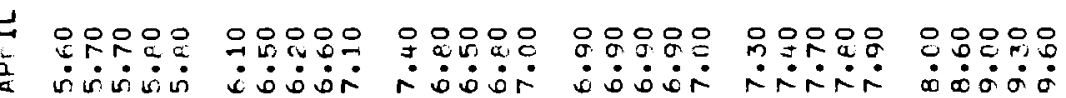

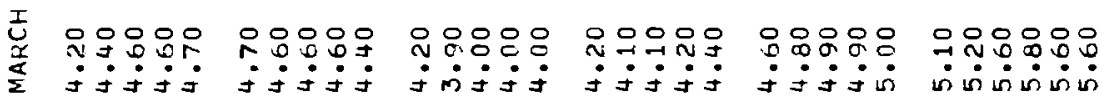

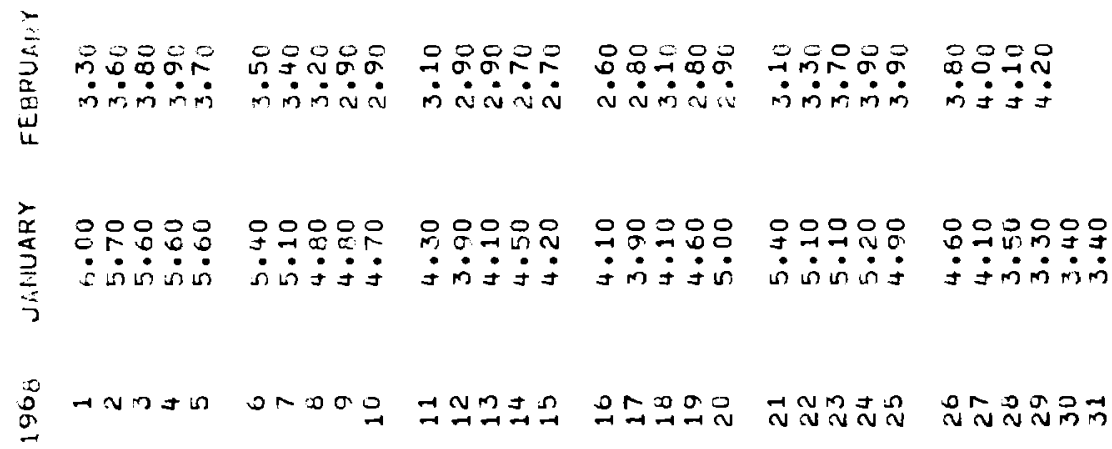




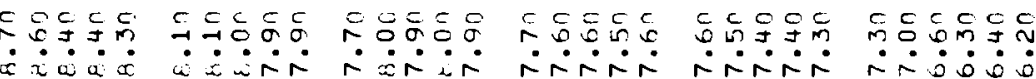

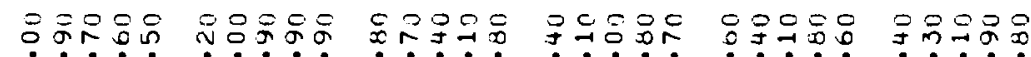

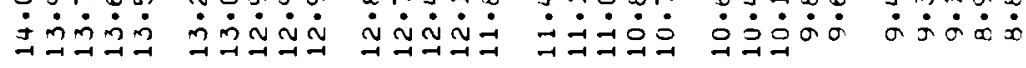

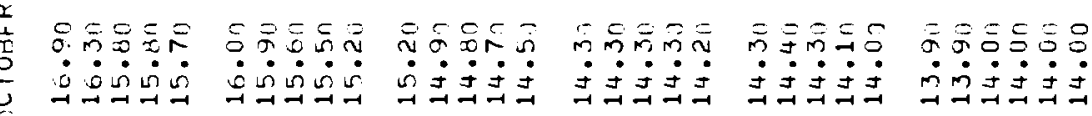

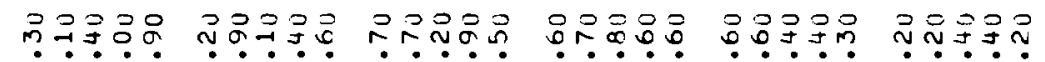

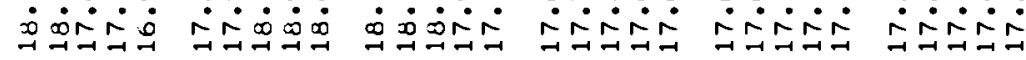

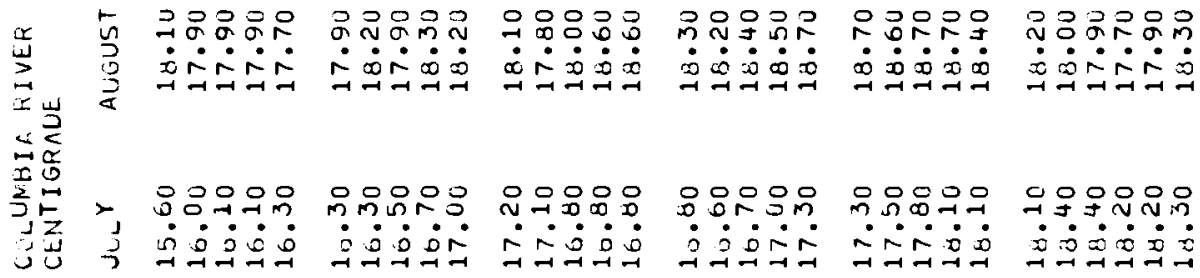

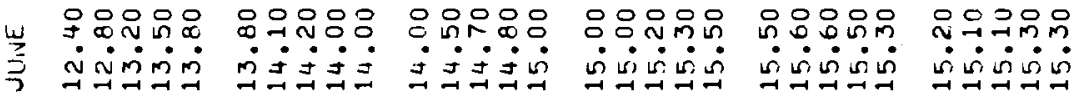

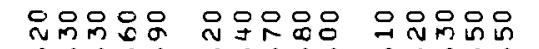

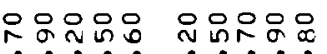
ํำํํํํำ

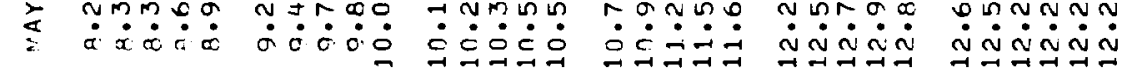

म

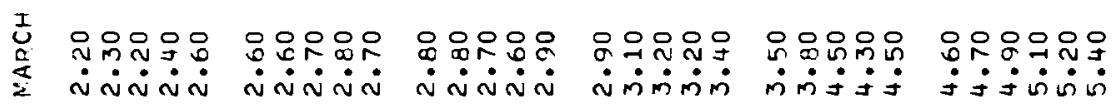

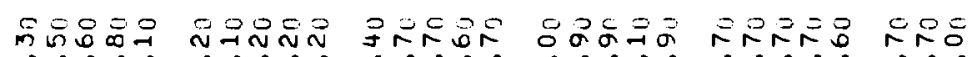

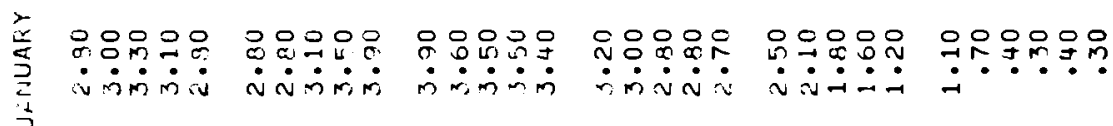

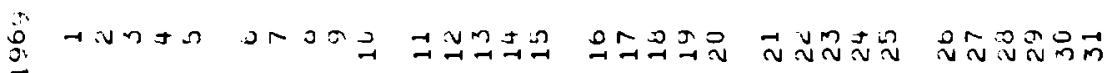




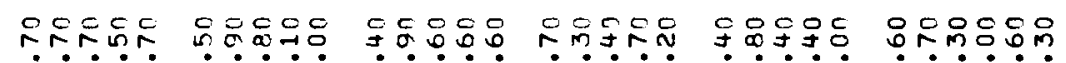

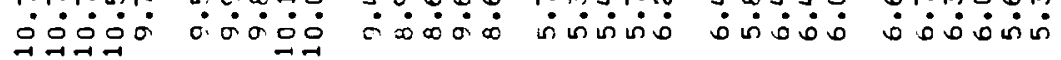

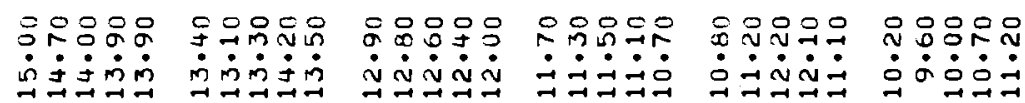

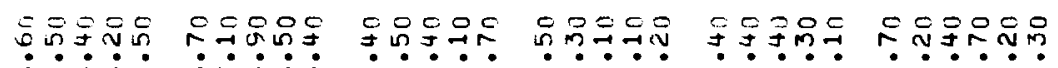

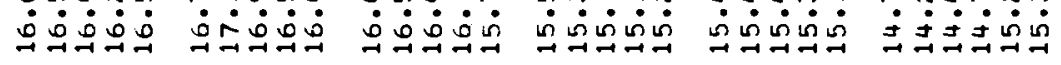

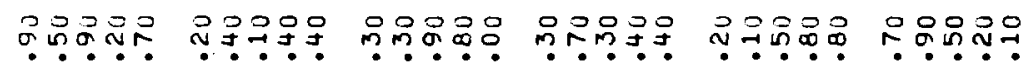

न

웅ㅇㅇㅇㅇㅇㅇㅛ

03000

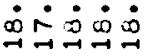

ำ

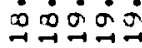

응워웅응

옥우웅요

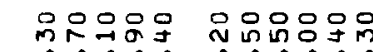

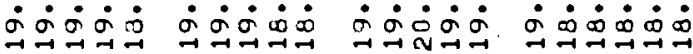

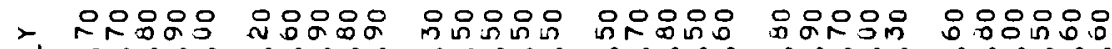

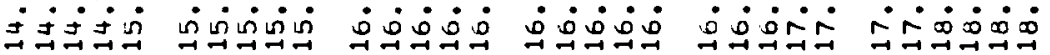

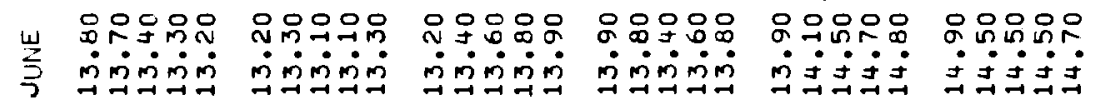

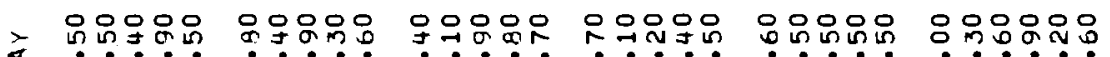

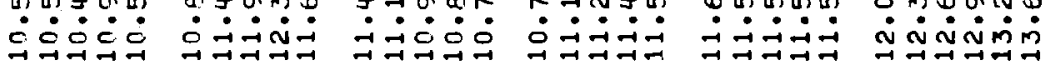

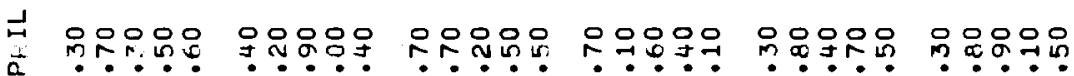

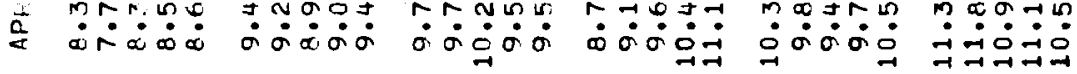

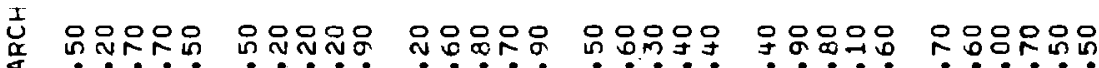

宏

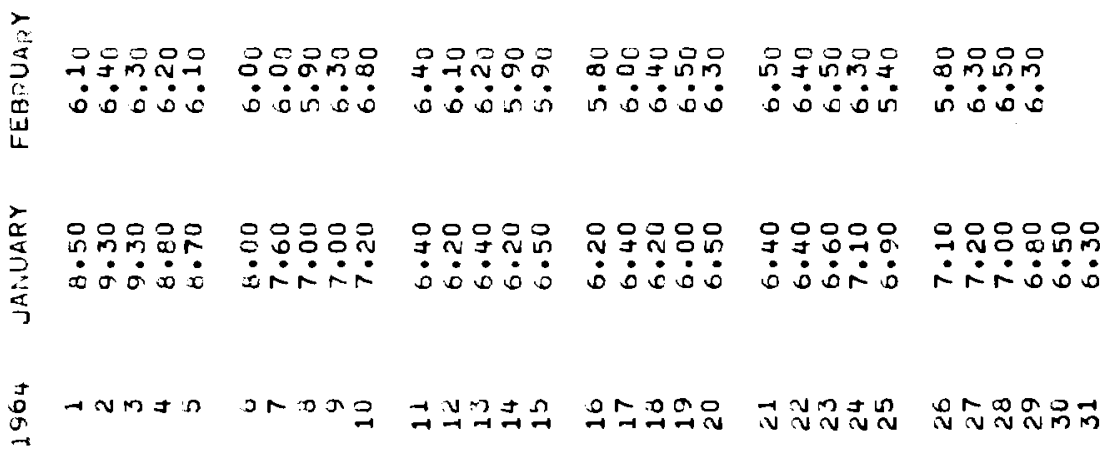




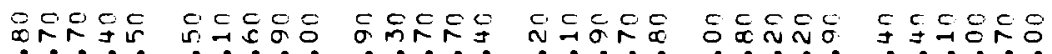

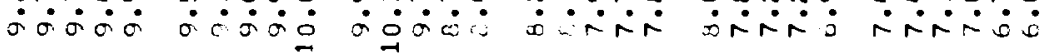

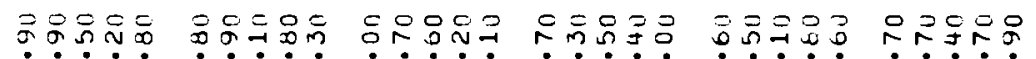

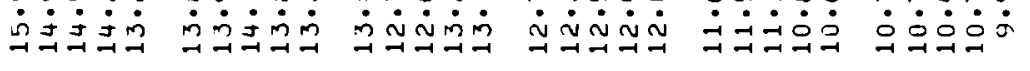

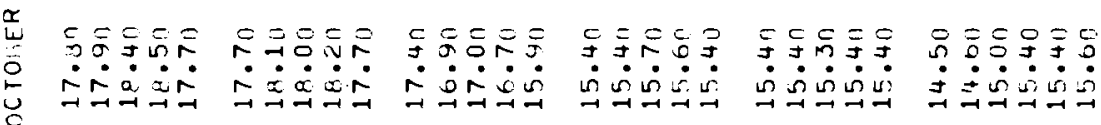

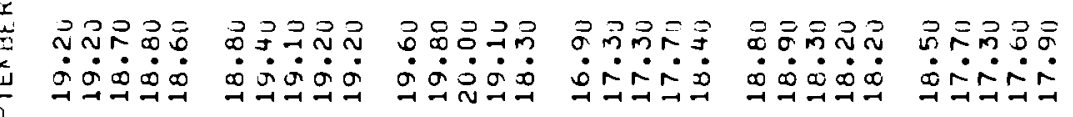

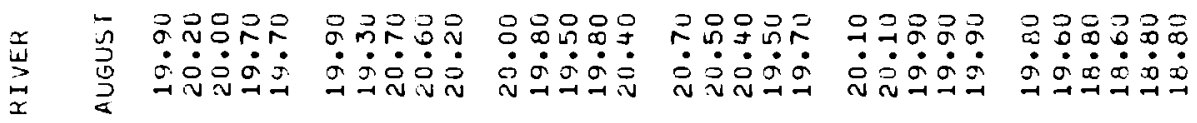

致崖

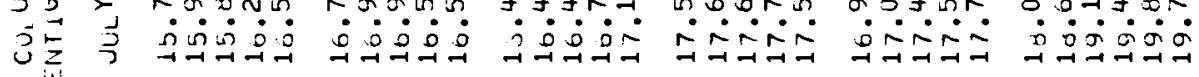

荤岕

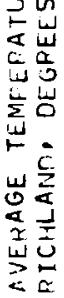

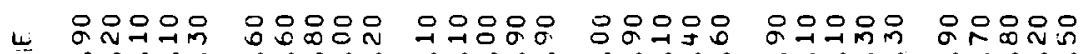

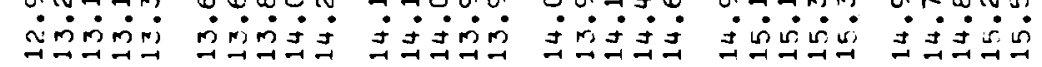

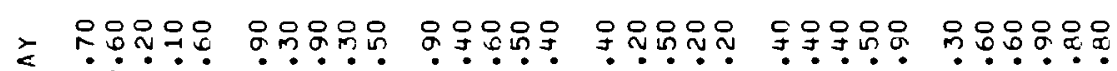

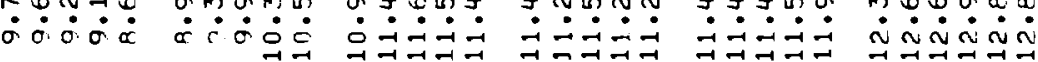

$\check{2}$

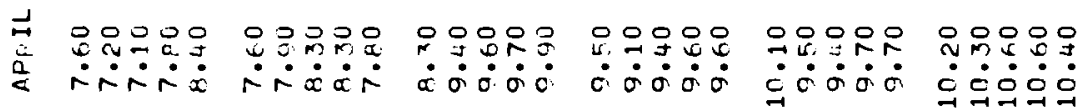

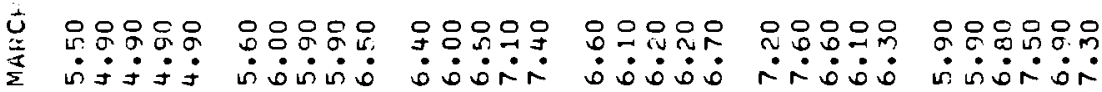

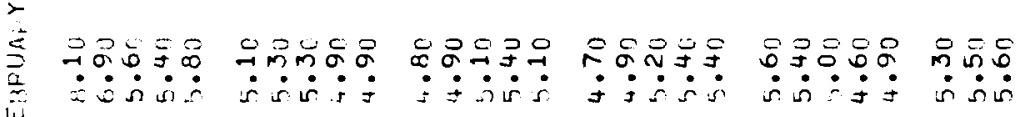

㟧

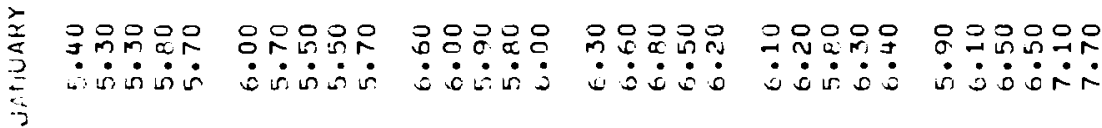

总 


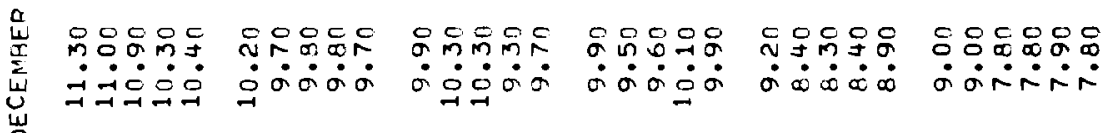

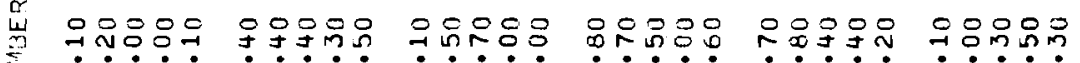

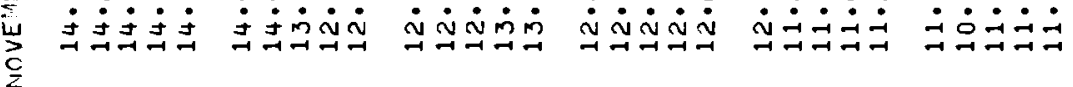

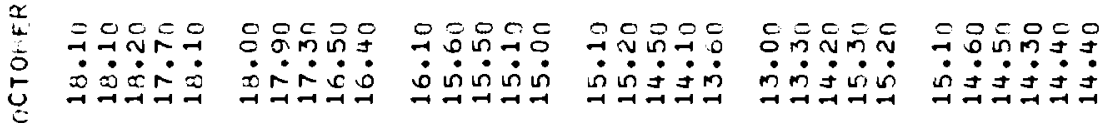

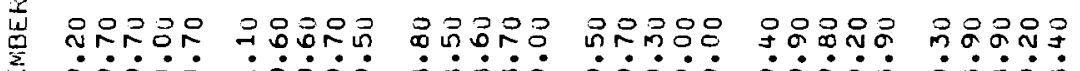

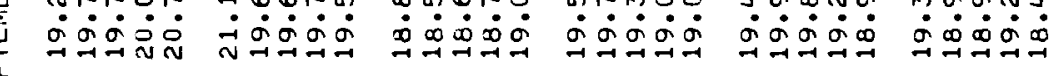

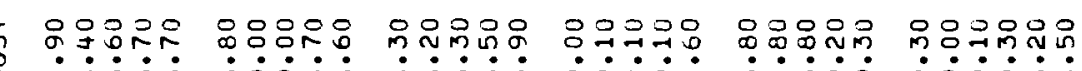

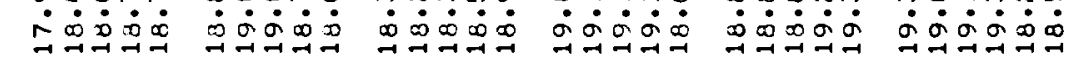

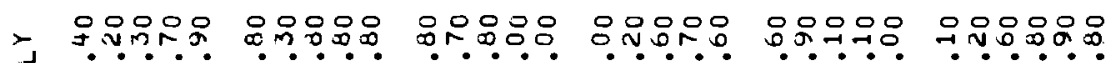

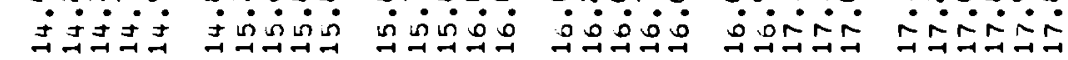

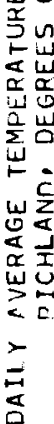

w

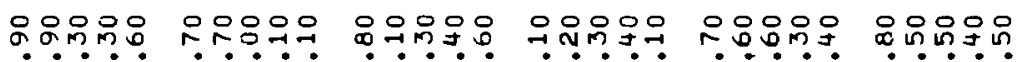

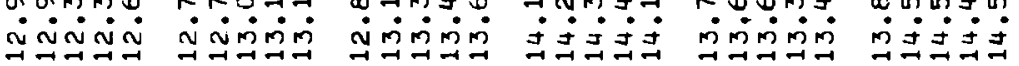

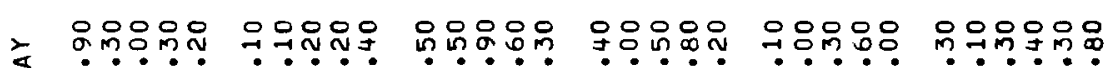

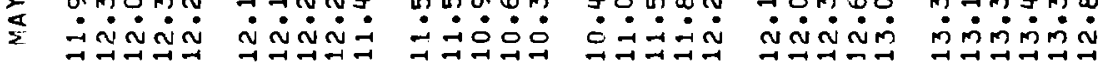

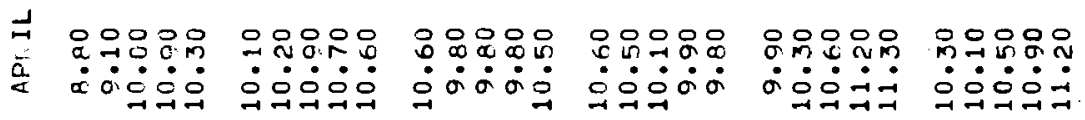

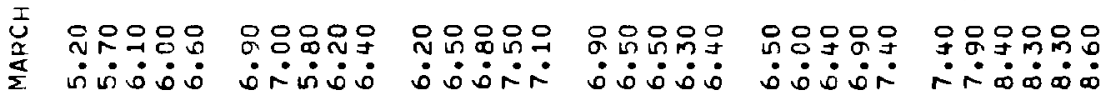

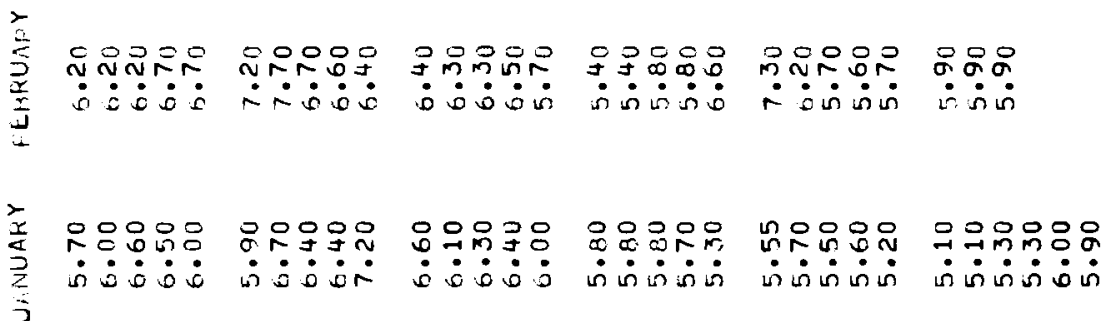

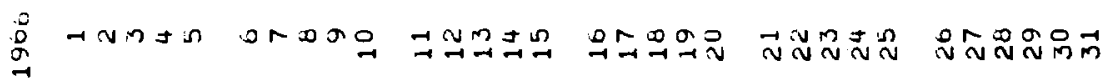




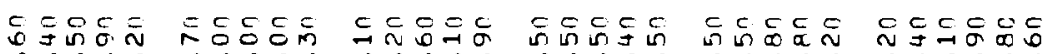

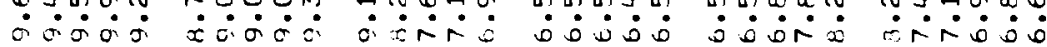

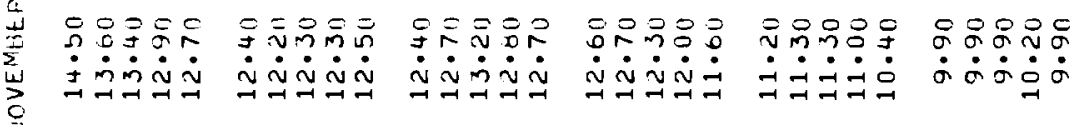

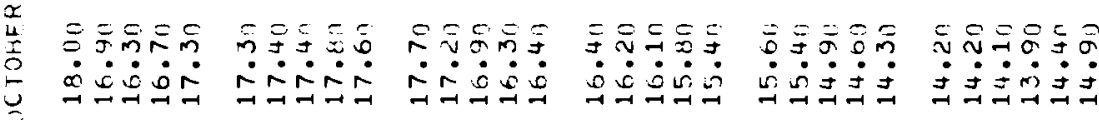

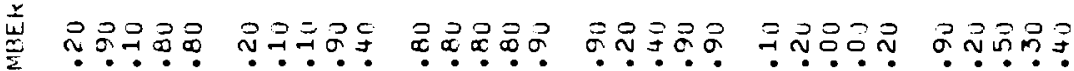

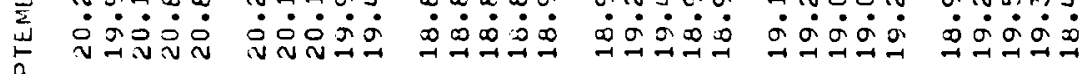

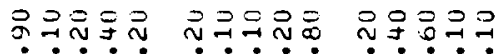

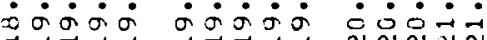

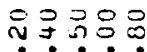

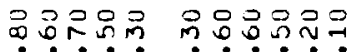

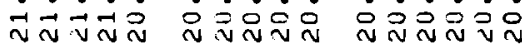

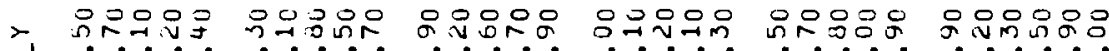

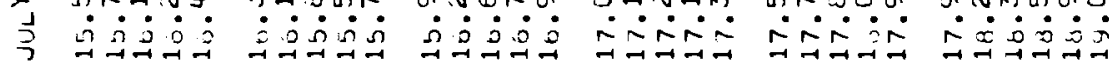

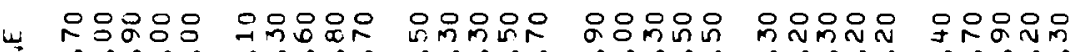

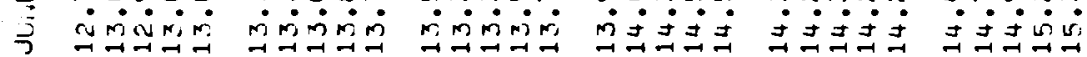

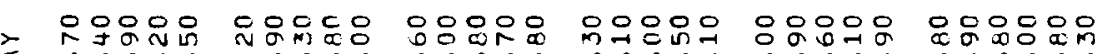

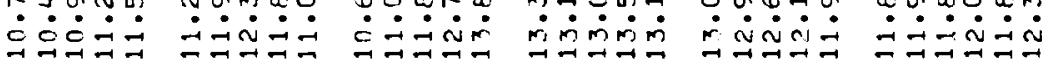

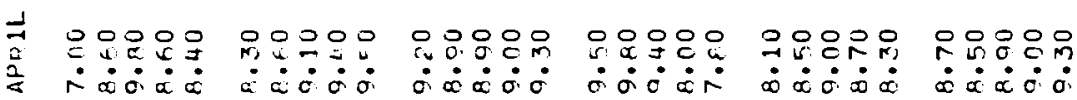

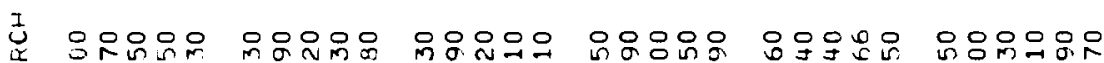

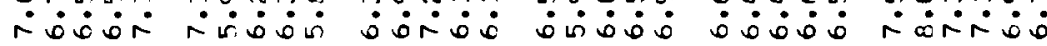

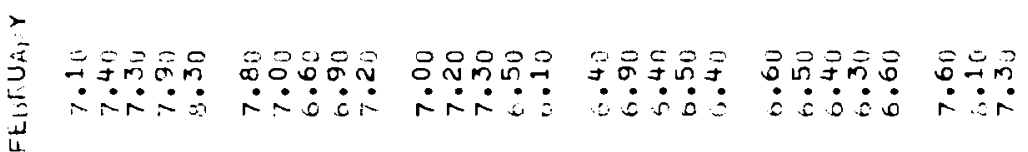

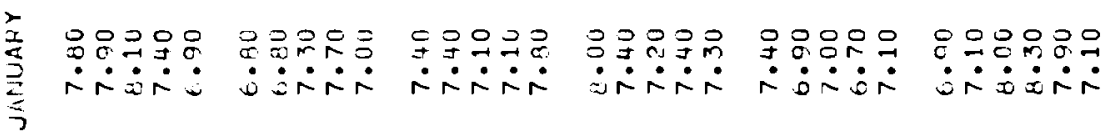

要 


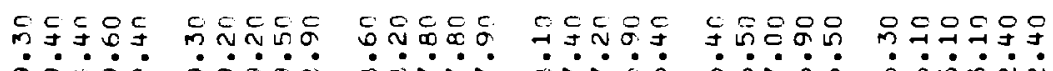

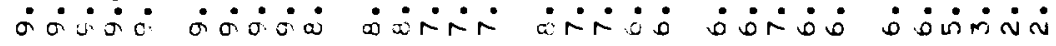

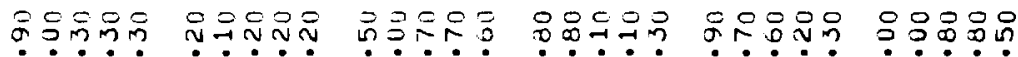

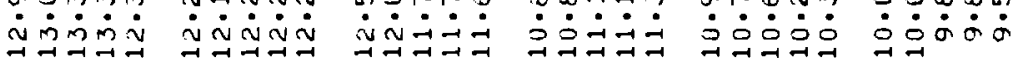

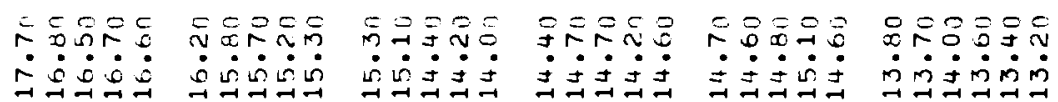

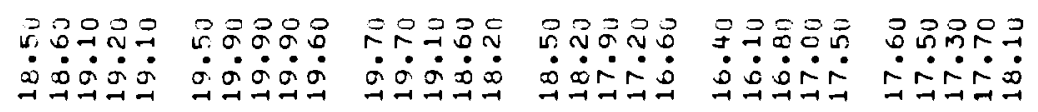

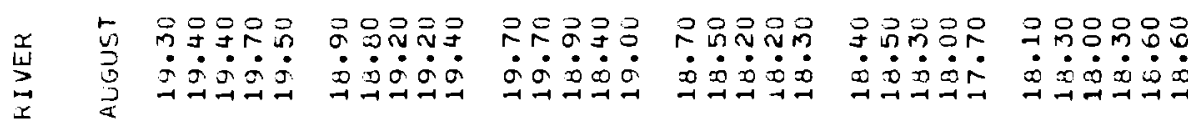

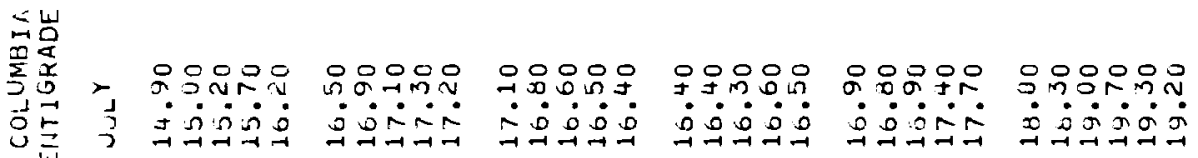

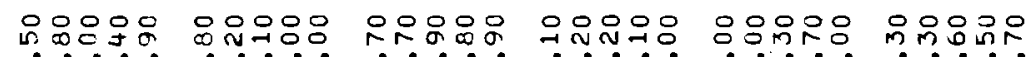

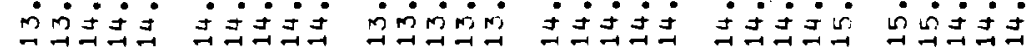

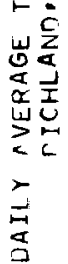

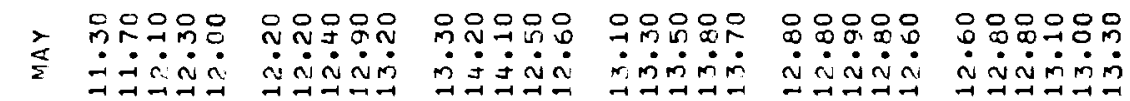

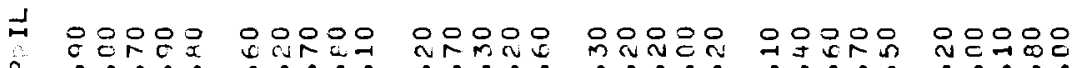

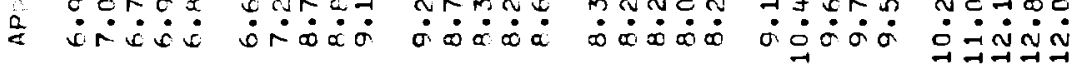

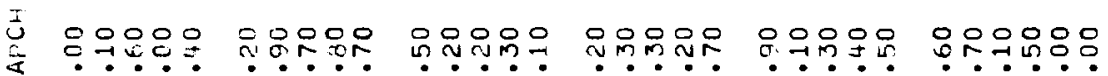

造

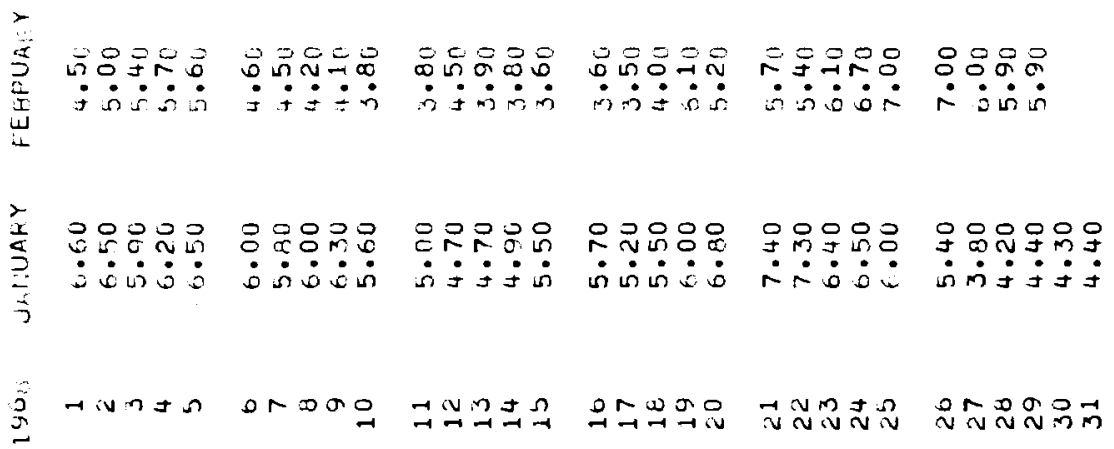




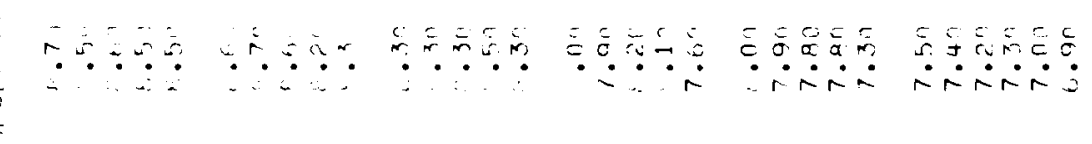

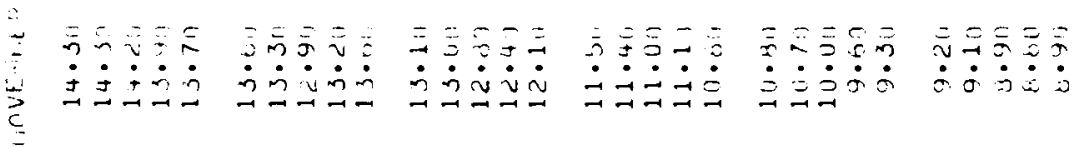

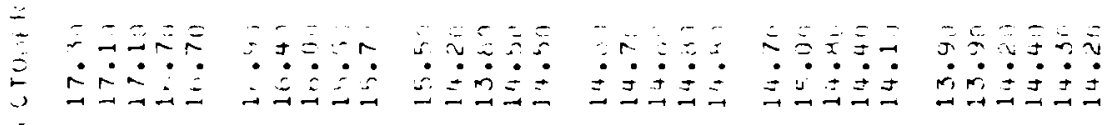

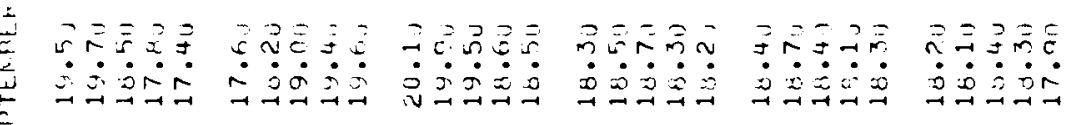
5

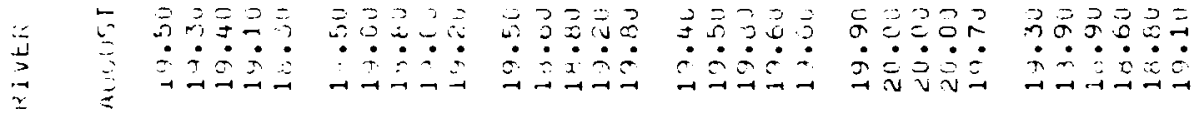

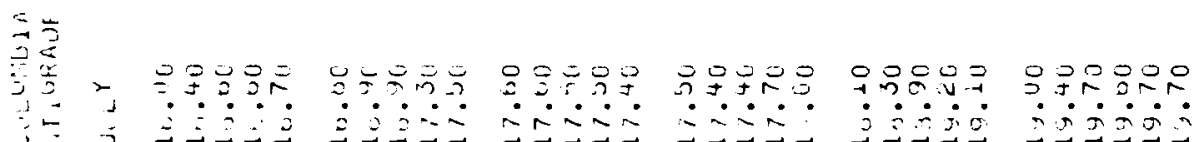

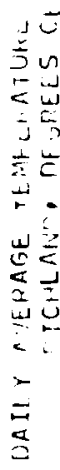

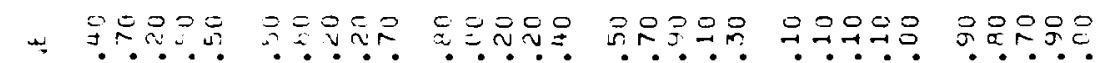
ᄀ

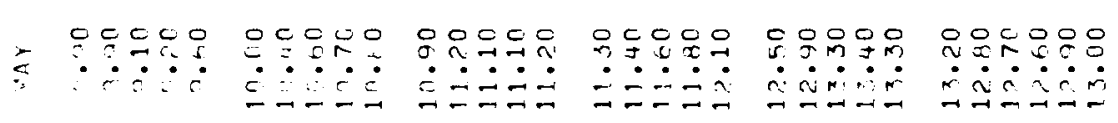

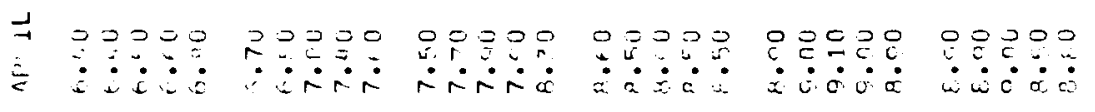

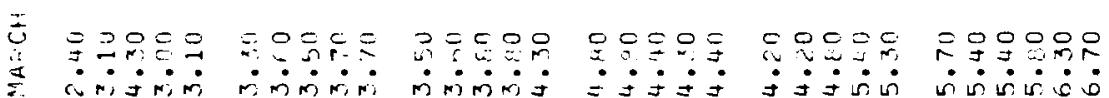

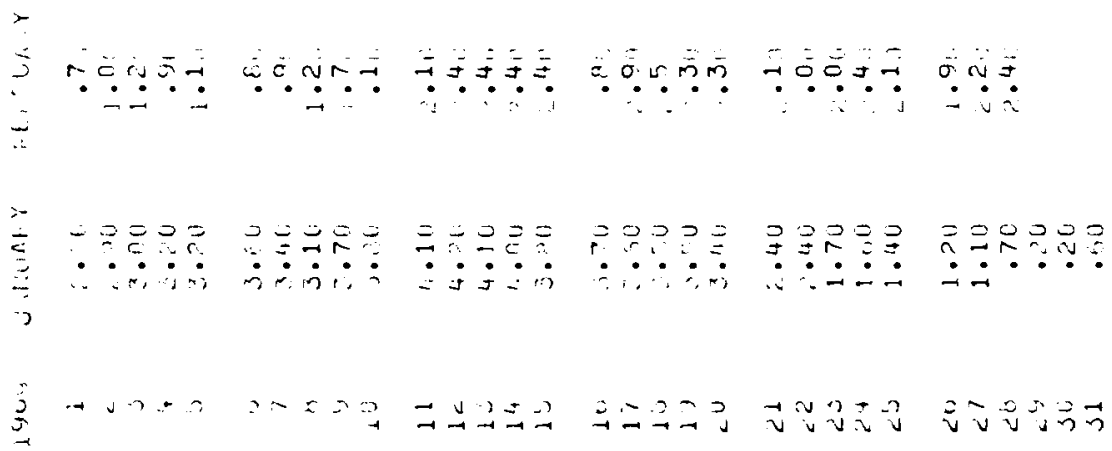




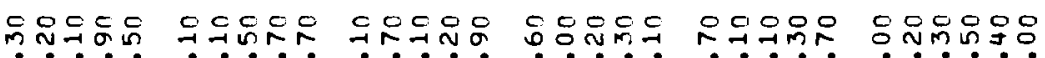

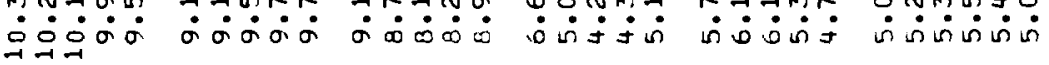

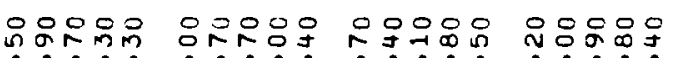

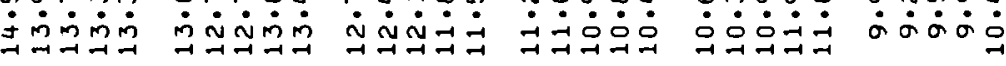

$\stackrel{x}{\underline{x}}$

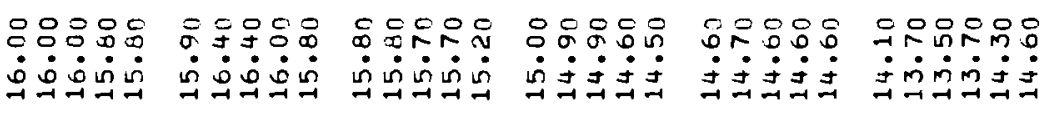

勇

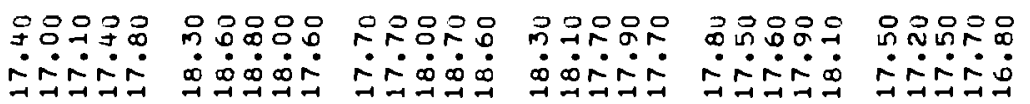

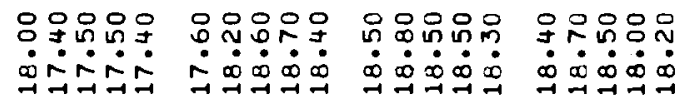

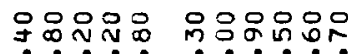

$\dot{\infty} \dot{\infty} \dot{\sigma} \dot{\sigma} \dot{0}$

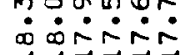

4

00000

过守守守

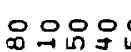

जिं

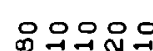

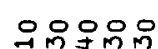

$\because$ mim

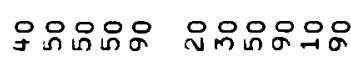

崖出

点的

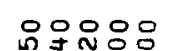

용용요

음유요용

웅워웅

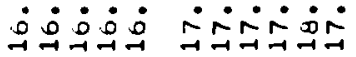

$\operatorname{mim}_{\rightarrow \rightarrow \infty} \min _{0}$

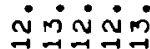

$\dot{m} \dot{\rightarrow} \rightarrow \dot{m} \rightarrow$

inimim

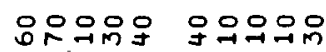

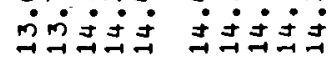

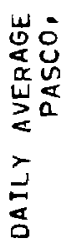

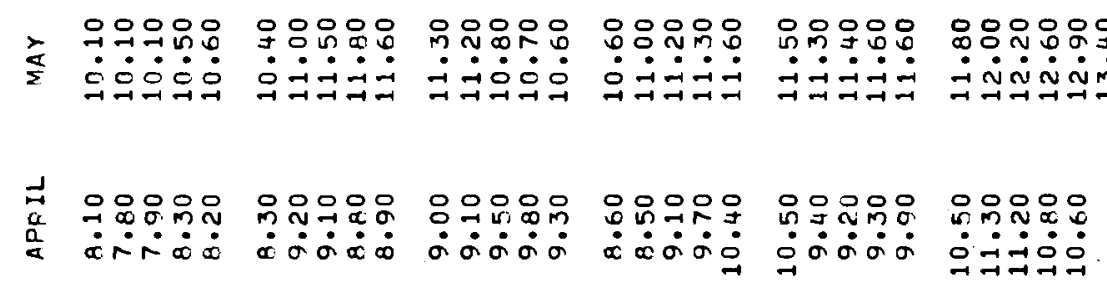

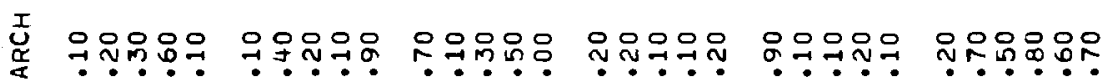

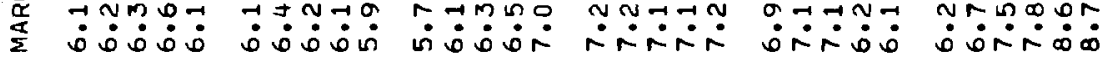

离

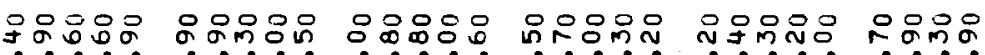

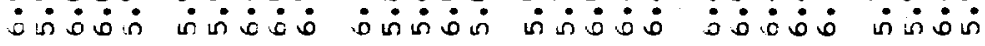

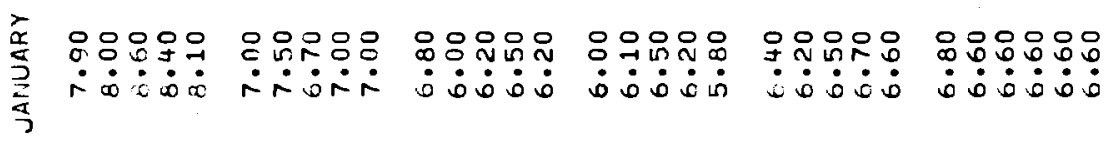

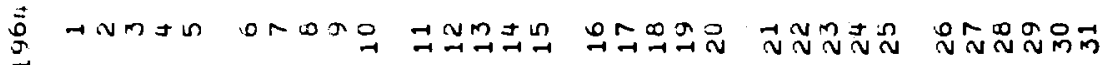




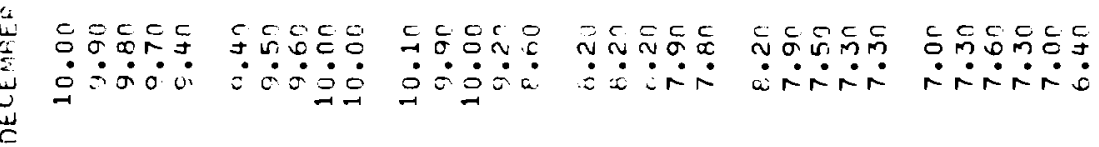

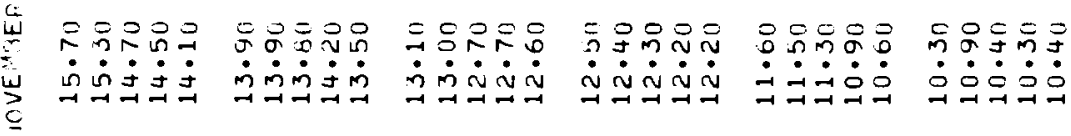

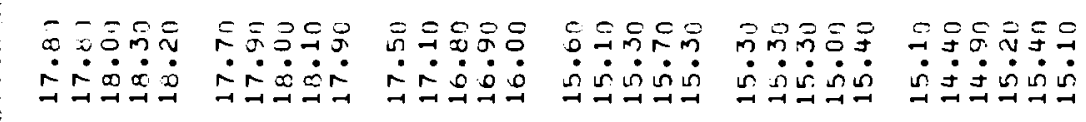

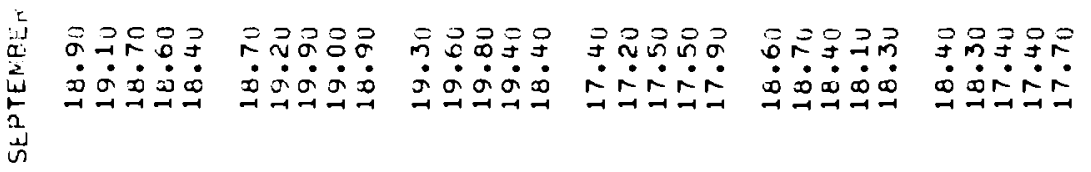

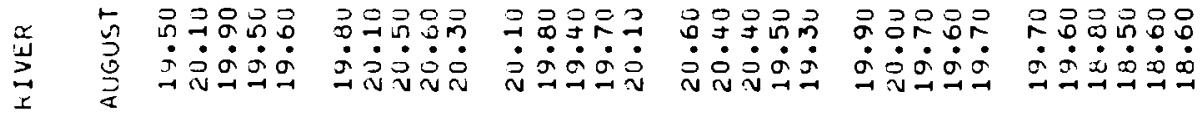

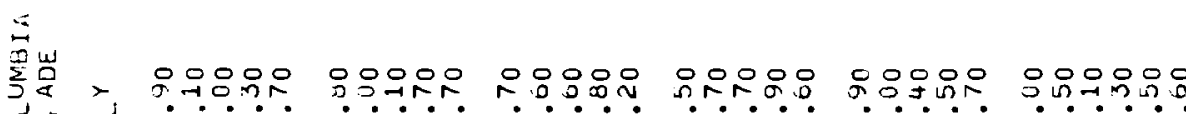

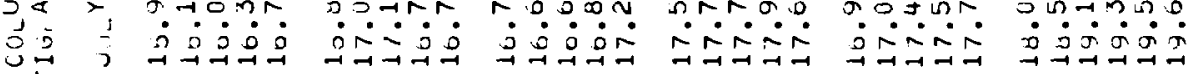

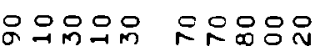

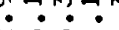
riming

\section{요요}

ำ

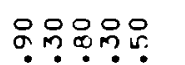

$\dot{0} \dot{0} \dot{0} \dot{0}$

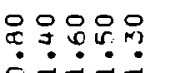

영ㅇํㅇำ

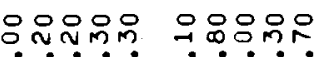

jंjim

ming

空

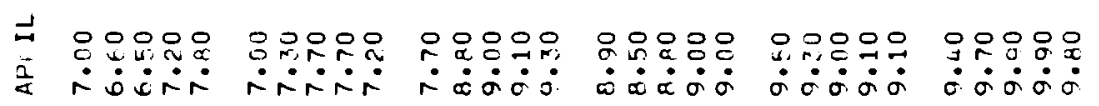

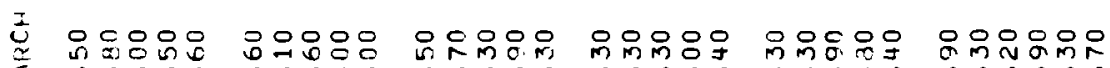
$\dot{0} \dot{\omega} \dot{0}$ जी

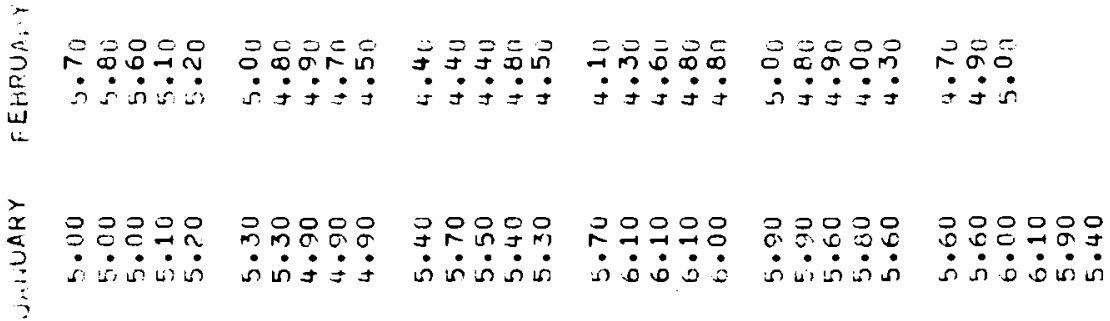

总 


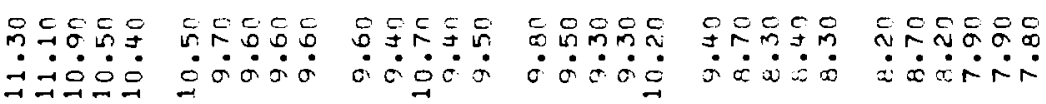

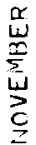

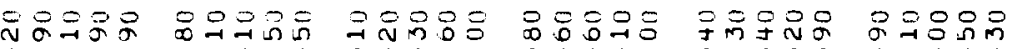

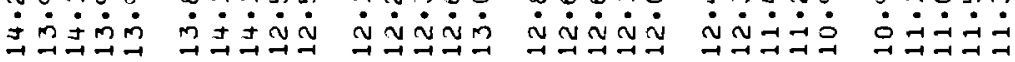

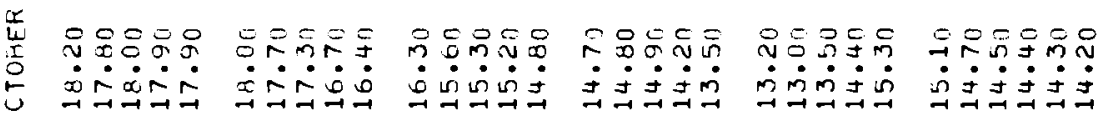

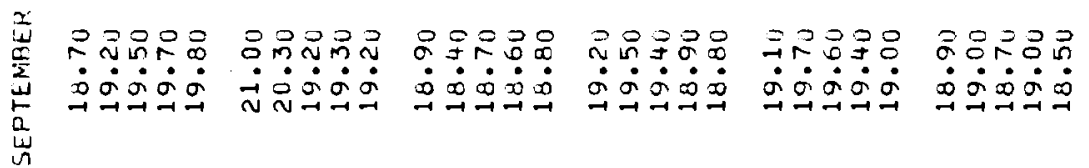

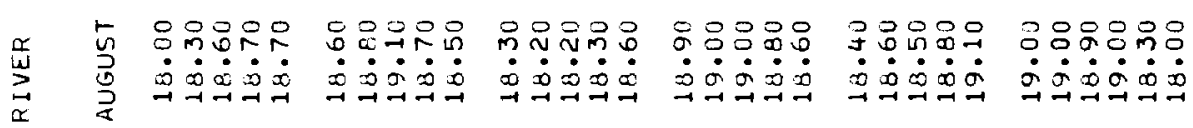

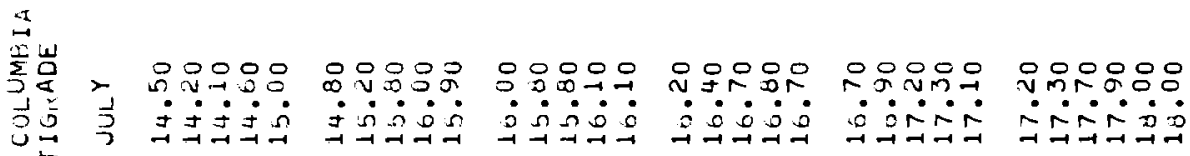

政

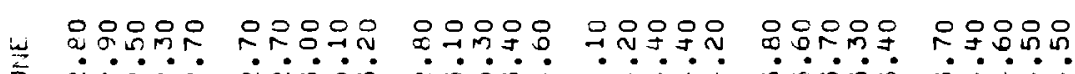

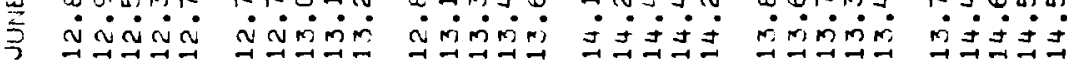

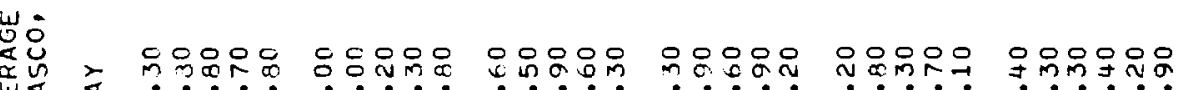

过主

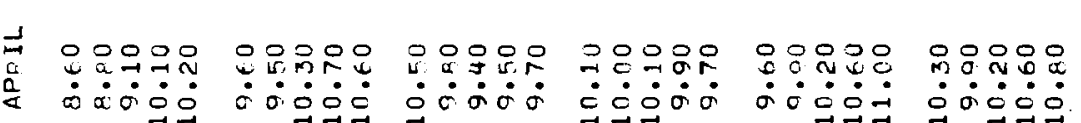

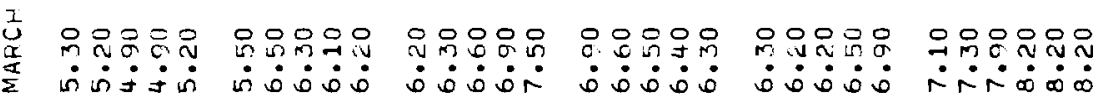

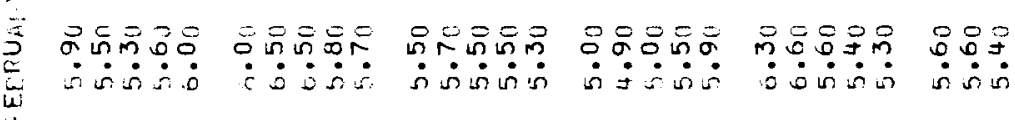

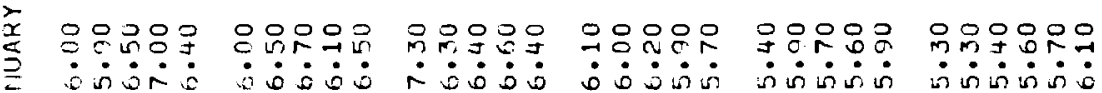

7

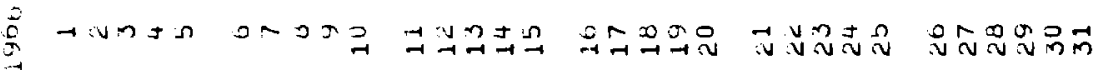




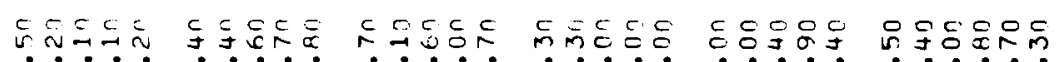

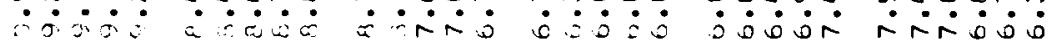

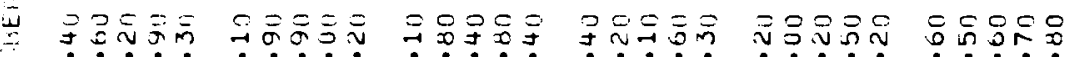

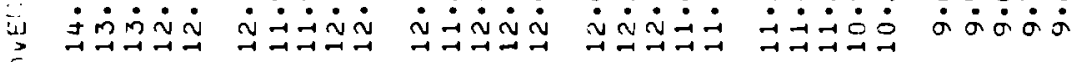

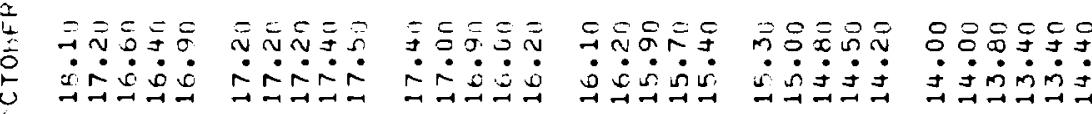

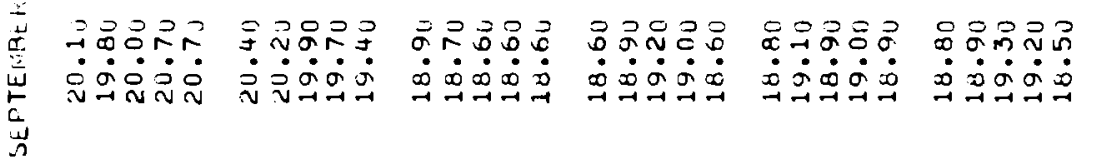

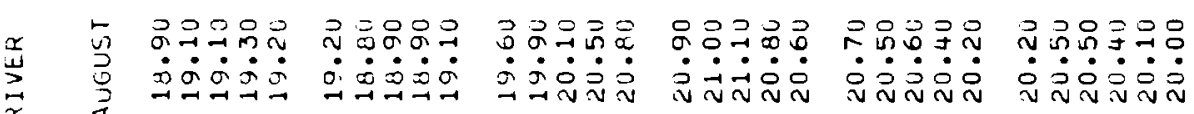

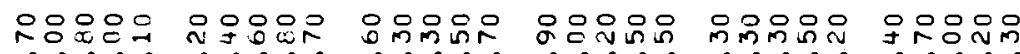

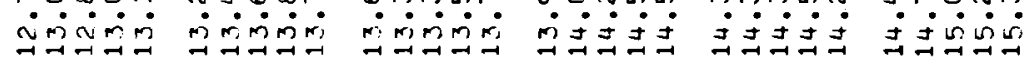

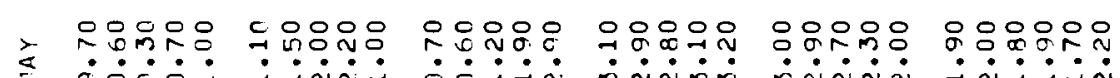

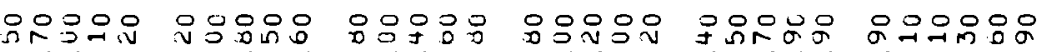

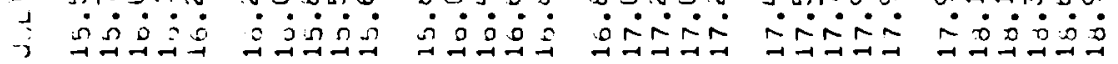
o

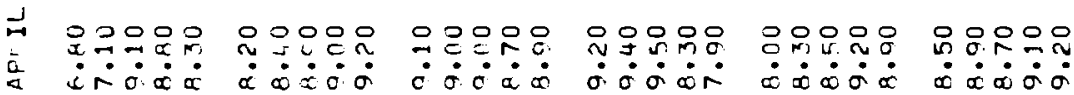

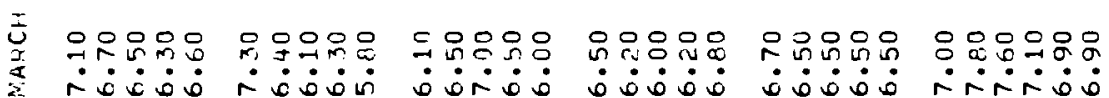

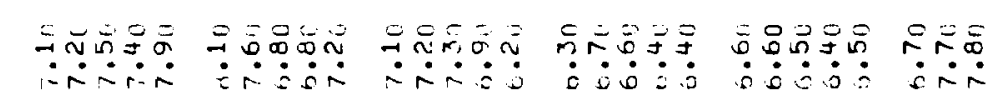

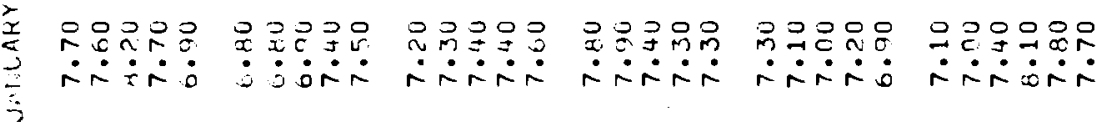

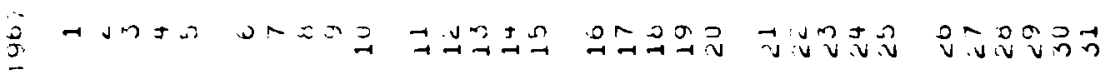


DAILY AVERAGE TEMPERATURE COLUMBIA KIVER PASCO, DEGREES CENTIGHADE

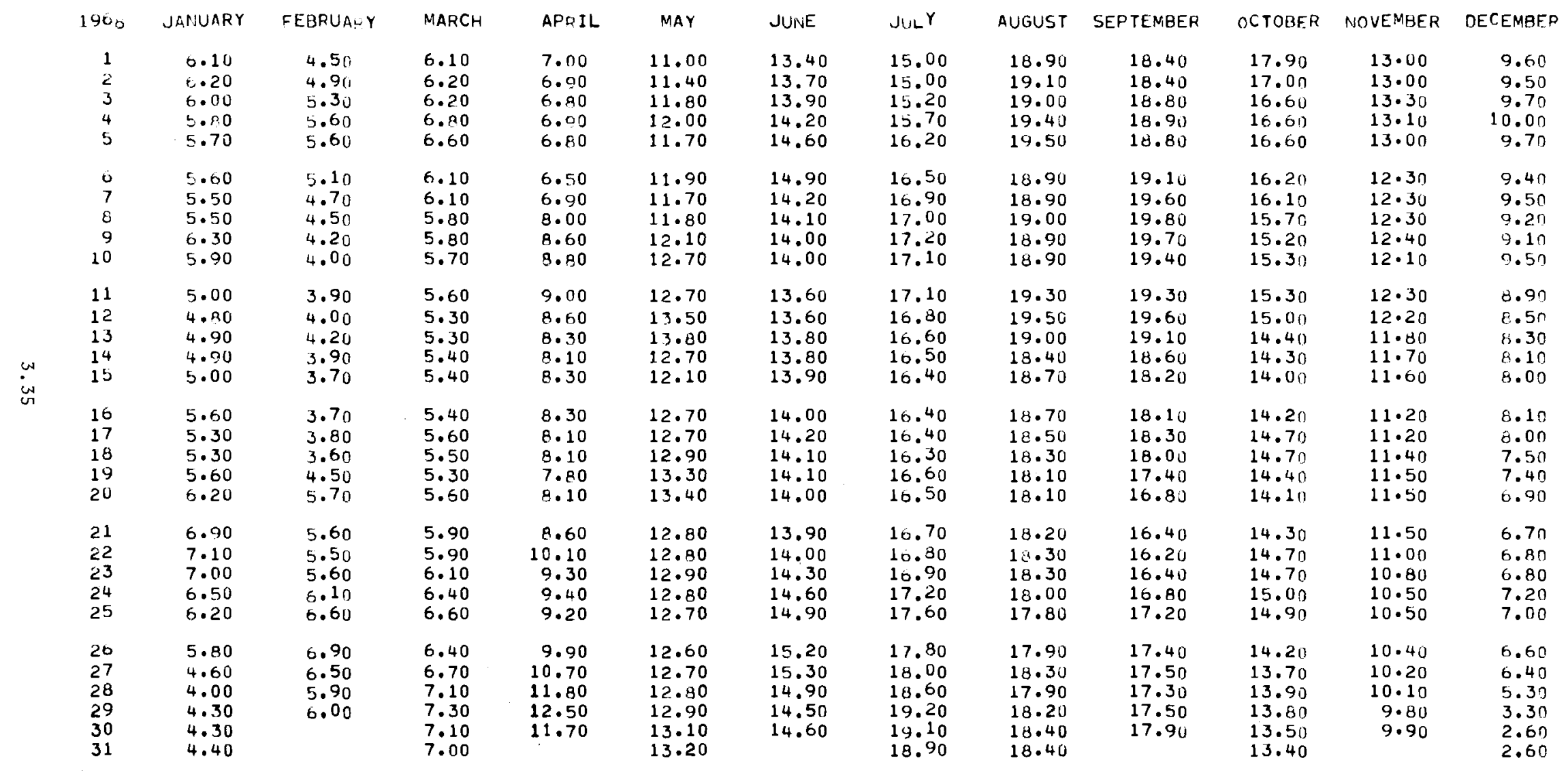




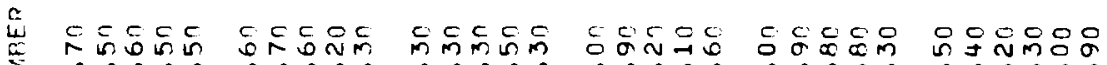

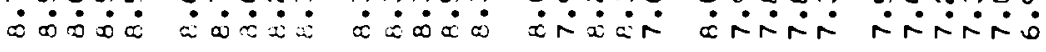

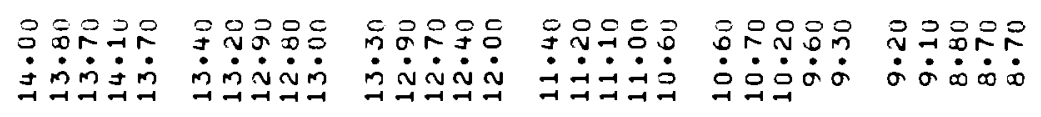

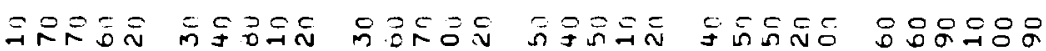

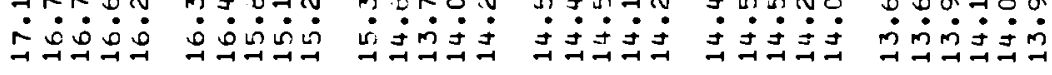

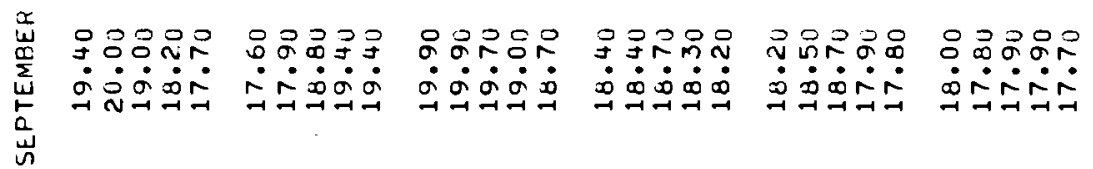

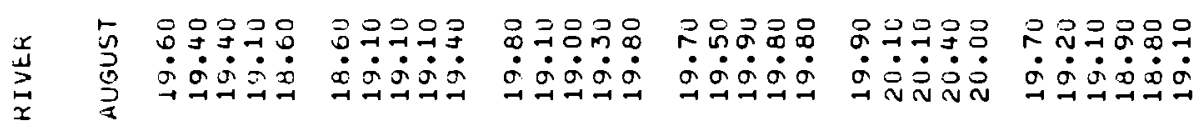

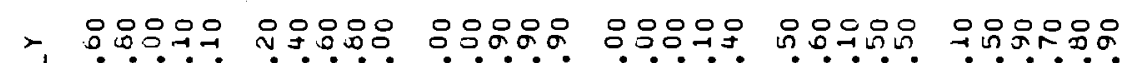

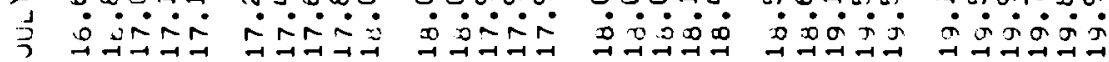

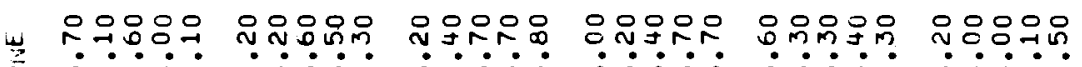

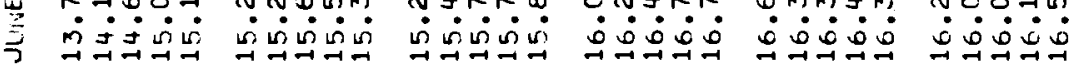

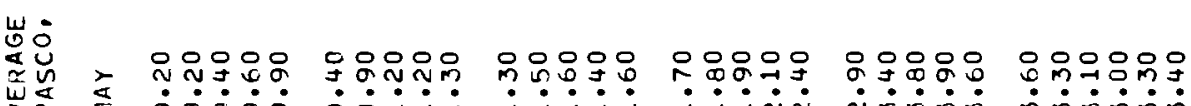

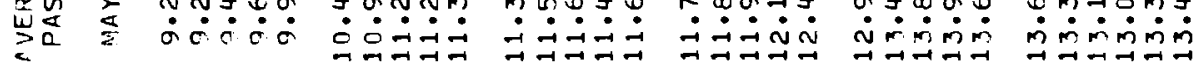

主

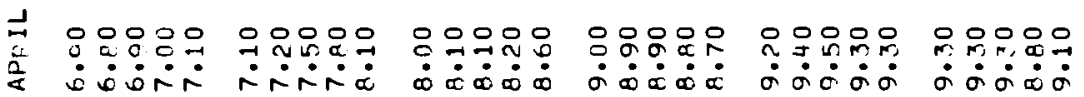

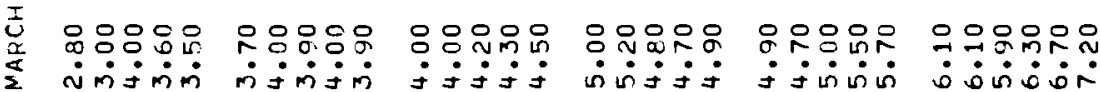

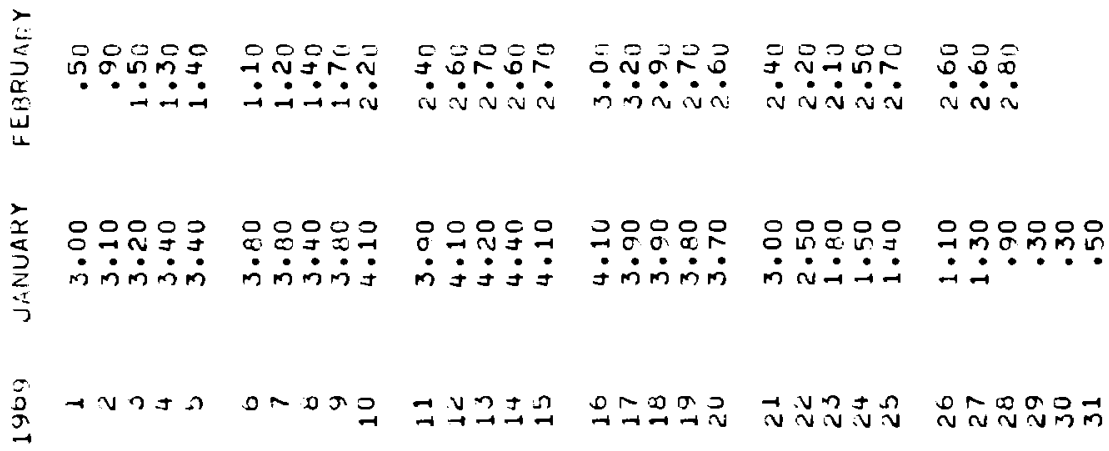




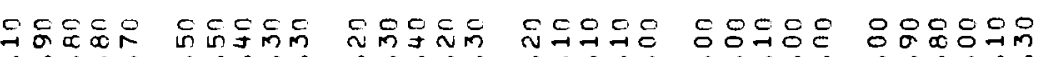

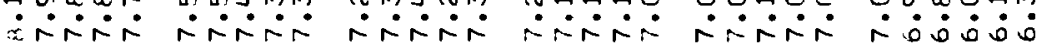

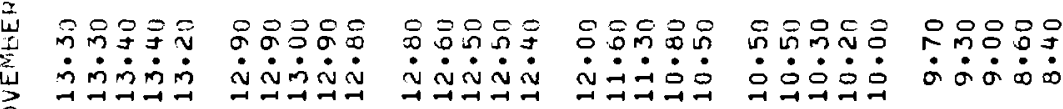

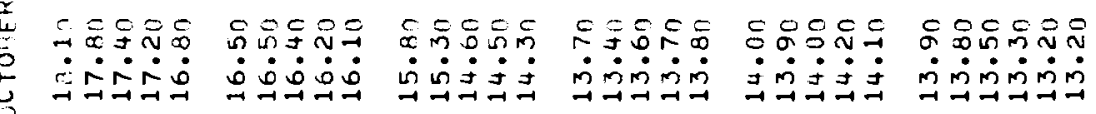

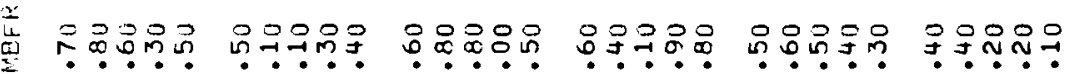

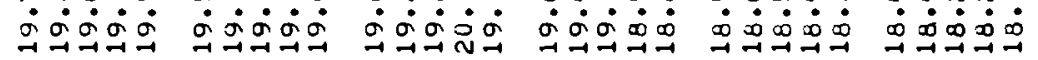

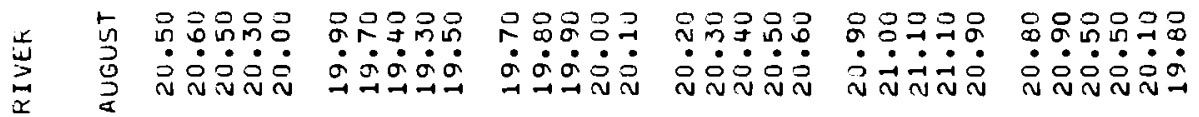

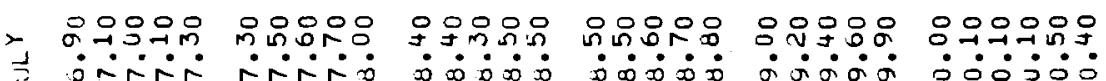

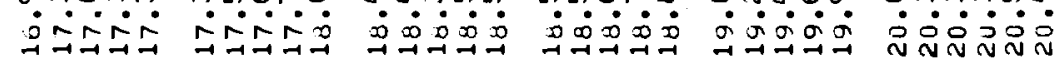

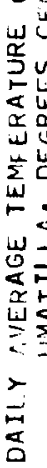

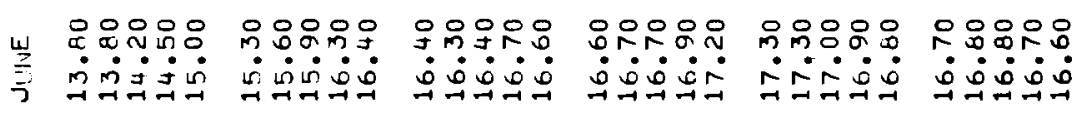

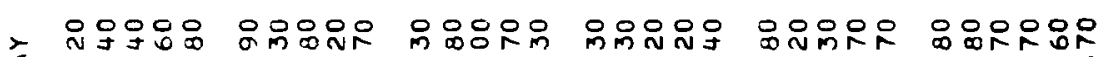

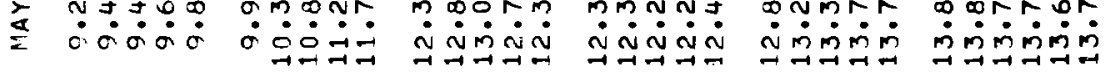

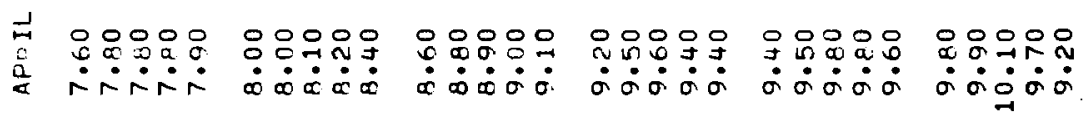

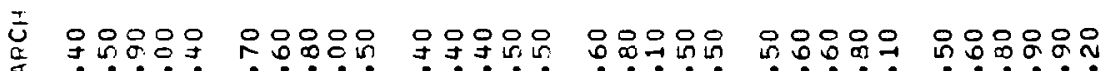

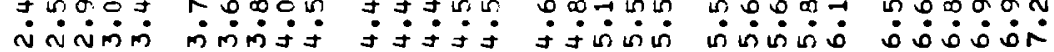

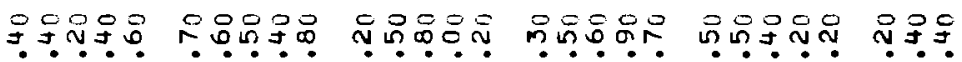

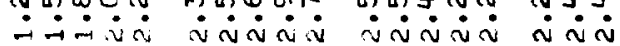

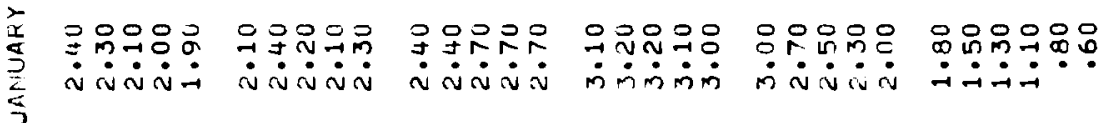

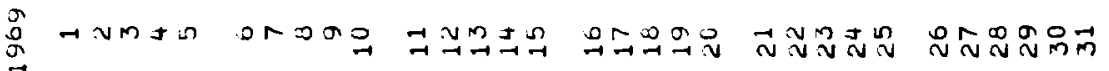




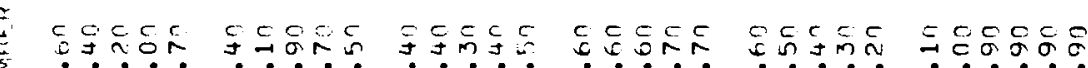

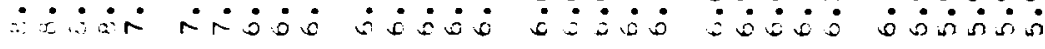

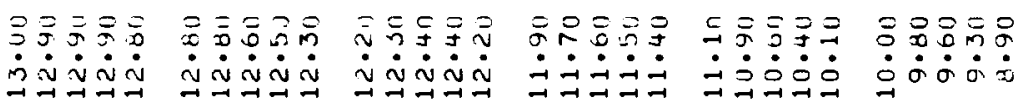

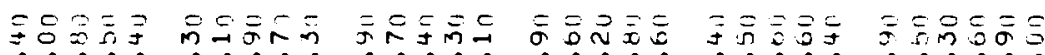

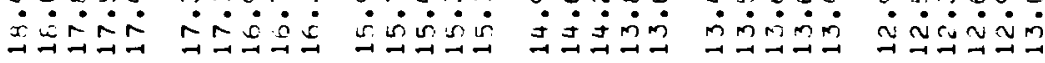

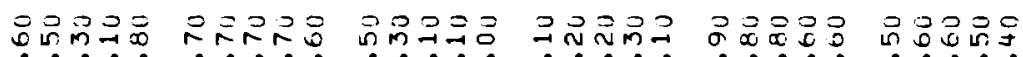

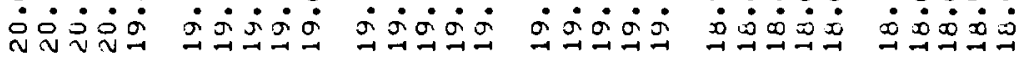

勇

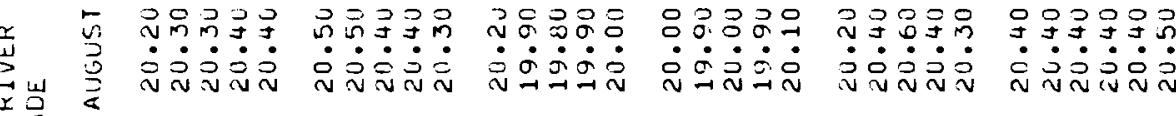

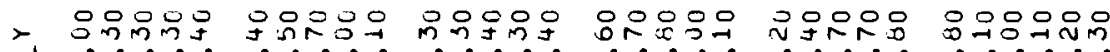

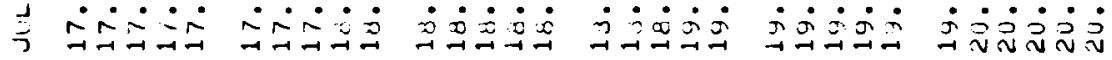

0000

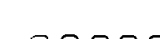

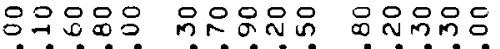

이이응

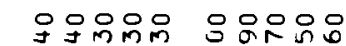

站

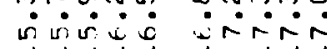

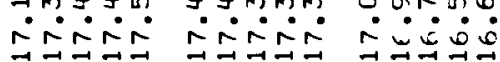

蓆

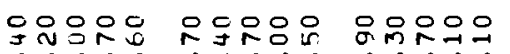

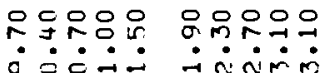

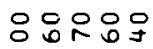

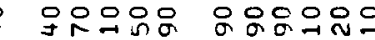

$\subseteq \subseteq \sigma^{\circ}$

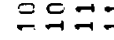

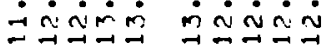

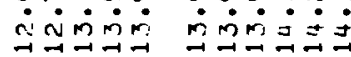

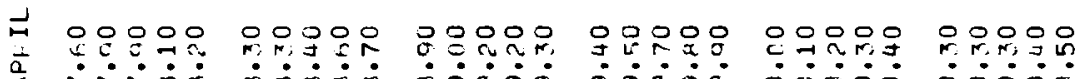

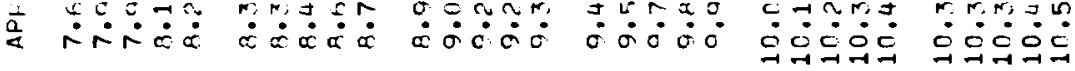

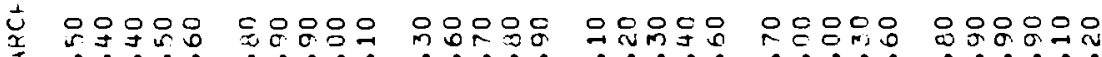

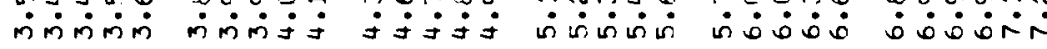

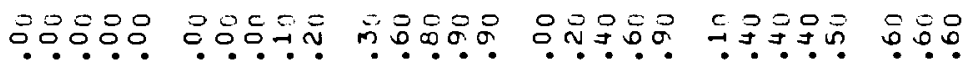

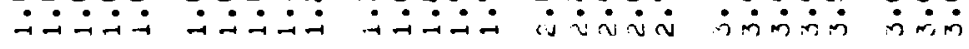

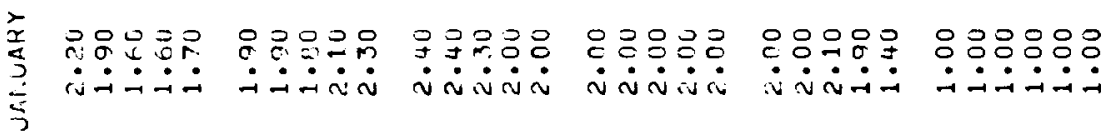

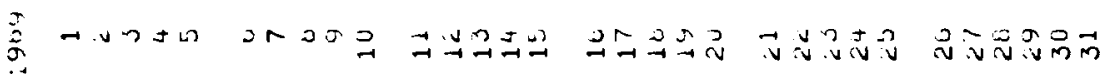




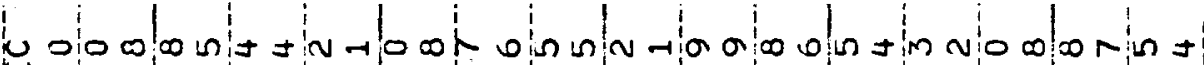

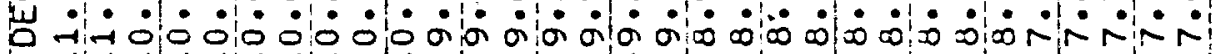
$-1-1-1-1-1-1-1-1-1-1$

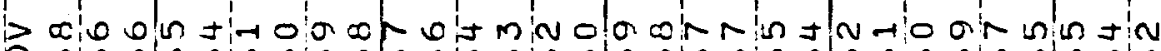

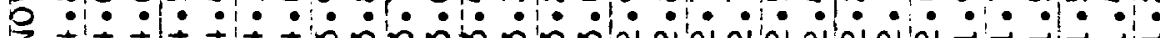

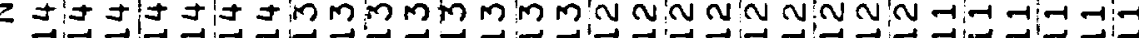

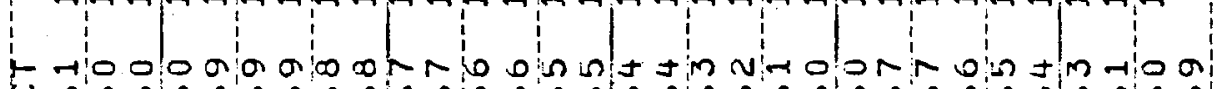

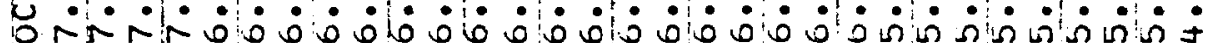

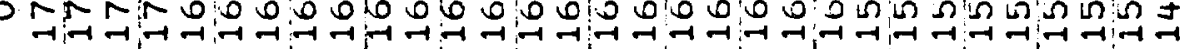

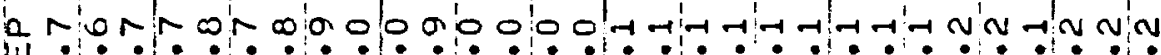

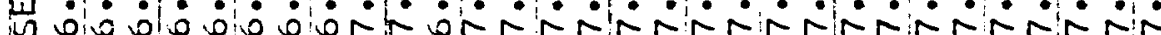

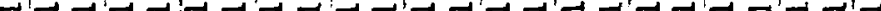

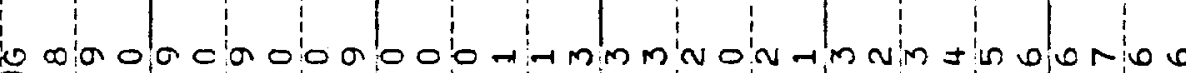
o mo o.

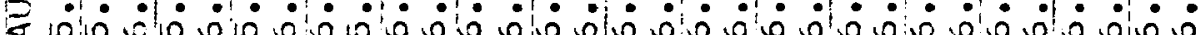

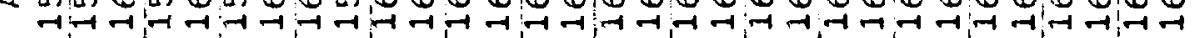

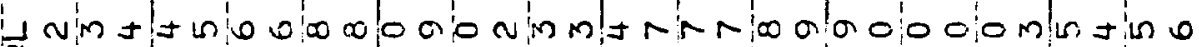
l=

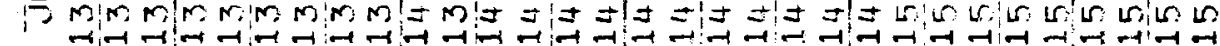
z ह 10 -

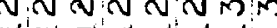

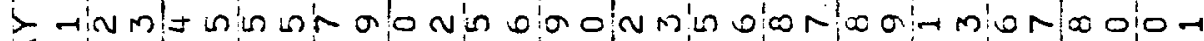

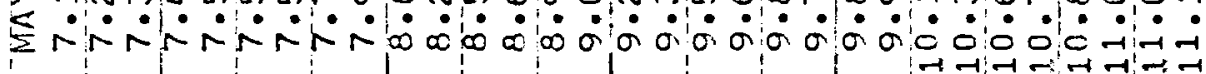

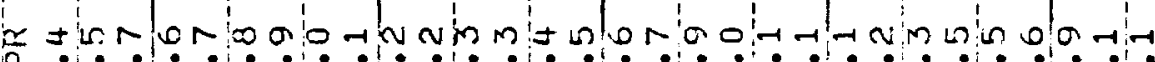

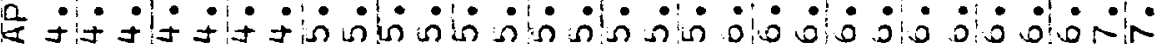

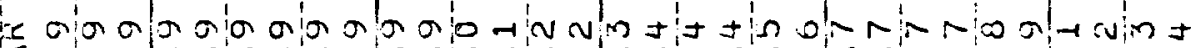

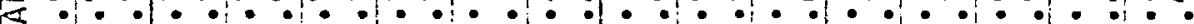

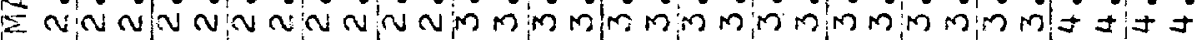

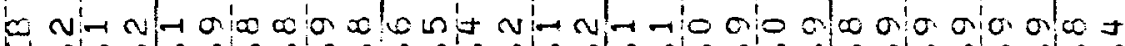

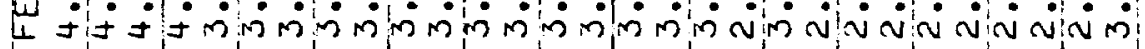

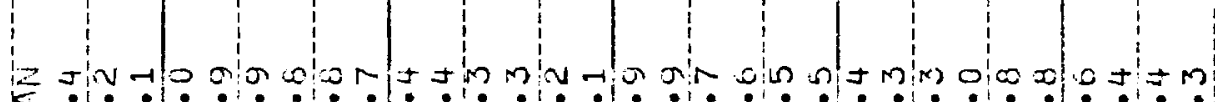
S

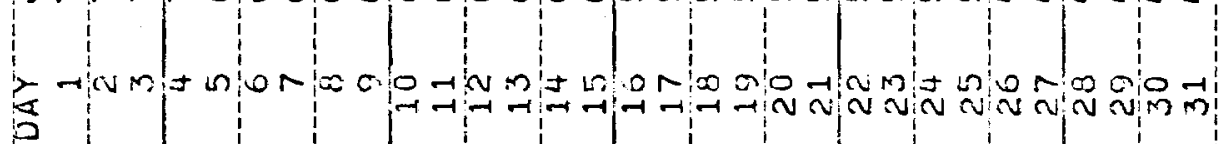




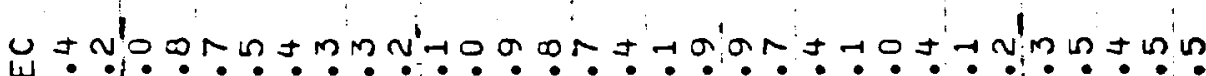
岩

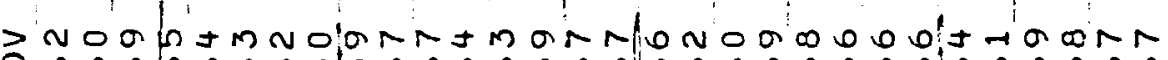

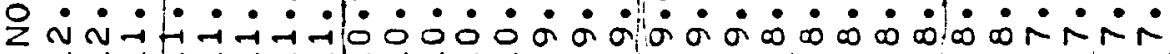

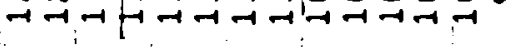

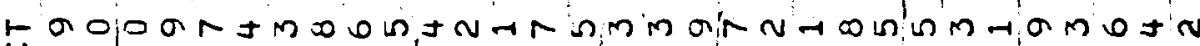
Ur

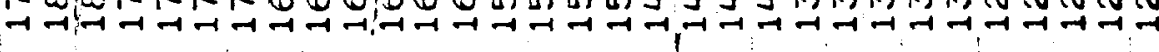

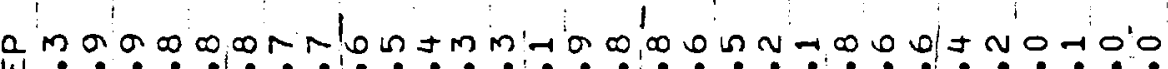
u $\dot{0} \dot{0} \dot{0} \dot{0} \dot{0} \dot{0} \dot{0} \dot{0} \dot{0} \dot{0} \dot{0} \dot{0} \dot{0} \dot{0} \dot{0} \dot{0}$ N onrmpina o o o onfl-

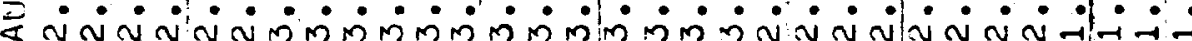

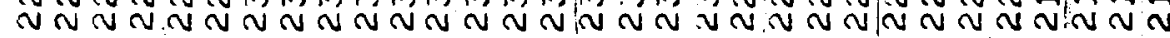

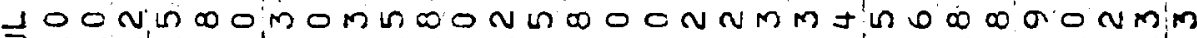

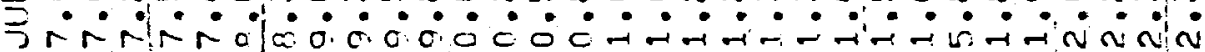
- -1- -

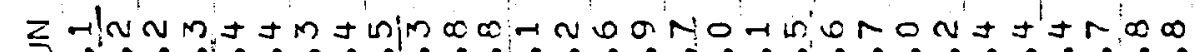

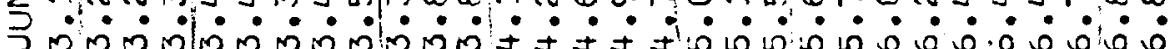
(1)-

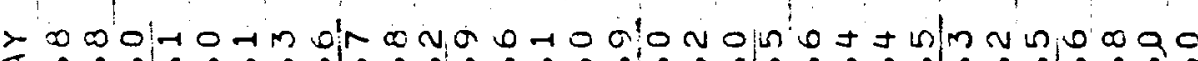

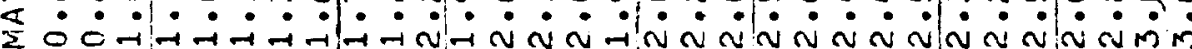

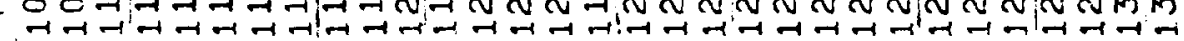

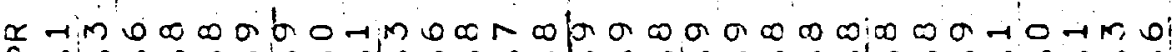

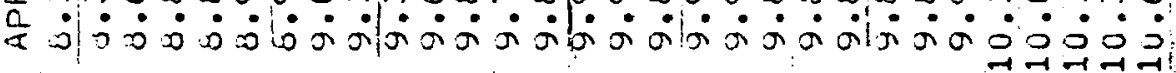
x oding

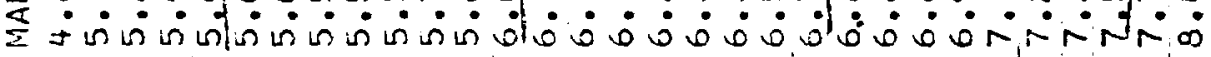

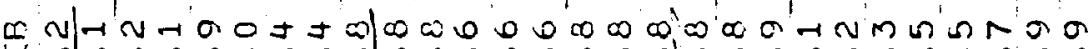
ú

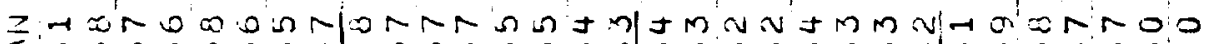

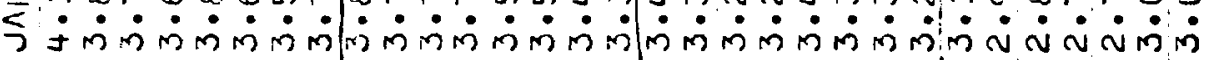

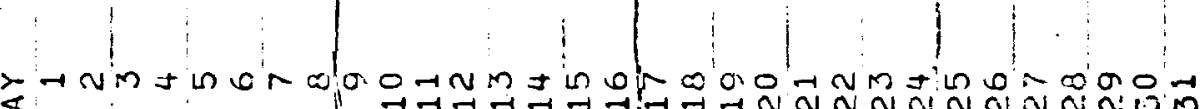
$\$$ 


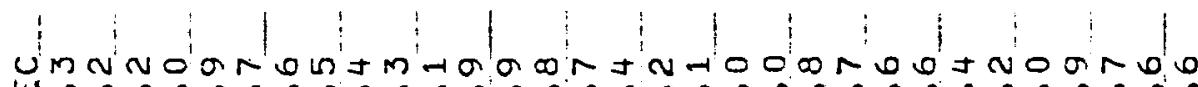

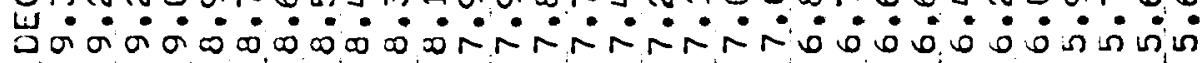

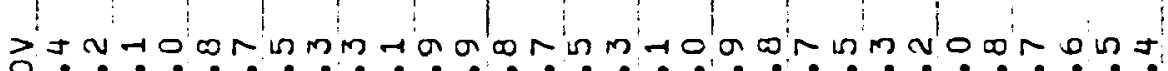

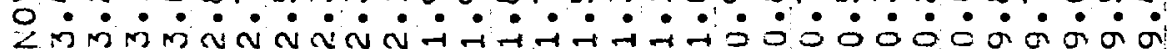

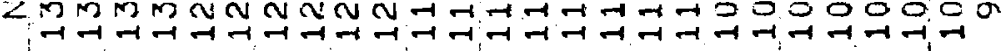

$\tilde{u}$ -

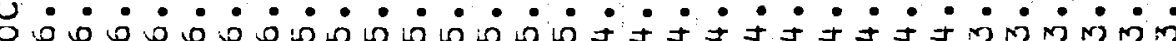

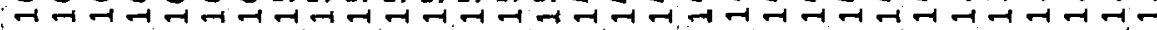

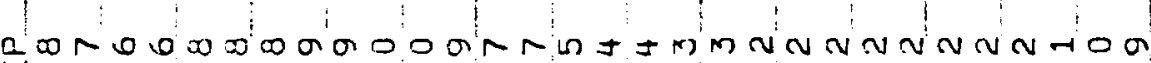

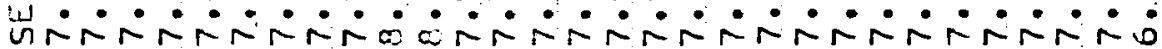

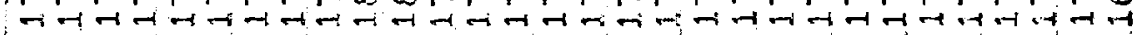

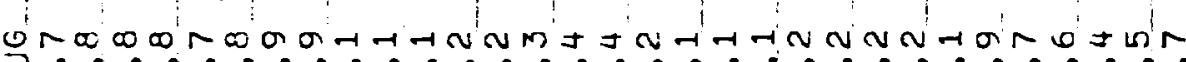

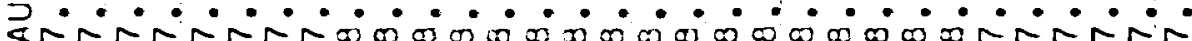

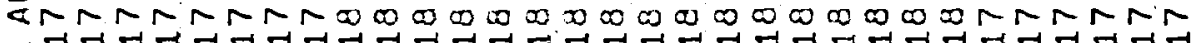

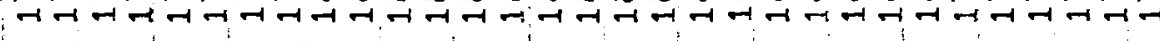
jorafuring

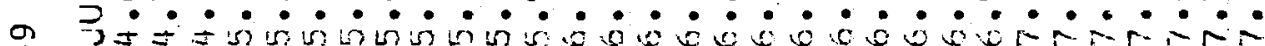

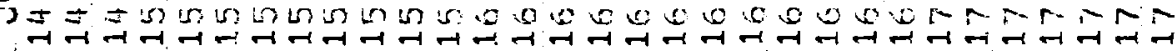
ZOM

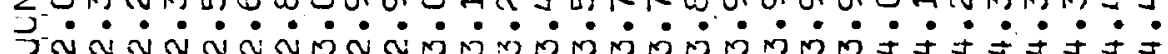

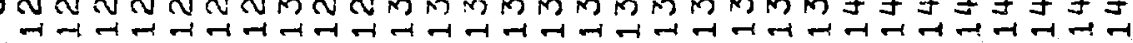

>

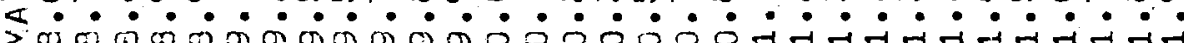

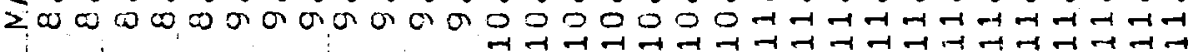

व

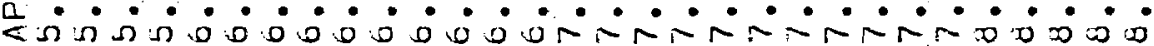
(

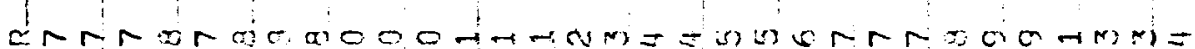

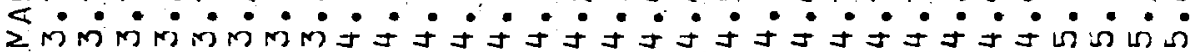

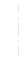

एक

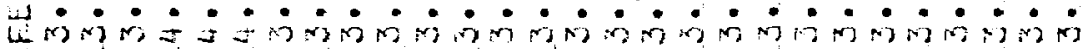

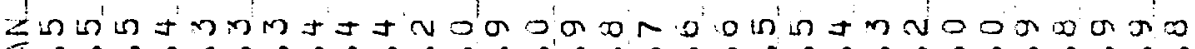
خ

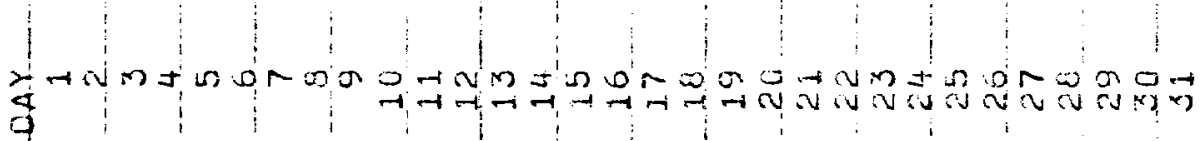


TABLE 4. Priest Rapids 20-Year Average of Daily Maximum Temperature in Degrees Centigrade for the Years 1947 Through 1967

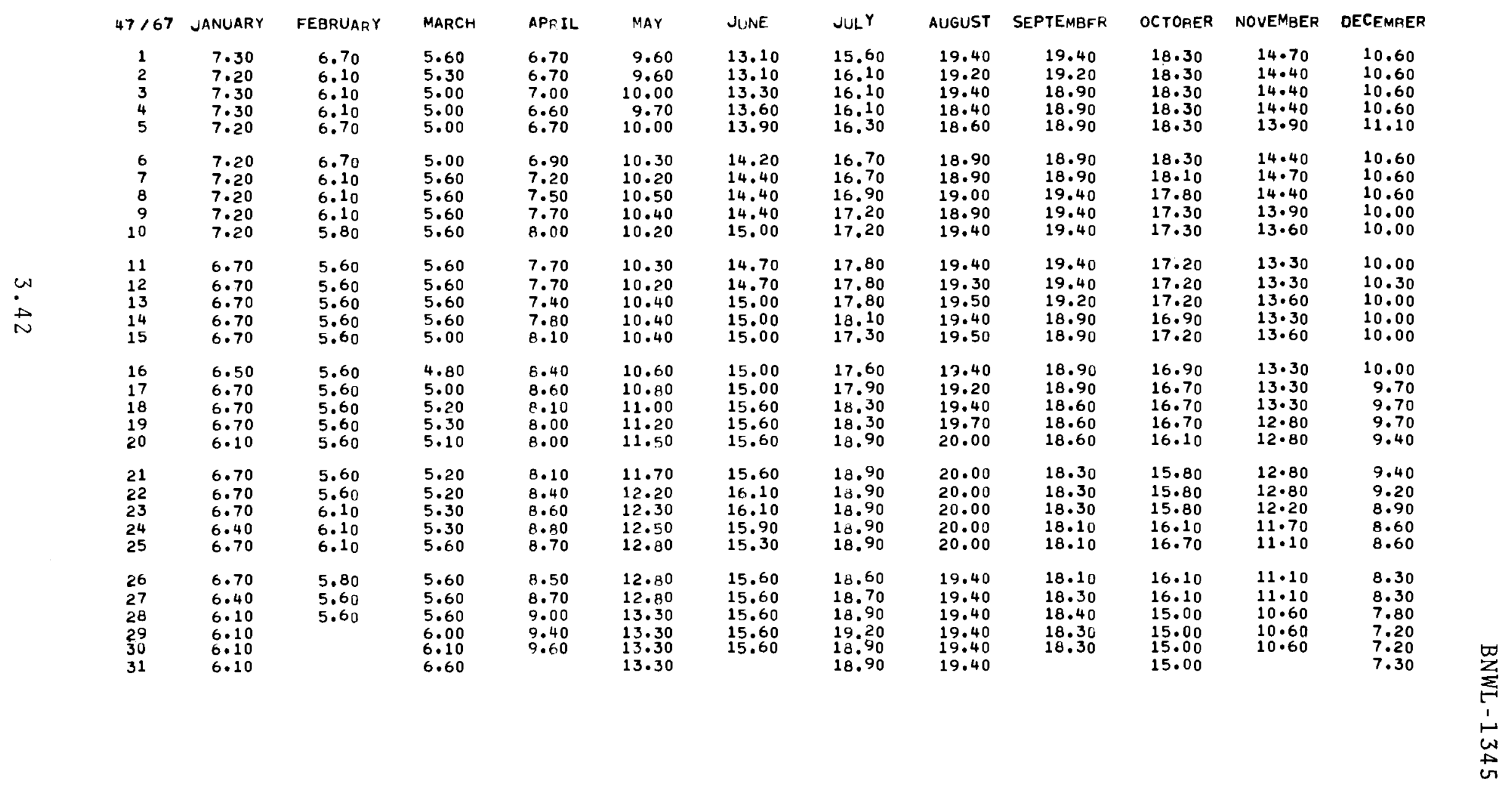


TABLE 5. Priest Rapids 20-Year Average of Daily Minimum Temperature in Degrees Centigrade for the Years 1947 'Through 1967

\begin{tabular}{|c|c|c|c|c|c|c|c|c|c|c|c|c|}
\hline $47 / 67$ & JANUARY & FEBRUARY & MARCH & APRIL & MAY & JUNE & JULY & AUGUST & SEPTEMBFR & OCTOBER & NOVEMBER & DECEMBER \\
\hline $\begin{array}{l}1 \\
2 \\
3 \\
4 \\
5\end{array}$ & $\begin{array}{l}3.60 \\
3.80 \\
4.10 \\
3.90 \\
3.90\end{array}$ & $\begin{array}{l}1.10 \\
1.10 \\
1.10 \\
1.10 \\
1.70\end{array}$ & $\begin{array}{l}1.90 \\
1.70 \\
1.90 \\
1.40 \\
1.40\end{array}$ & $\begin{array}{l}3.30 \\
3.60 \\
3.60 \\
3.60 \\
3.60\end{array}$ & $\begin{array}{l}5.60 \\
5.60 \\
6.10 \\
6.10 \\
6.10\end{array}$ & $\begin{array}{l}10.00 \\
10.00 \\
10.00 \\
10.30 \\
10.60\end{array}$ & $\begin{array}{l}11.70 \\
11.70 \\
12.20 \\
12.80 \\
12.80\end{array}$ & $\begin{array}{l}15.60 \\
15.60 \\
15.80 \\
15.80 \\
15.60\end{array}$ & $\begin{array}{l}16.40 \\
16.20 \\
16.30 \\
16.30 \\
16.50\end{array}$ & $\begin{array}{l}15.00 \\
14.70 \\
14.70 \\
14.40 \\
14.20\end{array}$ & $\begin{array}{l}12 \cdot 80 \\
12.50 \\
12.20 \\
12.20 \\
11.70\end{array}$ & $\begin{array}{l}8.60 \\
8.60 \\
8.30 \\
8.30 \\
8.10\end{array}$ \\
\hline $\begin{array}{r}6 \\
7 \\
8 \\
9 \\
10\end{array}$ & $\begin{array}{l}3.90 \\
3.90 \\
3.90 \\
3.90 \\
3.90\end{array}$ & $\begin{array}{l}1.70 \\
1.70 \\
1.70 \\
1.70 \\
1.90\end{array}$ & $\begin{array}{l}1.70 \\
1.70 \\
1.90 \\
1.70 \\
1.90\end{array}$ & $\begin{array}{l}3.90 \\
3.90 \\
4.00 \\
4.10 \\
4.10\end{array}$ & $\begin{array}{l}6.10 \\
6.60 \\
6.60 \\
6.90 \\
6.90\end{array}$ & $\begin{array}{l}10.60 \\
10.80 \\
10.80 \\
10.60 \\
10.60\end{array}$ & $\begin{array}{l}12.80 \\
13.30 \\
13.30 \\
13.30 \\
13.30\end{array}$ & $\begin{array}{l}15.60 \\
15.60 \\
15.80 \\
16.10 \\
16.10\end{array}$ & $\begin{array}{l}16.70 \\
16.70 \\
16.50 \\
16.40 \\
16.30\end{array}$ & $\begin{array}{l}14.20 \\
14.20 \\
15.00 \\
14.60 \\
14.40\end{array}$ & $\begin{array}{l}11.70 \\
11.60 \\
11.50 \\
11.40 \\
11.30\end{array}$ & $\begin{array}{l}7.80 \\
7.20 \\
7.20 \\
7.20 \\
7.20\end{array}$ \\
\hline $\begin{array}{l}11 \\
12 \\
13 \\
14 \\
15\end{array}$ & $\begin{array}{l}3.90 \\
3.90 \\
3.60 \\
3.30 \\
2.80\end{array}$ & $\begin{array}{l}1.90 \\
2.20 \\
1.90 \\
1.10 \\
1.10\end{array}$ & $\begin{array}{l}2.20 \\
1.70 \\
1.90 \\
1.90 \\
2.50\end{array}$ & $\begin{array}{l}4.20 \\
4.20 \\
4.20 \\
4.40 \\
4.40\end{array}$ & $\begin{array}{l}7.20 \\
7.80 \\
7.80 \\
7.90 \\
8.30\end{array}$ & $\begin{array}{l}11.10 \\
11.10 \\
11.10 \\
11.10 \\
11.10\end{array}$ & $\begin{array}{l}13.30 \\
13.30 \\
13.30 \\
13.30 \\
13.30\end{array}$ & $\begin{array}{l}16.10 \\
16.10 \\
16.70 \\
16.40 \\
16.40\end{array}$ & $\begin{array}{l}16.40 \\
16.50 \\
16.40 \\
16.40 \\
15.80\end{array}$ & $\begin{array}{l}14.10 \\
13.90 \\
13.90 \\
13.90 \\
13.90\end{array}$ & $\begin{array}{r}10.90 \\
10.90 \\
8.90 \\
8.60 \\
8.90\end{array}$ & $\begin{array}{l}6.80 \\
6.40 \\
6.20 \\
6.20 \\
6.10\end{array}$ \\
\hline $\begin{array}{l}16 \\
17 \\
18 \\
19 \\
20\end{array}$ & $\begin{array}{l}2.80 \\
3.10 \\
3.10 \\
3.10 \\
2.40\end{array}$ & $\begin{array}{l}1.10 \\
.80 \\
1.10 \\
1.10 \\
1.10\end{array}$ & $\begin{array}{l}2.80 \\
2.80 \\
2.80 \\
2.80 \\
2.80\end{array}$ & $\begin{array}{l}4.40 \\
4.40 \\
4.40 \\
4.70 \\
4.70\end{array}$ & $\begin{array}{l}8.30 \\
8.10 \\
8.10 \\
7.80 \\
8.10\end{array}$ & $\begin{array}{l}11.10 \\
11.10 \\
11.10 \\
11.10 \\
11.10\end{array}$ & $\begin{array}{l}13.60 \\
13.90 \\
13.90 \\
13.60 \\
13.90\end{array}$ & $\begin{array}{l}15.60 \\
15.60 \\
15.80 \\
15.80 \\
16.10\end{array}$ & $\begin{array}{l}15.60 \\
16.10 \\
16.10 \\
16.10 \\
15.90\end{array}$ & $\begin{array}{l}13.90 \\
14.00 \\
13.80 \\
13.60 \\
13.20\end{array}$ & $\begin{array}{l}8 \cdot 30 \\
8 \cdot 10 \\
8 \cdot 90 \\
8 \cdot 90 \\
8 \cdot 90\end{array}$ & $\begin{array}{l}6.10 \\
5.40 \\
5.30 \\
5.00 \\
4.70\end{array}$ \\
\hline $\begin{array}{l}21 \\
22 \\
23 \\
24 \\
25\end{array}$ & $\begin{array}{l}2.20 \\
2.40 \\
2.50 \\
2.60 \\
2.70\end{array}$ & $\begin{array}{l}1.40 \\
1.70 \\
1.90 \\
1.90 \\
1.70\end{array}$ & $\begin{array}{l}2.80 \\
2.80 \\
3.10 \\
2.80 \\
2.80\end{array}$ & $\begin{array}{l}5.00 \\
5.00 \\
5.30 \\
5.00 \\
5.00\end{array}$ & $\begin{array}{l}8.60 \\
8.60 \\
8.90 \\
8.90 \\
9.40\end{array}$ & $\begin{array}{l}11.10 \\
11.10 \\
11.40 \\
11.70 \\
11.70\end{array}$ & $\begin{array}{l}13.90 \\
13.90 \\
13.90 \\
14.40 \\
14.40\end{array}$ & $\begin{array}{l}15.80 \\
16.10 \\
16.10 \\
15.60 \\
15.60\end{array}$ & $\begin{array}{l}15.80 \\
15.90 \\
15.80 \\
16.10 \\
16.00\end{array}$ & $\begin{array}{l}12.80 \\
12.80 \\
12.80 \\
12.80 \\
12.80\end{array}$ & $\begin{array}{l}8 \cdot 90 \\
9.20 \\
9.10 \\
8.90 \\
8.70\end{array}$ & $\begin{array}{l}4.10 \\
4.20 \\
4.50 \\
4.40 \\
3.60\end{array}$ \\
\hline $\begin{array}{l}26 \\
27 \\
28 \\
29 \\
30 \\
31\end{array}$ & $\begin{array}{l}1.70 \\
1.70 \\
2.20 \\
2.20 \\
1.70 \\
1.10\end{array}$ & $\begin{array}{l}1.70 \\
1.70 \\
1.90\end{array}$ & $\begin{array}{l}2.80 \\
2.80 \\
3.30 \\
3.30 \\
3.30 \\
3.30\end{array}$ & $\begin{array}{l}5.00 \\
5.00 \\
5.00 \\
5.60 \\
5.60\end{array}$ & $\begin{array}{l}8.90 \\
9.40 \\
9.40 \\
9.40 \\
9.70 \\
9.70\end{array}$ & $\begin{array}{l}11.70 \\
11.40 \\
11.40 \\
11.70 \\
11.70\end{array}$ & $\begin{array}{l}14.40 \\
14.70 \\
14.40 \\
14.40 \\
15.00 \\
15.60\end{array}$ & $\begin{array}{l}15.60 \\
15.80 \\
15.80 \\
16.10 \\
16.10 \\
16.30\end{array}$ & $\begin{array}{l}16.10 \\
16.10 \\
15.60 \\
15.00 \\
14.70\end{array}$ & $\begin{array}{l}12.20 \\
12.80 \\
12.80 \\
12.20 \\
12.80 \\
12.80\end{array}$ & $\begin{array}{l}8 \cdot 40 \\
8 \cdot 30 \\
8 \cdot 30 \\
8 \cdot 30 \\
8 \cdot 30\end{array}$ & $\begin{array}{l}3.40 \\
3.30 \\
3.30 \\
3.60 \\
3.60 \\
3.60\end{array}$ \\
\hline
\end{tabular}


BNWL -1345

\section{LEAST-SQUARES FITTED CURVES}

The following least-squares fitted curves, Figures 6 through 31, were made using the observed temperatures, at Ice Harbor on the Snake River and various sites on the Columbia. Using the available analytical techniques of the Pacific Northwest Laboratory (Battelle-Northwest), it was possible to utilize this data in a least-squares curve fitting routine.

The general success of this method of least squares analyses has permitted the generation of a series of least-squares-fitted functional models to be used with daily flows, dew points, Langleys, air temperature, water temperature or other water quality data.

For temperature correlations, the function $\mathrm{T}=\mathrm{A}+\mathrm{B}$ sin $(C d+D)$ originally used in oceanography and described by Ward ${ }^{(2)}$ was adopted because of the excellence of representation of annual temperature data afforded by its use. In the operations research supporting this study, the data points were grouped by year from January 1 through December 31 using 365 or 366 days/year as appropriate. The data were then analyzed by means of a nonlinear, maximum-likelihood, leastsquares-fit routine capable of handling nonlinearities and saddle points reliably. This program makes use of numeric rounding for accuracy, ill-conditioned matrix inversion logic, and statistical determination of saddle points on the parametric surface. In addition, it contains a two-stage recovery logic. If the usual first order approximation fails, the routine tries the second order terms of the multivariate Taylor expansion. Failing this, the routine branches into a series of approximations best described as "reverse-and halve the last change" logic. The analysis of the data yields the best-fit parameters and, in addition, produces the root-meansquare error associated with that parameter estimate. 
Also included as output are a series of graphs drawn by the CALCOMP plotter from magnetic tapes generated by the computer. These plots include the observed data, the calculated curve, and another pair of curves of plus or minus one standard deviation on each side of the calculated curve.

If a function of the form $T=A+B \sin (C d+D)$ is fitted to annual temperature data, then $C \approx 2 \pi / 365.25 \approx 0.017$. As an added check on the goodness of fit, this parameter was allowed to vary in each case to see what value the computer would calculate.

Table 6 shows a series of values of four parameters for a series of data points from International Border, Northport site, to Beaver Terminal for calendar years 1964 through 1969. Included are calculations from data supplied by other agencies for Chief Joseph, Ice Harbor, McNary, and The Dalles. 
BNWL - 1345

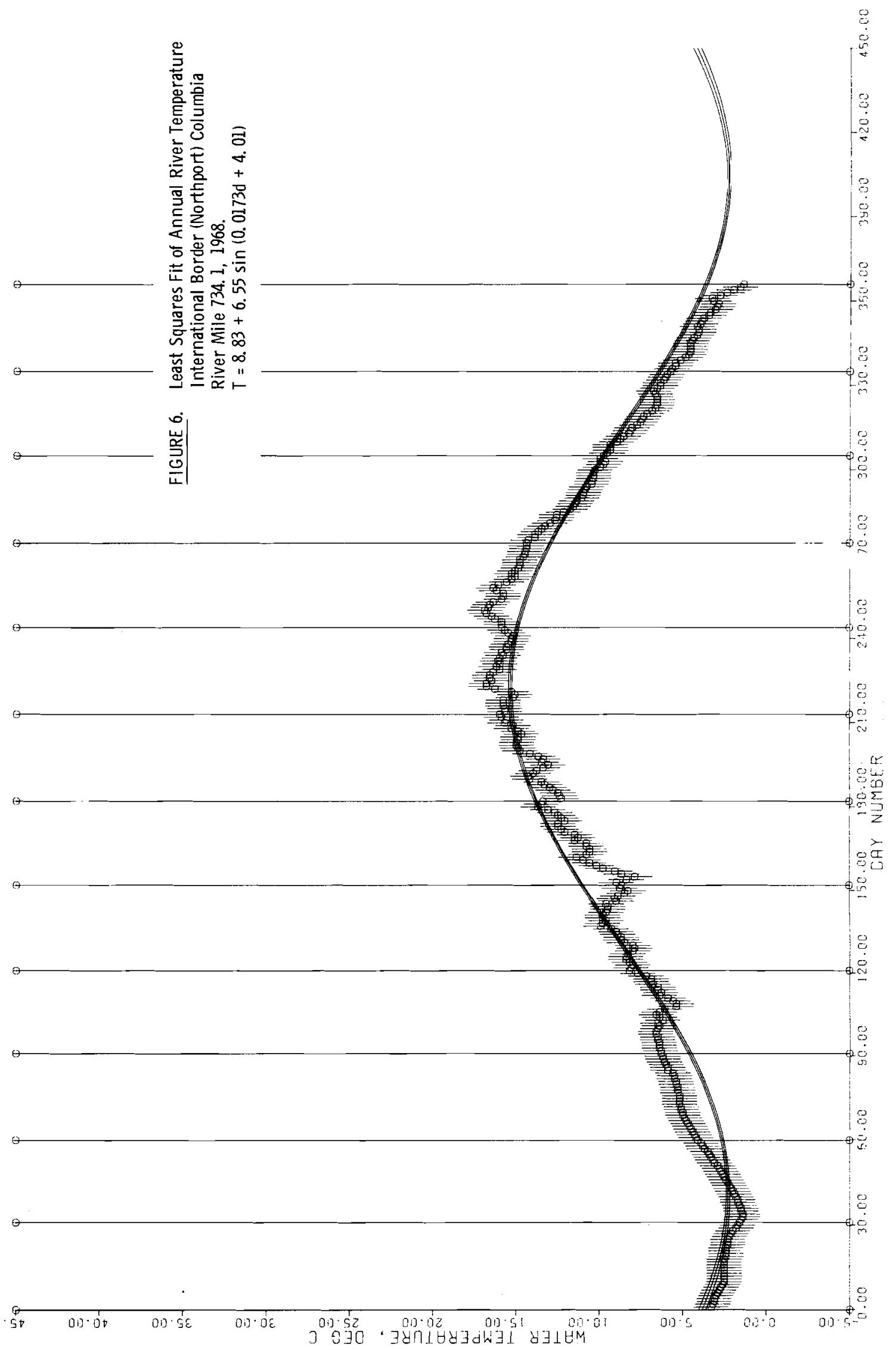

4.3 
BNWL - 1345

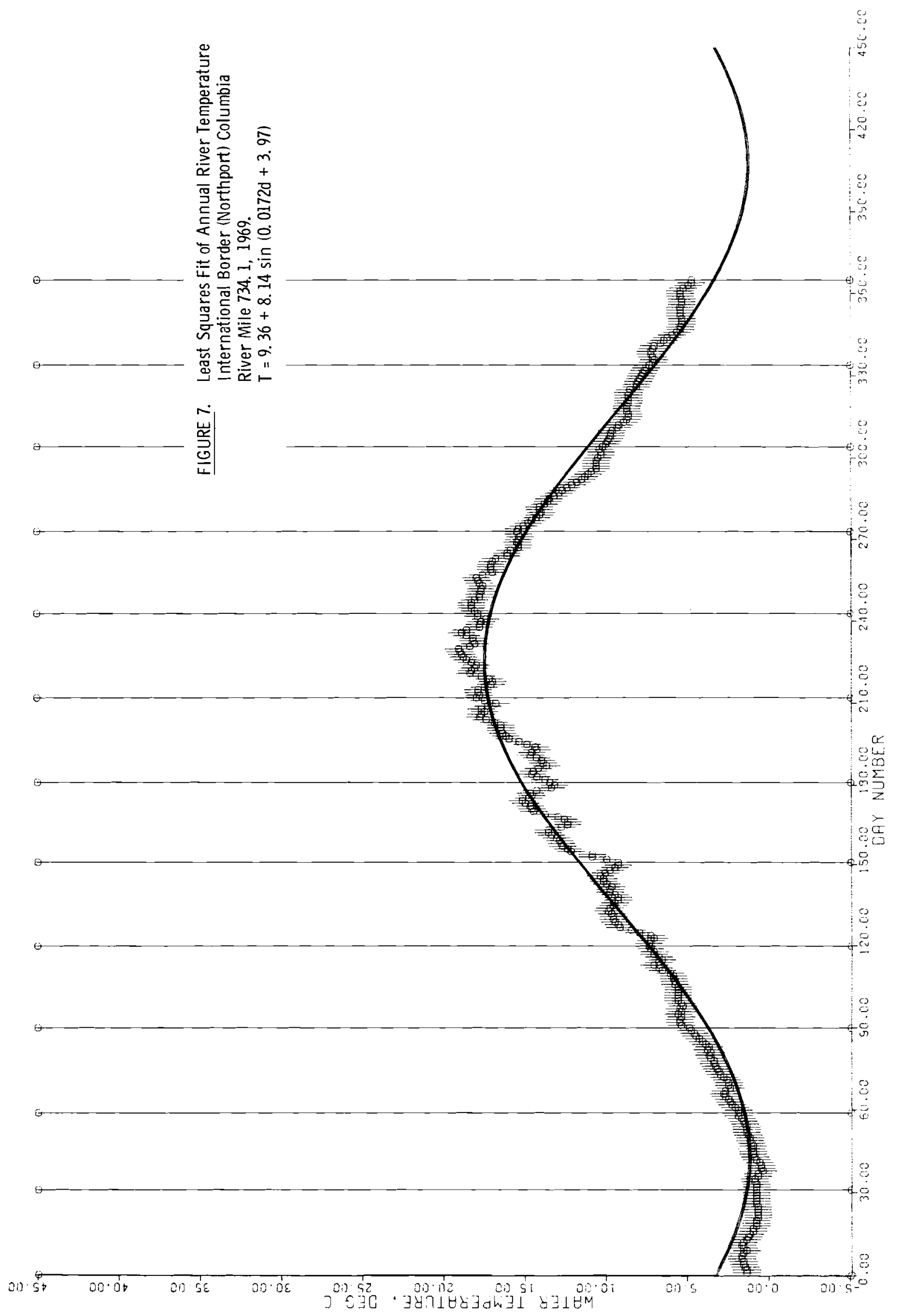

4.4 
BNWL- 1345

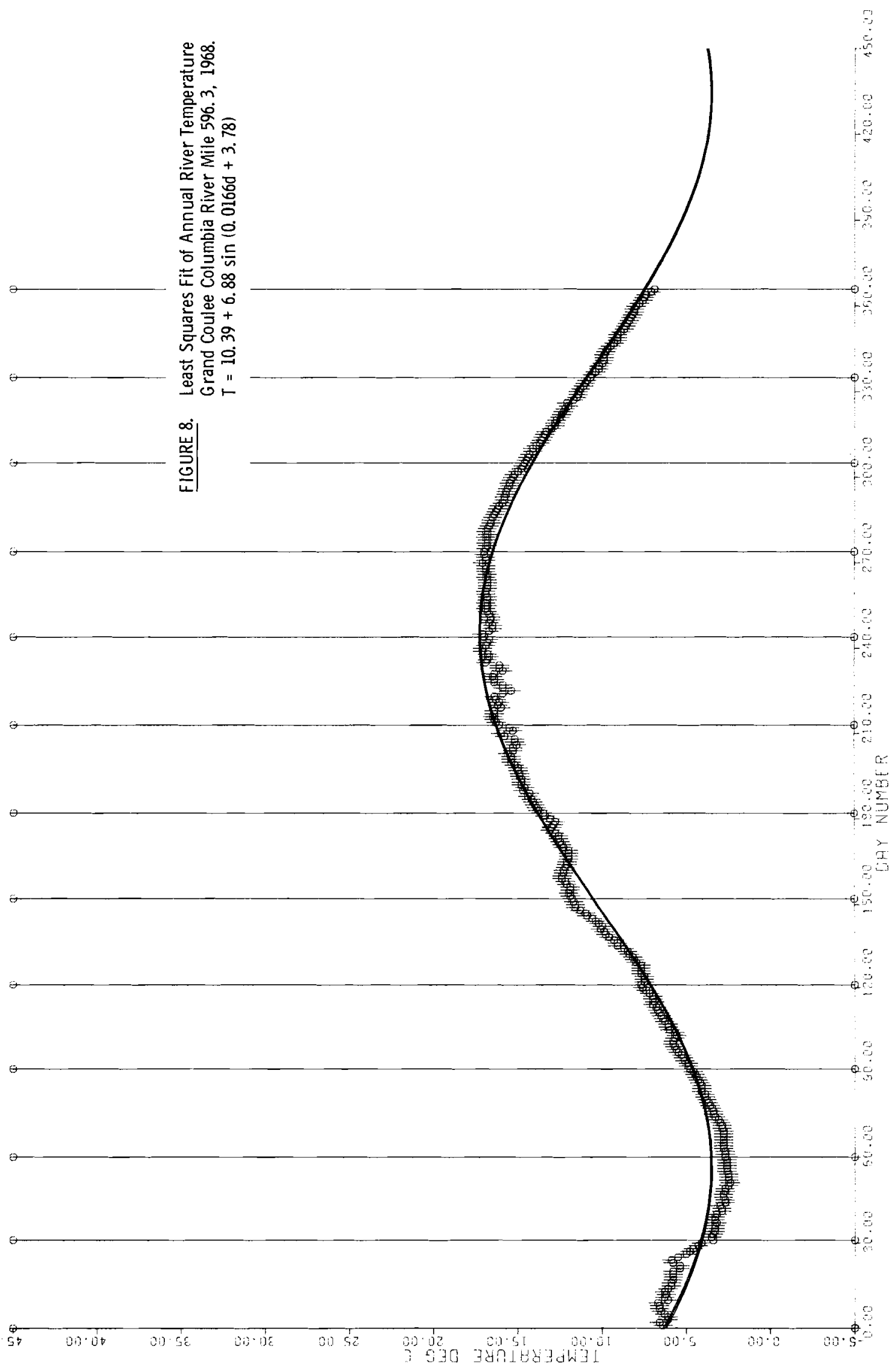

4.5 
BNWL - 1345

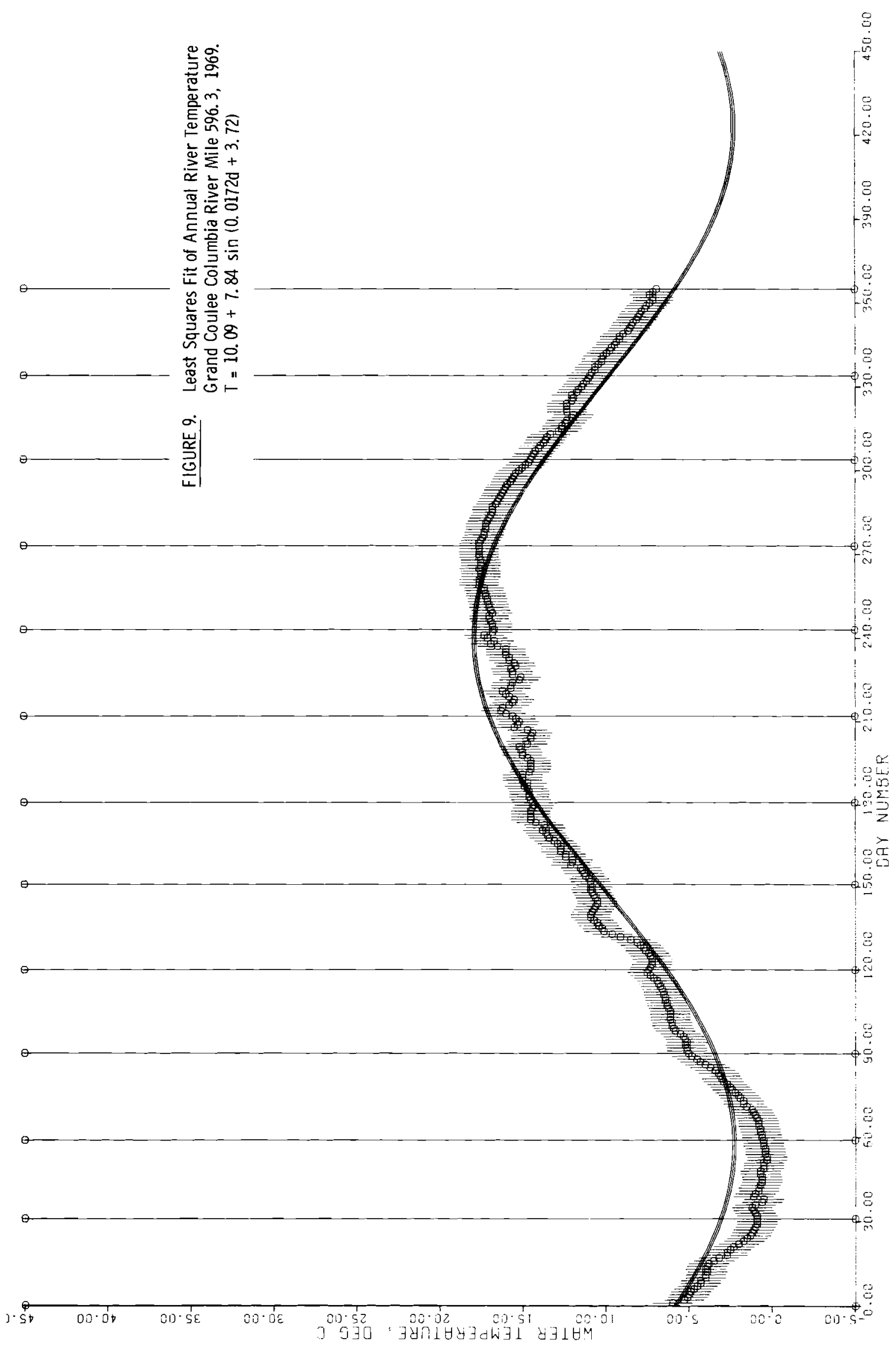

4.6 
BNWL - 1345

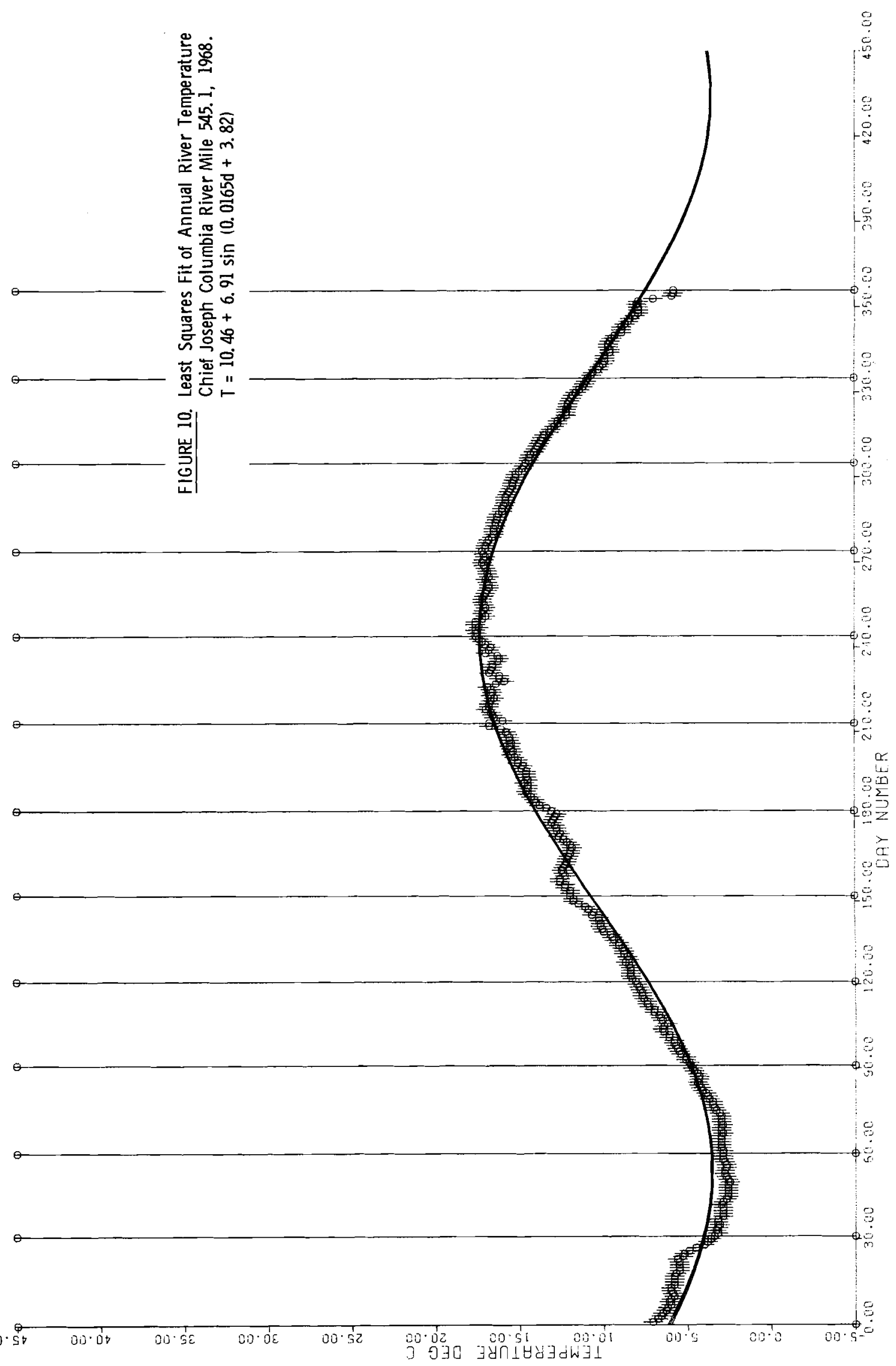

4.7 
BNWL -1345

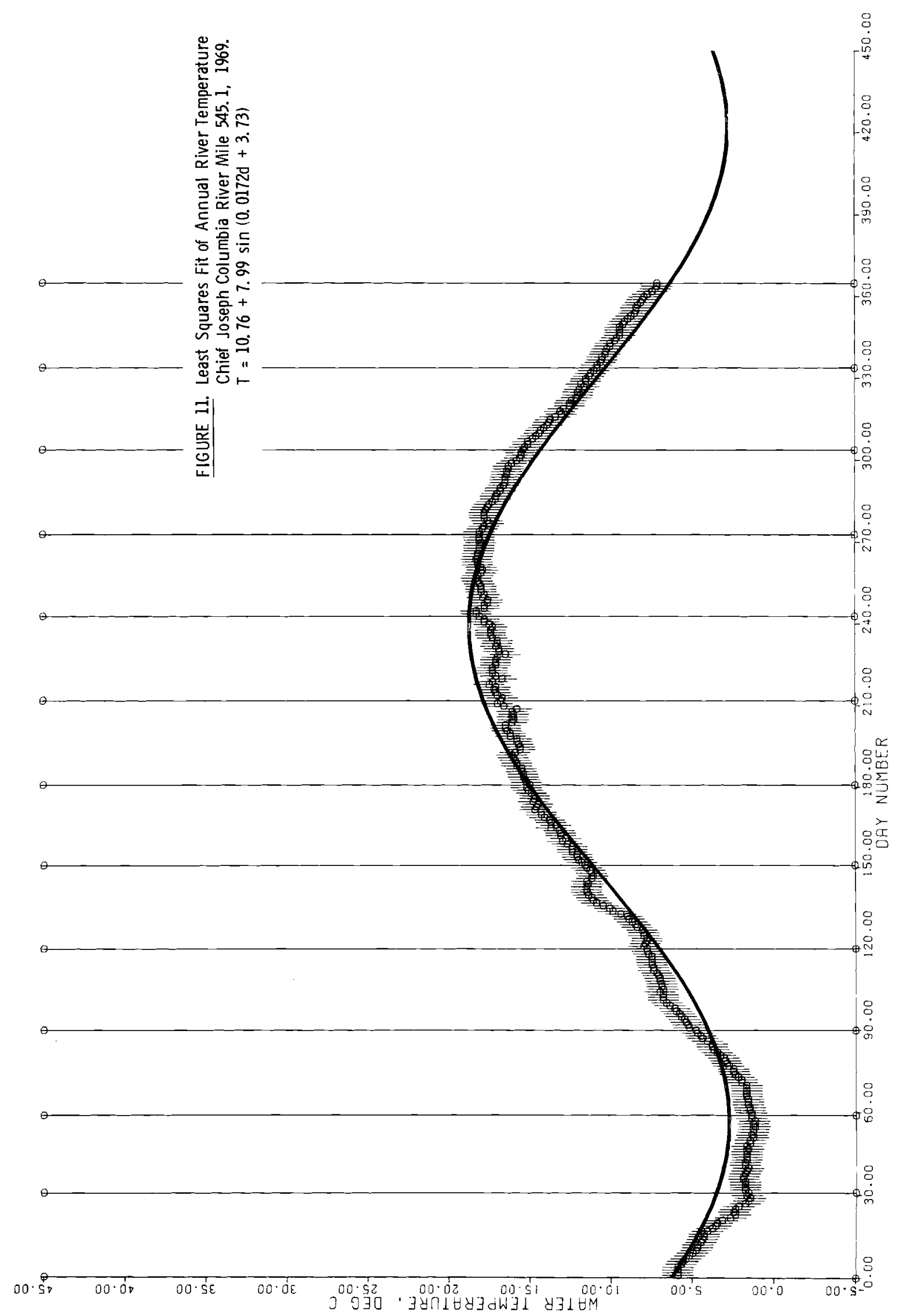


BNWL - 1345

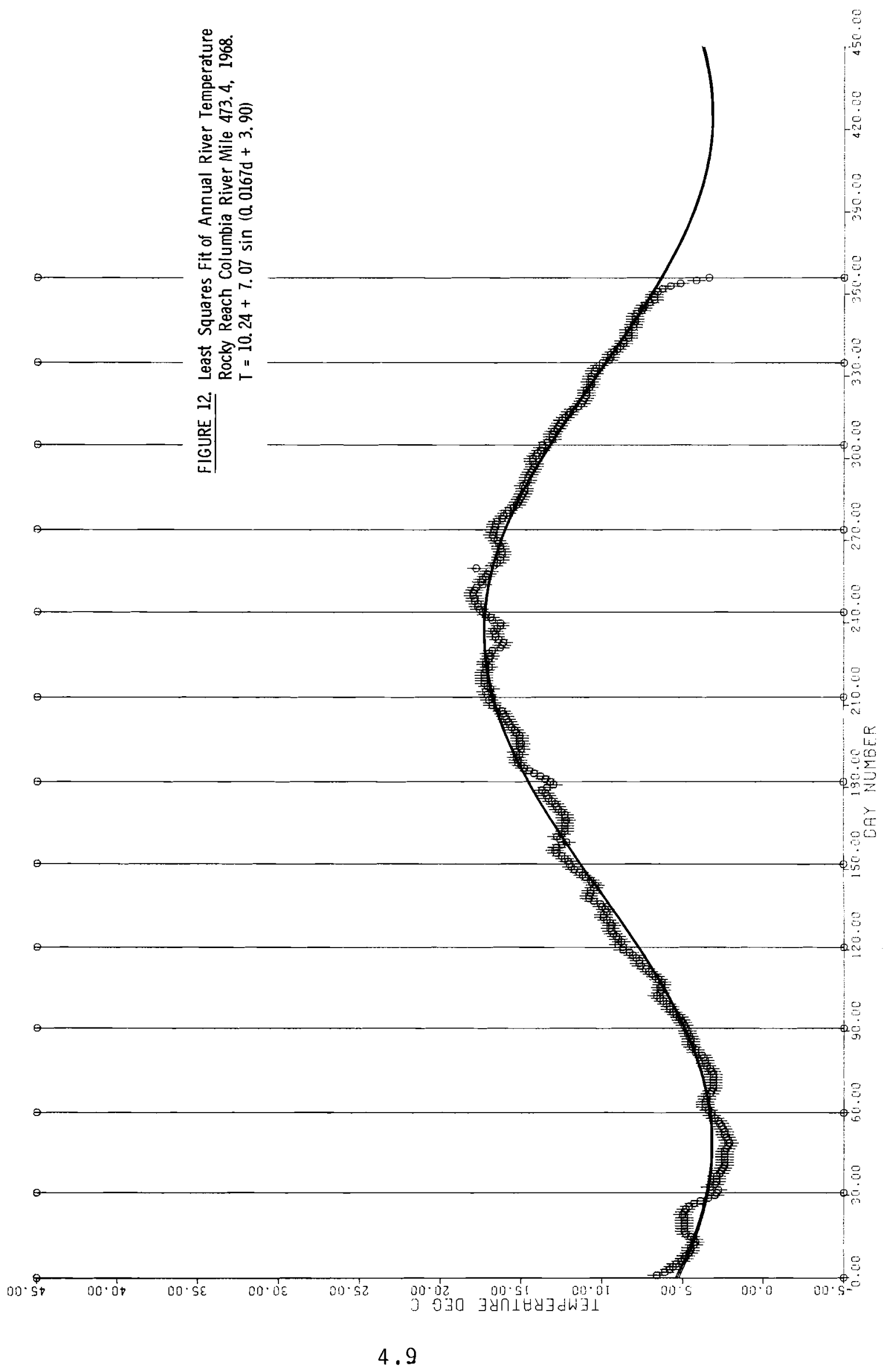


BNWL- 1345

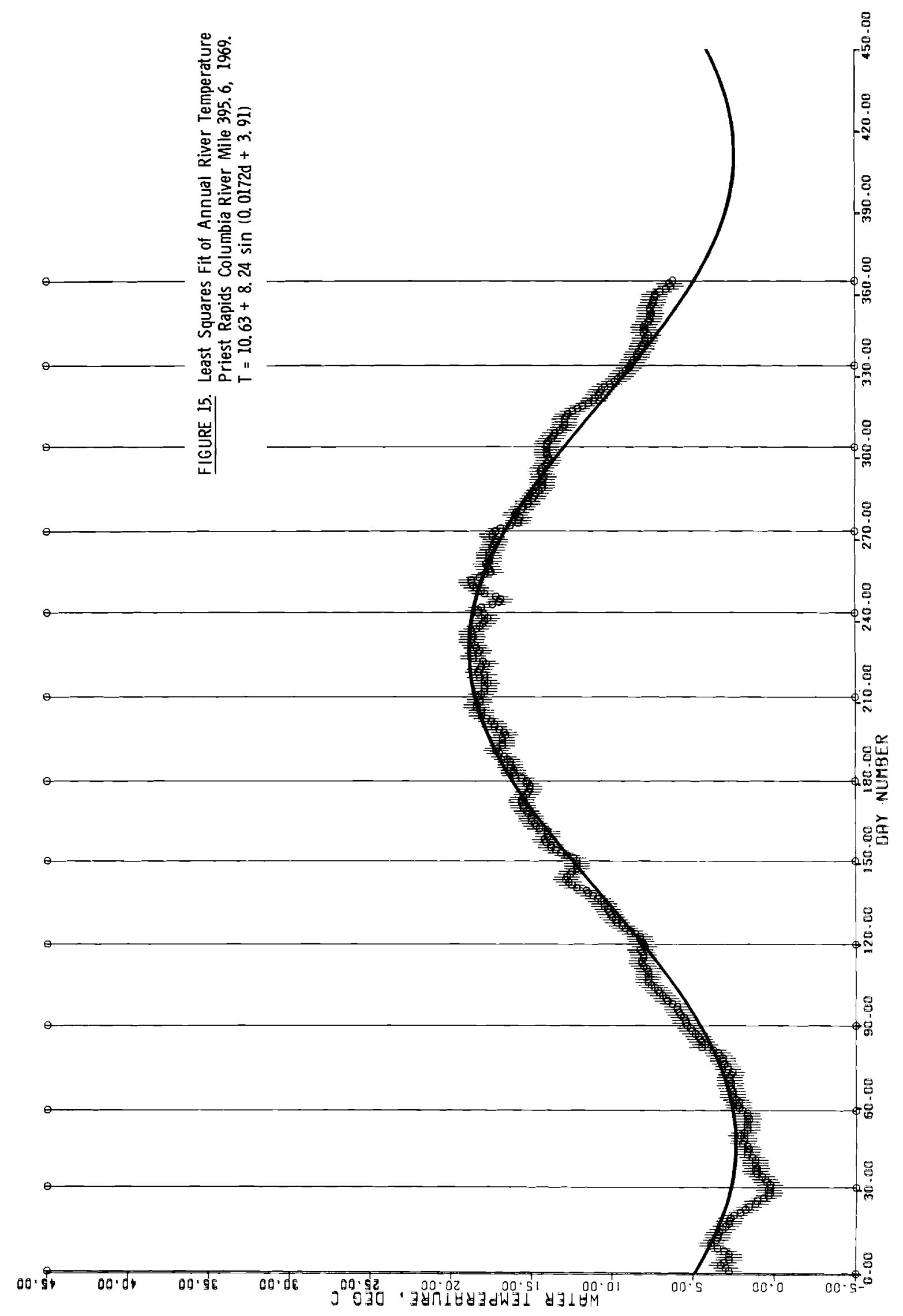




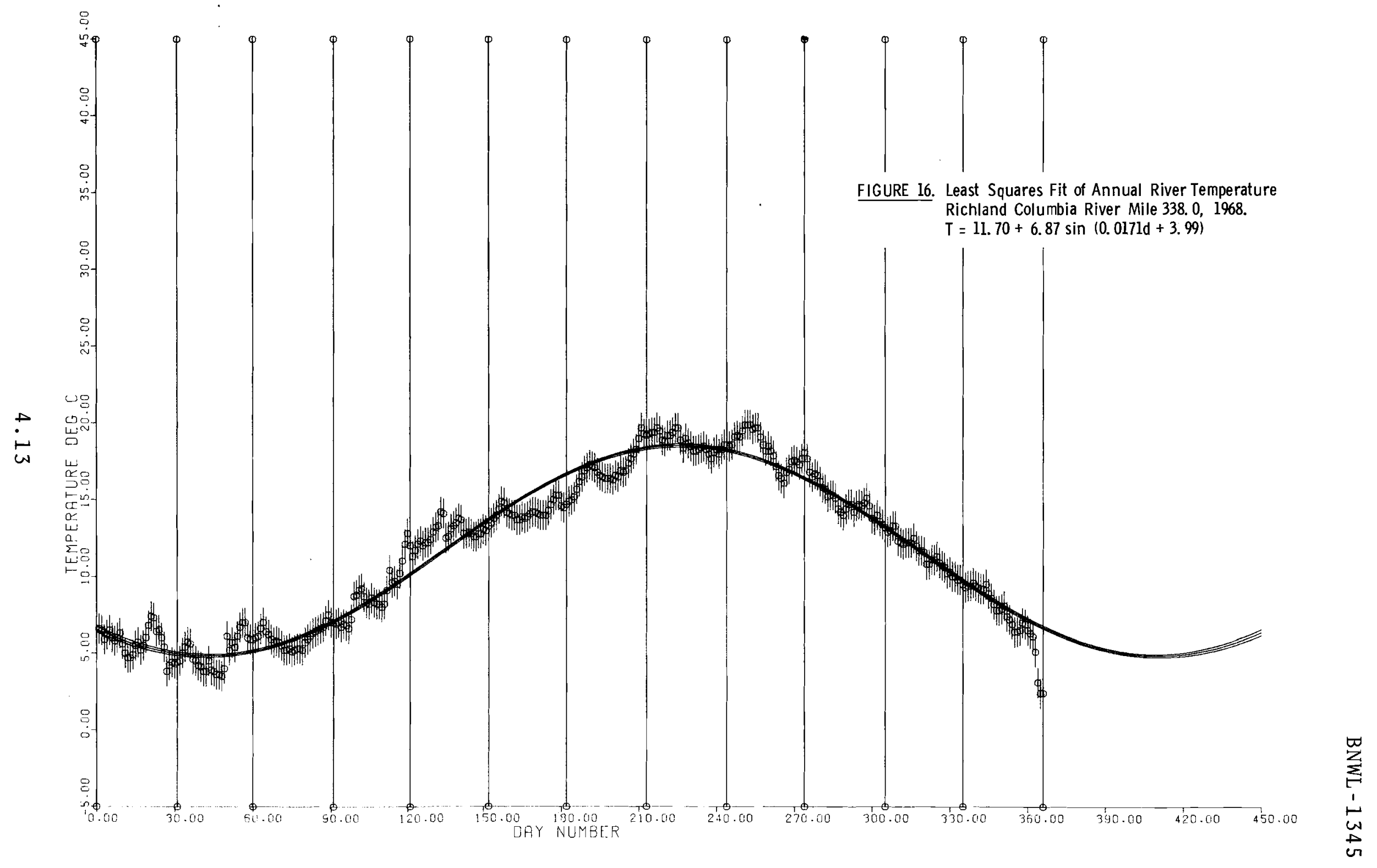


BNWL - 1345

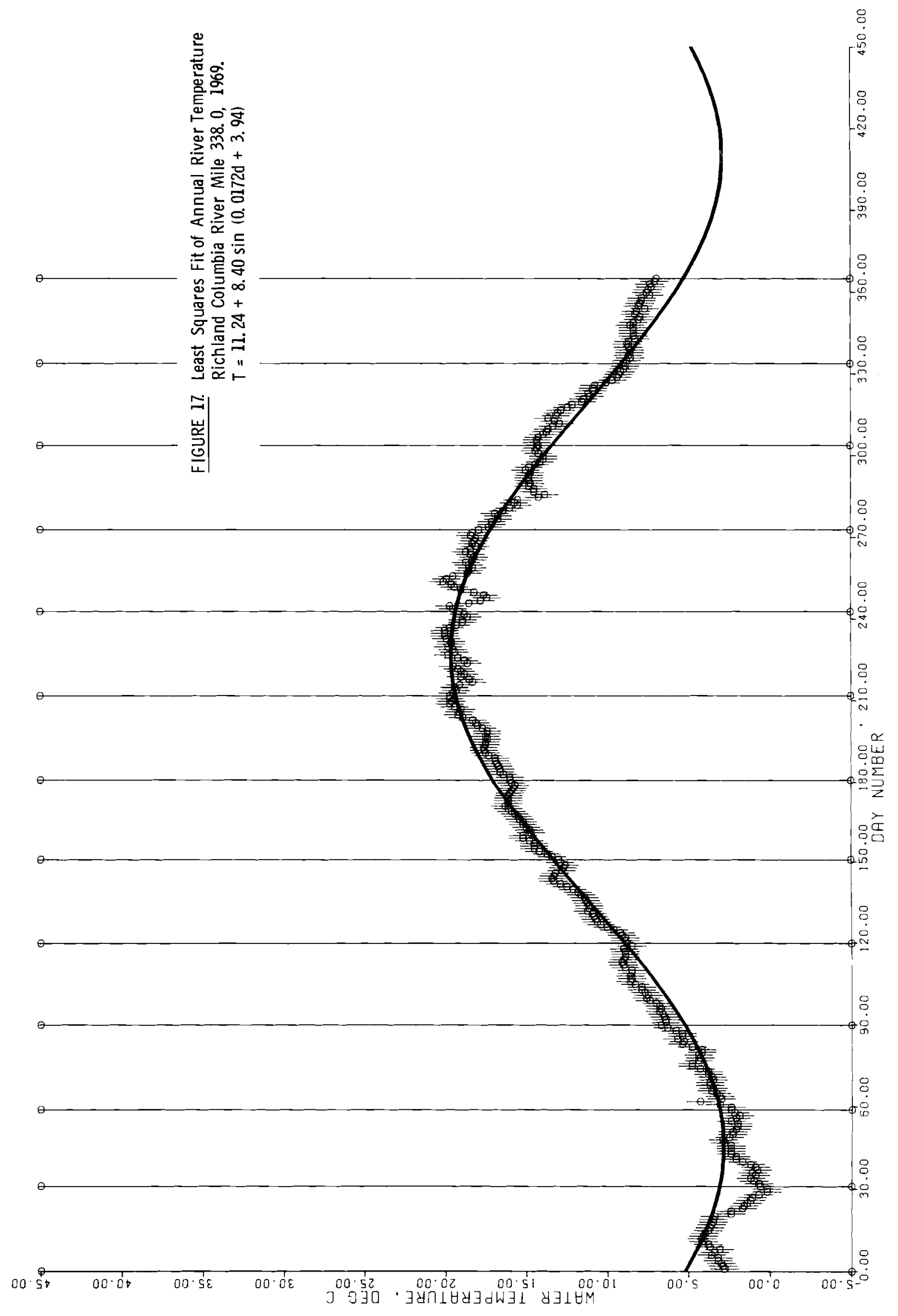


BNWL - 1345

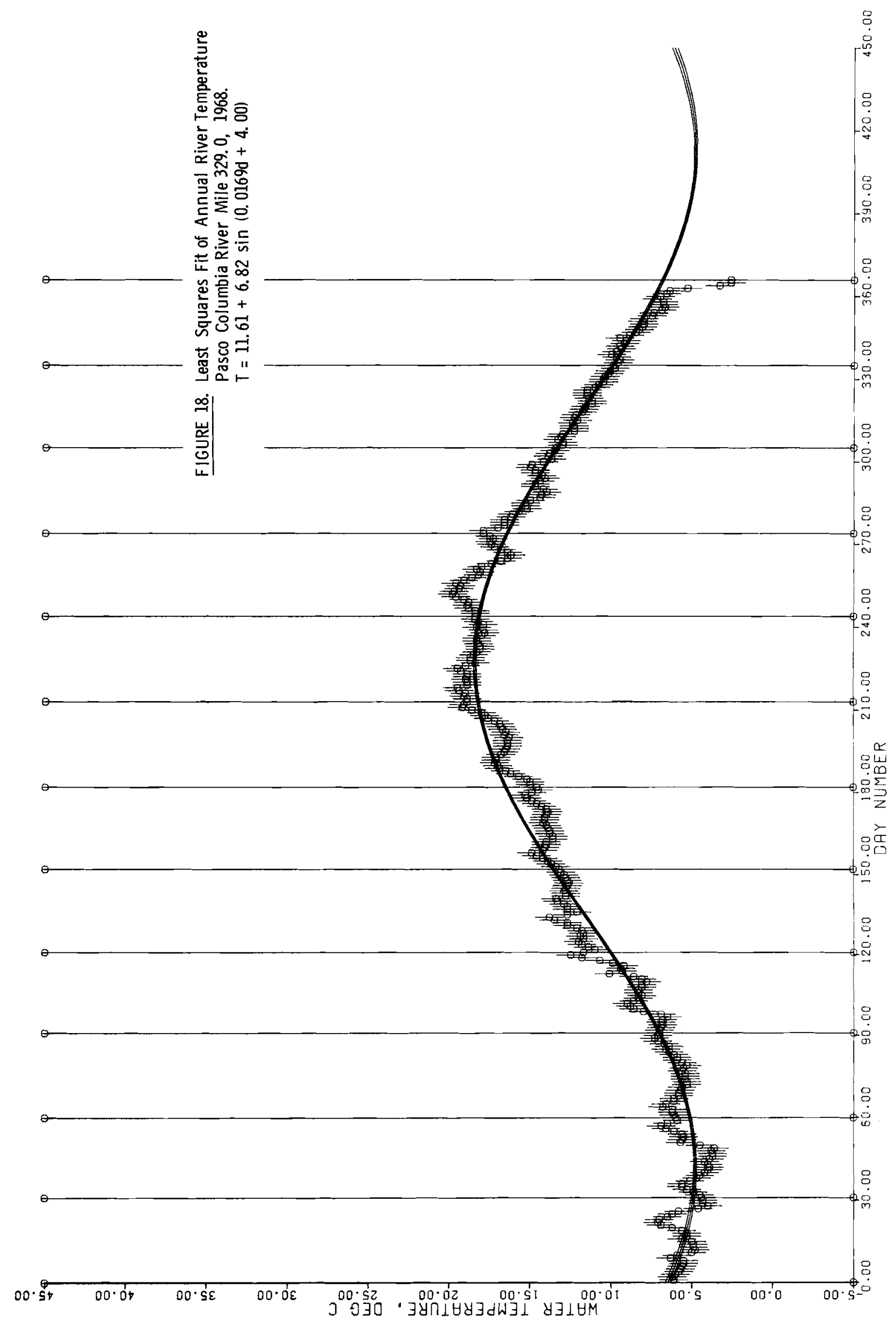




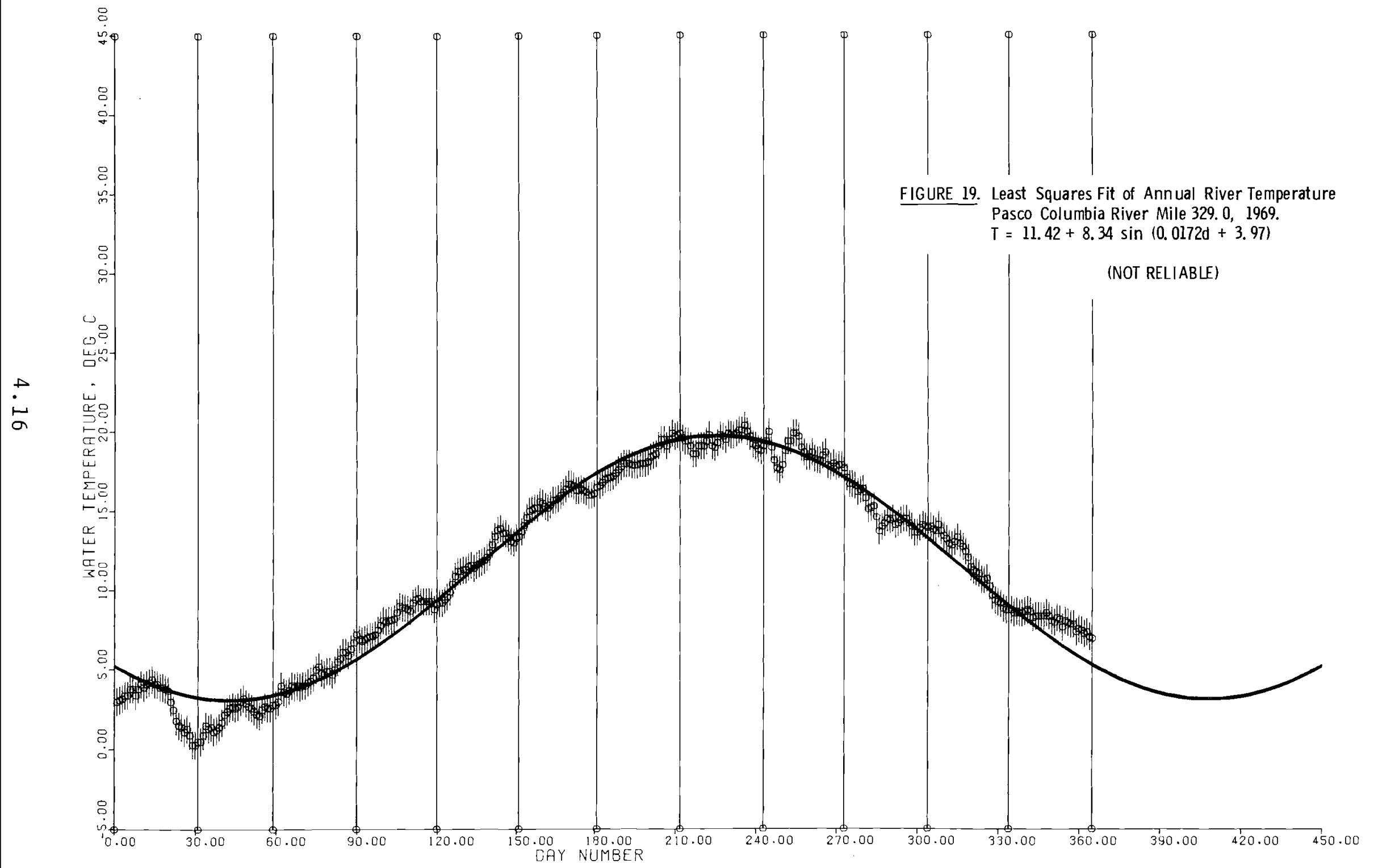

苜 


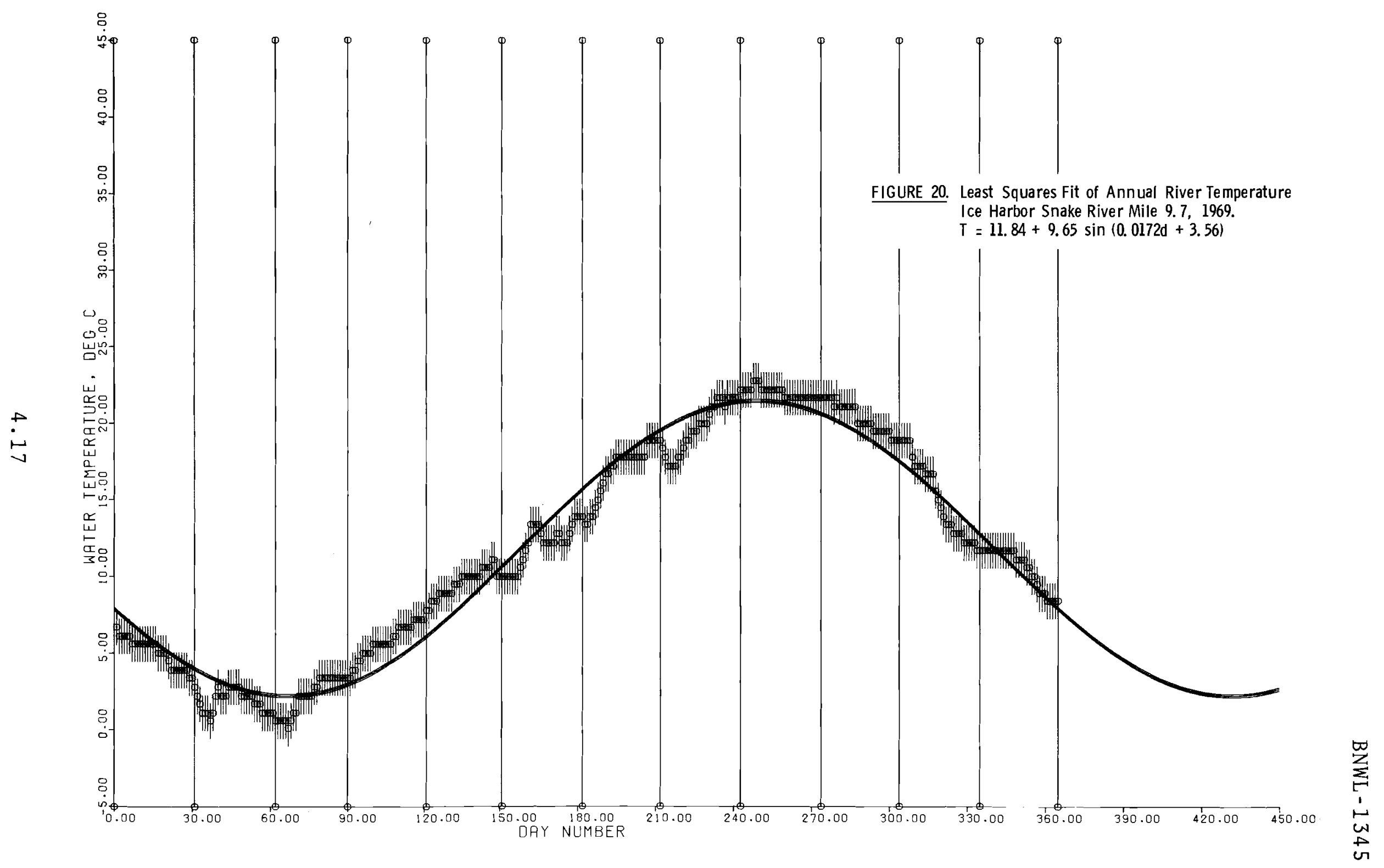


BNWL - 1345

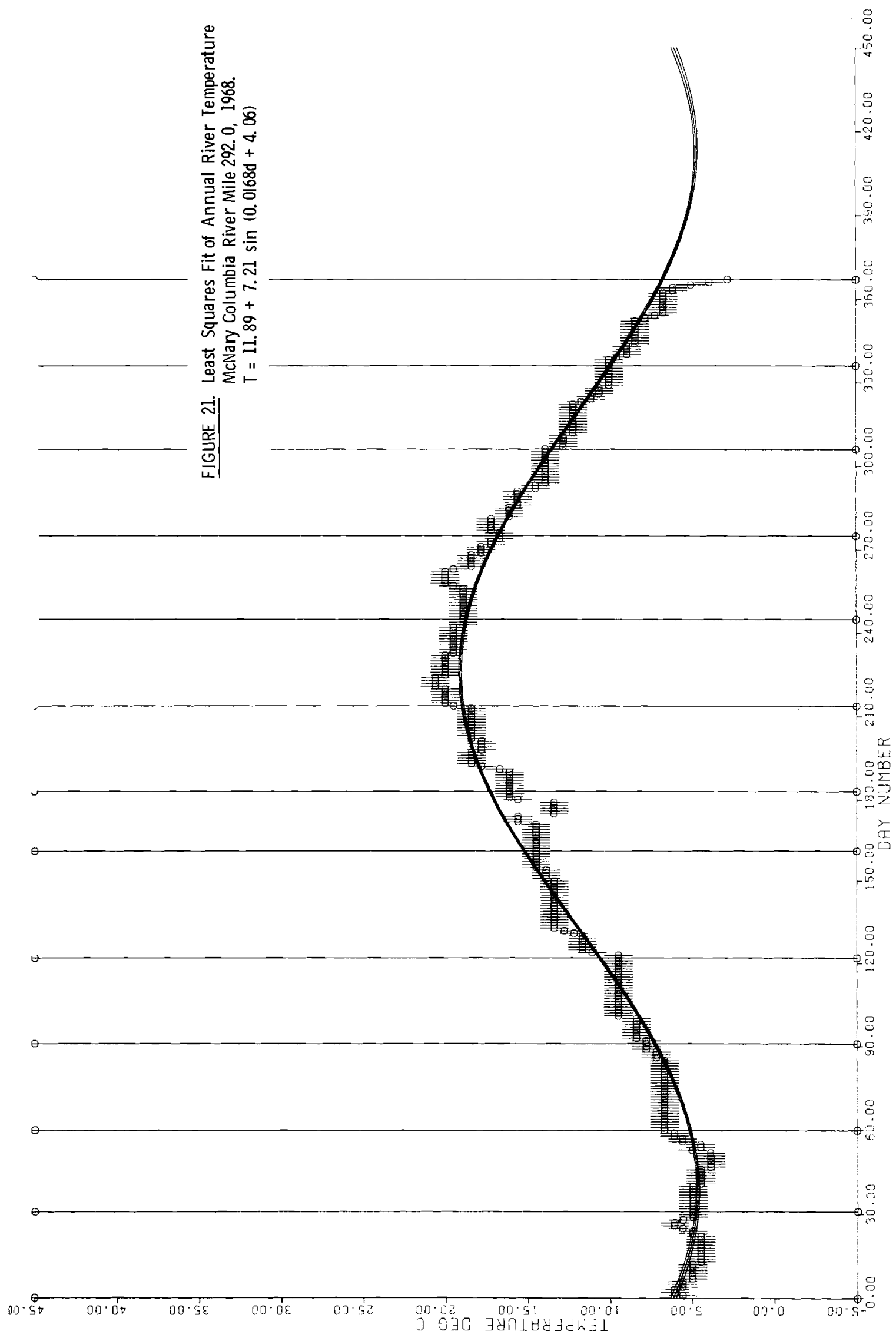

4.18 
BNWL - 1345

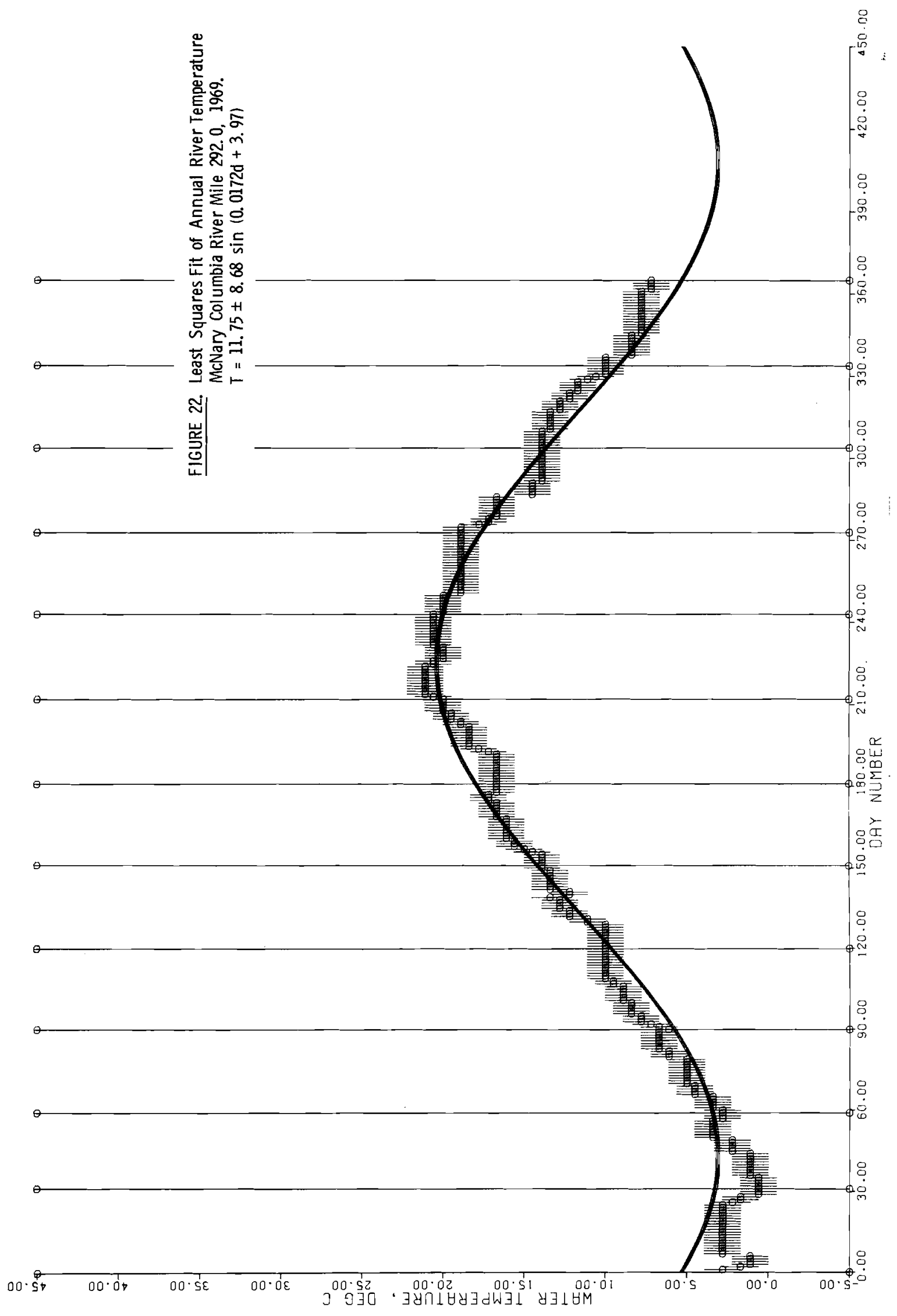

4.19 
BNWL - 1345

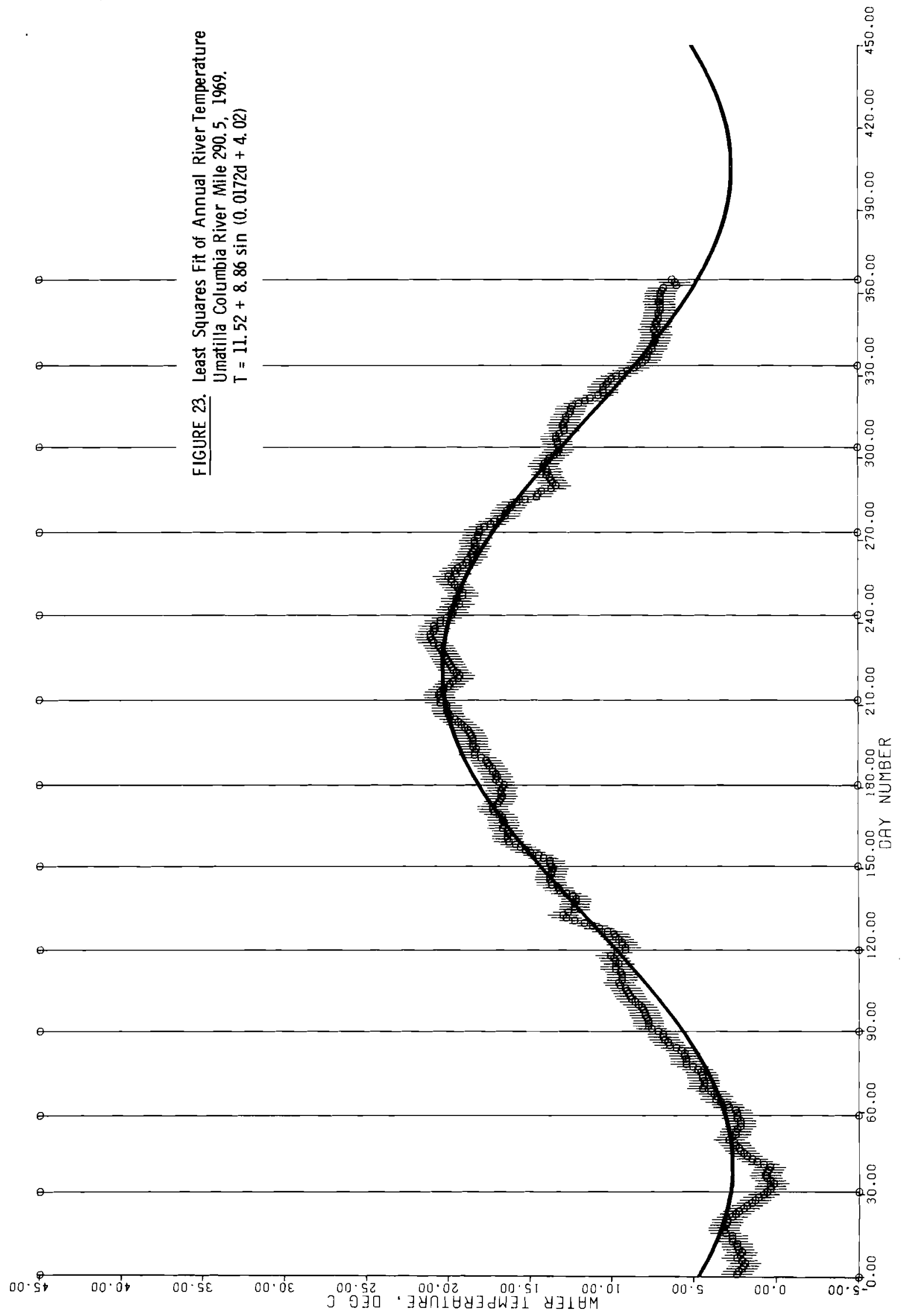


BNWL - 1345

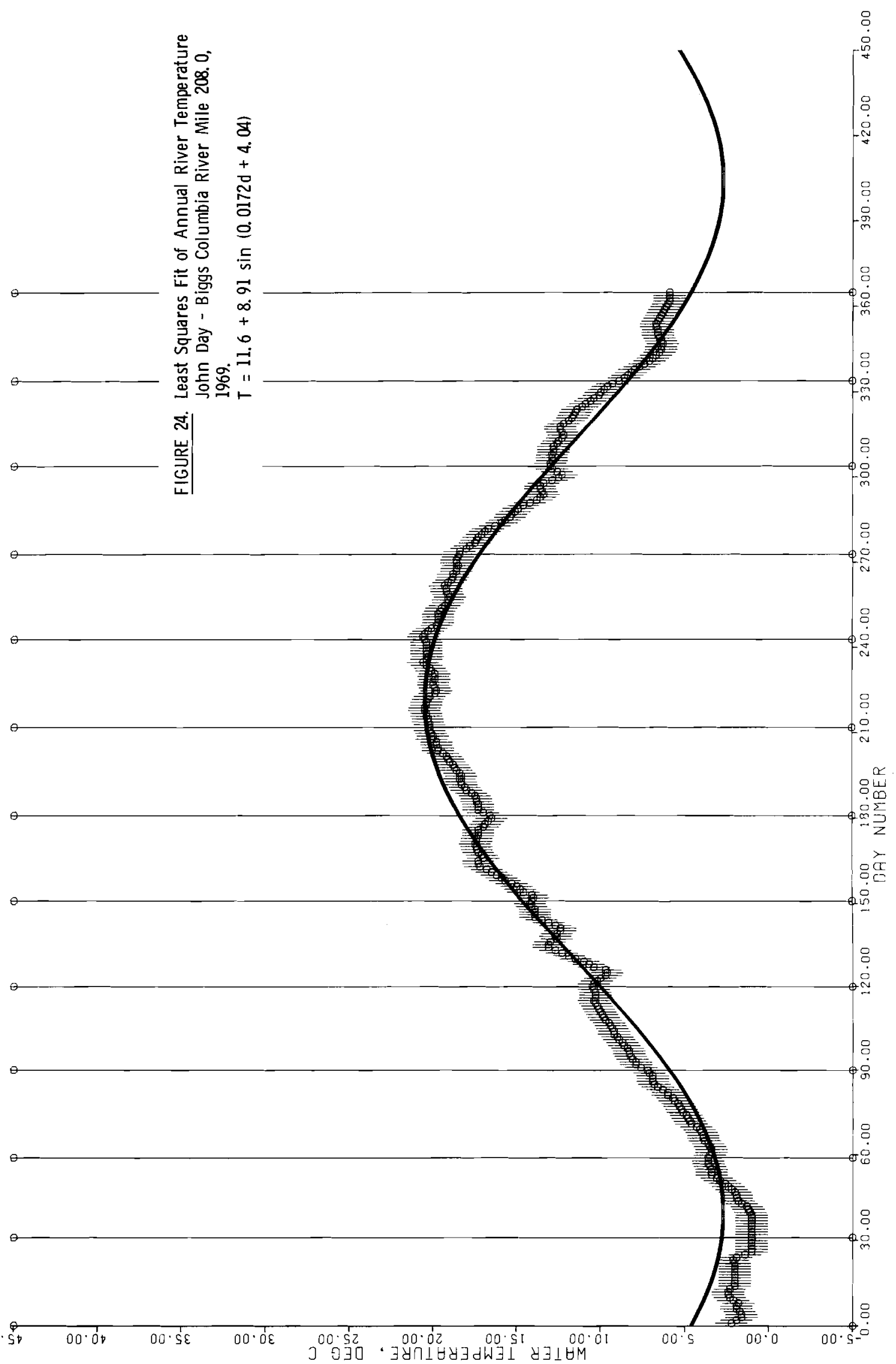

4.21 
BNWL - 1345

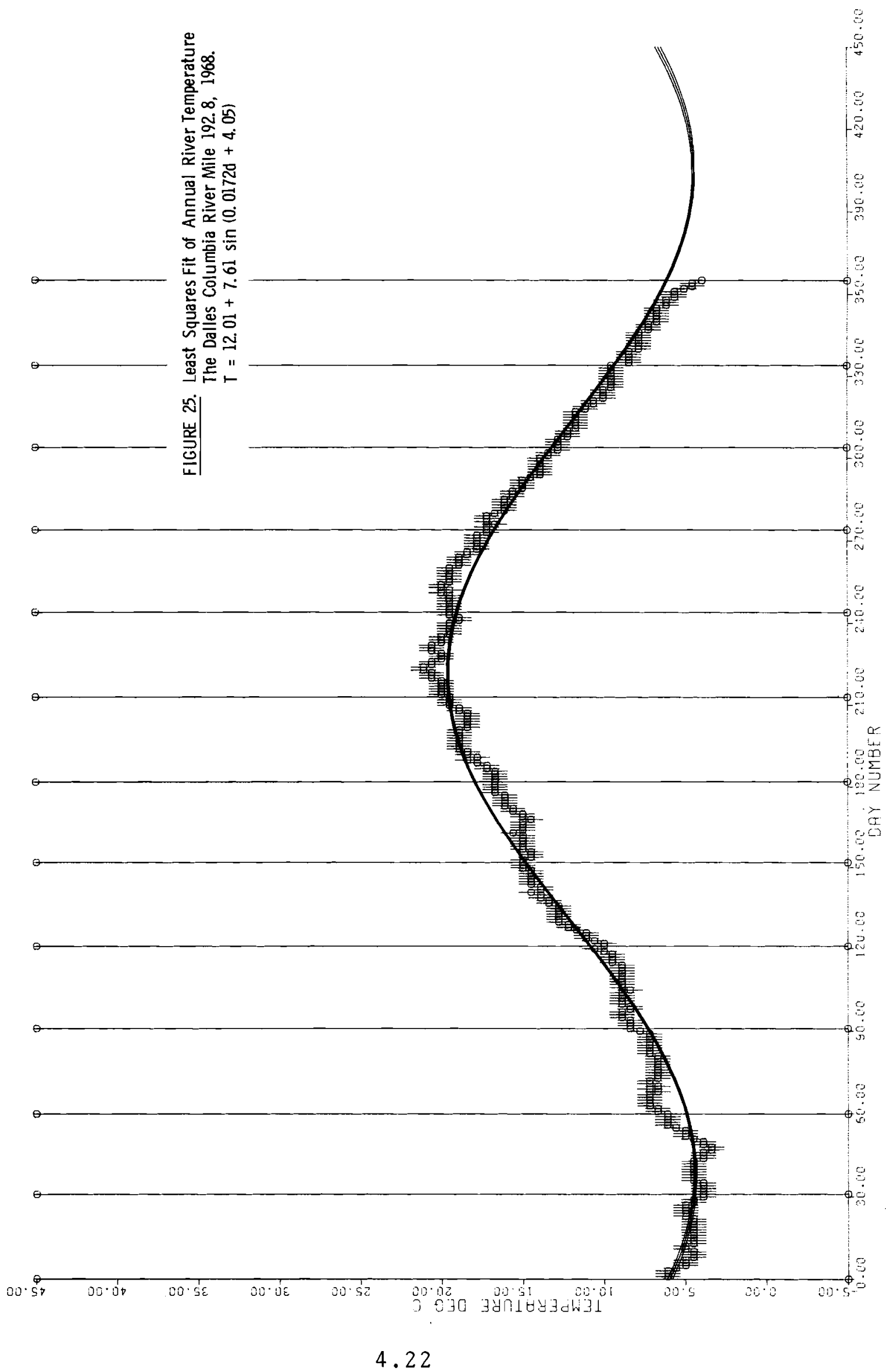


BNWL - 1345

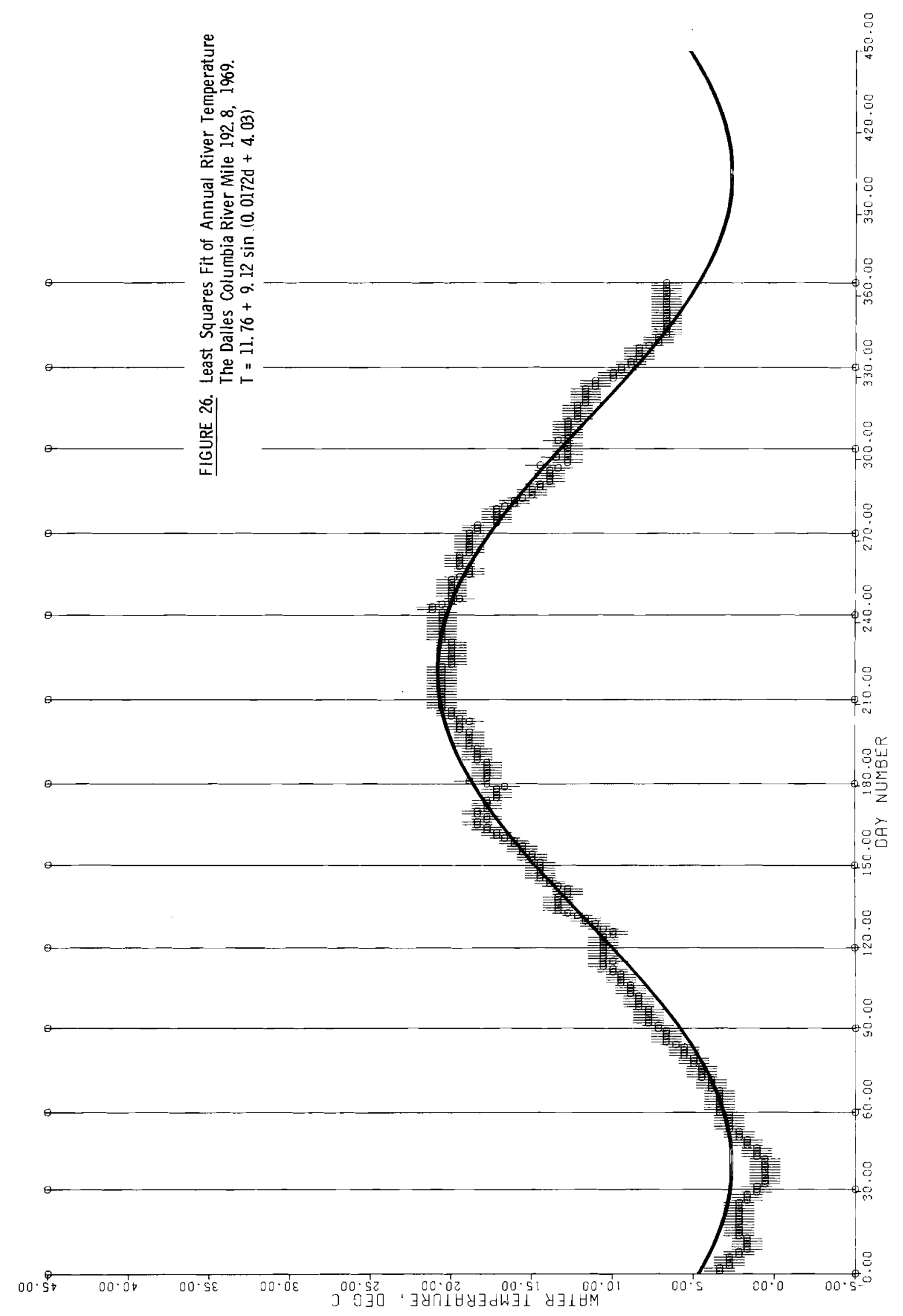


BNWL - 1345

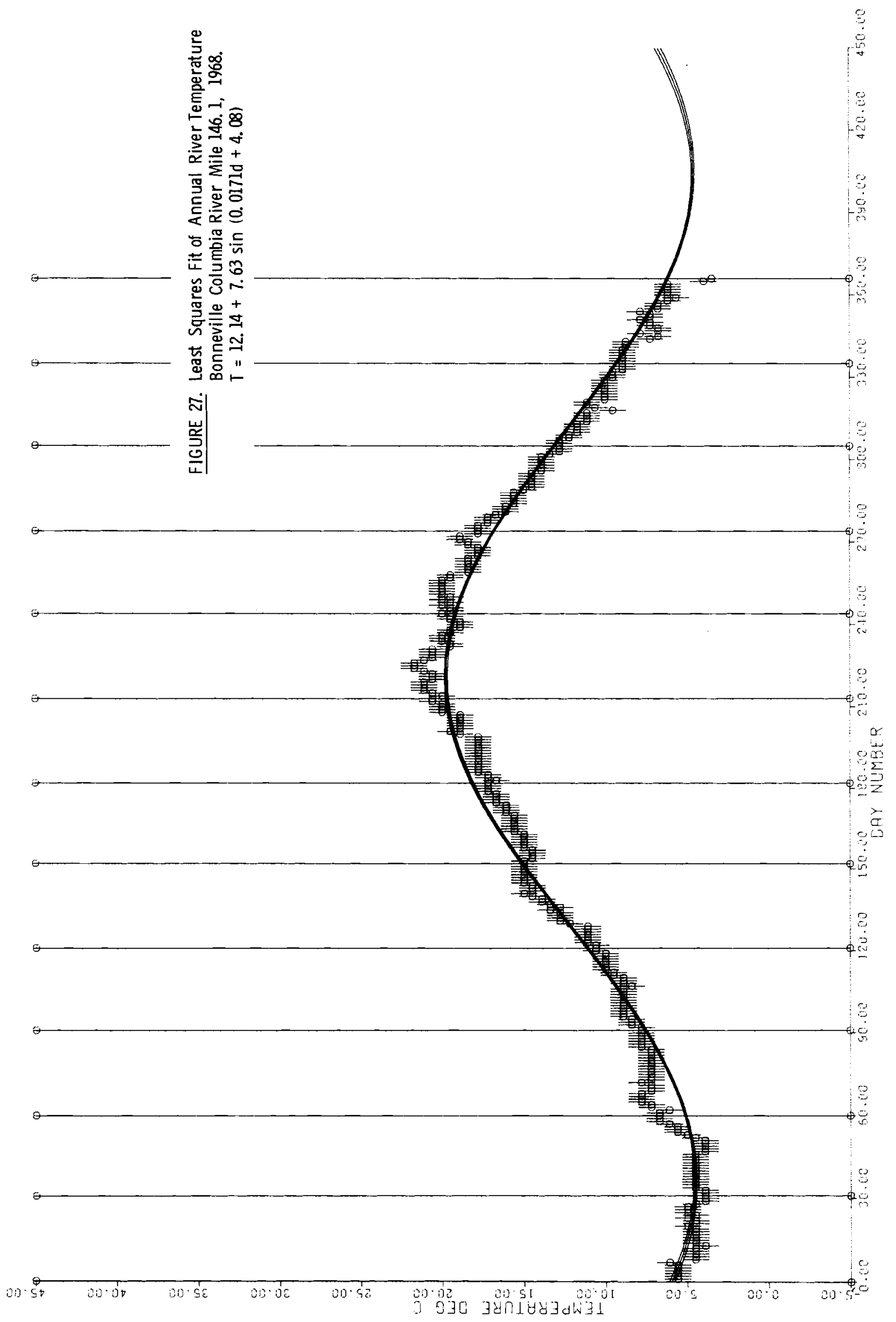

4.24 
BNWL - 1345

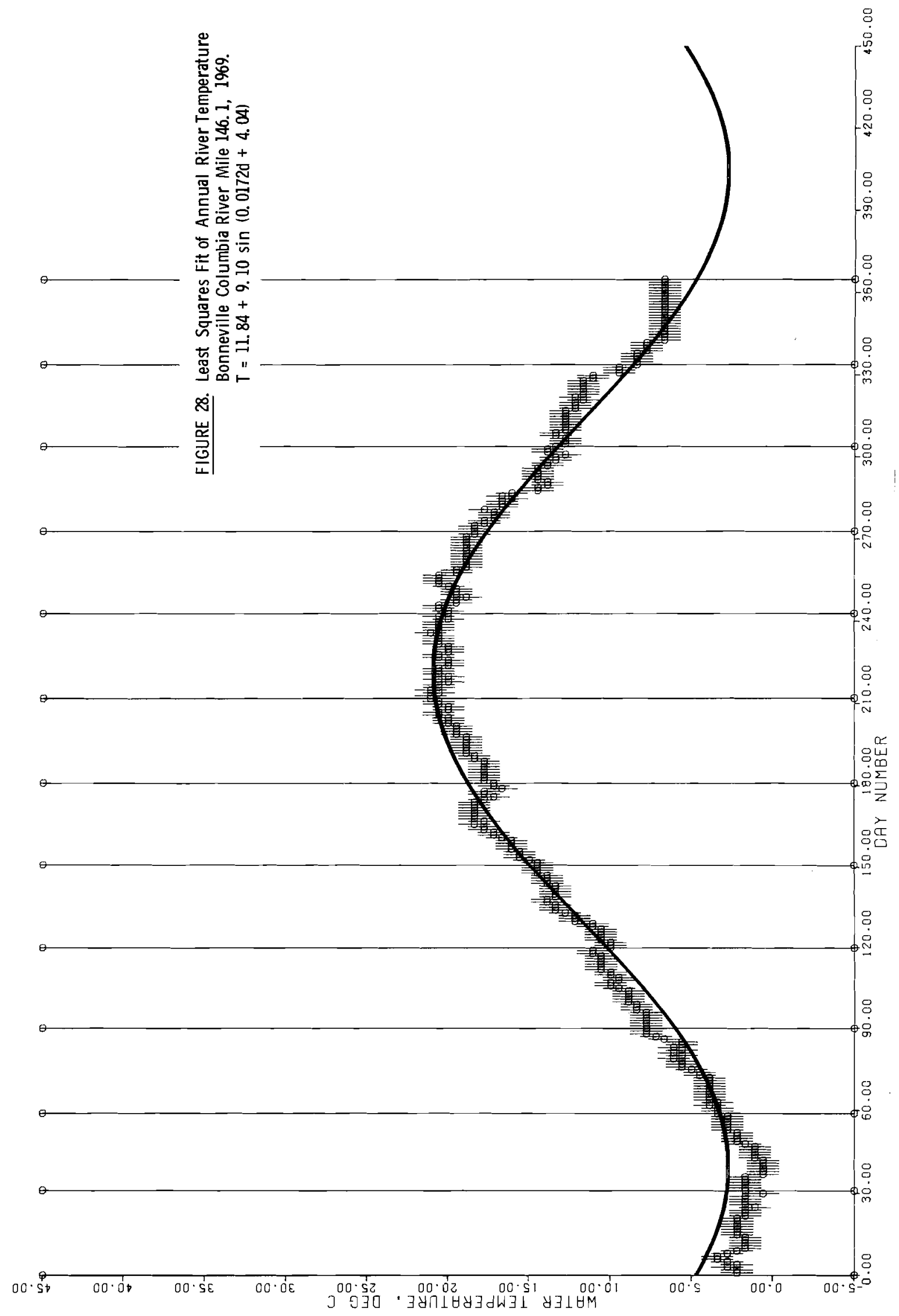

4.25 
BNWL - 1345

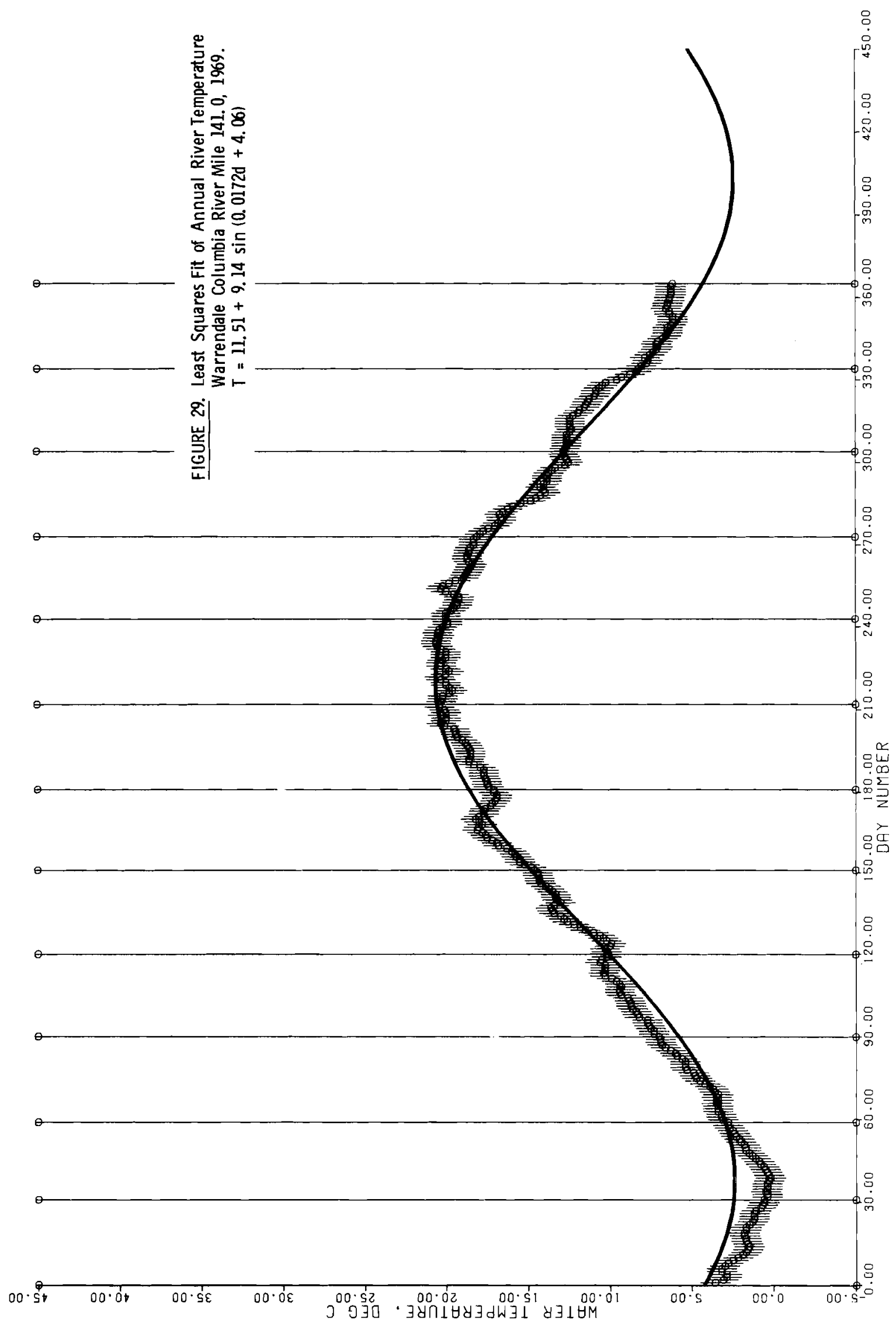


BNWL - 1345

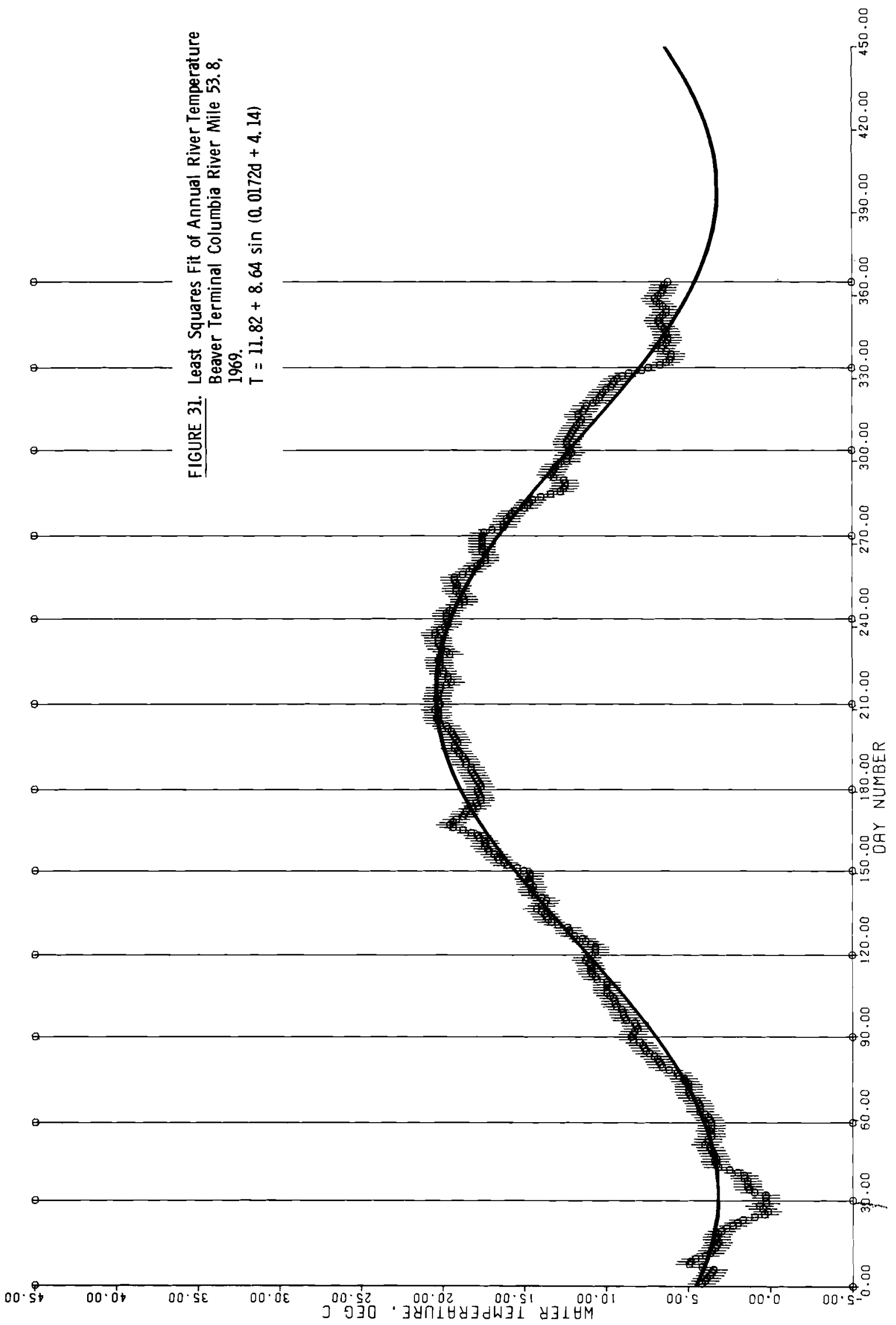

4.28 
TABLE 6. Least-Squares Fit Expressions Using the Single Function $\mathrm{T}=\overline{\mathrm{T}}+\mathrm{B}$ sin $(\mathrm{Cd}+\mathrm{D})$

\begin{tabular}{|c|c|c|c|c|c|c|}
\hline & \multicolumn{6}{|c|}{ January - December } \\
\hline & & $\begin{array}{c}\text { Temp, } \\
{ }^{\circ} \mathrm{C} \\
\end{array}$ & $\begin{array}{l}\text { Mode } 1 \\
\text { Mean } \\
\mathrm{A},{ }^{\circ} \mathrm{C} \\
\end{array}$ & $\begin{array}{l}\text { Comp. } \\
\text { Extreme } \\
\mathrm{B},{ }^{\circ} \mathrm{C} \\
\end{array}$ & $\begin{array}{l}\text { Daily } \\
\text { Shift-C } \\
\text { Radians } \\
\end{array}$ & $\begin{array}{l}\text { Peak } \\
\text { Shift-D } \\
\text { Radians } \\
\end{array}$ \\
\hline $\begin{array}{l}\text { International Border } \\
\text { (Northport) }\end{array}$ & $\begin{array}{l}1966 \\
1967 \\
1968 \\
1969\end{array}$ & $\mathrm{~T}=$ & $\begin{array}{l}9.29 \\
9.64 \\
8.83 \\
9.36\end{array}$ & $\begin{array}{l}6.66 \\
6.64 \\
6.56 \\
8.14\end{array}$ & $\begin{array}{l}0.0178 \\
0.0181 \\
0.0193 \\
0.0172\end{array}$ & $\begin{array}{l}3.70 \\
3.81 \\
4.01 \\
3.97\end{array}$ \\
\hline Grand Coulee & $\begin{array}{l}1964 \\
1965 \\
1966 \\
1967 \\
1968 \\
1969\end{array}$ & $\mathrm{~T}=$ & $\begin{array}{l}10.6 \\
10.4 \\
10.40 \\
10.98 \\
10.39 \\
10.09\end{array}$ & $\begin{array}{l}5.7 \\
7.2 \\
6.48 \\
6.95 \\
6.88 \\
7.84\end{array}$ & $\begin{array}{l}0.0180 \\
0.0164 \\
0.0165 \\
0.0173 \\
0.0166 \\
0.0172\end{array}$ & $\begin{array}{l}3.47 \\
3.75 \\
3.67 \\
3.51 \\
3.78 \\
3.92\end{array}$ \\
\hline Chief Joseph & $\begin{array}{l}1964 \\
1965 \\
1966 \\
1967 \\
1968 \\
1969\end{array}$ & $\mathrm{~T}=$ & $\begin{array}{l}10.50 \\
10.62 \\
10.71 \\
11.33 \\
10.46 \\
10.76\end{array}$ & $\begin{array}{l}6.52 \\
7.40 \\
6.51 \\
6.74 \\
6.49 \\
7.99\end{array}$ & $\begin{array}{l}0.0176 \mathrm{~d} \\
0.0165 \mathrm{~d} \\
0.0166 \mathrm{~d} \\
0.0175 \\
0.0165 \mathrm{~d} \\
0.0172\end{array}$ & $\begin{array}{l}3.46 \\
3.77 \\
3.69 \\
3.48 \\
3.82 \\
3.73\end{array}$ \\
\hline Rocky Reach & $\begin{array}{l}1964 \\
1965 \\
1966 \\
1967 \\
1968 \\
1969\end{array}$ & $\mathrm{~T}=$ & $\begin{array}{l}10.47 \\
10.46 \\
10.43 \\
11.05 \\
10.24 \\
10.45\end{array}$ & $\begin{array}{l}6.32 \\
7.29 \\
6.53 \\
6.98 \\
7.06 \\
8.26\end{array}$ & $\begin{array}{l}0.0180 \\
0.0169 \\
0.0166 \\
0.0177 \\
0.0167 \\
0.0172\end{array}$ & $\begin{array}{l}3.55 \\
3.77 \\
3.80 \\
3.58 \\
3.90 \\
3.83\end{array}$ \\
\hline Priest Rapids & $\begin{array}{l}1964 \\
1965 \\
1966 \\
1967 \\
1968 \\
1969\end{array}$ & $\mathrm{~T}=$ & $\begin{array}{l}10.57 \\
10.64 \\
10.65 \\
11.31 \\
10.56 \\
10.63\end{array}$ & $\begin{array}{l}6.48 \\
7.40 \\
6.63 \\
7.00 \\
7.04 \\
8.24\end{array}$ & $\begin{array}{l}0.0178 \\
0.0169 \\
0.0166 \\
0.0180 \\
0.0170 \\
0.0172\end{array}$ & $\begin{array}{l}3.72 \\
3.87 \\
3.95 \\
3.63 \\
3.94 \\
3.91\end{array}$ \\
\hline Richland & $\begin{array}{l}1964 \\
1965 \\
1966 \\
1967 \\
1968 \\
1969\end{array}$ & $\mathrm{~T}=$ & $\begin{array}{l}12.20 \\
12.12 \\
12.04 \\
12.63 \\
11.70 \\
11.24\end{array}$ & $\begin{array}{l}6.25 \\
6.99 \\
6.24 \\
6.69 \\
6.87 \\
8.39\end{array}$ & $\begin{array}{l}0.0176 \\
0.0172 \\
0.0163 \\
0.0183 \\
0.0171 \\
0.0172\end{array}$ & $\begin{array}{l}3.78 \\
3.86 \\
4.04 \\
3.63 \\
3.99 \\
3.94\end{array}$ \\
\hline Pasco & $\begin{array}{l}1964 \\
1965 \\
1966 \\
1967 \\
1968 \\
1969\end{array}$ & $\mathrm{~T}=$ & $\begin{array}{l}11.80 \\
11.88 \\
11.94 \\
12.53 \\
11.61 \\
11.42\end{array}$ & $\begin{array}{l}6.09 \\
7.27 \\
6.34 \\
6.67 \\
6.82 \\
8.34\end{array}$ & $\begin{array}{l}0.0177 \\
0.0170 \\
0.0164 \\
0.0185 \\
0.0169 \\
0.0172\end{array}$ & $\begin{array}{l}3.78 \\
3.90 \\
4.02 \\
3.60 \\
4.00 \\
3.97\end{array}$ \\
\hline Ice Harbor & $\begin{array}{l}1964 \\
1965 \\
1966 \\
1967 \\
1968 \\
1969\end{array}$ & $\mathrm{~T}=$ & $\begin{array}{l}11.31 \\
11.78 \\
12.41 \\
12.87 \\
12.09 \\
11.83\end{array}$ & $\begin{array}{l}8.53 \\
8.39 \\
9.08 \\
9.13 \\
8.59 \\
9.65\end{array}$ & $\begin{array}{l}0.0181 \\
0.0173 \\
0.0175 \\
0.0186 \\
0.0180 \\
0.0172\end{array}$ & $\begin{array}{l}3.83 \\
4.01 \\
4.00 \\
3.72 \\
4.00 \\
3.56\end{array}$ \\
\hline
\end{tabular}


BNWL - 1345

TABLE 6. (contd)

\begin{tabular}{|c|c|c|c|c|c|c|}
\hline & & & & & & \\
\hline & & $\begin{array}{l}\text { Temp, } \\
{ }^{\circ} \mathrm{C}\end{array}$ & $\begin{array}{l}\text { Mode I } \\
\text { Mean } \\
\mathrm{A},{ }^{\circ} \mathrm{C} \\
\end{array}$ & $\begin{array}{l}\text { Comp. } \\
\text { Extreme } \\
\mathrm{B},{ }^{\circ} \mathrm{C} \\
\end{array}$ & $\begin{array}{l}\text { Daily } \\
\text { Shift-C } \\
\text { Radians } \\
\end{array}$ & $\begin{array}{l}\text { Peak } \\
\text { Shift-D } \\
\text { Radians } \\
\end{array}$ \\
\hline McNary & $\begin{array}{l}1964 \\
1965 \\
1966 \\
1967 \\
1968 \\
1969\end{array}$ & $\begin{array}{l}T= \\
T= \\
T= \\
T= \\
T= \\
T=\end{array}$ & $\begin{array}{l}11.92 \\
11.97 \\
12.21 \\
12.90 \\
11.89 \\
11.75\end{array}$ & $\begin{array}{l}6.88 \\
7.64 \\
7.16 \\
7.33 \\
7.21 \\
8.69\end{array}$ & $\begin{array}{l}0.0178 \\
0.0165 \\
0.0165 \\
0.0182 \\
0.0168 \\
0.0172\end{array}$ & $\begin{array}{l}3.79 \\
4.09 \\
4.09 \\
3.67 \\
4.06 \\
3.97\end{array}$ \\
\hline Umatilla & 1969 & $T=$ & 11.52 & 8.86 & 0.0172 & 4.02 \\
\hline John Day - Biggs & 1969 & $T=$ & 11.60 & 8.91 & 0.0172 & 4.04 \\
\hline The Dalles & $\begin{array}{l}1964 \\
1965 \\
1966 \\
1967 \\
1968 \\
1969\end{array}$ & $\begin{array}{l}T= \\
T= \\
T= \\
T= \\
T=\end{array}$ & $\begin{array}{l}11.70 \\
11.87 \\
12.12 \\
12.91 \\
12.01 \\
11.76\end{array}$ & $\begin{array}{l}7.03 \\
7.74 \\
7.16 \\
7.56 \\
7.61 \\
9.12\end{array}$ & $\begin{array}{l}0.0178 \\
0.0166 \\
0.0166 \\
0.0182 \\
0.0172 \\
0.0172\end{array}$ & $\begin{array}{l}3.90 \\
4.11 \\
4.14 \\
3.75 \\
4.05 \\
4.03\end{array}$ \\
\hline Bonneville Dam & $\begin{array}{l}1964 \\
1965 \\
1966 \\
1967 \\
1968 \\
1969\end{array}$ & $\begin{array}{l}T= \\
T= \\
T= \\
T= \\
T= \\
T=\end{array}$ & $\begin{array}{l}11.62 \\
11.75 \\
11.93 \\
12.99 \\
12.14 \\
11.84\end{array}$ & $\begin{array}{l}6.97 \\
7.70 \\
7.20 \\
7.46 \\
7.63 \\
9.11\end{array}$ & $\begin{array}{l}0.0180 \\
0.0165 \\
0.0165 \\
0.0183 \\
0.0171 \\
0.0172\end{array}$ & $\begin{array}{l}3.89 \\
4.15 \\
4.17 \\
3.74 \\
4.08 \\
4.04\end{array}$ \\
\hline Warrendale & $\begin{array}{l}1968 \\
1969\end{array}$ & $T=$ & $\begin{array}{l}11.81 \\
11.51\end{array}$ & $\begin{array}{l}7.8 \\
9.14\end{array}$ & $\begin{array}{l}0.0170 \\
0.0172\end{array}$ & $\begin{array}{l}4.12 \\
4.06\end{array}$ \\
\hline Longview & 1969 & $\mathrm{~T}=$ & 11.51 & 8.23 & 0.0172 & 4.11 \\
\hline Beaver Terminal & 1969 & $\mathrm{~T}=$ & 11.82 & 8.64 & 0.0172 & 4.14 \\
\hline
\end{tabular}

January - December 


\section{ACKNOWLEDGEMENT}

An attempt to summarize the salient features of a program as long as the Division of Production supported study 02501010 , "Effects of Reactor Effluent on the Quality of Columbia River Water," would require a great deal of painstaking search to discover all of the research effort which should be individually acknowledged. In the present instance, which is limited to a summary of the temperature data alone, a great number of persons made contributions. The collection of meaningful data on a major river in al 1 sorts of weather, good and bad, and in many seasons of the year entails sacrifices often overlooked in the eagerness to derive meaningful conclusions of significance to an immediate problem. It is hoped that the information about the Columbia River which has been derived from this program is useful to the scientific community and of lasting value to persons seeking to optimize the value of the Columbia for the good of society.

The following persons because of unique circumstances made significant contributions in leadership and personal participation to this program.

R. F. Foster, who, recognizing the future necessity to create a meaningful relation of plant activities to the water quality of the Columbia River, initiated the program and was the person in charge until recent years.

J. P. Corley, in various capacities as research supervisor, principal investigator and consultant, collected the majority of data in the Hanford reach of the plant. This work involved great sacrifices of a personal nature in terms of time, discomfort and potential hazard to ensure consistency and confidence in the numerical information achieved. 
J. K. Soldat and R. B. HaZz, who, because of related work on radionuclide studies, rendered invaluable assistance in various aspects of the program.

C. T. Vaughn and his co-workers at the Grand coulee Project who through the years were most helpful in making special arrangements and data collections.

The total staff of the Regional Monitoring organizations including $W$. C. Horton, the present working leader, who provided personnel, equipment, and helpful assistance.

D. G. Daniels, J. R. Eliason, H. P. Foote and J. C. Sonnichsen who worked as an effective team in bringing advanced methods of remote imagery into effective use and ran the critical experiments which resulted in the information summarized in Appendix $C$.

D. A. Kottwitz who displayed unusual care, patience and diligence in the development of analytical programs without which the conclusions and observations of this report would not be possible.

To al1 persons, mentioned or not, who in the course of the past eight year gave willing assistance in order that this body of information be the state-of-the-art in the development of a factual data base. 
BNWL 1345

\section{REFERENCES AND BIBLIOGRAPHY}

\section{CITED REFERENCES}

1. R. T. Jaske and J. L. Spurgeon. "A Special Case, Thermal Digital Simulation of Waste Heat Discharges," Water Research, vol. 2, pp. 777-802. 1968.

2. J. C. Ward. "Anrual Variation of Stream Water Temperature," J. Sanit, Div., Am. Soc. Civil Engrs. December 1963.

3. R. T. Jaske and $J . B$. Geobel. "Effects of Dam Construction on Temperatures of Columbia River," J. Am. Water Works Assoc., vol. 59, no. 8, pp. 935-942. August 1967.

4. R. T. Jaske. "Thermal Modification of River Water Quality," Proceedings of the Sixth Biennial WSU Hydraulics Conference, Moscow, Idaho, November 30, 1967.

5. R. T. Jaske and D. G. Daniels. A Simulation of the Effects of the Hanford Plant at the Washington-oregon Border, BNWL-1344. Battelle-Northwest, Richland, Washington, August 1970.

6. R. T. Jaske. An Evaluation of the Use of Selective Discharges to Cool the Columbia River, BNWL-208. Batteile-Northwest, Richland, Washington, March 15, 1966. 
BNWL - 1345

\section{BIBLIOGRAPHY}
$B N W C-82$
J. P. Corley. Future River Temperatures at 181-N. May 11, 1965. (SECRET)
$B N W L-20$
R. T. Jaske. The Density Current Regime of Frankiin
D. Roosevelt Lake. March 16, 1965 .
$B N W L-390$
B. Duane. Maximum Likelihood Nonlinear Correlated Fields, (ProgramLikely). September 1967.
$B N W L-628$
R. T. Jaske. A Test Simulation of the Temperatures of the Deerfield River. December 1967 .
$B N W L-733$
R. T. Jaske. A Preliminary Evaluation of the Thermal Effects of the Ben Franklin Dam Project on Columbia River Temperatures Below the Hanford Plant. April 1968.

$B N W L-728$

R. T. Jaske. A Test Simulation of the Temperature of the Illinois River and a Prediction of the Effects of Dresden II and Dresden III Reactors. April 1968 .

$B N W L-999$

D. E. Peterson and R. T. Jaske. A Test Simulation of Potential Effects of Thermal Power Plants on Streams in the Upper Mississippi River Basin. December 1968 .

$B N W L-1228$

R. A. Burnett and D. G. Daniels. Evaluation of the Simpl-1 Hybrid Computer Concept on a Water Quality Benchmark Problem. November 1969.

\begin{tabular}{|c|c|}
\hline$B N W L-1277$ & $\begin{array}{l}\text { D. E. Peterson and } R \text {. T. Jaske. Simulation Mod- } \\
\text { eing of Thermal Effiuent in an Irrigation System. } \\
\text { January } 1970 .\end{array}$ \\
\hline$B N W L-1299$ & $\begin{array}{l}\text { D. E. Peterson and } R \text {. T. Jaske. Potential Thermal } \\
\text { Effects of an Expanding Power Industry: Ohio } \\
\text { River Basin I. February } 1970 .\end{array}$ \\
\hline$B N W L-1342$ & $\begin{array}{l}\text { D. G. Daniels, J. C. Sonnichsen and } R \text {. T. Jaske. } \\
\text { Simulation of Thermal Effluent Discharges in } \\
\text { Estuaries. June } 1970 .\end{array}$ \\
\hline$B N W L-1343$ & $\begin{array}{l}\text { R. W. Legan and } R \text {. T. Jaske. A Cost Analysis of } \\
\text { Improved Regulation of Columbia River to Increase } \\
\text { Minimum Flow in the Hanford Plant Vicinity. }\end{array}$ \\
\hline
\end{tabular}


EIV 201405

$B N W L-C C-492$

BNWL-CC-656

$B N W L-C C-807$

$B N W L-C C-979$
D. E. Peterson and R. T. Jaske. Potential Thermal Effects of an Expanding Power Industry: Upper Mississippi Basin. June 1970.

J.P. Corley. Comparison of Upstream and Downstream River Temperatures 1964-1965. Apriz 8, 1966. (SECRET)

J. P. Corley. Fiver Temperature Cycling at 181-D. May 13, 1966. (SECRET)

J. P. Corley. Inter-Reactor River Temperature Data. September 9, 1966. (SECRET)

R. F. Foster and R. T. Jaske. Progress Report on Research and Development Mission 14-Columbia River Studies. December 30, 1966. (Unpubiished Data)

BNWL-CC-1056 R. F. Foster and J. P. Corley. Environmental Effects of an Extended Hanford Plant Shutdown. February 28, 1967. (SECRET)

BNWL-CC-1236 R. T. Jaske. Columbia River Hudrograph. June 15, 1.67. (Unpubitished Data)

BNWL-CC-1261 R. T. Jaske. An Analysis of the Physical Factors Governing the Size and Gradients of the Hanford Effluent Plumes. July 1966 .

BNWL-CC-1355 R. T. Jaske. A Preliminary Appraisal of a Canal Lake cooling System. September 1967. (SECRET)

BNWL-CC-1628 J. P. Corley. Effluent Plume Temperature Data. June 3, 1968. (SECRET)

BNWL-CC-1931 J.F. Honstead and R. T. Jaske. 1967 Columbia River Temperature Analysis. November 1968. (SECRET)

BNWL-SA-1483 R. T. Jaske. Digital Simulation System for Prediction of water Quality. Wovember 1, 1967 . See also Proceedings of the Fourth International conference on Water Pollution Research, Prague, Czechoslovakia, April 1969.

BWHL-SA-2376 R. T. Jaske. Columbia River Trends - Fact and Fazzacy. May 8, 1969 . 
BNWL-SA-2536 R. T. Jaske. "Columbia River Temperature Trends Fact and Fallacy," Presented at the Fourth Annual Snake River Editors Conference, Walla Walla, Washington, May 8, 1969.

BNWL-SA-3193 "Improved Methods for Planning of Thermal Discharges Before Site Acquisition with a Specific Case Example on the Columbia River," IAE Symposiumon Environmental Aspects of Nuclear Power Stations, New York City, August 10-14, 1970.

DUN-959 W. N. Adachi and J.W. Ballowe. Columbia River Cooling Program CY 1965. Aprit 15,1966. (SECRET)

HW-69368 J.P. Corley, H. A. Kramer and J.K. Soldat. A Compization of Basic Data Relating to the Columbia River, Sections I-VI. December 1961. (Unpub. Data)

HW-80888 J.P. Corley. Effects of Hanford operations on Columbia River Temperature. December 3, 1965. (SECRET)

J. R. Adams. "Thermal Power, Aquatic Life, and Kilowatts on the Pacific Coast," Presented at the American Power Conference, Chicago, Illinois, April 1969.

F. A. Davidson. The Temperature Regime of the Columbia River from Priest Rapids, Washington to the Arrow Lakes in British Columbia. Public Utility District No. 2 of Grant County, Washington, January 1964.

R. E. Glover. Dispersion of Dissolved or Suspended Materials in Flowing Streams. U.S.G.S. Professional Paper 433-B, Washington D.C., 1962 .

J. P. Heaney. Mathematical Programming Model for Long-Range River Basin Planning with Emphasis on the Colorado River Basin. Northwestern University, Evanston, Illinois, 1968. (Ph.D. Dissertaion)

R. T. Jaske. "Heat as a Polzutant," Seminar WR 002.64, Oregon State University, Fall 1964.

R. T. Jaske. "Thermal Modification of River Water Quality," Proceedings of the 6th Biennial Washington State University Hydraulics Conference, october 1967.

R. T. Jaske for The Bureau of Commercial Fisheries. Final Report on Study Program to Determine the Potential Impact of Thermal Power Plants on Pacific Northwest Streams. October 1966. 
R. T. Jaske. "The Use of Digital Systems Modeling in the Evaluation of Regional Water Quality Involving Single or Multiple Releases," Am. Inst. Chem. Engrs., Water 1968. vol. 4, no. 90, pp. $1-9$.

R. T. Jaske. "An Independent View of the Thermal Effects and Radioactive Releases from Nuclear Power Plant," Presented at the University of Montana Seminar on Hydrologic Problems, January 15, 1969 .

R. T. Jaske. "Improved Methods for Evaluation of Thermal Discharge Practices and Alternative Actions," Presented at the 16 th Annual Technical Meeting of the Institute of Environmental Sciences, Boston, Massachusetts, April 12-16, 1970.

R. T. Jaske. "The Need for Advance Planning of Thermal Discharges Before Site Acquisition," Presented at the American Power Conference, Ilzinois Institute of Technology, Chicago, Ilzinois, Apriz 22-24, 1969.

R. T. Jaske. "Methods for Evaluating Effects of Transient Conditions in Heavily Loaded and Extensively Regulated Streams," Presented at the 1969 ASME/AICHE stream Polzution Abatement Conference at Rutgers University, June 10-12, 1969. (in press)

R. T. Jaske. "The Need for Advance Planning of Thermal Discharges Before Site Acquisition," Proceedings of American Power Conference, Nuclear News, September 1969. Also Selected Materials on Environmental Effects of Producing Electric Power, Joint Committee on Atomic Energy, Congress of the United States, August 1969.

R. T. Jaske. "The Use of Advanced Methods for Studies of Environmental Impact of Industrial Operations," Presented at the International Water Conference of the Engineers' Society of Western Pennsylvania, Pittsburgh, Pennsylvania, November 1969.

R. T. Jaske. "Improved Methods for Planning of Thermal Discharges Before Site Acquisition," Presented at the winter Meeting of the American Nuclear Society, San Francisco, California, November 30-December 4, 1969.

R. T. Jaske. "Comments Pertinent to the Use and Abuse of Energy in the American Economy," Presented at the Annual Meeting of the American Association for the Advancement of Science, Boston, Massachusetts, December 26-31, 1969.

R. T. Jaske, M. H. Karr and C. J. Touhilz. "Multiple Purpose Use of Thermal Condenser Discharges from Large Nuclear Systems to Supplement Inter-Regional Water Supply, "Presented at the 67 th National Meeting of the AICHE, AtZanta, Georgia, February 17,1970 . 
R. T. Jaske, J. F. Fletcher and K. R. Wise. "A National Estimate of Public and Industrial Heat Rejection Requirements by Decades Through the Year 2000," Presented at the 67th National Meeting of the AICHE, Atlanta, Georgia, February 17, 1970. Chemical Engineering Progress, March, 1970.

N. C. Matalas and W. J. Conover. Deviation of the Velocity Profile from a statistical Model of Turbulence, Water Resources Research, Washington D.C., Second Quarter 1965.

C. A. Oster, J. C. Sonnichsen and R. T. Jaske. "Numerical Solution to the Convective-Diffusion Equation," ASCE J. Sanitary Engineering Division, (in press).

G. E. Raines and R. T. Jaske. "The Hierarchical Systems Approach as Related to Simulation in the Water Resources Area, Presented at the Great Lakes Environmental Conference, Buffalo, New York, March 30-Apriz 2, 1970.

J. M. Raphael. The Effect of Wanapum and Priest Rapids Dams on the Temperature of the Columbia River. Public Utility District No. 2 of Grant County, Washington, September, 1961.

J. C. Sonnichsen and C. A. Oster. "Examination of the Thermocline," Journal of the Sanitary Engineering Division, Proceedings of the American Society of Civil Engineers, Apriz 1970.

Compization of Records of Surface Waters of the United States Through September 1950, Part 12, Pacific Slope Basins. Paper No. 1316, USGS Water Supply, Washington, D.C.

US Geological Survey Professional Paper. "Water Loss Investigations: Lake Hefner Report," Proceedings of the Symposium on Stream Flow Regulation for Quality Control Department of Health, Education and Welfare, Washington, D.C., June 1965.

Study Program to Determine the Potential Impact of Thermal Power Plants on Pacific Northwest Streams, Research Report to Bureau of Commercial Fisheries, Seattie, Washington. Battelle-Northwest, Richland, Washington, october, 1966.

Final Report on Nuclear Power Plant Siting in the Pacific Northwest, Battelie-Northwest for the Bonnevilie Power Administration, July 1, 1967.

New Washington State Water Quality Standards. Water PolZution Control Commission, Olympia, Washington, December 18, 1967. 
BNWL - 1345

Research Related to the Development of a Power Reactor site on the Lower Columbia River, Research Report to Portiand General Electric Company. Battelze-Northwest, Richland, Washington, June $15,1968$.

"Research Needs in the Civil Engineering Aspects of Power," Proceedings ASCE Specialty Conference, Washington State University, Pulzman, Washington, September 11-13, 1968.

Research Related to the Prediction of Temperature at a Power Reactor Site on the Lower Columbia River, Research Report to Portland General Electric Company. Battelle-Northwest, Richland, Washington, March 1, 1969.

A Three Dimensional Study of Parameters Related to the Current Distribution in Lake Roosevelt, Research Report to Office of Water Resources Research United States Department of the Interior, Washington, D.C. Battelze-Northwest, Richland, Washington, Apriz 23, 1969.

Temperature Prediction and Current Measurements Related to the Development of a Power Reactor Site on the Lower Columbia River in the Vicinity of Kalama, Washington, Research Report to Clark and Cowlitz County Public Utizity Districts. Battelze-

Northewst, Richland, Washingtion, June 3, 1969.

Prediction of Columbia River Temperatures Downstream from Grand Coulee for Wide Extremes of Flow and Weather Conditions, Contract report to Fish Passage Research, Bureau of Commercial Fisheries, U.S. Fish and WildIife Servive, U.S. Department of Interior, Washington, D.C.*

* Copy may be made avaizable by G. B. Colzins, Program Director. 
BNWL- 1345

APPENDIX A

RELATED STUDIES 


\section{APPENDIX A}

\section{RELATED STUDIES}

Tables A-1 and A-2 show weather factors and dewpoint at Hanford. Flow of the Columbia River from Trinidad, just below Rock Island Dam, to Priest Rapids for 1954 through 1969 is shown in Table A-3.

Figures A-1 through A- 8 depict the least-squares-fit curves of the Columbia River flow and the Hanford solar radiation, dewpoint and air temperature for 1964 and 1965. 
TABLE A-1. Weather Factors at Hanford

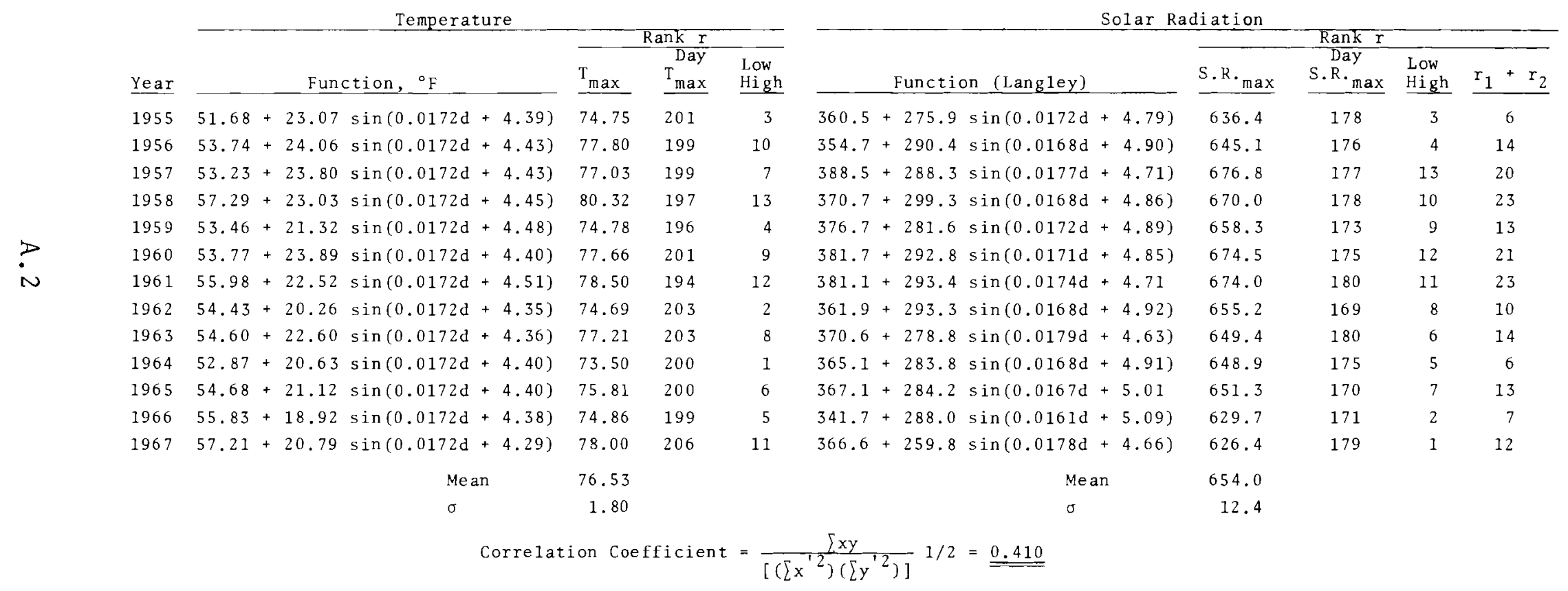


TABLE A-2. Dewpoint at Hanford Modeled to General Function: $\mathrm{T}=\overline{\mathrm{T}}+\mathrm{B} \sin (\mathrm{Cd}+\mathrm{D})$

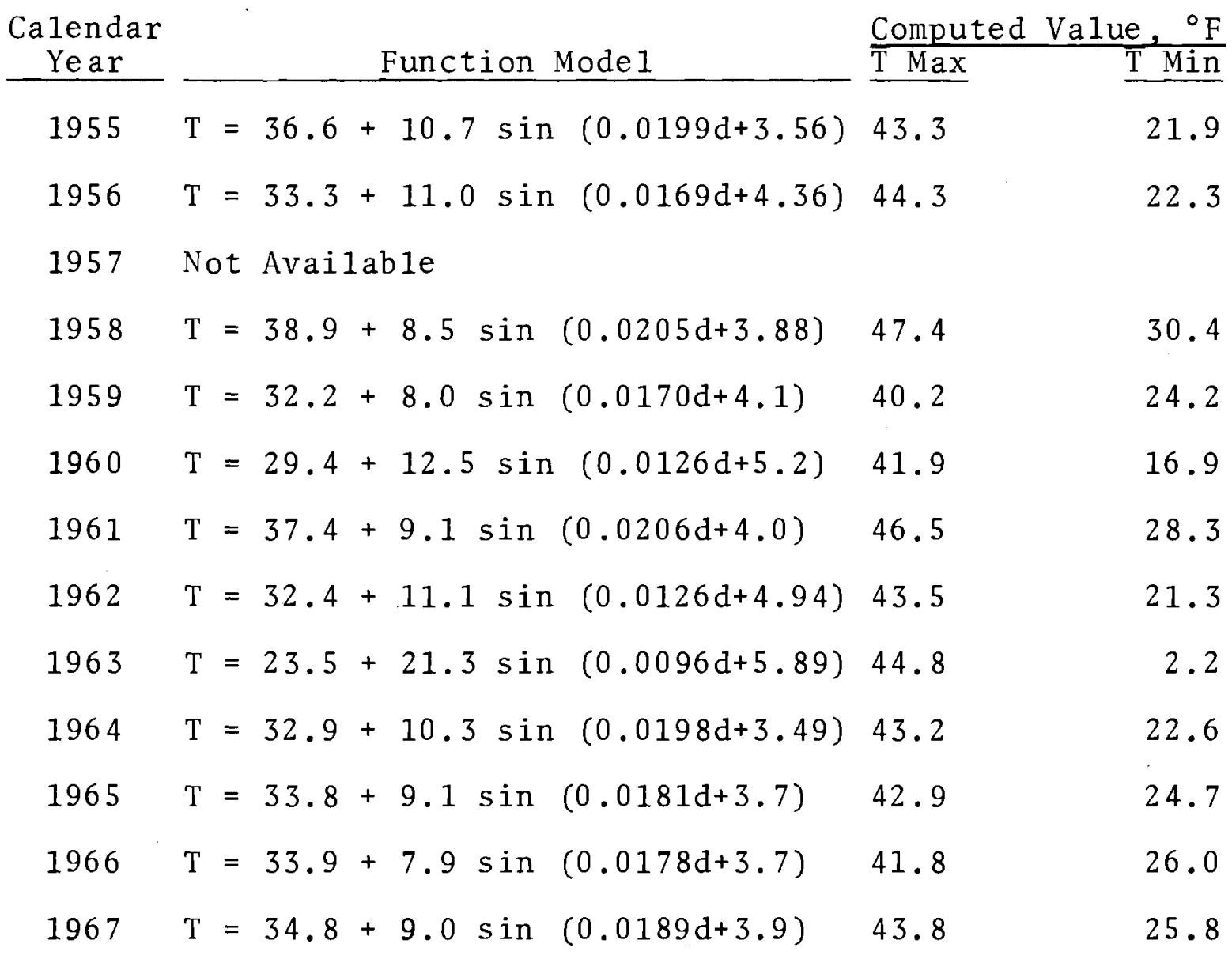


TABLE A-3. Columbia River Flow: Trinidad-Priest Rapids Flow $=A+B e^{-[(d-C) / E]^{2}}$

\begin{tabular}{|c|c|c|c|}
\hline Year & & Function, kcfs & $\begin{array}{l}\text { Volume in } \\
\text { Thousands Acre-Feet }\end{array}$ \\
\hline 1954 & 69.2 & $+351.8 e^{-[(d-177.1) / 46.8]^{2}}$ & 107,900 \\
\hline 1955 & $75 \cdot 5$ & $+341.0 \mathrm{e}^{-[(\mathrm{d}-185.3) / 31.1]^{2}}$ & 92,000 \\
\hline 1956 & 71.2 & $+373.8 \mathrm{e}^{-[(\mathrm{d}-159.7) / 45.2]^{2}}$ & 11,070 \\
\hline 1957 & 69.3 & $+331.0 \mathrm{e}^{-[(\mathrm{d}-154.2) / 30.0]^{2}}$ & 85,140 \\
\hline 1958 & 72.9 & $+288.2 \mathrm{e}^{-[(\mathrm{d}-159.6) / 29.9]^{2}}$ & 82,670 \\
\hline 1959 & 98.5 & $+295.4 \mathrm{e}^{-[(\mathrm{d}-172.3) / 40.4]^{2}}$ & 113,200 \\
\hline 1960 & 68.9 & $+220.9 \mathrm{e}^{-[(\mathrm{d}-170.7) / 52.0]^{2}}$ & 90,970 \\
\hline 1961 & 76.5 & $+407.1_{\mathrm{e}}^{-[(\mathrm{d}-163.8) / 27.4]}$ & 94,870 \\
\hline 1962 & 69.5 & $+199.8 \mathrm{e}^{-[(\mathrm{d}-167.3) / 46.2]^{2}}$ & 81,890 \\
\hline 1963 & 73.3 & $+207.8 \mathrm{e}^{-[(\mathrm{d}-173.1) / 35.3]}$ & 78,782 \\
\hline 1964 & 69.5 & $(d-176.7) / 35.1]^{2}$ & 92,990 \\
\hline 1965 & 76.7 & $(d-165.5) / 46.1]^{2}$ & 95,130 \\
\hline 1966 & 69.3 & $(d-172.0) / 43.2]^{2}$ & 83,310 \\
\hline 1967 & 81.5 & $(d-173.9) / 28.7]^{2}$ & 97,950 \\
\hline 1968 & 87.0 & $(d-177.8) / 30.8]^{2}$ & 86,530 \\
\hline 1969 & 90.8 & $+169.0 \mathrm{e}$ & 97,520 \\
\hline
\end{tabular}




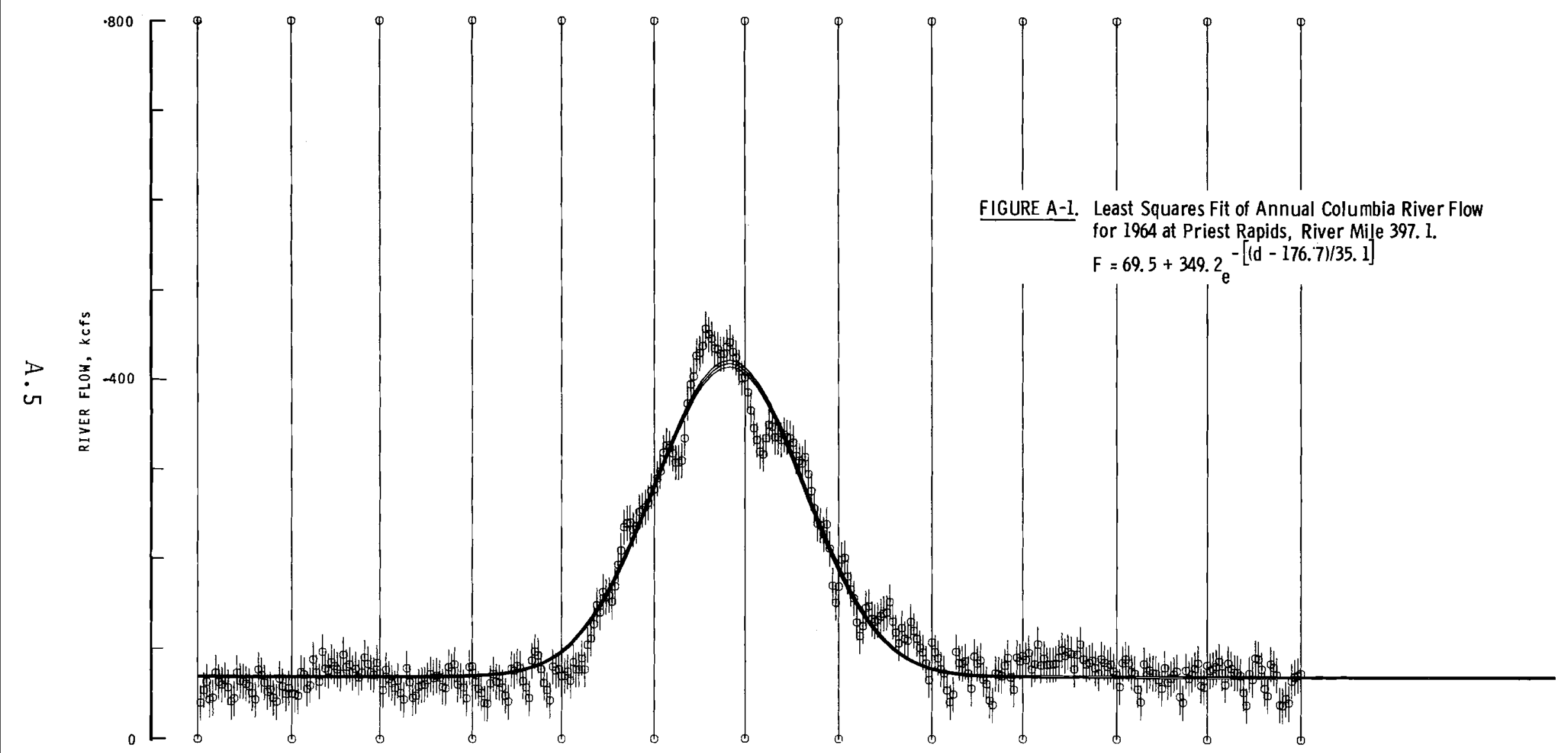

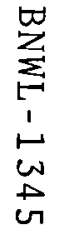


-BNWL - 1345

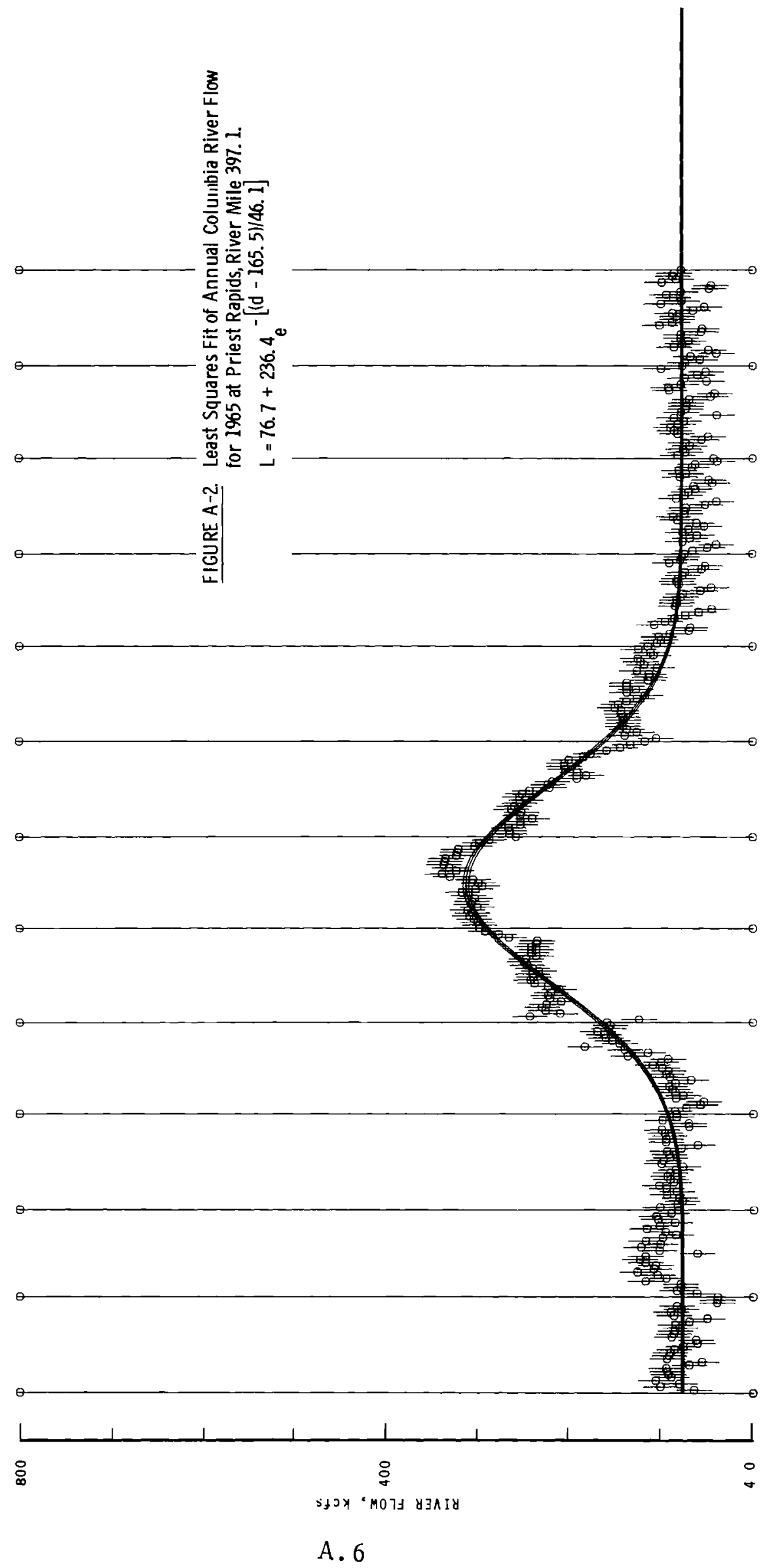




\section{BNWL - 1345}

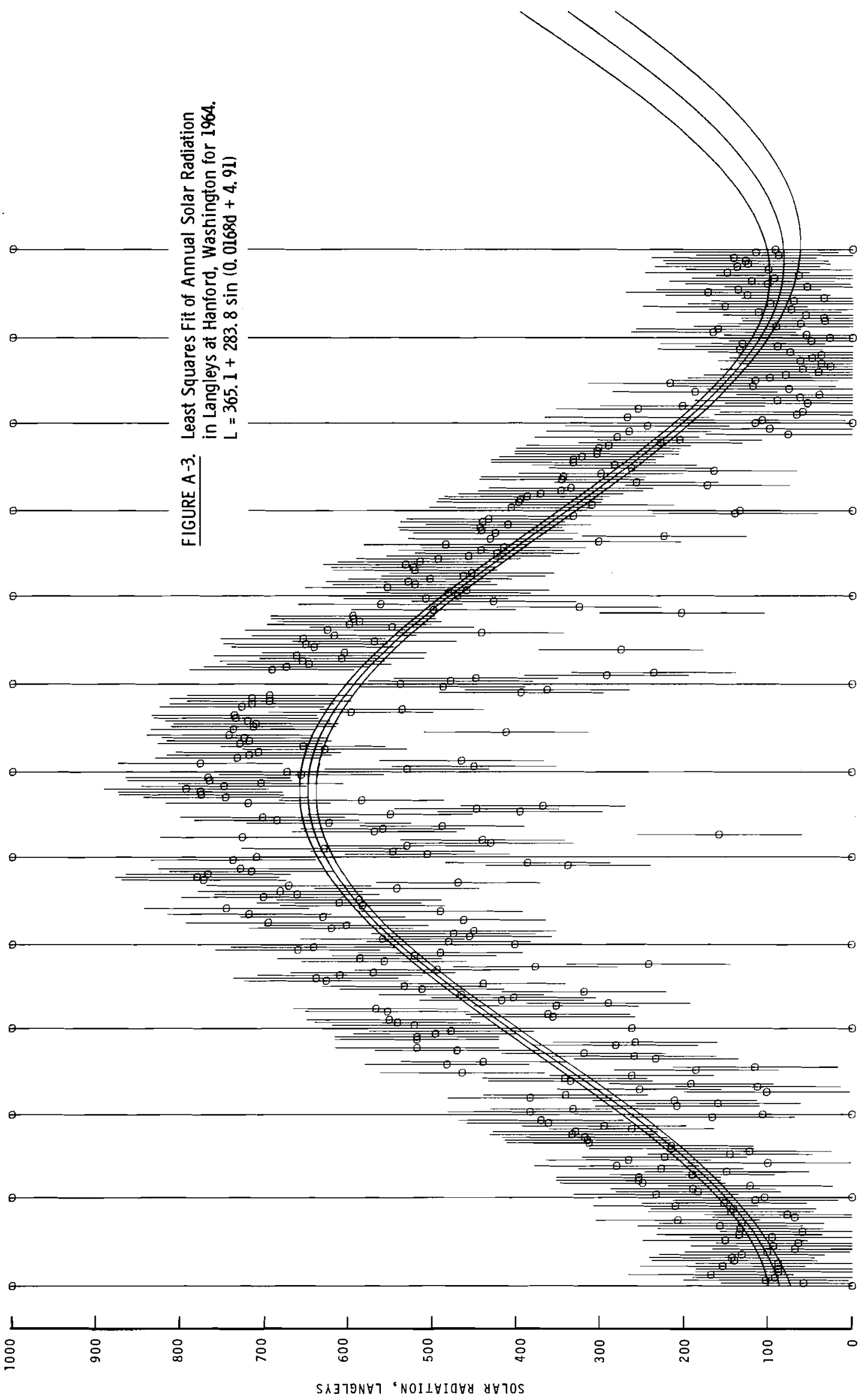

A. 7 


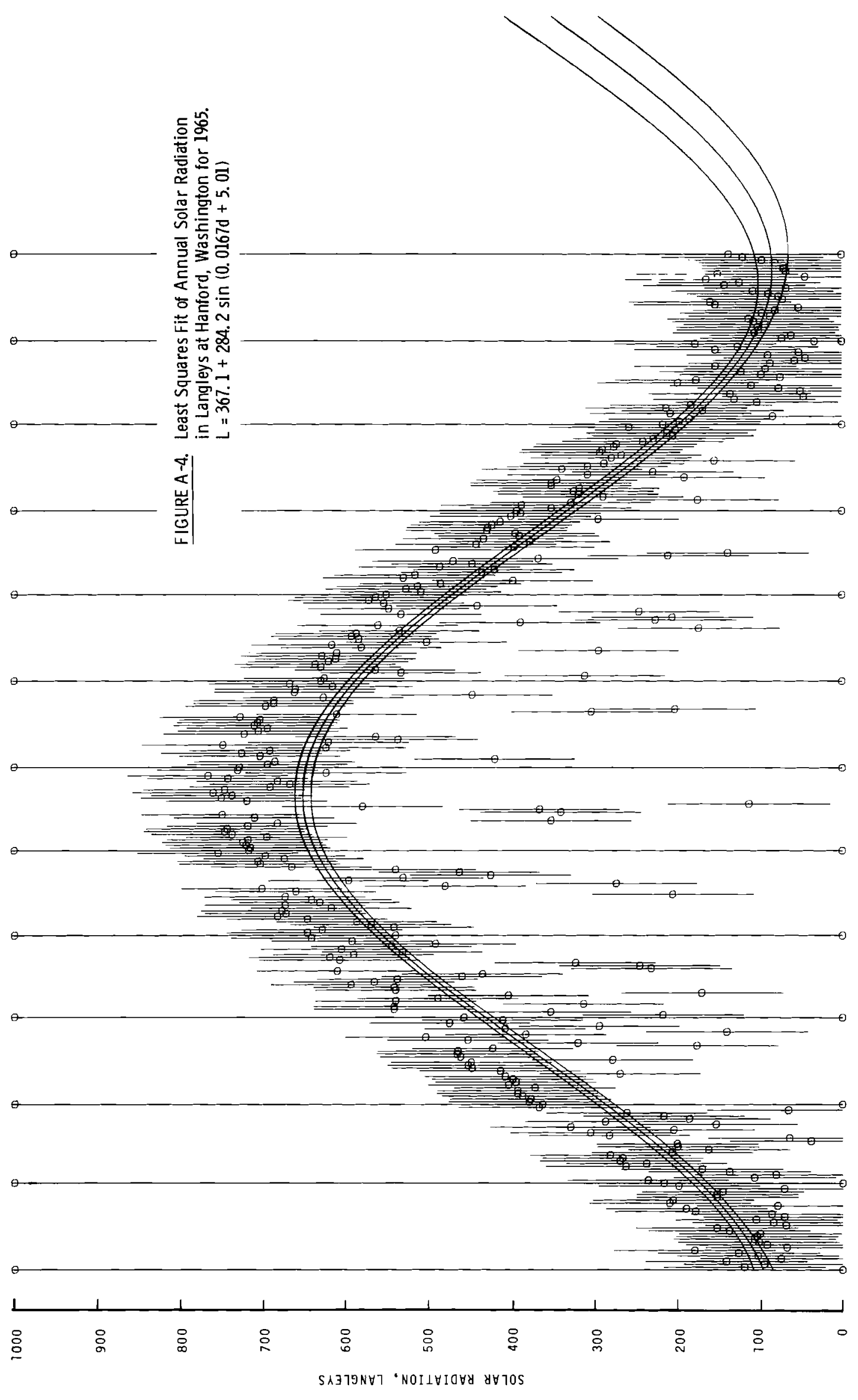

A. 8 


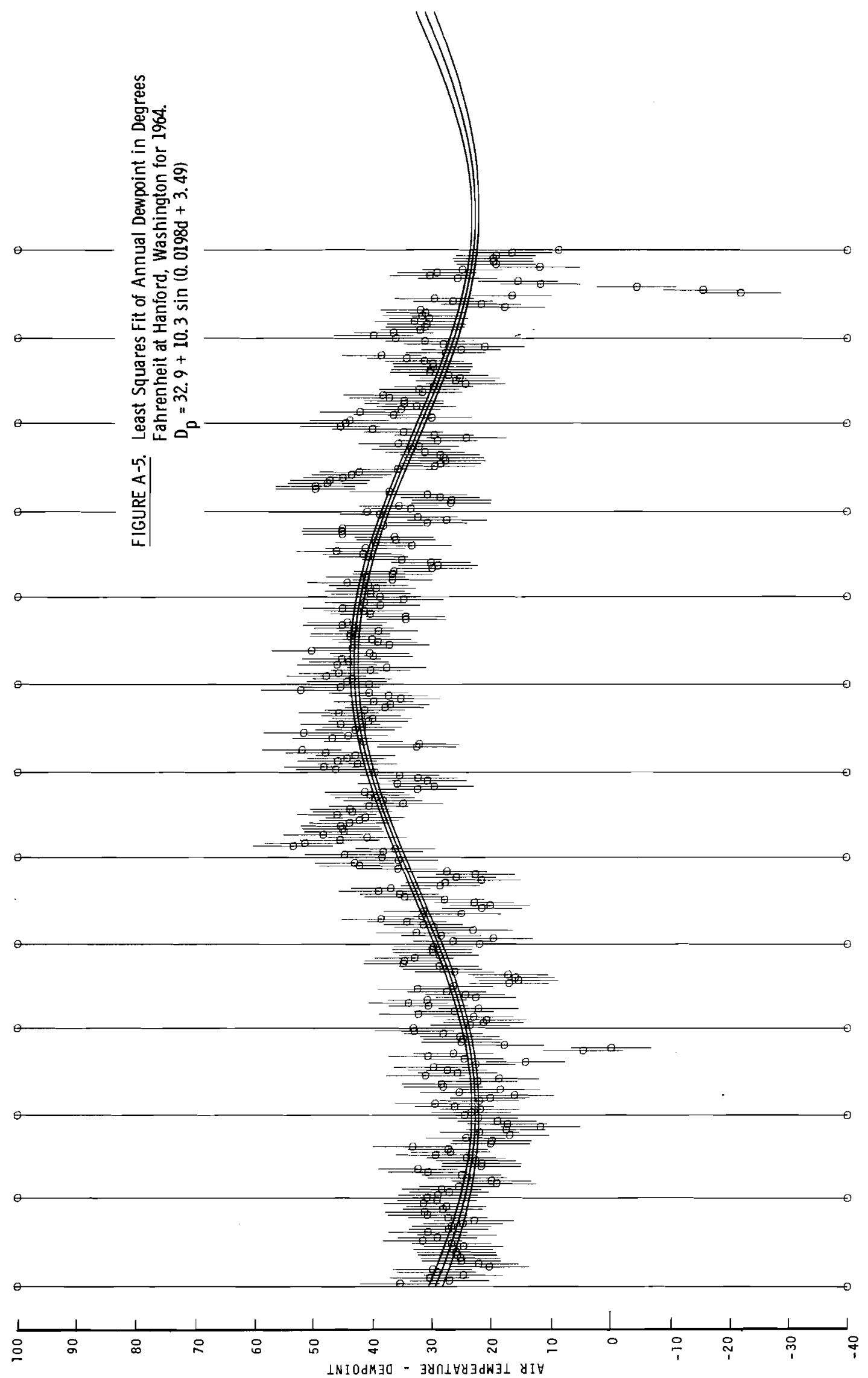

A. 9 
BNWL - 1345

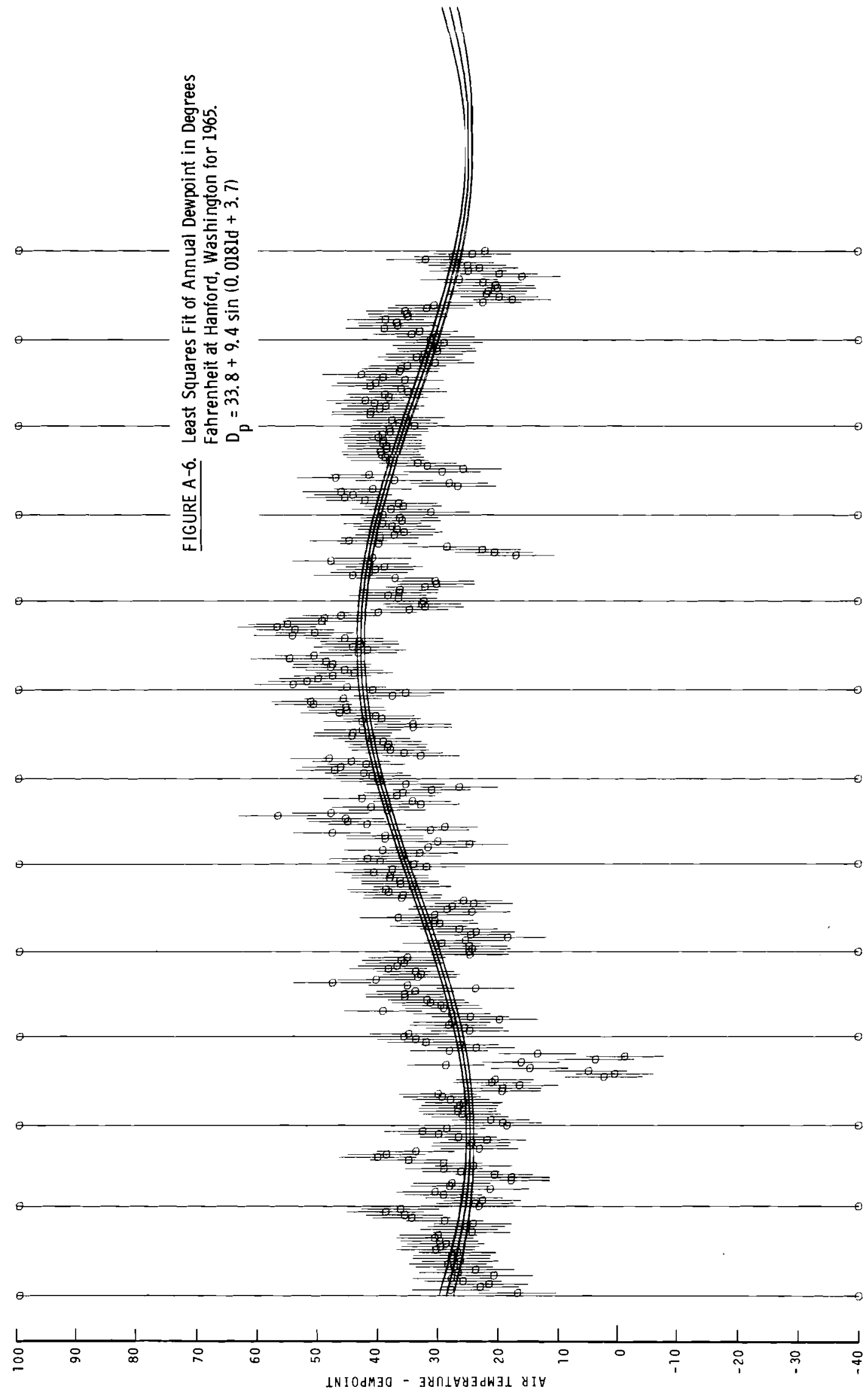

A. 10 
BNWL - 1345

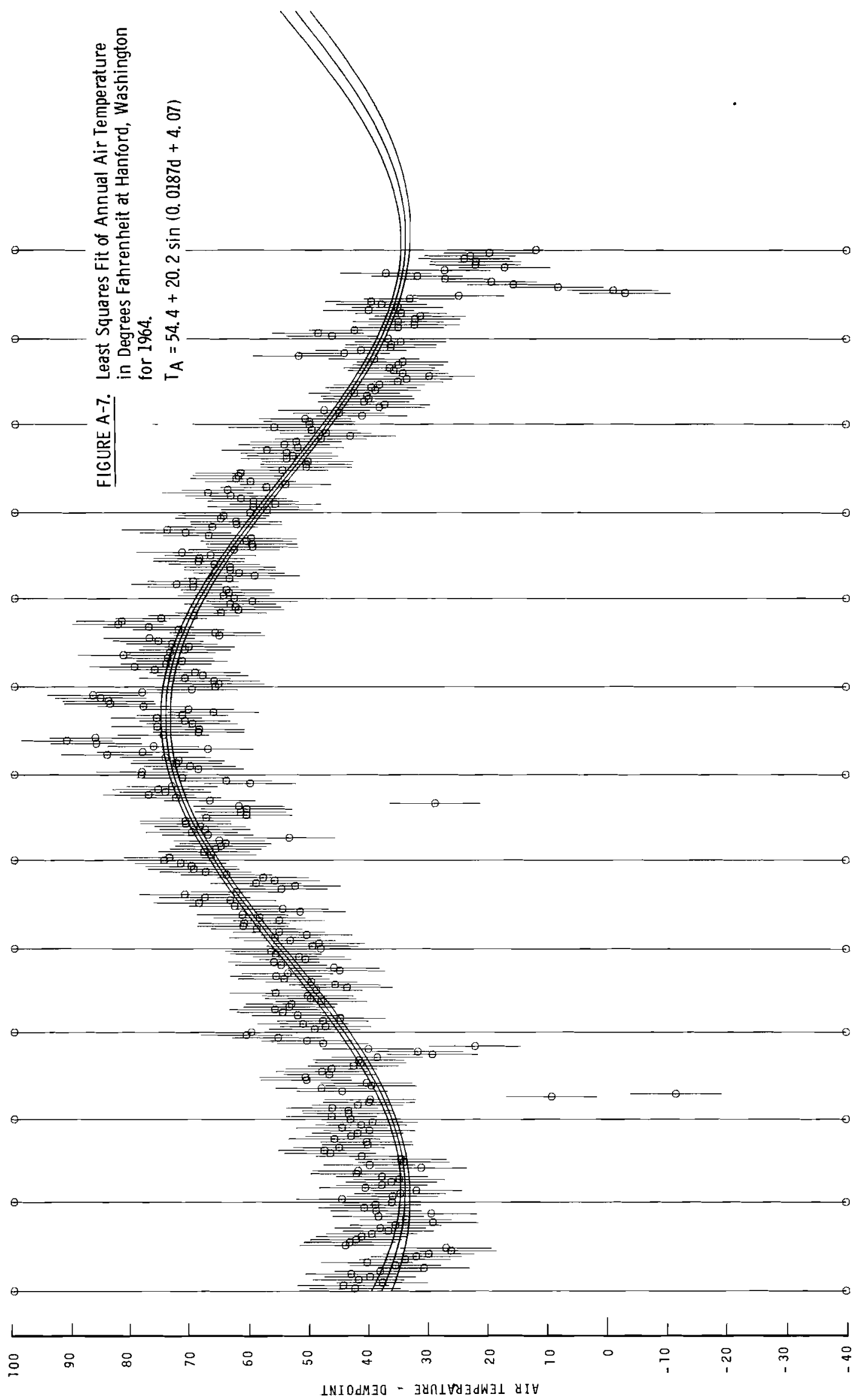

A. 11 


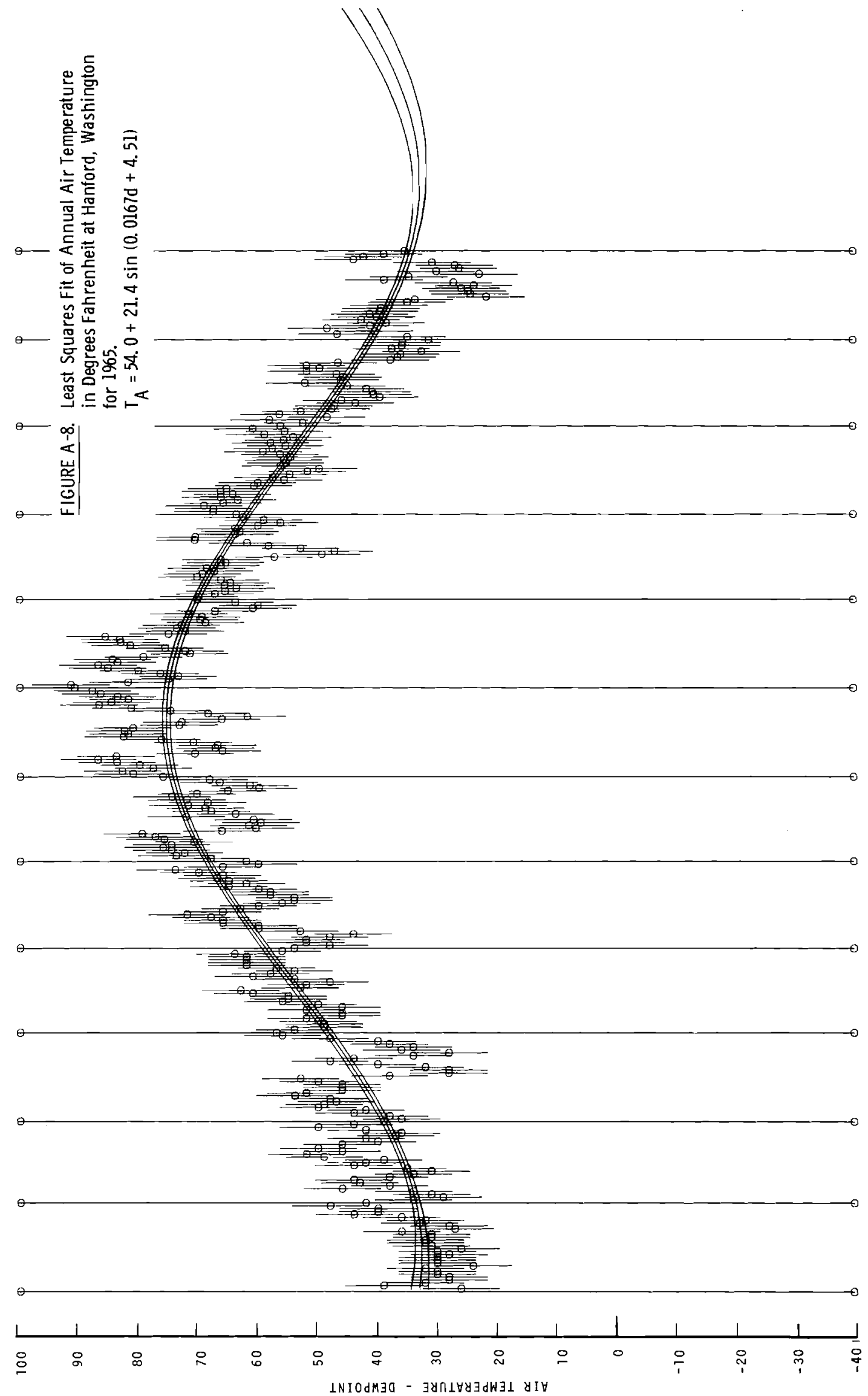

A. 12 
BNWL - 1345

\author{
APPENDIX B \\ LEAST-SQUARES FITTING PROGRAM LEARN \\ D. A. Kottwitz
}


BNWL - 1345

APPENDIX B

LEAST-SQUARES FITTING PROGRAM LEARN

D. A. Kottwitz

The nonlinear least-squares fitting program LEARN is a direct descendant of the computer program MISFIT, which was used in previously reported river studies. (B1) The principles on which LEARN is based are given in Reference B2, and instructions for practical use are given in Reference B3. In this appendix we shall concentrate on those features of particular interest in the present work.

The calculations reported here were performed over a period of several months by means of three closely related FORTRAN $V$ (UNIVAC 1108) versions, designated LEARN-I-A, (B2) LEARN-I-B, and LEARN-I-C. These evolving versions differ from the original in two major respects. First, the iterative portion of the program, in which the least-squares minimum is sought, has been extensively modified to increase the reliability and speed of convergence. This improvement in performance is due to the use of improved saddle-detection, step-reversal, step-reduction, and gradient techniques. The second major difference is an increase in the flexibility of control of the CALCOMP plotter output. The other important features of the program, including the basic structure and the statistical analyses, are unchanged.

Two variants of the temperature-fitting program are in use. In the first variant, all four parameters in the fitting function are adjustable; in the second, the frequency parameter is held fixed at a value specified by the user (normally about $2 \pi / 365.25=0.0172 \mathrm{radian} / \mathrm{day})$. Of course the four-parameter fit is better, but the frequency so obtained may not be "reas on ab le." 
In both variants of the temperature fitting program, the average temperature is explicitly calculated. For crosschecking this is done in three ways: (1) analytically from the fitted parameters, (2) approximately by summing the input data values, and (3) approximately by summing the corresponding discrete fitted values. The values obtained in this way usually agree to four or five significant figures.

\section{REFERENCES}

B1. R. T. Jaske and J. B. Goebel. "Effects of Dam Construction on Temperatures of Columbia River," J. Am. Water Works Assoc., vol. 59, pp.935-942. 1967 .

B2. B. H. Duane. Maximum Likelihood Nonlinear Correlated Fields (BNW Program LIKELY), BNWL-390. BattelleNorthwest, Richland, Washington, September 1967.

B3. G. D. Seybold. User's Aid: Programs LEARN and LIKELY, BNWL-1057. Battelle-Northwest, Richland, Washington, May 1969 . 
BNWL- 1345

APPENDIX C

SUMMARY OF EFFLUENT MIXING STUDIES 


\section{APPENDIX C}

\section{SUMMARY OF EFFLUENT MIXING STUDIES}

Commencing with the initiation of operations at Hanford, the dispersion of plant effluents into the Columbia River in relation to public safety has been the subject of intensive study. In the early days, the use of boat traverses or floats constituted the state-of-the-art in the development of early models which described effluent mixing. Early investigators such as Glover ${ }^{(\mathrm{C} 1)}$ and HONSTEAD (unpublished) produced satisfactory expressions for the one-dimensional description of radionuclide dispersion. This was relatively easy because the fine structure of mixing was unaffected by the effluent temperature and the means for measurement of radiation gave many orders of magnitude of concentration as compared with a relatively narrow span for thermal modifications.

In 1964 Corley initiated an intensive program to describe the thermal regimen of the river close-in to the reactor discharges. The results of this work are summarized in Figures $\mathrm{C}-1$ and $\mathrm{C}-2$. Hundreds of hours of boat time and a great deal of planning were required to coordinate the research operations with the plant operations and the regulated flow changes of the Columbia River. The results had to remain classified until the spring of 1969 when the office of Classification began a series of administrative actions which have permitted the disclosure of much previously classified documentation.

Beginning in 1966 and continuing to the present, BattelleNorthwest has developed remote imagery technology which has obsoleted the old methods of boat surveys except for the essential purposes of calibration and vertical concentration sounding. The mixing action at the point of discharge to a 


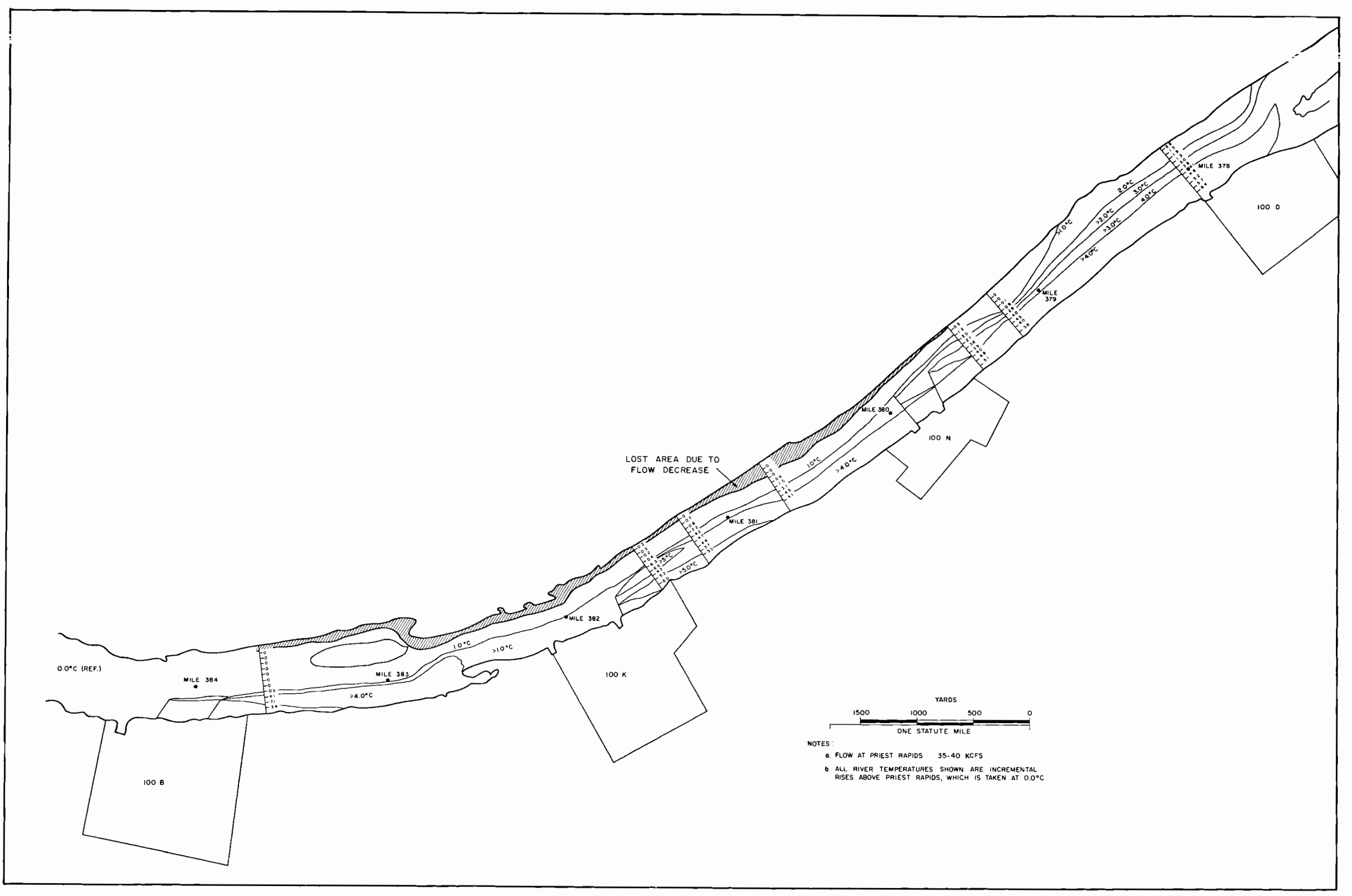

FIGURE C-1. Incremental Temperature Above Base River Temperature (Summary of Cross-Section Traverses, River Mile 378-384, All Reactors Operating, 4l,000 cfs) 


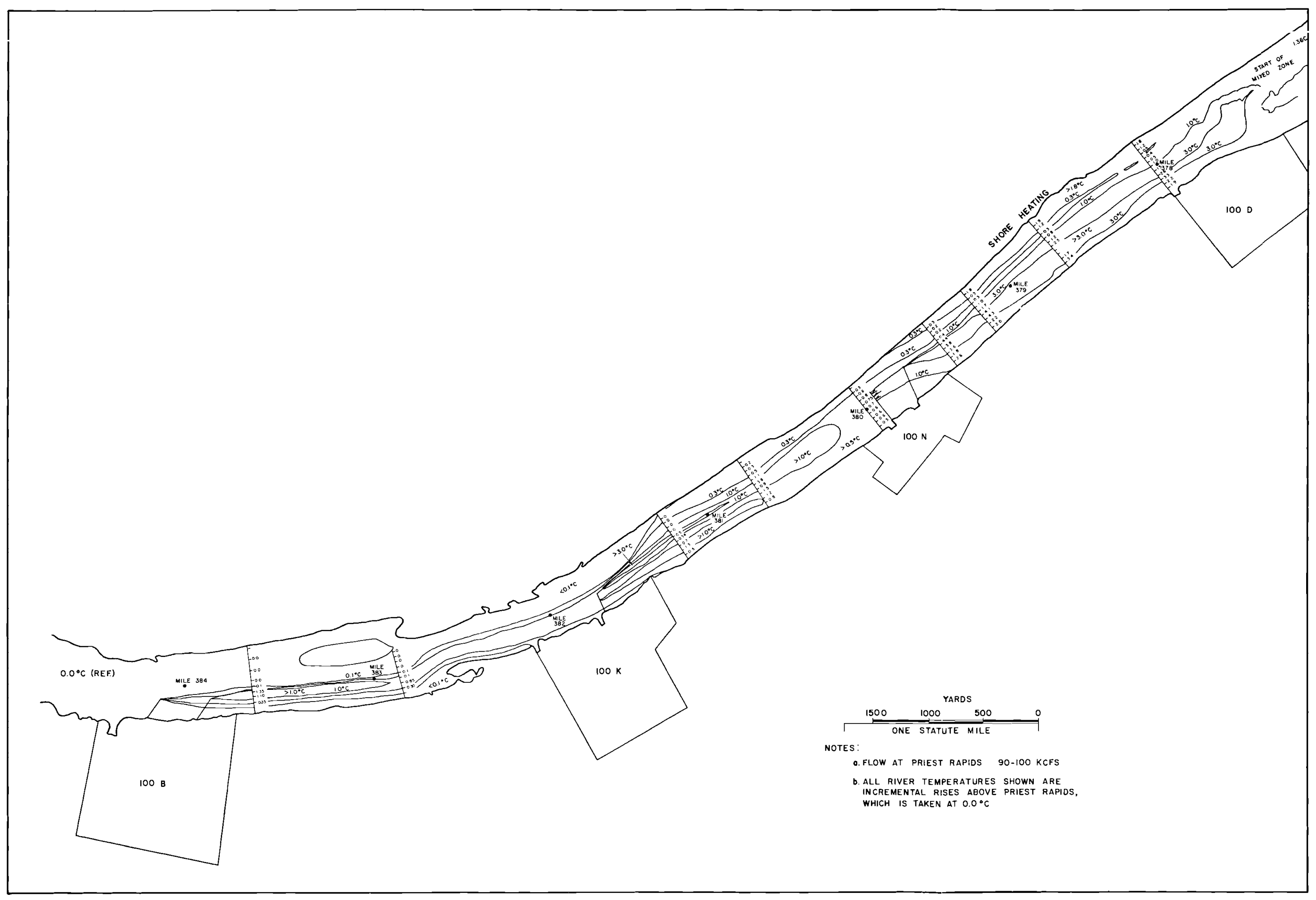

FIGURE C-2. Incremental Temperature Above Base River Temperature (Summary of Cross-Section

Traverses, River Mile 378-384, All

Reactors Operating, 88,000 cfs) 
distance of 300 yards downstream has always been too difficult to measure because of the combination of river and effluent turbulence and the size of the system under investigation. The use of remote imagery now permits real time analysis of thermal releases and the compression of data collection costs and time by orders of magnitude. Figures $\mathrm{C}-3, \mathrm{C}-4$ and $\mathrm{C}-5$ illustrate the type of real-time data which can be generated by a single time frame of imagery using advanced computer analysis techniques under development at Pacific Northwest Laboratory. These data also permitted advances in the development of mathematical models of dispersion in two dimensions and the first realistic attempts to model the mixing zone immediately at the point of discharge. The findings that cold effluent releases always disperse less rapidly than warmed releases remains to be corroborated by additional investigators, but this finding and that of the estimate of lateral dispersion discussed below would not have been possible without the use of advanced computer systems for the visual representation of remote imagery.

These determinations of temperature gradients are limited to two dimensions using present state-of-the-art technology and must still be supplemented by boat or buoy surveys to determine vertical current and concentration gradient profiles. However, using these two-dimensional methods and supplementing them with surface probing, information of the type shown in Figures $\mathrm{C}-3, \mathrm{C}-4$ and $\mathrm{C}-5$ can be developed. These figures are engineering drawings of the concentration isopleths in the mixing area below the discharge of the $100-\mathrm{K}$ production area at Hanford. The mathematical treatment of a series of data plots of this type on a real-time basis permits vastly improved insight into the physical mechanics of the mixing process. The significance of improved understanding of the physical mixing regimen cannot be overstated in the light of new standards limiting temperature differentials of mixing zones. 
BNWL- 1345

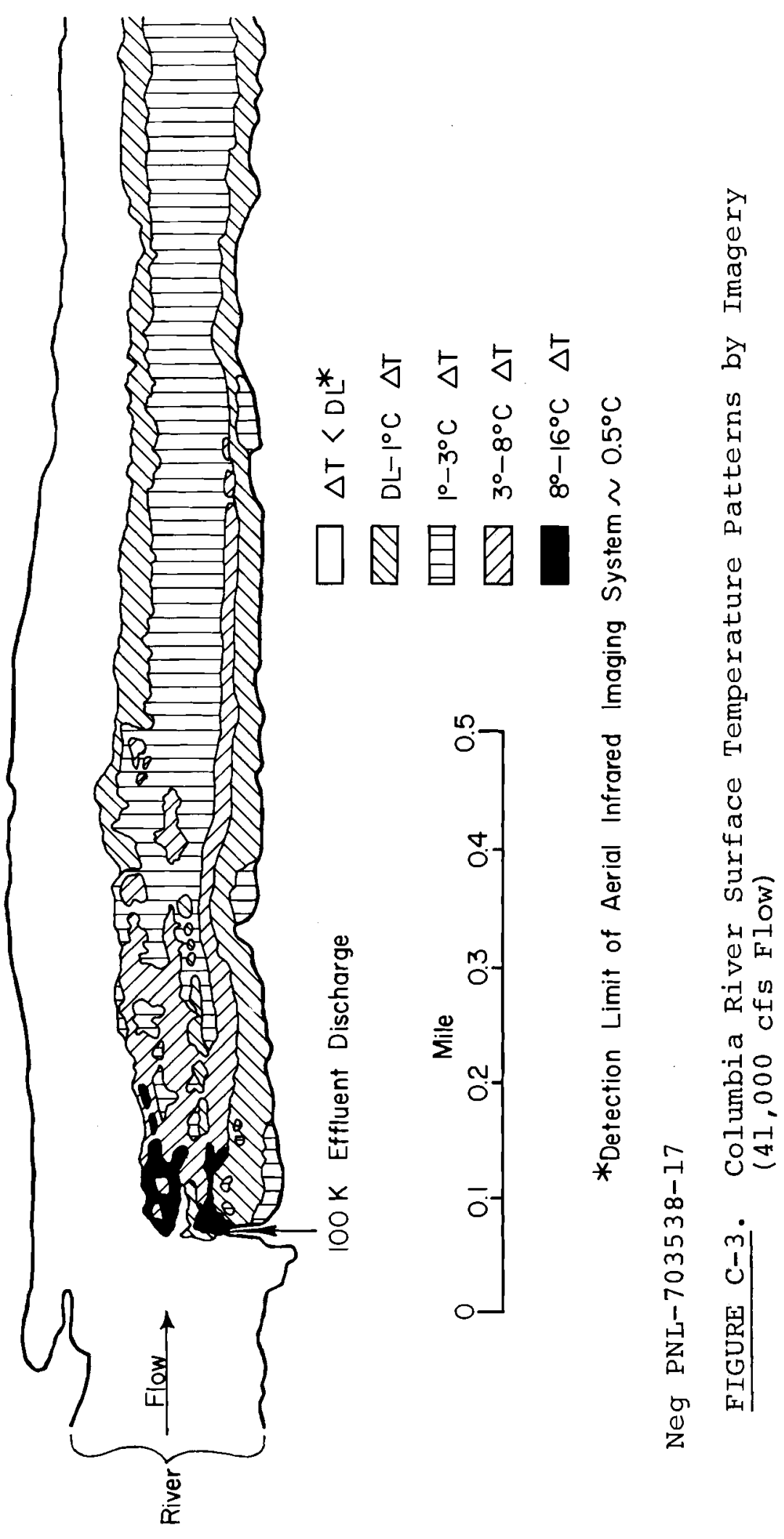

C. 5 
BNWL - 1345

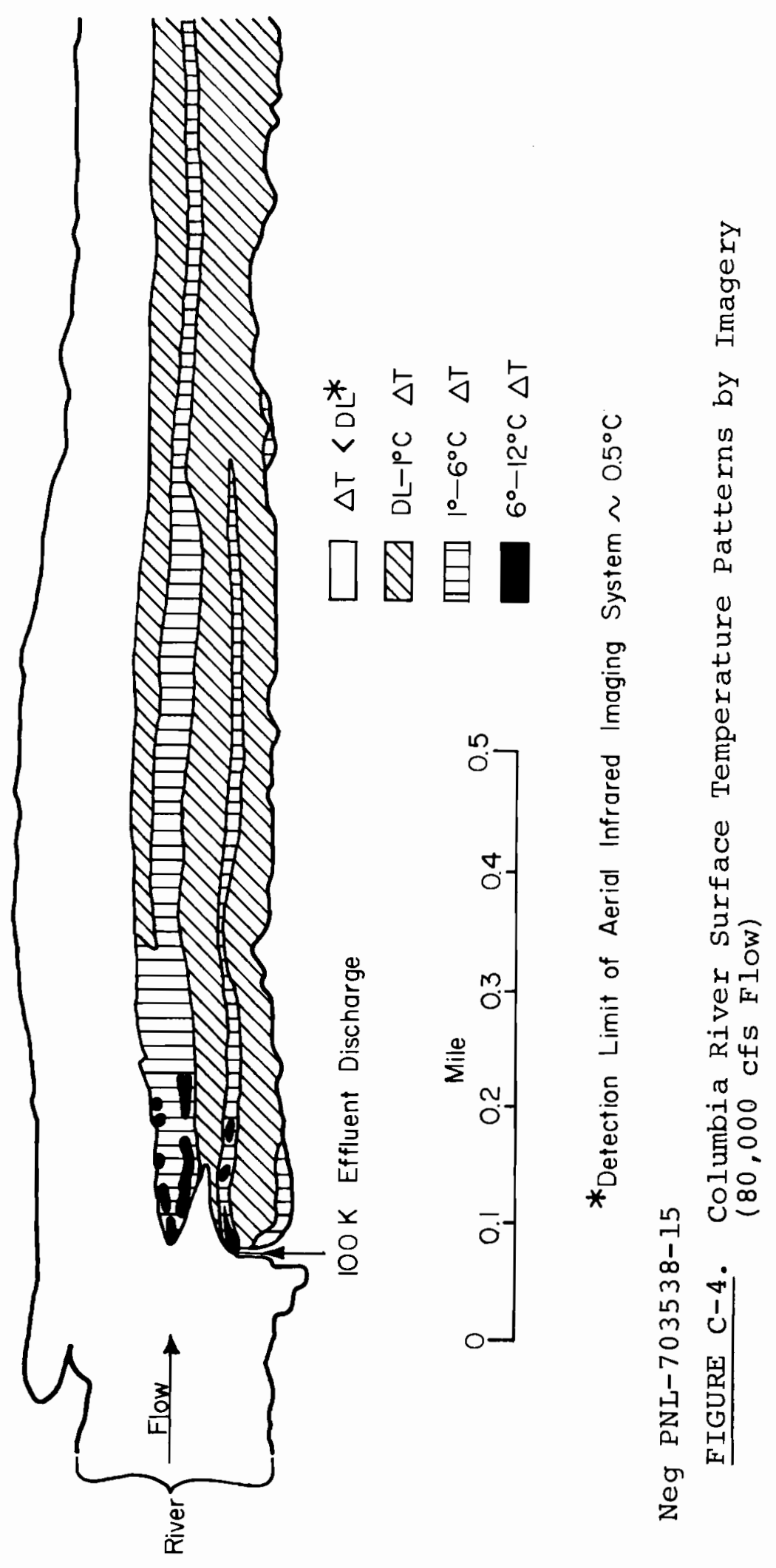

C. 6 
BNWL- 1345.

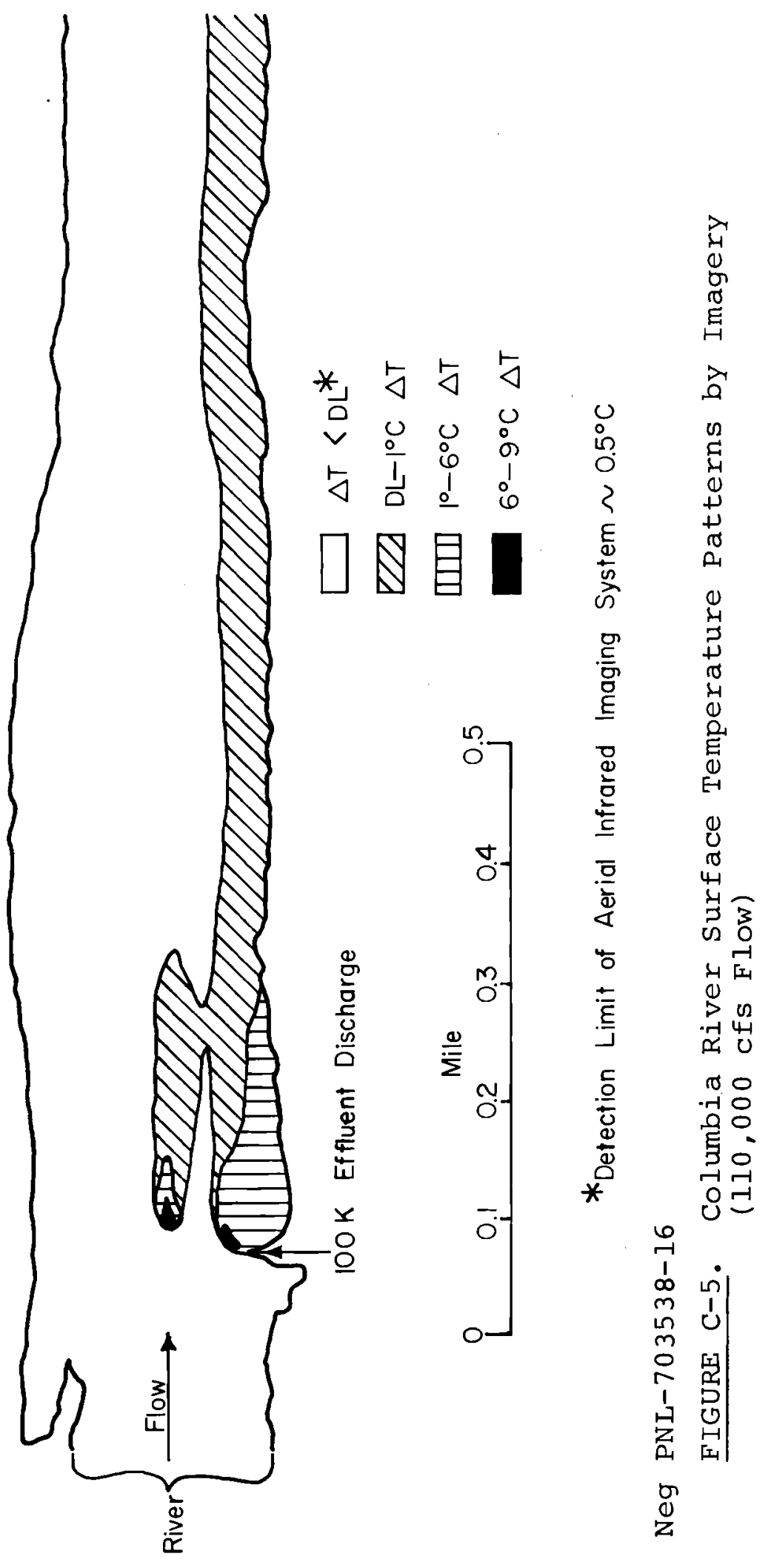

C. 7 
BNWL- 1345

\section{STUDY OF THE MIXING ZONE}

Below a few hundred meters from the point of release, the discharges in a relatively turbulent stream have been shown to follow the classical work of Taylor; however, the use of a single value of the dispersion coefficient for entire reaches of the sample stream can produce erroneous results. A solution of the Taylor expression:

$$
\frac{\partial \bar{c}}{\partial t}=-\frac{\bar{u} \partial \bar{c}}{\partial x}+D_{x} \frac{\partial^{2} \bar{c}}{\partial x^{2}}
$$

where $\bar{c}=$ the cross-sectional mean concentration

$\bar{u}=$ the mean flow velocity

$t=$ time

$x=$ distance in the direction of mean flow

$\mathrm{D}_{\mathrm{X}}=$ longitudinal dispersion coefficient

$\mathrm{K}_{\mathrm{x}}=\mathrm{a}$ scaling factor proportional to $\mathrm{C}_{\mathrm{O}}$, the original concentration can be written:

$$
\bar{c}(x, t)=\frac{K_{x}}{t^{112}} \exp -\frac{(x-\bar{u} t)^{2}}{4 D_{x} t}
$$

Then, estimates of the variables $K_{x}, D_{x}$, and $\bar{u}$ were obtained from a series of reaches for which real-time concentration data have been accumulated using a nonlinear least-squares fitting method developed at Pacific Northwest Laboratory $(\mathrm{C} 2)$ and modified by Kottwitz (unpublished). The special program called LEARN/DISPERS employs the general relationship:

$$
c=\frac{A_{1}}{\left.(t)^{d}\right)^{1 / 2}} \exp -\left[\frac{1}{4 t}\left(\frac{\left(x-A_{1} t\right)^{2}}{\left|A_{2}\right|}+\frac{\left(y-A_{3} t\right)^{2}}{\left|A_{4}\right|}+\frac{\left(z-A_{5} t\right)^{2}}{\left|A_{6}\right|}\right)\right]
$$

where $x, y, z=$ space coordinates

$A_{1}, A_{3}, A_{5}=$ convective speed parameters 


$$
\begin{aligned}
& \mathrm{A}_{2}, \mathrm{~A}_{4}, \mathrm{~A}_{6}= \text { dispersion coefficients } \\
& \mathrm{A}_{7}= \text { amplitude (concentration) parameter } \\
& \mathrm{d}=\text { number of space dimensions, e.g., } \\
& \quad \text { if } \mathrm{d}=1, \mathrm{y} \text { and } \mathrm{z} \text { terms are omitted } \\
& \quad \text { if } \mathrm{d}=2, \mathrm{z} \text { term is omitted } \\
& \quad \text { if } \mathrm{d}=3, \text { all terms are included. }
\end{aligned}
$$

The resulting program is capable of fitting separately a set of data for any number of discrete locations or fitting sequentially several sets of consecutive data sets. In addition to the numerical determination of the parameters, the program plots the results or graphical interpretation. An example of the output of such an analys is is shown in Figure C-6. The numerical values obtained indicated that the combination of remote sensing for concentration input and the use of improved evaluation of $D_{x}$ with relation to stream reach gives improved insight into predictive computations.

At present no well developed theory exists for predicting mixing in the lateral direction. Data summaries have been generally in the form which estimates the value of $D_{y}$ as follows:

$$
D_{y}=0.23 U_{x} h
$$

where $h=$ the depth of flow

$$
\mathrm{U}_{\mathrm{x}}=\text { mean velocity of a vertical transect. }
$$

In 1967, using boat data measured in the Hanford effluent plumes, Jaske ${ }^{(C 3)}$ developed an expression which correlated the spreading velocity as a function of downstream travel time $t$

$$
\frac{d y}{d t}=a \exp -(b x)
$$




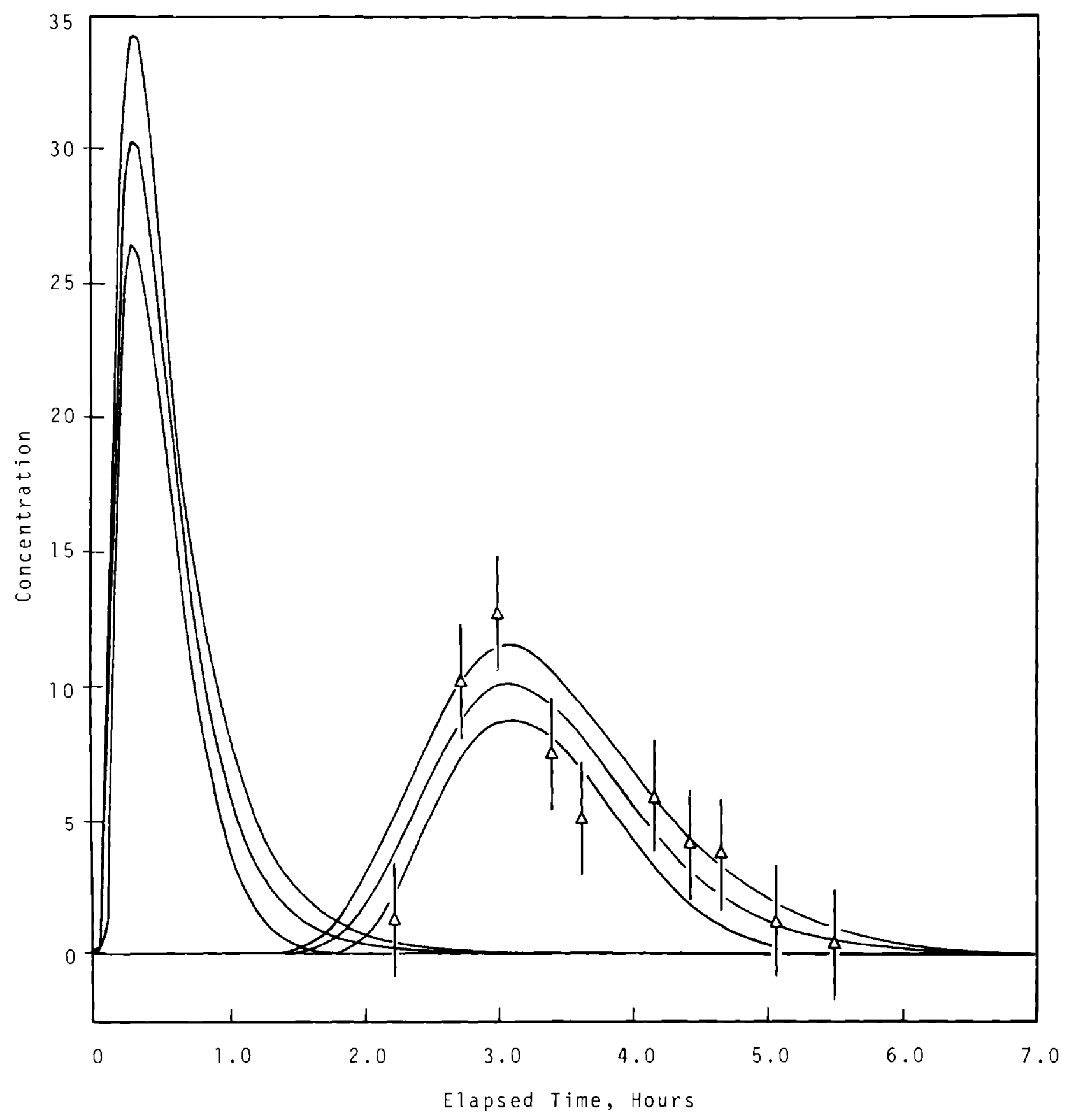

Neg PNL 703538-13

FIGURE C-6. Computer Generated Output of Best Fit to One-Dimensional Dispersion Using LEARN/DISPERS 
where $y, x=$ spacial parameters in consistent time frame

$$
t=\text { time }
$$

$\mathrm{a}, \mathrm{b}=$ experimental constants.

Using this expression, the external edge of the spreading mixing zone could be accurately predicted to the point where only one-dimensional effects became paramount.

Currently, using the dye imagery discussed above, a new approach has yielded additional insight into numerical values of the lateral dispersion coefficient $D_{y}$. If the assumption that the lateral distribution is Gaussian can be made, (generally true for reaches where momentum effects from the discharge jet or initial density differences are zero), the width of the plume at any distance ( $x$ ) from the point of release can be described as:

$$
\sigma_{(x)}=\sigma_{D}+\sigma_{M}
$$

where $\sigma_{(x)}=$ space variance at some distance from the release point

$\sigma_{D}=$ space variance due to the initial convective mixing

$$
\sigma_{M}=\text { dispersion represented by the diffusion process }
$$

then

$$
\sigma_{M}=(2 D y t)^{1 / 2}
$$

where $D_{y}=1$ ateral diffusion coefficient $\left(\mathrm{ft}^{2} / \mathrm{sec}\right)$.

Using the theory of convolution and making the further assumption that $95 \%$ or $4 \sigma$ of the tracer is the extent of the conservation of tracer to be included, it is possible to state:

$$
D_{y}=\frac{w_{2}^{2}-w_{1}^{2}}{32 \Delta t}
$$


where $W_{(x)}=$ plume width at a distance $x_{\text {from }} x_{0}$

$W_{0}=$ initial width at tracer release point

$x_{0}=$ origin of the diffusion process

then

$$
D_{y}=\frac{w_{2}^{2}-w_{1}^{2}}{32\left(\frac{\Delta x}{\bar{u}}\right)} \text { or } \frac{w^{2}(x)-w^{2}}{32\left(\frac{x-x_{0}}{\bar{u}}\right)}
$$

under the assumption that the conveyance velocity is uniform. Evaluations of the size of the spreading plume were made for both hot and cold conditions, all other effluent flow conditions being constant. The results are summarized in Table $\mathrm{C}-1$.

TABLE C-1. Summary of Lateral Diffusion Coefficients

\begin{tabular}{|c|c|c|c|c|}
\hline \multicolumn{2}{|c|}{ River Flow } & \multirow{2}{*}{$\begin{array}{l}\text { Effluent } \\
\text { Condition }\end{array}$} & \multirow{2}{*}{$\mathrm{D}_{\mathrm{y}} \mathrm{m}^{2} / \mathrm{sec}$} & \multirow[b]{2}{*}{$\mathrm{ft} / \mathrm{sec}$} \\
\hline (cumec) & $\mathrm{ft} / \mathrm{sec}$ & & & \\
\hline 1,420 & 50,108 & Cold & 0.177 & 1.9042 \\
\hline 2,850 & 100,560 & Cold & 0.184 & 1.9799 \\
\hline 2,270 & 80,100 & Hot & 0.492 & 5.2931 \\
\hline 3,130 & 110,450 & Hot & 0.680 & 7.3157 \\
\hline 4,120 & 145,380 & Hot & 0.271 & 2.9155 \\
\hline
\end{tabular}

The cases involving cold effluent or essentially neutral bouyant conditions reveal a lateral diffusion coefficient approximately equal to $0.18 \mathrm{~m}^{2} / \mathrm{sec}$. These results agree we 11 with the work performed independently by Glover. (C1) Under heated conditions, the lateral diffusion coefficient was generally higher. The assumption that dye particles are of zero size can be made, and for very low dye concentrations, (less than $10 \mathrm{ppb}$ ) the mixture of fluid and dye should have essentially the same density and kinematic viscosity as the normal, heated effluent. Therefore, the use of dye does not change the hydrodynamic character of the heated or cold plume 
to any measurable extent. This being the case, the results suggest that the thermal eddy diffusivity of the heated plume is greater than the natural eddy diffusivity of the river for the current ranges examined. This observation also suggests that as the momentum of the receiving water increases, the difference between the two coefficients will decrease asymptotically to a value identical with the eddy diffusivity of the river itself. These tentative conclusions appear to indicate that mixing from heated plums is greater in the cases where initial momentum differences between the receiving stream and the point of discharge are the greatest. This relationship has been qualitatively suggested in a number of publications and reports at Pacific Northwest Laboratory and is the basis for the recommendation that high velocity single point discharges are superior to canal discharges as a means of minimizing the temperatures of the mixing zone. This recommendation runs counter to standard design practice in many countries including the United States.

For a full treatment of this concept, please refer to "Dye Studies Through Use of Advancements in an Aerial Optical Mechanical Imaging System," by J. R. Eliason and H. P. Foote, BNWL-SA-3204, Apri1 1970, and "Dispersion Characteristics: Columbia River Between River Mile 383 and River Mile 355 ," by J. C. Sonnichsen, R. T. Jaske and D. A. Kottwitz, BNWL-1477 (in publication).

\section{REFERENCES}

C1. R. E. Glover. Dispersion of Dissolved or Suspended Materials in Flowing Streams. U.S.G.S. Professional Paper 433-B, Washington D.C., 1962 .

C2. B. Duane. Maximum Likelihood Nonlinear Correlated Fields, PROGRAM LIKELY, BNWL-390. Battelle-Northwest, Richland, Washington, September 1967.

C3. R. T. Jaske. Unpublished Data. Battelle-Northwest, Richland, Washington, June 1967. (Preliminary Report: "An Analysis of the Physical Factors Governing the Size and Temperature Gradients of the Hanford Effluent Plumes.") 
BNWL -1345

\author{
APPENDIX D \\ SECTION I \\ EFFECTS OF HANFORD OPERATIONS \\ ON COLUMBIA RIVER TEMPERATURES* \\ J. P. Corley
}

*Declassified Exerpts from HW-80888, dated December 3, 1964 


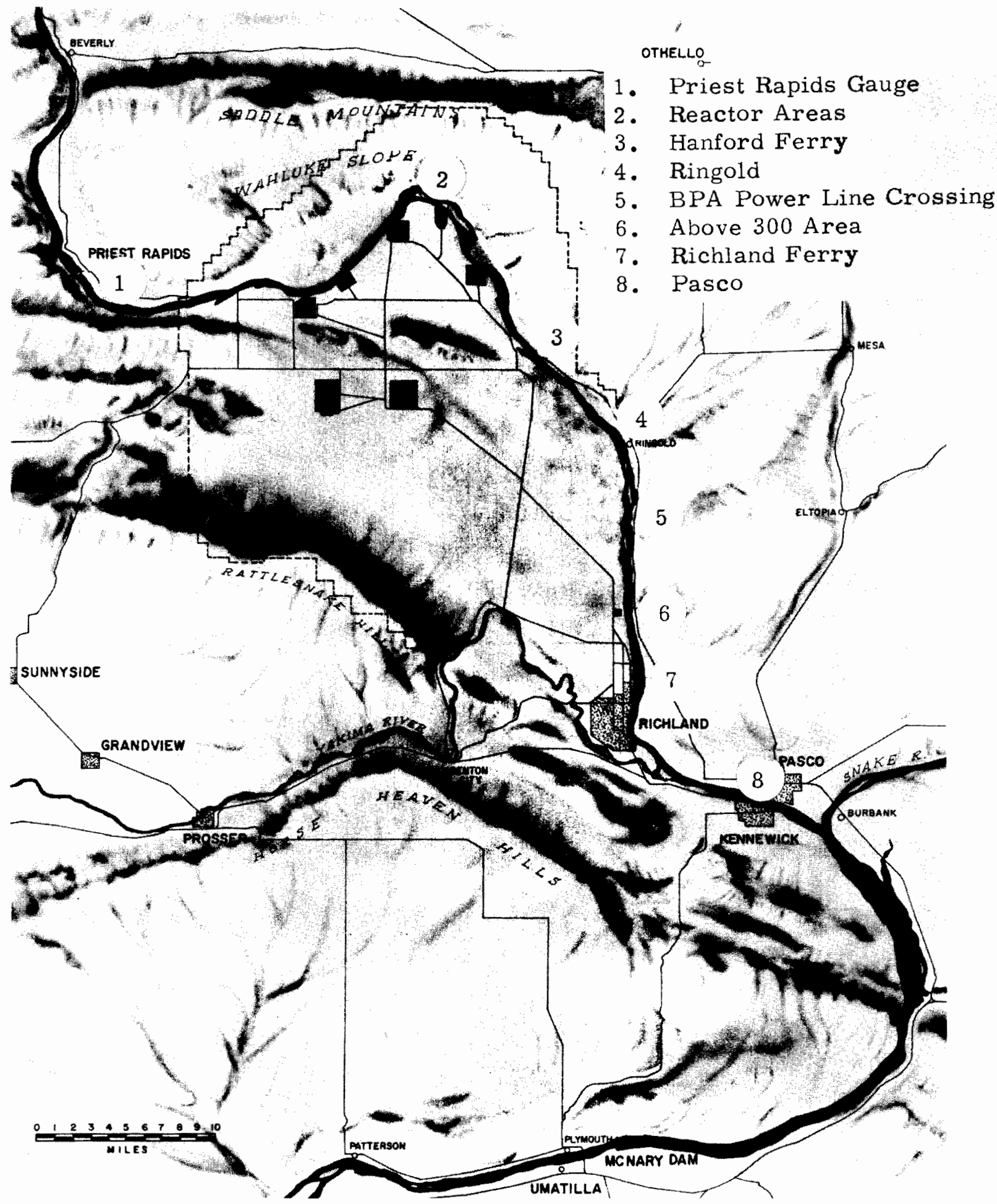

Neg 26119-1

Traverse Locations - Temperature Studies 


\section{APPENDIX D \\ SECTION I \\ EFFECTS OF HANFORD OPERATIONS \\ ON COLUMBIA RIVER TEMPERATURES}

J. P. Corley

\section{INTRODUCTION}

The effluent cooling water from the Hanford reactors differs markedly from its Columbia River source in its high temperature and chemical characteristics, as well as its radioactivity content. The effluents disperse slowly after discharge to the river; lateral variations being still measurable at Richland, some 30 miles downstream from the last reactor at $100-F$ Area. Direct measurement of these changes is difficult because of the uneven lateral distribution of the effluents, the variations with time due to rapidly varying water releases from Priest Rapids Dam, and external influences of weather and tributary and ground water inflows.

Columbia River temperatures are receiving increased attention by regulatory agencies (as well as potentially affected industries) because of the potential losses from increased temperatures of aquatic life, especially salmon. Increasing recognition of the thermal effects of dams in the rivers of the Pacific Northwest does not change the position of Hanford as the largest single man-made source of heat on the main stem of the Columbia. Accurate assessment of the actual impact of Hanford on river temperatures is, therefore, highly desirable. Related areas of local interest include local effluent distributions (for assessment of maximum concentration effects) and the accuracy with which Hanford production rates can be estimated from river temperatures (a plant security problem). In 
addition, detailed knowledge of changes in river chemical characteristics caused by reactor effluents is desired to evaluate the status of the river with respect to quality criteria for human consumption and for aquatic life.

A research and development project for study of the effects of reactor effluent on Columbia River water quality is being sponsored by the AEC Division of Production. Work was started in October 1962. The first effort was aimed at furnishing an immediate answer to a security question: how closely can Hanford production be estimated by measurement of river temperatures? An interim progress report (D1) gave the results of the preliminary investigation. During the calendar year 1963, the study was expanded to meet broader program objectives. This document is a progress report for the year, covering the temperature and effluent distribution phases of the program. Progress on the chemical characteristics phase of the program is documented separately. (D2) The frontispiece shows the section of the river under study.

\section{SUMMARY}

The first and major objective of the study program is determination of the net reactor heat effect on overall river temperatures. Progress during the year consisted primarily of instrument procurement and data collection. Investigation was started to determine the accuracy of existing upstream and downstream temperatures at the same locations, the heat losses from the effluent between the reactors and the river, the effluent trave 1 times, and the adequacy of meteorological data from the Meteorology Tower for use along the river. Specific items of interest found during the study included the probable inadequacy of any shoreline location as a direct 
monitoring point for river temperatures, and the probable existence of one or two points in every river cross section which would consistently give the mean cross-section temperature with adequate accuracy $\left( \pm 0.2^{\circ} \mathrm{C}\right)$.

Figures D.I 1 to D.I 3 show the envelopes of cross-section temperatures measured during 1963 at three locations. Note that the temperatures across each section were plotted in all cases as departures from the mean for a particular traverse. The mean temperatures varied from 5 to $20{ }^{\circ} \mathrm{C}$.

The $\mathrm{F}$ Area sewer temperature was recorded continuous $1 \mathrm{y}$ during September and October, 1963. This temperature cycled between 2 and $10{ }^{\circ} \mathrm{C}$ higher than the raw water temperature, and represents a total heat input to the river of less than $0.01{ }^{\circ} \mathrm{C}$ at all times. The data is summarized in Table D.I-11. Downstream River Flow Rate Estimation

No progress was made on this part of the study for 1 ack of a satisfactory base. Recorded gage elevations when translated to river flow rates showed consistent errors when compared with water release rates from Priest Rapids Dam as reported by Grant County P.U.D. The source of this error had not yet been found by the end of the calendar year. (Prior to issuing this report, the major source of error was found in a piece of mismatched equipment at the gage station. Corrections have been made and satisfactory base flow data established for proceeding with this part of the study.)

F1ow Time Measurement

A total of five instantaneous dye releases were made from various reactors from which time of travel measurements could be taken. The travel time data is summarized in Table D.I-1. The times of dye arrival were fairly close to those estimated from Reference D3. 
BNWL- 1345

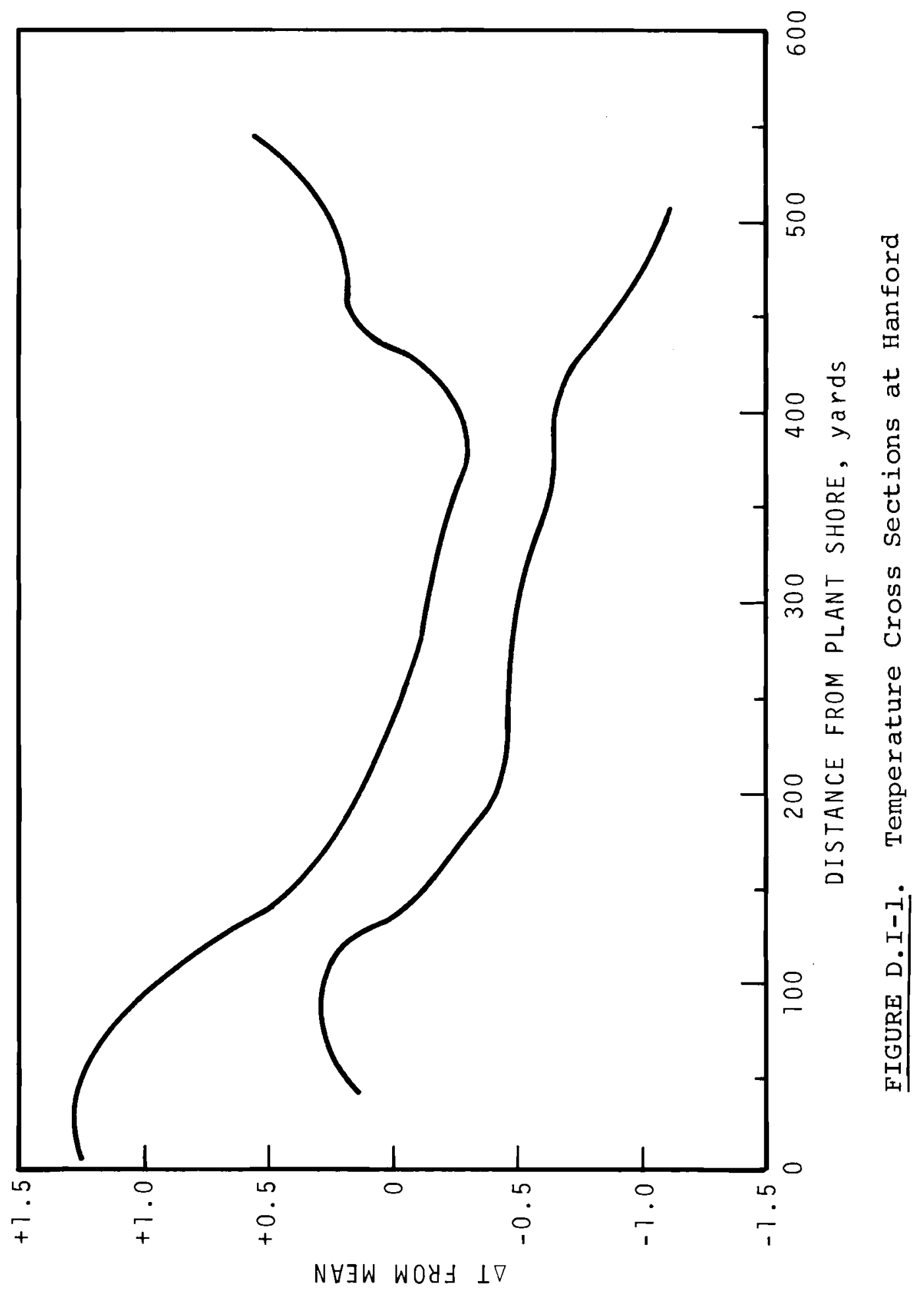

D. I . 4 
BNWL- 1345

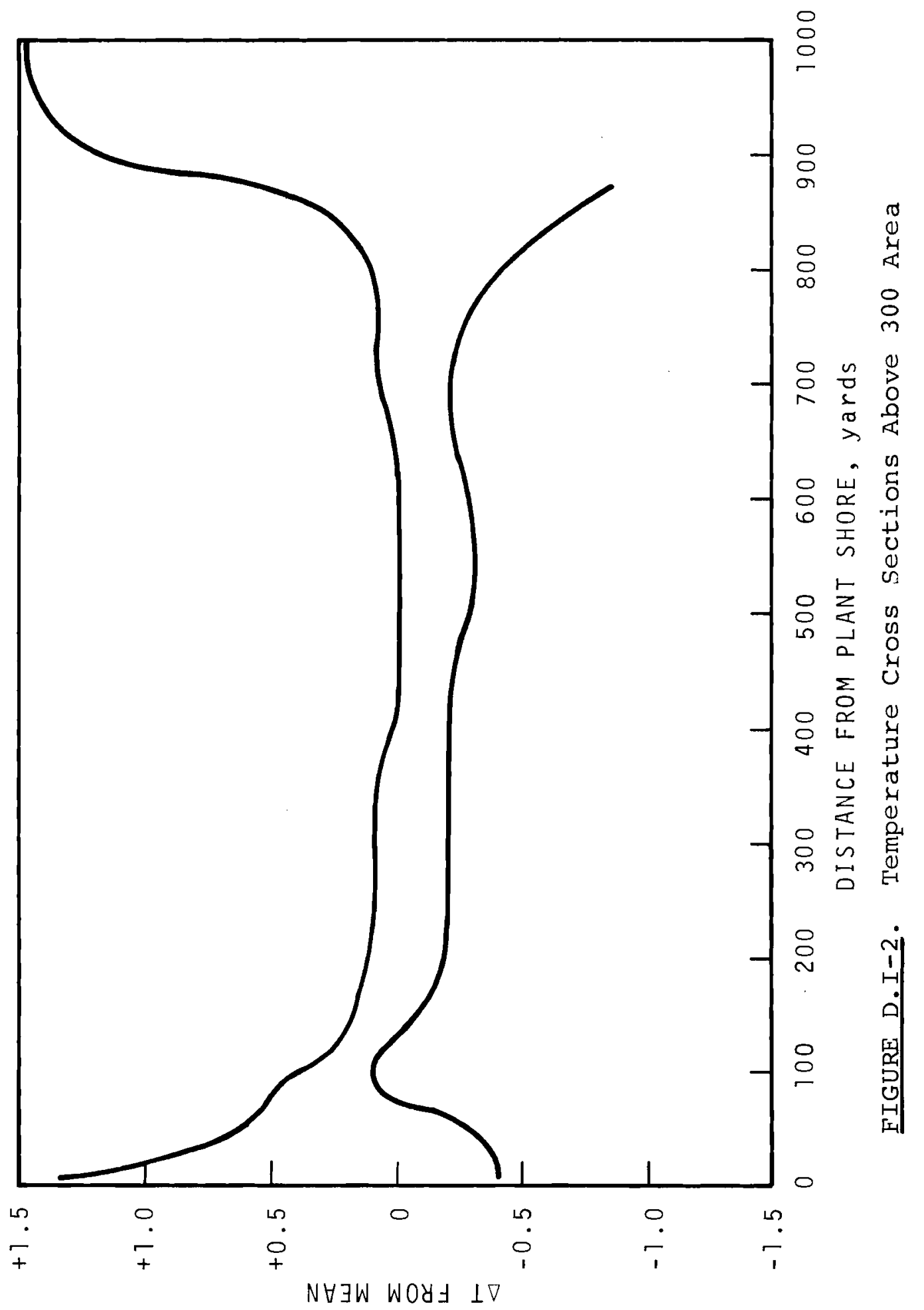

D. I. 5 
BNWL- 1345

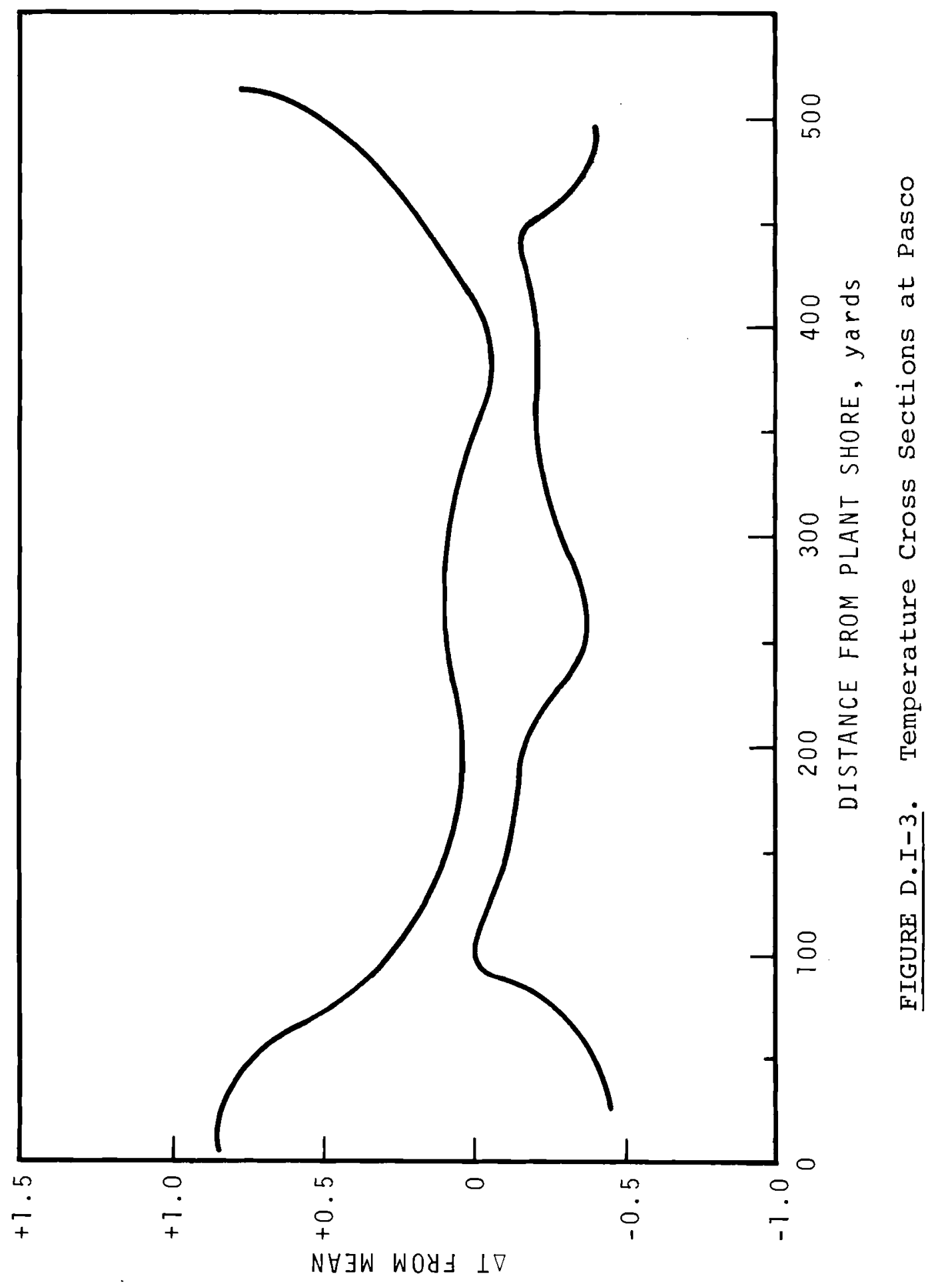

D. I. 6 
BNWL- 1345

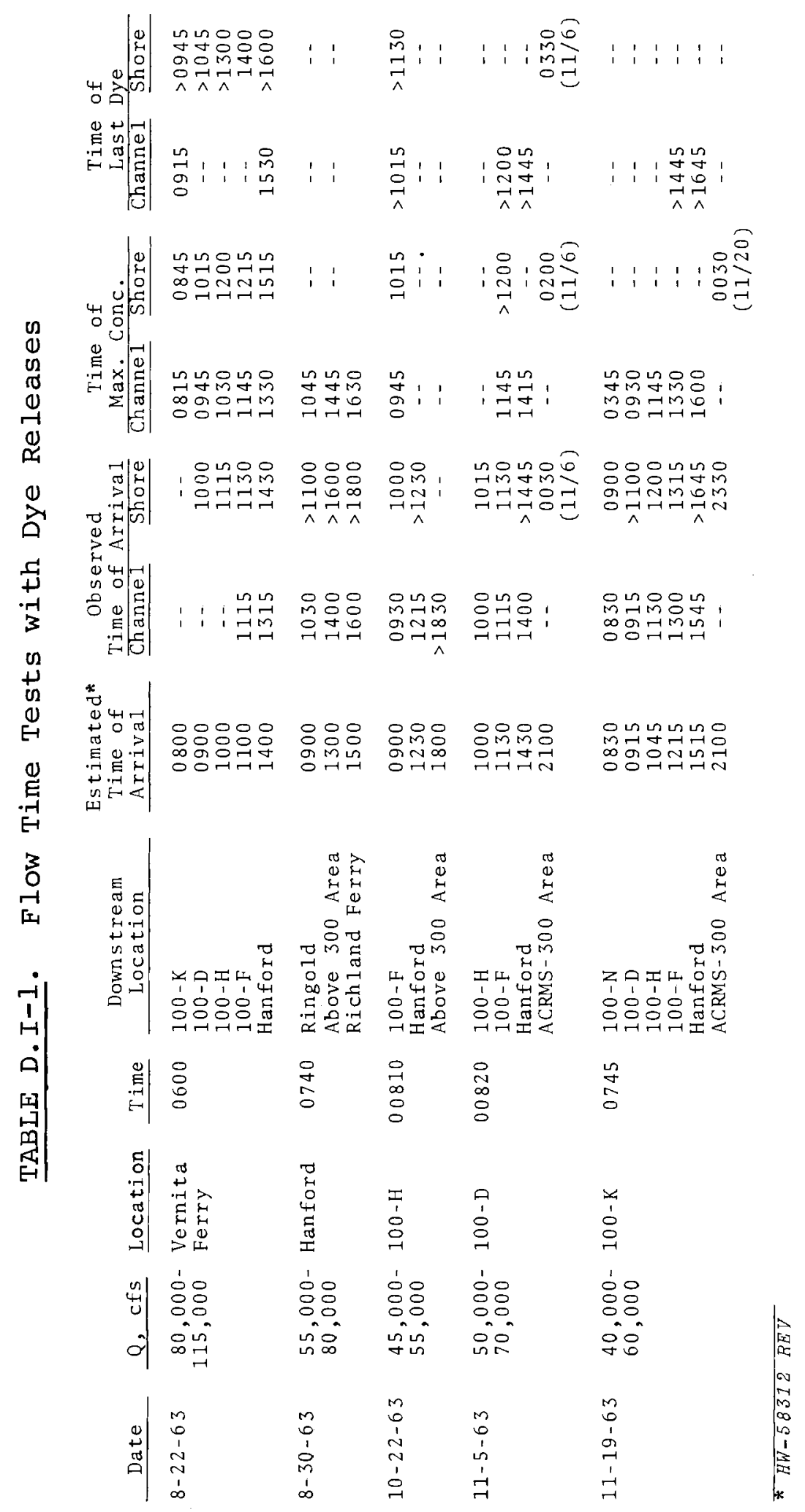

D. I. 7 
TABLE D.I-2. Cross-Section Mean Versus Recorded Temperatures

\begin{tabular}{|c|c|c|c|c|c|c|}
\hline Location & Date & Time & $\begin{array}{c}\text { Approximate } \\
\text { River Flow, cfs } \\
\end{array}$ & $\begin{array}{l}\text { Recorder } \\
\text { Temp, }{ }^{\circ} \mathrm{C} \\
\end{array}$ & $\begin{array}{l}\text { Mean River } \\
\text { Temp, }{ }^{\circ} \mathrm{C} \\
\end{array}$ & $\begin{array}{c}\Delta \mathrm{T} \quad \text { (Recorder } \\
\quad-\text { mean) } \\
\end{array}$ \\
\hline \multirow[t]{16}{*}{300 Area } & $11-29-62$ & 1330 & 80,000 & 10.2 & 10.2 & 0 \\
\hline & $2-12-63$ & 0800 & & 5.4 & 4.8 & +0.6 \\
\hline & $4-2-63$ & 1400 & 75,000 & 7.3 & 6.5 & +0.8 \\
\hline & $4-18-63$ & 1200 & 95,000 & 8.0 & 7.7 & +0.3 \\
\hline & $5-23-63$ & 1130 & 80,000 & 14.5 & 13.9 & +0.6 \\
\hline & $6-24-63$ & 1100 & 285,000 & 16.2 & 15.2 & +1.0 \\
\hline & $6-27-63$ & 1100 & 280,000 & 16.8 & 15.8 & +1.0 \\
\hline & $7-18-63$ & 1330 & 230,000 & 18.0 & 17.3 & +0.7 \\
\hline & $8-7-63$ & 1300 & 105,000 & 20.0 & 19.6 & +0.4 \\
\hline & $8-30-63$ & 1800 & 70,000 & 20.9 & 20.9 & 0 \\
\hline & $10-10-63$ & 1100 & 70,000 & 18.1 & 17.7 & +0.4 \\
\hline & $10-16-63$ & 1630 & 60,000 & 18.8 & 18.8 & 0 \\
\hline & $10-29-63$ & 1330 & 75,000 & 15.0 & 14.9 & +0.1 \\
\hline & $10-31-63$ & 1330 & 65,000 & 14.5 & 14.4 & +0.1 \\
\hline & $12-11-63$ & 1400 & 75,000 & 8.3 & 8.2 & +0.1 \\
\hline & $12-16-63$ & 0930 & 50,000 & 9.5 & 9.5 & 0 \\
\hline \multirow[t]{10}{*}{ Pasco } & $2-20-63$ & 1630 & 85,000 & 5.6 & 5.3 & +0.3 \\
\hline & $4-3-63$ & 1400 & 85,000 & 6.6 & 6.7 & -0.1 \\
\hline & $5-8-63$ & 1400 & 125,000 & 10.0 & 9.7 & +0.3 \\
\hline & $5-16-63$ & 1330 & 102,000 & 12.2 & 12.3 & -0.1 \\
\hline & $6-13-63$ & 1000 & 280,000 & 14.7 & 15.0 & -0.3 \\
\hline & $6-18-63$ & 1200 & 305,000 & 15.6 & 15.9 & -0.3 \\
\hline & $7-11-63$ & 1100 & 225,000 & 16.4 & 16.1 & +0.3 \\
\hline & $10-10-63$ & 1400 & 70,000 & 18.0 & 18.4 & -0.4 \\
\hline & $12-9-63$ & 1400 & 80,000 & 8.6 & 8.9 & -0.3 \\
\hline & $12-13-63$ & 1330 & 65,000 & 8.0 & 8.4 & -0.4 \\
\hline
\end{tabular}


TABLE D.I-3. River Temperature Traverses - Priest Rapids Gage (River Mile: 395.4)

\begin{tabular}{|c|c|c|c|c|c|c|c|c|c|c|c|}
\hline $\begin{array}{l}\text { Date: } \\
\text { Time: }\end{array}$ & $\begin{array}{l}1-3-63 \\
1120\end{array}$ & & $\begin{array}{l}\text { Date: } \\
\text { Time: }\end{array}$ & $\begin{array}{l}4-3-63 \\
1000\end{array}$ & & $\begin{array}{l}\text { Date: } \\
\text { Time: }\end{array}$ & $\begin{array}{l}5-21-6 \\
1200\end{array}$ & & $\begin{array}{l}\text { Date: } \\
\text { Time: }\end{array}$ & $\begin{array}{l}7-26-63 \\
1040\end{array}$ & \\
\hline $\begin{array}{r}\text { nistance } \\
\text { Plant } \\
\text { Sho. yd yd } \\
\end{array}$ & $\begin{array}{l}\text { Depth } \\
\mathrm{ft} \\
\end{array}$ & $\begin{array}{l}\text { Temp, } \\
{ }^{\circ} \mathrm{C} \\
\end{array}$ & $\begin{array}{l}\text { Distance } \\
\text { from P1ant } \\
\text { Shore, yd } \\
\end{array}$ & $\begin{array}{l}\text { Depth, } \\
\mathrm{ft} \\
\end{array}$ & $\begin{array}{l}\text { Temp } \\
{ }^{\circ} \mathrm{C} \\
\end{array}$ & $\begin{array}{l}\text { Distance } \\
\text { from Plant } \\
\text { Shore, yd } \\
\end{array}$ & $\begin{array}{l}\text { Depth } \\
\quad \mathrm{ft} \\
\end{array}$ & $\begin{array}{l}\text { Temp } \\
{ }^{\circ} \mathrm{C} \\
\end{array}$ & $\begin{array}{l}\text { Distance } \\
\text { from Plant } \\
\text { Shore, yd }\end{array}$ & $\begin{array}{l}\text { Depth, } \\
\text { ft } \\
\end{array}$ & $\begin{array}{c}\text { Temp, } \\
{ }^{\circ} \mathrm{C} \\
\end{array}$ \\
\hline 2 & 4 & 7.4 & 2 & 3 & 5.0 & 5 & 1 & 12.4 & 3 & 4 & 17.0 \\
\hline 50 & 28 & 7.5 & 40 & 27 & 5.0 & 40 & 27 & 11.8 & 45 & 30 & 17.1 \\
\hline 100 & 31 & 7.5 & 90 & 32 & 4.9 & 90 & 30 & 11.7 & 95 & 38 & 17.0 \\
\hline 150 & 35 & 7.5 & 140 & 32 & 5.0 & 140 & 30 & 11.7 & 145 & 38 & 16.9 \\
\hline 200 & 32 & 7.5 & 190 & 29 & 4.9 & 190 & 28 & 11.6 & 195 & 34 & 17.0 \\
\hline 250 & 29 & 7.4 & 240 & 27 & 5.0 & 240 & 25 & 11.0 & 245 & 33 & 17.0 \\
\hline 300 & 27 & 7.4 & 290 & 24 & 5.1 & 290 & 25 & 11.5 & 295 & 32 & 16.9 \\
\hline 350 & 20 & 7.4 & 340 & 21 & 5.1 & 340 & 22 & 11.5 & 345 & 27 & 17.0 \\
\hline \multirow[t]{2}{*}{380} & 3 & 7.5 & 390 & 3 & 5.0 & 390 & 13 & 11.7 & 395 & 17 & 17.0 \\
\hline & & & & & & 405 & 1 & 13.7 & 435 & 5 & 17.2 \\
\hline \multicolumn{3}{|c|}{$A_{t}=30,100 \mathrm{ft}^{2}$} & \multicolumn{3}{|c|}{$A_{t}=28,700 \mathrm{ft}^{2}$} & \multicolumn{3}{|c|}{$A_{t}=28,900 \mathrm{ft} t^{2}$} & \multicolumn{3}{|c|}{$A_{t}=37,400 \mathrm{ft}^{2}$} \\
\hline \multicolumn{3}{|c|}{$\mathrm{T}_{\mathrm{m}}=7.46^{\circ} \mathrm{C}$} & \multicolumn{3}{|c|}{$\mathrm{T}_{\mathrm{m}}=4.99^{\circ} \mathrm{C}$} & \multicolumn{3}{|c|}{$\mathrm{T}_{\mathrm{m}}=11.65^{\circ} \mathrm{C}$} & \multicolumn{3}{|c|}{$\mathrm{T}_{\mathrm{m}}=16.98^{\circ} \mathrm{C}$} \\
\hline \multicolumn{3}{|c|}{ Gage $=7.3{ }^{\circ} \mathrm{C}$} & \multicolumn{3}{|c|}{ Gage $=5.0^{\circ} \mathrm{C}$} & \multicolumn{3}{|c|}{ Gage $=11.65^{\circ} \mathrm{C}$} & \multicolumn{3}{|c|}{ Gage $=17.0{ }^{\circ} \mathrm{C}$} \\
\hline
\end{tabular}

NOTE: On Tables D.I-3 through D.I-10 the following nomenclature is used:

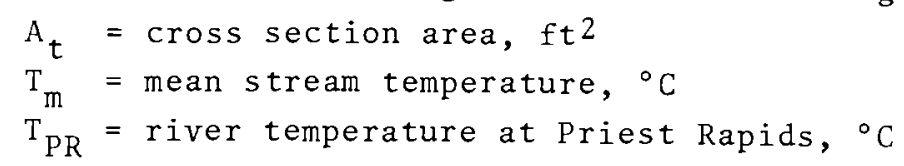


BNWL- 1345

TABLE D.I-4. River Temperature Traverses - Reactor Areas

\begin{tabular}{|c|c|c|c|c|c|c|c|c|}
\hline $\begin{array}{l}\text { Location: } \\
\text { River Mile: } \\
\text { Date: } \\
\text { Time }\end{array}$ & $\begin{array}{l}\text { Be 1ow } \\
381.5 \\
8-22-6 \\
0830\end{array}$ & $\begin{array}{l}1904-K \\
3\end{array}$ & $\begin{array}{l}\text { Location: } \\
\text { River Mile: } \\
\text { Date: } \\
\text { Time: }\end{array}$ & $\begin{array}{l}\text { Be 1ow } 1 \\
376.3 \\
8-22-63 \\
0940\end{array}$ & $\begin{array}{l}181-D \\
3\end{array}$ & $\begin{array}{l}\text { Location: } \\
\text { River Mile: } \\
\text { Date: } \\
\text { Time: }\end{array}$ & $\begin{array}{l}\text { Be 1 ow } \\
376.3 \\
8-22-6 \\
1000\end{array}$ & $181-\mathrm{D}$ \\
\hline $\begin{array}{l}\text { Distance } \\
\text { from Plant } \\
\text { Shore, yd } \\
\end{array}$ & $\begin{array}{c}\text { Depth, } \\
\mathrm{ft} \\
\end{array}$ & $\begin{array}{c}\text { Temp, } \\
{ }^{\circ} \mathrm{C} \\
\end{array}$ & $\begin{array}{l}\text { Distance } \\
\text { from Plant } \\
\text { Shore, yd }\end{array}$ & $\begin{array}{c}\text { Depth, } \\
\mathrm{ft} \\
\end{array}$ & $\begin{array}{l}\text { Temp, } \\
{ }^{\circ} \mathrm{C} \\
\end{array}$ & $\begin{array}{l}\text { Distance } \\
\text { from Plant } \\
\text { Shore, yd }\end{array}$ & $\begin{array}{c}\text { Depth, } \\
\mathrm{ft} \\
\end{array}$ & $\begin{array}{c}\text { Temp, } \\
{ }^{\circ} \mathrm{C}\end{array}$ \\
\hline 5 & 3 & 18.8 & 5 & 3 & 19.3 & 20 & 5 & 19.4 \\
\hline 100 & 8 & 19.0 & 15 & 4 & 19.3 & 70 & 8 & 19.4 \\
\hline 130 & 11 & 19.2 & 25 & 6 & 19.3 & 100 & 9 & 19.35 \\
\hline 175 & 19 & 18.9 & 55 & 8 & 19.3 & 115 & 10 & 19.4 \\
\hline 230 & 31 & 18.9 & 95 & 9 & 19.3 & 135 & 10 & 19.4 \\
\hline 270 & 25 & 19.5 & 115 & 10 & 19.35 & 155 & 10 & 19.4 \\
\hline 300 & 17 & 20.1 & 140 & 10 & 19.4 & 185 & 11 & 19.45 \\
\hline 355 & 12 & 18.8 & 185 & 11 & 19.4 & 200 & 11 & 19.5 \\
\hline 375 & 8 & 18.5 & 200 & 11 & 19.5 & 225 & 12 & 19.5 \\
\hline 430 & 6 & 18.4 & 220 & 12 & 19.5 & 245 & 12 & 19.5 \\
\hline \multirow[t]{12}{*}{450} & 4 & 18.4 & 290 & 11 & 19.5 & 275 & 12 & 19.5 \\
\hline & & & 310 & 11 & 19.5 & 295 & 11 & 19.5 \\
\hline & & & 335 & 10 & 19.5 & 320 & 10 & 19.45 \\
\hline & & & 365 & 11 & 19.45 & 345 & 11 & 19.4 \\
\hline & & & 395 & 12 & 19.4 & 365 & 11 & 19.3 \\
\hline & & & 415 & 13 & 19.3 & 390 & 12 & 19.25 \\
\hline & & & 445 & 13 & 19.2 & 415 & 13 & 19.15 \\
\hline & & & 470 & 12 & 19.1 & 440 & 13 & 19.0 \\
\hline & & & 495 & 11 & 19.0 & 475 & 12 & 18.9 \\
\hline & & & 525 & 8 & 18.9 & 495 & 11 & 18.8 \\
\hline & & & 550 & 6 & 18.9 & 525 & 8 & 18.7 \\
\hline & & & 570 & 4 & 18.8 & 570 & 4 & 18.7 \\
\hline & $\begin{array}{l}\mathrm{A}_{\mathrm{t}}=23, \\
\mathrm{~T}_{\mathrm{m}}=19 .\end{array}$ & $\begin{array}{l}800 \mathrm{ft}^{2} \\
04^{\circ} \mathrm{C}\end{array}$ & $\begin{array}{l}A_{t}=1 \\
T_{m}=1\end{array}$ & $\begin{array}{l}7,500 \mathrm{ft}^{2} \\
9.32{ }^{\circ} \mathrm{C}\end{array}$ & & $\begin{array}{l}A_{t}= \\
T_{m}=\end{array}$ & $\begin{array}{l}7,300 \mathrm{ft} \\
9.25^{\circ} \mathrm{C}\end{array}$ & \\
\hline $\mathrm{DA} / \mathrm{avg}$ & $\mathrm{PR}=18$ & $2^{\circ} \mathrm{C}$ & $\mathrm{T}_{\mathrm{PR}}=1$ & $8.2^{\circ} \mathrm{C}$ & & $\mathrm{T}_{\mathrm{PR}}=1$ & $8.2^{\circ} \mathrm{C}$ & \\
\hline
\end{tabular}


TABLE D.I-4. (contd) River Temperature Traverses Reactor Areas

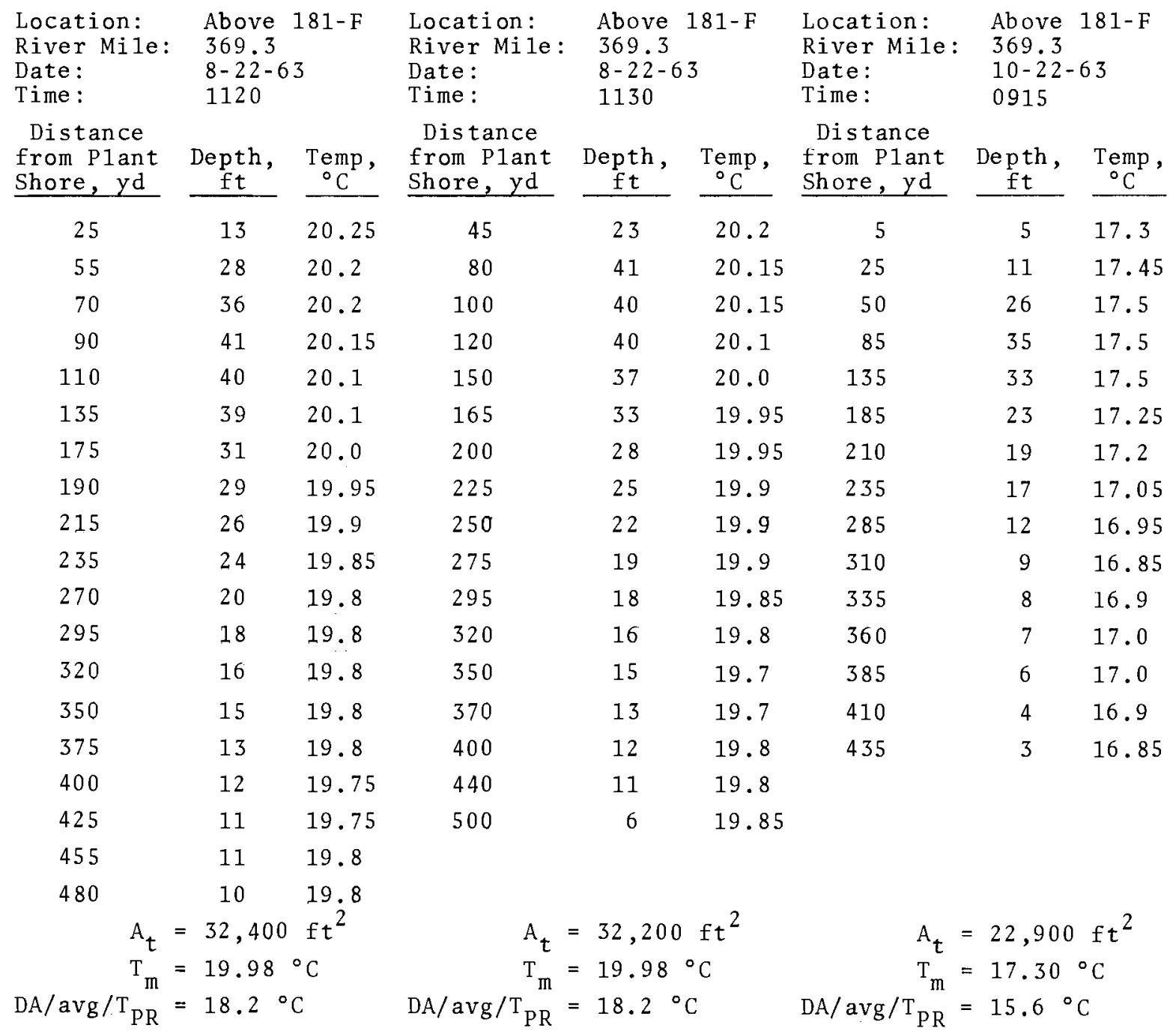


BNWL - 1345

\section{TABLE D.I-4. (contd) River Temperature Traverses - Reactor Areas}

\begin{tabular}{|c|c|c|c|c|c|c|c|c|}
\hline $\begin{array}{l}\text { Location: } \\
\text { River Mile: } \\
\text { Date: } \\
\text { Time: }\end{array}$ & $\begin{array}{l}\text { Above } 1 \\
369.3 \\
10-22-6 \\
0950\end{array}$ & $\begin{array}{l}181-F \\
63\end{array}$ & $\begin{array}{l}\text { Location: } \\
\text { River Mile: } \\
\text { Date: } \\
\text { Time: }\end{array}$ & $\begin{array}{l}\text { Above } 1 \\
369.3 \\
10-22-6 \\
1005\end{array}$ & $\begin{array}{l}181-F \\
63\end{array}$ & $\begin{array}{l}\text { Location: } \\
\text { River Mile: } \\
\text { Date: } \\
\text { Time: }\end{array}$ & $\begin{array}{l}\text { Above } \\
369.3 \\
10-29-6 \\
1045\end{array}$ & $\begin{array}{l}181-F \\
63\end{array}$ \\
\hline $\begin{array}{l}\text { Distance } \\
\text { from Plant } \\
\text { Shore, yd } \\
\end{array}$ & $\begin{array}{c}\text { Depth, } \\
\mathrm{ft} \\
\end{array}$ & $\begin{array}{l}\text { Temp, } \\
{ }^{\circ} \mathrm{C}, \\
\end{array}$ & $\begin{array}{l}\text { Distance } \\
\text { from Plant } \\
\text { Shore, yd }\end{array}$ & $\begin{array}{c}\text { Depth, } \\
\mathrm{ft} \\
\end{array}$ & $\begin{array}{l}\text { Temp, } \\
{ }^{\circ} \mathrm{C} \\
\end{array}$ & $\begin{array}{l}\text { Distance } \\
\text { from Plant } \\
\text { Shore, yd } \\
\end{array}$ & $\begin{array}{l}\text { Depth, } \\
\quad \mathrm{ft} \\
\end{array}$ & $\begin{array}{l}\text { Temp, } \\
{ }^{\circ} \mathrm{C} \\
\end{array}$ \\
\hline 5 & 5 & 17.5 & 5 & 5 & 17.55 & 5 & 5 & 15.5 \\
\hline 25 & 18 & 17.5 & 25 & 18 & 17.5 & 25 & 20 & 15.6 \\
\hline 50 & 24 & 17.5 & 50 & 18 & 17.5 & 50 & 29 & 15.6 \\
\hline 85 & 34 & 17.45 & 85 & 32 & 17.45 & 75 & 39 & 15.6 \\
\hline 135 & 36 & 17.35 & 135 & 40 & 17.4 & 125 & 36 & 15.5 \\
\hline 185 & 28 & 17.3 & 185 & 29 & 17.15 & 175 & 27 & 15.4 \\
\hline 210 & 23 & 17.2 & 210 & 24 & 17.0 & 200 & 22 & 15.3 \\
\hline 235 & 18 & 17.15 & 235 & 19 & 16.85 & 225 & 18 & 15.25 \\
\hline 285 & 14 & 17.05 & 260 & 15 & 16.85 & 250 & 16 & 15.2 \\
\hline 310 & 11 & 16.95 & 285 & 13 & 16.9 & 275 & 14 & 15.2 \\
\hline 335 & 9 & 16.9 & 310 & 10 & 16.95 & 300 & 11 & 15.15 \\
\hline 360 & 7 & 16.9 & 335 & 9 & 16.95 & 325 & 10 & 15.1 \\
\hline 385 & 6 & 16.85 & 360 & 8 & 16.95 & 350 & 9 & 15.05 \\
\hline 410 & 5 & 16.85 & 385 & 7 & 16.9 & 375 & 8 & 14.95 \\
\hline \multirow[t]{4}{*}{435} & 3 & 16.9 & 410 & 5 & 16.85 & 400 & 6 & 14.9 \\
\hline & & & 435 & 4 & 16.8 & 425 & 5 & 14.85 \\
\hline & & & 460 & 3 & 16.8 & 450 & 4 & 14.8 \\
\hline & & & & & & 475 & 4 & 14.8 \\
\hline$A_{t}$ & $=24,900$ & $f t^{2}$ & $A_{t}=$ & $=25,000$ & $\mathrm{ft}^{2}$ & $A_{t}$ & $=26,000$ & $f t^{2}$ \\
\hline $\mathrm{T}_{\mathrm{m}}$ & $=17.31^{\circ}$ & ${ }^{\circ} \mathrm{C}$ & $\mathrm{T}_{\mathrm{m}}=$ & $=17.21^{\circ}$ & ${ }^{\circ} \mathrm{C}$ & $\mathrm{T}_{\mathrm{m}}$ & $=15.38^{\circ}$ & ${ }^{\circ} \mathrm{C}$ \\
\hline $\mathrm{DA} / \mathrm{avg} / \mathrm{T} \mathrm{PR}$ & $=15.6^{\circ} \mathrm{C}$ & & $\mathrm{T}_{\mathrm{PR}}=$ & $15.6{ }^{\circ} \mathrm{C}$ & & $\mathrm{DA} / \mathrm{avg} / \mathrm{T}_{\mathrm{PR}}$ & $=14.1{ }^{\circ} \mathrm{C}$ & \\
\hline
\end{tabular}


BNWL- 1345

\section{TABLE D.I-4. (contd) River Temperature Traverses - Reactor Areas}

\begin{tabular}{|c|c|c|c|c|c|c|c|c|}
\hline $\begin{array}{l}\text { Location: } \\
\text { River Mile: } \\
\text { Date: } \\
\text { Time: }\end{array}$ & $\begin{array}{l}\text { Above } \\
369.3 \\
10-29- \\
1100\end{array}$ & $\begin{array}{l}181-F \\
63\end{array}$ & $\begin{array}{l}\text { Location: } \\
\text { River Mile: } \\
\text { Date: } \\
\text { Time: }\end{array}$ & $\begin{array}{l}\text { Above } \\
369.3 \\
11-5-6 \\
1045\end{array}$ & $181-F$ & $\begin{array}{l}\text { Location: } \\
\text { River Mile: } \\
\text { Date: } \\
\text { Time: }\end{array}$ & $\begin{array}{l}\text { Above } \\
369.3 \\
11-5-6 \\
1150\end{array}$ & $\begin{array}{l}181-F \\
3\end{array}$ \\
\hline $\begin{array}{l}\text { Distance } \\
\text { from Plant } \\
\text { Shore, yd } \\
\end{array}$ & $\begin{array}{c}\text { Depth, } \\
\mathrm{ft} \\
\end{array}$ & $\begin{array}{c}\text { Temp, } \\
{ }^{\circ} \mathrm{C} \\
\end{array}$ & $\begin{array}{l}\text { Distance } \\
\text { from P1ant } \\
\text { Shore, yd }\end{array}$ & $\begin{array}{c}\text { Depth, } \\
\mathrm{ft} \\
\end{array}$ & $\begin{array}{c}\text { Temp } \\
{ }^{\circ} \mathrm{C} \\
\end{array}$ & $\begin{array}{l}\text { Distance } \\
\text { from Plant } \\
\text { Shore, yd }\end{array}$ & $\begin{array}{c}\text { Depth, } \\
\mathrm{ft} \\
\end{array}$ & $\begin{array}{l}\text { Temp, } \\
{ }^{\circ} \mathrm{C} \\
\end{array}$ \\
\hline 5 & 4 & 15.7 & 5 & 5 & 14.4 & 10 & 11 & 14.65 \\
\hline 25 & 20 & 15.65 & 25 & 19 & 14.3 & 25 & 21 & 14.65 \\
\hline 50 & 26 & 15.65 & 50 & 27 & 14.25 & 50 & 34 & 14.65 \\
\hline 75 & 36 & 15.55 & 75 & 28 & 14.2 & 75 & 37 & 14.65 \\
\hline 125 & 37 & 15.45 & 100 & 38 & 14.15 & 100 & 38 & 14.65 \\
\hline 175 & 28 & 15.35 & 125 & 36 & 14.1 & 125 & 36 & 14.6 \\
\hline 200 & 23 & 15.3 & 150 & 32 & 13.95 & 150 & 31 & 14.55 \\
\hline 225 & 18 & 15.25 & 175 & 27 & 13.9 & 175 & 27 & 14.45 \\
\hline 250 & 16 & 15.2 & 200 & 21 & 13.75 & 200 & 21 & 14.35 \\
\hline 275 & 14 & 15.05 & 225 & 18 & 13.7 & 225 & 17 & 14.2 \\
\hline 300 & 11 & 15.0 & 250 & 15 & 13.65 & 250 & 15 & 14.25 \\
\hline 325 & 10 & 15.0 & 275 & 12 & 13.65 & 275 & 12 & 14.25 \\
\hline 350 & 9 & 14.95 & 300 & 10 & 13.6 & 300 & 10 & 14.2 \\
\hline 375 & 6 & 14.85 & 325 & 17 & 13.55 & 325 & 8 & 14.15 \\
\hline 400 & 6 & 14.85 & 350 & 14 & 13.45 & 350 & 7 & 14.15 \\
\hline 425 & 6 & 14.85 & 375 & 10 & 13.45 & 375 & 5 & 14.1 \\
\hline 450 & 4 & 14.8 & 400 & 7 & 13.45 & 400 & 4 & 14.05 \\
\hline \multirow[t]{2}{*}{475} & 3 & 14.8 & 425 & 5 & 13.5 & 425 & 4 & 14.05 \\
\hline & & & 450 & 5 & 13.5 & & & \\
\hline $\mathrm{DA} / \mathrm{avg} / \mathrm{T}_{\mathrm{PR}}^{\mathrm{T}_{\mathrm{m}}^{\mathrm{A}_{\mathrm{m}}}}$ & $\begin{array}{l}=26,100 \\
=15.35 \\
=14.1^{\circ}\end{array}$ & ${ }^{\circ} \mathrm{C}$ & $\mathrm{DA} / \mathrm{avg} / \mathrm{T}_{\mathrm{PR}}^{\mathrm{T}_{\mathrm{m}}}$ & $\begin{array}{l}=25,200 \\
=13.91^{\circ} \\
=13.0{ }^{\circ} \mathrm{C}\end{array}$ & ${ }_{\mathrm{C}}^{\mathrm{C} C}$ & $\begin{aligned} \mathrm{A}_{\mathrm{t}} & = \\
\mathrm{T}_{\mathrm{m}} & = \\
\mathrm{DA} / \mathrm{avg} / \mathrm{T}_{\mathrm{PR}} & =\end{aligned}$ & $\begin{array}{l}24,300 \\
14.49^{\circ} \\
13.0^{\circ} \mathrm{C}\end{array}$ & $f t^{2}$ \\
\hline
\end{tabular}




\section{TABLE D.I-4. (contd) River Temperature Traverses - Reactor Areas}

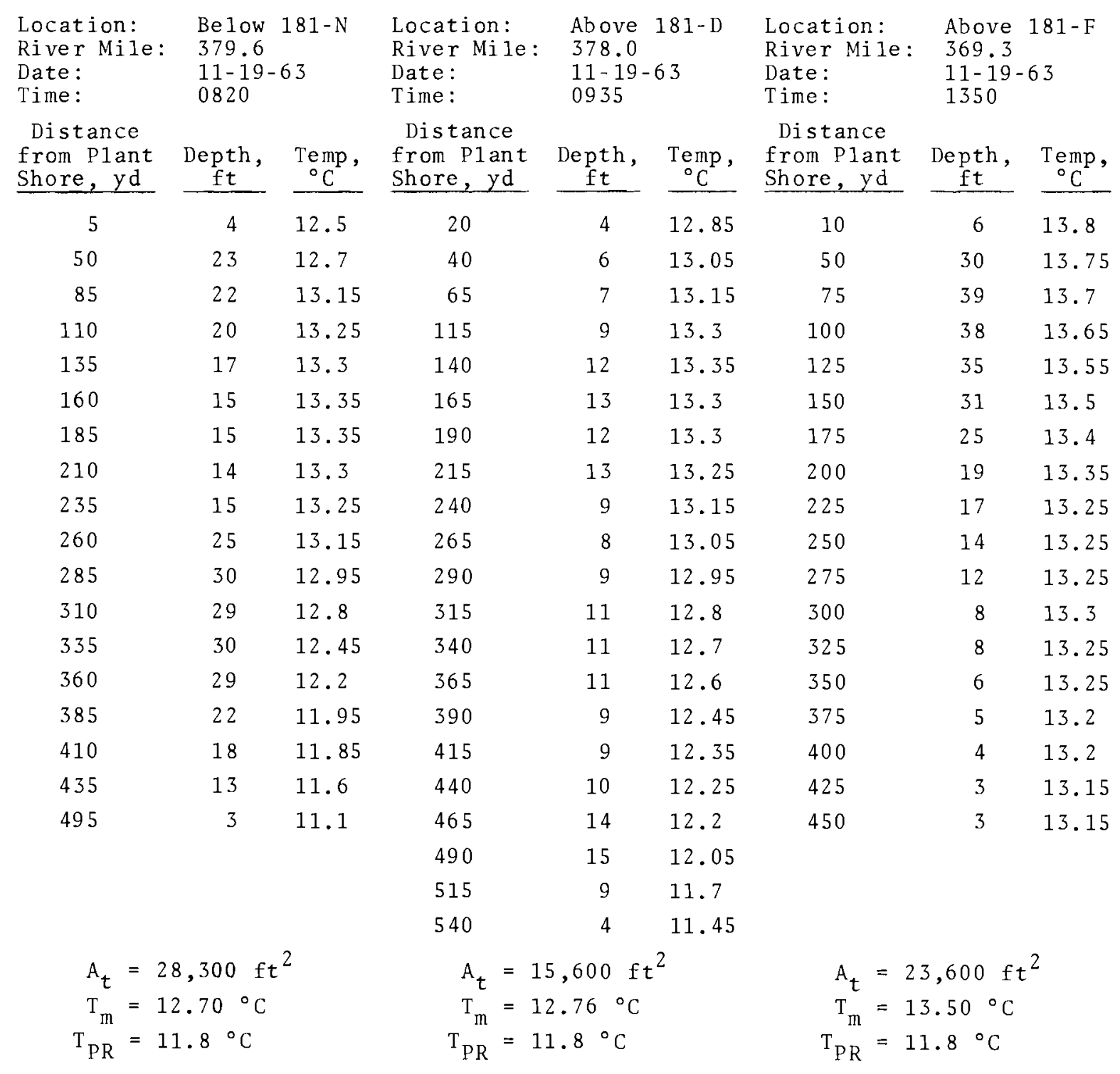


TABLE D.I-5. River Temperature Traverses - Hanford Ferry (River Mile: 361.6)

\begin{tabular}{|c|c|c|c|c|c|c|c|c|}
\hline \multirow{2}{*}{$\begin{array}{r}\text { Date } \\
\text { Time } \\
\text { Distance } \\
\text { from Plant } \\
\text { Shore, yd } \\
\end{array}$} & \multicolumn{2}{|c|}{$\begin{array}{l}1-3-63 \\
1325\end{array}$} & \multicolumn{3}{|c|}{$\begin{array}{l}\text { Date: } \\
\text { Time: }\end{array}$} & $\begin{array}{l}\text { Date: } \\
\text { Time: }\end{array}$ & \multicolumn{2}{|c|}{$\begin{array}{l}4-17-63 \\
1400\end{array}$} \\
\hline & $\begin{array}{c}\text { Depth, } \\
\mathrm{ft} \\
\end{array}$ & $\begin{array}{l}\text { Temp, } \\
{ }^{\circ} \mathrm{C} \\
\end{array}$ & $\begin{array}{l}\text { Distance } \\
\text { from Plant } \\
\text { Shore, yd } \\
\end{array}$ & $\begin{array}{c}\text { Depth, } \\
\mathrm{ft} \\
\end{array}$ & $\begin{array}{c}\text { Temp, } \\
{ }^{\circ} \mathrm{C} \\
\end{array}$ & $\begin{array}{l}\text { Distance } \\
\text { from Plant } \\
\text { Shore, yd }\end{array}$ & $\begin{array}{c}\text { Depth, } \\
\mathrm{ft}\end{array}$ & $\begin{array}{c}\text { Temp, } \\
{ }^{\circ} \mathrm{C}\end{array}$ \\
\hline 2 & 3 & 9.5 & 45 & 9 & 6.3 & 3 & 2 & 8.2 \\
\hline 50 & 10 & 9.5 & 95 & 11 & 6.4 & 35 & 9 & 8.2 \\
\hline 100 & 13 & 9.5 & 145 & 16 & 6.0 & 85 & 15 & 8.0 \\
\hline 150 & 14 & 9.4 & 195 & 17 & 6.0 & 135 & 16 & 7.9 \\
\hline 200 & 14 & 9.3 & 245 & 17 & 5.8 & 185 & 20 & 7.6 \\
\hline 250 & 15 & 9.3 & 295 & 18 & 5.1 & 235 & 19 & 7.5 \\
\hline 300 & 18 & 9.2 & 345 & 17 & 5.1 & 285 & 20 & 7.6 \\
\hline 350 & 23 & 9.1 & 395 & 14 & 4.8 & 335 & 19 & 7.5 \\
\hline 400 & 23 & 9.0 & 445 & 11 & 4.5 & 385 & 21 & 7.5 \\
\hline 450 & 20 & 8.9 & 495 & 2 & 4.5 & 435 & 17 & 7.4 \\
\hline 500 & 15 & 8.8 & & & & 485 & 7 & 7.4 \\
\hline 550 & 2 & 8.6 & & & & 505 & 1 & 7.5 \\
\hline $\begin{aligned} \mathrm{A}_{\mathrm{t}} & =25 \\
\mathrm{~T}_{\mathrm{m}} & =9 . \\
\mathrm{T}_{\mathrm{m}} & =7 .\end{aligned}$ & $\begin{array}{l}., 100 \mathrm{ft}^{2} \\
17^{\circ} \mathrm{C} \\
3{ }^{\circ} \mathrm{C}\end{array}$ & & $\begin{aligned} A_{t} & =1 \\
T_{m} & =5 \\
T_{m} & =3\end{aligned}$ & $\begin{array}{l}9,600 \mathrm{ft} \\
.53^{\circ} \mathrm{C} \\
8{ }^{\circ} \mathrm{C}\end{array}$ & & $\begin{aligned} A_{t} & =2 \\
T_{m} & =7 \\
& =6\end{aligned}$ & $\begin{array}{l}4,300 \mathrm{ft} \\
.64^{\circ} \mathrm{C} \\
6{ }^{\circ} \mathrm{C}\end{array}$ & \\
\hline $\mathrm{T}_{\mathrm{PR}}=7$ & $.3{ }^{\circ} \mathrm{C}$ & & $\mathrm{T}_{\mathrm{PR}}=3$ & $.8{ }^{\circ} \mathrm{C}$ & & $\mathrm{T}_{\mathrm{PR}}=6$ & $.6{ }^{\circ} \mathrm{C}$ & \\
\hline
\end{tabular}


BNWL- 1345

TABLE D.I-5. (contd) River Temperature Traverses Hanford Ferry (River Mile: 361.6)

\begin{tabular}{|c|c|c|c|c|c|c|c|c|}
\hline $\begin{array}{l}\text { Date: } \\
\text { Time: }\end{array}$ & $\begin{array}{l}6-20-63 \\
1130\end{array}$ & & $\begin{array}{l}\text { Date: } \\
\text { Time: }\end{array}$ & $\begin{array}{l}8-7-63 \\
1200\end{array}$ & & $\begin{array}{l}\text { Date: } \\
\text { Time: }\end{array}$ & $\begin{array}{l}8-22-63 \\
1235\end{array}$ & \\
\hline $\begin{array}{l}\text { Distance } \\
\text { from Plant } \\
\text { Shore, yd } \\
\end{array}$ & $\begin{array}{c}\text { Depth, } \\
\mathrm{ft} \\
\end{array}$ & $\begin{array}{l}\text { Temp, } \\
{ }^{\circ} \mathrm{C} \\
\end{array}$ & $\begin{array}{c}\text { Distance } \\
\text { from Plant } \\
\text { Shore, yd } \\
\end{array}$ & $\begin{array}{c}\text { Depth, } \\
\mathrm{ft} \\
\end{array}$ & $\begin{array}{l}\text { Temp, } \\
{ }^{\circ} \mathrm{C} \\
\end{array}$ & $\begin{array}{l}\text { Distance } \\
\text { from P1ant } \\
\text { Shore, yd } \\
\end{array}$ & $\begin{array}{c}\text { Depth, } \\
\mathrm{ft} \\
\end{array}$ & $\begin{array}{l}\text { Temp, } \\
{ }^{\circ} \mathrm{C}\end{array}$ \\
\hline 2 & 2 & 16.0 & 10 & 5 & 20.6 & 30 & 11 & 21.25 \\
\hline 50 & 20 & 16.1 & 40 & 12 & 20.5 & 65 & 16 & 21.15 \\
\hline 100 & 26 & 16.3 & 120 & 14 & 20.3 & 85 & 18 & 21.0 \\
\hline 150 & 31 & 16.1 & 175 & 19 & 20.0 & 115 & 20 & 20.95 \\
\hline 200 & 35 & 16.2 & 200 & 17 & 19.9 & 130 & 22 & 20.85 \\
\hline 250 & 32 & 16.1 & 225 & 21 & 19.8 & 150 & 22 & 20.75 \\
\hline 300 & 34 & 16.0 & 250 & 22 & 19.7 & 190 & 24 & 20.65 \\
\hline 350 & 33 & 16.0 & 275 & 21 & 19.65 & 200 & 24 & 20.6 \\
\hline 400 & 32 & 16.0 & 300 & 21 & 19.55 & 215 & 25 & 20.5 \\
\hline 450 & 32 & 16.0 & 325 & 20 & 19.5 & 250 & 25 & 20.45 \\
\hline 500 & 30 & 16.0 & 350 & 20 & 19.45 & 280 & 24 & 20.4 \\
\hline 550 & 18 & 16.1 & 375 & 19 & 19.4 & 315 & 24 & 20.35 \\
\hline 600 & 16 & 16.1 & 400 & 20 & 19.35 & 335 & 23 & 20.25 \\
\hline \multirow[t]{6}{*}{670} & 1 & 16.0 & 425 & 17 & 19.25 & 365 & 23 & 20.2 \\
\hline & & & 450 & 10 & 19.1 & 400 & 21 & 20.15 \\
\hline & & & 475 & 9 & 19.1 & 425 & 20 & 20.0 \\
\hline & & & 500 & 4 & 19.1 & 445 & 19 & 19.95 \\
\hline & & & 545 & 2 & 19.1 & 475 & 12 & 19.9 \\
\hline & & & & & & 490 & 9 & 19.85 \\
\hline \multicolumn{3}{|c|}{$A_{t}=53,100 \mathrm{ft}^{2}$} & \multicolumn{3}{|c|}{$A_{t}=24,150 \mathrm{ft}^{2}$} & \multicolumn{3}{|c|}{$A_{t}=29,700 \mathrm{ft}^{2}$} \\
\hline \multicolumn{3}{|c|}{$\mathrm{T}_{\mathrm{m}}=16.07{ }^{\circ} \mathrm{C}$} & $\mathrm{T}_{\mathrm{m}}=$ & $9.74{ }^{\circ} \mathrm{C}$ & & \multicolumn{3}{|c|}{$\mathrm{T}_{\mathrm{m}}=20.48^{\circ} \mathrm{C}$} \\
\hline \multicolumn{3}{|c|}{$\mathrm{T}_{\mathrm{PR}}=6.8{ }^{\circ} \mathrm{C}$} & $\mathrm{T}_{\mathrm{PR}}=$ & $8.6^{\circ} \mathrm{C}$ & & \multicolumn{3}{|c|}{$\mathrm{T}_{\mathrm{PR}}=18.2{ }^{\circ} \mathrm{C}$} \\
\hline
\end{tabular}

D. I. 16 
TABLE D.I-5. (contd)
River Temperature Traverses Hanford Ferry (River Mile: $361.6)$

\begin{tabular}{|c|c|c|}
\hline \multirow{2}{*}{$\begin{array}{r}\text { Date: } \\
\text { Time: } \\
\text { Distance } \\
\text { from Plant } \\
\text { Shore, yd } \\
\end{array}$} & \multicolumn{2}{|c|}{$\begin{array}{l}8-22-63 \\
1410\end{array}$} \\
\hline & $\begin{array}{c}\text { Depth, } \\
\mathrm{ft} \\
\end{array}$ & $\begin{array}{c}\text { Temp, } \\
{ }^{\circ} \mathrm{C}\end{array}$ \\
\hline 10 & 6 & 21.7 \\
\hline 25 & 10 & 21.7 \\
\hline 40 & 12 & 21.7 \\
\hline 80 & 17 & 21.7 \\
\hline 105 & 20 & 21.6 \\
\hline 135 & 23 & 21.25 \\
\hline 155 & 22 & 21.05 \\
\hline 190 & 24 & 20.85 \\
\hline 205 & 24 & 20.7 \\
\hline 230 & 26 & 20.6 \\
\hline 260 & 25 & 20.5 \\
\hline 285 & 24 & 20.4 \\
\hline 310 & 24 & 20.3 \\
\hline 335 & 23 & 20.3 \\
\hline 360 & 23 & 20.25 \\
\hline 380 & 23 & 20.25 \\
\hline 400 & 21 & 20.25 \\
\hline 430 & 20 & 20.3 \\
\hline 460 & 15 & 20.3 \\
\hline $\begin{array}{l}A_{t}= \\
T_{m}=\end{array}$ & $\begin{array}{l}.800 \mathrm{f} \\
.72{ }^{\circ} \mathrm{C}\end{array}$ & \\
\hline $\mathrm{T}_{\mathrm{PR}}=$ & $.2{ }^{\circ} \mathrm{C}$ & \\
\hline
\end{tabular}

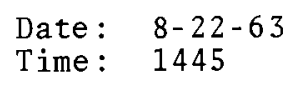

from Plant Depth, Temp, Shore, yd
Date: $8-22-63$

Time: 1555

Distance

from Plant Depth, Temp, Shore, yd $\mathrm{ft} \quad{ }^{\circ} \mathrm{C}$

5
35
70
85
110
140
170
215
250
280
295
345
370
390
420
440
470

$3 \quad 21.85$

$12 \quad 21.85$

$17 \quad 21.75$

$18 \quad 21.65$

$20 \quad 21.4$

23

22

21.1

$22 \quad 20.75$

$25 \quad 20.35$

$25 \quad 20.25$

$24 \quad 20.2$

$24 \quad 20.15$

$23 \quad 20.15$

$23 \quad 20.15$

$22 \quad 20.15$

$20 \quad 20.2$

$19 \quad 20.25$

$13 \quad 20.35$
5

85

110

135

160

185

200

215

240

265

285

315

355

395

420

455

475

510
21.75

$18 \quad 21.45$

$20 \quad 21.1$

$23 \quad 20.85$

$22 \quad 20.7$

$23 \quad 20.6$

$24 \quad 20.5$

$25 \quad 20.45$

$26 \quad 20.4$

$25 \quad 20.35$

$24 \quad 20.3$

$24 \quad 20.3$

$23 \quad 20.25$

$22 \quad 20.2$

$20 \quad 20.2$

$17 \quad 20.2$

$3 \quad 20.9$
$12 \quad 20.35$

$$
A_{t}=29,800 \mathrm{ft}^{2}
$$$$
A_{t}=22,700 f t^{2}
$$$$
\mathrm{T}_{\mathrm{m}}=20.59{ }^{\circ} \mathrm{C}
$$$$
A_{t}=29,300 \mathrm{ft}^{2}
$$$$
\mathrm{T}_{\mathrm{PR}}=18.2^{\circ} \mathrm{C}
$$$$
\begin{aligned}
\mathrm{T}_{\mathrm{m}} & =20.56^{\circ} \mathrm{C} \\
\mathrm{T}_{\mathrm{PR}} & =18.2^{\circ} \mathrm{C}
\end{aligned}
$$ 


\section{TABLE D.I-5. (contd) River Temperature Traverses - Hanford (River Mile: 361.0)}

\begin{tabular}{|c|c|c|c|c|c|c|c|c|}
\hline \multirow[b]{2}{*}{$\begin{array}{l}\text { Distance } \\
\text { from Plant } \\
\text { Shore, yd } \\
\end{array}$} & \multicolumn{2}{|l|}{$\begin{array}{l}9-25-63 \\
0930\end{array}$} & $\begin{array}{l}\text { Date: } \\
\text { Time: }\end{array}$ & \multicolumn{2}{|c|}{$\begin{array}{l}10-16-63 \\
0920\end{array}$} & $\begin{array}{l}\text { Date: } \\
\text { Time: }\end{array}$ & \multicolumn{2}{|c|}{$\begin{array}{l}10-16-63 \\
1000\end{array}$} \\
\hline & $\begin{array}{c}\text { Depth, } \\
\mathrm{ft} \\
\end{array}$ & $\begin{array}{l}\text { Temp, } \\
{ }^{\circ} \mathrm{C} \\
\end{array}$ & $\begin{array}{l}\text { Distance } \\
\text { from Plant } \\
\text { Shore, yd } \\
\end{array}$ & $\begin{array}{c}\text { Depth, } \\
\mathrm{ft} \\
\end{array}$ & $\begin{array}{l}\text { Temp, } \\
{ }^{\circ} \mathrm{C} \\
\end{array}$ & $\begin{array}{l}\text { Distance } \\
\text { from P1ant } \\
\text { Shore, yd } \\
\end{array}$ & $\begin{array}{c}\text { Depth, } \\
\mathrm{ft} \\
\end{array}$ & $\begin{array}{c}\text { Temp, } \\
{ }^{\circ} \mathrm{C}\end{array}$ \\
\hline 5 & 3 & 19.85 & 5 & 3 & 18.75 & 5 & 3 & 19.0 \\
\hline 25 & 7 & 19.75 & 50 & 13 & 18.65 & 25 & 7 & 18.85 \\
\hline 50 & 10 & 19.7 & 75 & 16 & 18.4 & 75 & 17 & 18.65 \\
\hline 75 & 13 & 19.6 & 95 & 18 & 18.35 & 125 & 23 & 18.6 \\
\hline 100 & 15 & 19.5 & 125 & 23 & 18.3 & 165 & 21 & 18.3 \\
\hline 125 & 17 & 19.4 & 175 & 18 & 18.0 & 200 & 15 & 18.1 \\
\hline 150 & 18 & 19.25 & 205 & 14 & 17.75 & 225 & 12 & 18.05 \\
\hline 175 & 20.5 & 18.95 & 230 & 11 & 17.7 & 250 & 9 & 17.9 \\
\hline 200 & 19.5 & 18.75 & 255 & 9 & 17.65 & 275 & 8 & 17.8 \\
\hline 225 & 15 & 18.7 & 285 & 7 & 17.6 & 300 & 8 & 17.9 \\
\hline 250 & 11 & 18.65 & 305 & 7 & 17.55 & 325 & 8 & 17.75 \\
\hline 275 & 8.5 & 18.6 & 350 & 7 & 17.5 & 350 & 8 & 17.6 \\
\hline 300 & 7 & 18.6 & 385 & 7 & 17.45 & 375 & 8 & 17.55 \\
\hline 325 & 6.5 & 18.55 & 400 & 7 & 17.4 & 405 & 8 & 17.5 \\
\hline 350 & 7 & 18.45 & 425 & 7 & 17.3 & 425 & 7 & 17.5 \\
\hline 375 & 7 & 18.4 & 450 & 6 & 17.2 & 445 & 7 & 17.45 \\
\hline 400 & 7 & 18.35 & 505 & 2 & 16.85 & 475 & 7 & 17.5 \\
\hline 425 & 7 & 18.35 & & & & 500 & 3 & 17.35 \\
\hline 465 & 7 & 18.35 & & & & & & \\
\hline 515 & 6 & 18.4 & & & & & & \\
\hline 540 & 3 & 18.9 & & & & & & \\
\hline $\mathrm{DA} / \mathrm{avg} / \mathrm{T}_{\mathrm{PR}}^{\mathrm{T}_{\mathrm{m}}}{ }_{\mathrm{P}}^{\mathrm{T}_{\mathrm{t}}}$ & $\begin{array}{l}=17,000 \\
=18.92 \\
=17.9^{\circ}\end{array}$ & ${ }^{\circ} \mathrm{Ct}$ & $\begin{array}{r}\mathrm{T}_{\mathrm{n}} \\
\mathrm{T}_{\mathrm{PF}}\end{array}$ & $\begin{array}{l}=15,7 \\
=17.94 \\
=16.6\end{array}$ & ${ }^{\circ} \mathrm{C}$ & $\mathrm{T}_{\mathrm{Pl}}^{\mathrm{T}_{\mathrm{r}}}$ & $\begin{array}{l}=17,10 \\
=18.22 \\
=16.6\end{array}$ & ${ }^{0} \mathrm{ft}^{2}$ \\
\hline
\end{tabular}


TABLE D.I-5. (contd) River Temperature Traverses Hanford (River Mile: 361.0)

\begin{tabular}{|c|c|c|c|c|c|c|c|c|}
\hline $\begin{array}{l}\text { Date: } \\
\text { Time: }\end{array}$ & $\begin{array}{l}10-16-6 \\
1030\end{array}$ & & $\begin{array}{l}\text { Date: } \\
\text { Time: }\end{array}$ & $\begin{array}{l}10-16-6 \\
1120\end{array}$ & & $\begin{array}{l}\text { Date: } \\
\text { Time: }\end{array}$ & $\begin{array}{l}10-16-6 \\
1200\end{array}$ & \\
\hline $\begin{array}{l}\text { Distance } \\
\text { from P1ant } \\
\text { Shore, yd } \\
\end{array}$ & $\begin{array}{c}\text { Depth, } \\
\mathrm{ft}\end{array}$ & $\begin{array}{c}\text { Temp, } \\
{ }^{\circ} \mathrm{C} \\
\end{array}$ & $\begin{array}{l}\text { Distance } \\
\text { from Plant } \\
\text { Shore, yd } \\
\end{array}$ & $\begin{array}{l}\text { Depth, } \\
\quad \mathrm{ft} \\
\end{array}$ & $\begin{array}{c}\text { Temp, } \\
{ }^{\circ} \mathrm{C} \\
\end{array}$ & $\begin{array}{l}\text { Distance } \\
\text { from Plant } \\
\text { Shore, yd }\end{array}$ & $\begin{array}{c}\text { Depth, } \\
\mathrm{ft}\end{array}$ & $\begin{array}{c}\text { Temp, } \\
{ }^{\circ} \mathrm{C}\end{array}$ \\
\hline 5 & 3 & 19.1 & 10 & 6 & 19.2 & 10 & 6 & 19.4 \\
\hline 25 & 7 & 19.05 & 25 & 13 & 19.1 & 25 & 6 & 19.4 \\
\hline 70 & 14 & 18.95 & 75 & 16 & 19.0 & 50 & 9 & 19.5 \\
\hline 125 & 23 & 18.6 & 125 & 22 & 18.75 & 75 & 14 & 19.5 \\
\hline 175 & 18 & 18.35 & 150 & 20 & 18.6 & 100 & 18 & 19.35 \\
\hline 200 & 15 & 18.3 & 175 & 17 & 18.5 & 125 & 19 & 19.2 \\
\hline 225 & 11 & 18.2 & 200 & 15 & 18.35 & 150 & 19 & 18.95 \\
\hline 250 & 10 & 18.15 & 225 & 9 & 18.2 & 175 & 19 & 18.8 \\
\hline 275 & 8 & 18.05 & 250 & 8 & 18.15 & 200 & 14 & 18.65 \\
\hline 300 & 7 & 18.05 & 275 & 6 & 18.1 & 225 & 9 & 18.5 \\
\hline 325 & 7 & 18.0 & 300 & 6 & 18.05 & 250 & 7 & 18.5 \\
\hline 350 & 7 & 17.95 & 325 & 6 & 18.0 & 275 & 6 & 18.45 \\
\hline 375 & 7 & 17.9 & 350 & 6 & 18.0 & 300 & 6 & 18.4 \\
\hline 400 & 7 & 17.75 & 375 & 6 & 17.95 & 325 & 6 & 18.4 \\
\hline 425 & 7 & 17.75 & 400 & 8 & 17.9 & 350 & 6 & 18.3 \\
\hline 455 & 6 & 17.7 & 425 & 6 & 17.85 & 375 & 6 & 18.3 \\
\hline 475 & 7 & 17.5 & 450 & 5 & 17.65 & 400 & 6 & 18.25 \\
\hline \multirow[t]{3}{*}{495} & 4 & 17.6 & 470 & 3 & 17.5 & 425 & 6 & 18.15 \\
\hline & & & & & & 450 & 5 & 18.1 \\
\hline & & & & & & 475 & 3 & 18.0 \\
\hline $\begin{aligned} \mathrm{A}_{\mathrm{t}} & =1 \\
\mathrm{~T}_{\mathrm{m}} & =1 \\
\mathrm{~T}_{\mathrm{PR}} & =1\end{aligned}$ & $\begin{array}{l}6,300 \mathrm{ft} \\
8.33^{\circ} \mathrm{C} \\
6.6{ }^{\circ} \mathrm{C}\end{array}$ & & $\begin{aligned} \mathrm{A}_{\mathbf{t}} & = \\
\mathrm{T}_{\mathrm{m}} & = \\
\mathrm{T}_{\mathrm{PR}} & =\cdots\end{aligned}$ & $\begin{array}{l}5,300 \mathrm{ft} \\
8.51{ }^{\circ} \mathrm{C} \\
6.6{ }^{\circ} \mathrm{C}\end{array}$ & & $\begin{aligned} \mathrm{A}_{\mathrm{t}} & = \\
\mathrm{T}_{\mathrm{m}} & = \\
\mathrm{T}_{\mathrm{PR}} & =\end{aligned}$ & $\begin{aligned} \mathrm{T}_{\mathrm{m}} & =18.85{ }^{\circ} \mathrm{C} \\
{ }_{\mathrm{T}} \mathrm{PR} & =16.6{ }^{\circ} \mathrm{C}\end{aligned}$ & \\
\hline
\end{tabular}




\section{TABLE D.I-5. (contd) River Temperature Traverses - Hanford (River Mile: 361.0)}

\begin{tabular}{|c|c|c|c|c|c|c|c|c|}
\hline \multirow{2}{*}{$\begin{array}{r}\text { Date: } \\
\text { Time: } \\
\text { Distance } \\
\text { from Plant } \\
\text { Shore, yd } \\
\end{array}$} & \multicolumn{2}{|c|}{$\begin{array}{l}10-16-63 \\
1245\end{array}$} & $\begin{array}{l}\text { Date: } \\
\text { Time: }\end{array}$ & \multicolumn{2}{|c|}{$\begin{array}{l}10-22-63 \\
1110\end{array}$} & $\begin{array}{l}\text { Date: } \\
\text { Time: }\end{array}$ & \multicolumn{2}{|c|}{$\begin{array}{l}10-22-63 \\
1230\end{array}$} \\
\hline & $\begin{array}{l}\text { Depth, } \\
\mathrm{ft} \\
\end{array}$ & $\begin{array}{c}\text { Temp, } \\
{ }^{\circ} \mathrm{C} \\
\end{array}$ & $\begin{array}{l}\text { Distance } \\
\text { from Plant } \\
\text { Shore, yd } \\
\end{array}$ & $\begin{array}{l}\text { Depth, } \\
\mathrm{ft} \\
\end{array}$ & $\begin{array}{l}\text { Temp, } \\
{ }^{\circ} \mathrm{C} \\
\end{array}$ & $\begin{array}{l}\text { Distance } \\
\text { from Plant } \\
\text { Shore, yd } \\
\end{array}$ & $\begin{array}{l}\text { Depth, } \\
\mathrm{ft} \\
\end{array}$ & $\begin{array}{l}\text { Temp, } \\
{ }^{\circ} \mathrm{C} \\
\end{array}$ \\
\hline 5 & 3 & 19.6 & 10 & 4 & 17.6 & 10 & 4 & 17.65 \\
\hline 15 & 6 & 19.65 & 25 & 5 & 17.8 & 25 & 5 & 17.7 \\
\hline 50 & 10 & 19.7 & 50 & 7 & 17.85 & 80 & 17 & 17.85 \\
\hline 75 & 11 & 19.6 & 95 & 14 & 17.7 & 120 & 21 & 17.55 \\
\hline 125 & 20 & 19.3 & 120 & 16 & 17.55 & 170 & 17 & 17.3 \\
\hline 175 & 20 & 19.1 & 170 & 18 & 17.4 & 195 & 13 & 17.15 \\
\hline 200 & 18 & 18.9 & 195 & 14 & 16.85 & 220 & 10 & 16.95 \\
\hline 225 & 13 & 18.8 & 220 & 9 & 16.95 & 245 & 8 & 16.95 \\
\hline 250 & 9 & 18.7 & 245 & 7 & 16.9 & 270 & 5 & 16.9 \\
\hline 385 & 7 & 18.45 & 270 & 5 & 16.85 & 295 & 5 & 16.9 \\
\hline 415 & 7 & 18.4 & 295 & 5 & 16.85 & 320 & 5 & 16.85 \\
\hline 435 & 7 & 18.35 & 320 & 5 & 16.7 & 345 & 5 & 16.85 \\
\hline 465 & 6 & 18.3 & 345 & 6 & 16.65 & 370 & 5 & 16.8 \\
\hline \multirow[t]{4}{*}{475} & 5 & 18.3 & 370 & 6 & 16.8 & 395 & 6 & 16.75 \\
\hline & & & 395 & 6 & 16.55 & 420 & 6 & 16.65 \\
\hline & & & 420 & 5 & 16.5 & 445 & 4 & 16.55 \\
\hline & & & 450 & 4 & 16.5 & 470 & 3 & \\
\hline \multicolumn{3}{|c|}{$A_{t}=15,500 \mathrm{ft}^{2}$} & \multicolumn{3}{|c|}{$A_{t}=12,000 \mathrm{ft}^{2}$} & \multicolumn{3}{|c|}{$A_{t}=12,800 \mathrm{ft}^{2}$} \\
\hline \multicolumn{3}{|c|}{$\mathrm{T}_{\mathrm{m}}=19.00^{\circ} \mathrm{C}$} & \multicolumn{3}{|c|}{$\mathrm{T}_{\mathrm{m}}=17.27{ }^{\circ} \mathrm{C}$} & \multicolumn{3}{|c|}{$\mathrm{T}_{\mathrm{m}}=17.30^{\circ} \mathrm{C}$} \\
\hline${ }^{\mathrm{T}} \mathrm{PR}=$ & $6.6{ }^{\circ} \mathrm{C}$ & & ${ }^{T}{ }_{P R}=1$ & $5.6{ }^{\circ} \mathrm{C}$ & & $\mathrm{T}_{\mathrm{PR}}=$ & $5.6{ }^{\circ} \mathrm{C}$ & \\
\hline
\end{tabular}


TABLE D.I-5. (contd) River Temperature Traverses Hanford (River Mile: 361.0)

\begin{tabular}{|c|c|c|c|c|c|c|c|c|}
\hline $\begin{array}{l}\text { Date: } \\
\text { Time: }\end{array}$ & $\begin{array}{l}10-29-6 \\
1130\end{array}$ & & $\begin{array}{l}\text { Date: } \\
\text { Time: }\end{array}$ & $\begin{array}{l}10-31-6 \\
1115\end{array}$ & & $\begin{array}{l}\text { Date: } \\
\text { Time: }\end{array}$ & $\begin{array}{l}11-5-63 \\
1300\end{array}$ & \\
\hline $\begin{array}{l}\text { Distance } \\
\text { from Plant } \\
\text { Shore, yd }\end{array}$ & $\begin{array}{c}\text { Depth, } \\
\mathrm{ft}\end{array}$ & $\begin{array}{c}\text { Temp, } \\
{ }^{\circ} \mathrm{C} \\
\end{array}$ & $\begin{array}{l}\text { Distance } \\
\text { from Pl ant } \\
\text { Shore, yd }\end{array}$ & $\begin{array}{l}\text { Depth, } \\
\mathrm{ft} \\
\end{array}$ & $\begin{array}{l}\text { Temp, } \\
{ }^{\circ} \mathrm{C} \\
\end{array}$ & $\begin{array}{l}\text { Distance } \\
\text { from P1ant } \\
\text { Shore, yd } \\
\end{array}$ & $\begin{array}{c}\text { Depth } \\
\mathrm{ft}\end{array}$ & $\begin{array}{c}\text { Temp, } \\
{ }^{\circ} \mathrm{C} \\
\end{array}$ \\
\hline 5 & 3 & 15.75 & 5 & 3 & 15.1 & 5 & 2 & 14.2 \\
\hline 20 & 6 & 15.8 & 30 & 6 & 15.3 & 50 & 10 & 14.3 \\
\hline 40 & 12 & 16.0 & 50 & 11 & 15.4 & 70 & 13 & 14.35 \\
\hline 65 & 15 & 16.1 & 70 & 15 & 15.35 & 95 & 10 & 14.35 \\
\hline 85 & 17 & 15.9 & 95 & 21 & 15.05 & 120 & 20 & 14.35 \\
\hline 115 & 19 & 15.7 & 120 & 21 & 14.85 & 145 & 20 & 14.3 \\
\hline 140 & 19 & 15.55 & 145 & 19 & 14.55 & 170 & 18 & 14.25 \\
\hline 175 & 20 & 15.4 & 170 & 17 & 14.6 & 195 & 12 & 14.05 \\
\hline 195 & 14 & 15.2 & 195 & 13 & 14.5 & 220 & 8 & 14.05 \\
\hline 215 & 11 & 15.1 & 220 & 9 & 14.55 & 245 & 6 & 14.0 \\
\hline 245 & 9 & 15.05 & 245 & 7 & 14.45 & 270 & 5 & 14.0 \\
\hline 270 & 8 & 15.0 & 270 & 6 & 14.45 & 295 & 5 & 13.9 \\
\hline 295 & 7 & 15.0 & 295 & 6 & 14.4 & 320 & 5 & 13.9 \\
\hline 320 & 7 & 14.95 & 320 & 6 & 14.4 & 345 & 6 & 13.85 \\
\hline 340 & 7 & 14.9 & 345 & 6 & 14.4 & 370 & 6 & 13.8 \\
\hline 365 & 7 & 14.85 & 370 & 6 & 14.2 & 395 & 6 & 13.75 \\
\hline 395 & 7 & 14.7 & 395 & 6 & 14.2 & 420 & 5 & 13.6 \\
\hline 425 & 6 & 14.7 & 420 & 5 & 14.2 & 445 & 5 & 13.55 \\
\hline 465 & 3 & 14.7 & 445 & 4 & 14.2 & 450 & 3 & 13.55 \\
\hline$A_{t}=1$ & $4,700 \mathrm{ft}$ & & $A_{t}=$ & $3,600 \mathrm{ft}$ & & $A_{t}=1$ & $1,300 \mathrm{ft}^{2}$ & \\
\hline $\mathrm{T}_{\mathrm{m}}=1$ & $5.35{ }^{\circ} \mathrm{C}$ & & $\mathrm{T}_{\mathrm{m}}=$ & $4.70^{\circ} \mathrm{C}$ & & $\mathrm{T}_{\mathrm{m}}=1$ & $4.12{ }^{\circ} \mathrm{C}$ & \\
\hline $\mathrm{T}_{\mathrm{PR}}=1$ & $4.1^{\circ} \mathrm{C}$ & & $\mathrm{T}_{\mathrm{PR}}=$ & $3.7^{\circ} \mathrm{C}$ & & $\mathrm{T}_{\mathrm{PR}}=1$ & $3.0^{\circ} \mathrm{C}$ & \\
\hline
\end{tabular}


TABLE D.I-5. (contd) River Temperature Traverses -

Hanford (River Mile: 361.0)

\section{Date: $\quad 11-19-63$ \\ Time: 1550}

Distance

from Plant Depth, Temp,

Shore, yd

25

50

75

110

125

150

175

200

225

255

275

300

325

350

375

400

425

450

475

$$
\begin{aligned}
\mathrm{A}_{\mathrm{t}} & =12,800 \mathrm{ft}^{2} \\
\mathrm{~T}_{\mathrm{m}} & =13.60{ }^{\circ} \mathrm{C} \\
\mathrm{T}_{\mathrm{PR}} & =11.8{ }^{\circ} \mathrm{C}
\end{aligned}
$$

14.4

6

14.4

14.45

14.35

14.0

13.45

13.3

13.2

13.2

13.2

13.2

13. 15

13. 1

13.05

$6 \quad 13.05$

613.0

$5 \quad 12,85$

$4 \quad 12.45$

112.15

12.15

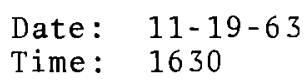

Distance

from Plant Depth, Temp,

Shore, yd

\section{5}

20

25

35

80

110

140

165

185

210

230

260

280

310

335

355

380

405

440

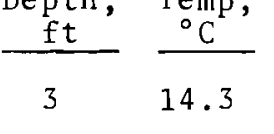

$4.5 \quad 14.3$

$5 \quad 14.35$

7

15

17

19

17

$13.5 \quad 13.45$

$10.5 \quad 13.35$

$7.5 \quad 13.35$

7

13.3

6

6

6.513 .25

6.513 .2

6

6

13.15

13.05

4.513 .0
Date: $\quad 12-17-63$

Time: 1230

Distance

from Plant Depth, Temp, Shore, yd

5

25

50

75

100

125

150

175

200

225

250

275

300

325

350

375

400

425

450

$$
\begin{aligned}
\mathrm{A}_{\mathrm{t}} & =12,600 \mathrm{ft}^{2} \\
\mathrm{~T}_{\mathrm{m}} & =9.31{ }^{\circ} \mathrm{C} \\
\mathrm{T}_{\mathrm{PR}} & =7.1{ }^{\circ} \mathrm{C}
\end{aligned}
$$


BNWL- 1345

TABLE D.I-6. River Temperature Traverses - Ringold (River Mile: 354.4)

\begin{tabular}{|c|c|c|c|c|c|c|c|c|}
\hline $\begin{array}{l}\text { Date: } \\
\text { Time: }\end{array}$ & $\begin{array}{l}2-26-63 \\
1110\end{array}$ & & $\begin{array}{l}\text { Date: } \\
\text { Time: }\end{array}$ & $\begin{array}{l}6-24-63 \\
1320\end{array}$ & & $\begin{array}{l}\text { Date: } \\
\text { Time: }\end{array}$ & $\begin{array}{l}8-30-63 \\
1030\end{array}$ & \\
\hline $\begin{array}{l}\text { Distance } \\
\text { from Plant } \\
\text { Shore, yd } \\
\end{array}$ & $\begin{array}{c}\text { Depth, } \\
\mathrm{ft} \\
\end{array}$ & $\begin{array}{l}\text { Temp, } \\
{ }^{\circ} \mathrm{C} \\
\end{array}$ & $\begin{array}{l}\text { Distance } \\
\text { from Plant } \\
\text { Shore, yd } \\
\end{array}$ & $\begin{array}{l}\text { Depth, } \\
\mathrm{ft} \\
\end{array}$ & $\begin{array}{c}\text { Temp, } \\
{ }^{\circ} \mathrm{C} \\
\end{array}$ & $\begin{array}{l}\text { Distance } \\
\text { from Plant } \\
\text { Shore, yd } \\
\end{array}$ & $\begin{array}{c}\text { Depth, } \\
\mathrm{ft} \\
\end{array}$ & $\begin{array}{c}\text { Temp, } \\
{ }^{\circ} \mathrm{C} \\
\end{array}$ \\
\hline 30 & 7 & 5.7 & 2 & 3 & 16.2 & 25 & 4 & 20.8 \\
\hline 80 & 12 & 5.6 & 50 & 14 & 15.6 & 80 & 14 & 20.6 \\
\hline 130 & 10 & 6.0 & 100 & 15 & 15.4 & 130 & 12 & 20.5 \\
\hline 180 & 7 & 5.7 & 150 & 19 & 15.5 & 180 & 9 & 20.45 \\
\hline 230 & 7 & 5.6 & 200 & 24 & 15.4 & 230 & 9 & 20.4 \\
\hline 280 & 7 & 5.5 & 250 & 26 & 15.4 & 280 & 9 & 20.3 \\
\hline 330 & 4 & 5.6 & 300 & 22 & 15.3 & 330 & 6 & 20.3 \\
\hline 380 & 6 & 5.4 & 350 & 20 & 15.2 & 380 & 8 & 20.25 \\
\hline 430 & 6 & 5.5 & 400 & 19 & 15.1 & 405 & 8 & 20.2 \\
\hline 480 & 10 & 5.2 & 450 & 18 & 15.1 & 430 & 8 & 20.2 \\
\hline 530 & 20 & 5.0 & 500 & 18 & 15.1 & 455 & 10 & 20.2 \\
\hline 580 & 7 & 4.8 & 550 & 20 & 15.1 & 480 & 12 & 20.1 \\
\hline \multirow[t]{7}{*}{630} & 3 & 6.2 & 600 & 24 & 15.1 & 505 & 17 & 20.1 \\
\hline & & & 650 & 30 & 15.1 & 530 & 22 & 20.1 \\
\hline & & & 700 & 34 & 15.1 & 555 & 16 & 20.1 \\
\hline & & & 750 & 21 & 15.2 & 580 & 9 & 20.1 \\
\hline & & & 800 & 18 & 15.3 & 605 & 7 & 20.0 \\
\hline & & & 850 & 10 & 15.4 & 630 & 5 & 20.0 \\
\hline & & & 900 & 8 & 15.5 & 675 & 4 & 20.0 \\
\hline \multicolumn{3}{|c|}{$A_{t}=15,400 \mathrm{ft}^{2}$} & \multicolumn{3}{|c|}{$A_{t}=54,300 \mathrm{ft}^{2}$} & \multicolumn{3}{|c|}{$A_{t}=20,600 \mathrm{ft}^{2}$} \\
\hline \multicolumn{3}{|c|}{$\mathrm{T}_{\mathrm{m}}=5.44^{\circ} \mathrm{C}$} & \multicolumn{3}{|c|}{$\mathrm{T}_{\mathrm{m}}=15.26{ }^{\circ} \mathrm{C}$} & \multicolumn{3}{|c|}{$\mathrm{T}_{\mathrm{m}}=20.30^{\circ} \mathrm{C}$} \\
\hline $\mathrm{T}_{\mathrm{PR}}=3$ & $80^{\circ} \mathrm{C}$ & & \multicolumn{3}{|c|}{$\mathrm{T}_{\mathrm{PR}}=14.6{ }^{\circ} \mathrm{C}$} & \multicolumn{3}{|c|}{$\mathrm{T}_{\mathrm{PR}}=18.0{ }^{\circ} \mathrm{C}$} \\
\hline
\end{tabular}


TABLE D.I-6. (contd) River Temperature Traverses Ringold (River Mile: 354.4)

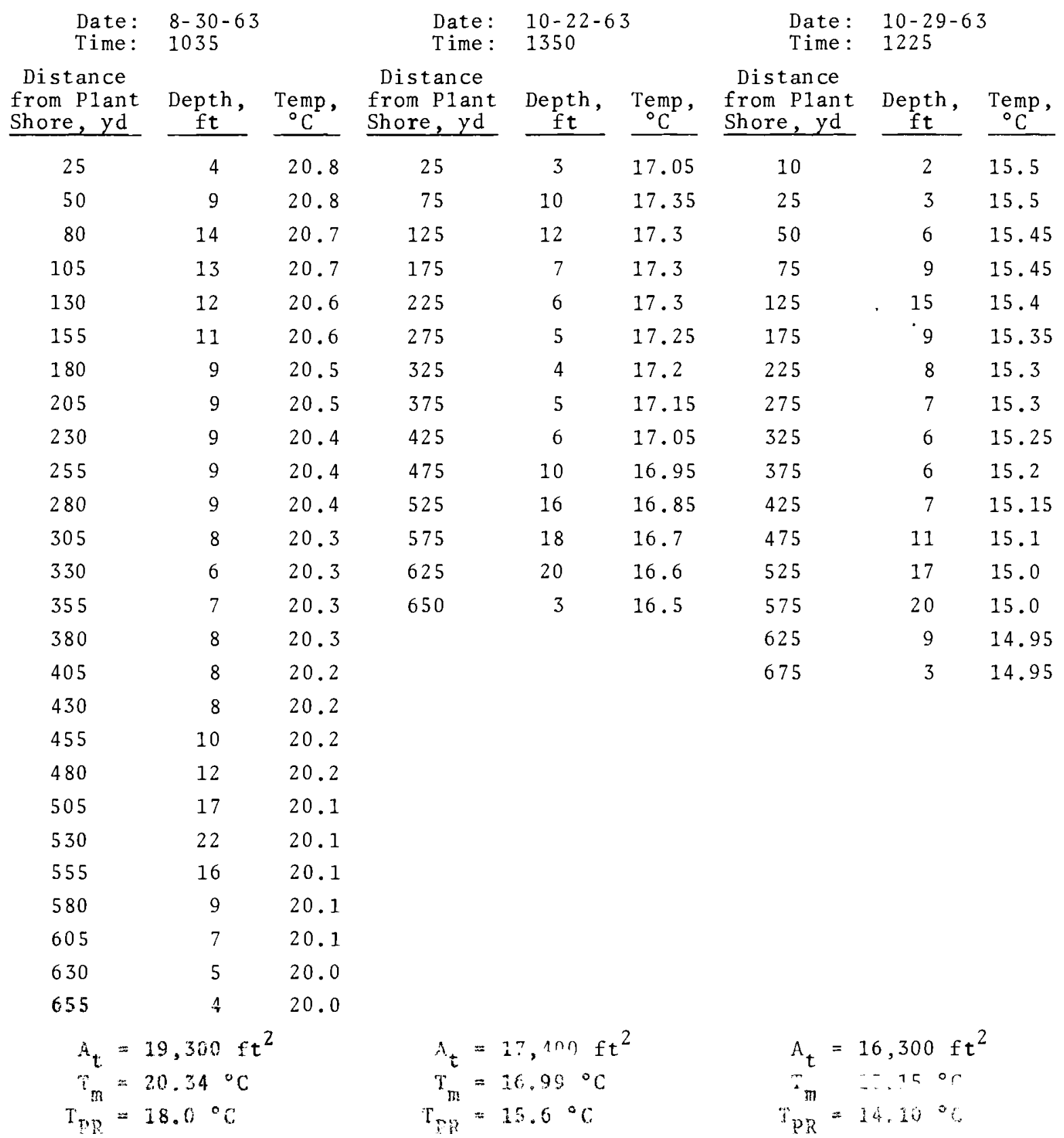


TABLE D.I-6. (contd) River Temperature Traverses Ringold (River Mile: 354.4 )

Date: $\quad 10-31-63$

Time : $\quad 1200$

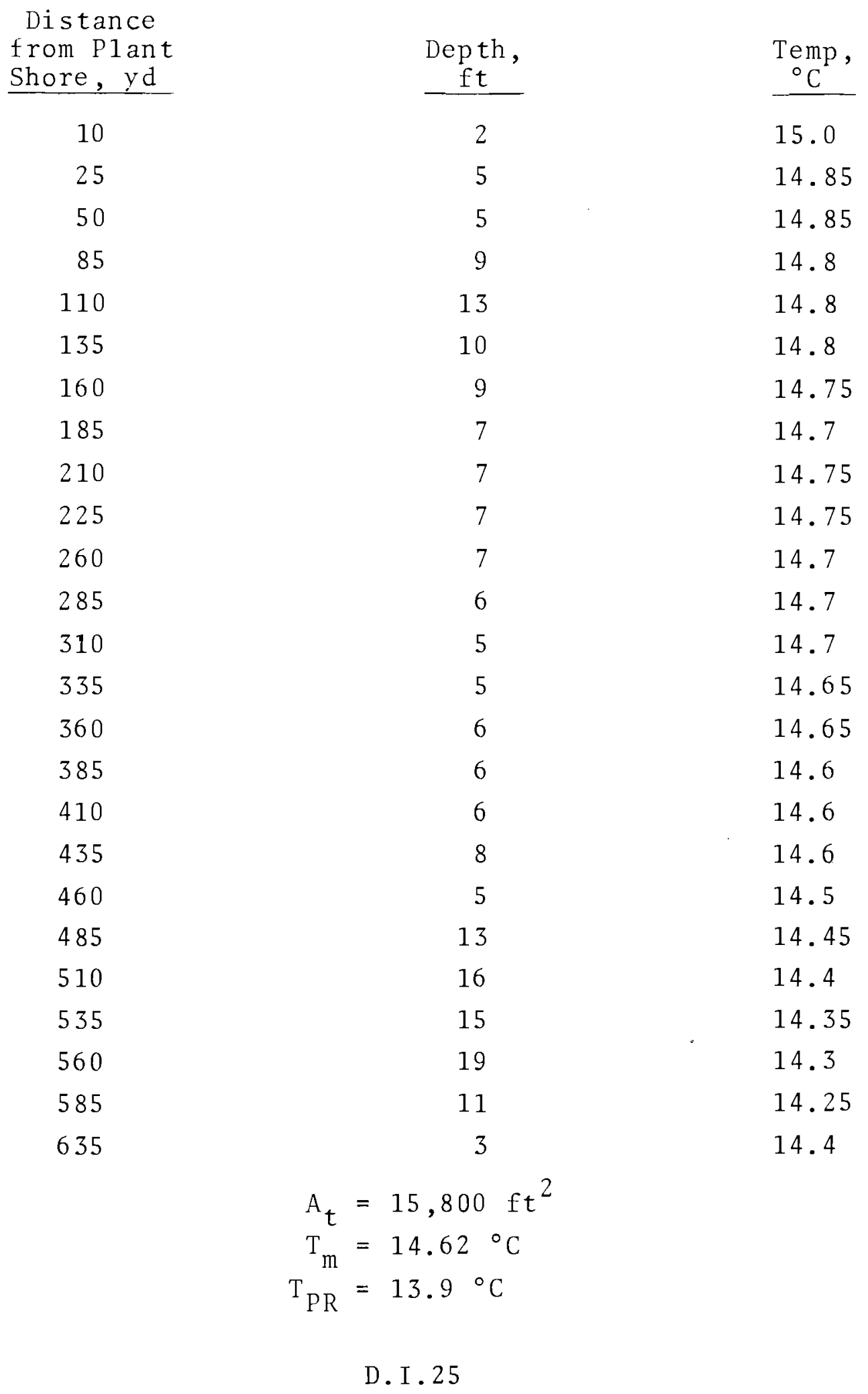


TABLE D.I-7. River Temperature Traverses Power Line Crossing

(River Mile: 350.5 )

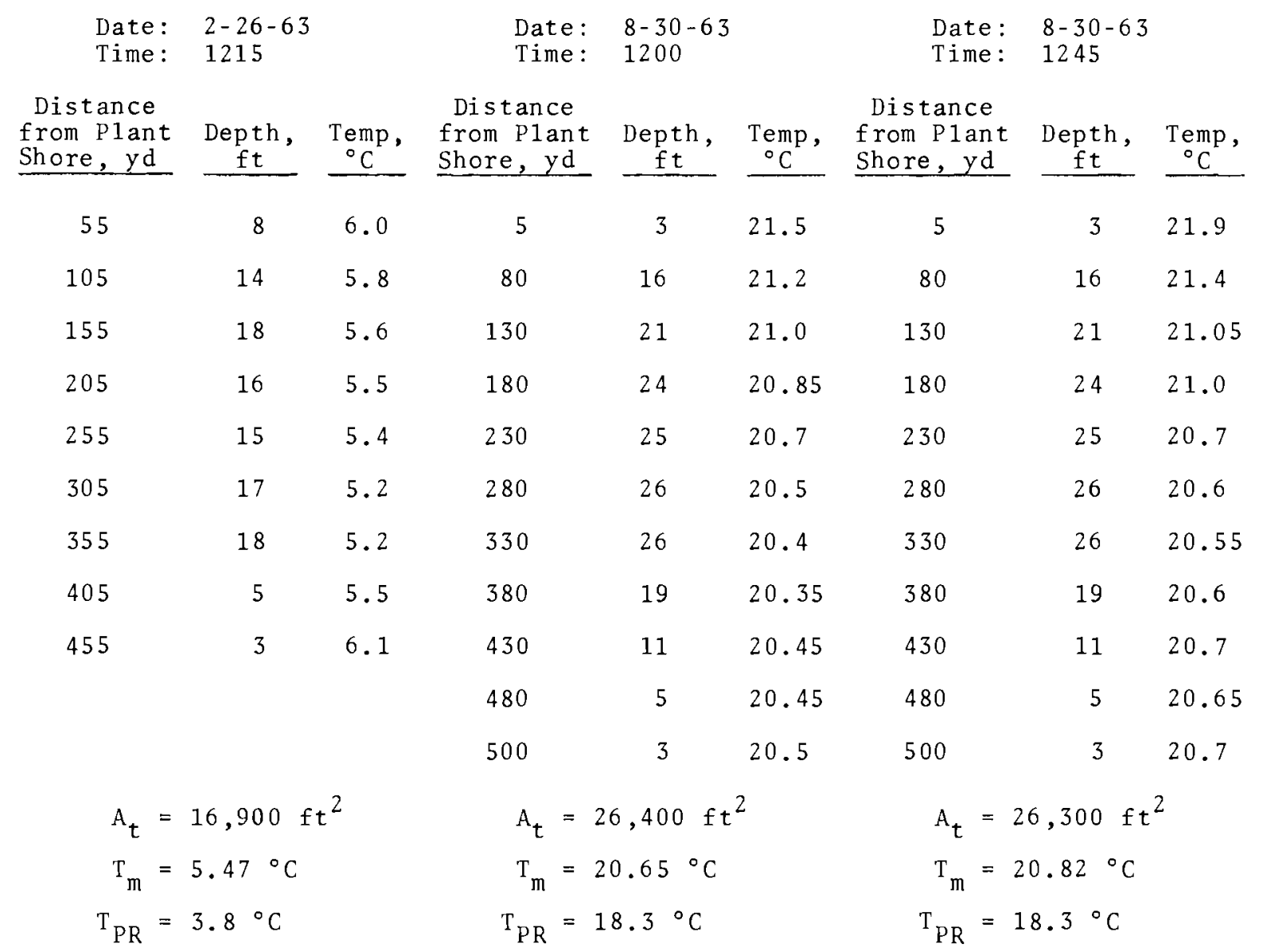


TABLE D.I-7. (contd) River Temperature Traverses Power Line Crossing

(River Mile: 350.5 )

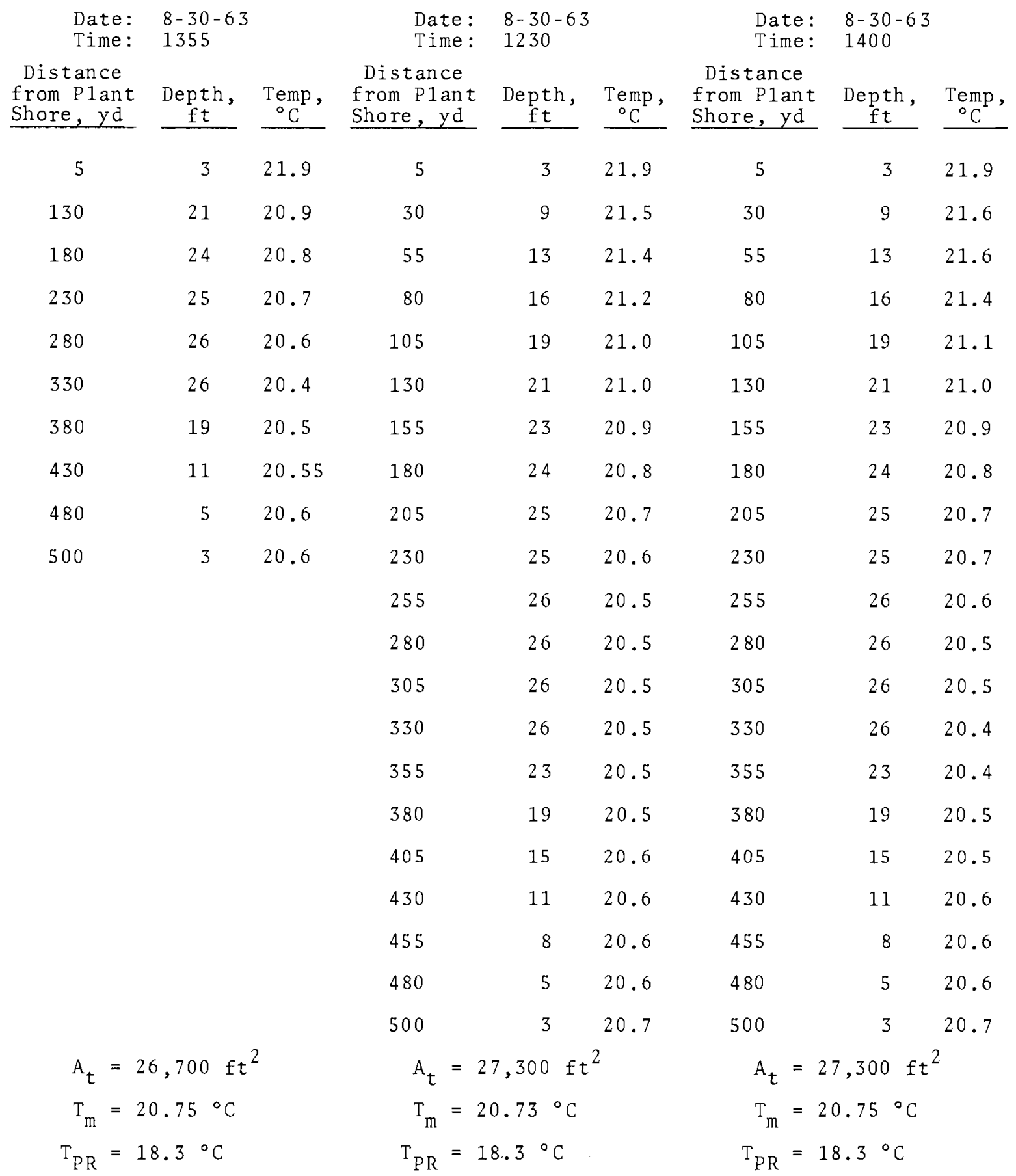

D. I. 27 
TABLE D.I-7. (contd) River Temperature Traverses Power Line Crossing

(River Mile: 350.5)

\begin{tabular}{|c|c|c|c|c|c|c|c|c|}
\hline \multirow{2}{*}{$\begin{array}{r}\text { Date: } \\
\text { Time: } \\
\text { Distance } \\
\text { from P1ant } \\
\text { Shore, yd } \\
\end{array}$} & \multicolumn{2}{|c|}{$\begin{array}{l}10-16-63 \\
1340\end{array}$} & $\begin{array}{l}\text { Date: } \\
\text { Time: }\end{array}$ & \multicolumn{2}{|c|}{$\begin{array}{l}10-16-63 \\
1410\end{array}$} & $\begin{array}{l}\text { Date: } \\
\text { Time: }\end{array}$ & \multicolumn{2}{|c|}{$\begin{array}{l}10-16-63 \\
1415\end{array}$} \\
\hline & $\begin{array}{c}\text { Depth, } \\
\text { ft } \\
\end{array}$ & $\begin{array}{l}\text { Temp } \\
{ }^{\circ} \mathrm{C} \\
\end{array}$ & $\begin{array}{l}\text { Distance } \\
\text { from Plant } \\
\text { Shore, yd } \\
\end{array}$ & $\begin{array}{c}\text { Depth, } \\
\mathrm{ft} \\
\end{array}$ & $\begin{array}{l}\text { Temp } \\
{ }^{\circ} \mathrm{C} \\
\end{array}$ & $\begin{array}{l}\text { Distance } \\
\text { from Plant } \\
\text { Shore, yd } \\
\end{array}$ & $\begin{array}{l}\text { Depth } \\
\mathrm{ft} \\
\end{array}$ & $\begin{array}{c}\text { Temp, } \\
{ }^{\circ} \mathrm{C} \\
\end{array}$ \\
\hline 10 & 6 & 19.4 & 10 & 6 & 19.4 & 5 & 3 & 19.7 \\
\hline 25 & 8 & 19.25 & 25 & 8 & 19.25 & 25 & 12 & 19.3 \\
\hline 50 & 11 & 19.2 & 50 & 15 & 19.1 & 75 & 18 & 19.05 \\
\hline 75 & 18 & 19.1 & 75 & 18 & 19.0 & 100 & 20 & 19.0 \\
\hline 100 & 22 & 18.95 & 100 & 24 & 18.95 & 125 & 24 & 18.95 \\
\hline 125 & 23 & 18.85 & 125 & 23 & 18.9 & 150 & 24 & 18.85 \\
\hline 150 & 23 & 18.8 & 150 & 23 & 18.85 & 175 & 25 & 18.8 \\
\hline 175 & 24 & 18.75 & 175 & 24 & 18.75 & 200 & 25 & 18.75 \\
\hline 200 & 24 & 18.7 & 200 & 26 & 18.7 & 225 & 26 & 18.6 \\
\hline 225 & 25 & 18.65 & 225 & 26 & 18.6 & 250 & 27 & 18.5 \\
\hline 250 & 26 & 18.6 & 250 & 27 & 18.55 & 275 & 27 & 18.45 \\
\hline 275 & 26 & 18.5 & 275 & 28 & 18.5 & 300 & 27 & 18.45 \\
\hline 300 & 26 & 18.4 & 300 & 26 & 18.45 & 325 & 27 & 18.45 \\
\hline 325 & 26 & 18.4 & 325 & 27 & 18.45 & 350 & 26 & 18.45 \\
\hline 350 & 26 & 18.4 & 350 & 26 & 18.4 & 375 & 19 & 18.5 \\
\hline 375 & 26 & 18.4 & 375 & 23 & 18.4 & 400 & 11 & 18.55 \\
\hline 400 & 12 & 18.4 & 400 & 14 & 18.4 & 425 & 10 & 18.7 \\
\hline \multirow[t]{2}{*}{425} & 8 & 18.3 & 425 & 6 & 18.3 & 450 & 6 & 18.75 \\
\hline & & & & & & 475 & 5 & 18.85 \\
\hline \multicolumn{3}{|c|}{$A_{t}=27,200 \mathrm{ft}^{2}$} & \multicolumn{3}{|c|}{$A_{t}=27,200 \mathrm{ft}^{2}$} & \multicolumn{3}{|c|}{$A_{t} 27,700 \mathrm{ft}^{2}$} \\
\hline \multicolumn{3}{|c|}{$\mathrm{T}_{\mathrm{m}}=18.66^{\circ} \mathrm{C}$} & \multicolumn{3}{|c|}{$\mathrm{T}_{\mathrm{m}}=18.66{ }^{\circ} \mathrm{C}$} & \multicolumn{3}{|c|}{$\mathrm{T}_{\mathrm{m}}=18.71{ }^{\circ} \mathrm{C}$} \\
\hline \multicolumn{3}{|c|}{$\mathrm{T}_{\mathrm{PR}}=16.6^{\circ} \mathrm{C}$} & \multicolumn{3}{|c|}{$\mathrm{T}_{\mathrm{PR}}=16.6{ }^{\circ} \mathrm{C}$} & \multicolumn{3}{|c|}{$\mathrm{T}_{\mathrm{PR}}=16.6^{\circ} \mathrm{C}$} \\
\hline
\end{tabular}




\section{TABIE D.I-7. (contd) River Temperature Traverses - Power Line Crossing (River Mile: 350.5 )}

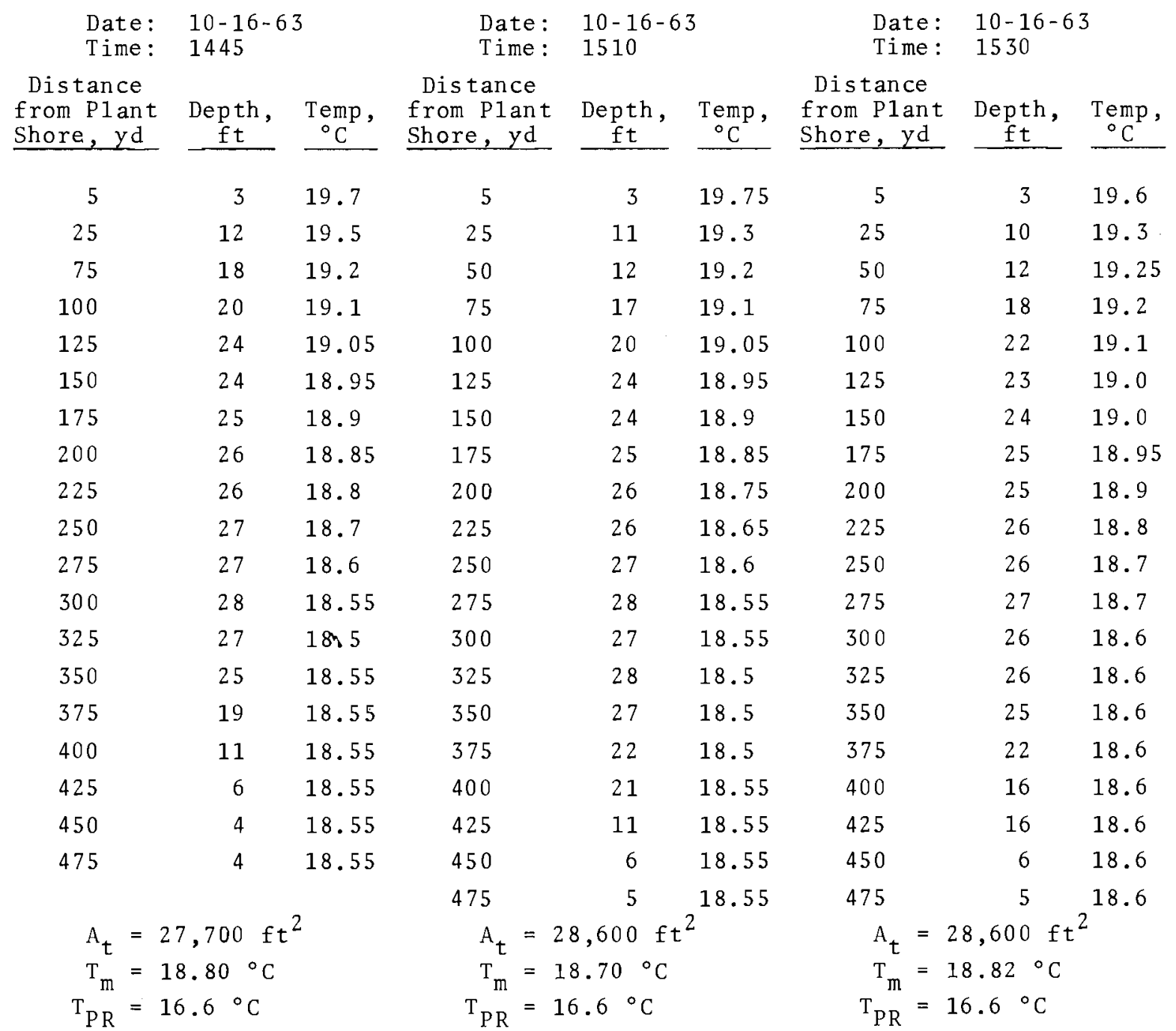


TABLE D.I-7. (contd) River Temperature Traverses Power Line Crossing

(River Mile: 350.5 )

\begin{tabular}{|c|c|c|c|c|c|c|c|c|}
\hline $\begin{array}{l}\text { Date: } \\
\text { Time: }\end{array}$ & $\begin{array}{l}10-29-6 \\
1250\end{array}$ & & $\begin{array}{l}\text { Date: } \\
\text { Time: }\end{array}$ & $\begin{array}{l}10-29-6 \\
1255\end{array}$ & & $\begin{array}{l}\text { Date: } \\
\text { Time: }\end{array}$ & $\begin{array}{l}10-31- \\
1235\end{array}$ & \\
\hline $\begin{array}{l}\text { Distance } \\
\text { from Plant } \\
\text { Shore, yd }\end{array}$ & $\begin{array}{c}\text { Depth, } \\
\mathrm{ft} \\
\end{array}$ & $\begin{array}{c}\text { Temp } \\
{ }^{\circ} \mathrm{C} \\
\end{array}$ & $\begin{array}{l}\text { Distance } \\
\text { from Plant } \\
\text { Shore, yd }\end{array}$ & $\begin{array}{c}\text { Depth, } \\
\mathrm{ft} \\
\end{array}$ & $\begin{array}{c}\text { Temp, } \\
{ }^{\circ} \mathrm{C} \\
\end{array}$ & $\begin{array}{l}\text { Distance } \\
\text { from Plant } \\
\text { Shore, yd }\end{array}$ & $\begin{array}{c}\text { Depth, } \\
\mathrm{ft} \\
\end{array}$ & $\begin{array}{c}\text { Temp } \\
{ }^{\circ} \mathrm{C} \\
\end{array}$ \\
\hline 5 & 3 & 15.35 & 5 & 2 & 15.4 & 5 & 3 & 14.7 \\
\hline 25 & 12 & 15.35 & 25 & 8 & 15.45 & 30 & 8 & 14.75 \\
\hline 50 & 11 & 15.4 & 50 & 10 & 15.45 & 55 & 12 & 14.8 \\
\hline 75 & 19 & 15.35 & 75 & 17 & 15.45 & 80 & 13 & 14.8 \\
\hline 100 & 22 & 15.35 & 100 & 21 & 15.4 & 105 & 23 & 14.75 \\
\hline 125 & 24 & 15.3 & 125 & 23 & 15.35 & 130 & 23 & 14.7 \\
\hline 150 & 24 & 15.25 & 150 & 25 & 15.3 & 155 & 23 & 14.65 \\
\hline 175 & 25 & 15.2 & 175 & 25 & 15.25 & 180 & 24 & 14.6 \\
\hline 200 & 26 & 15.2 & 200 & 25 & 15.15 & 205 & 25 & 14.55 \\
\hline 225 & 26 & 15.15 & 225 & 26 & 15.15 & 230 & 24 & 14.5 \\
\hline 250 & 27 & 15.1 & 250 & 27 & 15.1 & 255 & 27 & 14.4 \\
\hline 275 & 28 & 15.05 & 275 & 28 & 15.05 & 280 & 26 & 14.35 \\
\hline 300 & 27 & 15.0 & 300 & 28 & 15.0 & 305 & 26 & 14.3 \\
\hline 325 & 27 & 14.95 & 325 & 27 & 14.95 & 330 & 26 & 14.3 \\
\hline 350 & 27 & 14.9 & 350 & 27 & 14.9 & 355 & 23 & 14.3 \\
\hline 375 & 20 & 14.85 & 375 & 24 & 14.8 & 380 & 22 & 14.25 \\
\hline 400 & 15 & 14.8 & 400 & 15 & 14.75 & 405 & 6 & 14.2 \\
\hline 425 & 9 & 14.8 & 425 & 9 & 14.8 & 430 & 4 & 14.15 \\
\hline 450 & 5 & 14.75 & 450 & 5 & 14.8 & 455 & 4 & 14.15 \\
\hline 475 & 3 & 14.75 & 475 & 4 & 14.85 & 480 & 4 & 14.15 \\
\hline 500 & 3 & 15.1 & 500 & 3 & 14.85 & & & \\
\hline $\begin{aligned} A_{t} & =2 \\
T_{m} & = \\
T_{P R} & =\end{aligned}$ & $\begin{array}{l}8,400 \mathrm{ft} \\
5.12{ }^{\circ} \mathrm{C} \\
4.1{ }^{\circ} \mathrm{C}\end{array}$ & & $\begin{aligned} \mathrm{A}_{\mathrm{t}} & = \\
\mathrm{T}_{\mathrm{m}} & = \\
\mathrm{T}_{\mathrm{PR}} & =\end{aligned}$ & $\begin{array}{l}7,900 \mathrm{ft} \\
5.08^{\circ} \mathrm{C} \\
4.1{ }^{\circ} \mathrm{C}\end{array}$ & & $\begin{aligned} \mathrm{A}_{\mathrm{t}} & = \\
\mathrm{T}_{\mathrm{m}} & = \\
\mathrm{T}_{\mathrm{PR}} & =\end{aligned}$ & $\begin{array}{l}5,800 \mathrm{f} \\
4.48^{\circ} \mathrm{C} \\
3.7^{\circ} \mathrm{C}\end{array}$ & \\
\hline
\end{tabular}

D. I. 30 
BNWL -1345

TABLE D.I-8. River Temperature Traverses - Above 300 Area (River Mile: 344.9)

\begin{tabular}{|c|c|c|c|c|c|c|c|c|}
\hline \multirow{2}{*}{$\begin{array}{r}\text { Date: } \\
\text { Time: } \\
\text { Distance } \\
\text { from Plant } \\
\text { Shore, yd } \\
\end{array}$} & \multicolumn{2}{|l|}{$\begin{array}{l}2-12-63 \\
0730\end{array}$} & $\begin{array}{l}\text { Date: } \\
\text { Time: }\end{array}$ & $\begin{array}{l}4-2-63 \\
1300\end{array}$ & \multicolumn{2}{|r|}{$\begin{array}{l}\text { Date: } \\
\text { Time: }\end{array}$} & \multicolumn{2}{|l|}{$\begin{array}{l}4-18-63 \\
1110\end{array}$} \\
\hline & $\begin{array}{c}\text { Depth, } \\
\mathrm{ft} \\
\end{array}$ & $\begin{array}{c}\text { Temp } \\
{ }^{\circ} \mathrm{C}\end{array}$ & $\begin{array}{l}\text { Distance } \\
\text { from Plant } \\
\text { Shore, yd }\end{array}$ & $\begin{array}{c}\text { Depth, } \\
\mathrm{ft} \\
\end{array}$ & $\begin{array}{c}\text { Temp, } \\
{ }^{\circ} \mathrm{C} \\
\end{array}$ & $\begin{array}{l}\text { Distance } \\
\text { from Plant } \\
\text { Shore, yd }\end{array}$ & $\begin{array}{c}\text { Depth, } \\
\mathrm{ft} \\
\end{array}$ & $\begin{array}{l}\text { Temp, } \\
{ }^{\circ} \mathrm{C} \\
\end{array}$ \\
\hline 5 & 3 & 5.2 & 3 & 5 & 7.9 & 2 & 1 & 8.2 \\
\hline 30 & 14 & 5.1 & 50 & 12 & 7.2 & 50 & 15 & 7.8 \\
\hline 80 & 21 & 5.1 & 85 & 13 & 7.0 & 85 & 21 & 7.9 \\
\hline 125 & 32 & 5.0 & 135 & 30 & 6.6 & 125 & 31 & 7.8 \\
\hline 180 & 30 & 5.0 & 185 & 25 & 6.5 & 175 & 32 & 7.7 \\
\hline 230 & 18 & 4.8 & 235 & 20 & 6.4 & 225 & 18 & 7.7 \\
\hline 275 & 15 & 4.9 & 285 & 10 & 6.4 & 275 & 18 & 7.7 \\
\hline 325 & 13 & 4.7 & 335 & 12 & 6.4 & 325 & 13 & 7.7 \\
\hline 375 & 10 & 4.7 & 385 & 8 & 6.4 & 375 & 9 & 7.7 \\
\hline 425 & 10 & 4.8 & 435 & 7 & 6.3 & 425 & 9 & 7.7 \\
\hline 465 & 11 & 4.8 & 485 & 6 & 6.3 & 475 & 8 & 7.7 \\
\hline 520 & 11 & 4.6 & 535 & 4 & 6.3 & 525 & 9 & 7.7 \\
\hline 575 & 12 & 4.6 & 585 & 8 & 6.3 & 575 & 10 & 7.7 \\
\hline 630 & 14 & 4.6 & 635 & 10 & 6.3 & 625 & 12 & 7.7 \\
\hline 680 & 16 & 4.6 & 684 & 11 & 6.3 & 675 & 12 & 7.6 \\
\hline 740 & 15 & 4.7 & 735 & 11 & 6.3 & 725 & 13 & 7.6 \\
\hline 785 & 10 & 4.5 & 785 & 13 & 6.5 & 775 & 10 & 7.6 \\
\hline 830 & 3 & 4.6 & 835 & 8 & 6.5 & 875 & 3 & 8.3 \\
\hline & & & 865 & 3 & 8.0 & & & \\
\hline $\begin{array}{l}\mathrm{A}_{\mathrm{t}}=38,00 \\
\mathrm{~T}_{\mathrm{m}}=4.84 \\
\text { PRTR Recor } \\
\mathrm{T}_{\mathrm{PR}}=4.15\end{array}$ & $\begin{array}{l}\mathrm{ft} \mathrm{t}^{2} \\
\mathrm{C} \\
{ }^{\circ} \mathrm{r}=5.4\end{array}$ & ${ }^{\circ} \mathrm{C}$ & $\begin{array}{l}\mathrm{A}_{\mathrm{t}}=31,100 \\
\mathrm{~T}_{\mathrm{m}}=6.52^{\circ} \\
\text { PRTR Record } \\
\mathrm{T}_{\mathrm{PR}}=5.0^{\circ}\end{array}$ & $\begin{array}{l}\text { er }=7.2 \\
\text { C }\end{array}$ & ${ }^{\circ} \mathrm{C}$ & $\begin{array}{l}\mathrm{A}_{\mathrm{t}}=36,700 \\
\mathrm{~A}_{\mathrm{m}}=7.72 \\
\text { PRTR Recor } \\
\mathrm{T}_{\mathrm{PR}}=6.7\end{array}$ & er $=8.0$ & ${ }^{\circ} \mathrm{C}$ \\
\hline
\end{tabular}




\section{TABLE D.I-8. (contd) River Temperature Traverses - Above 300 Area \\ (River Mile: 344.9)}

\begin{tabular}{|c|c|c|c|c|c|c|c|c|}
\hline $\begin{array}{l}\text { Date: } \\
\text { Time: }\end{array}$ & $\begin{array}{l}5-23-63 \\
1100\end{array}$ & & $\begin{array}{l}\text { Date: } \\
\text { Time: }\end{array}$ & $\begin{array}{l}6-24-63 \\
1040\end{array}$ & & $\begin{array}{l}\text { Date: } \\
\text { Time: }\end{array}$ & $\begin{array}{l}6-27-63 \\
1100\end{array}$ & \\
\hline $\begin{array}{l}\text { Distance } \\
\text { from Plant } \\
\text { Shore, yd } \\
\end{array}$ & $\begin{array}{c}\text { Depth, } \\
\mathrm{ft}\end{array}$ & $\begin{array}{l}\text { Temp, } \\
{ }^{\circ} \mathrm{C} \\
\end{array}$ & $\begin{array}{l}\text { Distance } \\
\text { from P1 ant } \\
\text { Shore, yd } \\
\end{array}$ & $\begin{array}{c}\text { Depth, } \\
\mathrm{ft} \\
\end{array}$ & $\begin{array}{l}\text { Temp } \\
{ }^{\circ} \mathrm{C} \\
\end{array}$ & $\begin{array}{l}\text { Distance } \\
\text { from Plant } \\
\text { Shore, yd } \\
\end{array}$ & $\begin{array}{l}\text { Depth, } \\
\quad \mathrm{ft} \\
\end{array}$ & $\begin{array}{l}\text { Temp, } \\
{ }^{\circ} \mathrm{C} \\
\end{array}$ \\
\hline 2 & 2 & 15.3 & 2 & 3 & 15.7 & 5 & 5 & 16.3 \\
\hline 50 & 13 & 14.6 & 37 & 20 & 15.5 & 85 & 27 & 16.1 \\
\hline 100 & 21 & 14.2 & 87 & 27 & 15.3 & 185 & 38 & 15.75 \\
\hline 150 & 32 & 13.9 & 137 & 40 & 15.3 & 285 & 24 & 15.7 \\
\hline 200 & 32 & 13.8 & 187 & 38 & 15.1 & 385 & 20 & 15.65 \\
\hline 250 & 18 & 13.8 & 237 & 27 & 15.1 & 485 & 16 & 15.65 \\
\hline 300 & 14 & 13.7 & 287 & 24 & 15.1 & 585 & 19 & 15.65 \\
\hline 350 & 13 & 13.7 & 337 & 23 & 15.1 & 685 & 21 & 15.65 \\
\hline 400 & 9 & 13.7 & 387 & 20 & 15.1 & 785 & 23 & 15.65 \\
\hline 450 & 8 & 13.7 & 437 & 18 & 15.1 & 885 & 21 & 15.85 \\
\hline 500 & 8 & 13.7 & 487 & 16 & 15.1 & 935 & 13 & 15.85 \\
\hline 550 & 8 & 13.6 & 537 & 18 & 15.1 & 955 & 3 & 15.9 \\
\hline 600 & 9 & 13.6 & 587 & 19 & 15.1 & & & \\
\hline 650 & 13 & 13.7 & 637 & 19 & 15.1 & & & \\
\hline 700 & 9 & 13.8 & 687 & 21 & 15.1 & & & \\
\hline 750 & 11 & 13.8 & 737 & 21 & 15.0 & & & \\
\hline 800 & 11 & 14.0 & 787 & 23 & 15.1 & & & \\
\hline \multirow[t]{5}{*}{840} & 3 & 15.3 & 837 & 22 & 15.1 & & & \\
\hline & & & 887 & 21 & 15.1 & & & \\
\hline & & & 937 & 19 & 15.2 & & & \\
\hline & & & 987 & 13 & 15.2 & & & \\
\hline & & & 1027 & 2 & 16.4 & & & \\
\hline \multicolumn{3}{|c|}{$A_{t}=33,900 \mathrm{ft}^{2}$} & $A_{t}=66,900$ & $f t^{2}$ & & \multicolumn{3}{|c|}{$A_{t}=64,900 \mathrm{ft}^{2}$} \\
\hline \multicolumn{3}{|c|}{$\mathrm{T}_{\mathrm{m}}=13.91{ }^{\circ} \mathrm{C}$} & $\begin{array}{l}\mathrm{T}_{\mathrm{m}}=15.16 \\
\mathrm{PRTR} \text { Record }\end{array}$ & ${ }^{\circ} \mathrm{C}$ & 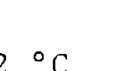 & \multicolumn{3}{|c|}{$\mathrm{T}_{\mathrm{m}}=15.77^{\circ} \mathrm{C}$} \\
\hline \multicolumn{3}{|c|}{$\mathrm{T}_{\mathrm{PR}}=12.3{ }^{\circ} \mathrm{C}$} & $\mathrm{T}_{\mathrm{PR}}=14.6$ & & & \multicolumn{3}{|c|}{$\mathrm{T}_{\mathrm{PR}}=14.9^{\circ} \mathrm{C}$} \\
\hline
\end{tabular}

D. I. 32 
BNWL- 1345

\section{TABLE D.I-8. (contd) River Temperature Traverses - Above 300 Area (River Mile: 344.9)}

\begin{tabular}{|c|c|c|c|c|c|c|c|c|}
\hline $\begin{array}{l}\text { Date: } \\
\text { Time: }\end{array}$ & $\begin{array}{l}7-18-63 \\
1330\end{array}$ & & $\begin{array}{l}\text { Date: } \\
\text { Time: }\end{array}$ & $\begin{array}{l}8-30-63 \\
1500\end{array}$ & & $\begin{array}{l}\text { Date: } \\
\text { Time: }\end{array}$ & $\begin{array}{l}10-10-63 \\
1100\end{array}$ & \\
\hline $\begin{array}{l}\text { Distance } \\
\text { from P1 ant } \\
\text { Shore, yd }\end{array}$ & $\begin{array}{c}\text { Depth, } \\
\mathrm{ft} \\
\end{array}$ & $\begin{array}{c}\text { Temp, } \\
{ }^{\circ} \mathrm{C}\end{array}$ & $\begin{array}{l}\text { Distance } \\
\text { from Plant } \\
\text { Shore, yd } \\
\end{array}$ & $\begin{array}{c}\text { Depth, } \\
f t \\
\end{array}$ & $\begin{array}{l}\text { Temp, } \\
{ }^{\circ} \mathrm{C} \\
\end{array}$ & $\begin{array}{l}\text { Distance } \\
\text { from Plant } \\
\text { Shore, yd } \\
\end{array}$ & $\begin{array}{c}\text { Depth, } \\
\mathrm{ft} \\
\end{array}$ & $\begin{array}{l}\text { Temp, } \\
{ }^{\circ} \mathrm{C}\end{array}$ \\
\hline 5 & 5 & 17.9 & 25 & 9 & 21.5 & 5 & 3 & 17.6 \\
\hline 75 & 23 & 17.6 & 50 & 16 & 21.4 & 50 & 16 & 17.7 \\
\hline 175 & 38 & 17.3 & 75 & 19 & 21.3 & 80 & 19 & 17.8 \\
\hline 275 & 20 & 17.3 & 100 & 22 & 21.2 & 130 & 28 & 17.8 \\
\hline 375 & 15 & 17.25 & 125 & 27 & 21.2 & 180 & 33 & 17.7 \\
\hline 475 & 14 & 17.25 & 150 & 32 & 21.0 & 230 & 25 & 17.7 \\
\hline 575 & 15 & 17.2 & 175 & 33 & 21.0 & 280 & 19 & 17.7 \\
\hline 675 & 17 & 17.2 & 200 & 33 & 21.0 & 330 & 17 & 17.6 \\
\hline 775 & 20 & 17.2 & 225 & 26 & 20.9 & 380 & 12 & 17.6 \\
\hline 875 & 17 & 17.25 & 250 & 19 & 20.9 & 430 & 10 & 17.6 \\
\hline 975 & 10 & 17.5 & 275 & 19 & 20.9 & 480 & 9 & 17.6 \\
\hline 1005 & 5 & 17.7 & 300 & 19 & 20.8 & 630 & 12 & 17.6 \\
\hline & & & 325 & 17 & 20.8 & 880 & 5 & 17.5 \\
\hline & & & 350 & 14 & 20.8 & 920 & 3 & 17.4 \\
\hline & & & 375 & 12 & 20.8 & & & \\
\hline & & & 400 & 10 & 20.8 & & & \\
\hline & & & 425 & 10 & 20.8 & & & \\
\hline & & & 450 & 10 & 20.8 & & & \\
\hline & & & 475 & 9 & 20.7 & & & \\
\hline & & & 500 & 9 & 20.7 & & & \\
\hline & & & 525 & 10 & 20.7 & & & \\
\hline & & & 550 & 10 & 20.7 & & & \\
\hline & & & 575 & 11 & 20.7 & & & \\
\hline & & & 600 & 11 & 20.7 & & & \\
\hline & & & 625 & 12 & 20.8 & & & \\
\hline & & & 650 & 13 & 20.8 & & & \\
\hline & & & 675 & 13 & 20.8 & & & \\
\hline & & & 700 & 13 & 20.8 & & & \\
\hline & & & 725 & 14 & 20.8 & & & \\
\hline & & & 750 & 14 & 20.9 & & & \\
\hline & & & 775 & 13 & 20.9 & & & \\
\hline & & & 800 & 11 & 20.9 & & & \\
\hline & & & 825 & 9 & 20.9 & & & \\
\hline & & & 850 & 7 & 21.0 & & & \\
\hline & & & 875 & 5 & 21.2 & & & \\
\hline & & & 900 & 4 & 21.5 & & & \\
\hline$A_{t}=55,400$ & $f t^{2}$ & & $A_{t}=40,000$ & $f t^{2}$ & & $A_{t}=37,900$ & $f t^{2}$ & \\
\hline $\mathrm{T}_{\mathrm{m}}=17.32$ & ${ }^{\circ} \mathrm{C}$ & & $\mathrm{T}_{\mathrm{m}}=20.94$ & ${ }^{\circ} \mathrm{C}$ & & $\mathrm{T}_{\mathrm{m}}=17.66$ & ${ }^{\circ} \mathrm{C}$ & \\
\hline $\begin{array}{l}\text { PRTR Record } \\
\mathrm{T}_{\mathrm{DP}}=16.8\end{array}$ & $\begin{array}{l}\text { er }=18.0 \\
{ }^{\circ} \mathrm{C}\end{array}$ & ${ }^{\circ} \mathrm{C}$ & $\begin{array}{l}\text { PRTR Record } \\
T_{\text {DD }}=18.00\end{array}$ & $\begin{array}{l}\mathrm{er}=20.9 \\
{ }^{\circ} \mathrm{C}\end{array}$ & ${ }^{\circ} \mathrm{C}$ & $\begin{array}{l}\text { PRTR Record } \\
T_{D P}=17.3\end{array}$ & $\begin{array}{l}\mathrm{er}=18.1 \\
{ }^{\circ} \mathrm{C}\end{array}$ & $1{ }^{\circ} \mathrm{C}$ \\
\hline
\end{tabular}


BNWL -1345

TABLE D.I-8. (contd)

River Temperature Traverses -

Above 300 Area

(River Mile: 344.9)

\begin{tabular}{|c|c|c|c|c|c|c|c|c|}
\hline \multirow{2}{*}{$\begin{array}{r}\text { Date: } \\
\text { Time: } \\
\text { Distance } \\
\text { from P1ant } \\
\text { Shore, yd } \\
\end{array}$} & \multicolumn{2}{|c|}{$\begin{array}{l}10-16-63 \\
1615\end{array}$} & $\begin{array}{l}\text { Date: } \\
\text { Time: }\end{array}$ & \multicolumn{2}{|c|}{$\begin{array}{l}10-29-63 \\
1330\end{array}$} & $\begin{array}{l}\text { Date: } \\
\text { T: a: }\end{array}$ & \multicolumn{2}{|c|}{$\begin{array}{l}10-31-63 \\
\div 40\end{array}$} \\
\hline & $\begin{array}{c}\text { Depth, } \\
\mathrm{ft} \\
\end{array}$ & $\begin{array}{l}\text { Temp, } \\
{ }^{\circ} \mathrm{C} \\
\end{array}$ & $\begin{array}{l}\text { Distance } \\
\text { from Plant } \\
\text { Shore, yd } \\
\end{array}$ & $\begin{array}{c}\text { Depth, } \\
\mathrm{ft} \\
\end{array}$ & $\begin{array}{l}\text { Temp, } \\
{ }^{\circ} \mathrm{C} \\
\end{array}$ & $\begin{array}{l}\text { Distance } \\
\text { from Plant } \\
\text { Shore, yd }\end{array}$ & $\begin{array}{c}\text { Depth, } \\
\quad \mathrm{ft} \\
\end{array}$ & $\begin{array}{l}\text { Temp, } \\
{ }^{\circ} \mathrm{C} \\
\end{array}$ \\
\hline 10 & 8 & 19.3 & 20 & 5 & 15.15 & 5 & 3 & 14.6 \\
\hline 25 & 8 & 19.3 & 50 & 7 & 15.15 & 25 & 9 & 14.65 \\
\hline 50 & 13 & 19.25 & 100 & 12 & 15.2 & 70 & 18 & 14.65 \\
\hline 70 & 16 & 19.2 & 150 & 33 & 15.05 & 120 & 29 & 14.65 \\
\hline 95 & 30 & 19.05 & 200 & 27 & 15.0 & 170 & 31 & 14.5 \\
\hline 170 & 31 & 18.75 & 250 & 18 & 15.0 & 220 & 17 & 14.45 \\
\hline 195 & 19 & 18.7 & 300 & 14 & 14.95 & 270 & 14 & 14.4 \\
\hline 220 & 15 & 18.7 & 350 & 12 & 14.9 & 320 & 11 & 14.45 \\
\hline 245 & 14 & 18.7 & 400 & 10 & 14.9 & 370 & 8 & 14.35 \\
\hline 270 & 14 & 18.7 & 450 & 10 & 14.85 & 420 & 7 & 14.3 \\
\hline 295 & 12 & 18.65 & 500 & 10 & 14.85 & 470 & 7 & 14.35 \\
\hline 320 & 12 & 18.65 & 550 & 9 & 14.85 & 520 & 8 & 14.3 \\
\hline 355 & 9 & 18.6 & 600 & 12 & 14.8 & 570 & 8 & 14.25 \\
\hline 370 & 8 & 18.65 & 650 & 13 & 14.8 & 620 & 12 & 14.3 \\
\hline 395 & 7 & 18.65 & 700 & 14 & 14.75 & 670 & 12 & 14.2 \\
\hline 420 & 7 & 18.7 & 750 & 15 & 14.75 & 720 & 14 & 14.2 \\
\hline 445 & 6 & 18.7 & 800 & 14 & 14.75 & 770 & 12 & 14.1 \\
\hline 470 & 6 & 18.7 & 850 & 4 & 14.8 & 820 & 12 & 14.2 \\
\hline 495 & 6 & 18.7 & & & & 870 & 3 & 14.3 \\
\hline 520 & 6 & 18.7 & & & & & & \\
\hline 545 & 7 & 18.7 & & & & & & \\
\hline 570 & 7 & 18.7 & & & & & & \\
\hline 595 & 8 & 18.7 & & & & & & \\
\hline 620 & 9 & 18.5 & & & & & & \\
\hline 670 & 11 & 18.65 & & & & & & \\
\hline 720 & 12 & 18.65 & & & & & & \\
\hline 770 & 14 & 18.6 & & & & & & \\
\hline 820 & 11 & 18.6 & & & & & & \\
\hline 870 & 10 & 18.7 & & & & & & \\
\hline 900 & 6 & 18.7 & & & & & & \\
\hline $\begin{array}{l}\mathrm{A}_{\mathrm{t}}=33,600 \\
\mathrm{~T}_{\mathrm{m}}=18.77 \\
\text { PRTR Record } \\
\mathrm{T}_{\mathrm{PR}}=16.6\end{array}$ & $\begin{array}{l}\mathrm{ft}^{2} \\
{ }^{\circ} \mathrm{C} \\
\mathrm{er}=18.8 \\
{ }^{\circ} \mathrm{C}\end{array}$ & $3^{\circ} \mathrm{C}$ & $\begin{array}{l}\mathrm{A}_{\mathrm{t}}=34,800 \\
\mathrm{~T}_{\mathrm{m}}=14.92 \\
\text { PRTR Record } \\
\mathrm{T}_{\mathrm{PR}}=14.1\end{array}$ & $\begin{array}{l}\mathrm{ft}^{2} \\
{ }^{\circ} \mathrm{C} \\
\mathrm{r}=15 . \\
{ }^{\circ} \mathrm{C}\end{array}$ & ${ }^{\circ} \mathrm{C}$ & $\begin{array}{l}\mathrm{A}_{\mathrm{t}}=33,400 \\
\mathrm{~T}_{\mathrm{m}}=14.4^{\circ} \\
\text { PRTR Record } \\
\mathrm{T}_{\mathrm{PR}}=13.7\end{array}$ & $\begin{array}{l}f t^{2} \\
e r=14 .\end{array}$ & ${ }^{\circ} \mathrm{C}$ \\
\hline
\end{tabular}


TABLE D.I-8. (contd)
River Temperature Traverses Above 300 Area

(River Mile: 344.9)
Date: $\quad 12-11-63$

Time: 1400
Date: $\quad 12-16-63$

Time: 0930
Distance

from Plant Depth, Temp,

Shore, yd

$$
5
$$

50

75

125

175

225

275

325

375

425

475

525

575

625

675

725

775

805

825 $\mathrm{ft}$

$3 \quad 7.75$

11

18

7.85

8.15

35

31

18

8.25

8.3

8.25

15

8.3

13

10

8.3

8.25

10

10

8.2

8.2

9

10

12

12

15

8.15

8.15

8.1

8.1

8.05

11

8.0

12

8.0

7.9
Distance

from Plant Depth, Temp, Shore, yd

\section{5}

25

50

75

125

175

225

275

325

375

425

475

525

575

625

675

725

775

825

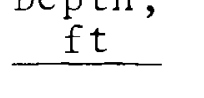

49.15

$8 \quad 8.95$

$\begin{array}{ll}18 & 9.5\end{array}$

$17 \quad 9.75$

$33 \quad 9.8$

$30 \quad 9.65$

$17 \quad 9.55$

$14 \quad 9.5$

129.45

$9 \quad 9.45$

$10 \quad 9.45$

$10 \quad 9.4$

$9 \quad 9.4$

$10 \quad 9.35$

$12 \quad 9.35$

$13 \quad 9.3$

$13 \quad 9.25$

$12 \quad 9.15$

$A_{t}=35,000 \mathrm{ft}^{2}$

$\mathrm{T}_{\mathrm{m}}=9.49^{\circ} \mathrm{C}$

PRTR Recorder $=9.5{ }^{\circ} \mathrm{C}$

$\mathrm{T}_{\mathrm{PR}}=7.1^{\circ} \mathrm{C}$ 
TABLE D.I-9. River Temperature Traverses - Richland Ferry (River Mile: 340.9 )

\begin{tabular}{|c|c|c|c|c|c|c|c|c|}
\hline $\begin{array}{l}\text { Date: } \\
\text { Time: }\end{array}$ & $\begin{array}{l}2-12-63 \\
0935\end{array}$ & & $\begin{array}{l}\text { Date } \\
\text { Time: }\end{array}$ & $\begin{array}{l}4-18-63 \\
0915\end{array}$ & & $\begin{array}{l}\text { Date: } \\
\text { Time: }\end{array}$ & $\begin{array}{l}5-9-63 \\
1345\end{array}$ & \\
\hline $\begin{array}{l}\text { Distance } \\
\text { from P1ant } \\
\text { Shore, yd }\end{array}$ & $\begin{array}{c}\text { Depth, } \\
\mathrm{ft} \\
\end{array}$ & $\begin{array}{l}\text { Temp, } \\
{ }^{\circ} \mathrm{C} \\
\end{array}$ & $\begin{array}{l}\text { Distance } \\
\text { from Plant } \\
\text { Shore, yd }\end{array}$ & $\begin{array}{c}\text { Depth, } \\
\mathrm{ft}\end{array}$ & $\begin{array}{l}\text { Temp, } \\
{ }^{\circ} \mathrm{C} \\
\end{array}$ & $\begin{array}{l}\text { Distance } \\
\text { from Plant } \\
\text { Shore, yd }\end{array}$ & $\begin{array}{c}\text { Depth, } \\
\mathrm{ft} \\
\end{array}$ & $\begin{array}{c}\text { Temp, } \\
{ }^{\circ} \mathrm{C}\end{array}$ \\
\hline 5 & 4 & 5.2 & 2 & 1 & 7.7 & 2 & 3 & 10.4 \\
\hline 50 & 14 & 5.0 & 50 & 11 & 7.6 & 50 & 17 & 10.0 \\
\hline 100 & 15 & 5.0 & 100 & 15 & 7.5 & 100 & 17 & 10.0 \\
\hline 140 & 13 & 5.0 & 150 & 12 & 7.5 & 150 & 15 & 9.9 \\
\hline 185 & 11 & 4.9 & 200 & 8 & 7.5 & 200 & 11 & 9.9 \\
\hline 235 & 8 & 4.6 & 250 & 7 & 7.5 & 250 & 9 & 9.9 \\
\hline 295 & 23 & 5.0 & 300 & 16 & 7.6 & 300 & 17 & 10.0 \\
\hline 340 & 25 & 4.9 & 350 & 24 & 7.5 & 350 & 21 & 9.9 \\
\hline 390 & 23 & 4.9 & 400 & 26 & 7.5 & 400 & 28 & 9.9 \\
\hline 455 & 12 & 4.9 & 450 & 20 & 7.5 & 450 & 26 & 9.8 \\
\hline 505 & 10 & 4.7 & 500 & 11 & 7.5 & 500 & 17 & 9.8 \\
\hline 540 & 10 & 4.7 & 550 & 10 & 7.5 & 550 & 14 & 9.8 \\
\hline 595 & 10 & 4.7 & 600 & 7 & 7.5 & 600 & 15 & 9.8 \\
\hline 640 & 10 & 4.9 & 650 & 10 & 7.5 & 650 & 15 & 9.7 \\
\hline 695 & 11 & 4.9 & 700 & 13 & 7.5 & 700 & 16 & 9.8 \\
\hline 750 & 17 & 4.2 & 750 & 16 & 7.5 & 750 & 19 & 9.8 \\
\hline 805 & 25 & 5.0 & 800 & 22 & 7.5 & 800 & 25 & 9.8 \\
\hline 840 & 26 & 4.8 & 850 & 23 & 7.5 & 850 & 26 & 9.8 \\
\hline 850 & 25 & 4.7 & 900 & 13 & 7.5 & 900 & 23 & 10.0 \\
\hline 885 & 16 & 4.9 & 955 & 2 & 8.5 & 1000 & 3 & 10.5 \\
\hline 915 & 6 & 4.4 & & & & & & \\
\hline $\begin{aligned} \mathrm{A}_{\mathrm{t}} & = \\
\mathrm{T}_{\mathrm{m}} & = \\
\mathrm{T}_{\mathrm{PR}} & =\end{aligned}$ & $\begin{array}{l}41,400 \mathrm{ft} \\
4.87{ }^{\circ} \mathrm{C} \\
4.15^{\circ} \mathrm{C}\end{array}$ & & $\begin{aligned} \mathrm{A}_{\mathrm{t}} & = \\
\mathrm{T}_{\mathrm{m}} & = \\
\mathrm{T}_{\mathrm{PR}} & =\end{aligned}$ & $\begin{array}{l}35,300 \mathrm{f} \\
7.53{ }^{\circ} \mathrm{C} \\
6.7{ }^{\circ} \mathrm{C}\end{array}$ & & $\begin{aligned} \mathrm{A}_{\mathrm{t}} & = \\
\mathrm{T}_{\mathrm{m}} & = \\
\mathrm{T}_{\mathrm{PR}} & =\end{aligned}$ & $\begin{array}{l}52,000 \\
9.90{ }^{\circ} \mathrm{C} \\
8.8{ }^{\circ} \mathrm{C}\end{array}$ & \\
\hline
\end{tabular}


BNWL- 1345

TABLE D.I-9. (contd) River Temperature Traverses Richland Ferry

(River Mile: 340.9 )

\begin{tabular}{|c|c|c|c|c|c|c|c|c|}
\hline $\begin{array}{l}\text { Date: } \\
\text { Time: }\end{array}$ & $\begin{array}{l}5-16-63 \\
1045\end{array}$ & & $\begin{array}{l}\text { Date: } \\
\text { Time: }\end{array}$ & $\begin{array}{l}6-18-63 \\
1340\end{array}$ & & $\begin{array}{l}\text { Date: } \\
\text { Time: }\end{array}$ & $\begin{array}{l}6-26-63 \\
1340\end{array}$ & \\
\hline $\begin{array}{l}\text { Distance } \\
\text { from P1ant } \\
\text { Shore, yd } \\
\end{array}$ & $\begin{array}{c}\text { Depth, } \\
\mathrm{ft} \\
\end{array}$ & $\begin{array}{l}\text { Temp, } \\
{ }^{\circ} \mathrm{C} \\
\end{array}$ & $\begin{array}{l}\text { Distance } \\
\text { from Plant } \\
\text { Shore, yd } \\
\end{array}$ & $\begin{array}{c}\text { Depth } \\
\mathrm{ft} \\
\end{array}$ & $\begin{array}{c}\text { Temp, } \\
{ }^{\circ} \mathrm{C}\end{array}$ & $\begin{array}{l}\text { Distance } \\
\text { from P1ant } \\
\text { Shore, yd } \\
\end{array}$ & $\begin{array}{c}\text { Depth, } \\
\mathrm{ft} \\
\end{array}$ & $\begin{array}{l}\text { Temp, } \\
{ }^{\circ} \mathrm{C}\end{array}$ \\
\hline 3 & 1 & 12.4 & 2 & 5 & 16.5 & 2 & 3 & 16.2 \\
\hline 43 & 13 & 12.0 & 50 & 21 & 16.2 & 50 & 18 & 16.0 \\
\hline 93 & 15 & 11.9 & 100 & 22 & 16.1 & 100 & 20 & 15.9 \\
\hline 143 & 13 & 11.8 & 150 & 20 & 16.1 & 150 & 19 & 15.9 \\
\hline 193 & 10 & 11.8 & 210 & 17 & 16.0 & 200 & 17 & 15.9 \\
\hline 243 & 7 & 11.9 & 245 & 15 & 16.0 & 250 & 13 & 15.8 \\
\hline 293 & 13 & 11.8 & 305 & 32 & 16.0 & 300 & 28 & 15.9 \\
\hline 343 & 24 & 11.7 & 350 & 33 & 16.1 & 350 & 31 & 15.8 \\
\hline 393 & 25 & 11.5 & 400 & 27 & 15.9 & 400 & 29 & 15.8 \\
\hline 443 & 18 & 11.6 & 450 & 19 & 15.9 & 450 & 21 & 15.7 \\
\hline 493 & 13 & 11.6 & 500 & 16 & 15.9 & 500 & 16 & 15.7 \\
\hline 543 & 10 & 11.6 & 550 & 14 & 15.9 & 550 & 14 & 15.7 \\
\hline 593 & 8 & 11.6 & 610 & 15 & 15.9 & 600 & 14 & 15.7 \\
\hline 643 & 10 & 11.6 & 650 & 16 & 16.0 & 650 & 15 & 15.7 \\
\hline 693 & 12 & 11.6 & 700 & 17 & 15.9 & 700 & 17 & 15.8 \\
\hline 743 & 18 & 11.6 & 750 & 19 & 15.9 & 750 & 17 & 15.7 \\
\hline 793 & 24 & 11.6 & 800 & 25 & 15.9 & 800 & 21 & 15.7 \\
\hline 843 & 23 & 11.7 & 850 & 29 & 15.9 & 850 & 27 & 15.6 \\
\hline 893 & 24 & 11.6 & 900 & 32 & 15.9 & 900 & 30 & 15.7 \\
\hline 943 & 15 & 11.9 & 950 & 33 & 16.0 & 950 & 30 & 15.7 \\
\hline \multirow[t]{3}{*}{993} & 3 & 12.3 & 1000 & 31 & 16.0 & 1000 & 27 & 15.8 \\
\hline & & & 1050 & 15 & 16.1 & 1050 & 10 & 15.9 \\
\hline & & & 1100 & 1.5 & 16.8 & 1100 & 2 & 16.2 \\
\hline \multicolumn{3}{|c|}{$\begin{aligned} \mathrm{T}_{\mathrm{m}} & =11.71{ }^{\circ} \mathrm{C} \\
\mathrm{T}_{\mathrm{PR}} & =10.6{ }^{\circ} \mathrm{C}\end{aligned}$} & \multicolumn{3}{|c|}{$\begin{aligned} \mathrm{T}_{\mathrm{m}}^{\mathrm{T}} & =15.99{ }^{\circ} \mathrm{C} \\
\mathrm{T}_{\mathrm{PR}} & =14.8{ }^{\circ} \mathrm{C}\end{aligned}$} & \multicolumn{3}{|c|}{$\begin{aligned} \mathrm{T}_{\mathrm{m}}^{\tau} & =15.77{ }^{\circ} \mathrm{C} \\
\mathrm{T}_{\mathrm{PR}} & =14.9^{\circ} \mathrm{C}\end{aligned}$} \\
\hline
\end{tabular}

D. I. 37 


\section{TABIE D.I-9. (contd) River Temperature Traverses - Richland Ferry \\ (River Mile: 340.9 )}

\begin{tabular}{|c|c|c|c|c|c|c|c|c|}
\hline $\begin{array}{l}\text { Date: } \\
\text { Time: }\end{array}$ & $\begin{array}{l}8-30-63 \\
1700\end{array}$ & & $\begin{array}{l}\text { Date: } \\
\text { Time: }\end{array}$ & $\begin{array}{l}8-30-63 \\
1800\end{array}$ & & $\begin{array}{l}\text { Date: } \\
\text { Time: }\end{array}$ & $10-10-6$ & \\
\hline $\begin{array}{l}\text { Distance } \\
\text { from Plant } \\
\text { Shore, yd }\end{array}$ & $\begin{array}{c}\text { Depth, } \\
\mathrm{ft} \\
\end{array}$ & $\begin{array}{c}\text { Temp, } \\
{ }^{\circ} \mathrm{C} \\
\end{array}$ & $\begin{array}{l}\text { Distance } \\
\text { from Plant } \\
\text { Shore, yd }\end{array}$ & $\begin{array}{l}\text { Depth, } \\
\mathrm{ft} \\
\end{array}$ & $\begin{array}{c}\text { Temp, } \\
{ }^{\circ} \mathrm{C} \\
\end{array}$ & $\begin{array}{l}\text { Distance } \\
\text { from Plant } \\
\text { Shore, yd }\end{array}$ & $\begin{array}{c}\text { Depth, } \\
\mathrm{ft} \\
\end{array}$ & $\begin{array}{l}\text { Temp, } \\
{ }^{\circ} \mathrm{C} \\
\end{array}$ \\
\hline 5 & 3 & 21.3 & 5 & 3 & 21.5 & 5 & 3 & 17.95 \\
\hline 50 & 17 & 21.2 & 50 & 17 & 21.2 & 45 & 13 & 17.95 \\
\hline 90 & 17 & 21.1 & 90 & 17 & 21.2 & 95 & 15 & 18.0 \\
\hline 140 & 15 & 21.1 & 140 & 15 & 21.1 & 145 & 13 & 18.0 \\
\hline 190 & 11 & 21.1 & 190 & 11 & 21.0 & 195 & 10 & 17.95 \\
\hline 240 & 9 & 21.1 & 240 & 9 & 21.0 & 245 & 11 & 17.95 \\
\hline 290 & 17 & 21.0 & 290 & 17 & 20.9 & 295 & 23 & 17.9 \\
\hline 340 & 21 & 20.9 & 340 & 21 & 20.8 & 345 & 25 & 17.85 \\
\hline 390 & 28 & 20.8 & 390 & 28 & 20.8 & 395 & 23 & 17.85 \\
\hline 440 & 26 & 20.8 & 440 & 26 & 20.8 & 445 & 15 & 17.85 \\
\hline 490 & 17 & 20.8 & 490 & 17 & 20.8 & 495 & 11 & 17.85 \\
\hline 540 & 14 & 20.8 & 540 & 14 & 20.8 & 545 & 10 & 17.85 \\
\hline 590 & 15 & 20.9 & 590 & 15 & 20.8 & 595 & 10 & 17.85 \\
\hline 640 & 15 & 20.9 & 640 & 15 & 20.8 & 645 & 11 & 17.85 \\
\hline 690 & 16 & 20.9 & 690 & 16 & 20.8 & 695 & 12 & 17.85 \\
\hline 740 & 19 & 20.9 & 740 & 19 & 20.8 & 745 & 16 & 17.8 \\
\hline 790 & 25 & 20.9 & 790 & 25 & 20.8 & 795 & 23 & 17.8 \\
\hline 840 & 26 & 20.9 & 840 & 26 & 20.7 & 845 & 26 & 17.8 \\
\hline 890 & 23 & 20.9 & 890 & 23 & 20.8 & 895 & 16 & 17.7 \\
\hline 940 & 15 & 21.0 & 940 & 15 & 21.0 & 945 & 5 & 17.6 \\
\hline 990 & 4 & 21.0 & 990 & 4 & 21.1 & & & \\
\hline $\begin{aligned} \mathrm{A}_{\mathrm{t}} & =5 \\
\mathrm{~T}_{\mathrm{m}} & =2 \\
\mathrm{~T}_{\mathrm{PR}} & =1\end{aligned}$ & $\begin{array}{l}2,500 \mathrm{ft} \\
0.93^{\circ} \mathrm{C} \\
8.0^{\circ} \mathrm{C}\end{array}$ & & $\begin{aligned} \mathrm{A}_{\mathrm{t}} & = \\
\mathrm{T}_{\mathrm{m}} & = \\
\mathrm{T}_{\mathrm{PR}} & =\end{aligned}$ & $\begin{array}{l}2,500 \mathrm{ft} \\
0.91{ }^{\circ} \mathrm{C} \\
8.0^{\circ} \mathrm{C}\end{array}$ & & $\begin{aligned} \mathrm{A}_{\mathrm{t}} & =4 \\
\mathrm{~T}_{\mathrm{m}} & =1 \\
\mathrm{~T}_{\mathrm{PR}} & =1\end{aligned}$ & $\begin{array}{l}2,800 \mathrm{ft} \\
7.86{ }^{\circ} \mathrm{C} \\
7.3{ }^{\circ} \mathrm{C}\end{array}$ & \\
\hline
\end{tabular}


TABLE D.I-9. (contd) River Temperature Traverses Richland Ferry (River Mile 340.9)

\begin{tabular}{|c|c|c|c|c|c|c|c|c|}
\hline \multirow{2}{*}{$\begin{array}{r}\text { Date: } \\
\text { Time: } \\
\text { Distance } \\
\text { from Plant } \\
\text { Shore, yd } \\
\end{array}$} & \multicolumn{2}{|c|}{$\begin{array}{l}10-16-63 \\
1715\end{array}$} & $\begin{array}{l}\text { Date: } \\
\text { Time: }\end{array}$ & \multicolumn{2}{|c|}{$\begin{array}{l}10-16-63 \\
1830\end{array}$} & $\begin{array}{l}\text { Date: } \\
\text { Time: }\end{array}$ & \multicolumn{2}{|c|}{$\begin{array}{l}10-29-63 \\
1445\end{array}$} \\
\hline & $\begin{array}{c}\text { Depth, } \\
\text { ft }\end{array}$ & $\begin{array}{c}\text { Temp, } \\
{ }^{\circ} \mathrm{C} \\
\end{array}$ & $\begin{array}{l}\text { Distance } \\
\text { from Plant } \\
\text { Shore, yd } \\
\end{array}$ & $\begin{array}{c}\text { Depth, } \\
\mathrm{ft} \\
\end{array}$ & $\begin{array}{c}\text { Temp, } \\
{ }^{\circ} \mathrm{C} \\
\end{array}$ & $\begin{array}{l}\text { Distance } \\
\text { from Plant } \\
\text { Shore, yd } \\
\end{array}$ & $\begin{array}{c}\text { Depth, } \\
\mathrm{ft} \\
\end{array}$ & $\begin{array}{l}\text { Temp, } \\
{ }^{\circ} \mathrm{C} \\
\end{array}$ \\
\hline 25 & 10 & 18.85 & 25 & 11 & 18.7 & 5 & 3 & 15.1 \\
\hline 75 & 13 & 18.85 & 75 & 12 & 18.6 & 50 & 12 & 15.1 \\
\hline 125 & 12 & 18.8 & 125 & 14 & 18.6 & 100 & 14 & 15.1 \\
\hline 175 & 11 & 18.8 & 175 & 11 & 18.6 & 150 & 14 & 15.1 \\
\hline 200 & 8 & 18.7 & 200 & 8 & 18.55 & 200 & 13 & 15.05 \\
\hline 275 & 5 & 18.75 & 275 & 9 & 18.5 & 250 & 11 & 15.05 \\
\hline 325 & 23 & 18.8 & 325 & 24 & 18.5 & 300 & 8 & 15.05 \\
\hline 375 & 24 & 18.65 & 375 & 24 & 18.5 & 350 & 4 & 15.05 \\
\hline 425 & 25 & 18.6 & 425 & 22 & 18.5 & 400 & 21 & 15.05 \\
\hline 475 & 17 & 18.6 & 475 & 15 & 18.5 & 450 & 27 & 14.95 \\
\hline 525 & 11 & 18.6 & 525 & 9 & 18.5 & 500 & 26 & 14.95 \\
\hline 575 & 8 & 18.6 & 575 & 9 & 18.5 & 550 & 16 & 14.95 \\
\hline 625 & 6 & 18.6 & 625 & 7 & 18.5 & 600 & 7 & 14.95 \\
\hline 675 & 7 & 18.6 & 675 & 6 & 18.5 & 650 & 9 & 14.9 \\
\hline 725 & 9 & 18.6 & 725 & 8 & 18.5 & 700 & 10 & 14.9 \\
\hline 775 & 10 & 18.6 & 775 & 11 & 18.5 & 750 & 11 & 14.9 \\
\hline 825 & 15 & 18.6 & 825 & 17 & 18.5 & 800 & 12 & 14.95 \\
\hline 850 & 17 & 18.6 & 875 & 22 & 18.5 & 850 & 22 & 14.9 \\
\hline 875 & 19 & 18.6 & 925 & 25 & 18.5 & 900 & 26 & 14.9 \\
\hline 900 & 21 & 18.55 & 975 & 25 & 18.5 & 950 & 23 & 14.8 \\
\hline 925 & 23 & 18.55 & 1025 & 22 & 18.4 & 1000 & 10 & 14.7 \\
\hline 950 & 25 & 18.5 & & & & 1020 & 3 & 14.8 \\
\hline 975 & 25 & 18.5 & & & & & & \\
\hline 1025 & 24 & 18.6 & & & & & & \\
\hline $\begin{aligned} \mathrm{A}_{\mathrm{t}} & = \\
\mathrm{T}_{\mathrm{m}} & = \\
\mathrm{T}_{\mathrm{PR}} & =\end{aligned}$ & $\begin{array}{l}3,300 \mathrm{ft} \\
8.64{ }^{\circ} \mathrm{C} \\
6.6{ }^{\circ} \mathrm{C}\end{array}$ & & $\begin{aligned} \mathrm{A}_{\mathrm{t}} & = \\
\mathrm{T}_{\mathrm{m}} & = \\
\mathrm{T}_{\mathrm{PR}} & =\end{aligned}$ & $\begin{array}{l}3,700 \mathrm{ft} \\
8.51{ }^{\circ} \mathrm{C} \\
6.6{ }^{\circ} \mathrm{C}\end{array}$ & & $\begin{aligned} A_{t} & = \\
T_{m} & = \\
T_{P R} & =\end{aligned}$ & $\begin{array}{l}4,300 \mathrm{f} \\
4.96^{\circ} \mathrm{C} \\
4.1{ }^{\circ} \mathrm{C}\end{array}$ & \\
\hline
\end{tabular}




$$
0 t^{*} I^{*}
$$

\begin{tabular}{|c|c|c|c|c|c|}
\hline \multicolumn{3}{|c|}{$\begin{aligned} J_{0} \tau \cdot L & ={ }^{y d} d_{L} \\
J_{0} L \varepsilon \cdot 6 & =u_{L}\end{aligned}$} & \multicolumn{3}{|c|}{$\begin{aligned} J_{0} \cdot t I & ={ }^{\mathrm{d} d} \mathrm{~L} \\
J_{0} 80^{\circ} \mathrm{S} I & ={ }_{L}\end{aligned}$} \\
\hline \multicolumn{3}{|c|}{$z^{7 f} 000^{6}\left[t={ }^{7} \forall\right.$} & \multicolumn{3}{|c|}{$z^{7 F} 00 \varepsilon^{6} \Delta t={ }^{7} H$} \\
\hline$s \cdot 8$ & s & ५५ 6 & $s 8 \cdot t \tau$ & $\varepsilon$ & $020 I$ \\
\hline$S L \cdot 8$ & $t \tau$ & $\varsigma £ 6$ & $\varsigma 8 \cdot t \tau$ & $t z$ & $0 \mathrm{~S} 6$ \\
\hline$\varsigma 8 \cdot 8$ & I & $\varsigma 88$ & $S 6^{\circ} \circ \mathrm{I}$ & 92 & 006 \\
\hline$z \cdot 6$ & 02 & 098 & $\mathrm{~s} 0^{\circ} \mathrm{sI}$ & $6 \mathrm{~L}$ & $0 \mathrm{~s} 8$ \\
\hline$t \cdot 6$ & $t z$ & $0 โ 8$ & $S 0^{\circ} \mathrm{SI}$ & I I & 008 \\
\hline$t \cdot 6$ & 02 & $09 L$ & $\varsigma 0^{\circ} \varsigma \mathrm{I}$ & 6 & $0 S \angle$ \\
\hline$s t \cdot 6$ & $t \tau$ & $0[L$ & $\varsigma 0^{\circ} \mathrm{SI}$ & 8 & $00 L$ \\
\hline$s t \cdot 6$ & I I & 099 & $S 0^{\circ} \varsigma I$ & 0[ & $0 \sin$ \\
\hline$s t \cdot 6$ & 8 & $0[9$ & $\varsigma 0^{\circ} \varsigma \tau$ & $z I$ & 009 \\
\hline$s t \cdot 6$ & $s \cdot L$ & 095 & $\varsigma 0^{\circ} \varsigma \mathrm{L}$ & $\mathrm{Iz}$ & oss \\
\hline$s t \cdot 6$ & 6 & 0 IS & $I \cdot S I$ & $\angle Z$ & $00 \mathrm{~s}$ \\
\hline$s \cdot 6$ & $2 I$ & $09 t$ & $S I \cdot S I$ & 92 & ost \\
\hline$s \cdot 6$ & 22 & $0[t$ & $\tau \cdot s[$ & $t z$ & $00 t$ \\
\hline$s \cdot 6$ & $\varepsilon z$ & $09 \varepsilon$ & $\tau \cdot s I$ & $\mathrm{~s}$ & $0 S \mathcal{L}$ \\
\hline$s \cdot 6$ & 92 & OLE & $S I \cdot S I$ & 8 & $00 \varepsilon$ \\
\hline$s t \cdot 6$ & $\mathrm{~s}$ & 092 & $S[\cdot S I$ & I I & $0 \varsigma z$ \\
\hline$s \cdot 6$ & 8 & $0 \tau z$ & $S I \cdot S I$ & $\varepsilon I$ & $00 z$ \\
\hline $5 \cdot 6$ & I I & 09[ & $z \cdot s I$ & $t I$ & OS I \\
\hline$s t \cdot 6$ & $t I$ & $0 \tau \tau$ & $Z \cdot S I$ & $t \mathrm{I}$ & $00 \mathrm{~L}$ \\
\hline$t \cdot 6$ & $2 \mathrm{I}$ & 09 & $S Z^{\cdot} \varsigma I$ & $2 I$ & $0 \mathrm{~S}$ \\
\hline $56 \cdot 8$ & 6 & $0 I$ & $\varepsilon \cdot s[$ & $L$ & $S I$ \\
\hline$\frac{\partial_{\sigma}}{d u \partial_{L}}$ & 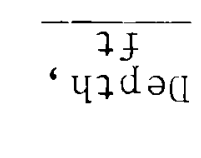 & 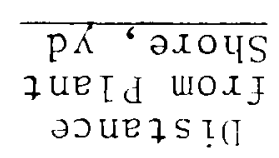 & 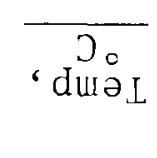 & 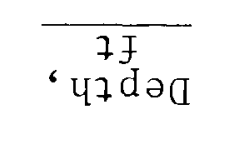 & 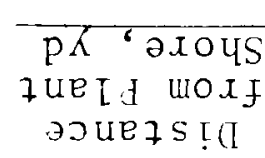 \\
\hline & $\begin{array}{r}0 z \cup[ \\
\because 9-9[-z I\end{array}$ & $\begin{array}{l}: \text { :uLL } \\
: \partial \mathcal{Z} \mathbb{L}\end{array}$ & & $\begin{array}{r}00 S I \\
29-6 \bar{c}-0[\end{array}$ & 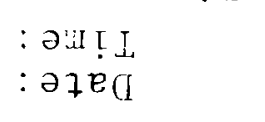 \\
\hline
\end{tabular}


TABLE D.I-10. River Temperature Traverses -

Pasco Water Plant

(River Mile: 328.7 )

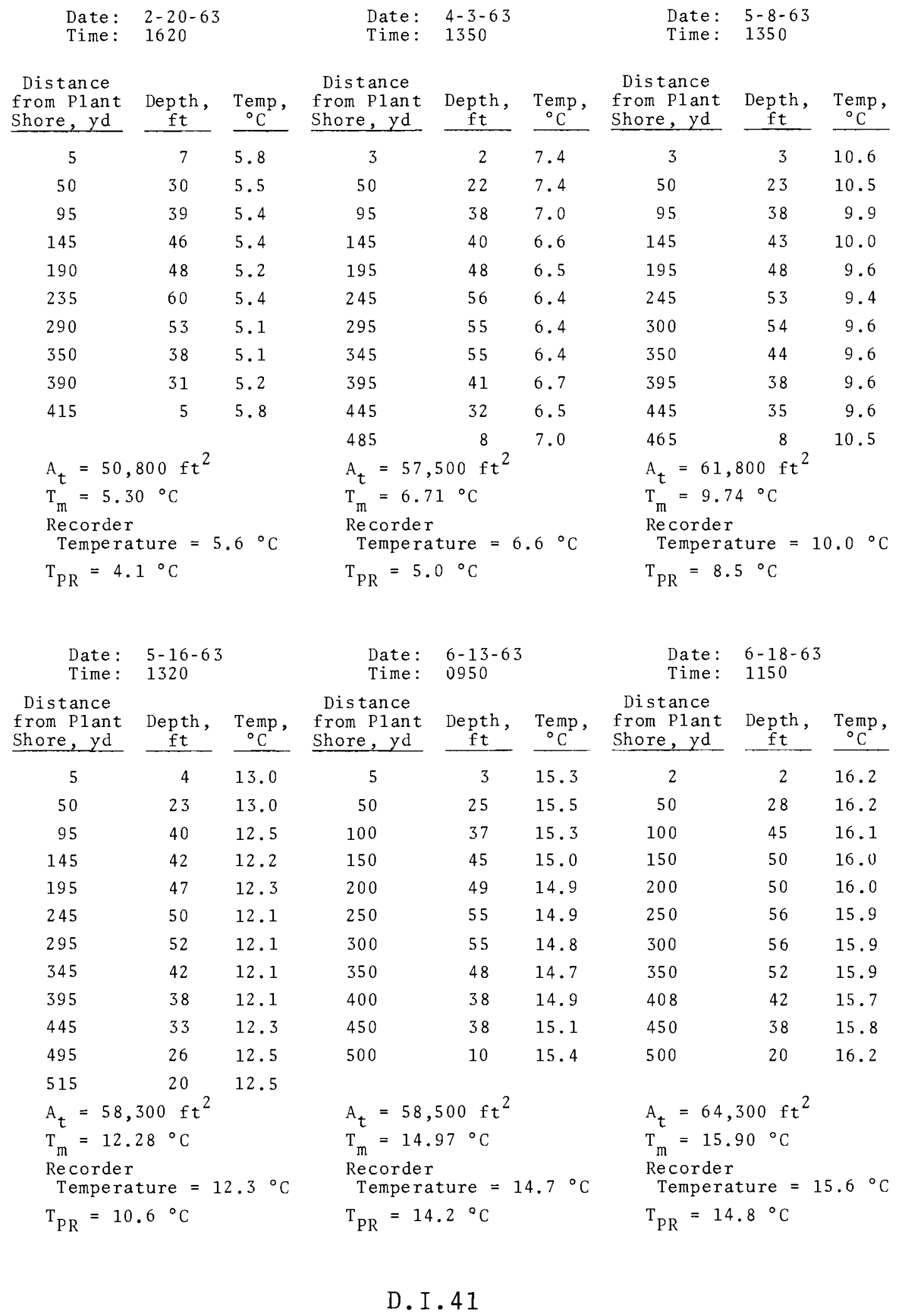




\section{TABLE D.I-10. (contd) River Temperature Traverses - Pasco Water Plant \\ (River Mile: 328.7 )}

\begin{tabular}{|c|c|c|c|c|c|c|c|c|}
\hline \multirow{2}{*}{$\begin{array}{r}\text { Date: } \\
\text { Time: } \\
\text { Distance } \\
\text { from Plant } \\
\text { Shore, yd } \\
\end{array}$} & \multicolumn{2}{|l|}{$\begin{array}{l}7-11-63 \\
1100\end{array}$} & $\begin{array}{l}\text { Date: } \\
\text { Time: }\end{array}$ & \multicolumn{2}{|c|}{$\begin{array}{l}10-10-63 \\
1400\end{array}$} & $\begin{array}{l}\text { Date: } \\
\text { Time: }\end{array}$ & \multicolumn{2}{|c|}{$\begin{array}{l}12-9-63 \\
1400\end{array}$} \\
\hline & $\begin{array}{c}\text { Depth, } \\
\mathrm{ft} \\
\end{array}$ & $\begin{array}{c}\text { Temp , } \\
{ }^{\circ} \mathrm{C} \\
\end{array}$ & $\begin{array}{l}\text { Distance } \\
\text { from P1 ant } \\
\text { Shore, yd }\end{array}$ & $\begin{array}{c}\text { Depth, } \\
\mathrm{ft} \\
\end{array}$ & $\begin{array}{c}\text { Temp, } \\
{ }^{\circ} \mathrm{C} \\
\end{array}$ & $\begin{array}{l}\text { Distance } \\
\text { from Plant } \\
\text { Shore, yd }\end{array}$ & $\begin{array}{c}\text { Depth, } \\
\mathrm{ft}\end{array}$ & $\begin{array}{c}\text { Temp, } \\
{ }^{\circ} \mathrm{C} \\
\end{array}$ \\
\hline 5 & 8 & 16.6 & 5 & 5 & 18.4 & 10 & 10 & 8.35 \\
\hline 100 & 37 & 16.3 & 50 & 25 & 18.4 & 25 & 23 & 8.45 \\
\hline 200 & 49 & 16.05 & 105 & 38 & 18.45 & 50 & 24 & 8.4 \\
\hline 300 & 55 & 15.95 & 155 & 42 & 18.45 & 75 & 25 & 8.4 \\
\hline 400 & 38 & 15.9 & 205 & 50 & 18.5 & 95 & 38 & 8.9 \\
\hline 500 & 38 & 16.0 & 265 & 56 & 18.45 & 120 & 48 & 8.9 \\
\hline \multirow[t]{13}{*}{535} & 10 & 16.25 & 305 & 55 & 18.45 & 145 & 52 & 8.9 \\
\hline & & & 355 & 52 & 18.4 & 170 & 54 & 8.95 \\
\hline & & & 405 & 38 & 18.35 & 195 & 52 & 9.0 \\
\hline & & & 455 & 27 & 18.35 & 220 & 62 & 9.0 \\
\hline & & & 505 & 7 & 18.35 & 245 & 66 & 9.0 \\
\hline & & & & & & 270 & 68 & 9.0 \\
\hline & & & & & & 295 & 58 & 9.0 \\
\hline & & & & & & 320 & 54 & 8.9 \\
\hline & & & & & & 345 & 47 & 8.9 \\
\hline & & & & & & 370 & 43 & 8.85 \\
\hline & & & & & & 395 & 40 & 8.8 \\
\hline & & & & & & 420 & 30 & 8.7 \\
\hline & & & & & & 445 & 22 & 8.5 \\
\hline \multicolumn{3}{|c|}{$A_{t}=62,800 \mathrm{ft}^{2}$} & \multicolumn{3}{|c|}{$A_{t}=57,400 \mathrm{ft}^{2}$} & \multicolumn{3}{|c|}{$A_{t}=59,700 \mathrm{ft}^{2}$} \\
\hline \multicolumn{3}{|c|}{$\mathrm{T}_{\mathrm{m}}=16.08^{\circ} \mathrm{C}$} & \multicolumn{3}{|c|}{$\mathrm{T}_{\mathrm{m}}^{\mathrm{L}}=18.43^{\circ} \mathrm{C}$} & \multicolumn{3}{|c|}{$\mathrm{T}_{\mathrm{m}}^{\mathrm{C}}=8.88^{\circ} \mathrm{C}$} \\
\hline \multicolumn{3}{|c|}{$\begin{array}{l}\text { Recorder } \\
\text { Temperature }=16.4\end{array}$} & \multicolumn{3}{|c|}{$\begin{array}{l}\text { Recorder } \\
\text { Temperature }=\end{array}$} & \multicolumn{3}{|c|}{ Recorder } \\
\hline \multicolumn{3}{|c|}{$\mathrm{T}_{\mathrm{PR}}=15.8{ }^{\circ} \mathrm{C}$} & \multicolumn{3}{|c|}{$\mathrm{T}_{\mathrm{PR}}=17.3^{\circ} \mathrm{C}$} & \multicolumn{3}{|c|}{$\mathrm{T}_{\mathrm{PR}}=8.4{ }^{\circ} \mathrm{C}$} \\
\hline
\end{tabular}




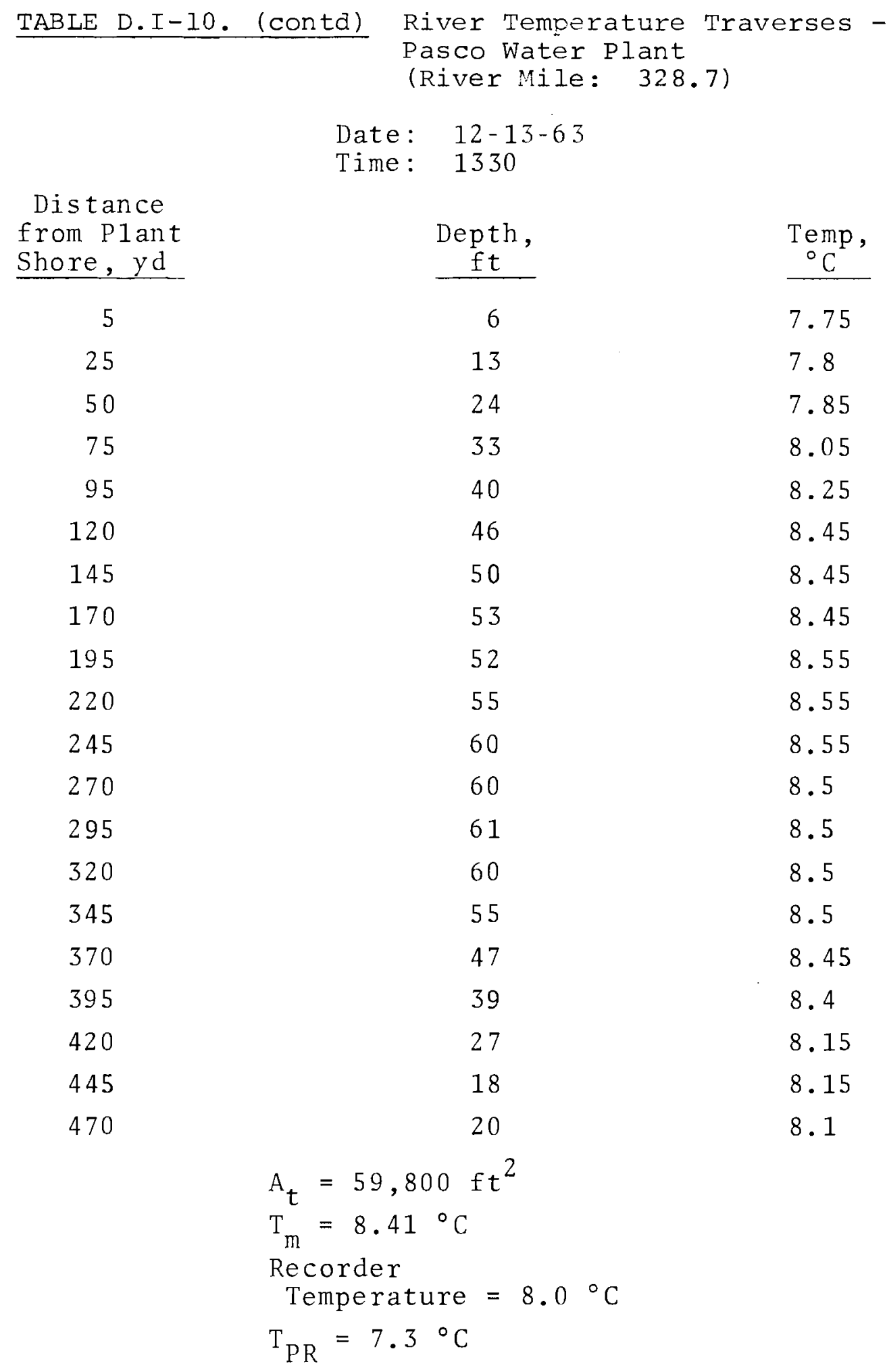

D. I. 43 
BNWL- 1345

TABLE D.I-11. F-Area Fewer Temperatures

\begin{tabular}{|c|c|c|}
\hline Date & $\begin{array}{l}\text { Ave rage Raw } \\
\text { Water Temp, }{ }^{\circ} \mathrm{C} \\
\end{array}$ & $\begin{array}{l}\text { Weighted Average } \\
\text { Sewer Temp, }{ }^{\circ} \mathrm{C}\end{array}$ \\
\hline $\begin{array}{l}8-29-63 \\
8-30 \\
8-31\end{array}$ & $\begin{array}{l}20.0 \\
20.3 \\
20.7\end{array}$ & $\begin{array}{l}24 \\
24.5 \\
25\end{array}$ \\
\hline $\begin{array}{l}9-1 \\
9-2 \\
9-3 \\
9-4 \\
9-5 \\
9-6 \\
9-7 \\
9-8 \\
9-13 \\
9-14 \\
9-15 \\
9-16 \\
9-17 \\
9-18 \\
9-19 \\
9-20 \\
9-21 \\
9-25 \\
9-26 \\
9-27 \\
9-30\end{array}$ & $\begin{array}{l}20.6 \\
20.1 \\
20.3 \\
20.0 \\
20.7 \\
20.7 \\
21.0 \\
21.2 \\
20.9 \\
20.7 \\
20.5 \\
20.0 \\
20.2 \\
20.3 \\
19.7 \\
19.9 \\
20.3 \\
20.4 \\
20.6 \\
20.8 \\
21.0\end{array}$ & $\begin{array}{l}24.5 \\
24.5 \\
25.5 \\
25 \\
26 \\
25 \\
25 \\
25.5 \\
26 \\
25 \\
24 \\
24 \\
24 \\
24 \\
23 \\
25.5 \\
23.5 \\
24 \\
24 \\
24 \\
24\end{array}$ \\
\hline $\begin{array}{l}10-1 \\
10-2 \\
10-3 \\
10-4 \\
10-5 \\
10-6 \\
10-7 \\
10-11 \\
10-12 \\
10-15 \\
10-16 \\
10-17 \\
10-18 \\
10-19 \\
10-20\end{array}$ & $\begin{array}{l}20.6 \\
20.0 \\
20.0 \\
19.9 \\
19.8 \\
20.4 \\
20.3 \\
19.2 \\
19.1 \\
18.9 \\
18.8 \\
18.5 \\
18.3 \\
18.8 \\
18.8\end{array}$ & $\begin{array}{l}24 \\
24.5 \\
25 \\
22.5 \\
22.5 \\
22.5 \\
23 \\
24 \\
21.5 \\
21.5 \\
22 \\
22 \\
22 \\
22 \\
22.5\end{array}$ \\
\hline $\begin{array}{l}10-21-63 \\
10-22 \\
10-23 \\
10-24 \\
10-25 \\
10-26 \\
10-27 \\
10-28 \\
10-29 \\
10-30 \\
10-31\end{array}$ & $\begin{array}{l}18.5 \\
17.8 \\
17.5 \\
17.3 \\
17.0 \\
16.5 \\
17.0 \\
16.5 \\
15.8 \\
15.5 \\
15.1\end{array}$ & $\begin{array}{l}22 \\
21 \\
20.5 \\
20.5 \\
19.5 \\
19.5 \\
19.5 \\
19.5 \\
19 \\
19 \\
18.5\end{array}$ \\
\hline $\begin{array}{l}11-1 \\
11-2 \\
11-3\end{array}$ & $\begin{array}{l}15.1 \\
15.0 \\
15.3\end{array}$ & $\begin{array}{l}18 \\
17.5 \\
17.5\end{array}$ \\
\hline
\end{tabular}

D. I. 44 
TABLE D.I-12. Temperatures in Effluent Bubbles and Plumes

\begin{tabular}{|c|c|c|c|c|c|c|}
\hline $\begin{array}{l}\text { Outfali } \\
\text { Location }\end{array}$ & $\begin{array}{c}\text { Depth, } \\
\text { ft } \\
\end{array}$ & Date & Time & $\begin{array}{l}\text { Maximum } \\
\text { Temp, }{ }^{\circ} \mathrm{C}\end{array}$ & $\begin{array}{l}\text { Upstream } \\
\text { Temp, }{ }^{\circ} \mathrm{C}\end{array}$ & $\begin{array}{c}\text { Maximum Temp } \\
\text { at } 100 \text { yd } \\
\text { Downstream } \\
\end{array}$ \\
\hline \multirow[t]{4}{*}{ B } & 1 & $11-26-63$ & $1230-$ & 17.2 & 10.6 & -- \\
\hline & 4 & & 1330 & 17.7 & 10.6 & 14.1 \\
\hline & 8 & & & 16.3 & 10.6 & - \\
\hline & 15 & & & 19.5 & 10.6 & -- \\
\hline \multirow[t]{2}{*}{ C } & 3 & $11-26-63$ & 1330 & 19.5 & -- & 14.4 \\
\hline & 10 & & & 17.6 & -- & -- \\
\hline \multirow[t]{2}{*}{$\mathrm{D}$} & 1 & $11-26$ & 1430 & 28.1 & & \\
\hline & 10 & & & 22.3 & 13.8 & 16.9 \\
\hline \multirow[t]{3}{*}{$\mathrm{F}$} & 1 & $10-16-63$ & 0800 & 26.1 & 18.0 & - \\
\hline & 1 & $12-3-63$ & 1330 & 23.8 & 10.8 & - \\
\hline & 5 & & & 20.3 & -- & \\
\hline $\mathrm{H}$ & 1 & $12-3-63$ & 1130 & 16.7 & 11.0 & - \\
\hline $\mathrm{K}$ (shore) & 3 & $11-26-63$ & 1400 & 17.9 & 11.3 & 12.9 \\
\hline (main & 3 & & & 21.7 & & 12.8 \\
\hline bubb le ) & 15 & & & 23.7 & & \\
\hline
\end{tabular}




\section{TABLE D.I-13. Equilibrium Dye Concentrations}

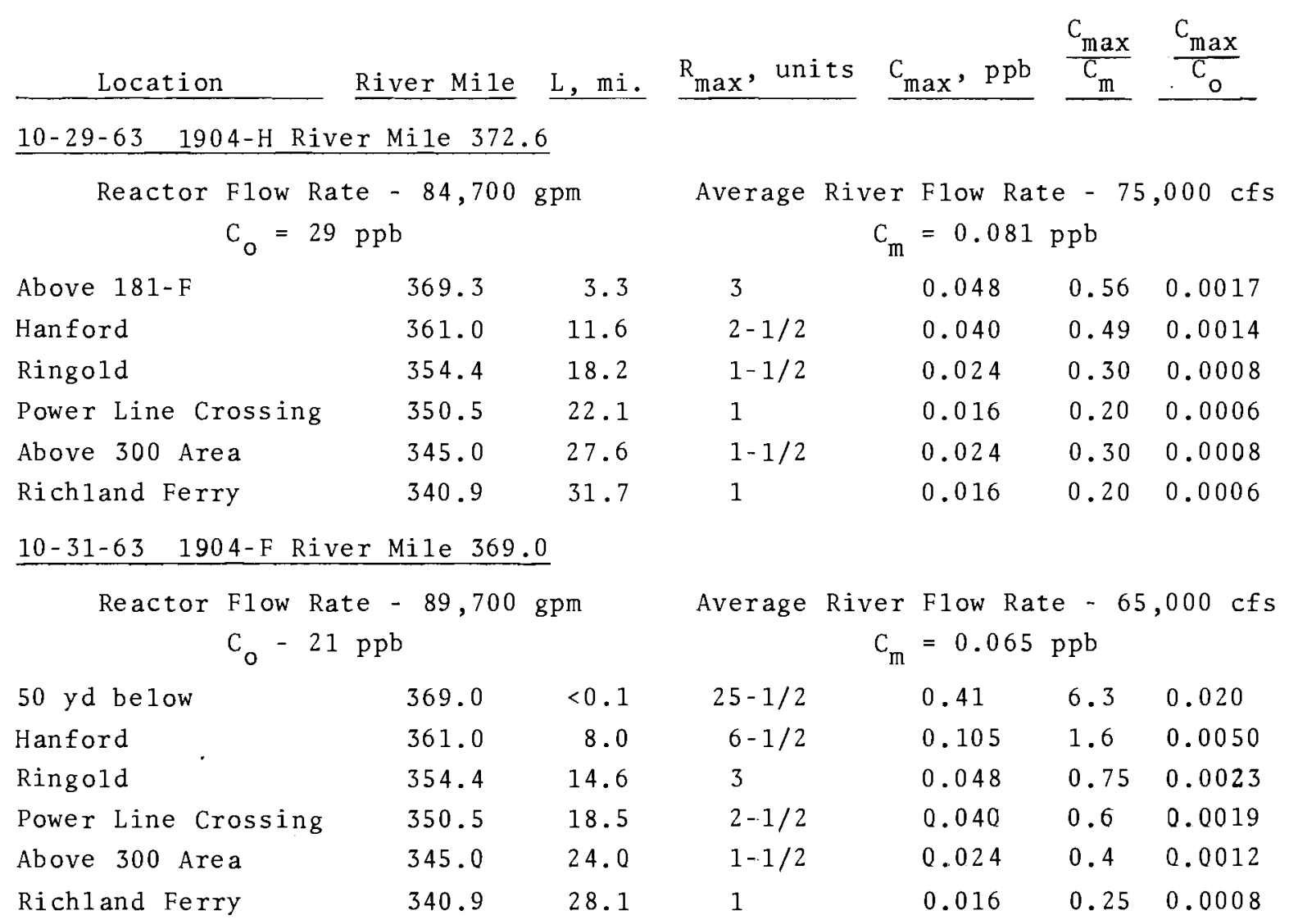


BNWL- 1345

\section{REFERENCES}

D1. J. P. Corley. Effects of Hanford operations on Columbia River Temperatures - Interim Report, HW-76210. February 13, 1963. (SECRET)

D2. J. P. Corley. Effects of Hanford operations on Columbia River Chemical Characteristics, HW-84537. December 10, 1964. (Unpublished Data)

D3. J.K. Soldat. Columbia River Flow-Time Calculations, HW-58312. July 1, 1963 . 
BNWL -1345

APPENDIX D

SECTION II

INTER-REACTOR RIVER TEMPERATURE DATA *

J. P. Corley

* Declassified excerpts from Batte11e-Northwest internal report BNWL-CC-807, dated September 9, 1966. 


\title{
INTER-REACTOR RIVER TEMPERATURE DATA
}

\author{
J. P. Corley
}

\section{Tab1e D.II-1}

This table shows direct temperature measurements made just downstream from the ends of the reactor outfall lines. The procedure genera11y was to probe the river at the selected distance to find the maximum temperature in the cross section. Temperatures were measured with a resistance thermometer.

In most cases, readings were taken at several depths at the indicated point to determine the degree of mixing, and the depth of the maximum reading. Although not shown, the depth readings show little difference vertically at 400 -yard distances, even at the lower river flow rates. At 100-yard distances and low river flow rates, vertical mixing is not complete and the maximum reading frequently occurs at some depth beneath the water surface.

In the table, $Q_{R}$ is river flow rate as measured at the Priest Rapids gage station, adjusted for time of wave trave1 to the outfall whenever the flow rate was changing rapidly. ${ }_{\mathrm{T}}$ is the river temperature measured just upstream from the end of the outfall line. $\Delta T$ equals $T_{\max }$ minus $T_{R}$.

$B$ and $C$ outfall lines, like the $D$ and $D R$ lines, are less than 400 yards apart. The 400 -yard distances, therefore, were measured from the downstream line, $C$ and DR respectively.

Except where noted by an approximation symbol, the accuracy of the data is believed to be:

$$
\begin{gathered}
\mathrm{T}_{\mathrm{R}} \text { and } \mathrm{T}_{\max } \text { at } 400 \text { yards }= \pm 0.2{ }^{\circ} \mathrm{C} \\
\mathrm{Q}_{\mathrm{R}}= \pm 5 \%
\end{gathered}
$$


BNWL - 1345

Table D.II-2

Sets of traverse markers have been set up at 400 yards, $1 / 2$ mile, and 1 mile below each of the reactor outfalls, and above $N$ and $D$ Area river pump intakes. Data for all these locations is given in the several sections of Table D.II-2. Time has not permitted measurements at a11 points on any one date.

River traverses for temperature measurements only were made with the resistance thermometer probe suspended from the boat at a water depth of about 2 feet. When temperature measurements were taken in conjunction with dye tests, a continuous sample line was used with the intake about 2 feet beneath the water and the resistance thermometer installed through a fitting into the sample line. A stadimeter was used for lateral distance measurement, and a fathometer for water depth. The instruments, procedure and some early data have been discussed in more detail in $\mathrm{HW}-80888$.*

In the tables, those reactors operating upstream from the traverse location are indicated. Where an asterisk appears, the reactor was shut down and effluent flow rate had been reduced; but, the temperature of the effluent was stil1 well above river temperature.

River flow rate was determined, as in Table D.II-1 from the Priest Rapids gage record, as was the base river temperature, $\mathrm{T}_{\mathrm{PR}} \cdot \mathrm{A}_{t}$, the cross-sectional area, was calculated from the tabulated distance and depth measurements, and the mean river temperature $T_{m}$ for the cross section was calculated by the meansection method as:

$$
\mathrm{T}_{\mathrm{m}}=\frac{\sum\left(\mathrm{w} \times \mathrm{d}_{\text {ave }} \mathrm{x} \mathrm{T}_{\text {ave }}\right)}{\sum\left(\mathrm{w} \times \mathrm{d}_{\text {ave }}\right)}
$$

* J.P. Corley, Effects of Hanford operations on Columbia River Temperatures - Interim Report No. 2, HW-80888. December 3, 1964. (original classified)

$$
\text { D. I I. } 2
$$


In some of the tables, a column is shown headed, for example, "B Effluent Units." This is the measured relative concentration of effluent from the named reactor at the cross section. In al1 such tables, Rhodamine B dye was being pumped continuously into the reactor outfall. The units shown are actually dial readings on the Turner Fluorometer used to measure dye concentrations in river water. Since the addition rate and apparent dye loss varied between tests, it is not possible to compare directly the indicated units values for different days. However, for the same date at different locations, the indicated units should be comparable.

Accuracy of the temperature data is the same as for Table D.II-1 data, except where the notation "est." appears. Depth accuracy is generally +2 feet, although frequent difficulties with fathometer equipment may be reflected in several traverses for which the depths and calculated areas do not reconcile with other data from the same location. The most frequent errors in the data occur in the lateral distance measurement. It is difficult to maintain a straight line with the boat at uniform traverse speed in the high and fluctuating water velocities usually found in this section of the river.

No attempt has been made here to reconcile al1 the traverses at a given 1ocation; the only changes in the raw data have been calibration corrections. It is believed that showing the actual run-to-run variations will permit the user of this data to do so more intel1igent $1 y$. 
BNWL-1345

TABLE D.II-1. Effluent Plume Temperatures

TABLE D.II-1. Effluent Plume Temperatures

\begin{tabular}{|c|c|c|c|c|c|c|}
\hline \multirow[b]{2}{*}{ Outfal1 } & \multirow[b]{2}{*}{$\mathrm{Q}_{\mathrm{R}}, \operatorname{cfs}$} & \multirow[b]{2}{*}{${ }^{\mathrm{T}} \mathrm{R},{ }^{\circ} \mathrm{C}$} & \multicolumn{2}{|c|}{$100 \mathrm{yd}$} & \multicolumn{2}{|c|}{$400 \mathrm{yd}$} \\
\hline & & & $\mathrm{T}_{\max ,}{ }^{\circ} \mathrm{C}$ & $\Delta \mathrm{T},{ }^{\circ} \mathrm{C}$ & $\mathrm{T}_{\max ,}{ }^{\circ} \mathrm{C}$ & $\Delta \mathrm{T},{ }^{\circ} \mathrm{C}$ \\
\hline $1904-B-C$ & $\begin{array}{r}41,000 \\
54,000 \\
87,000 \\
115,000 \\
160,000 \\
189,000 \\
255,000\end{array}$ & $\begin{array}{c}7.15 \\
6.45 \\
10.5 \\
4.6 \\
9.35 \\
16.7 \\
10.45\end{array}$ & $\begin{array}{c}11.1 \\
10.6 \\
14.4 \\
8.3 \\
10.95 \\
17.85 \\
11.65\end{array}$ & $\begin{array}{l}3.95 \\
4.15 \\
2.6 \\
3.7 \\
1.6 \\
1.15 \\
1.15\end{array}$ & $\begin{array}{c}7.65 \\
9.45 \\
11.8 \\
6.4 \\
- \\
17.95 \\
11.4\end{array}$ & $\begin{array}{l}3.6 \\
3.0 \\
1.3 \\
1.8 \\
- \\
1.25 \\
0.95\end{array}$ \\
\hline $1904-\mathrm{K}$ & $\begin{array}{r} \\
75,000 \\
71,000 \\
\sim 80,000 \\
153,000 \\
241,000 \\
277,000\end{array}$ & $\begin{array}{c}3.80 \\
7.45 \\
5.45 \\
9.1 \\
10.95 \\
12.8\end{array}$ & $\begin{array}{c}10.15 \\
10.15 \\
9.45 \\
9.9 \\
12.95 \\
-\end{array}$ & $\begin{array}{l}6.35 \\
2.7 \\
4.0 \\
0.8 \\
2.0 \\
-\end{array}$ & $\begin{array}{c}9.35 \\
9.5 \\
7.85 \\
\quad- \\
12.3 \\
14.15\end{array}$ & $\begin{array}{c}5.55 \\
2.05 \\
2.40 \\
- \\
1.35 \\
1.35\end{array}$ \\
\hline $1904-D-D R$ & $\begin{array}{r}77,000 \\
108,000 \\
140,000 \\
237,000 \\
277,000\end{array}$ & $\begin{array}{c}9.1 \\
6.15 \\
18.05 \\
11.3 \\
13.2\end{array}$ & $\begin{array}{c}13.7 \\
8.3 \\
20.35 \\
12.6 \\
-\end{array}$ & $\begin{array}{l}4.6 \\
2.15 \\
2.3 \\
1.3 \\
-\end{array}$ & $\begin{array}{r}12.5 \\
7.25 \\
19.90 \\
12.2 \\
14.05\end{array}$ & $\begin{array}{l}3.4 \\
1.1 \\
1.85 \\
0.9 \\
0.85\end{array}$ \\
\hline $1908-\mathrm{N}$ & 140,000 & 3.55 & 6.8 & 3.25 & 6.05 & 2.5 \\
\hline
\end{tabular}




\section{TABLE D.II-2. River Temperature Traverses - 400 Yards Below C \\ (River Mile: 383.8)}

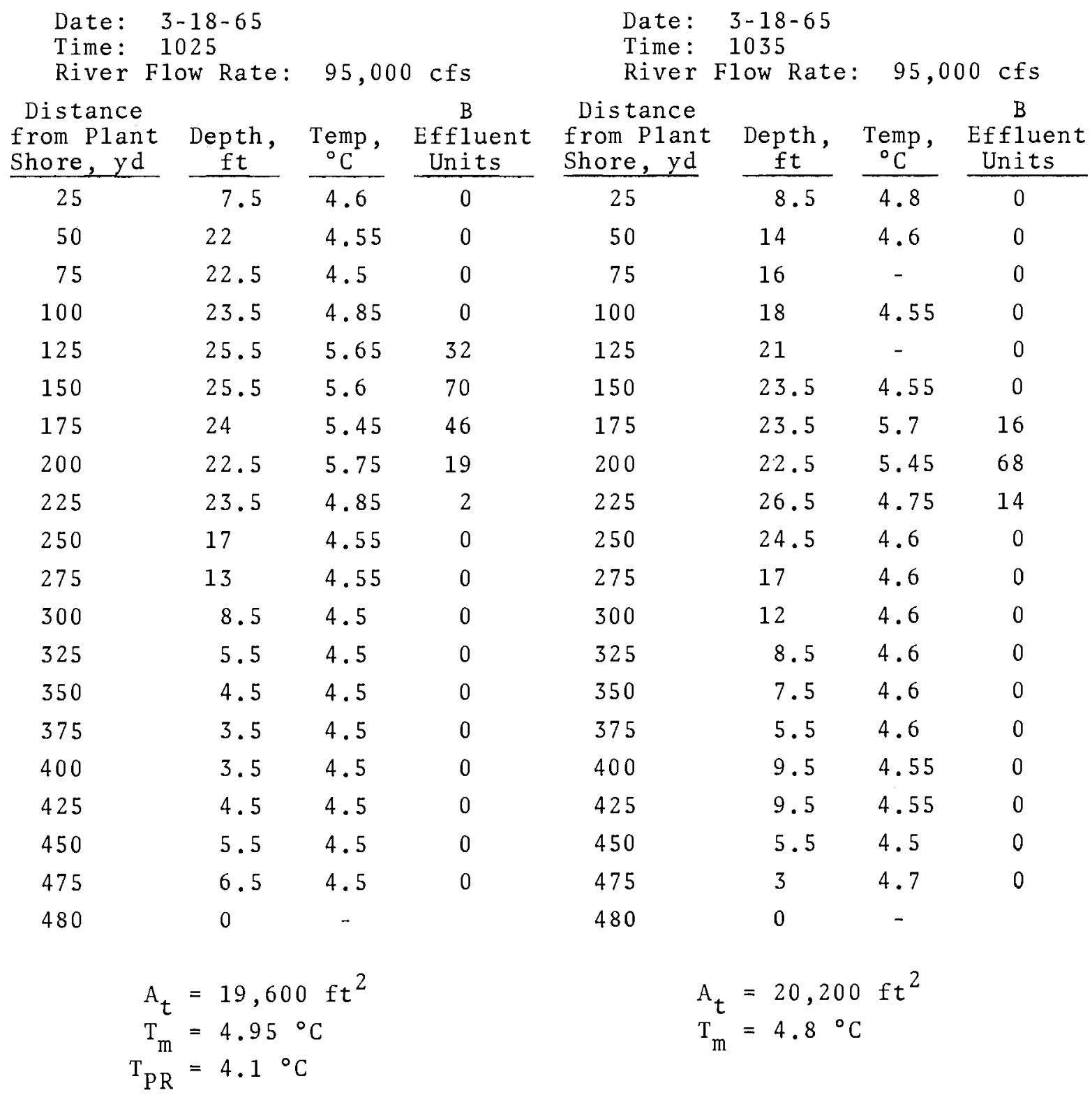

NOTE: On Tables D.II-2 through D.II-15 the following nomenclature is used:

$A_{t}=$ cross section area, $f t^{2}$

$\mathrm{T}_{\mathrm{m}}=$ mean stream temperature, ${ }^{\circ} \mathrm{C}$

$\mathrm{T}_{\mathrm{PR}}=$ river temperature at Priest Rapids, ${ }^{\circ} \mathrm{C}$ 


\section{TABLE D.II-2. (contd)}

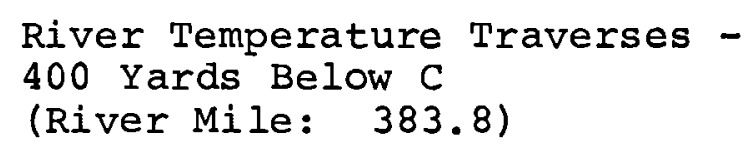

Date: $\quad 5-26-66$

Time: 1105

River Flow Rate: 175,000 cfs

Distance

from Plant Depth, Temp,

$\frac{\text { Shore, yd }}{0} \frac{\mathrm{ft}}{2} \frac{{ }^{\circ} \mathrm{C}}{12.5}$

$\begin{array}{lll}25 & 7 & 12.2\end{array}$

$\begin{array}{lll}45 & 20 & 11.85\end{array}$

$\begin{array}{lll}70 & 27 & 12.8\end{array}$

$95 \quad 30 \quad 13.0$

120

145

170

195

220

245

270

295

320

345

370

395

420

445

470

495

520

545

570

580

30

11.85

11.75

11.75

11.75

11.75

11.75

11.75

11.75

11.75

11.75

11.8

11.8

11.85

11.9

12.1

12.25

12.6

13.4

13.45

$-$

$$
\begin{aligned}
\mathrm{A}_{\mathrm{t}} & =24,600 \mathrm{ft}^{2} \\
\mathrm{~T}_{\mathrm{m}} & =12.05{ }^{\circ} \mathrm{C} \\
\mathrm{T}_{\mathrm{PR}} & =11.8{ }^{\circ} \mathrm{C}
\end{aligned}
$$

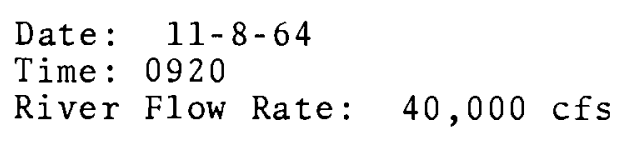

Distance

from Plant Depth, Temp, Effluent $\frac{\text { Shore, yd }}{30}$

$\frac{\mathrm{ft}}{9.5} \frac{{ }^{\circ} \mathrm{C}}{11.6} \frac{\text { Units }}{0}$

50

$10.5 \quad 15.0 \quad 25$

75

$13 \quad 15.0 \quad 52$

100

$13 \quad 18.6 \quad 71$

125

$11.5 \quad 17.1$

29

150

$8 \quad 15.6$

$7.5 \quad 13.9$

$13.5 \quad 12.0$

$13 \quad 11.6$

$11.5 \quad 11.6$

$8.5 \quad 11.5$

5.511 .3

290

330

0

0
5
0
0
0
0
0


TABLE D.II-3. River Temperature Traverses - 0.5 Mile Below C [River Mile: 383.5 (Plant shore to island on ly) ]

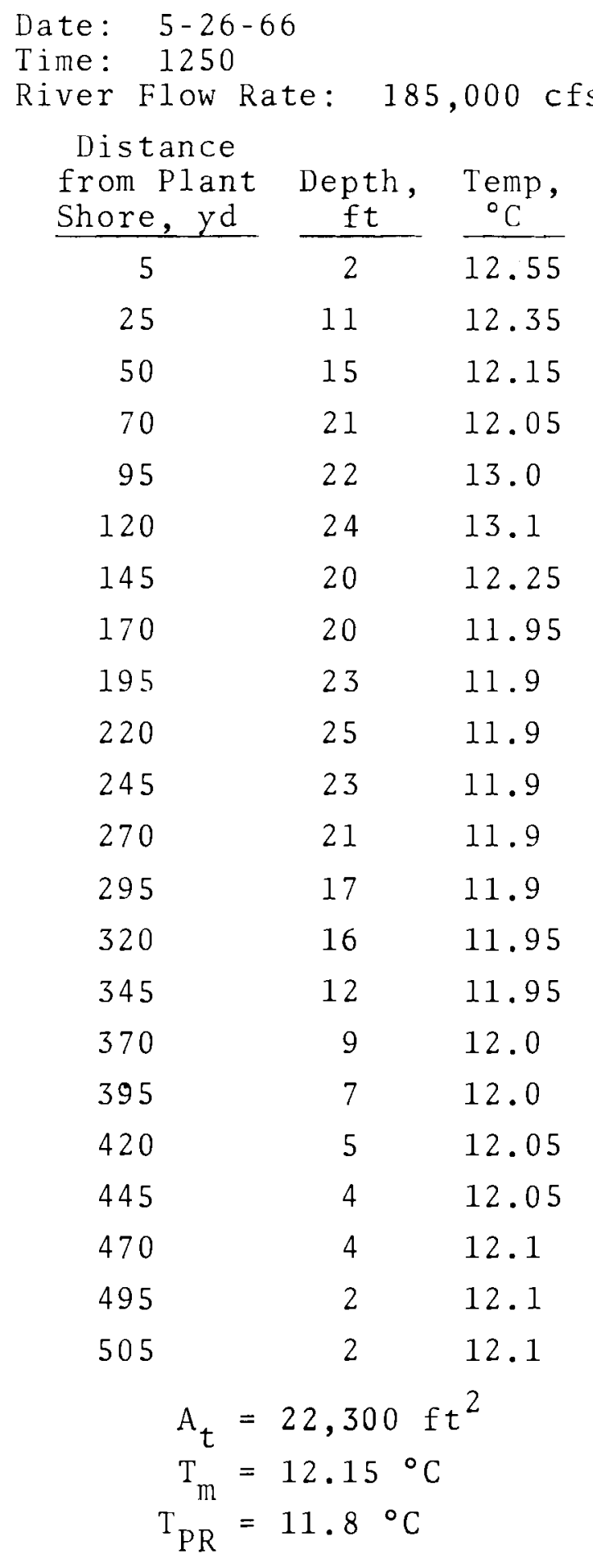

Date: $\quad 5-26-66$

Time: 1300

River Flow Rate: 185,000 cfs Distance from Plant Depth, Temp, Shore, yd

$\begin{array}{rrl}5 & 2 & 12.5 \\ 25 & - & 12.45 \\ 50 & - & - \\ 70 & 18 & 12.4 \\ 95 & 21 & 12.2 \\ 120 & 25 & 12.9 \\ 145 & 22 & 12.9 \\ 170 & 24 & 12.3 \\ 195 & 25 & 11.95 \\ 220 & 25 & 11.75 \\ 245 & 25 & 11.75 \\ 270 & 22 & 11.75 \\ 295 & 20 & 11.75 \\ 320 & 18 & 11.75 \\ 345 & 16 & 11.8 \\ 370 & 15 & 11.8 \\ 395 & 13 & 11.8 \\ 420 & 12 & 11.8 \\ 445 & 9 & 11.85 \\ 470 & 5 & 11.95 \\ 495 & 4 & 11.9 \\ 505 & 2 & 12.0\end{array}$

$$
\begin{aligned}
& A_{t}=25,500 \mathrm{ft}^{2} \\
& T_{m}=12.1{ }^{\circ} \mathrm{C}
\end{aligned}
$$


TABLE D.II-4. River Temperature Traverses - 1 Mile Below C (River Mile: 383.0)

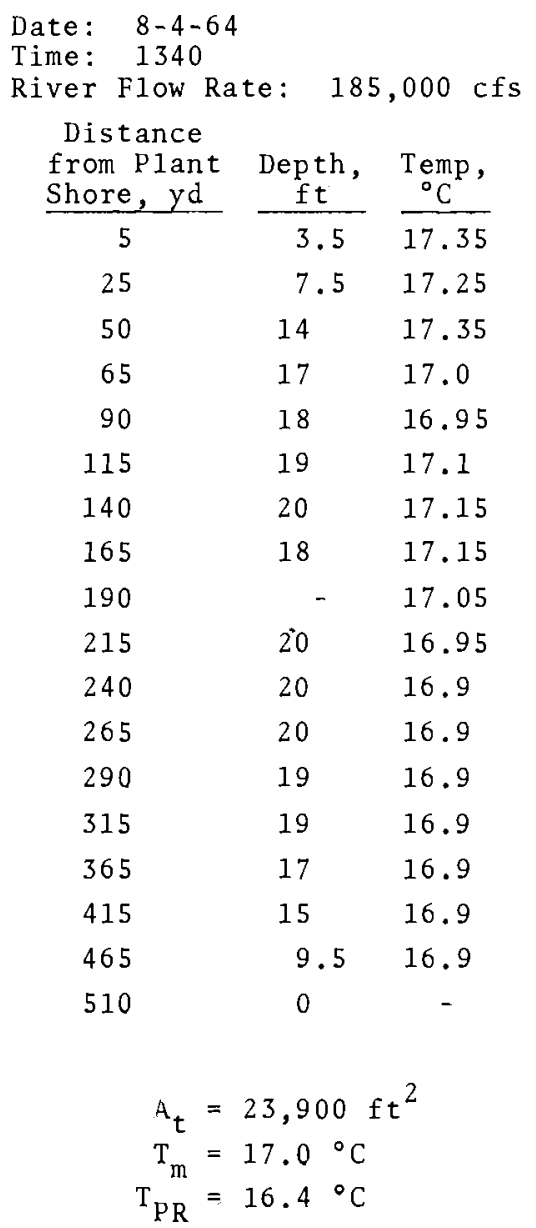

\begin{tabular}{|c|c|c|c|c|c|c|}
\hline \multicolumn{3}{|l|}{$\begin{array}{ll}\text { Date: } & 8-6-64 \\
\text { Time: } & 0830\end{array}$} & \multicolumn{4}{|c|}{$\begin{array}{ll}\text { Date: } & 8-6-64 \\
\text { Time: } & 0840\end{array}$} \\
\hline $\begin{array}{l}\text { Distance } \\
\text { from Plant } \\
\text { Shore, yd }\end{array}$ & $\begin{array}{c}\text { Depth, } \\
\mathrm{ft} \\
\end{array}$ & $\begin{array}{l}\text { Temp, } \\
{ }^{\circ} \mathrm{C} \\
\end{array}$ & $\begin{array}{l}\text { Dist } \\
\text { from } \\
\text { Shore } \\
\end{array}$ & $\begin{array}{l}\text { nce } \\
1 \text { ant } \\
y d\end{array}$ & $\begin{array}{c}\text { Depth, } \\
\mathrm{ft} \\
\end{array}$ & $\begin{array}{l}\text { Temp } \\
{ }^{\circ} \mathrm{C} \\
\end{array}$ \\
\hline 25 & 11.5 & 16.85 & 25 & & - & 16.5 \\
\hline 50 & 12 & - & 50 & & 14 & 16.8 \\
\hline 75 & 13 & 16.95 & 75 & & 15 & 16.85 \\
\hline 100 & 14 & - & 100 & & 16 & 17.0 \\
\hline 125 & 15 & 17.05 & 125 & & 18 & 17.25 \\
\hline 150 & 15.5 & - & 150 & & - & - \\
\hline 175 & 16 & 17.45 & 175 & & - & 17.5 \\
\hline 200 & 17 & 17.55 & 200 & & 19 & 17.45 \\
\hline 225 & 17 & 17.5 & 225 & & 19 & 17.3 \\
\hline 250 & 18 & 17.3 & 250 & & 17 & 17.15 \\
\hline 275 & 17 & 17.05 & 275 & & 16 & 17.0 \\
\hline 300 & 15 & 16.95 & 300 & & 17 & 16.95 \\
\hline 325 & 15.5 & - & 325 & & 17 & 16.95 \\
\hline 350 & 16 & 16.9 & 350 & & 15 & 16.9 \\
\hline 375 & 15.5 & - & 375 & & 14 & 16.9 \\
\hline 400 & 15 & 16.9 & 400 & & 12 & 16.9 \\
\hline 425 & 11 & - & 425 & & 9.5 & 16.9 \\
\hline 450 & 6.5 & 16.95 & 450 & & 7.5 & 16.9 \\
\hline 490 & 0 & -- & 490 & & 0 & - \\
\hline $\begin{aligned} \mathrm{A}_{\mathrm{t}} & = \\
\mathrm{T}_{\mathrm{m}} & = \\
\mathrm{T}_{\mathrm{PR}} & =\end{aligned}$ & $\begin{array}{l}18,900 \\
17.1{ }^{\circ} \mathrm{C} \\
16.4{ }^{\circ} \mathrm{C}\end{array}$ & & & $\begin{array}{l}A_{t}= \\
T_{m}=\end{array}$ & $\begin{array}{l}18,900 \\
17.1{ }^{\circ} \mathrm{C}\end{array}$ & \\
\hline
\end{tabular}

D.II. 8 


\section{TABLE D.II-4. (contd) River Temperature Traverses - 1 Mile Below C (River Mile: 383.0 )}

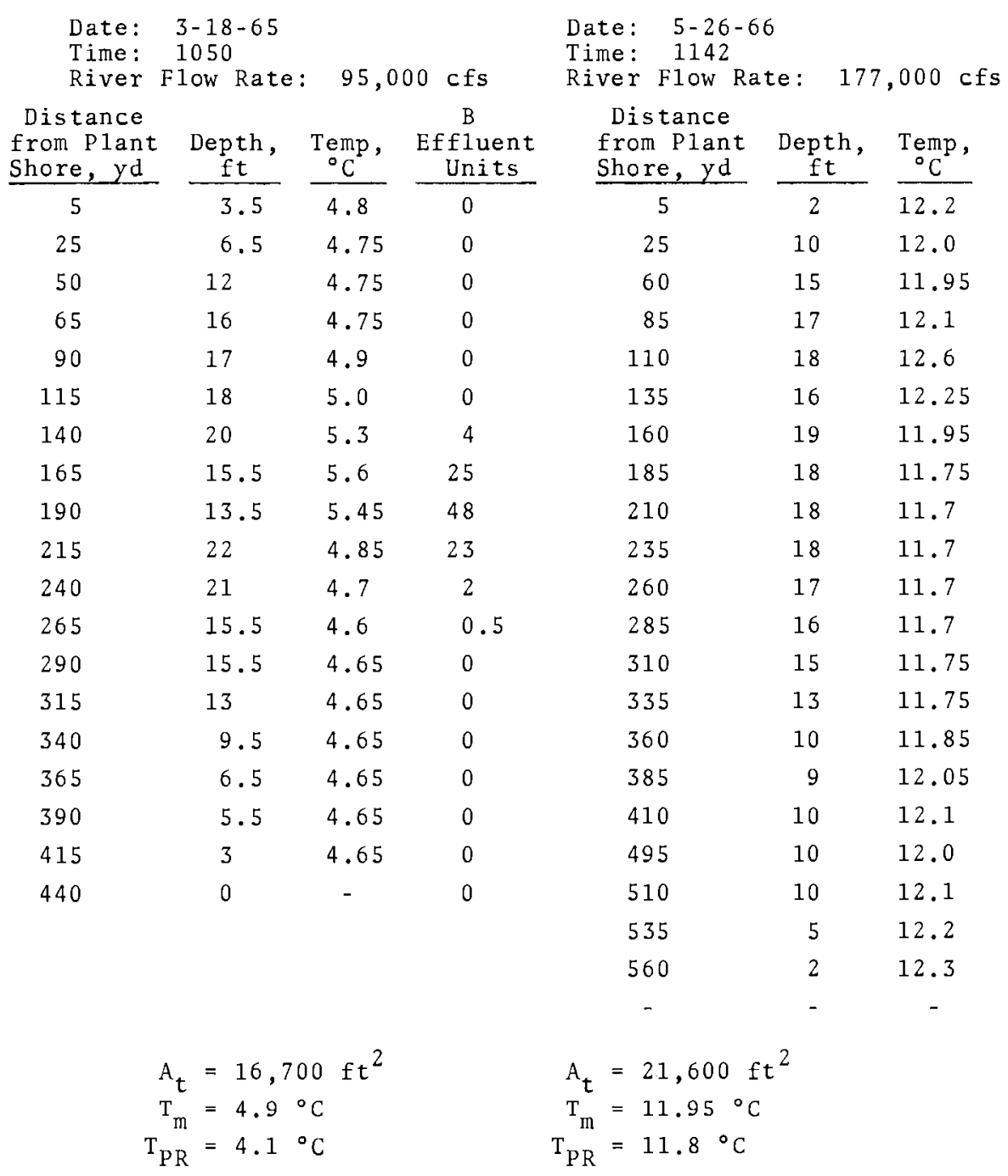


TABLE D.II-5. River Temperature Traverses 400 Yards Below $\mathrm{K}$

(River Mile: 381.5)

Date: 3-4-66

Time: 1315

River Flow Rate: 115,000 cfs

Distance from Plant

Shore, yd

5

25

40

65

90

115

140

165

190

215

240

265

290

315

340

365

390

415

440

450

470 $\frac{\begin{array}{c}\text { Depth, } \\ \mathrm{ft}\end{array}}{3.5}$

8.5

10.5

10.5

10.5

12

16

20

26.5

31.5

31.5

29

28

26.5

16

9.5

9.5

9.5

4.5

3.5

0 $\frac{\begin{array}{c}\text { Temp } \\ { }^{\circ} \mathrm{C}\end{array}}{5.1}$

4.0

4.0

4.0

4.9

4. 7

4.2

3.8

3.9

4.7

6.0

3.6

3.5

3.5

3.5

3.5

3.5

3.5

3.5

3.6

$$
\begin{aligned}
\mathrm{A}_{\mathrm{t}} & =22,900 \mathrm{ft}^{2} \\
\mathrm{~T}_{\mathrm{m}} & =4.15{ }^{\circ} \mathrm{C} \\
\mathrm{T}_{\mathrm{PR}} & =3.4{ }^{\circ} \mathrm{C}
\end{aligned}
$$

D. I I 10 


\section{TABLE D.II-5. (contd) River Temperature Traverses - 400 Yards Below K \\ (River Mile: 381.5)}

$\begin{array}{ll}\text { Date: } & 4-3-66 \\ \text { Time: } & 1015\end{array}$

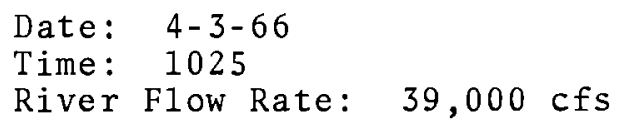

$$
\begin{aligned}
& \mathrm{A}_{t}=21,900 \mathrm{ft}^{2} \\
& \mathrm{~T}_{\mathrm{m}}=10.0^{\circ} \mathrm{C} \text { (est.) }
\end{aligned}
$$


TABLE D.II-5. (contd) River Temperature Traverses 400 Yards Below 1904-K

(River Mile: 381.5 )

Date: $\quad 5-20-66$

Time: 1105

River Flow Rate: 195,000 cfs

Distance

from Plant

Shore, yd

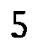

25

50

75

100

125

150

175

200

225

250

275

300

325

350

375

400

425

450

475

490

515

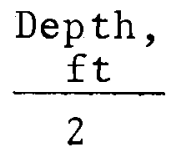

11

16

15

15

16

21

26

36.5

39

40

36.5

34.5

31

25

16

17

15

7

4

2

0
Temp,

$\frac{{ }^{\circ} \mathrm{C}}{12.8}$

11.1

11.1

11.15

11.45

11.55

11.4

13.35

13.3

11.1

10.95

10.95

11.0

11.0

11.05

11.1

11.15

11.2

11.65

12.3

12.7

$$
\begin{aligned}
A_{t} & =31,600 \mathrm{ft}^{2} \\
T_{m} & =11.45^{\circ} \mathrm{C} \\
T_{P R} & =10.6{ }^{\circ} \mathrm{C}
\end{aligned}
$$


TABLE D.II-6. River Temperature Traverses $0.5 \mathrm{Mile}$ Below $\mathrm{K}$

(River Mile: 381.3 )

Date: $\quad 8-4-64$

Time: 1405

River Flow Rate: 185,000 cfs

Distance

from Plant

Shore, yd

50

80

105

130

155

180

205

230

255

280

305

355

380

405

430

480

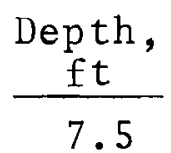

15

17

18

21

23.5

24.5

31.5

37.5

37.5

36.5

35.5

34.5

29

18

0 $\frac{\begin{array}{c}\text { Temp } \\ { }^{\circ} \mathrm{C}\end{array}}{17.1}$

17.05

17.2

17.2

17.1

17.15

17.45

18.05

18.05

17.5

17.0

16.8

16.8

16.8

$$
\begin{aligned}
\mathrm{A}_{\mathrm{t}} & =30,900 \mathrm{ft}^{2} \\
\mathrm{~T}_{\mathrm{m}} & =17.2{ }^{\circ} \mathrm{C} \\
\mathrm{T}_{\mathrm{PR}} & =16.4{ }^{\circ} \mathrm{C}
\end{aligned}
$$


TABEL D.II-6. (contd) River Temperature Traverses 0.5 Mile Below $\mathrm{K}$

(River Mile: 381.3)

\begin{tabular}{r} 
Date: \\
Time: \\
River \\
Dist \\
from \\
Shore \\
\hline 25 \\
50 \\
75 \\
100 \\
125 \\
150 \\
175 \\
200 \\
225 \\
250 \\
275 \\
300 \\
325 \\
350 \\
375 \\
400 \\
425 \\
450 \\
500 \\
525
\end{tabular}

Date: $\quad 9-1-64$

Time: 0830

River Flow Rate: 80,000 cfs (est.)

Distance

$\mathrm{KW}$

Effluent

from Plant

$\frac{\begin{array}{l}\text { from Plant } \\ \text { Shore, yd }\end{array}}{25} \frac{\begin{array}{c}\text { Depth, } \\ \text { ft }\end{array}}{5.5} \frac{\begin{array}{l}\text { Temp, } \\ { }^{\circ} \mathrm{C}\end{array}}{16.8} \frac{\begin{array}{c}\text { Effluent } \\ \text { Units }\end{array}}{2}$

50

75

100

125

150

175

200

225

250

275

300

325

350

375

400

425

450

500

525 $\begin{array}{lll}7.5 & 17.05 & 2\end{array}$

$\begin{array}{lll}8.5 & 17.2 & 8\end{array}$

$\begin{array}{lll}8.5 & 17.6 & 14\end{array}$

$11.5 \quad 17.3 \quad 9$

$14 \quad 17.1 \quad 2$

$14 \quad 17.2 \quad 3$

$22.5 \quad 17.2 \quad 5$

$\begin{array}{lll}26.5 & 17.8 & 13\end{array}$

$30.5 \quad 17.75 \quad 15$

$24.5 \quad 17.6 \quad 13$

$\begin{array}{lll}28 & 17.1 & 6\end{array}$

$27 \quad 16.9$

$25.5 \quad 16.75$ -

$20 \quad 16.6 \quad 0$

$\begin{array}{lll}10.5 & 16.5 & 0\end{array}$

$19 \quad 16.5 \quad 0$

$\begin{array}{lll}18 & 16.5 & 0\end{array}$

$\begin{array}{lll}5.5 & 16.5 & 0\end{array}$

0 - $\quad 0$

$$
\begin{aligned}
\mathrm{A}_{\mathrm{t}} & =25,600 \mathrm{ft}^{2} \\
\mathrm{~T}_{\mathrm{m}} & =17.05{ }^{\circ} \mathrm{C} \\
\mathrm{T}_{\mathrm{PR}} & =16.2{ }^{\circ} \mathrm{C}
\end{aligned}
$$


TABLE D.II-6. (contd) River Temperature Traverses 0.5 Mile Below $\mathrm{K}$

(River Mile: 381.3 )

Date: $\quad 11-28-64$

Time: 0830

River Flow Rate: 41,000 cfs

Distance

from Plant

Shore, yd

55

70

95

120

145

170

195

220

245

270

295

320

345

370

395

420

445

470

480

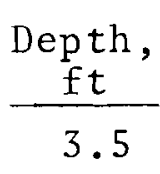

5.5

9.5

7.5

10.5

17.5

24.5

25.0

25.5

25.5

25.5

20

10.5

$$
7.0
$$

.

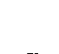

3.5

0

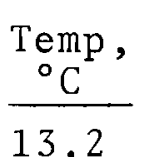

13.2

12.6

12.75

12.6

13.0

13.0

12.55

12.4

12.7

11.9

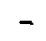

$-$

8.95

8.9

8.9

8.9

8.95

-
KW

Effluent

Units

20

30

34

36

35

35

36

31

34

37

31

16

7

0

0

0

0

0

0

$$
\begin{aligned}
\mathrm{A}_{\mathrm{t}} & =17,700 \mathrm{ft}^{2} \\
\mathrm{~T}_{\mathrm{m}} & =11.75^{\circ} \mathrm{C} \\
\mathrm{T}_{\mathrm{PR}} & =9.4{ }^{\circ} \mathrm{C}
\end{aligned}
$$

D. I I. 15 
TABLE D.II-6. (contd) River Temperature Traverses 0.5 Mile Below $\mathrm{K}$

(River Mile: 381.3)

\begin{tabular}{|c|c|c|c|c|c|c|c|}
\hline $\begin{array}{lr}\text { Date: } & 3 \\
\text { Time: } & 1 \\
\text { River } & \text { Fl }\end{array}$ & $\begin{array}{l}-18-65 \\
130 \\
\text { ow Rate: }\end{array}$ & 95,00 & $\mathrm{cfs}$ & $\begin{array}{l}\text { Date: } \\
\text { Time: } \\
\text { River F }\end{array}$ & $\begin{array}{l}-18-65 \\
140 \\
\text { Ow Rate: }\end{array}$ & 95,0 & $0 \mathrm{cfs}$ \\
\hline $\begin{array}{l}\text { Distance } \\
\text { from P1ant } \\
\text { Shore, yd }\end{array}$ & $\begin{array}{c}\text { Depth, } \\
\mathrm{ft} \\
\end{array}$ & $\begin{array}{c}\text { Temp, } \\
{ }^{\circ} \mathrm{C} \\
\end{array}$ & $\begin{array}{c}\text { B } \\
\text { Effluent } \\
\text { Units } \\
\end{array}$ & $\begin{array}{l}\text { Distance } \\
\text { from Plant } \\
\text { Shore, yd }\end{array}$ & $\begin{array}{c}\text { Depth, } \\
\mathrm{ft} \\
\end{array}$ & $\underset{{ }^{\circ} \mathrm{C} p}{\text { Temp }}$ & $\begin{array}{c}\text { B } \\
\text { Effluent } \\
\text { Units } \\
\end{array}$ \\
\hline 5 & - & - & 2 & 5 & - & 5.55 & 2 \\
\hline 25 & 3.5 & 5.2 & 2 & 25 & 5.5 & 5.2 & 2 \\
\hline 50 & 8.5 & 5.3 & 2 & 50 & 4.5 & 5.25 & 2 \\
\hline 75 & 8.5 & 5.2 & 2 & 75 & 7.5 & 5.4 & 3 \\
\hline 100 & 10.5 & 5.5 & 2 & 100 & - & 5.7 & 3 \\
\hline 125 & 9.5 & 6.1 & 3 & 125 & 7.5 & 5.7 & 7 \\
\hline 150 & 12 & 6.0 & 3 & 150 & 8.5 & 5.3 & 10 \\
\hline 175 & 15 & 5.6 & 10 & 175 & 12 & 5.7 & 18 \\
\hline 200 & 24.5 & 5.4 & 14 & 200 & 17 & 6.7 & 20 \\
\hline 225 & 30.5 & 6.4 & 16 & 225 & 27 & 7.1 & 18 \\
\hline 250 & 33.5 & 7.5 & 19 & 250 & 32.5 & 6.2 & 14 \\
\hline 275 & 32.5 & 7.8 & 16 & 275 & 31.5 & 4.75 & 9 \\
\hline 300 & 29 & 5.6 & 9 & 300 & 33.5 & 4.65 & 3 \\
\hline 325 & 27 & 5.2 & 3 & 325 & 29 & 4.6 & 1 \\
\hline 350 & 23.5 & 5.0 & 3 & 350 & 29 & 4.6 & 1 \\
\hline 375 & 15 & 4.85 & 1 & 375 & 22 & 4.6 & 0 \\
\hline 400 & 7.5 & 4.8 & 1 & 400 & 8.5 & 4.6 & 0 \\
\hline 425 & 10.5 & 4.75 & 1 & 425 & 9.5 & 4.6 & 0 \\
\hline 450 & 9.5 & 4.7 & 0 & 450 & 7.5 & 4.7 & 0 \\
\hline 475 & 5.5 & 4.75 & 0 & 475 & 5.5 & 4.7 & 0 \\
\hline 480 & 0 & - & 0 & 480 & 0 & - & 0 \\
\hline $\begin{array}{l}\mathrm{A}_{\mathrm{t}} \\
\mathrm{T}_{\mathrm{m}} \\
\mathrm{T}_{\mathrm{PR}}\end{array}$ & $\begin{array}{l}=23,40 \\
=5.85 \\
=4.2^{\circ}\end{array}$ & ${ }^{0} \mathrm{Ct} t^{2}$ & & & $\begin{array}{l}=22,50 \\
=5.25\end{array}$ & $\begin{array}{l}0 \mathrm{ft} \\
{ }^{\circ} \mathrm{C}\end{array}$ & \\
\hline
\end{tabular}


TABLE D.II-6. (contd) River Temperature Traverses 0.5 Mile Below $\mathrm{K}$ (River Mile: 381.3 )

Date: $4-8-65$

Time: 1043

River F1ow Rate: 65,000 cfs Distance (est.)

\begin{tabular}{|c|c|c|}
\hline $\begin{array}{l}\text { from Plant } \\
\text { Shore, yd }\end{array}$ & $\begin{array}{c}\text { Depth } \\
\mathrm{ft}\end{array}$ & $\begin{array}{l}\text { Temp } \\
{ }^{\circ} \mathrm{C}\end{array}$ \\
\hline 20 & 4.8 & 7.95 \\
\hline 40 & 7.2 & 7.5 \\
\hline 90 & 10.2 & 7.55 \\
\hline 140 & 15.3 & 7.05 \\
\hline 190 & 23.3 & 7.75 \\
\hline 240 & 29.5 & 7.55 \\
\hline 290 & 28.8 & 6.0 \\
\hline 340 & 22.3 & 6.0 \\
\hline 390 & 10 & 6.0 \\
\hline 440 & 7.5 & 6.0 \\
\hline 455 & 5.5 & 6.4 \\
\hline 470 & 0 & - \\
\hline
\end{tabular}

Date: $\quad 3-4-66$

Time: 1325

River Flow Rate: 115,000 cfs

\section{Distance}

from Plant Depth, Temp, $\frac{\text { Shore, yd }}{25} \frac{\mathrm{ft}}{3.5} \frac{{ }^{\circ} \mathrm{C}}{4.1}$

70

$9.5 \quad 4.0$

95

120

170

195

220

245

270

295

320

345

370

395

420

445

470

495

510
$10.5 \quad 4.3$

12

4.4

14

3.9

16

4.0

20

4.1

$24.5 \quad 5.9$

29

5.5

29

4.0

29

3.5

29

3.5

26.5

3.5

19

3.5

13

3.5

12

3.5

10.53 .6

$2 \quad 3.6$

0

$$
\begin{aligned}
\mathrm{A}_{\mathrm{t}} & =23,200 \mathrm{ft}^{2} \\
\mathrm{~T}_{\mathrm{m}} & =6.8{ }^{\circ} \mathrm{C} \\
\mathrm{T}_{\mathrm{PR}} & =5.4{ }^{\circ} \mathrm{C}
\end{aligned}
$$


TABLE D.II-6. (contd) River Temperature Traverses.0.5 Mile Below $\mathrm{K}$

(River Mile: 381.3)

\begin{tabular}{|c|c|c|c|c|c|c|c|}
\hline $\begin{array}{lr}\text { Date: } & 4 \\
\text { Time: } & 1 \\
\text { River } & \text { F1 }\end{array}$ & $\begin{array}{l}-3-66 \\
055 \\
\text { ow Rate: }\end{array}$ & 39,00 & $0 \mathrm{cfs}$ & $\begin{array}{l}\text { Date: } \\
\text { Time: } \\
\text { River }\end{array}$ & $\begin{array}{l}-3-66 \\
105 \\
\text { ow Rate: }\end{array}$ & 39,0 & $\mathrm{cfs}$ \\
\hline $\begin{array}{l}\text { Distance } \\
\text { from Plant } \\
\text { Shore, yd } \\
\end{array}$ & $\begin{array}{c}\text { Depth } \\
\mathrm{ft} \\
\end{array}$ & $\begin{array}{c}\text { Temp } \\
{ }^{\circ} \mathrm{C} \\
\end{array}$ & $\begin{array}{c}\mathrm{KE} \\
\text { Effluent } \\
\text { Units } \\
\end{array}$ & $\begin{array}{l}\text { Distance } \\
\text { from Plant } \\
\text { Shore, yd }\end{array}$ & $\begin{array}{c}\text { Depth } \\
\mathrm{ft} \\
\end{array}$ & $\begin{array}{l}\text { Temp, } \\
{ }^{\circ} \mathrm{C} \text {, } \\
\end{array}$ & $\begin{array}{c}\mathrm{KE} \\
\text { Effluent } \\
\text { Units } \\
\end{array}$ \\
\hline 55 & 4 & 11.75 & 22 & 55 & 3 & - & - \\
\hline 65 & 8 & 12.2 & 56 & 65 & 6 & - & 43 \\
\hline 90 & 8 & 12.2 & 44 & 90 & 8 & 12.05 & 37 \\
\hline 115 & 10 & 11.5 & 30 & 115 & 10 & 12.25 & 20 \\
\hline 140 & 15 & 10.9 & 15 & 140 & 14 & 11.45 & 16 \\
\hline 165 & 15 & 10.8 & 37 & 165 & 16 & 10.5 & 28 \\
\hline 190 & 16 & 11.0 & 50 & 190 & 28 & 11.0 & 45 \\
\hline 215 & 23 & 11.1 & 42 & 215 & 31 & 10.9 & 40 \\
\hline 240 & 30 & 10.3 & 32 & 240 & 33.5 & 10.8 & 35 \\
\hline 265 & 33.5 & 9.5 & 27 & 265 & 31 & 10.1 & 29 \\
\hline 290 & 36.5 & 8.5 & 17 & 290 & 31 & 9.8 & 28 \\
\hline 315 & 28 & 7.1 & 3 & 315 & 26 & 8.45 & 12 \\
\hline 340 & 16 & 6.8 & 0 & 340 & 21 & 6.95 & 0 \\
\hline 365 & 10 & 6.7 & 0 & 365 & 19 & 6.95 & 0 \\
\hline 390 & 11 & 6.8 & 0 & 390 & 15 & 7.55 & 0 \\
\hline 415 & 9 & 6.8 & 0 & 415 & 8 & 7.25 & 0 \\
\hline 440 & 5 & - & 0 & 440 & 10 & 7.15 & 0 \\
\hline 455 & 4 & 8.0 & 0 & 455 & 4 & 8.45 & 0 \\
\hline 470 & 0 & - & 0 & 470 & 0 & - & 0 \\
\hline $\begin{array}{r}\mathrm{T}_{\mathrm{m}} \\
\mathrm{T}_{\mathrm{PR}}\end{array}$ & $\begin{array}{l}=20,40 \\
=9.35 \\
=6.3^{\circ}\end{array}$ & $\mathrm{Ct}^{2}$ & & $\mathrm{~T}_{\mathrm{F}}$ & $\begin{array}{l}=20,50 \\
=9.6^{\circ} \\
=6.2^{\circ}\end{array}$ & $f t^{2}$ & \\
\hline
\end{tabular}




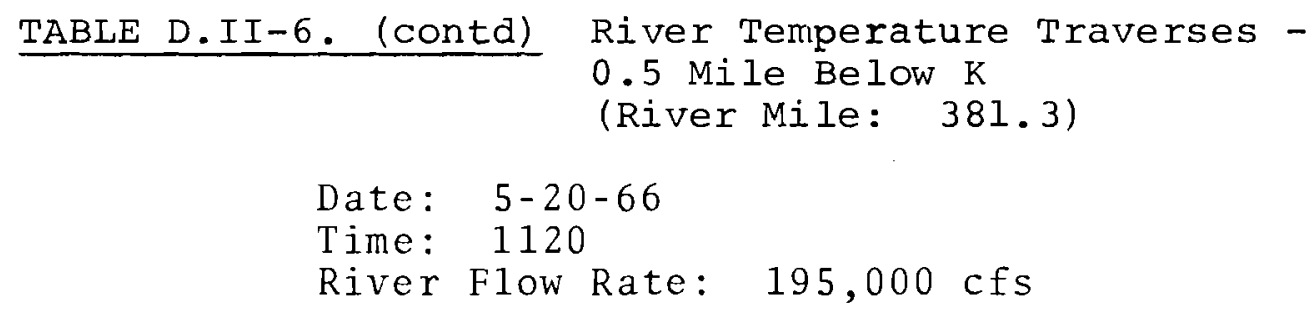

5

25

70

95

120

145

170

195

220

245

270

295

320

345

370

395

420

445

470

495

520

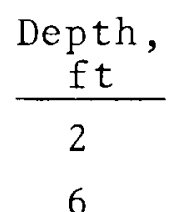

14

16

16

19

22

23

30

36.5

36.5

36.5

36.5

35.5

29

18

17

15

11

3

0
Temp,

$\frac{{ }^{\circ} \mathrm{C}}{11.9}$

11.4

11.3

11.8

11.8

11.45

11.45

12.45

12.6

12.45

11.3

11.0

10.9

10.85

10.9

10.95

10.95

11.0

11.7

12.45

$$
\begin{aligned}
\mathrm{A}_{\mathrm{t}} & =32,000 \mathrm{ft}^{2} \\
\mathrm{~T}_{\mathrm{m}} & =11.45^{\circ} \mathrm{C} \\
\mathrm{T}_{\mathrm{PR}} & =10.6{ }^{\circ} \mathrm{C}
\end{aligned}
$$

D. II. 19 
TABLE D.II-7. River Temperature Traverses - 1 Mile Below K (River Mile: 380.8 )

\begin{tabular}{|c|c|c|c|c|c|c|c|c|}
\hline \multicolumn{3}{|c|}{$\begin{array}{ll}\text { Date: } & 8-4-64 \\
\text { Time: } & 1415 \\
\text { River Flow Rate: }\end{array}$} & \multicolumn{3}{|l|}{$\begin{array}{ll}\text { Date: } & 8-6-64 \\
\text { Time: } & 0855\end{array}$} & \multicolumn{3}{|c|}{$\begin{array}{ll}\text { Date: } & 8-6-64 \\
\text { Time: } & 0905\end{array}$} \\
\hline $\begin{array}{l}\text { Distance } \\
\text { from Plant } \\
\text { Shore, yd }\end{array}$ & $\begin{array}{c}\text { Depth, } \\
f t \\
\end{array}$ & $\begin{array}{l}\text { Temp } \\
{ }^{\circ} \mathrm{C} \\
\end{array}$ & $\begin{array}{l}\text { Distance } \\
\text { from Plant } \\
\text { Shore, yd } \\
\end{array}$ & $\begin{array}{c}\text { Depth, } \\
\mathrm{ft} \\
\end{array}$ & $\begin{array}{l}\text { Temp, } \\
{ }^{\circ} \mathrm{C} \\
\end{array}$ & Distance & $\begin{array}{c}\text { Depth, } \\
\mathrm{ft} \\
\end{array}$ & $\begin{array}{l}\text { Temp, } \\
{ }^{\circ} \mathrm{C} \\
\end{array}$ \\
\hline 50 & 5.5 & 16.95 & 70 & 3.5 & 17.1 & 70 & 3.5 & 17.05 \\
\hline 75 & 6.5 & 16.95 & 95 & 6.5 & 17.0 & 95 & - & - \\
\hline 100 & 7.5 & 16.95 & 120 & 13 & 17.1 & 120 & 8.5 & 17.25 \\
\hline 125 & 14 & 17.15 & 145 & 17 & 17.2 & 145 & - & - \\
\hline 150 & 20 & 17.2 & 170 & 19 & 17.3 & 170 & 16 & 17.4 \\
\hline 175 & 28 & 17.4 & 195 & 29 & 17.5 & 195 & 21 & 17.55 \\
\hline 200 & 34.5 & 17.7 & 220 & 40 & 18.05 & 220 & 39 & 17.65 \\
\hline 225 & 36.5 & 17.85 & 245 & 40 & 18.2 & 245 & 40 & 18.1 \\
\hline 250 & 39 & 17.5 & 270 & - & - & 270 & 40 & 18.2 \\
\hline 275 & 40 & 17.05 & 295 & 37.5 & 17.65 & 295 & 37.5 & 17.85 \\
\hline 300 & 40 & 16.8 & 320 & 37.5 & 17.3 & 320 & 36.5 & 17.25 \\
\hline 325 & 39 & 16.7 & 345 & 38.5 & 17.0 & 345 & 36.5 & 17.0 \\
\hline 350 & 39 & 16.65 & 370 & 36.5 & 16.9 & 370 & 36.5 & 16.85 \\
\hline 375 & 38.5 & 16.65 & 395 & 36.5 & 16.85 & 395 & 38.5 & 16.8 \\
\hline 400 & 34.5 & 16.7 & 420 & 31.5 & 16.8 & 420 & 34.5 & 16.8 \\
\hline 425 & 28 & 16.75 & 445 & 27 & 16.8 & 445 & 16 & 16.75 \\
\hline 450 & 19 & 16.95 & 470 & 12 & 16.75 & 470 & 10.5 & 16.75 \\
\hline 475 & 10.5 & - & 530 & 0 & - & 530 & 0 & - \\
\hline 500 & 5.5 & - & & & & & & \\
\hline 540 & 0 & - & & & & & & \\
\hline $\begin{aligned} \mathrm{A}_{\mathrm{t}} & =34,3 \\
\mathrm{~T}_{\mathrm{m}} & =17.1 \\
\mathrm{~T}_{\mathrm{PR}} & =16.4\end{aligned}$ & $\begin{array}{l}00 \mathrm{ft}^{2} \\
5{ }^{\circ} \mathrm{C} \\
{ }^{\circ} \mathrm{C}\end{array}$ & & $\begin{aligned} \mathrm{A}_{\mathrm{t}} & =34,2 \\
\mathrm{~T}_{\mathrm{m}} & =17.3 \\
\mathrm{~T}_{\mathrm{PR}} & =16.4\end{aligned}$ & $\begin{array}{l}00 \mathrm{ft}^{2} \\
5^{\circ} \mathrm{C} \\
{ }^{\circ} \mathrm{C}\end{array}$ & & $\begin{array}{l}\mathrm{A}_{\mathrm{t}}=31,9 \\
\mathrm{~T}_{\mathrm{m}}=17.3\end{array}$ & $\begin{array}{l}00 \mathrm{ft}^{2} \\
5{ }^{\circ} \mathrm{C}\end{array}$ & \\
\hline
\end{tabular}




\section{TABLE D.II-7. (contd) River Temperature Traverses - 1 Mile Below $\mathrm{K}$ (River Mile: 380.8)}

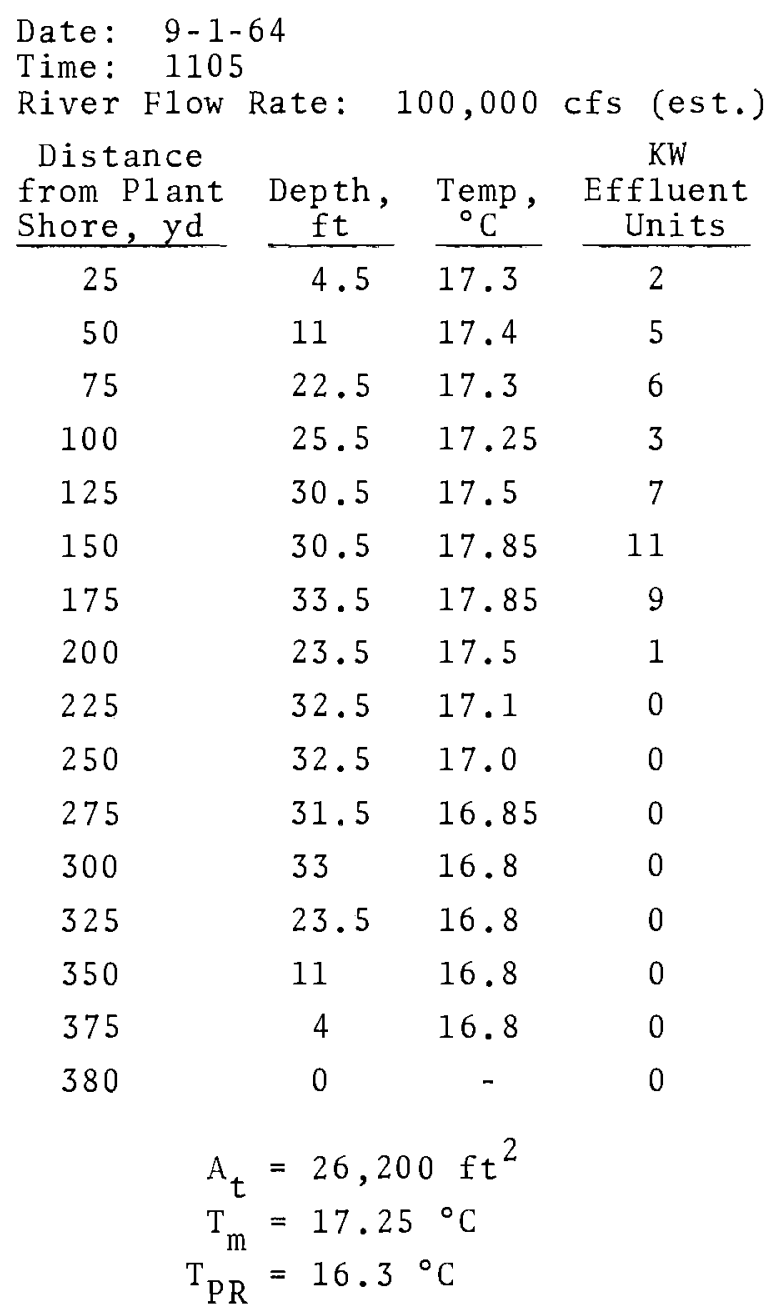

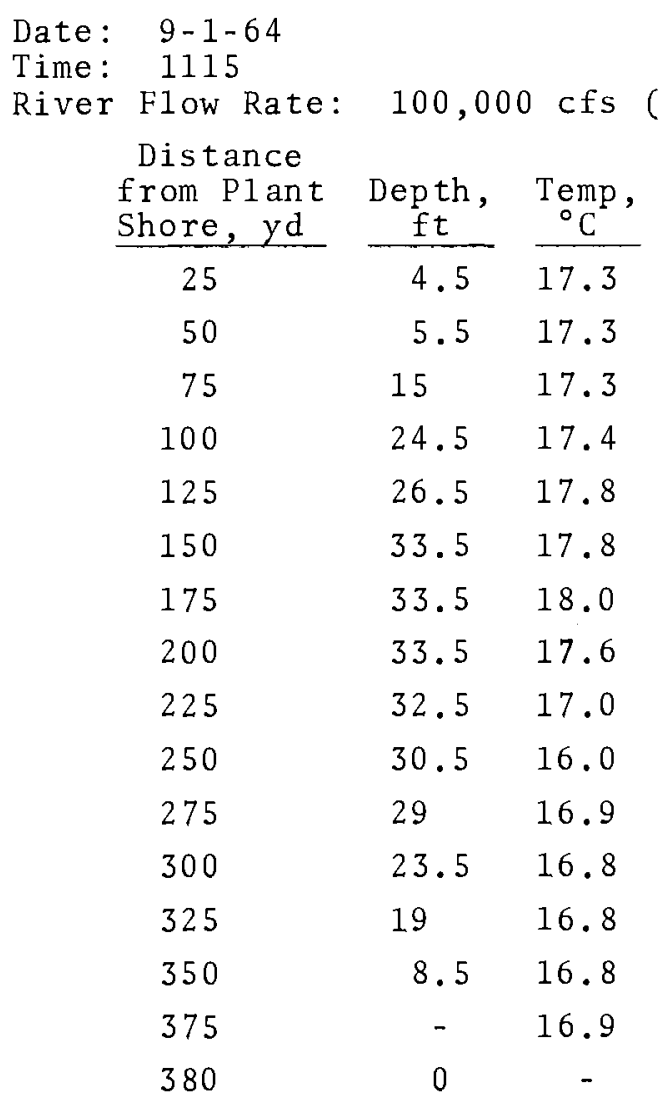

$$
\begin{aligned}
& \mathrm{A}_{\mathrm{t}}=23,800 \mathrm{ft}^{2} \\
& \mathrm{~T}_{\mathrm{m}}=17.3^{\circ} \mathrm{C}
\end{aligned}
$$




\section{TABIE D.II-7. (contd) River Temperature Traverses - 1 Mile Below $\mathrm{K}$ \\ (River Mile: 380.8 )}

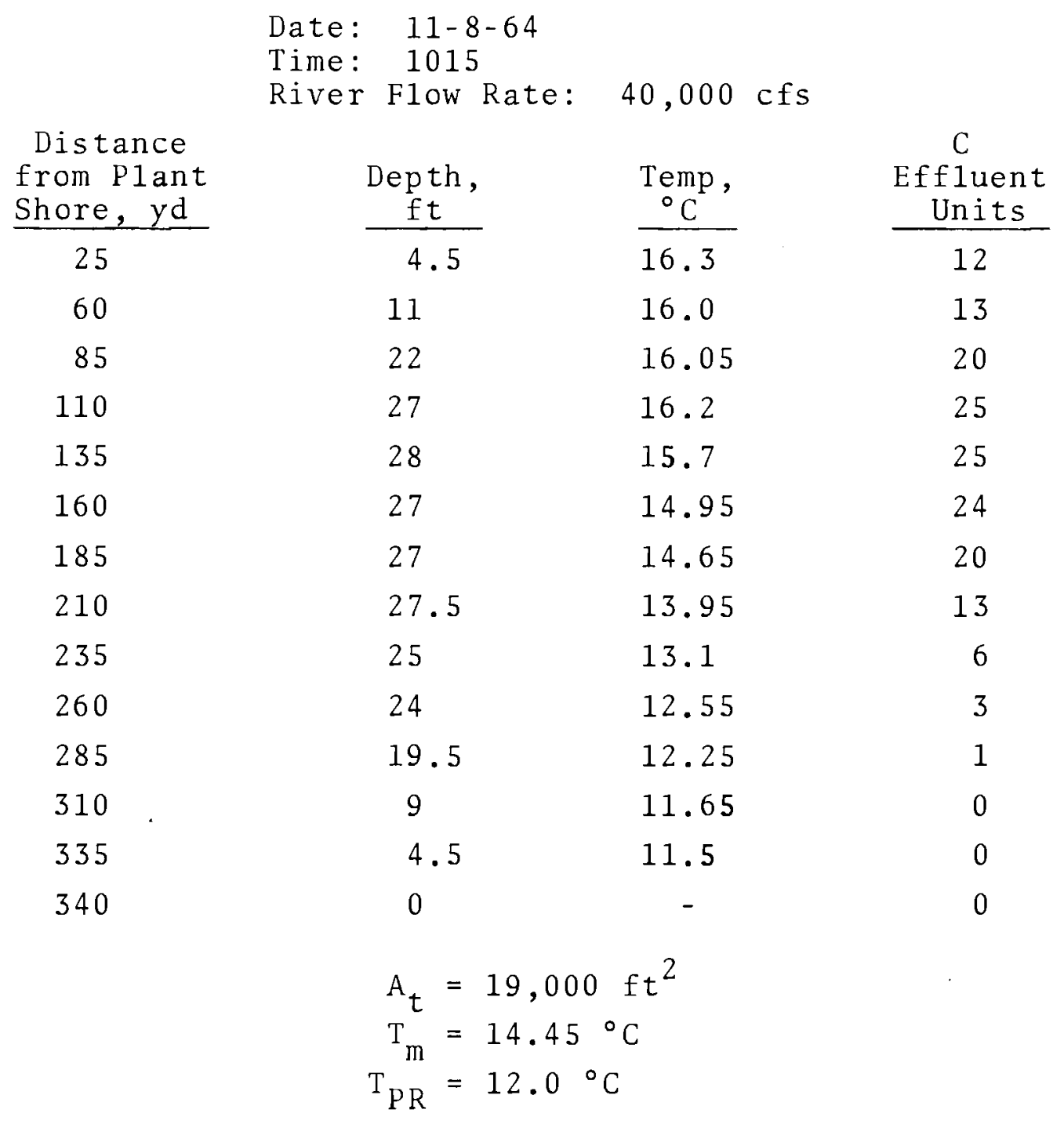

D. I I. 22 
TABLE D.II-7. (contd) River Temperature Traverses 1 Mile Below $\mathrm{K}$

(River Mile: 380.8 )

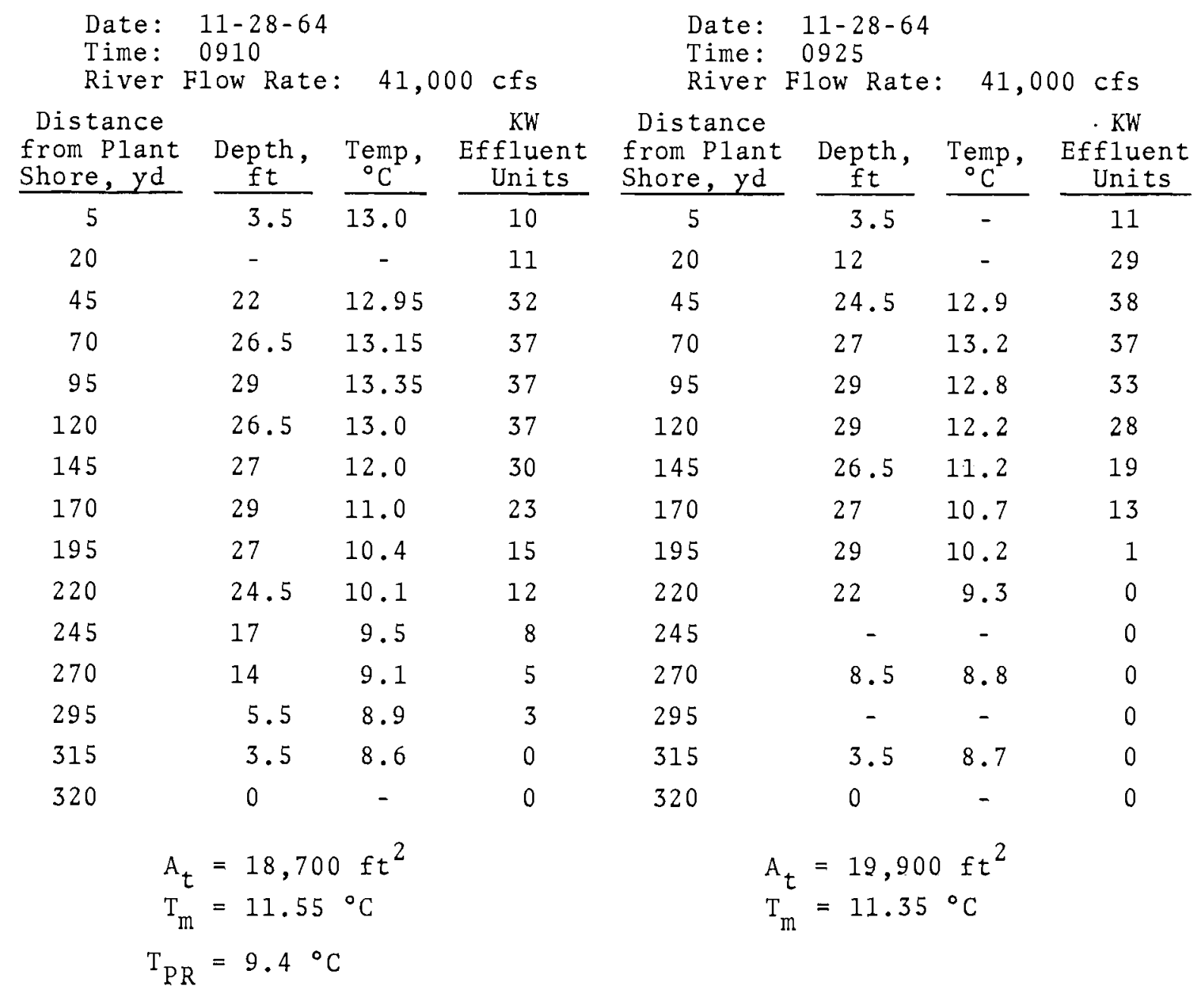


TABLE D.II-7. (contd) River Temperature Traverses 1 Mile Below $\mathrm{K}$

(River Mile: 380.8 )

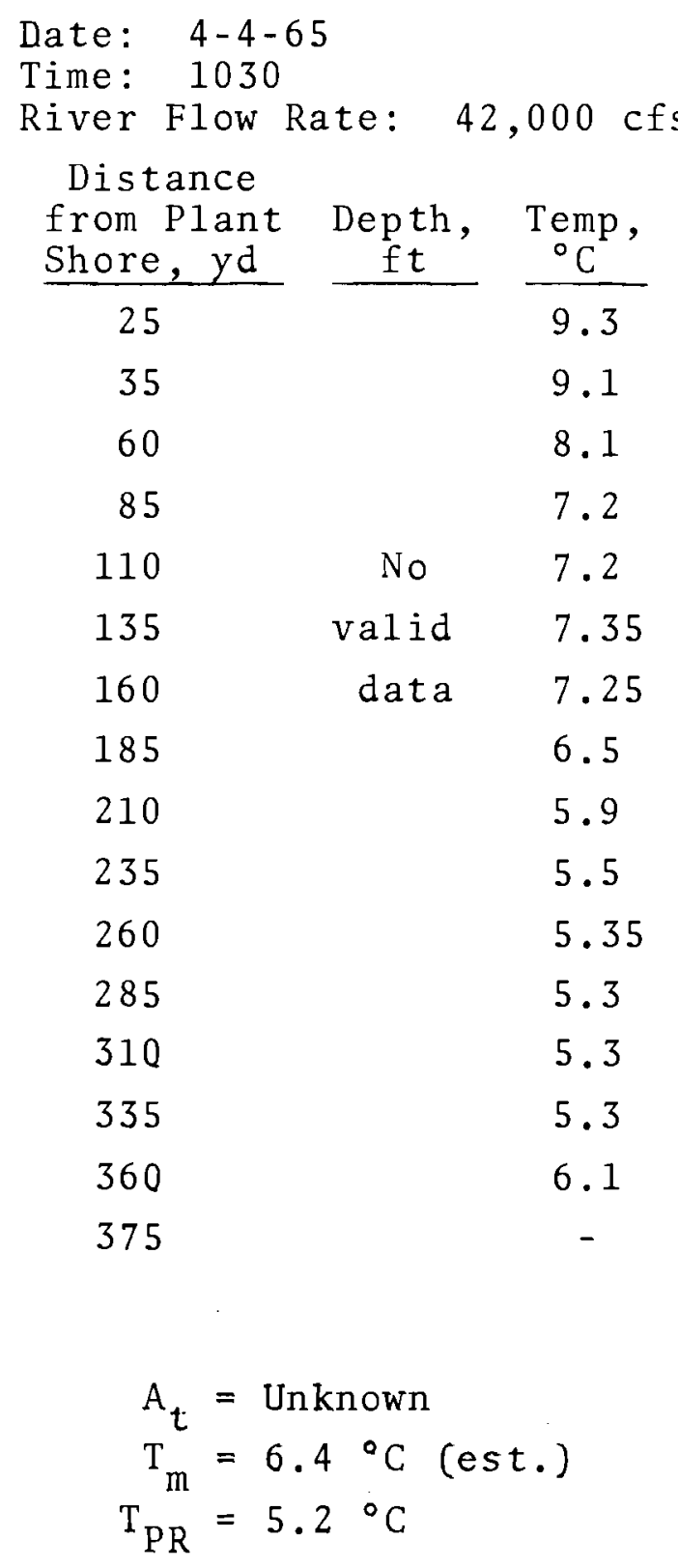

Date: $\quad 3-4-66$

Time: 1440

River Flow Rate: 115,000 cfs

Distance

from Plant Depth, Temp, $\frac{\text { Shore, yd }}{65} \frac{\mathrm{ft}}{3.5} \frac{{ }^{\circ} \mathrm{C}}{4.4}$

$90 \quad 6.5 \quad 4.2$

$115 \quad 14 \quad 4.3$

$\begin{array}{lll}140 & 15 & 4.4\end{array}$

$\begin{array}{lll}165 & 29 & 4.1\end{array}$

$\begin{array}{lll}190 & 32.5 & 4.0\end{array}$

$\begin{array}{lll}215 & 31.5 & 5.1\end{array}$

$240 \quad 32.5 \quad 5.2$

$265 \quad 31.5 \quad 4.1$

$\begin{array}{lll}290 & 30.5 & 3.6\end{array}$

$\begin{array}{lll}315 & 29 & 3.6\end{array}$

$\begin{array}{lll}340 & 28 & 3.5\end{array}$

$\begin{array}{lll}365 & 19 & 3.6\end{array}$

$\begin{array}{lll}390 & 14 & 3.6\end{array}$

$\begin{array}{lll}415 & 5.5 & 3.6\end{array}$

$\begin{array}{lll}425 & 3.5 & 3.7\end{array}$

$455 \quad 0$

$$
\begin{aligned}
\mathrm{A}_{\mathrm{t}} & =24,000 \mathrm{ft}^{2} \\
\mathrm{~T}_{\mathrm{m}} & =4.1^{\circ} \mathrm{C} \\
\mathrm{T}_{\mathrm{PR}} & =3.4^{\circ} \mathrm{C}
\end{aligned}
$$

D. I I . 24 
TABLE D.II-7. (contd) River Temperature Traverses I Mile Below $\mathrm{K}$

(River Mile: 380.8 )

Date: $\quad 5-20-66$

Time: 1145

River Flow Rate: 195,000 cfs

Distance

from Plant

Shore, yd

5

25

50

75

100

125

150

175

200

225

250

275

300

325

350

375

400

425

450

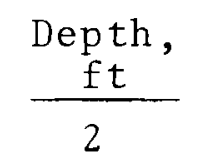

Temp,

(5)

5

6

6

8

$\frac{{ }^{\circ} \mathrm{C}}{11.6}$

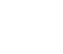

11.7

11.45

5

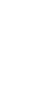

\section{BNW -1345}

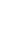


TABLE D.II-8. River Temperature Traverses - Above N (River Mile: 380.0 )

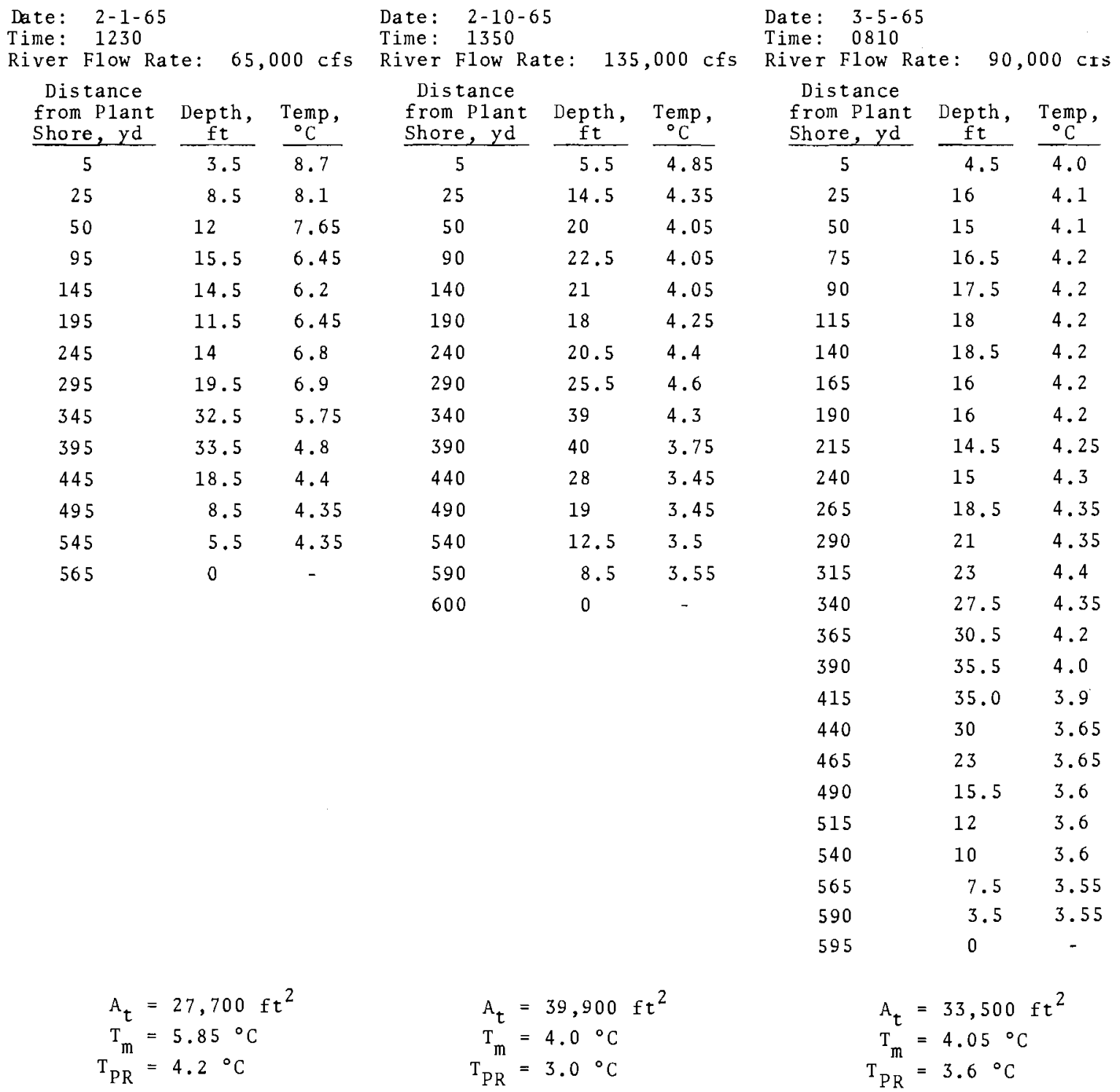

D. II. 26 
1BLE D.II-9. River Temperature Traverses - 100 Yards Below N (River Mile: 379.6)

Date: 6-5-64

Time: 0914

River Flow Rate: 325,000 cfs

Distance

from Plant

Shore, yd

5

100

Depth,

125

$\frac{\mathrm{ft}}{5.5}$

Temp,

150

41

$\frac{{ }^{\circ} \mathrm{C}}{15.3}$

175

39.5

12.6

200

35.5

12.65

225

33.5

12.75

250

$-$

12.9

12.95

275

32.5

13.0

37.5

12.95

300

41

12.9

325

45.5

12.75

350

46.5

12.65

47.5

12.6

375

46.5

12.5

400

41

12.5

425

35.5

12.5

450

26.5

12.5

475

24.5

12.5

500

22

12.55

525

19

12.55

550

17

12.6

575

12

600

7.5

12.7

620

0

$$
\begin{aligned}
\mathrm{A}_{\mathrm{t}} & =56,000 \mathrm{ft}^{2} \\
\mathrm{~T}_{\mathrm{m}} & =12.85{ }^{\circ} \mathrm{C} \\
\mathrm{T}_{\mathrm{PR}} & =12.0{ }^{\circ} \mathrm{C}
\end{aligned}
$$


TABLE D.II-10. River Temperature Traverses - 400 Yards Below N (River Mile: 379.4)

\begin{tabular}{|c|c|c|c|c|c|c|c|}
\hline $\begin{array}{ll}\text { Date: } & 8-27 \\
\text { Time: } & 1142 \\
\text { River Flow }\end{array}$ & $\begin{array}{l}64 \\
\text { ate: }\end{array}$ & $0,000 \mathrm{c}$ & fs (est.) & $\begin{array}{lr}\text { Date: } & 8-2 \\
\text { Time: } & 114 \\
\text { River Flow }\end{array}$ & $\begin{array}{l}7^{-64} \\
\text { Rate: }\end{array}$ & 10,000 & cfs (est.) \\
\hline $\begin{array}{l}\text { Distance } \\
\text { from Plant } \\
\text { Shore, yd } \\
\end{array}$ & $\begin{array}{c}\text { Depth, } \\
\mathrm{ft} \\
\end{array}$ & $\begin{array}{l}\text { Temp, } \\
{ }^{\circ} \mathrm{C} \\
\end{array}$ & $\begin{array}{c}N \\
\text { Effluent } \\
\text { Units } \\
\end{array}$ & $\begin{array}{l}\text { Distance } \\
\text { from Plant } \\
\text { Shore, yd }\end{array}$ & $\begin{array}{c}\text { Depth, } \\
\mathrm{ft}\end{array}$ & $\begin{array}{c}\text { Temp, } \\
{ }^{\circ} \mathrm{C} \\
\end{array}$ & $\begin{array}{c}\mathrm{N} \\
\text { Effluent } \\
\text { Units } \\
\end{array}$ \\
\hline 5 & 5.5 & 19.3 & 3 & 5 & 3.5 & 18.9 & 2 \\
\hline 25 & 12 & 19.0 & 3 & 25 & 8.5 & 18.7 & 2 \\
\hline 50 & 18 & 18.9 & 3 & 50 & 17 & 18.5 & 1 \\
\hline 85 & 25.5 & 18.45 & 0 & 85 & 26.5 & 18.35 & 1 \\
\hline 110 & 28 & 18.4 & 0 & 110 & 28 & 18.5 & 2 \\
\hline 135 & 25.5 & 18.4 & 2 & 135 & 26.5 & 18.6 & 4 \\
\hline 160 & 24.5 & 19.1 & 49 & 160 & 25.5 & 19.0 & 29 \\
\hline 185 & 22.5 & 19.1 & 60 & 185 & 23.5 & 19.35 & 65 \\
\hline 210 & 21 & 18.8 & 22 & 210 & 22 & 19.3 & 73 \\
\hline 235 & 23.5 & 18.4 & 3 & 235 & 21 & 18.9 & 50 \\
\hline 260 & - & 18.25 & 1 & 260 & 22.5 & 18.4 & 1 \\
\hline 285 & 30.5 & 18.15 & 1 & 285 & 30.0 & 18.1 & 0 \\
\hline 310 & 31.5 & 18.0 & 1 & 310 & 31.5 & 18.1 & 0 \\
\hline 335 & 31.5 & 18.0 & 0 & 335 & 30.5 & 18.05 & 0 \\
\hline 360 & 27 & 18.0 & 0 & 360 & 30.5 & 18.0 & 0 \\
\hline 385 & 17 & 17.95 & 0 & 385 & 29.0 & 17.9 & 0 \\
\hline 410 & 13 & 17.9 & 0 & 410 & 21 & 17.9 & 0 \\
\hline 435 & 10.5 & 17.9 & 0 & 435 & 14 & 17.9 & 0 \\
\hline 460 & 18 & 17.9 & 0 & 460 & 9.5 & 17.9 & 0 \\
\hline 485 & 7.5 & 17.9 & 0 & 485 & 7.5 & 17.95 & 0 \\
\hline 505 & 7.5 & 18.0 & 0 & 505 & 7.5 & 18.0 & 0 \\
\hline 520 & 0 & - & 0 & 520 & 0 & - & 0 \\
\hline$T_{T}$ & $\begin{aligned} \mathrm{t} & =32,0 \\
\mathrm{~m} & =18.3 \\
\mathrm{R} & =17.3 \\
\mathrm{~N} & =25^{\circ}\end{aligned}$ & $\begin{array}{l}00 \mathrm{ft}^{2} \\
5^{\circ} \mathrm{C} \\
{ }^{\circ} \mathrm{C}\end{array}$ & & & $\begin{aligned} t_{t} & =32, \\
{ }_{m} & =18 . \\
{ }_{R} & =17 .\end{aligned}$ & $\begin{array}{l}00 \mathrm{ft}^{2} \\
{ }^{\circ} \mathrm{C} \\
{ }^{\circ} \mathrm{C}\end{array}$ & \\
\hline
\end{tabular}




\section{TABLE D.II-10. (contd) River Temperature Traverses - 400 Yards Below N \\ (River Mile: 379.4 )}

\begin{tabular}{|c|c|c|c|c|c|c|c|}
\hline $\begin{array}{lc}\text { Date: } & 9-1-6 \\
\text { Time: } & 1150 \\
\text { River F1ow }\end{array}$ & ate: & 5,000 & fs (est.) & $\begin{array}{lr}\text { Date: } & 9- \\
\text { Time: } & 12 \\
\text { River } & \text { Flor }\end{array}$ & $\begin{array}{l}64 \\
\text { Rate: }\end{array}$ & 05,000 & cfs (est.) \\
\hline $\begin{array}{l}\text { Distance } \\
\text { from Plant } \\
\text { Shore, yd } \\
\end{array}$ & $\begin{array}{c}\text { Depth, } \\
\mathrm{ft} \\
\end{array}$ & $\begin{array}{l}\text { Temp, } \\
{ }^{\circ} \mathrm{C} \\
\end{array}$ & $\begin{array}{c}N \\
\text { Effluent } \\
\text { Units } \\
\end{array}$ & $\begin{array}{r}\text { Dist ance } \\
\text { from Plan } \\
\text { Shore, yd } \\
\end{array}$ & $\begin{array}{c}\text { Depth, } \\
\mathrm{ft}\end{array}$ & $\begin{array}{l}\text { Temp, } \\
{ }^{\circ} \mathrm{C} \\
\end{array}$ & $\begin{array}{c}N \\
\text { Effluent } \\
\text { Units }\end{array}$ \\
\hline 5 & 4.5 & 18.0 & 8 & 5 & 4.5 & 18.1 & 9 \\
\hline 25 & 5.5 & 18.0 & 9 & 25 & 7.5 & 18.0 & 8 \\
\hline 50 & 7.5 & 17.9 & 8 & 50 & 20 & 17.7 & 7 \\
\hline 90 & 24.5 & 17.6 & 6 & 90 & 27 & 17.5 & 6 \\
\hline 115 & 28 & 17.8 & 4 & 115 & 22.5 & 17.5 & 2 \\
\hline 140 & 26.5 & 17.8 & 1 & 140 & 26 & 17.6 & 1 \\
\hline 190 & 23.5 & 17.8 & - & 190 & 22.5 & 17.8 & - \\
\hline 215 & 22 & 17.55 & 1 & 215 & 22 & 17.75 & 2 \\
\hline 240 & 24.5 & 17.35 & 1 & 240 & 22.5 & 17.7 & 2 \\
\hline 265 & 23.5 & 17.2 & 0 & 265 & 29 & 17.5 & 2 \\
\hline 290 & 32.5 & 17.2 & 0 & 290 & 31.5 & 17.3 & 2 \\
\hline 315 & 32.5 & 17.2 & 0 & 315 & 32.5 & 17.2 & 2 \\
\hline 340 & 32.5 & 17.15 & 0 & 340 & 32.5 & 17.15 & 2 \\
\hline 365 & 32.5 & 17.05 & 0 & 365 & 22.5 & 17.1 & 2 \\
\hline 390 & 20 & 17.0 & 0 & 390 & 20 & 17.0 & 2 \\
\hline 415 & 15 & 17.0 & 1 & 415 & 12 & 17.0 & 1 \\
\hline 440 & 12 & 16.95 & 1 & 440 & 10.5 & 17.0 & 1 \\
\hline 465 & 9.5 & 16.9 & 0 & 465 & 8.5 & 17.0 & 1 \\
\hline 490 & 7.5 & 16.95 & 0 & 490 & 6.5 & 17.0 & 1 \\
\hline 515 & 7.5 & 17.1 & 0 & 515 & 4.5 & 17.3 & 1 \\
\hline 530 & 3.5 & 17.2 & 0 & 530 & - & 17.3 & 0 \\
\hline 535 & 0 & - & 0 & 535 & 0 & - & 0 \\
\hline $\begin{array}{l}\mathrm{A}_{\mathrm{t}} \\
\mathrm{T}_{\mathrm{m}} \\
\mathrm{T}_{\mathrm{PR}}\end{array}$ & $\begin{array}{l}=32,30 \\
=17.4 \\
=16.3\end{array}$ & $\mathrm{ft}^{2}$ & & & $\begin{array}{l}=31,2 \\
=17.4\end{array}$ & ${ }^{0} \mathrm{ft} \mathrm{t}^{2}$ & \\
\hline
\end{tabular}


TABLE D.II-10. (contd) River Temperature Traverses 400 Yards Below $\mathrm{N}$

(River Mile: 379.4)

Date: 9-4-64

Time: 1038

River Flow Rate: 65,000 cfs (est.)

Distance

from Plant Depth, Temp,

Shore, yd

25

50

75

100

125

150

175

200

225

250

275

300

325

350

375

400

425

450

465

490

$$
\begin{aligned}
\mathrm{A}_{\mathrm{t}} & =24,100 \mathrm{ft}^{2} \\
\mathrm{~T}_{\mathrm{m}} & =17.65{ }^{\circ} \mathrm{C} \\
\mathrm{T}_{\mathrm{PR}} & =16.0{ }^{\circ} \mathrm{C} \\
\mathrm{T}_{\mathrm{N}} & =30{ }^{\circ} \mathrm{C} \text { (est.) }
\end{aligned}
$$

Date: $9-4-64$

Time: 1043

River Flow Rate: 65,000 cfs (est.)

Distance from Plant Depth, Temp, $\frac{\text { Shore, yd }}{5}-\frac{\mathrm{ft}}{2} \frac{{ }^{\circ} \mathrm{C}}{18.65}$

$\begin{array}{lll}25 & 4.5 & 18.4\end{array}$

$\begin{array}{lll}50 & 13 & 18.2\end{array}$

75

100

125

150

175

200

225

250

275

300

325

350

375

400

425

450

465

490
$22.5 \quad 17.85$

$24.5 \quad 17.9$

$23.5 \quad 18.4$

$21 \quad 18.85$

$19 \quad 19.0$

$18 \quad 18.6$

$21.5 \quad 17.9$

$28 \quad 17.3$

$28 \quad 16.9$

$28 \quad 16.75$

$29 \quad 16.65$

$21 \quad 16.6$

$13 \quad 16.55$

$9.5 \quad 16.55$

6.516 .55

$4.5 \quad 16.6$

$3.5 \quad 16.75$

0

$$
\begin{aligned}
& \mathrm{A}_{\mathrm{t}}=25,200 \mathrm{ft} \\
& \mathrm{T}_{\mathrm{m}}=17.55^{\circ} \mathrm{C}
\end{aligned}
$$


TABLE D.II-10. (contd) River Temperature Traverses 400 Yards Below $\mathrm{N}$

(River Mile: 379.4)

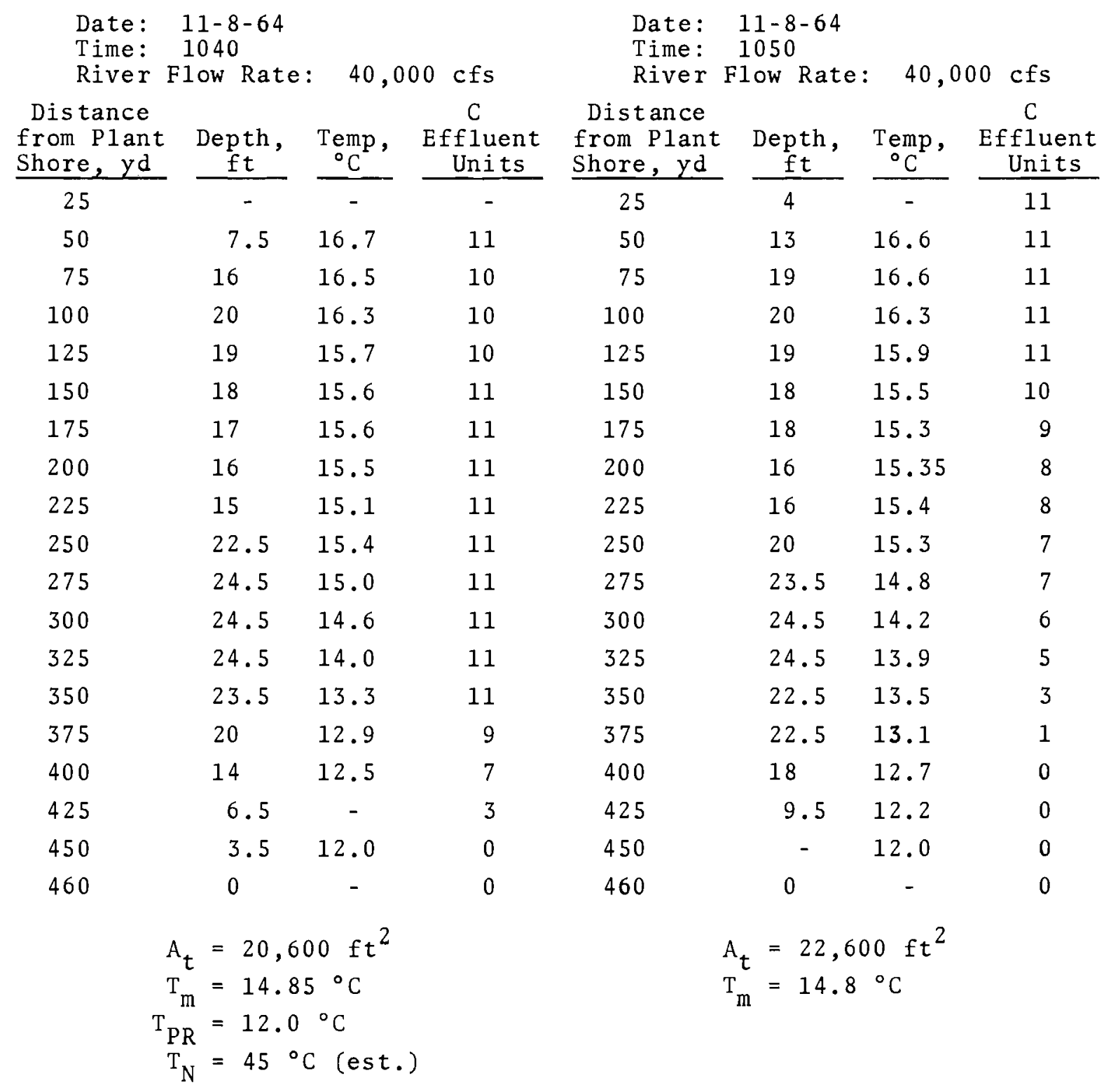

D. II . 31 


\section{TABLE D.II-10 (contd) River Temperature Traverses - 400 Yards Below $\mathrm{N}$ (River Mile: 379.4 )}

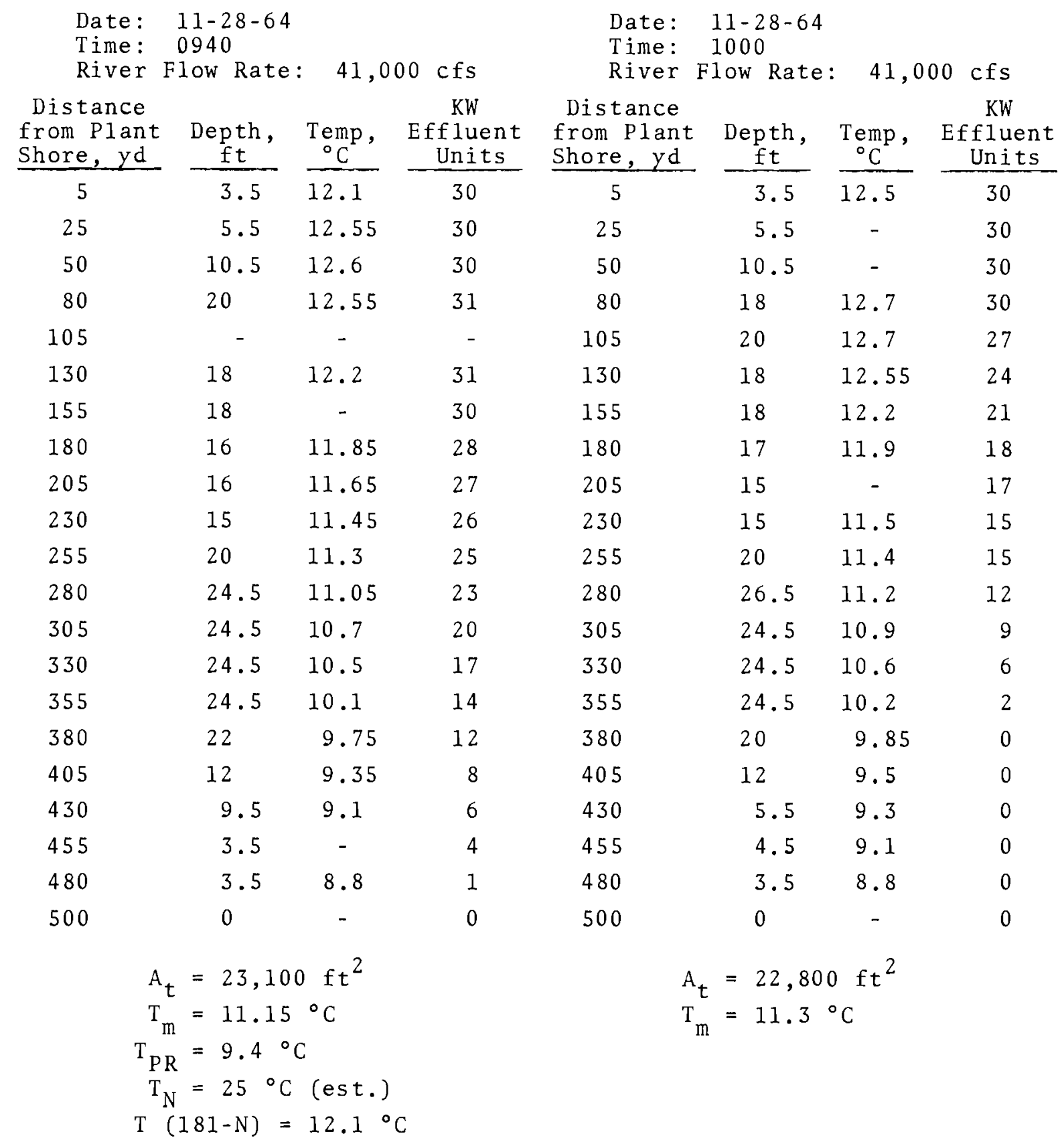




\section{TABLE D.II-10. (contd) River Temperature Traverses - 400 Yards Below $\mathrm{N}$ \\ (River Mile: 379.4)}

\begin{tabular}{|c|c|c|c|c|c|c|c|}
\hline $\begin{array}{l}\text { Date: } \\
\text { Time: } \\
\text { River }\end{array}$ & $\begin{array}{l}3-18-65 \\
1200 \\
10 w \text { Rate }\end{array}$ & 95, & $00 \mathrm{cfs}$ & $\begin{array}{l}\text { Date: } \\
\text { Time: } \\
\text { River }\end{array}$ & $\begin{array}{l}3-18-65 \\
1210 \\
\text { Flow Rate: }\end{array}$ & 95, & $00 \mathrm{cfs}$ \\
\hline $\begin{array}{l}\text { Distance } \\
\text { from Plant } \\
\text { Shore, yd }\end{array}$ & $\begin{array}{c}\text { Depth, } \\
\mathrm{ft} \\
\end{array}$ & $\begin{array}{c}\text { Temp, } \\
{ }^{\circ} \mathrm{C} \\
\end{array}$ & $\begin{array}{l}\mathrm{KW} \\
\text { Effluent } \\
\text { Units } \\
\end{array}$ & $\begin{array}{l}\text { Distance } \\
\text { from Plant } \\
\text { Shore, yd }\end{array}$ & $\begin{array}{c}\text { Depth, } \\
\mathrm{ft}\end{array}$ & $\begin{array}{l}\text { Temp, } \\
{ }^{\circ} \mathrm{C} \\
\end{array}$ & $\begin{array}{c}\text { KW } \\
\text { Effluent } \\
\text { Units } \\
\end{array}$ \\
\hline 5 & 2 & 6.4 & 10 & 5 & 3.5 & 6.3 & 10 \\
\hline 25 & 12 & 6.2 & 9 & 25 & 10.5 & 6.0 & 10 \\
\hline 50 & 12 & 6.2 & 9 & 50 & 18 & 5.9 & 10 \\
\hline 75 & 25.5 & 6.3 & 14 & 75 & 22.5 & 6.0 & 11 \\
\hline 100 & 25.5 & 6.25 & 14 & 100 & 25.5 & 6.15 & 14 \\
\hline 125 & 25.5 & 6.3 & 14 & 125 & 26.5 & 6.15 & 14 \\
\hline 150 & 24.5 & 6.25 & 14 & 150 & 24.5 & 6.1 & 14 \\
\hline 175 & 24.5 & 6.2 & 14 & 175 & 23.5 & 6.1 & 14 \\
\hline 200 & 20 & 6.15 & 14 & 200 & 22 & 5.95 & 14 \\
\hline 225 & 26.5 & 6.1 & 14 & 225 & 20 & 5.85 & 13 \\
\hline 250 & 31.5 & 6.05 & 14 & 250 & 34.5 & 5.7 & 13 \\
\hline 275 & 23.5 & 6.1 & 13 & 275 & 33.5 & 5.2 & 11 \\
\hline 300 & 32.5 & 5.8 & 10 & 300 & 31.5 & 5.1 & 8 \\
\hline 325 & 31.5 & 5.4 & 6 & 325 & 31.5 & 5.0 & 5 \\
\hline 350 & 29 & 5.1 & 5 & 350 & 29 & 4.9 & 3 \\
\hline 375 & 23.5 & 5.05 & 5 & 375 & 20 & 4.9 & 2 \\
\hline 400 & 15 & 4.9 & 3 & 400 & 24.5 & 4.9 & 1 \\
\hline 425 & 10.5 & 4.8 & 1 & 425 & 15 & 4.8 & 1 \\
\hline 450 & 7.5 & 4.8 & 1 & 450 & 13 & 4.8 & 1 \\
\hline 475 & 6.5 & 4.8 & 1 & 475 & 14 & 4.9 & 1 \\
\hline 500 & 5.5 & 4.8 & 0 & 500 & 5.5 & 4.8 & 1 \\
\hline 515 & 3.5 & 5.1 & 0 & 515 & 3.5 & 5.15 & 0 \\
\hline 520 & 0 & - & 0 & 520 & 0 & - & 0 \\
\hline $\begin{array}{l}\mathrm{A}_{\mathrm{t}} \\
\mathrm{T}_{\mathrm{m}} \\
\mathrm{T}_{\mathrm{PR}}\end{array}$ & $\begin{array}{l}=31,500 \\
=5.8{ }^{\circ} \\
=4.2{ }^{\circ} \mathrm{C}\end{array}$ & $\mathrm{ft}^{2}$ & & \multicolumn{4}{|c|}{$\begin{array}{l}\mathrm{A}_{\mathrm{t}}=33,400 \mathrm{ft}^{2} \\
\mathrm{~T}_{\mathrm{m}}=5.55^{\circ} \mathrm{C}\end{array}$} \\
\hline
\end{tabular}




\section{TABLE D.II-10. (contd) River Temperature Traverses - 400 Yards Below $\mathrm{N}$ \\ (River Mile: 379.4 )}

Date: $\quad 3-31-66$

Time: 1055

River Flow Rate: 85,000 cfs

Distance

from Plant

Shore, yd

5

25

50

85

110

135

160

185

210

235

260

285

310

333

360

385

410

435

460

485

490

495

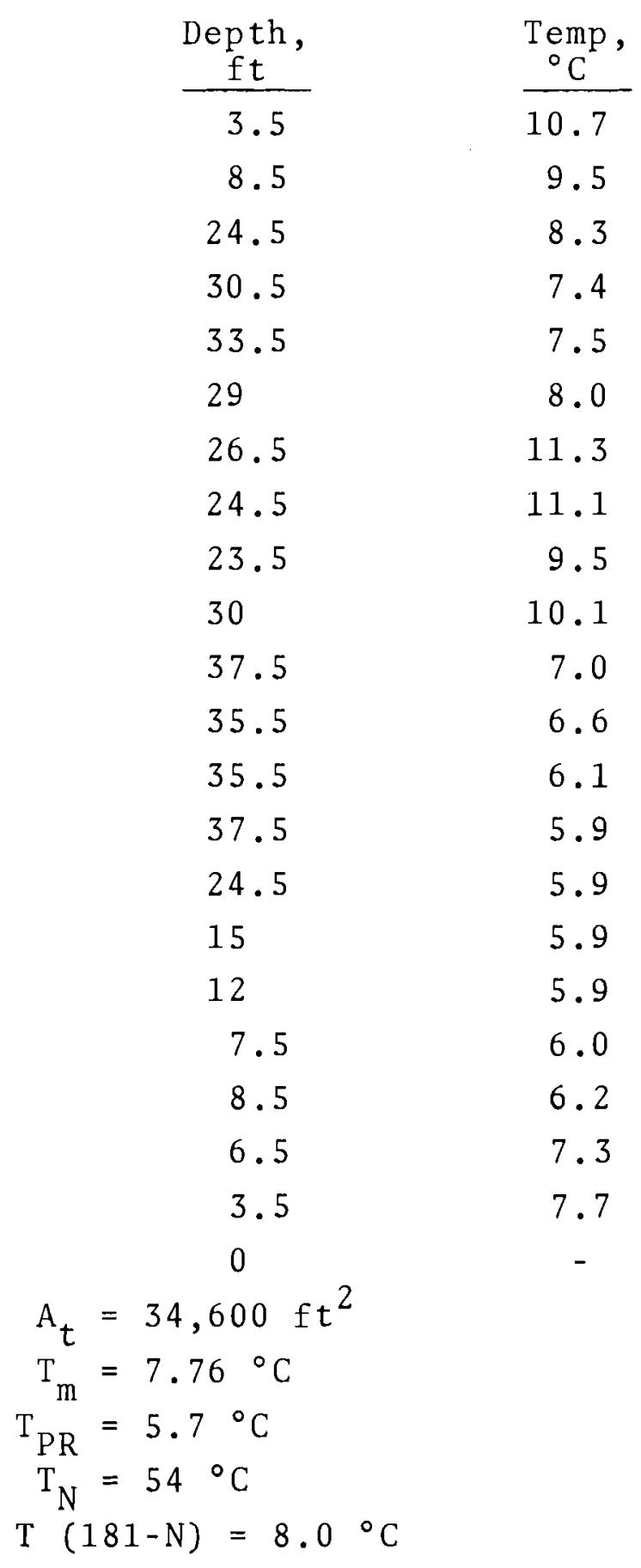

D. I I. 34 
TABLE D.II-11. River Temperature Traverses - 0.5 Mile Below $\mathrm{N}$ (River Mile: 379.2)

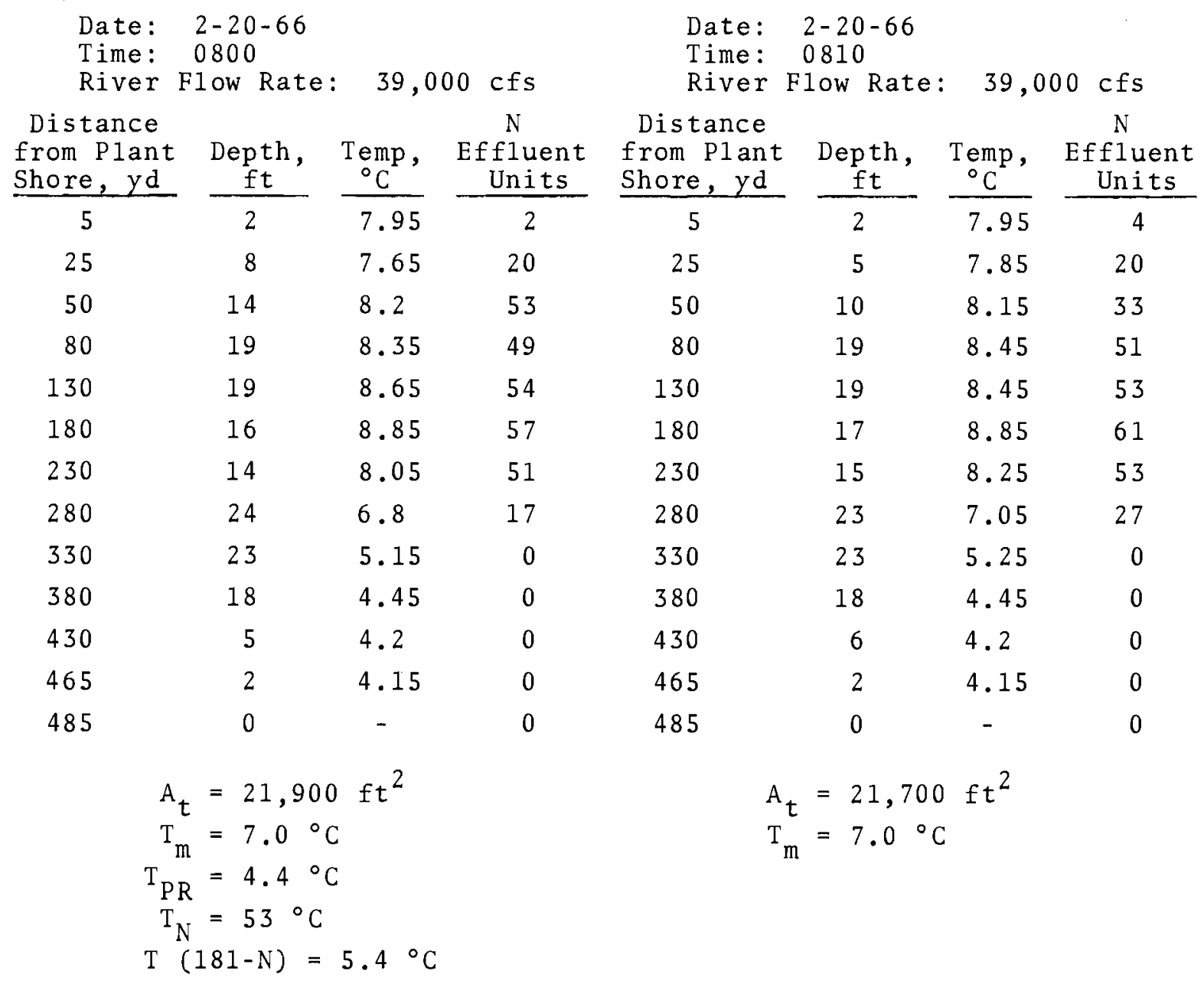


TABLE D.II-11. (contd) River Temperature Traverses 0.5 Mile Below $\mathrm{N}$

(River Mile: 379.2)

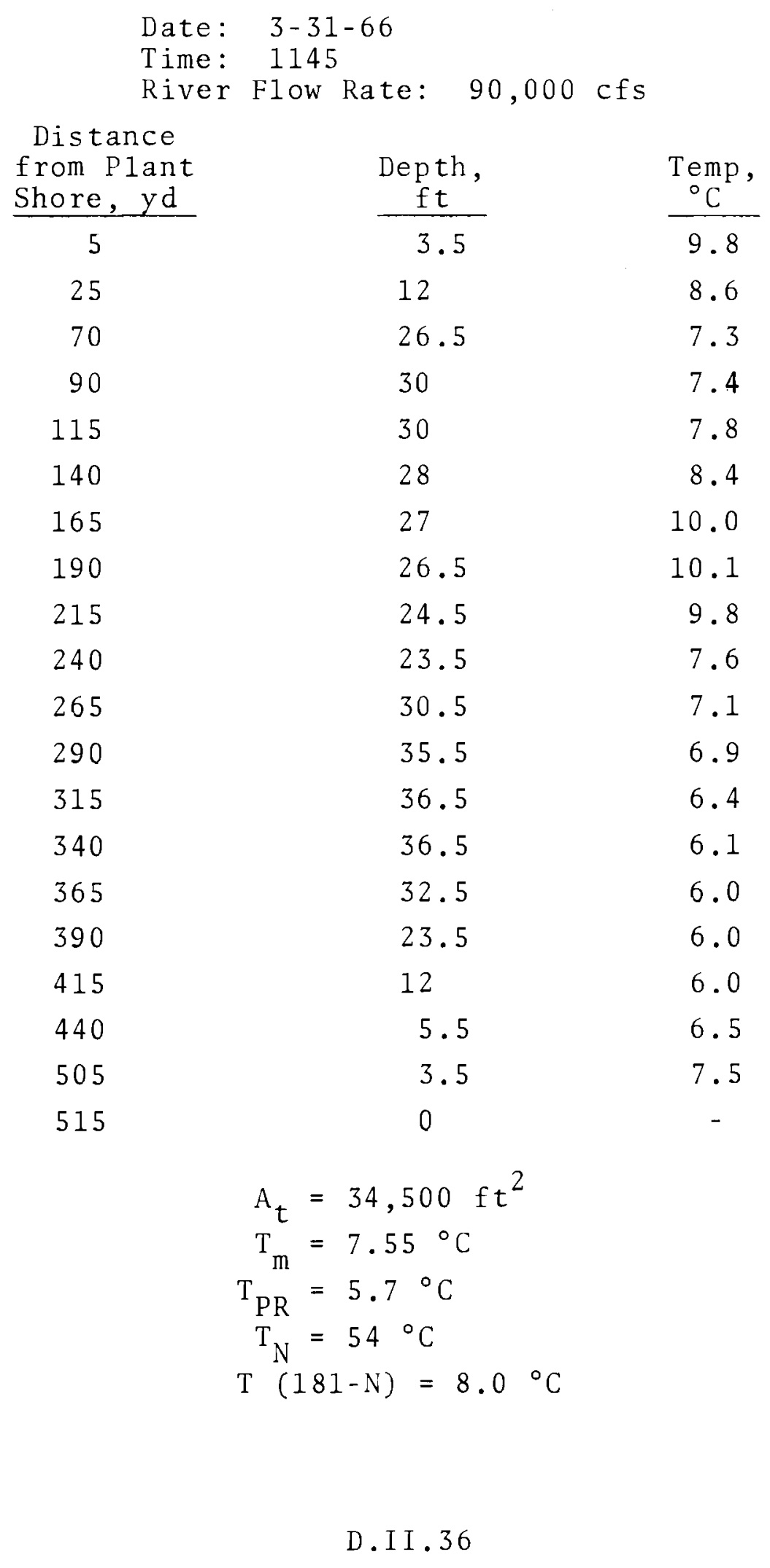


TABLE D.II-12. River Temperature Traverses - 1 Mile Below N (River Mile: 378.7)

\begin{tabular}{|c|c|c|c|c|c|c|c|c|}
\hline \multirow{2}{*}{$\begin{array}{r}\text { Date: } \\
\text { Time: } \\
\text { River } \\
\text { Distance } \\
\text { from Plant } \\
\text { Shore, yd } \\
\end{array}$} & $\begin{array}{l}2-20-66 \\
0825 \\
\text { Flow Rate: }\end{array}$ & \multicolumn{2}{|c|}{$39,000 \mathrm{cfs}$} & \multicolumn{2}{|c|}{$\begin{array}{l}\text { Date: } \\
\text { Time: } \\
\text { River }\end{array}$} & $\begin{array}{l}2-20-66 \\
0837\end{array}$ & & $000 \mathrm{cfs}$ \\
\hline & $\begin{array}{c}\text { Depth, } \\
\mathrm{ft} \\
\end{array}$ & $\begin{array}{c}\text { Temp, } \\
{ }^{\circ} \mathrm{C} \\
\end{array}$ & $\begin{array}{c}\mathrm{N} \\
\text { Effluent } \\
\text { Units } \\
\end{array}$ & $\begin{array}{l}\text { Dista } \\
\text { from } \\
\text { Shore }\end{array}$ & $\begin{array}{l}\text { ance } \\
\text { Pl ant } \\
\text { yd } \\
\end{array}$ & $\begin{array}{c}\text { Depth, } \\
\text { ft } \\
\end{array}$ & $\begin{array}{c}\text { Temp, } \\
{ }^{\circ} \mathrm{C} \\
\end{array}$ & $\begin{array}{c}\mathrm{N} \\
\text { Effluent } \\
\text { Units } \\
\end{array}$ \\
\hline 5 & 2 & 6.95 & 6 & 5 & & 2 & 6.95 & 14 \\
\hline 25 & 5 & 7.6 & 19 & 25 & & 4 & 7.65 & 22 \\
\hline 50 & 8 & 7.85 & 37 & 50 & & 6 & 7.95 & 30 \\
\hline 70 & 11 & 8.0 & 33 & 70 & & 11 & 8.15 & 35 \\
\hline 120 & 13 & 8.0 & 37 & 120 & & 15 & 8.15 & 42 \\
\hline 170 & 17 & 8.05 & 34 & 170 & & 16 & 8.05 & 37 \\
\hline 220 & 15 & 8.45 & 48 & 220 & & 16 & 8.35 & 51 \\
\hline 270 & 16 & 7.35 & 21 & 270 & & 15 & 7.05 & 29 \\
\hline 320 & 19 & 6.8 & 4 & 320 & & 19 & 5.9 & 10 \\
\hline 370 & 20 & 5.4 & 0 & 370 & & 20 & 4.95 & 0 \\
\hline 420 & 8 & 4.85 & 0 & 420 & & 8 & 4.5 & 0 \\
\hline 470 & 6 & 4.45 & 0 & 470 & & 7 & 4.35 & 0 \\
\hline 500 & 2 & 4.25 & 0 & 500 & & - & 4.25 & 0 \\
\hline 515 & 0 & - & 0 & 515 & & 0 & - & 0 \\
\hline $\begin{array}{r}\mathrm{A}_{\mathrm{t}} \\
\mathrm{T}_{\mathrm{m}} \\
\mathrm{T}_{\mathrm{PR}} \\
\mathrm{T}_{\mathrm{N}}\end{array}$ & $\begin{array}{l}=19,100 \\
=7.0{ }^{\circ} \mathrm{C} \\
=4.4{ }^{\circ} \mathrm{C} \\
=53{ }^{\circ} \mathrm{C}\end{array}$ & $\mathrm{ft}^{2}$ & & & $A_{t}$ & $\begin{array}{l}=19,20 \\
=6.9\end{array}$ & $0 \mathrm{ft}^{2}$ & \\
\hline
\end{tabular}


TABLE D.II-12. (contd) River Temperature Traverses 1 Mile Below $\mathrm{N}$

(River Mile: 378.7)

Date: $3-31-66$

Time: 1205

River F1ow Rate: 90,000 cfs

Distance

from Plant

$\frac{\text { Shore, yd }}{5}$

25

40

60

85

110

135

160

185

210

235

260

285

310

335

360

385

410

435

460

485

510

525

540

$\begin{array}{cc}\begin{array}{c}\text { Depth, } \\ \text { ft }\end{array} & \begin{array}{c}\text { Temp, } \\ { }^{\circ} \mathrm{C}\end{array} \\ 9.5 & 10.0 \\ 9.5 & 9.1 \\ 12 & 8.2 \\ 17 & 7.6 \\ 21 & 7.4 \\ 28 & 7.4 \\ 27 & 7.8 \\ 26.5 & 8.1 \\ 26.5 & 9.3 \\ 27 & 9.5 \\ 25.5 & 8.2 \\ 26.5 & 7.1 \\ 31.5 & 6.8 \\ 32.5 & 6.5 \\ 33.5 & 6.2 \\ 33.5 & 6.1 \\ 30 & 6.0 \\ 22.5 & 6.0 \\ 18 & - \\ 12 & 6.1 \\ 7.5 & 6.4 \\ 4.5 & 6.9 \\ 3.5 & 7.6 \\ 0 & -\end{array}$

$$
\begin{array}{ll}
\mathrm{A}_{\mathrm{t}}=35,100 \mathrm{ft} & \mathrm{T}_{\mathrm{PR}}=5.7{ }^{\circ} \mathrm{C} \\
\mathrm{T}_{\mathrm{m}}=7.24{ }^{\circ} \mathrm{C} & \mathrm{T}_{\mathrm{N}}=54^{\circ} \mathrm{C}
\end{array}
$$




$$
\begin{aligned}
& \text { J。 } s z=N_{I} \\
& \text { J० } \nabla \cdot 9 \mathrm{I}=\mathrm{dd}_{\mathrm{I}} \\
& \text { J。 } S \varepsilon^{\cdot} \angle I={ }^{\text {UI }} \mathrm{I} \\
& z^{7 f} 00 \varepsilon^{\prime} 6 z={ }^{7} \mathrm{~V}
\end{aligned}
$$

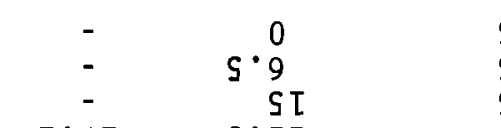

$\tau \cdot L I \quad S \cdot z z \quad 00$

$S 6 \cdot 9 T \quad S \cdot \nabla 2 \quad S \angle D$

6.9 $S \cdot \varepsilon Z \quad$ OSt

$6^{\circ} 9 \mathrm{I} \quad 8 \mathrm{I} \quad \mathrm{SZD}$

$6.9 \mathrm{I} \quad 6 \mathrm{I} \quad 00 \mathrm{t}$

S6.9 I $\mathrm{IZ} \quad \mathrm{SLE}$

$0^{\circ} \angle I \quad$ IZ $\quad 0 S \varepsilon$

$I \cdot L I \quad I Z \quad S Z \varepsilon$

$S Z \cdot \angle \mathrm{I} \quad 6 \mathrm{~T} \quad 00 \varepsilon$

$S \sigma^{\circ} \angle I \quad \angle I \quad s \angle Z$

$59^{\circ} \angle I \quad 8 I \quad 0 S Z$

$58^{\circ} \angle \tau \quad$ TZ $\quad 5 Z Z$

$0.8 \mathrm{~T} \quad 22 \quad 002$

$\mathrm{I} \cdot 8 \mathrm{~L} \quad \mathrm{~S} \cdot 22 \quad \mathrm{SLT}$

$S 8^{\circ} \angle I \quad Z Z \quad$ OSI

$9^{\circ} \mathrm{LI} \quad 6 \mathrm{I} \quad \mathrm{SZT}$

$S t^{\circ} \angle I \quad L I \quad 00 \mathrm{~T}$

$S E^{\circ} \angle I \quad L I \quad S L$

$\varepsilon \cdot \angle I \quad 6 I \quad 0 S$

$\begin{array}{lll}S E \cdot L T & 9 T & S Z \\ S S \cdot L T & S \cdot 8 & S\end{array}$

כ० $\frac{7 f}{p K \text { 'әлоYS }}$

' duә

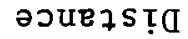

Sรว $000^{\circ} \mathrm{s}$ : :

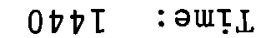
๑-๑-8: :

$$
\begin{aligned}
& \text { ว० } 0 \cdot z \tau=\frac{y d_{L}}{} \\
& \text { ว。 } \nu^{\cdot \varepsilon \tau}={ }_{L}^{\omega_{L}} \\
& z^{7}=006^{\circ} \tau t={ }^{7} V
\end{aligned}
$$

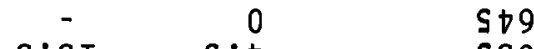

$\varepsilon \cdot \varepsilon I \quad S \cdot t \quad s \varepsilon 9$

$\varepsilon \cdot \varepsilon \mathrm{L} \quad \mathrm{S}^{\circ} \mathrm{OL} \quad$ OL

$\varepsilon \cdot \varepsilon I \quad L I \quad$ S8S

$\varepsilon \cdot \varepsilon \mathrm{L} \quad 02 \quad 095$

$\varepsilon \cdot \varepsilon \tau$ S.9Z SES

$\varepsilon \cdot \varepsilon \mathrm{L} \quad S^{\circ}[\varepsilon \quad$ OTS

$\varepsilon \cdot \varepsilon \mathrm{L} \quad 0 \varepsilon \quad 58 \mathrm{D}$

$\varepsilon \cdot \varepsilon I$ S. $52 \quad 090$

$S \varepsilon \cdot \varepsilon L \quad S \cdot S Z \quad S E D$

t. $\varepsilon \mathrm{S}$ S. SZ OTD

St $\varepsilon \mathrm{I}$ S. 92 S

$9^{\circ} \varepsilon I \quad S \cdot 92 \quad 09 \varepsilon$

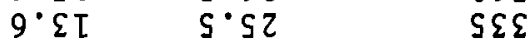

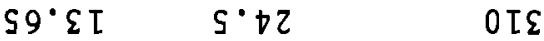

$L \cdot \varepsilon \tau$ S. $\varepsilon z \quad$ S8z

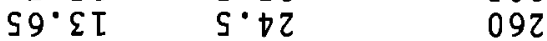

$9^{\circ} \varepsilon \tau \quad 8 Z \quad$ SEZ

$\mathrm{S}^{\circ} \varepsilon \mathrm{I} \quad 82 \quad 0 \mathrm{TZ}$

$t \cdot \varepsilon I \quad 62 \quad$ S8T

$\varepsilon \cdot \varepsilon \tau \quad L Z \quad 09 T$

$S T \cdot \varepsilon \tau$ S. $t \tau$

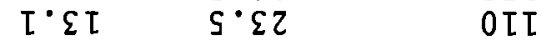

So. $2 \mathrm{~L}$ S. 22

$0 . \varepsilon \tau \quad S \cdot \varepsilon 2 \quad 09$

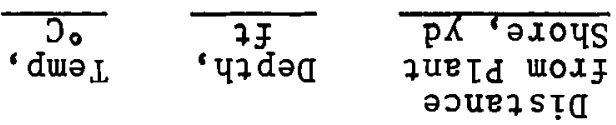

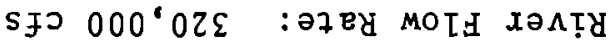

$0 \triangleright 60$ : әயप L

$\downarrow 9-s-9:$ :

\section{$(0.8 L \varepsilon \quad:$

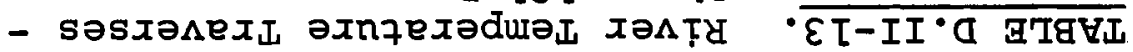


BNWL - 1345

TABLE D.II-13. (contd) River Temperature Traverses Above 181-D

(River Mile: 378.0)

Date: $\quad 8-6-64$

Time: 0940

River Flow Rate: 155,000 cfs

Distance

\begin{tabular}{|c|c|c|}
\hline $\begin{array}{l}\text { from Plant } \\
\text { Shore, yd }\end{array}$ & $\begin{array}{c}\text { Depth, } \\
\mathrm{ft}\end{array}$ & $\begin{array}{c}\text { Temp } \\
{ }^{\mathrm{C}} \mathrm{C} \\
\end{array}$ \\
\hline 25 & 13.5 & 17.45 \\
\hline 60 & 16.5 & 17.5 \\
\hline 85 & 16 & 17.55 \\
\hline 110 & 15.5 & 17.65 \\
\hline 135 & 16.5 & 17.8 \\
\hline 160 & 19 & 17.85 \\
\hline 185 & 19.5 & 17.95 \\
\hline 210 & 20.5 & 18.0 \\
\hline 235 & 20.5 & 17.95 \\
\hline 260 & 18.5 & 17.9 \\
\hline 285 & 17.5 & 17.7 \\
\hline 310 & 18 & 17.55 \\
\hline 335 & 17 & 17.5 \\
\hline 360 & 19 & 17.3 \\
\hline 385 & 19 & 17.2 \\
\hline 410 & 17 & 17.1 \\
\hline 435 & 20.5 & 16.95 \\
\hline 460 & 23.5 & 16.95 \\
\hline 485 & 23.5 & . \\
\hline 510 & 23 & 16.85 \\
\hline 535 & 16 & 16.8 \\
\hline 560 & 11 & 16.8 \\
\hline 580 & 0 & - \\
\hline
\end{tabular}

$A_{t}=29,000 \mathrm{ft}^{2}$

$\mathrm{T}_{\mathrm{m}}=17.45^{\circ} \mathrm{C}$

$\mathrm{T}_{\mathrm{PR}}=16.4{ }^{\circ} \mathrm{C}$
Date: $\quad 8-6-64$

Time: $\quad 1050$

River F1ow Rate: 155,000 cfs

Distance

from Plant Depth, Temp, Shore, yd $\mathrm{ft}{ }^{\circ} \mathrm{C}$,

$\begin{array}{lll}25 & 10.5 & 17.8\end{array}$

$\begin{array}{lll}60 & 16 & 17.65\end{array}$

$\begin{array}{lll}85 & 15 & 17.7\end{array}$

$\begin{array}{lll}110 & 15 & 17.8\end{array}$

$\begin{array}{lll}135 & 17 & 17.95\end{array}$

$160 \quad 20 \quad 18.1$

$\begin{array}{lll}185 & 20 & 18.15\end{array}$

$\begin{array}{lll}210 & 20 & 18.1\end{array}$

$235 \quad 20 \quad 18.0$

$\begin{array}{lll}260 & 17 & 17.9\end{array}$

$\begin{array}{lll}285 & 15 & 17.85\end{array}$

$\begin{array}{lll}310 & 17 & 17.75\end{array}$

$\begin{array}{lll}335 & 19 & 17.65\end{array}$

$\begin{array}{lll}360 & 19 & 17.55\end{array}$

$\begin{array}{lll}385 & 19 & 17.4\end{array}$

$\begin{array}{lll}410 & 17 & 17.35\end{array}$

$\begin{array}{lll}435 & 17 & 17.25\end{array}$

$460 \quad 17 \quad 17.2$

$\begin{array}{lll}485 & 22 & 17.15\end{array}$

$\begin{array}{lll}510 & 23.5 & 17.15\end{array}$

$\begin{array}{lll}535 & 22.5 & 17.1\end{array}$

$\begin{array}{rrr}560 & 12 & - \\ 580 & 0 & -\end{array}$

$A_{t}=28,000 \mathrm{ft}^{2}$

$\mathrm{T}_{\mathrm{m}}=17.6^{\circ} \mathrm{C}$

$\mathrm{T}_{\mathrm{PR}}=16.4{ }^{\circ} \mathrm{C}$

D. I I. 40 


\section{TABLE D.II-13. (contd) River Temperature Traverses - Above 181-D (River Mile: 378.0 )}

\begin{tabular}{|c|c|c|c|c|c|c|c|}
\hline $\begin{array}{lc}\text { Date: } & 8-27- \\
\text { Time: } & 1200 \\
\text { River Flow R }\end{array}$ & $\begin{array}{l}64 \\
\text { ate : }\end{array}$ & 0,000 & Es (est.) & $\begin{array}{lr}\text { Date: } & 8-27 \\
\text { Time: } & 1215 \\
\text { River } & \text { Flow }\end{array}$ & $\begin{array}{l}64 \\
\text { Rate : }\end{array}$ & 0,000 & Es (est.) \\
\hline $\begin{array}{l}\text { Distance } \\
\text { from Plant } \\
\text { Shore, yd }\end{array}$ & $\begin{array}{c}\text { Depth, } \\
\mathrm{ft} \\
\end{array}$ & $\begin{array}{c}\text { Temp, } \\
{ }^{\circ} \mathrm{C} \\
\end{array}$ & $\begin{array}{c}\mathrm{N} \\
\text { Effluent } \\
\text { Units } \\
\end{array}$ & $\begin{array}{l}\text { Distance } \\
\text { from Plant } \\
\text { Shore, yd } \\
\end{array}$ & $\begin{array}{c}\text { Depth, } \\
\mathrm{ft} \\
\end{array}$ & Temp, & $\begin{array}{c}\mathrm{N} \\
\text { Effluent } \\
\text { Units } \\
\end{array}$ \\
\hline 5 & 3.5 & 19.1 & 2 & 5 & 3.5 & 18.8 & 2 \\
\hline 25 & 10.5 & 18.9 & 2 & 25 & 12 & 18.7 & 2 \\
\hline 40 & - & - & 2 & 40 & 12 & 18.5 & 1 \\
\hline 65 & 13 & - & 1 & 65 & 12 & 18.45 & 1 \\
\hline 90 & 13 & 18.4 & 1 & 40 & 10.5 & 18.4 & 1 \\
\hline 115 & 13 & 18.4 & 2 & 110 & 13 & 18.4 & 2 \\
\hline 140 & 16 & 18.5 & 9 & 140 & 15 & 18.5 & 8 \\
\hline 165 & 16 & 18.6 & 20 & 165 & 16 & 18.6 & 19 \\
\hline 190 & 16 & 18.7 & 28 & 190 & 16 & 18.7 & 29 \\
\hline 215 & 16 & 18.7 & 31 & 215 & 16 & 18.7 & 28 \\
\hline 240 & 15 & 18.6 & 18 & 240 & 13 & 18.35 & 13 \\
\hline 265 & 13 & 18.4 & 7 & 265 & 13 & 18.25 & 6 \\
\hline 290 & 13 & 18.25 & 2 & 290 & 13 & 18.15 & 2 \\
\hline 315 & 15 & 18.2 & 1 & 315 & 15 & 18.1 & 1 \\
\hline 340 & 15 & 18.15 & 1 & 340 & 15 & 18.1 & 0 \\
\hline 365 & 15 & 18.1 & 1 & 365 & 15 & 18.0 & 0 \\
\hline 390 & 15 & 18.1 & 1 & 390 & 13 & 18.0 & 0 \\
\hline 415 & 15 & 18.05 & 0 & 415 & 13 & 18.0 & 0 \\
\hline 440 & 13 & 18.0 & 0 & 440 & 15 & 18.05 & 0 \\
\hline 465 & 15 & 18.0 & 0 & 465 & 20 & 18.0 & 0 \\
\hline 490 & 20 & 18.05 & 0 & 490 & 20 & 18.1 & 0 \\
\hline 515 & 20 & 18.1 & 0 & 515 & 15 & 18.15 & 0 \\
\hline 540 & 17 & 18.1 & 0 & 540 & 6.5 & 18.2 & 0 \\
\hline 565 & 5.5 & 18.3 & 0 & 565 & 5.5 & - & 0 \\
\hline 575 & 0 & - & 0 & 575 & 0 & - & 0 \\
\hline & $\begin{array}{l}\mathrm{A}_{\mathrm{t}}=24 \\
\mathrm{~T}_{\mathrm{m}}=18 \\
\mathrm{~T}_{\mathrm{PR}}=1 \\
\mathrm{~T}_{\mathrm{N}}=25\end{array}$ & $\begin{array}{l}.400 \mathrm{ft} \\
.35{ }^{\circ} \mathrm{C} \\
7.3{ }^{\circ} \mathrm{C} \\
{ }^{\circ} \mathrm{C} \quad \mathrm{es}\end{array}$ & & & $\begin{array}{l}\mathrm{t}=22, \\
\mathrm{~m}=18.2 \\
\mathrm{PR}=17 \\
\mathrm{~N}=25\end{array}$ & $\begin{array}{l}00 \mathrm{ft}^{2} \\
5{ }^{\circ} \mathrm{C} \\
3{ }^{\circ} \mathrm{C} \\
\mathrm{C}\end{array}$ & \\
\hline
\end{tabular}


TABLE D.II-13. (contd) River Temperature Traverses Above 181-D

(River Mile: 378.0 )

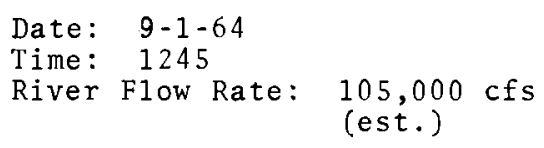

$$
\begin{aligned}
& \mathrm{A}_{\mathrm{t}}=26,700 \mathrm{ft}^{2} \\
& \mathrm{~T}_{\mathrm{m}}=17.7{ }^{\circ} \mathrm{C} \\
& \mathrm{T}_{\mathrm{PR}}=16.3^{\circ} \mathrm{C}
\end{aligned}
$$

\begin{tabular}{|c|c|c|c|}
\hline $\begin{array}{l}\text { Distance } \\
\text { from Plant } \\
\text { Shore, yd } \\
\end{array}$ & $\begin{array}{l}\text { Depth, } \\
\mathrm{ft}\end{array}$ & $\begin{array}{l}\text { Temp, } \\
{ }^{\circ} \mathrm{C} \\
\end{array}$ & $\begin{array}{c}C \\
\text { Effluent } \\
\text { Units } \\
\end{array}$ \\
\hline 5 & 5.5 & 16.6 & 6 \\
\hline 30 & 7.5 & 16.1 & 6 \\
\hline 55 & 7.5 & 16.0 & 6 \\
\hline 80 & 7.5 & 15.85 & 6 \\
\hline 105 & 8.5 & 15.65 & 6 \\
\hline 130 & 9.5 & 15.55 & 6 \\
\hline 155 & 10.5 & 15.35 & 7 \\
\hline 180 & 12 & 15.25 & 7 \\
\hline 205 & 12 & 14.95 & 7 \\
\hline 230 & 8.5 & 14.7 & 7 \\
\hline 255 & 9.5 & 14.5 & 7 \\
\hline 280 & 9.5 & 14.35 & 7 \\
\hline 305 & 10.5 & 14.3 & 7 \\
\hline 330 & 10.5 & 14.1 & 7 \\
\hline 355 & 11.5 & 13.95 & 7 \\
\hline 380 & 11.5 & 13.8 & 7 \\
\hline 405 & 10.5 & 13.6 & 7 \\
\hline 430 & 9.5 & 13.45 & 6 \\
\hline 455 & 7.5 & 13.35 & 6 \\
\hline 480 & 8.5 & 13.2 & 5 \\
\hline 505 & 7.5 & 13.05 & 5 \\
\hline 530 & 13 & 12.55 & 4 \\
\hline 555 & 9.5 & - & 3 \\
\hline 580 & 3.5 & 11.65 & 0 \\
\hline 590 & 0 & - & 0 \\
\hline (Long) & & & \\
\hline
\end{tabular}

$$
\begin{aligned}
& \mathrm{A}_{\mathrm{t}}=16,200 \mathrm{ft}^{2} \\
& \mathrm{~T}_{\mathrm{m}}=14.3{ }^{\circ} \mathrm{C} \\
& \mathrm{T}_{\mathrm{PR}}=12.0{ }^{\circ} \mathrm{C} \\
& \mathrm{T}_{\mathrm{N}}=45^{\circ} \mathrm{C} \text { (est.) }
\end{aligned}
$$


TABLE D.II-13. (contd) River Temperature Traverses Above 181-D (River Mile: 378.0)

Date: $\quad 11-28-64$

Time: 1020

River Flow Rate: $41,000 \mathrm{cfs}$

Distance

from Plant

Shore, yd

5

30

55

80

105

130

155

180

205

230

255

280

305

330

355

380

405

430

455

480

505

530

535

$$
\begin{aligned}
A_{t} & =14,900 \mathrm{ft}^{2} \\
T_{m} & =11.3{ }^{\circ} \mathrm{C}
\end{aligned}
$$

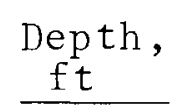

$$
3.5
$$

8

6.5

6.5

7.5

9.5

11.5

12

11.5

10

8.0

8.5

10

10.5

10.5

8

8

8.5

14.5

14

12.5

2

0

$\begin{array}{crr}\begin{array}{cr}\text { Temp } \\ { }^{\circ} \mathrm{C}\end{array} & & \begin{array}{r}\mathrm{KW} \\ \text { Efflu } \\ \text { Uni }\end{array} \\ \begin{array}{c}12.5 \\ 12.6\end{array} & & 24 \\ 12.7 & & 29 \\ 12.7 & & 29 \\ 12.65 & & 29 \\ 12.5 & & 28 \\ 12.3 & & 26 \\ 12.0 & & 25 \\ 11.75 & & 23 \\ 11.6 & & 21 \\ 11.4 & & 20 \\ 11.3 & & 19 \\ 11.25 & & 18 \\ 11.05 & & 17 \\ 10.85 & & 15 \\ 10.7 & & 14 \\ 10.55 & & 13 \\ 10.45 & & 12 \\ 10.35 & & 10 \\ 10.0 & & 8 \\ 9.5 & & 5 \\ 8.9 & & 0 \\ - & & \\ & & \end{array}$

$\mathrm{T}_{\mathrm{PR}}=9.4{ }^{\circ} \mathrm{C}$

$\mathrm{T}_{\mathrm{N}}=25^{\circ} \mathrm{C}$ (est.) 


\section{TABLE D.II-13. (contd) River Temperature Traverses - Above 181-D \\ (River Mile: 378.0 )}

\begin{tabular}{|c|c|c|c|c|c|c|c|}
\hline \multirow{2}{*}{$\begin{array}{r}\text { Date: } \\
\text { Time: } \\
\text { River } \\
\text { Distance } \\
\text { from Plant } \\
\text { Shore, yd } \\
\end{array}$} & $\begin{array}{l}3-18-65 \\
1230 \\
\text { Flow Rate: }\end{array}$ & \multicolumn{2}{|c|}{$95,000 \mathrm{cfs}$} & \multicolumn{2}{|c|}{$\begin{array}{ll}\text { Date: } & 3-18-65 \\
\text { Time: } & 1240\end{array}$} & \multicolumn{2}{|c|}{$95,000 \mathrm{cfs}$} \\
\hline & $\begin{array}{c}\text { Depth, } \\
\mathrm{ft} \\
\end{array}$ & $\begin{array}{c}\text { Temp } \\
{ }^{\circ} \mathrm{C} \\
\end{array}$ & $\begin{array}{c}\text { B } \\
\text { Effluent } \\
\text { Units } \\
\end{array}$ & $\begin{array}{l}\text { Distance } \\
\text { from Plant } \\
\text { Shore, yd } \\
\end{array}$ & $\begin{array}{l}\text { Depth, } \\
\mathrm{ft} \\
\end{array}$ & $\begin{array}{l}\text { Temp, } \\
{ }^{\circ} \mathrm{C}\end{array}$ & $\begin{array}{c}\mathrm{B} \\
\text { Effluent } \\
\text { Units } \\
\end{array}$ \\
\hline 5 & 3 & 6.5 & 13 & 5 & 3.5 & 6.5 & 13 \\
\hline 25 & 8.5 & 6.45 & 13 & 25 & 6.5 & 6.45 & 13 \\
\hline 50 & 10 & 6.3 & 12 & 50 & 8.5 & 6.25 & 13 \\
\hline 75 & 12 & 6.2 & 12 & 75 & 10.5 & 6.0 & 13 \\
\hline 100 & 10 & 6.15 & 13 & 100 & 10.5 & 6.0 & 13 \\
\hline 125 & 12 & 6.15 & 13 & 125 & 15 & 6.0 & 13 \\
\hline 150 & 15 & 6.05 & 13 & 150 & 16 & 5.95 & 13 \\
\hline 175 & 16 & 6.0 & 12 & 175 & 16 & 5.95 & 13 \\
\hline 200 & 16 & 5.95 & 12 & 200 & 17 & 5.95 & 13 \\
\hline 225 & 14 & 5.95 & 12 & 225 & 13 & 5.9 & 13 \\
\hline 250 & 12 & 5.95 & 12 & 250 & 12 & 5.8 & 13 \\
\hline 275 & 12 & 5.95 & 12 & 275 & 14 & 5.6 & 11 \\
\hline 300 & 14 & 5.85 & 11 & 300 & 14 & 5.45 & 9 \\
\hline 325 & 15 & 5.8 & 11 & 325 & 15 & 5.3 & 8 \\
\hline 350 & 15 & 5.7 & 10 & 350 & 15 & 5.2 & 7 \\
\hline 375 & 14 & 5.55 & 9 & 375 & 14 & 5.1 & 6 \\
\hline 400 & 12 & 5.35 & 7 & 400 & 14 & 5.05 & 6 \\
\hline 425 & 13 & 5.25 & 6 & 425 & 19 & 4.95 & 5 \\
\hline 450 & 17 & 5.15 & 5 & 450 & 18 & 4.9 & 3 \\
\hline 475 & 18 & 5.1 & 5 & 475 & 16 & 4.9 & 1 \\
\hline 500 & 17 & 5.0 & 4 & 500 & 22 & 5.0 & 1 \\
\hline 525 & 8.5 & 5.0 & 2 & 525 & 12 & 5.2 & 0 \\
\hline 550 & 3.5 & 5.2 & 0 & 550 & 3.5 & - & 0 \\
\hline 555 & 0 & - & 0 & 555 & 0 & & \\
\hline & $\begin{array}{l}\mathrm{A}_{\mathrm{t}}=2 \mathrm{~J} \\
\mathrm{~T}_{\mathrm{m}}=5 \\
\mathrm{~T}_{\mathrm{PR}}=\end{array}$ & $\begin{array}{l}1,200 \mathrm{f} \\
.7{ }^{\circ} \mathrm{C} \\
4.2{ }^{\circ} \mathrm{C}\end{array}$ & & & $\begin{array}{l}\mathrm{A}_{\mathrm{t}}=22 \\
\mathrm{~T}_{\mathrm{m}}=5 \\
\mathrm{~T}_{\mathrm{PR}}=\end{array}$ & $\begin{array}{l}.600 \mathrm{f} \\
.5{ }^{\circ} \mathrm{C} \\
.2{ }^{\circ} \mathrm{C}\end{array}$ & \\
\hline
\end{tabular}


TABLE D.II-13. (contd) River Temperature Traverses Above 181-D

(River Mile: 378.0 )

Date: $\quad 4-8-65$

Time: 1120

River Flow Rate: $65,000 \mathrm{cfs}$ (est.)
Date: $2-13-66$

Time: 0745

River Flow Rate: 42,000 cfs
Distance

from Plant

Shore, yd

$\begin{array}{rrl}5 & 2 & 9.75 \\ 40 & 14 & 8.55 \\ 90 & 12 & 8.05 \\ 140 & 17 & 7.85 \\ 190 & 17 & 7.6 \\ 240 & 14 & 7.5 \\ 290 & 14 & 7.25 \\ 340 & 15 & 6.95 \\ 390 & 14 & 6.65 \\ 440 & 18 & 6.4 \\ 490 & 19 & 6.4 \\ 540 & 2 & 7.05 \\ 545 & 0 & -\end{array}$

Distance

from Plant Depth, Temp, Shore, yd ft ${ }^{\circ} \mathrm{C}$

$\begin{array}{rrl}5 & 3 & 6.8 \\ 40 & 9 & 7.15 \\ 65 & 8 & 7.1 \\ 90 & 9 & 7.1 \\ 115 & 13 & 7.0 \\ 140 & - & 6.9 \\ 190 & - & 6.9 \\ 215 & 8 & 6.85 \\ 240 & 9 & 6.6 \\ 265 & 8 & 6.3 \\ 290 & 10 & 6.0 \\ 315 & 10 & 5.7 \\ 340 & 11 & 5.25 \\ 365 & 10.5 & 5.15 \\ 390 & 10 & 5.1 \\ 415 & 9 & 5.9 \\ 440 & 10 & 4.8 \\ 465 & 15 & 4.65 \\ 490 & 14 & 4.45 \\ 515 & 6 & 4.2 \\ 525 & 3 & 4.2 \\ 530 & 0 & -\end{array}$

$$
\begin{aligned}
& \mathrm{A}_{\mathrm{t}}=14,900 \mathrm{ft}^{2} \\
& \mathrm{~T}_{\mathrm{m}}=5.95{ }^{\circ} \mathrm{C} \\
& \mathrm{T}_{\mathrm{PR}}=4.6{ }^{\circ} \mathrm{C} \\
& \mathrm{T}_{\mathrm{N}}=53{ }^{\circ} \mathrm{C}
\end{aligned}
$$


BNWL - 1345

\section{TABLE D.II-13. (contd) River Temperature Traverses - Above 181-D \\ (River Mile: 378.0 )}

\begin{tabular}{|c|c|c|c|c|c|c|c|}
\hline \multirow{2}{*}{$\begin{array}{r}\text { Date: } \\
\text { Time: } \\
\text { River } \\
\text { Distance } \\
\text { from Plant } \\
\text { Shore, yd } \\
\end{array}$} & $\begin{array}{l}2-20-66 \\
0905 \\
\text { F1 ow Rate: }\end{array}$ & \multicolumn{2}{|c|}{$38,000 \mathrm{cfs}$} & \multicolumn{2}{|c|}{$\begin{array}{ll}\text { Date: } & 2-20-66 \\
\text { Time: } & 0915\end{array}$} & \multicolumn{2}{|c|}{$38,000 \mathrm{cfs}$} \\
\hline & $\begin{array}{l}\text { Depth, } \\
\mathrm{ft}\end{array}$ & $\begin{array}{l}\text { Temp, } \\
{ }^{\circ} \mathrm{C} \\
\end{array}$ & $\begin{array}{c}\mathrm{N} \\
\text { Effluent } \\
\text { Units } \\
\end{array}$ & $\begin{array}{l}\text { Distance } \\
\text { from P1ant } \\
\text { Shore, yd } \\
\end{array}$ & $\begin{array}{l}\text { Depth, } \\
\mathrm{ft}\end{array}$ & $\begin{array}{l}\text { Temp, } \\
{ }^{\circ} \mathrm{C} \\
\end{array}$ & $\begin{array}{l}\mathrm{N} \\
\text { Eff1uent } \\
\text { Units } \\
\end{array}$ \\
\hline 5 & 2 & 7.45 & 19 & 5 & 2 & 7.65 & 23 \\
\hline 25 & 7 & 7.8 & 29 & 25 & 8 & 7.85 & 25 \\
\hline 50 & 8 & 8.05 & 38 & 50 & 8 & - & - \\
\hline 60 & 8 & 8.05 & 39 & 60 & 8 & 8.15 & 36 \\
\hline 90 & 6 & 8.05 & 40 & 90 & 7 & 8.15 & 41 \\
\hline 140 & 10 & 8.15 & 39 & 140 & 9 & 8.15 & 39 \\
\hline 190 & 10 & 8.4 & 50 & 190 & 11 & 8.3 & 46 \\
\hline 215 & - & - & - & 215 & 11 & 7.95 & 49 \\
\hline 240 & 8 & 7.55 & 26 & 240 & 9 & 7.55 & 39 \\
\hline 290 & 8 & 6.95 & 15 & 290 & 9 & 6.55 & 20 \\
\hline 340 & 10 & 6.2 & 6 & 340 & 10 & 5.9 & 11 \\
\hline 390 & 10 & 5.65 & 2 & 390 & 9 & 5.2 & 2 \\
\hline 440 & 7 & 5.15 & 0 & 440 & 9 & 4.95 & 1 \\
\hline 490 & 13 & 4.85 & 0 & 490 & 12 & 4.85 & 0 \\
\hline 540 & 6 & 4.5 & 0 & 540 & 5 & 4.45 & 0 \\
\hline 550 & 2 & 4.45 & 0 & 550 & - & 4.45 & 0 \\
\hline 555 & 0 & - & 0 & 555 & 0 & - & 0 \\
\hline & $\begin{array}{l}\mathrm{A}_{\mathrm{t}}=14, \\
\mathrm{~T}_{\mathrm{m}}=6.7 \\
\mathrm{~T}_{\mathrm{PR}}=4 . \\
\mathrm{T}_{\mathrm{N}}=53\end{array}$ & $\begin{array}{l}100 \mathrm{ft}^{2} \\
7{ }^{\circ} \mathrm{C} \\
5^{\circ} \mathrm{C} \\
{ }^{\circ} \mathrm{C}\end{array}$ & & & $\begin{array}{l}A_{t}=14, \\
T_{m}=6.6\end{array}$ & $\begin{array}{l}, 600 \mathrm{f} \\
6{ }^{\circ} \mathrm{C}\end{array}$ & \\
\hline
\end{tabular}


TABLE D.II-13. (contd) River Temperature Traverses Above 181-D

(River Mile: 378.0 )

Date: $\quad 3-17-66$

Time: 1040

River Flow Rate: 68,000 cfs

Distance

from Plant Depth, Temp, Shore, yd $\mathrm{ft}$

${ }^{\circ} \mathrm{C}$

7.95

7.55

7.35

7.65

8.2

7.9

6.8

6.0

5.6

5.05

4.85

4.7

4.65

540

550
Date: $\quad 3-31-66$

Time: 1300

River Flow Rate: 90,000 cfs

Distance from Plant Depth, Temp, Shore, $y d$ ft ${ }^{\circ} \mathrm{C}$

5
40
65
90
140
165
190
215
240
265
290
340
365
390
415
440
465
490
515
530
540

$3.5 \quad 8.9$

$\begin{array}{ll}17 & 7.7\end{array}$

$13 \quad 7.5$

$15 \quad 7.5$

$21 \quad 8.3$

$21 \quad 9.1$

$21 \quad 8.9$

$14 \quad 7.7$

$17 \quad 7.3$

$18 \quad 7.1$

$20 \quad 6.9$

$20 \quad 6.5$

$16 \quad 6.4$

$17 \quad 6.3$

226.2

$24.5 \quad 6.1$

20 -

$16 \quad 6.2$

$10.3 \quad 6.4$

$3.5 \quad 7.5$
$A_{t}=18,900 \mathrm{ft}^{2}$

$\mathrm{T}_{\mathrm{m}}=6.4{ }^{\circ} \mathrm{C}$

$\mathrm{T}_{\mathrm{PR}}=4.4{ }^{\circ} \mathrm{C}$

$\mathrm{T}_{\mathrm{N}}=55^{\circ} \mathrm{C}$ 
TABLE D.II-13. (contd) River Temperature Traverses Above 181-D

(River Mile: 378.0 )

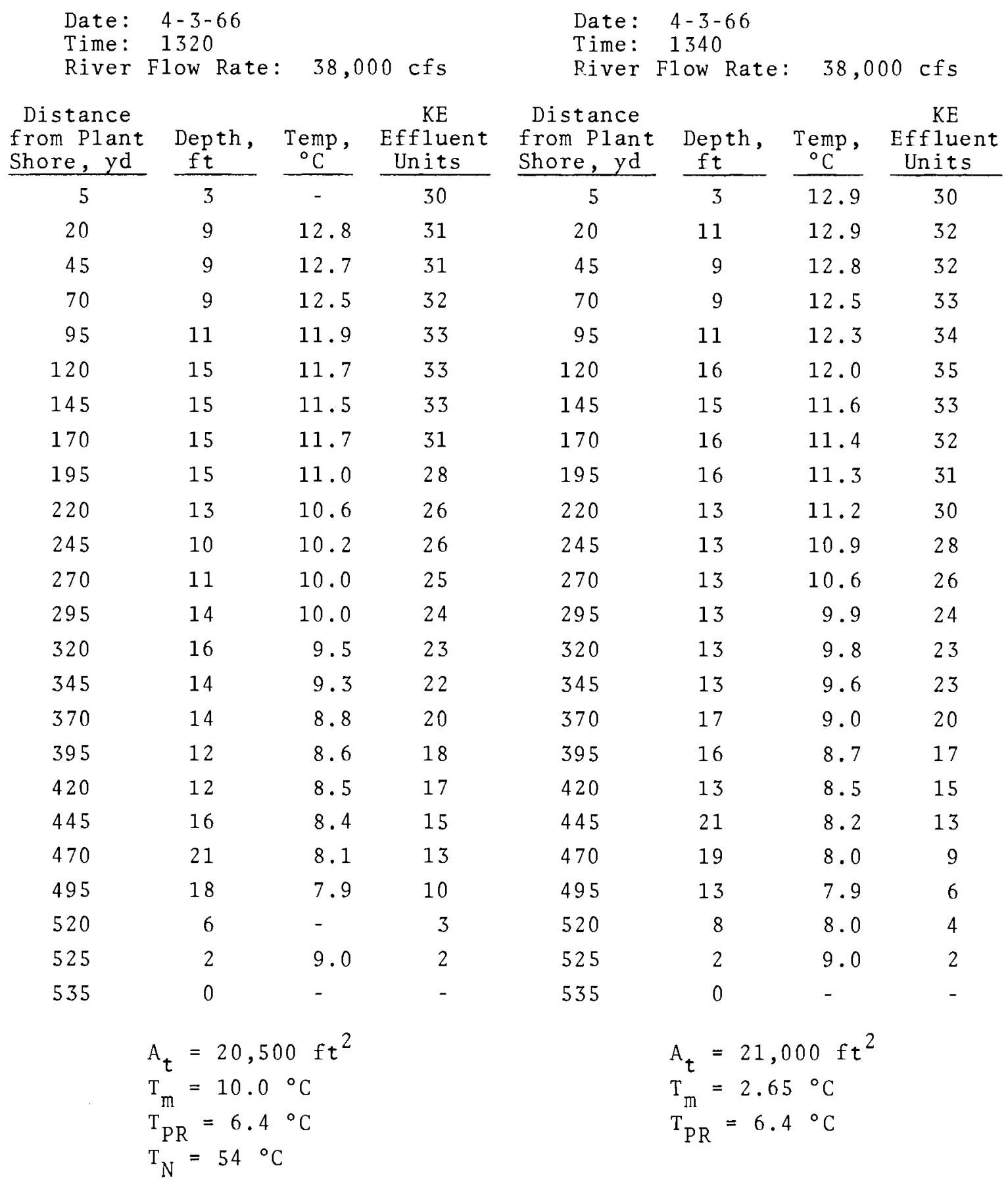


TABLE D.II-14. River Temperature Traverses 0.5 Mile Below 1904-DR

(River Mile: 376.8 )

Date: $\quad 8-14-64$

Time: 1020

River Flow Rate: 130,000 cfs

\begin{tabular}{|c|c|c|c|}
\hline $\begin{array}{l}\text { Distance } \\
\text { from P1 ant } \\
\text { Shore, yd } \\
\end{array}$ & $\begin{array}{l}\text { Depth, } \\
\mathrm{ft} \\
\end{array}$ & $\begin{array}{l}\text { Temp, } \\
{ }^{\circ} \mathrm{C} \\
\end{array}$ & $\begin{array}{c}\text { DR } \\
\text { Effluent } \\
\text { Units } \\
\end{array}$ \\
\hline 70 & 3.5 & 18.1 & 0 \\
\hline 95 & 7.5 & 18.1 & 0 \\
\hline 120 & 7.5 & 18.0 & 0 \\
\hline 145 & 11.5 & 17.85 & 0 \\
\hline 170 & 12 & 17.85 & 0 \\
\hline 195 & 18 & 17.85 & 0 \\
\hline 220 & 21 & 17.95 & 1 \\
\hline 245 & 34.5 & 18.45 & 24 \\
\hline 370 & 49.5 & 18.65 & 23 \\
\hline 295 & 49.5 & 18.7 & 22 \\
\hline 320 & 49.5 & 18.6 & 15 \\
\hline 345 & 52.5 & 18.3 & 4 \\
\hline 370 & 55 & 17.85 & 3 \\
\hline 395 & 17 & 17.4 & 0 \\
\hline 420 & 16 & 17.15 & 0 \\
\hline 470 & 20 & 17.1 & 0 \\
\hline 495 & 12 & 17.1 & 0 \\
\hline 515 & 5.5 & 17.5 & 0 \\
\hline 520 & 0 & - & 0 \\
\hline$A_{t}=33$ & $f t^{2}$ & $\mathrm{~T}_{\mathrm{PR}}=$ & $.8{ }^{\circ} \mathrm{C}$ \\
\hline
\end{tabular}


TABLE D.II-14. (contd) River Temperature Traverses 0.5 Mile Below 1904-DR

(River Mile: 376.8 )

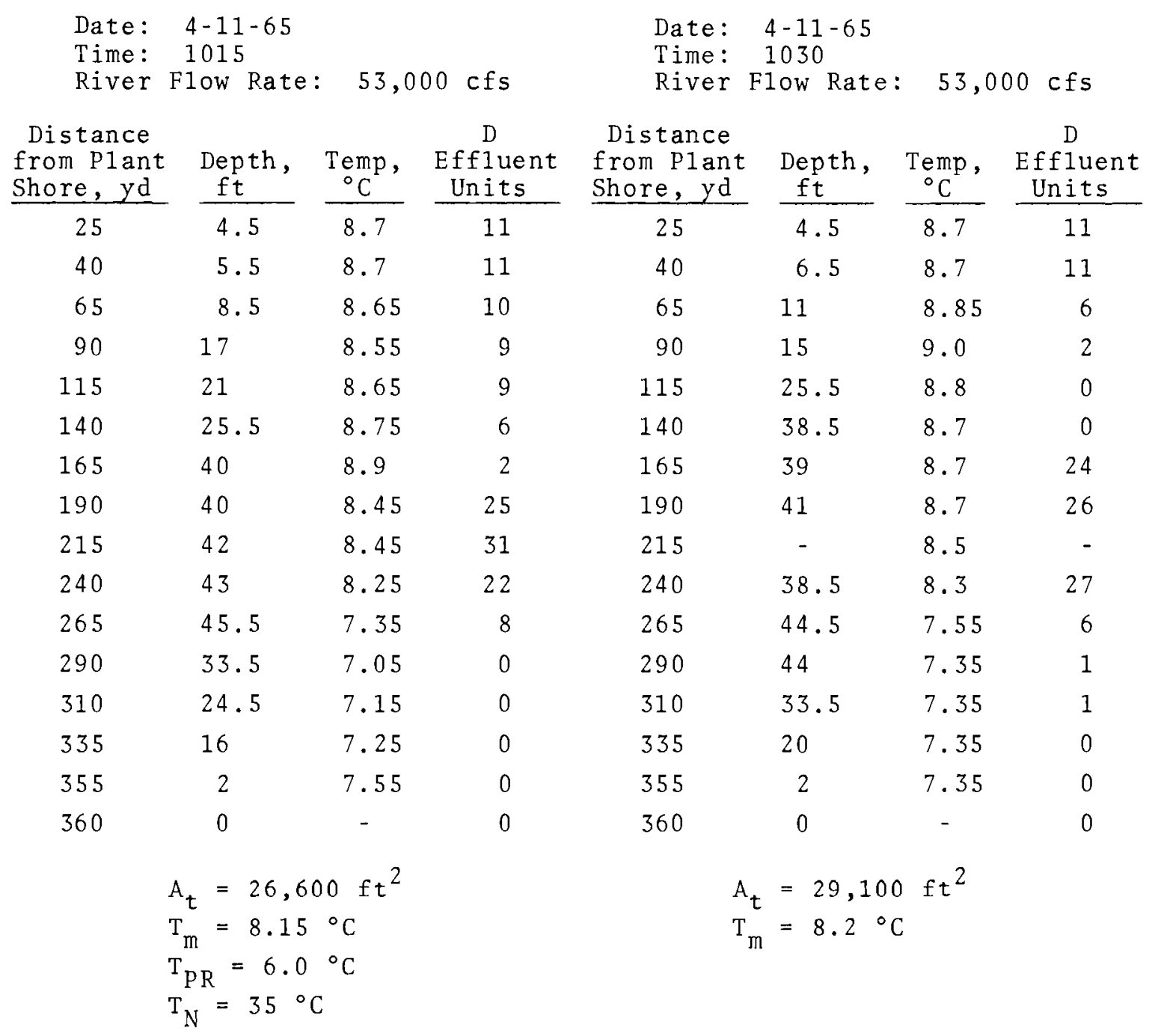

D. I I. 50 


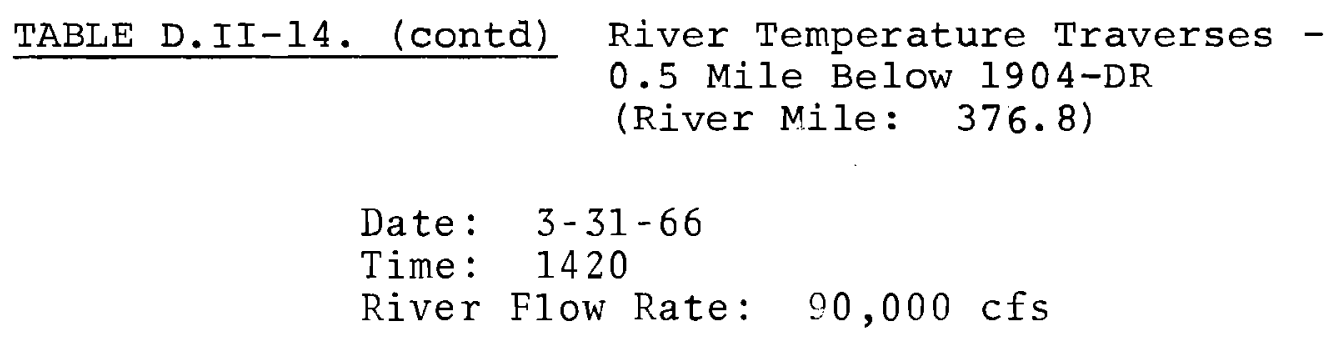

Distance from Plant Shore, yd

15

35

60

85

110

135

160

185

210

235

260

285

310

335

360

390

415

435

440

$$
\begin{gathered}
\text { Depth } \\
\mathrm{ft}
\end{gathered}
$$

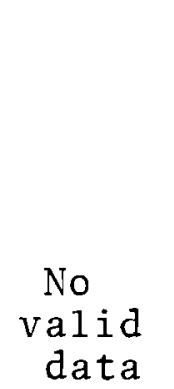

$$
\begin{aligned}
& \mathrm{A}_{\mathrm{t}}=\text { Unknown } \\
& \mathrm{T}_{\mathrm{m}}=7.8{ }^{\circ} \mathrm{C} \text { (est.) } \\
& \mathrm{T}_{\mathrm{PR}}=5.7{ }^{\circ} \mathrm{C} \\
& \mathrm{T}_{\mathrm{N}}=54{ }^{\circ} \mathrm{C}
\end{aligned}
$$

\begin{tabular}{c}
$\begin{array}{c}\text { Temp } \\
{ }^{\circ} \mathrm{C}\end{array}$ \\
\hline 9.2 \\
8.7 \\
8.5 \\
8.1 \\
7.9 \\
7.8 \\
7.8 \\
8.9 \\
9.1 \\
9.1 \\
9.0 \\
8.0 \\
6.6 \\
6.3 \\
6.3 \\
6.3 \\
6.4 \\
6.4 \\
-
\end{tabular}

Temp

9.2

8.7

8.5

8.1

7.9

7.8

8.9

9.1

9.0

8.0

6.6

6.3

6.3

6.4

6.4 


\section{TABLE D.II-15. River Temperature Traverses - 1 Mile Below DR \\ (River Mile: 376.3 )}

\begin{tabular}{|c|c|c|c|}
\hline $\begin{array}{l}\text { Date: } \\
\text { Time: } \\
\text { River }\end{array}$ & $\begin{array}{l}\text { 8-14-64 } \\
1030 \\
\text { Flow Rate: }\end{array}$ & 130,000 & $\operatorname{cfs}$ \\
\hline $\begin{array}{l}\text { Distance } \\
\text { from Plant } \\
\text { Shore, yd } \\
\end{array}$ & $\begin{array}{l}\text { Depth, } \\
\mathrm{ft}\end{array}$ & $\begin{array}{c}\text { Temp } \\
{ }^{\circ} \mathrm{C} \\
\end{array}$ & $\begin{array}{c}\text { DR } \\
\text { Effluent } \\
\text { Units } \\
\end{array}$ \\
\hline $\begin{array}{r}15 \\
65 \\
90 \\
115 \\
140 \\
165 \\
190 \\
215 \\
240 \\
265 \\
290 \\
315 \\
340 \\
365 \\
390 \\
415 \\
440 \\
465 \\
515 \\
540 \\
560 \\
565\end{array}$ & $\begin{array}{r}5 \\
32 \\
36 \\
32 \\
32 \\
26 \\
24 \\
22 \\
21 \\
22 \\
21 \\
20 \\
21 \\
22 \\
22 \\
23 \\
24 \\
22 \\
26 \\
13 \\
4 \\
0\end{array}$ & $\begin{array}{l}18.1 \\
18.3 \\
18.4 \\
18.5 \\
18.6 \\
18.6 \\
18.5 \\
18.5 \\
18.4 \\
18.35 \\
18.25 \\
18.15 \\
17.95 \\
17.9 \\
17.9 \\
17.95 \\
17.8 \\
17.6 \\
17.3 \\
17.3 \\
17.3 \\
-\end{array}$ & $\begin{array}{r}0 \\
10 \\
12 \\
13 \\
14 \\
14 \\
13 \\
11 \\
11 \\
8 \\
7 \\
5 \\
5 \\
5 \\
6 \\
1 \\
0 \\
0 \\
0 \\
0 \\
0 \\
0\end{array}$ \\
\hline \multicolumn{4}{|c|}{$\begin{array}{l}\mathrm{A}_{\mathrm{t}}=38,000 \mathrm{ft}^{2} \\
\mathrm{~T}_{\mathrm{m}}=18.1{ }^{\circ} \mathrm{C} \\
\mathrm{T}_{\mathrm{PR}}=16.8{ }^{\circ} \mathrm{C}\end{array}$} \\
\hline
\end{tabular}




\section{TABLE D.II-15. (contd) River Temperature Traverses - 1 Mile Below DR \\ (River Mile: 376.3 )}

\begin{tabular}{|c|c|c|c|c|c|c|c|}
\hline $\begin{array}{l}\text { Date: } \\
\text { Time: } \\
\text { River }\end{array}$ & $\begin{array}{l}8-27-64 \\
1245 \\
\text { Flow Rate }\end{array}$ & 115 , & $000 \mathrm{cfs}$ & $\begin{array}{l}\text { Date: } \\
\text { Time: } \\
\text { River }\end{array}$ & $\begin{array}{l}8-27-64 \\
1255 \\
\text { F1ow Rate: }\end{array}$ & 115 & $000 \mathrm{cfs}$ \\
\hline $\begin{array}{l}\text { Distance } \\
\text { from Plant } \\
\text { Shore, yd }\end{array}$ & $\begin{array}{l}\text { Depth, } \\
\mathrm{ft}\end{array}$ & $\begin{array}{l}\text { Temp, } \\
{ }^{\circ} \mathrm{C}\end{array}$ & $\begin{array}{c}\mathrm{N} \\
\text { Effluent } \\
\text { Units } \\
\end{array}$ & $\begin{array}{l}\text { Distance } \\
\text { from Plant } \\
\text { Shore, yd }\end{array}$ & $\begin{array}{l}\text { Depth, } \\
\mathrm{ft}\end{array}$ & $\begin{array}{c}\text { Temp, } \\
{ }^{\circ} \mathrm{C} \\
\end{array}$ & $\begin{array}{c}N \\
\text { Effluent } \\
\text { Units } \\
\end{array}$ \\
\hline 5 & 3.5 & 19.8 & 5 & 5 & 4.5 & 19.0 & 2 \\
\hline 30 & 8.5 & 19.3 & 2 & 30 & 15 & 19.0 & 7 \\
\hline 55 & 22 & 19.2 & 5 & 55 & 26.5 & 19.0 & 14 \\
\hline 80 & 25.5 & 19.1 & 12 & 80 & 29 & 18.9 & 14 \\
\hline 105 & 25.5 & 19.0 & 12 & 105 & 25.5 & 18.9 & 14 \\
\hline 130 & 25.5 & 19.1 & 15 & 130 & 24.5 & 18.9 & 12 \\
\hline 155 & 22.5 & 19.1 & 15 & 155 & 22.5 & 18.85 & 9 \\
\hline 180 & 27 & 19.1 & 16 & 180 & 22.5 & 18.8 & 9 \\
\hline 205 & 20 & 19.1 & 17 & 205 & 22.5 & 18.8 & 11 \\
\hline 230 & 20 & 19.1 & 17 & 230 & 22.5 & 18.8 & 13 \\
\hline 255 & 18 & 18.95 & 14 & 255 & 22.5 & 18.8 & 12 \\
\hline 280 & 18 & 18.8 & 9 & 280 & 24.5 & 18.7 & 11 \\
\hline 305 & 18 & 18.7 & 8 & 305 & 24.5 & 18.6 & 10 \\
\hline 330 & 19 & 18.65 & 8 & 330 & 25.5 & 18.35 & 6 \\
\hline 355 & 19 & 18.55 & 7 & 355 & 26.5 & 18.3 & 5 \\
\hline 380 & 19 & 18.6 & 8 & 380 & 26.5 & 18.2 & 3 \\
\hline 405 & 18 & 18.5 & 8 & 405 & 26.5 & 18.1 & 2 \\
\hline 430 & 26.5 & 18.45 & 5 & 430 & 29 & 18.1 & 2 \\
\hline 470 & 20 & 18.2 & 1 & 470 & 15 & 18.0 & 1 \\
\hline 495 & 10.5 & 18.2 & 1 & 495 & 7.5 & 18.1 & 0 \\
\hline 515 & 4.5 & 18.1 & 0 & 515 & - & - & 0 \\
\hline 520 & 0 & - & 0 & 520 & 0 & - & 0 \\
\hline & $\begin{array}{l}=29,900 \\
=18.85 \\
=17.3 \\
R=25{ }^{\circ} \mathrm{C}\end{array}$ & $\begin{array}{l}0 \mathrm{ft}^{2} \\
{ }^{\circ} \mathrm{C} \\
{ }^{\circ} \mathrm{C} \\
\text { (est.) }\end{array}$ & & \multicolumn{4}{|c|}{$\begin{array}{l}\mathrm{A}_{\mathrm{t}}=33,800 \mathrm{ft} \\
\mathrm{T}_{\mathrm{m}}=18.55^{\circ} \mathrm{C} \\
\mathrm{T}_{\mathrm{PR}}=17.3{ }^{\circ} \mathrm{C}\end{array}$} \\
\hline
\end{tabular}

D. II. 53 
BNWL -1345

TABLE D.II-15. (contd) River Temperature Traverses I Mile Below DR

(River Mile: 376.3)

\begin{tabular}{|c|c|c|c|c|c|c|c|}
\hline \multirow[b]{2}{*}{$\begin{array}{l}\text { Distance } \\
\text { from Plant } \\
\text { Shore, yd } \\
\end{array}$} & $\begin{array}{l}9-1-64 \\
1300 \\
\text { F1ow Rate : }\end{array}$ & \multicolumn{2}{|c|}{$\begin{array}{l}110,000 \text { cfs } \\
\text { (est.) }\end{array}$} & \multicolumn{2}{|c|}{$\begin{array}{ll}\text { Date: } & 9-1-64 \\
\text { Time: } & 1310\end{array}$} & \multicolumn{2}{|c|}{$\begin{array}{l}110,000 \text { cfs } \\
\text { (est.) }\end{array}$} \\
\hline & $\begin{array}{l}\text { Depth, } \\
\text { ft }\end{array}$ & $\begin{array}{l}\text { Temp, } \\
\text { o } \mathrm{C} \\
\end{array}$ & $\begin{array}{c}\text { KW } \\
\text { Effluent } \\
\text { Units } \\
\end{array}$ & $\begin{array}{l}\text { Distance } \\
\text { from Plant } \\
\text { Shore, yd }\end{array}$ & $\begin{array}{l}\text { Depth, } \\
\mathrm{ft}\end{array}$ & $\begin{array}{l}\text { Temp } \\
{ }^{\circ} \mathrm{C} \\
\end{array}$ & $\begin{array}{c}\mathrm{KW} \\
\text { Effluent } \\
\text { Units } \\
\end{array}$ \\
\hline 5 & 4 & 17.3 & 10 & 5 & 4 & - & 10 \\
\hline 25 & 7 & 17.1 & 10 & 25 & 20 & 17.1 & 10 \\
\hline 50 & 14 & 17.2 & 9 & 50 & 27 & 17.15 & 9 \\
\hline 100 & 25 & 17.4 & 8 & 100 & 26 & 17.2 & 8 \\
\hline 125 & 23.5 & 17.5 & 7 & 125 & 24.5 & 17.3 & 6 \\
\hline 150 & 22.5 & 17.5 & 6 & 150 & 24.5 & 17.45 & 5 \\
\hline 175 & 22.5 & 17.55 & 6 & 175 & 24.5 & 17.55 & 4 \\
\hline 200 & 21.5 & 17.75 & 6 & 200 & 24.5 & 17.7 & 3 \\
\hline 225 & 21 & 17.8 & 5 & 225 & 23.5 & 17.8 & 3 \\
\hline 250 & 20 & 17.9 & 4 & 250 & 24.5 & 17.8 & 3 \\
\hline 275 & 21 & 17.95 & 3 & 275 & 22.5 & 17.8 & 3 \\
\hline 300 & 21 & 18.0 & 3 & 300 & 22.5 & 17.9 & 3 \\
\hline 325 & 21 & 18.05 & 2 & 325 & 23.5 & 17.9 & 3 \\
\hline 350 & 21 & 18.1 & 2 & 350 & 24.5 & 17.9 & 2 \\
\hline 375 & 22.5 & 18.1 & 2 & 375 & 24.5 & 17.9 & 2 \\
\hline 400 & 24.5 & 18.1 & 2 & 400 & 24.5 & 17.9 & 2 \\
\hline 425 & 31 & 18.0 & 2 & 425 & 26 & 17.9 & 2 \\
\hline 450 & - & 18.0 & 2 & 450 & 29 & 17.95 & 2 \\
\hline 475 & 31 & 18.25 & 2 & 475 & 28 & 18.0 & 1 \\
\hline 500 & 32.5 & 18.3 & - & 500 & 7 & 18.15 & 1 \\
\hline 525 & 20 & 18.4 & - & 525 & 5 & 18.8 & 1 \\
\hline 540 & 3 & 18.8 & 1 & 540 & 3 & 18.8 & 1 \\
\hline 545 & 0 & - & - & 545 & 0 & - & - \\
\hline 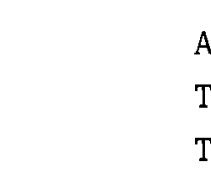 & $\begin{aligned} t & =33,900 \\
m & =17.9^{\circ} \\
P R & =16.3\end{aligned}$ & $\begin{array}{l}{ }^{0} \mathrm{ft} \\
{ }^{\circ} \mathrm{C}\end{array}$ & & & $\begin{array}{l}t_{t}=33,000 \\
r_{m}=17.75\end{array}$ & $\mathrm{ft}^{2}$ & \\
\hline
\end{tabular}

D. I I. 54 


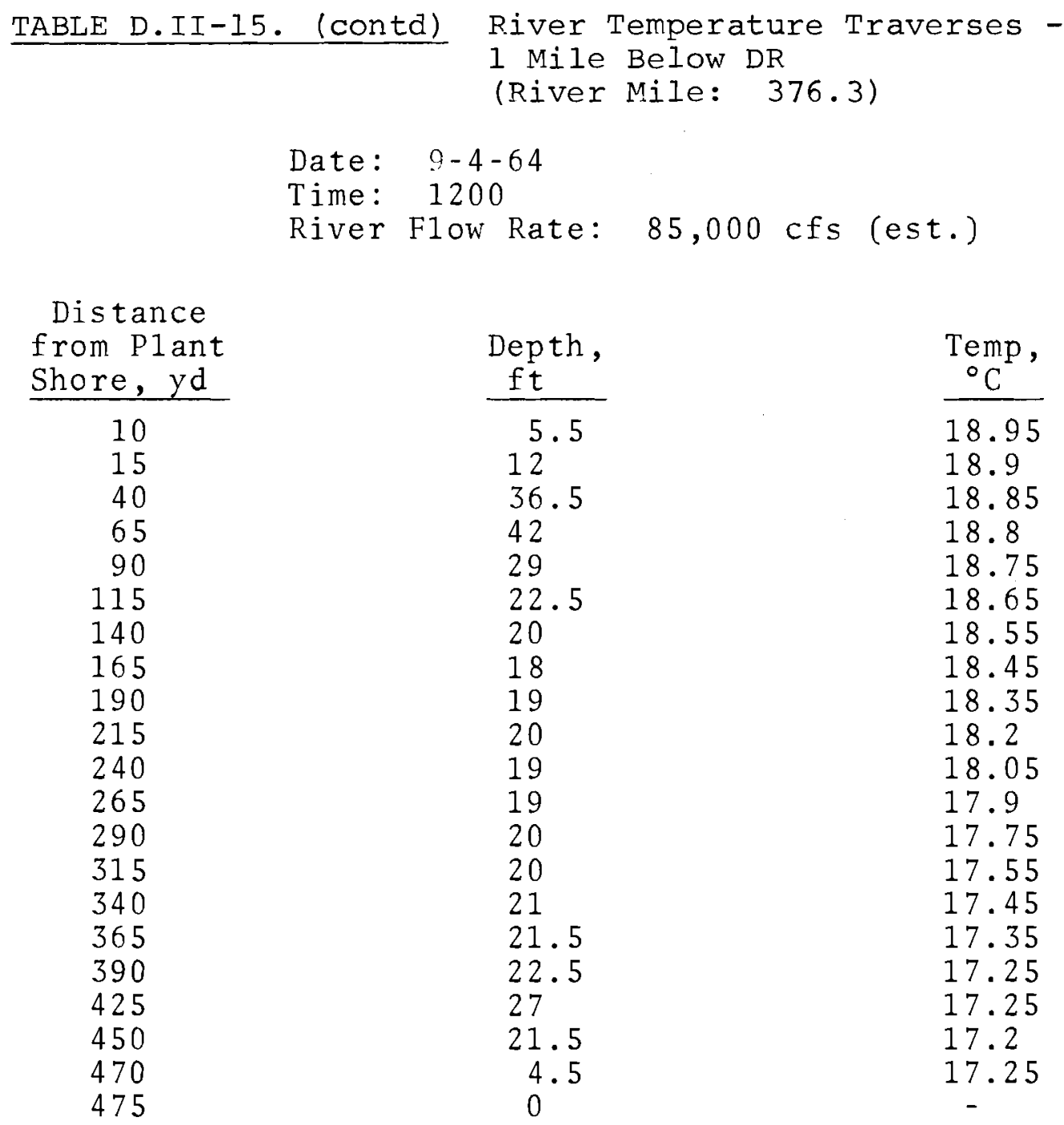

$$
\begin{aligned}
& \mathrm{A}_{\mathrm{t}}=31,200 \mathrm{ft}^{2} \\
& \mathrm{~T}_{\mathrm{m}}=17.7{ }^{\circ} \mathrm{C} \\
& \mathrm{T}_{\mathrm{PR}}=16.1{ }^{\circ} \mathrm{C} \\
& \mathrm{T}_{\mathrm{N}}=30{ }^{\circ} \mathrm{C} \text { (est.) }
\end{aligned}
$$

D. I I. 55 
BNWL - 1345

TABLE D.II-15. (contd) River Temperature Traverses 1 Mile Below DR

(River Mile: 376.3 )

Date: $\quad 9-9-64$

Time: 1145

River Flow Rate: $100,000 \mathrm{cfs}$ (est.)

Distance

from Plant Depth, Temp,

$\frac{\text { Shore, } y d}{10} \frac{\mathrm{ft}}{3.5} \frac{{ }^{\circ} \mathrm{C}}{19.75}$

$\begin{array}{lll}35 & 28 & 19.5\end{array}$

$\begin{array}{lll}60 & 21 & 19.35\end{array}$

$\begin{array}{lll}85 & 22.5 & 19.25\end{array}$

$\begin{array}{lll}110 & 27 & 19.2\end{array}$

$\begin{array}{lll}135 & 24.5 & 19.2\end{array}$

160

185

210

235

260

285

310

335

360

385

410

440

465

485

490
$22.5 \quad 19.15$

18.95

18.8

18.7

18.6

18.55

18.4

18.1

17.7

17.2

17.0

16.9

17.0
Date: $9-9-64$

Time: 1155

River F1ow Rate: $100,000 \mathrm{cfs}$ (est.)
Distance

from Plant Depth, Temp, $\frac{\text { Shore, yd }}{10} \frac{\mathrm{ft}}{-} \frac{{ }^{\circ} \mathrm{C}}{19.75}$

$\begin{array}{lll}35 & 15 & 19.7\end{array}$

$\begin{array}{lll}60 & 15 & 19.55\end{array}$

$\begin{array}{lll}85 & 24.5 & 19.45\end{array}$

$\begin{array}{lll}110 & 25.5 & 19.25\end{array}$

$\begin{array}{lll}135 & 24.5 & 19.15\end{array}$

160

185

210

235

260

285

310

335

360

385

410

440

465

485

490
$22.5 \quad 19.0$

$22 \quad 18.8$

$22.5 \quad 18.55$

$22 \quad 18.2$

$21 \quad 18.15$

$22 \quad 18.45$

$22.5 \quad 18.6$

$22.5 \quad 18.35$

$22.5 \quad 17.95$

$23.5 \quad 17.75$

$24.5 \quad 17.35$

$29 \quad 17.1$

$25.5 \quad 16.9$

$3.5 \quad 15.85$

$$
\begin{aligned}
& \mathrm{A}_{\mathrm{t}}=32,400 \mathrm{ft}^{2} \\
& \mathrm{~T}_{\mathrm{m}}=18.55^{\circ} \mathrm{C} \\
& \mathrm{T}_{\mathrm{PR}}=16.1{ }^{\circ} \mathrm{C} \\
& \mathrm{T}_{\mathrm{N}}=35^{\circ} \mathrm{C} \text { (est.) }
\end{aligned}
$$

$$
\begin{aligned}
& \mathrm{A}_{\mathrm{t}}=30,200 \mathrm{ft}^{2} \\
& \mathrm{~T}_{\mathrm{m}}=18.35{ }^{\circ} \mathrm{C} \\
& \mathrm{T}_{\mathrm{PR}}=16.1{ }^{\circ} \mathrm{C}
\end{aligned}
$$

D. I I . 56 


\section{TABLE D.II-15. (contd) River Temperature Traverses - $1 \mathrm{Mile}$ Below DR (River Mile: 376.3 )}

Date: $\quad 9-9-64$

Time: 1200

River Flow Rate: $100,000 \mathrm{cfs}$ (est.)

Distance

from Plant Depth, Temp, Shore, yd $\mathrm{ft} \quad{ }^{\circ} \mathrm{C}$

$\begin{array}{rll}10 & 4.5 & 19.65 \\ 35 & 24.5 & 19.4 \\ 60 & 15 & 19.3 \\ 85 & 25.5 & 19.25 \\ 110 & 27 & 19.15 \\ 135 & 24.5 & 19.1 \\ 160 & 22.5 & 19.0 \\ 185 & 21 & 18.9 \\ 210 & 22 & 18.7 \\ 235 & 22.5 & 18.55 \\ 260 & 20 & 18.3 \\ 285 & 21 & 18.1 \\ 310 & 22 & 18.0 \\ 335 & 22 & 17.85 \\ 360 & 22.5 & 17.6 \\ 385 & 23.5 & 17.3 \\ 410 & 24.5 & 17.15 \\ 440 & 29 & 16.95 \\ 465 & 19 & 16.9 \\ 485 & 3.5 & 16.95 \\ 490 & 0 & -\end{array}$

$$
\begin{aligned}
& \mathrm{A}_{\mathrm{t}}=31,100 \mathrm{ft}^{2} \\
& \mathrm{~T}_{\mathrm{m}}=18.3^{\circ} \mathrm{C} \\
& \mathrm{T}_{\mathrm{PR}}=16.1{ }^{\circ} \mathrm{C}
\end{aligned}
$$

Date: $9-9-64$

Time : 1300

River Flow Rate: 110,000 cfs

Distance

from Plant Depth, Temp, Shore $y$

5

35

60

85

110

135

160

185

210

235

260

285

310

335

360

385

410

440

465

485

490

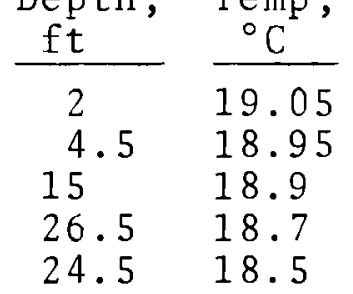

$28 \quad 18.3$

$23.5 \quad 18.3$

$22.5 \quad 18.25$

$20 \quad 18.15$

$20 \quad 18.05$

$21 \quad 17.95$

$20 \quad 17.8$

$20 \quad 17.8$

$21 \quad 17.8$

$21 \quad 17.65$

$22 \quad 17.55$

$22.5 \quad 17.5$

$24.5 \quad 17.1$

$29 \quad 17.0$

$\begin{array}{ll}18 & 16.95\end{array}$

$3.5 \quad 17.0$

$$
\begin{aligned}
& \mathrm{A}_{\mathrm{t}}=30,600 \mathrm{ft}^{2} \\
& \mathrm{~T}_{\mathrm{m}}=17.9{ }^{\circ} \mathrm{C} \\
& \mathrm{T}_{\mathrm{PR}}=16.2{ }^{\circ} \mathrm{C}
\end{aligned}
$$


TABLE D.II-15. (contd) River Temperature Traverses 1 Mile Below DR

(River Mile: 376.3 )

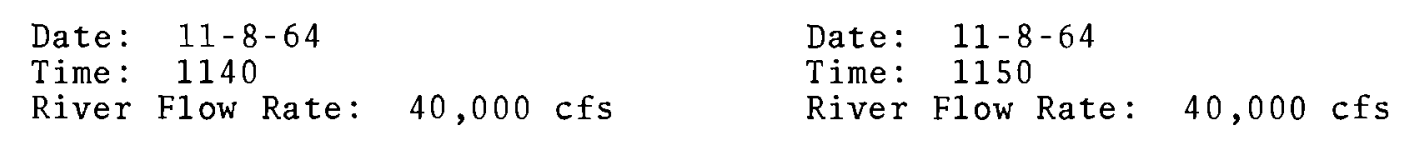

River Flow Rate: 40,000 cfs

$\begin{array}{ll}\text { Date: } & 11-8-64 \\ \text { Time: } & 1150\end{array}$

River Flow Rate: 40,000 cfs

\begin{tabular}{|c|c|c|c|c|c|c|c|}
\hline $\begin{array}{l}\text { Distance } \\
\text { from P1ant } \\
\text { Shore, yd }\end{array}$ & $\begin{array}{l}\text { Depth, } \\
\mathrm{ft}\end{array}$ & $\begin{array}{l}\text { Temp, } \\
{ }^{\circ} \mathrm{C} \\
\end{array}$ & $\begin{array}{c}\mathrm{C} \\
\text { Effluent } \\
\text { Units } \\
\end{array}$ & $\begin{array}{l}\text { Distance } \\
\text { from P1ant } \\
\text { Shore, yd }\end{array}$ & $\begin{array}{l}\text { Depth, } \\
\mathrm{ft}\end{array}$ & $\begin{array}{c}\text { Temp, } \\
{ }^{\circ} \mathrm{C} \\
\end{array}$ & $\begin{array}{c}\mathrm{C} \\
\text { Effluent } \\
\text { Units } \\
\end{array}$ \\
\hline 5 & 3.5 & - & 5 & 5 & - & 15.4 & 6 \\
\hline 30 & 13 & - & 5 & 30 & 27 & 15.1 & 6 \\
\hline 55 & 18 & 15.6 & 5 & 55 & 22 & 15.1 & 6 \\
\hline 80 & 22.5 & 15.55 & 5 & 80 & 18 & 15.1 & 6 \\
\hline 105 & 22.5 & 15.3 & 5 & 105 & 19 & 15.3 & 6 \\
\hline 130 & 21 & 15.2 & 5 & 130 & 20 & 15.2 & 6 \\
\hline 155 & 19 & 15.2 & 5 & 155 & 24.5 & 15.1 & 6 \\
\hline 180 & 18 & 15.2 & 5 & 180 & 22.5 & 15.1 & 6 \\
\hline 205 & 18 & 15.3 & 5 & 205 & 23.5 & 15.05 & 6 \\
\hline 230 & 18 & 15.3 & 5 & 230 & 24.5 & 15.0 & 6 \\
\hline 255 & 17 & 15.3 & 5 & 255 & 24.5 & 15.0 & 6 \\
\hline 280 & 18 & 15.35 & 5 & 280 & 24.5 & 15.0 & 6 \\
\hline 305 & 18 & 15.35 & 5 & 305 & 24.5 & 15.0 & 6 \\
\hline 330 & 19 & 15.3 & 5 & 330 & 24.5 & 15.25 & 6 \\
\hline 355 & 19 & 15.3 & 5 & 355 & 20 & 15.25 & 6 \\
\hline 380 & 19 & 15.3 & 5 & 380 & 21 & 15.0 & 6 \\
\hline 405 & 20 & 15.2 & 5 & 405 & 21 & 15.0 & 6 \\
\hline 430 & 21 & 15.2 & 5 & 430 & 22.5 & 14.95 & 6 \\
\hline 455 & 23.5 & 15.25 & 5 & 455 & 24.5 & 14.9 & 6 \\
\hline 480 & 17 & 15.25 & 4 & 480 & - & - & 6 \\
\hline 500 & 6.5 & 15.0 & 0 & 500 & 4.5 & - & 6 \\
\hline 505 & 0 & - & 0 & 505 & 0 & - & - \\
\hline
\end{tabular}

$$
\begin{array}{ll}
\mathrm{A}_{\mathrm{t}}=27,300 \mathrm{ft}^{2} & \mathrm{~A}_{\mathrm{t}}=30,400 \mathrm{ft}^{2} \\
\mathrm{~T}_{\mathrm{m}}=15.3{ }^{\circ} \mathrm{C} & \mathrm{T}_{\mathrm{m}}=15.05{ }^{\circ} \mathrm{C} \\
\mathrm{T}_{\mathrm{PR}}=12.0{ }^{\circ} \mathrm{C} & \mathrm{T}_{\mathrm{PR}}=12.0{ }^{\circ} \mathrm{C} \\
\mathrm{T}_{\mathrm{N}}=45{ }^{\circ} \mathrm{C} \text { (est.) } &
\end{array}
$$


TABLE D.II-15. (contd) River Temperature Traverses 1 Mile Below DR

(River Mile: 376.3 )

\begin{tabular}{|c|c|c|c|c|c|c|}
\hline \multicolumn{2}{|c|}{$\begin{array}{l}\text { Date: } 11-28-64 \\
\text { Time: } 1120 \\
\text { River Flow Rate: }\end{array}$} & \multicolumn{5}{|c|}{$\begin{array}{ll}\text { Date: } & 2-28-65 \\
\text { Time: } & 1005\end{array}$} \\
\hline $\begin{array}{l}\text { Distance } \\
\text { from Plant } \\
\text { Shore, yd } \\
\end{array}$ & $\begin{array}{l}\text { Depth, } \\
\mathrm{ft}\end{array}$ & $\begin{array}{l}\text { Temp, } \\
{ }^{\circ} \mathrm{C} \\
\end{array}$ & $\begin{array}{c}\mathrm{KW} \\
\text { Effluent } \\
\text { Units } \\
\end{array}$ & $\begin{array}{l}\text { Distance } \\
\text { from P1ant } \\
\text { Shore, yd } \\
\end{array}$ & $\begin{array}{l}\text { Depth, } \\
\mathrm{ft} \\
\end{array}$ & $\begin{array}{l}\text { Temp, } \\
{ }^{\circ} \mathrm{C} \\
\end{array}$ \\
\hline 5 & 3.5 & 12.2 & 25 & 5 & 4.5 & 5.0 \\
\hline 30 & 10.5 & - & 25 & 30 & 7.5 & 5.65 \\
\hline 55 & 14 & 12.4 & 25 & 55 & 14 & 5.6 \\
\hline 80 & 22 & 12.4 & 25 & 80 & 23.5 & 5.55 \\
\hline 105 & 22 & 12.45 & 25 & 105 & 26.5 & 5.55 \\
\hline 130 & 21 & 12.5 & 25 & 130 & 25.5 & 5.5 \\
\hline 155 & 19 & 12.6 & 25 & 155 & 23.5 & 5.45 \\
\hline 180 & 18 & 12.65 & 25 & 180 & 21 & 5.25 \\
\hline 205 & 19 & 12.5 & 24 & 205 & 22 & 5.15 \\
\hline 230 & 19 & 12.4 & 24 & 230 & 22 & 5.15 \\
\hline 255 & 18 & 12.3 & 23 & 255 & 22.5 & 5.15 \\
\hline 280 & 20 & 12.2 & 23 & 280 & 20 & 5.15 \\
\hline 305 & 20 & 12.15 & 23 & 305 & 21 & 5.05 \\
\hline 330 & 21 & 11.85 & 22 & 330 & 22 & 4.95 \\
\hline 355 & 22 & 11.7 & 22 & 355 & 22 & 4.85 \\
\hline 380 & 22.5 & 11.6 & 22 & 380 & 22 & 4.85 \\
\hline 405 & 22.5 & 11.5 & 22 & 405 & 22.5 & 4.85 \\
\hline 430 & 23.5 & 11.45 & 22 & 430 & 25.5 & 4.8 \\
\hline 450 & 24.5 & 11.2 & 22 & 465 & 29 & 4.75 \\
\hline 475 & 20 & 11.3 & 22 & 490 & 19 & 4.7 \\
\hline 495 & 3.5 & 11.2 & 22 & 510 & 4.5 & 4.6 \\
\hline 500 & 0 & - & - & 515 & 0 & - \\
\hline $\begin{array}{l}\mathrm{A}_{\mathrm{t}} \\
\mathrm{T}_{\mathrm{m}} \\
\mathrm{T}_{\mathrm{PF}} \\
\mathrm{T}_{\mathrm{N}}\end{array}$ & $\begin{array}{l}=28,100 \\
=12.05 \\
=9.4^{\circ} \\
=25^{\circ} \mathrm{C}\end{array}$ & $\begin{array}{l}f t^{2} \\
{ }^{\circ} \mathrm{C} \\
\mathrm{C} \\
(\text { est.) }\end{array}$ & & $\begin{array}{l}\mathrm{A}_{\mathrm{t}}=31 \\
\mathrm{~T}_{\mathrm{m}}=5 \\
\mathrm{~T}_{\mathrm{PR}}=3 \\
\mathrm{~T}_{\mathrm{N}}=50\end{array}$ & $\begin{array}{l}800 \mathrm{ft}^{2} \\
{ }^{\circ} \mathrm{C} \\
{ }^{\circ} \mathrm{C} \\
{ }^{\circ} \mathrm{C}\end{array}$ & \\
\hline
\end{tabular}

D. I I. 59 
TABLE D.II-15. (contd) River Temperature Traverses 1 Mile Below DR

(River Mile: 376.3 )

Date: $\quad 2-28-65$

Time: 1040

River Flow Rate: 100,000 cfs
Date: $\quad 3-6-65$

Time: 0920

River Flow Rate: 85,000 cfs
Distance

from Plant Shore, yd

5

35

60

85

110

135

160

185

210

235

260

285

310

335

360

385

410

435

470

495

515

520

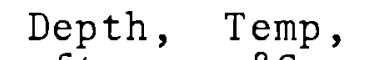

$\frac{\mathrm{ft}}{4.5} \frac{{ }^{\circ} \mathrm{C}}{5.4}$

$14 \quad 5.4$

$18 \quad 5.4$

$25.5 \quad 5.35$

26.5

25.5

23.5

20

23.5

22.5

21

22

24.5

27

25.5

23.5

24.5

25.5

29

24.5

4.5

0
5.35

5.3

$-$

5.1

5.1

5.0

4.95

4.8

$-$

4.7

4.55

4.4

4.25

4.25

4.2
Distance

from Plant Depth, Temp, $\frac{\text { Shore, } y d}{5} \frac{\mathrm{ft}}{3.5} \frac{{ }^{\circ} \mathrm{C}}{5.8}$

$\begin{array}{lll}20 & 7.5 & 5.8\end{array}$

45

70

95

120

145

170

195

220

245

270

295

320

345

370

395

420

445

480

505

525

530

$14 \quad 5.9$

$22 \quad 5.85$

$24.5 \quad 5.7$

$27 \quad 5.6$

$23.5 \quad 5.55$

$22 \quad 5.55$

$21 \quad 5.5$

$22 \quad 5.5$

$21 \quad 5.5$

$21 \quad 5.45$

$20 \quad 5.4$

$21 \quad 5.35$

$22 \quad 5.25$

- 5.2

$22.5 \quad 5.15$

$22.5 \quad 5.1$

23.5

25.5

16

4.5 -

0 -

$$
\begin{aligned}
& \mathrm{A}_{\mathrm{t}}=34,400 \mathrm{ft}^{2} \\
& \mathrm{~T}_{\mathrm{m}}=4.95{ }^{\circ} \mathrm{C} \\
& \mathrm{T}_{\mathrm{PR}}=3.2{ }^{\circ} \mathrm{C}
\end{aligned}
$$

$$
\begin{aligned}
& \mathrm{A}_{\mathrm{t}}=25,800 \mathrm{ft}^{2} \\
& \mathrm{~T}_{\mathrm{m}}=5.5{ }^{\circ} \mathrm{C} \text { (est.) } \\
& \mathrm{T}_{\mathrm{PR}}=3.6{ }^{\circ} \mathrm{C}
\end{aligned}
$$


TABLE D.II-15. (contd) River Temperature Traverses 1 Mile Below DR

(River Mile: 376.3 )

Date: $\quad 4-11-65$

Time: 1040

River Flow Rate: 55,000 cfs

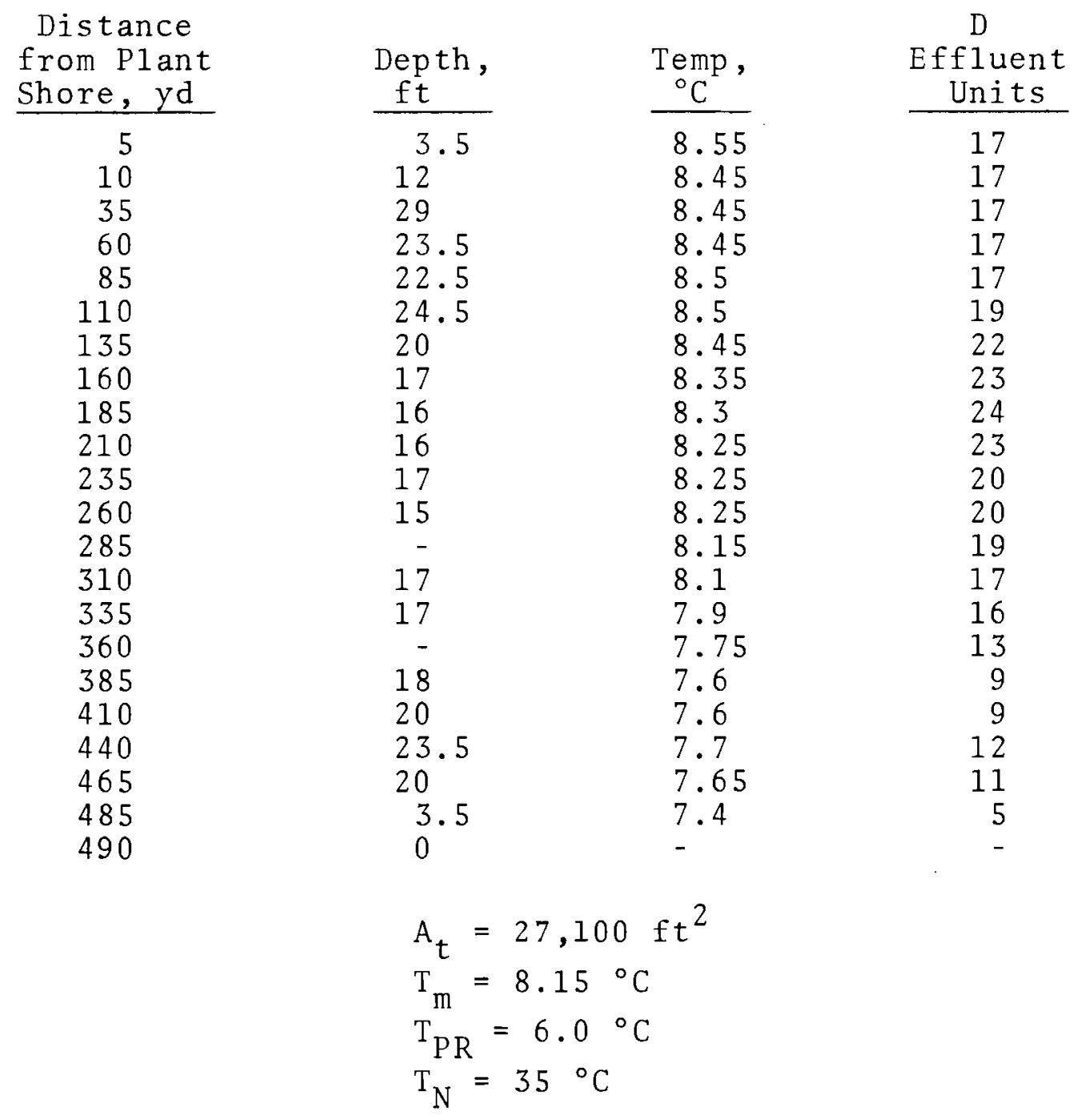


TABLE D.II-15. (contd) River Temperature Traverses 1 Mile Below DR

(River Mile: 376.3 )

Date: 2-13-66

Time: 0915

River Flow Rate: 42,000 cfs River Flow Rate: 48,000 cfs
Date: $\quad 2-13-66$

Time: 1140

\begin{tabular}{|c|c|c|}
\hline $\begin{array}{l}\text { Distance } \\
\text { from Plant } \\
\text { Shore, yd } \\
\end{array}$ & $\begin{array}{l}\text { Depth, } \\
\mathrm{ft}\end{array}$ & $\begin{array}{c}\text { Temp, } \\
{ }^{\circ} \mathrm{C} \\
\end{array}$ \\
\hline 15 & 2 & 7.05 \\
\hline 25 & 16 & 7.05 \\
\hline 50 & 35.5 & 7.05 \\
\hline 75 & 33.5 & 7.05 \\
\hline 100 & 34.5 & 7.1 \\
\hline 125 & 21 & 7.15 \\
\hline 150 & 18 & 7.15 \\
\hline 175 & 16 & 7.1 \\
\hline 200 & 15 & 7.05 \\
\hline 225 & 15 & 6.85 \\
\hline 250 & - & 6.85 \\
\hline 275 & - & 6.8 \\
\hline 300 & 15 & 6.75 \\
\hline 325 & 16 & 6.7 \\
\hline 350 & 17 & 6.6 \\
\hline 375 & 18 & 6.3 \\
\hline 400 & 19 & 6.0 \\
\hline 425 & 19 & 5.7 \\
\hline 455 & 21 & - \\
\hline 480 & 23 & 5.6 \\
\hline 500 & 3 & 6.1 \\
\hline 505 & 0 & - \\
\hline
\end{tabular}

$$
\begin{aligned}
& \mathrm{A}_{\mathrm{t}}=28,600 \mathrm{ft}^{2} \\
& \mathrm{~T}_{\mathrm{m}}=6.7{ }^{\circ} \mathrm{C} \\
& \mathrm{T}_{\mathrm{PR}}=4.6{ }^{\circ} \mathrm{C} \\
& \mathrm{T}_{\mathrm{N}}=53{ }^{\circ} \mathrm{C}
\end{aligned}
$$

Distance

from Plant Depth, Temp, $\frac{\text { Shore, } y d}{5} \frac{f t}{2} \frac{{ }^{\circ} \mathrm{C}}{7.3}$

5

15

40

65

90

115

140

165

190

215

240

265

290

315

340

365

390

415

445

470

490

495

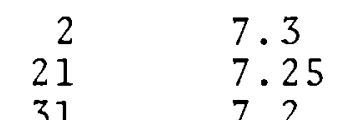

$31 \quad 7.2$

$34 \quad 7.2$

$29 \quad 7.2$

$20 \quad 7.15$

$17 \quad 6.95$

$16 \quad 6.9$

$14.5 \quad 6.9$

$13 \quad 6.9$

$14 \quad 6.95$

$15 \quad 6.95$

$15 \quad 6.4$

156.7

$15 \quad 6.55$

$17 \quad 6.3$

$17 \quad 6.0$

$19 \quad 5.7$

$24 \quad 5.65$

$21 \quad 5.55$

$3 \quad 5.55$

$$
\begin{aligned}
& \mathrm{A}_{\mathrm{t}}=27,400 \mathrm{ft}^{2} \\
& \mathrm{~T}_{\mathrm{m}}=6.7{ }^{\circ} \mathrm{C} \\
& \mathrm{T}_{\mathrm{PR}}=4.4{ }^{\circ} \mathrm{C}
\end{aligned}
$$


TABLE D.II-15. (contd) River Temperature Traverses 1 Mile Below DR

(River Mile: 376.3 )

Date: $2-20-66$

Time: 0950

River Flow Rate: 38,000 cfs
Date: $\quad 2-20-66$

Time : 1000

River Flow Rate: 38,000 cfs

\begin{tabular}{|c|c|c|c|c|c|c|c|}
\hline $\begin{array}{l}\text { Distance } \\
\text { from P1ant } \\
\text { Shore, yd } \\
\end{array}$ & $\begin{array}{l}\text { Depth, } \\
\mathrm{ft}\end{array}$ & $\begin{array}{l}\text { Temp, } \\
{ }^{\circ} \mathrm{C} \\
\end{array}$ & $\begin{array}{c}N \\
\text { Effluent } \\
\text { Units } \\
\end{array}$ & $\begin{array}{l}\text { Distance } \\
\text { from P1ant } \\
\text { Shore, yd } \\
\end{array}$ & $\begin{array}{l}\text { Depth, } \\
\mathrm{ft}\end{array}$ & $\begin{array}{l}\text { Temp, } \\
{ }^{\circ} \mathrm{C} \\
\end{array}$ & $\begin{array}{c}N \\
\text { Effluent } \\
\text { Units } \\
\end{array}$ \\
\hline 5 & 3 & 7.45 & 25 & 5 & 3 & 7.55 & 28 \\
\hline 15 & 13 & 7.4 & 25 & 15 & 16 & 7.6 & 28 \\
\hline 65 & 29 & 7.55 & 27 & 65 & 29 & 7.65 & 28 \\
\hline 115 & 19 & 7.7 & 29 & 115 & 23 & 7.6 & 30 \\
\hline 165 & 15 & 7.55 & 27 & 165 & 16 & 7.55 & 28 \\
\hline 215 & 16 & 7.55 & 26 & 215 & 15 & 7.65 & 30 \\
\hline 265 & 15 & 7.55 & 28 & 265 & 16 & 7.55 & 29 \\
\hline 315 & 17 & 7.45 & 26 & 315 & 16 & 7.45 & 29 \\
\hline 365 & 16 & 7.15 & 24 & 365 & 18 & 6.85 & 26 \\
\hline 415 & 18 & 6.95 & 17 & 415 & 18 & 6.4 & 17 \\
\hline 445 & 22 & 6.3 & 14 & 445 & 16 & 6.0 & 13 \\
\hline 470 & 18 & 6.15 & 12 & 470 & 9 & 5.9 & 12 \\
\hline 490 & 3 & 6.0 & 12 & 490 & 3 & 6.0 & 12 \\
\hline 495 & 0 & - & - & 495 & 0 & - & - \\
\hline $\begin{array}{l}\mathrm{A}_{\mathrm{t}} \\
\mathrm{T}_{\mathrm{n}} \\
\mathrm{T}_{\mathrm{P}} \\
\mathrm{T}_{\mathrm{N}}\end{array}$ & $\begin{array}{l}=25,50 \\
=7.3^{\circ} \\
=4.6 \\
=53{ }^{\circ} \mathrm{C}\end{array}$ & $f t^{2}$ & & $\begin{array}{l}A_{t} \\
T_{m}\end{array}$ & $\begin{array}{l}=25,60 \\
=7.3\end{array}$ & $f t^{2}$ & \\
\hline
\end{tabular}


TABLE D.II-15. (contd). River Temperature Traverses 1 Mile Below DR

(River Mile: 376.3 )

Date: $\quad 3-17-66$

Time: 1105

River Flow Rate: 70,000 cfs

Distance

\begin{tabular}{|c|c|c|}
\hline $\begin{array}{l}\text { from Plant } \\
\text { Shore, yd }\end{array}$ & $\begin{array}{l}\text { Depth, } \\
\mathrm{ft}\end{array}$ & $\begin{array}{l}\text { Temp, } \\
{ }^{\circ} \mathrm{C} \\
\end{array}$ \\
\hline 10 & 2 & 9.0 \\
\hline 35 & 26 & 8.85 \\
\hline 85 & 28 & 8.7 \\
\hline 135 & 24 & 8.35 \\
\hline 185 & 19 & 7.8 \\
\hline 235 & 18 & 7.75 \\
\hline 285 & 18 & 7.9 \\
\hline 335 & 20 & 8.05 \\
\hline 385 & 21 & 6.85 \\
\hline 435 & 22 & 6.4 \\
\hline 465 & 26 & 5.75 \\
\hline 490 & 12 & 5.65 \\
\hline 510 & 3 & 5.65 \\
\hline 515 & 0 & - \\
\hline
\end{tabular}

Date: $\quad 3-17-66$

Time: 1120

River F1ow Rate: 70,000 cfs

$$
\begin{aligned}
& \mathrm{A}_{\mathrm{t}}=30,900 \mathrm{ft}^{2} \\
& \mathrm{~T}_{\mathrm{m}}=7.7{ }^{\circ} \mathrm{C} \\
& \mathrm{T}_{\mathrm{PR}}=4.3^{\circ} \mathrm{C} \\
& \mathrm{T}_{\mathrm{N}}=55^{\circ} \mathrm{C}
\end{aligned}
$$

Distance

from Plant Depth, Temp, $\frac{\text { Shore, } y d}{10} \frac{\mathrm{ft}}{2} \frac{{ }^{\circ} \mathrm{C}}{9.0}$

$\begin{array}{lll}35 & 15 & 9.05\end{array}$

$\begin{array}{lll}85 & 25 & 8.9\end{array}$

$\begin{array}{lll}135 & 24 & 8.8\end{array}$

185

235

285

335

385

435

465

490

510

515
$17 \quad 8.9$

$18 \quad 8.45$

$18 \quad 7.85$

$19 \quad 6.95$

$21 \quad 6.4$

$24 \quad 6.55$

$26 \quad 6.55$

$15 \quad 6.4$

$3 \quad 5.65$

$$
\begin{aligned}
& \mathrm{A}_{\mathrm{t}}=29,200 \mathrm{ft}^{2} \\
& \mathrm{~T}_{\mathrm{m}}=7.8{ }^{\circ} \mathrm{C} \\
& \mathrm{T}_{\mathrm{PR}}=4.3^{\circ} \mathrm{C}
\end{aligned}
$$

D. I I . 64 
BNWL - 1345

\section{TABLE D.II-15. (contd) River Temperature Traverses - 1 Mile Below DR \\ (River Mile: 376.3 )}

Date: $\quad 3-17-66$

Time: 1235

River Flow Rate: 73,000 cfs

Distance

from Plant Depth, Temp, Shore, yd

5
35
85
135
185
235
285
335
385
435
465
490
510
515

5
35

85

135

235

285

335

385

435

465

490

510

515

\begin{tabular}{rll}
$\mathrm{ft}$ & & ${ }^{\circ} \mathrm{C}$ \\
\cline { 1 - 1 } 2 & & 8.95 \\
21 & & 8.85 \\
27 & & 8.65 \\
25 & & 8.35 \\
20 & & 8.15 \\
19 & & 7.5 \\
18 & & 7.35 \\
20 & & 7.15 \\
21 & & 6.95 \\
22 & & 6.3 \\
25 & & 5.9 \\
13 & & 5.65 \\
3 & & 5.6 \\
0 & & -
\end{tabular}

Date: $\quad 3-31-66$

Time: 1445

River Flow Rate: 90,000 cfs

Distance

from Plant Depth, Temp, Shore, yd

$\begin{array}{rrr}5 & 2 & 8.7 \\ 10 & & 8.8 \\ 35 & & 8.9 \\ 60 & & 8.1 \\ 85 & & 8.2 \\ 10 & & 8.4 \\ 35 & \text { No } & 8.3 \\ 60 & \text { valid } & 8.3 \\ 85 & \text { data } & 8.2 \\ 10 & & 8.0 \\ 35 & & 7.9 \\ 60 & & 8.1 \\ 85 & & 7.9 \\ 10 & & 8.4 \\ 60 & & 8.1 \\ 10 & & 7.1 \\ 45 & & 6.5 \\ 70 & & 6.5 \\ 90 & 3 & 6.5 \\ 95 & 0 & -\end{array}$

$$
\begin{aligned}
& \mathrm{A}_{\mathrm{t}}=31,500 \mathrm{ft}^{2} \\
& \mathrm{~T}_{\mathrm{m}}=7.6{ }^{\circ} \mathrm{C} \\
& \mathrm{T}_{\mathrm{PR}}=4.3^{\circ} \mathrm{C}
\end{aligned}
$$

$$
\begin{aligned}
& \mathrm{A}_{\mathrm{t}}=\text { Unknown } \\
& \mathrm{T}_{\mathrm{m}}=7.9{ }^{\circ} \mathrm{C} \text { (est.) } \\
& \mathrm{T}_{\mathrm{PR}}=5.7{ }^{\circ} \mathrm{C} \\
& \mathrm{T}_{\mathrm{N}}=54{ }^{\circ} \mathrm{C}
\end{aligned}
$$


TABLE D.II-15. (contd) River Temperature Traverses 1 Mile Below DR

(River Mile: 376.3)

Date: $\quad 4-3-66$

Time : 1430

River Flow Rate: 39,000 cfs

\begin{tabular}{|c|c|c|c|}
\hline $\begin{array}{l}\text { Distance } \\
\text { from Plant } \\
\text { Shore, yd }\end{array}$ & $\begin{array}{l}\text { Depth, } \\
\mathrm{ft}\end{array}$ & $\begin{array}{l}\text { Temp, } \\
{ }^{\circ} \mathrm{C} \\
\end{array}$ & $\begin{array}{c}\text { KE } \\
\text { Effluent } \\
\text { Units } \\
\end{array}$ \\
\hline $\begin{array}{r}10 \\
35 \\
60 \\
85 \\
110 \\
135 \\
160 \\
185 \\
210 \\
235 \\
260 \\
285 \\
310 \\
335 \\
360 \\
385 \\
410 \\
420 \\
445 \\
465 \\
470\end{array}$ & $\begin{array}{l}\text { No } \\
\text { valid } \\
\text { data }\end{array}$ & $\begin{array}{l}11.5 \\
11.5 \\
11.5 \\
11.6 \\
11.5 \\
11.5 \\
11.55 \\
11.5 \\
11.35 \\
11.25 \\
11.25 \\
11.2 \\
11.3 \\
11.45 \\
10.95 \\
10.65 \\
10.25 \\
10.1 \\
9.8 \\
9.8 \\
-\end{array}$ & $\begin{array}{l}26 \\
26 \\
26 \\
27 \\
27 \\
27 \\
26 \\
26 \\
26 \\
26 \\
26 \\
26 \\
25 \\
26 \\
26 \\
24 \\
23 \\
21 \\
20 \\
20 \\
-\end{array}$ \\
\hline \multicolumn{4}{|c|}{$\begin{array}{l}\mathrm{A}_{\mathrm{t}}=\text { Unknown } \\
\mathrm{T}_{\mathrm{m}}=11.1{ }^{\circ} \mathrm{C} \text { (est.) } \\
\mathrm{T}_{\mathrm{PR}}=6.6{ }^{\circ} \mathrm{C} \\
\mathrm{T}_{\mathrm{N}}=54{ }^{\circ} \mathrm{C}\end{array}$} \\
\hline
\end{tabular}


BNWL -1345

\section{APPENDIX E \\ ENVIRONMENTAL EFFECTS \\ OF AN EXTENDED HANFORD PLANT SHUTDOWN}

Abstracted from unpublished notes by R. B. Hall, J. P. Corley, J. K. Soldat and R. T. Jaske 
BNWL - 1345

\section{APPENDIX E}

ENVIRONMENTAL EFFECTS

OF AN EXTENDED HANFORD PLANT SHUTDOWN

\section{INTRODUCTION}

The work stoppage and consequent shutdown of the plutonium production facilities in the summer of 1966 resulted in a marked reduction in the discharge of radionuclides and heat to the Columbia River and, to a lesser extent, to the discharge of effluents from the Chemical Separations Plants to the atmosphere and to the ground. The extent to which this curtailment of effluent discharge resulted in reductions in the levels of radioactive materials in the environment and in the thermal and chemical impacts on the Columbia River is of substantial interest to the Division of Production and Operational Safety and to the Research Divisions sponsoring studies on the river. Because of this broad interest, the Pacific Northwest Laboratory was asked $(E 1)$ to prepare a comprehensive report assessing the impact of the shutdown. This report is in response to that request.

On July 8, 1966, one reactor (KE) was already down. $\mathrm{N}$ Reactor was also shut down on July 8 . The other reactors ( $B, C, D$ and $K W$ ) were shut down by early morning of July 9 , because of the work stoppage. All reactors remained down until one reactor (D) resumed operation on August 23, one (KW) on August 24, and three (B, C, and KE) on August 25. Cooling water flow in the five production reactors was reduced to a fraction of normal during the outage.

The river flow rate, illustrated in Figure E-1, dropped from levels near the maximum for the year to levels near normal for the fall and winter months during the outage period. 
BNWL - 1345

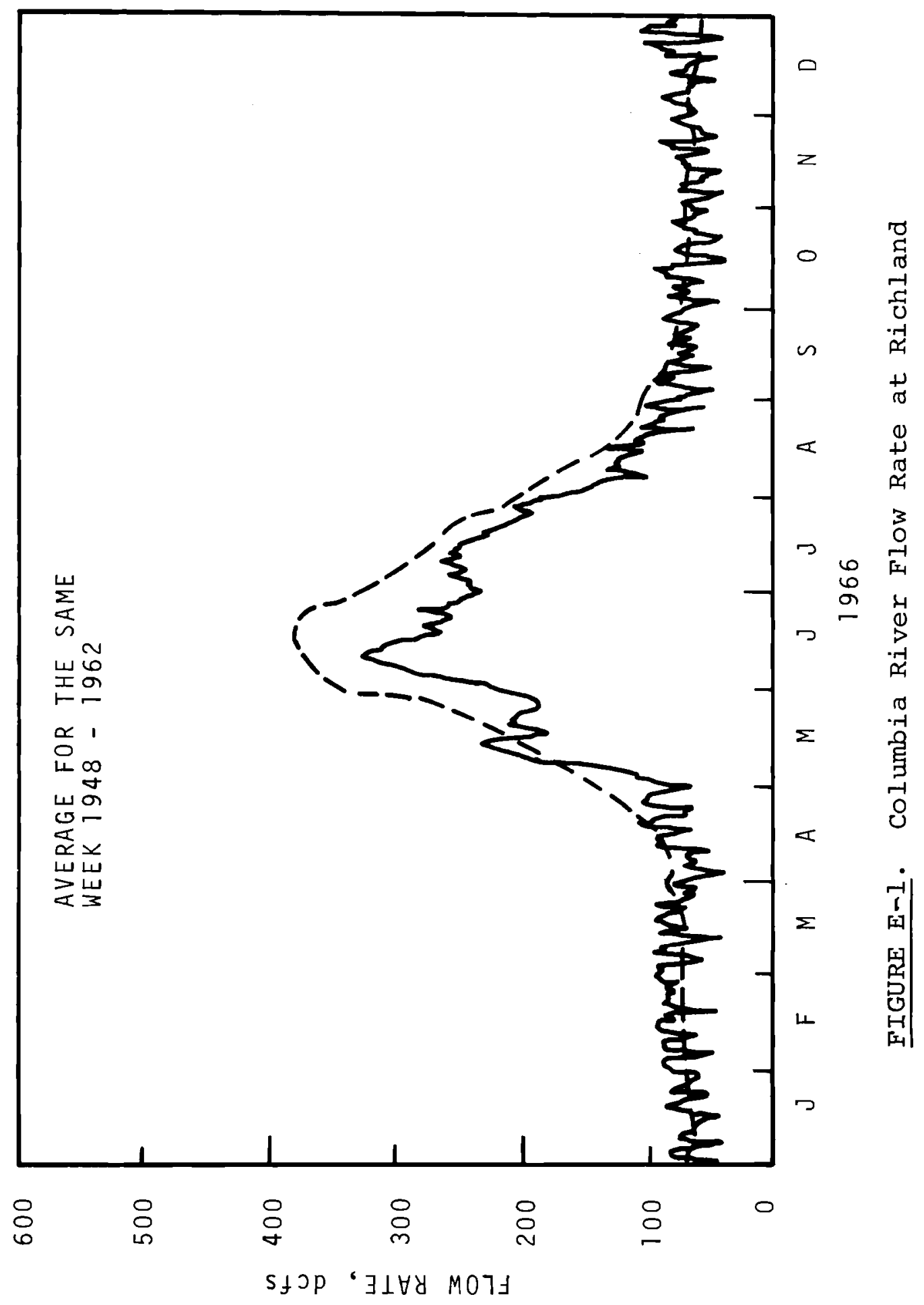

E. 2 
Under ordinary conditions, the concentration of reactor effluent in the Columbia River increases markedly during this time because of the reduced flow available for dilution.

A shortage of personnel also forced curtailment of chemical separations processing from July 8 to August 22. No fue 1 processing was done at the Purex P1ant in 200 East Area, but the Redox Plant continued to process material already present in the system. The total radioiodine release did not reach a minimum until september. Water flow was maintained to open pond areas to prevent spread of contamination by the wind. Liquid discharges to the several waste cribs also continued, though of less than normal volume.

The environmental parameters and the operating history during the strike period were such that significant. changes were expected only in those environmental measurements directly related to reactor effluents, i.e., river temperature, radioactivity, and chemical content.

The Pacific Northwest Laboratory has, for several years, carried on programs for the AEC that involve the presence and movement of radionuclides through various physical, chemical, and biological systems of the Columbia River and the impact of reactor heat. The simultaneous shutdown of all of the production reactors for an extended period of time caused a unique perturbation that afforded an opportunity to develop additional information on the dynamics of the flux of radionuclides through these systems and on natural heating of the river. Insofar as possible, both regular and special sampling and analysis were undertaken during the period of the shutdown to capitalize on this unusual opportunity.

A full treatment of this is carried in declassified report BNWL-CC-1056. 
BNWL -1345

\section{SUMMARY AND CONCLUSIONS}

With the shutdown of the reactors during the work stoppage, formation of radionuclides in the coolant system ceased. Small releases of longer-1ived nuclides such as ${ }^{46} \mathrm{Sc},{ }^{54} \mathrm{Mn},{ }^{65} \mathrm{Zn}$, and ${ }^{60}$ Co continued however, owing to erosion and desorption from films on reactor fuels and piping.

Within a few days after the shutdown, the concentrations of reactor-produced radionuclides in the Columbia River dropped to very low levels (in most cases, below the detection levels of analytical procedures used routinely for surveillance purposes). However, the quantities of ${ }^{65} \mathrm{Zn},{ }^{54} \mathrm{Mn},{ }^{46} \mathrm{Sc}$, and ${ }^{60} \mathrm{Co}$ in river water did not drop as much as would be expected from the reactor effluent data. It was evident that significant amounts of these nuclides, retained in the bed of the river, were recycled to the water through continued scouring and leaching of the sediments. Samples of sediments from behind NcNary Dam indicated that small amounts of radionuclides were leached from the top layers during the shutdown period. During the shutdown, more of the radioactive material transported by the river water was associated with particulates than was present in solution.

In the absence of "fresh" radioactive materials from the reactors, the dose rate from the shoreline within the reservation remained low (ordinarily it increases as river flow diminishes during the summer).

Concentrations of radionuclides in invertebrate organisms of the river decreased by one or two orders of magnitude during the shutdown. The change was greatest in the case of ${ }^{32} \mathrm{P}$ and ${ }^{51} \mathrm{Cr}$. The levels of ${ }^{65} \mathrm{Zn}$ decreased less than those of other nuclides, suggesting recycling of zinc within the river ecosystem. 
BNWL - 1345

Because of the reactor shutdown, the concentration of ${ }^{32} \mathrm{p}$ in juvenile fish decreased by as much as two orders of magnitude. For the flesh of adult fish, the change was about one order of magnitude. The loss was greatest among herbivorous species. In the case of whitefish, the decrease amounted to about 75 percent. The levels of ${ }^{65} \mathrm{Zn}$ in the fish changed much less than those of ${ }^{32} \mathrm{P}$.

Ordinarily, the concentration of $65 \mathrm{Zn}$ in Willapa Bay oysters and in milk from local farms irrigated with Columbia River water increases slightly during the summer. In 1966 , the concentration diminished slightly in both of these products.

The radiation dose to Tri-City residents from drinking water was nil during the shutdown period. In effect, this reduced the annual dose to the GI tract for 1966 by about 10 percent for both Richland and Pasco.

Following startup of the reactors late in August, the concentrations of most radionuclides in the effluent, the local river water, and the invertebrate organisms returned to "normal" within a few days time. However, recharging of the storage capacity of the river bed was indicated for ${ }^{65} \mathrm{Zn}$ and some other nuclides by a greater-than-normal time of recovery to preflood concentrations in river water. In the case of adult fish, nearly a month was required before the usual seasonal trends in the concentrations of radionuclides were re-established. The maximum levels obtained during the fall were undoubtedly lower than would have been reached in the absence of the shutdown.

To the casual observer, the effect of the shutdown on Columbia River temperatures was obscured by natural fluctuations of greater magnitude during this period. The real effects are about as predicted, however. Because of the relatively high velocity and turbulence of the river within the reservation, the natural temperature rise (without reactor 
heat) between Priest Rapids and Richland is substantially higher than in impounded portions of the river during the summer months. This could easily lead the uninformed observer to an overestimation of the effects of the Hanford reactors.

The chemical separations plants do not ordinarily release enough ${ }^{131}$ I or other nuclides to the environment to be quantitatively measurable with routine procedures in air, milk, and other samples taken offsite. For this reason, any effect of the shutdown on the levels of airborne contaminants beyond the project boundaries was not evident.

The relatively small and brief decrease in the discharge of 1 iquid waste to ground at the 200 Areas had essentially no effect on the level of contamination in the ground water.

\section{EFFECTS OF A COMPLETE REACTOR SHUTDOWN ON COLUMBIA}

\section{RIVER TEMPERATURES}

During the reactor shutdown, normal temperature monitoring activities were carried out to provide a comparative basis for reference purposes. In addition, several spot surveys were taken at established river mile locations to double check the routine records and examine the transverse temperature distribution of the river under natural conditions.

Several conclusions were developed as a result of these measurements. They are as follows:

1. The normal temperature rise (without reactor heat) between Priest Rapids and Richland is a good deal higher than would be expected from temperature prediction for impounded portions of the river. It is apparent that the increased velocity of the river in this reach increases the heat conduction to a point where the overall exchange coefficient reaches values on the order of 200 to $300 \mathrm{Btu} /\left(\right.$ day) $\left({ }^{\circ} \mathrm{F}\right)$. Figure E-2 is illustrative of typical values for a lake. 
BNWL -1345

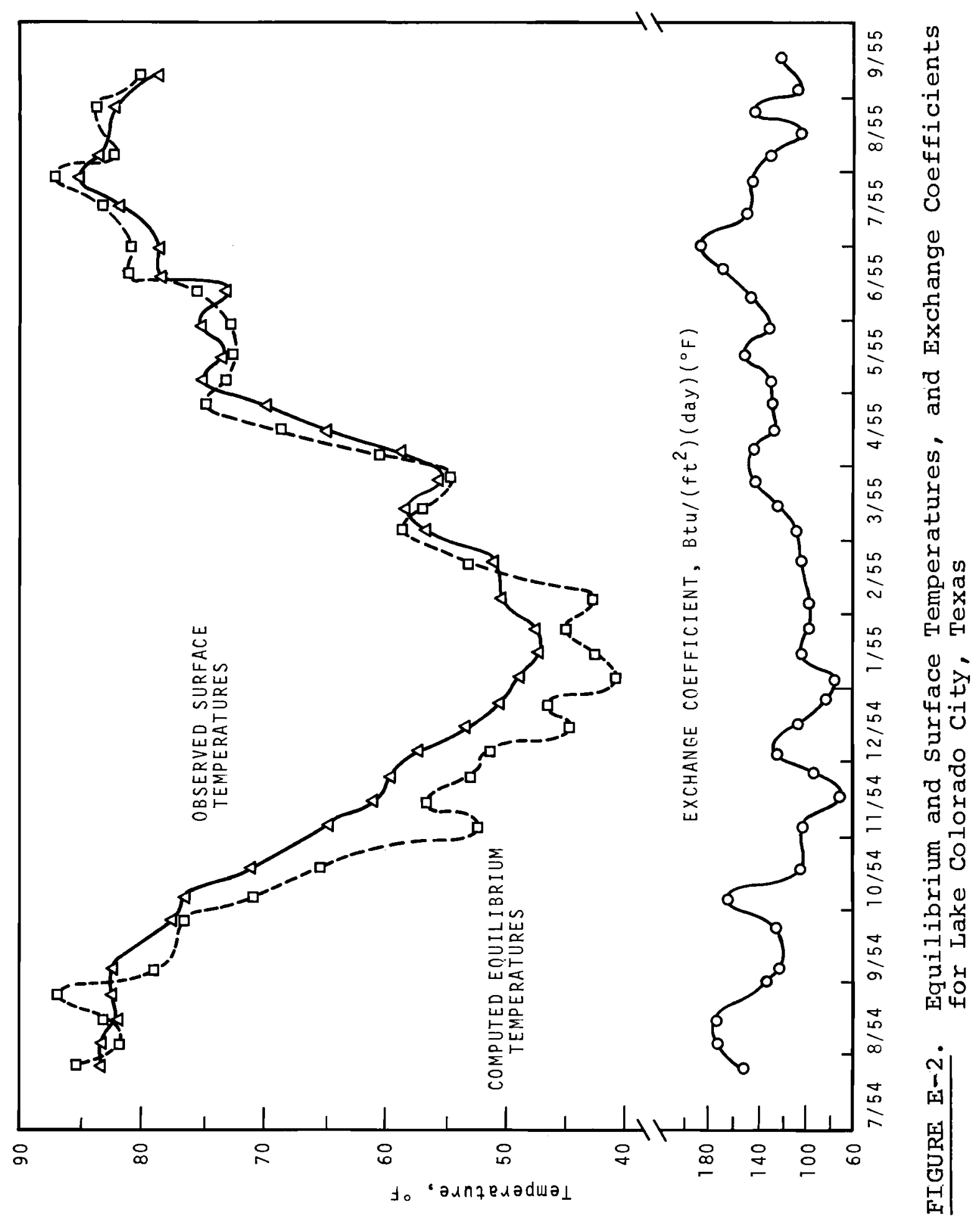


2. Studies of traverse measurements indicate a very low temperature differential across the river. Elevated temperatures were noted on both river banks. Other than bank heating effects, no individual run exceeded a temperature differential of $0.5^{\circ} \mathrm{C}$.

3. Inasmuch as the run of the river portions on the Columbia system frequently show temperature differentials higher than impounded reaches, it would easily be possible for the uninformed observer to over estimate the effects of the Hanford Plant.

Routine data taken during the shutdown period are illustrated in Figure E-3. Superimposed on the routine data are computed values of river temperature derived by subtracting known reactor input information from the measured values. During the high flow portion of the season, between June 1 and the commencement of the shutdown, reactor temperature inputs did not exceed $0.43{ }^{\circ} \mathrm{C}$ on any one day and averaged about $0.35^{\circ} \mathrm{C}$ for the period. The measured rise in the plant reach, taking into account the travel time, averaged approximately $0.65^{\circ} \mathrm{C}$ in June, $0.75{ }^{\circ} \mathrm{C}$ in July, and $0.85^{\circ} \mathrm{C}$ through the 25 th of August. As the reactors came back on stream the river flows had decreased substantially and temperature increases from plant contribution are noted in the figure. By comparison, essentially little temperature differential of any consequence was noted between Richland and Pasco, a distance of some nine miles, having a total river surface area approximately equal to that between $100 \mathrm{~B}$ Area and the city of Richland. It is apparent that the exchange coefficients for the different portions of the river are affected by the current velocity as would be expected by film coefficient correlations. Wind direction undoubtedly plays an important part in absolute computations of surface 


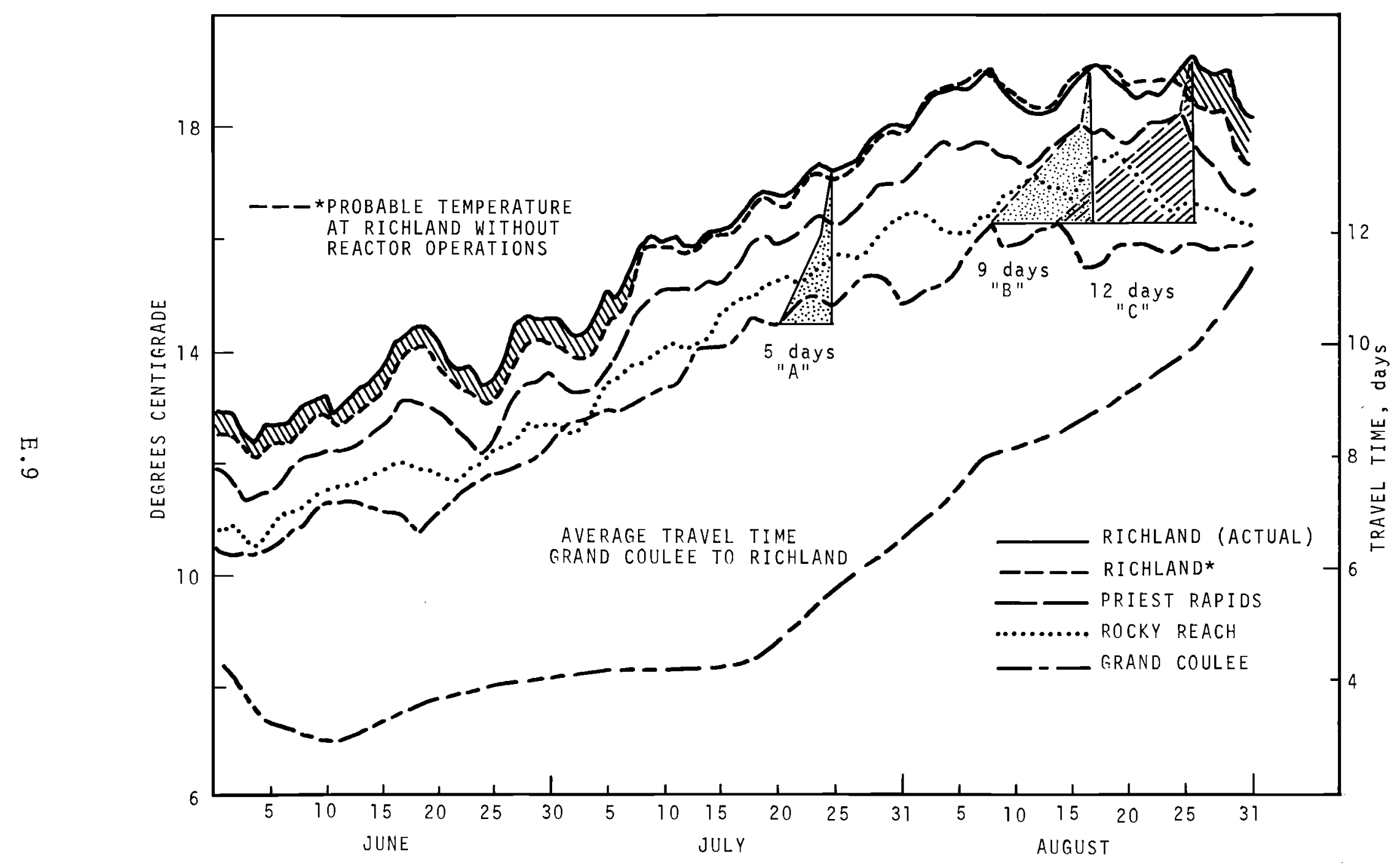

FIGURE E-3. Daily Average Columbia River Temperature 
coefficients since vector addition of air velocity would be required to compare rapidly moving stream coefficients with those measured on stagnant lakes. As indicated above, coefficients of 200 to 300 were routinely observed in the plant reaches of the Columbia as compared with the values of 150 for impounded reaches.

As an added item of interest, temperatures measured at Grand Coulee (discharge) and Rocky Reach are also illustrated in Figure E-3. During July when the average travel time from Grand Coulee to Richland was about $51 / 3$ days, the temperature differential between these points never exceeded $2.6{ }^{\circ} \mathrm{C}$, of which approximately $0.75^{\circ} \mathrm{C}$ represents the plant reach increment. Subtraction leaves $1.85^{\circ} \mathrm{C}$ as the rise between Grand Coulee and Priest Rapids during the example period illustrated (A on Figure E-3). Since the surface area of the impounded river between Grand Coulee and Priest Rapids exceeds the surface area of the local reach by a factor of 4 , it is safe to conclude that exchange coefficients for the impounded river will probably be 50 to $60 \%$ of the values for the run-of-the-river reaches. Application of these values to the Richland-to-Pasco reach yields a small, appropriate temperature rise. Examples $B$ and $C$ on Figure E-3 provide a further justification for the conclusions drawn from the data. Most of the input information pertinent to the analysis is available in theoretical form; however, it is rare that an opportunity is given to evaluate theoretical computations with excellent instrumentation on a local basis.

Figure E-4 shows the plotted values for the individual traverse measurements. Thus unusual bank heating noticed in the Hanford-Ringold reach is evident in the data plots. It has been speculated that warm ground water may account for some of this temperature differential. If this were the case, it would be expected that the majority of the temperature rise in the 


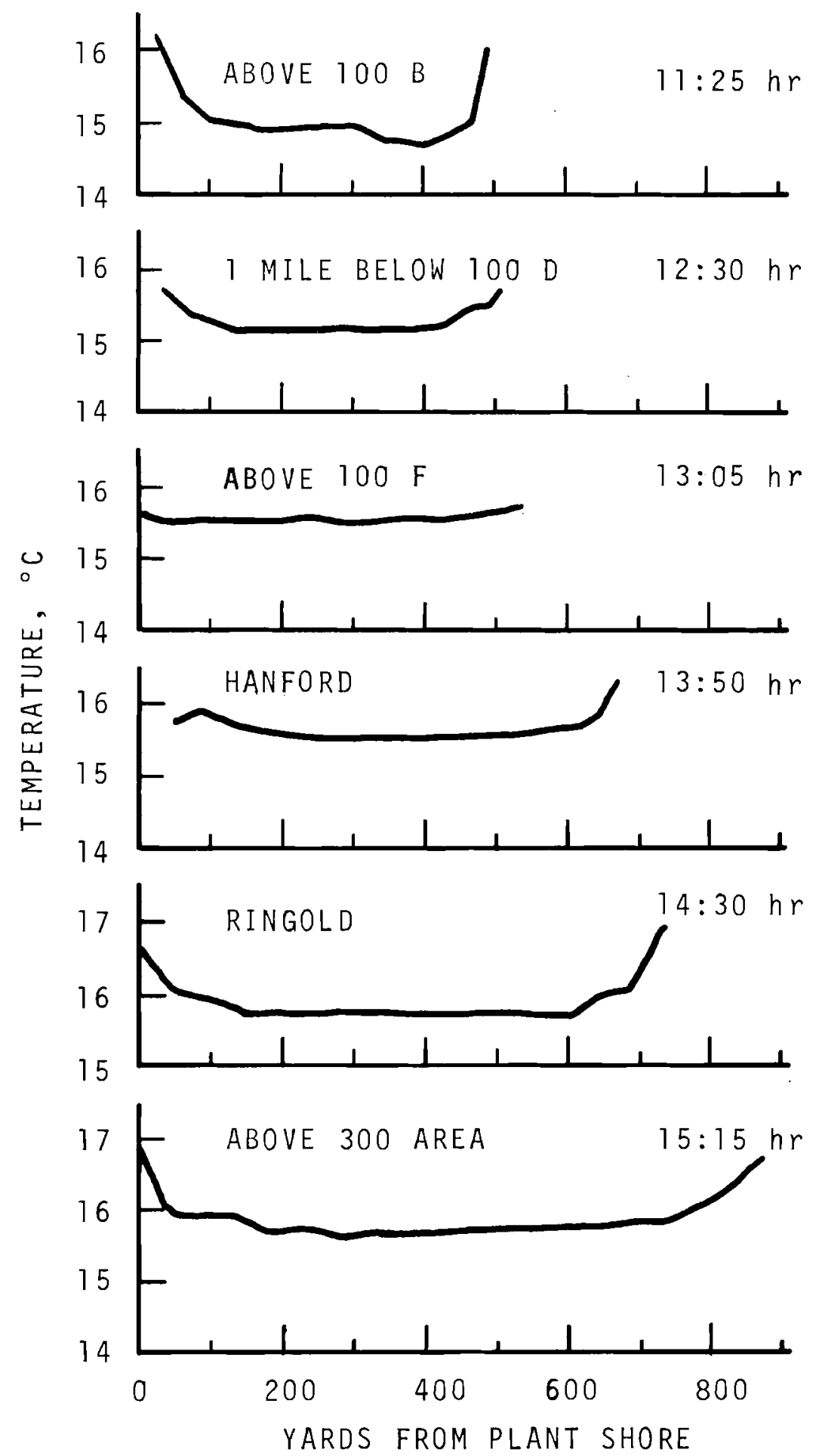

FIGURE E-4. River Traverse Data for July 13, 1966 (River Flow, 254,000 cfs) 
plant reach would occur in this area. The evidence does not strongly support this condition. Ground water inputs can not be ruled out on the basis of present data, however.

EFFECTS OF AN EXTENDED REACTOR OUTAGE ON CHEMICAL CONCENTRATIONS IN THE COLUMBIA RIVER

The only chemicals used routinely by the Hanford plants in sufficient amounts to cause a measurable change in the chemical characteristics of the river are hexavalent chromium and nitrate ion. These chemical species are monitored routinely in the river water below the reactors at Richland. Several other normal chemical characteristics, such as pH, dissolved oxygen, sulfate ion, phosphate, etc., are also measured routinely in the vicinity of the reactors, but no effects of the plant effluents on them have been observed.

Sodium dichromate is used as a corrosion inhibitor in the reactor cooling water. The resulting concentration in the Columbia River is ordinarily in the range of 2 to $7 \mathrm{ppb}$, depending on river flow. During the reactor shutdown, the reduced coolant flow contributed much lower quantities to the river and estimates place the concentration of hexavalent chromium at Richland in the range of 0.2 and $0.7 \mathrm{ppb}$ on the basis of effluent and river flows. Inasmuch as the analytical method used by the laboratory is not sensitive to concentrations below about 1 ppb, positive values were not expected during the shutdown period. Figure $E-5$ shows the results of the hexavalent chromium measurements for 1966. The result of 4 ppb obtained from a water sample collected during the first week of August was confirmed by laboratory recheck, but remains an unexplained anomaly. 
BNWL -1345

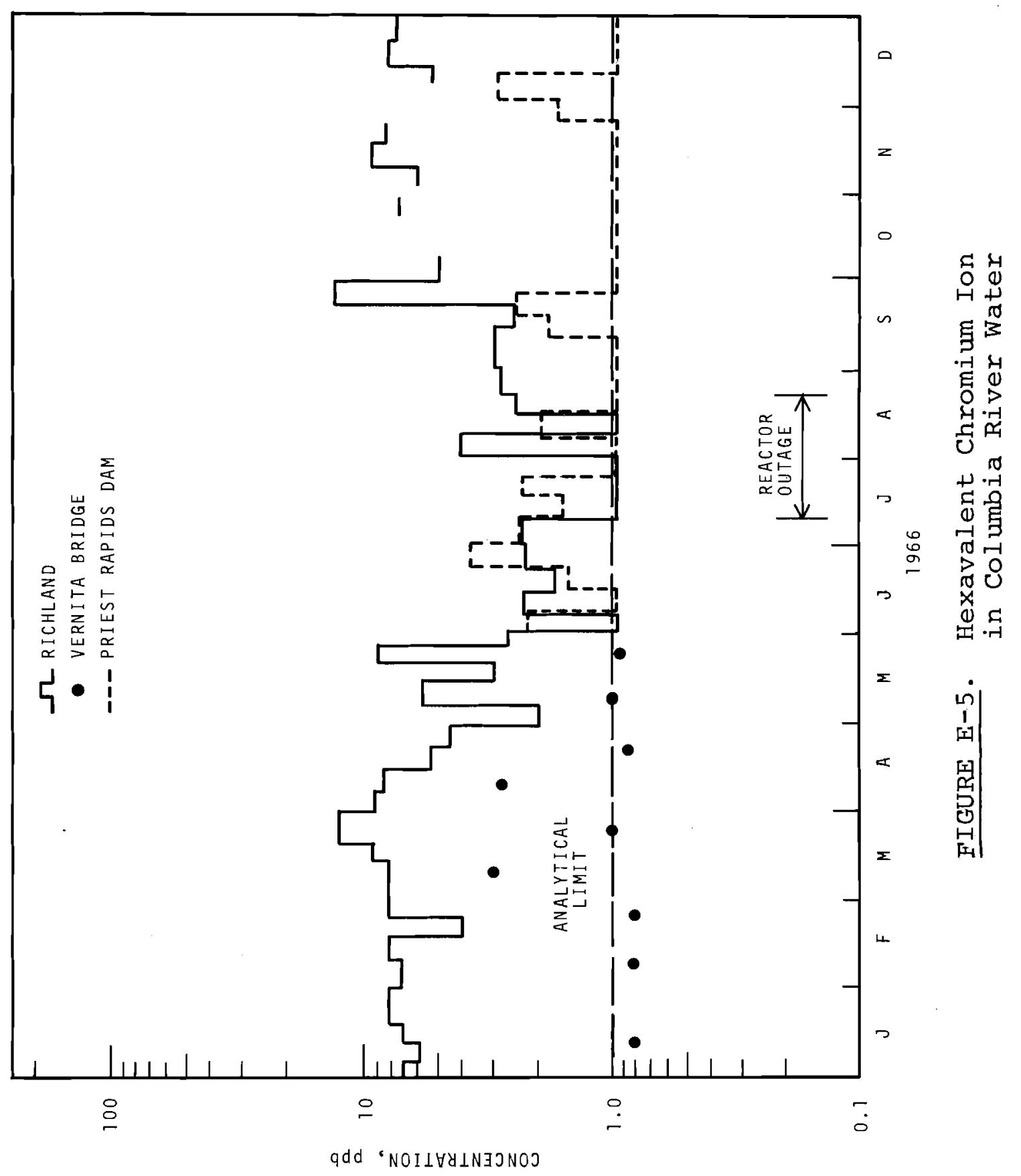

E. 13 
BNWL -1345

The largest potential source of nitrate ion to the Columbia River from Hanford is the chemical separations plants. However, the nitrate must travel with the ground water for many years before reaching the river and even under low river flow conditions, the increment is not obvious. Because of the relatively large amount of natural nitrate ion in the river, the large reservoir of nitrate ion in the ground water beneath the reservation, and the very long travel time between the separations plants and the river, no detectable effect of the work stoppage on the nitrate ion in the Columbia can be expected.

\section{REFERENCES}

E1. P. G. Holsted. Unpujilished Data. AEC Richland operations Operations office, August 12, 1966. (Letter: to R. S. Paul).

E2. J. P. Corley, H. A. Kramer, and J.K. Soldat. "Section 4-Chemical Characteristics," A Compilation of Basic Data Relating to the Columbia River, HW-69368. December 1961. 


\section{$\underline{\text { DISTRIBUTION }}$}

No. of

Copies

OFFSITE

1 AEC Chicago Patent Group

G. H. Lee

2 AEC Division of Biology and Medicine

C. Osterberg

John Wolfe

1 AEC Division of Reactor Development and Technology

I. C. Roberts

177 AEC Division of Technical Information Extension

3 AEC, Washington Division of Operational Safety

A. A. Schoen

3 AEC, Washington Division of Production

F. P. Baranowski

K. B. Fox

W. J. Lindsey

1 AEC, Washington Office of the General Manager

E. J. Bloch

1 American Institute of Chemical Engineers 900 W. Orange Grove Road Tucson, Arizona 84704

Lawrence Cecil

1 Atomic Energy of Canada Limited Chalk River Nuclear Laboratories Chalk River, Ontario, Canada

$$
\text { J. C. Biggs }
$$

1 Benton County Public Utility District No. 1 524 South Auburn Street P. O. Box 6270 Kennewick, Washington 99336

Thomas E. Black, Manager
No. of

Copies

4 Bonneville Power Administration 1002 N. E. Holladay

Portland, Oregon 97208

C. R. Foleen

F. A. Limpert

W. L. Morse

E. C. Starr

1 British Columbia Hydro and Power Authority 970 Burrard Street Vancouver, B.C., Canada

T. F. Daggs

1 Chelan County Public Utility District (Rocky Reach Dam) 327 North Wenatchee Avenue Wenatchee, Washington 98801

Howard C. Elmore, Manager

1 Chelan County Public Utility District

P. O. Box 1231

Wenatchee, Washington

J. H. Schrengohst

1 City of Richland

M. W. Fuller

1 Clallam County, PUD No. 1

P. O. Box 951

Port Angeles, Washington 98362

M. D. Parrett

2 Clark County Public Utility District

1200 Fort Vancouver Drive

Vancouver, Washington 98663

F. H. Lehman

George W. Watters, Manager

1 Colorado River Board

State of California

909 South Broadway

Los Angeles, California 90015

H. F. Pellegrin

1 Computer Sciences Corporation

J. L. Spurgeon 
No. of

Copies

1 Commonwea1th Edison Company 72 West Adams Street Chicago, I11inois

W. B. Behnke, Jr.

1 Corne11 University

Ithaca, New York 14850

L. B. Dworsky

1 Corlitz County Public Utility District

960 Commerce Avenue Longview, Washington 98632

R. L. McKinney

1 Department of Commerce and Economic Development Office of Nuclear Energy Development

General Administration B $1 \mathrm{dg}$. Olympia, Washington 98501

L. B. Bradley

2 Department of Energy, Mines and Resources

Ottawa 4, Ontario, Canada

E. R. Tinney

1 Department of Energy, Mines and Resources

Canada Center for Inland Waters P. O. Box 5050

Burlington, Ontario, Canada

J. P. Bruce

1 Department of Lands, Forests and Water Resources

Water Resources Service

Parliament Buildings

Victoria, B.C., Canada

V. Raudsepp

1 Douglas County Public Utility

District

(We11s Dam)

1151 North Main

East Wenatchee

Wenatchee, Washington 98801

Fred Lieberg, Manager

2 Duke Power Company

422 South Church Street Charlotte, North Carolina 28201

W. S. Lee

W. H. Owen
No. of

Copies

1 Edison Electric Institute 750 Third Ave.

New York, New York 10017

G. E. Watkins

1 Eugene Water \& Electric Board P. O. Box 1112

Eugene, Oregon 97401

Byron Price

3 Federal Power Commission

610 S. Canal Street

Chicago, Illinois 60607

L . B. Young

1 Federal Power Commission

441 G. Street NW

Washington, D.C. 20426

F. S. Brown

1 Federal Water Quality

Administration

Southeast Water Laboratory

Athens, Georgia 30601

W. M. Sanders, I I I

5 Federal water Quality

Administration

1921 Jeff Davis Highway

Arlington, Virginia

R. G. Burd

W. A. Cawley

Arnold Joseph

Joseph I. Lewis

J. E. McLean

6 Federa1 Water Quality Administration

Columbia Basin Project

570 Pittock Block

Portland, Oregon 97205

$\mathrm{J}$. L. Agee (3)

J. Vlastilicia

J.R. Yearsley

R. W. Zeller

1 Federal Water Quality

Administration

Robert A. Taft Center

4676 Columbia Parkway

Cincinnati, Ohio

J. M. Symons 
No. of

Copies

2 Federal Water Quality

Administration

Pacific Northwest Water

Laboratory

Corvallis, Oregon 97330

A. F. Bartsch, Director

B. E. Tichenor

1 Foundation of Canada Engineering

Corporation Limited

2200 Yonge Street

Toronto 12 , Canada

H. R. Kivisild

1 Governor's Science Resource Advisory Board

State Office Building

Annapolis, Maryland 21401

L. W. Fredrick

3 Grant County Public Utility

District

(Wanapum and Priest Rapids Dams)

30 C Street, S. W.

Ephrata, Washington 98823

R. R. Ries

1 Great Lakes Basin Commission

Ann Arbor, Michigan 48105

L. T. Crook

1 Illinois State Water Survey Champaign, Illinois

W. C. Ackermann

1 Internationa1 Joint Commission Chairman, U.S. Section Washington, D.C. 20000

M. E. We1sh

1 Manhattan College

Parkway and West 242 nd

New York, New York 10000

D. J. O'Connor

1 NAPCA, Division Process

Control Engineer

5710 Wooster Pike

Cincinnati, Ohio 45227

R. P. Hangebrouck

1 New York State Department

of Conservation

A1bany, New York

N. L. Barbarossa
No of

Copies

1 New York State Department

of Hea1th

84 Holland Avenue

Albany, New York

L. G. Hetling

1 Northwest Public Power

Association

Vancouver, Washington 98660

Henry Curtis, Manager

2 Oak Ridge National Laboratory Building 9201-3

P. O. Box $Y$

Oak Ridge, Tennessee 37830

S. E. Beal1, Jr.

M. M. Yarosh

1 Ontario Water Resources

Commission

801 Bay Street

Toronto, Ontario, Canada

W. A. Steggles

1 Pacific Northwest River

Basins Commission

Trans America Tile Company

Building

110 E. 13th Street

Vancouver, Washington 98661

Done1 Lane

2 Pacific Power and Light

Public Service Building

Portland, Oregon 97204

E. B. Hedberg

R. B. Lisbakken

1 Pend Oreille County Public Utility District (Box Canyon Dam)

$130 \mathrm{~N}$. Washington Avenue

Newport, Washington

George Kennett, Manager

1 Philadelphia Electric Company 1000 Chestnut Street

Philadelphia, Pennsylvania 19105

J. L. Everett

2 Portland General Electric

621 S. W. Alder

Portland, Oregon 97205

L. E. Hode1

A. J. Porter 
No. of

Copies

3 Puget Sound Power \& Light Co. Puget Power Building Bellevue, Washington 98004

Max Blumeyer

D. H. Knight

F. M. Treffinger

1 Robert S. Kerr Water Research Laboratory

Federal Water Quality

Administration

P. O. Box 1198

Ada, Oklahoma 74820

William G. Galegar

1 Sargent and Lundy 140 South Dearborn

Chicago, Illinois 60603

Tor Kolflat

3 Seattle City Light

1015 Third Avenue

Seattle, Washington 98104

John Nelson, Manager

R. G. Sheehan

R. L. Skone

1 State Sanitary Authority

State Office Building

Portland, Oregon 97201

Kenneth H. Spies

1 State University of New York Department of Atmospheric

Science

1400 Washington Avenue

Albany, New York 12203

Ronald Stewart

2 Tennessee Valley Authority

410 Edney Building

Chat tanooga, Tennessee

Milo Churchil1

Norris, Tennessee

Rex Elder (1)

1 Texas Electric Service Co. Box 970

Fort Worth, Texas 76101

Howard Drew
No. of

Copies

2 U.S. Army Corps of Engineers, North Central Division

536 South Clark

Chicago, Illinois 60605

M. L. Dixon

R. J. Lykowski

1 U.S. Army Corps of Engineers, North Pacific Division Portland, Oregon 97008

G. H. Fernald

3 U.S. Army Corps of Engineers, North Pacific Division

417 Custom House

Portland, Oregon

David M. Rockwood

1 U.S. Army Corps of Engineers, North Pacific Division Walla Walla District Office Walla Walla, Washington

Melvin Ord

1 U.S. Army Corps of Engineers, Ohio River Division

550 Main Street

Cincinnati, Ohio 45202

Glenn R. Von Gunten

2 U.S. Army Corps of Engineers, Portland District

P.0.Box 2946

Portland, Oregon 97208

Clarence Pederson

Henry Stewart

2 U.S. Army Corps of Engineers, Seattle District 1519 Alaskan Way South Seattle, Washington 98134

Patrick Keough

Norman McDonald

1 U.S. Department of Interior Bureau of sport Fisheries and Wildlife 710 N.E. Holliday Portland, Oregon 97205

L. E. Perry 
No. of Copies

2 U.S. Department of Interior Bureau of Commercial Fisheries 2725 Montlake Blvd. Seattle, Washington 98102

G. B. Col1ins

G. R. Snyder

4 U.S. Department of Interior Bureau of Reclamation 6728 Pomona Street Boise, Idaho

J. C. Peterson

R. H. Schirk

Denver Federal Center

Denver, Colorado 80225

B. P. Bellport

A. M. Kahan

1 U.S. Department of Interior Bureau of Reclamation Office of Chief Engineer Building 53, Denver Federal Center

Denver, Colorado 80225

G. E. Burnett

1 U.S. Department of Interior Office of Regional Coordinator 1002 N.E. Holladay

Portland, Oregon 97208

$$
\text { L. B. Day }
$$

1 U.S. Department of Interior Office of Water Resources Research Washington, D.C. 20242

$$
\text { E. D. Eaton }
$$

1 U.S. Department of Interior Water Resources Division 2300 john F. Kennedy Bldg. Boston, Massachusetts 02203

$$
\text { C. E. Knox }
$$

1 U.S. Geological Survey Hydraulics Section Washington Building, Room 227 Arlington Towers Arlington, Virginia

Fred Kilpatrick

1 U.S. Geological Survey 345 Middlefield Road Menlo Park, California 94025

W. D. Simons
No. of

Copies

4 U.S. Geological Survey 830 N.E. Holladay Street Portland, Oregon 97208

N. A. Kallio (2)

S. F. Kapustka (2)

1 University of Idaho Water Resources Research Institute Moscow, Idaho 83843

C. C. Warnick

1 University of Oregon Institute of International Studies and Overseas Administration Eugene, Oregon 97403 John Gange

2 University of Texas Environmental Health Engineering Engineering Laboratories Bldg. 305 Austin, Texas 78712

W. Eckenfelder

E. F. Gloyna

1 University of Western Ontario Department of Geology London, Ontario, Canada

E. G. Pleva

1 University of WisconsinMilwaukee

Center for Great Lakes Studies Milwaukee, Wiscons in 53201

C. H. Mortimer

1 University of Wyoming Laramie, Wyoming 82070

$$
\text { F. Trelease }
$$

3 Vanderbilt University Dept. of Environmental and Water Resources Eng. Nashville, Tennessee 37203

$$
\text { F. L. Parker }
$$

1 Virginia Electric and Power Company Richmond, Virginia 23201

J. D. Ristroph 
No. of

\section{Copies}

2 Washington Department of Fisheries

General Adminstration Bldg. Room 115

Olympia, Washington 98501

A1 Lasater

E. H. Lemier

3 Washington Public Power Supply System

132 Vista Way

Kennewick, Washington 99336

Owen Hurd, Manager

5 Washington State Department of Ecology

335 General Administration B1dg.

Olympia, Washington 98502

Maurice Ahlquist

John A. Biggs

Fred Hahn

George Hansen

Robert Stockman

1 Washington State Department of Health

Olympia, Washington

E. C. Jensen

1 Washington State University Water Resources Research

Pullman, Washington

Howard Copp

2 Washington Water Power

East 1411 Mission

Spokane, Washington

H. W. Harding

M. F. Hatch

1 Water Resources Research Institute

Oregon State University

Corvallis, Oregon

John Seaders

1 Wisconsin State Department of Natural Resources

Division of Conservation Box 4500

Madison, Wisconsin 53701

E. Schneberger
No. of

Copies

1 Yankee Atomic Electric Co.

441 Stuart Street

Boston, Massachusetts 02116

J. W. Lebourveau

1 Kirby Billingsley

1825 Mulberry Lane

Wenatchee, Washington

ONS ITE-HANFORD

1 AEC Chicago Patent Group

R. K. Sharp (Richland)

11 AEC Richland Operations Office

W. Devine

C. L. Robinson

M. R. Schne11er

M. W. Tiernan (5)

Technical Information Files (3)

3 Battelle Memorial Institute

R. J. Anderson

F. A. Butrico

G. E. Raines

9 Douglas United Nuclear

R. S. Be 11

L. B. Brinkman

D. W. Diediker

W. J. Dowis

R. T. Jessen

C. W. Kuhlman

R. S. Peterson

O. C. Schroeder

DUN File

5 Atlantic Richfield Hanford

R. E. Isaacson

H. P. Shaw

R. E. Tom 1 inson

J. H. Warren

ARHCO File

5 WADCO Corporation

J. F. Fletcher

D. E. Peterson

F. H. Shade 1

J. C. Sonnicissen

Document Control 
No of

Copies

130 Battel1e-Northwest

W. L. Bathke

J. P. Corley

G. L. Culp

G. M. Dalen

D. E. Deonigi

R. F. Foster

J. C. Fox

J. J. Fuquay

W. A. Haney

J. F. Honstead

R. T. Jaske (100)

D. A. Kottwitz

R. W. Legan

D. E. Olesen

W. A. Reardon

W. D. Richmond

A. J. Scott

W. L. Templeton

C. J. Touhi11

D. G. Watson

W. A. Watts

M. A. Wolf

L. E. Yandon

J. R. Young

Technical Information (5)

Technical Publications (2) 